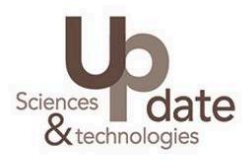

\title{
LES TERRES AGRICOLES FACE À L'URBANISATION
}

De la donnée à l'action, quels rôles pour linformation?

Roel Plant, Pierre Maurel, Éric Barbe, Jane Brennan, coordinateurs

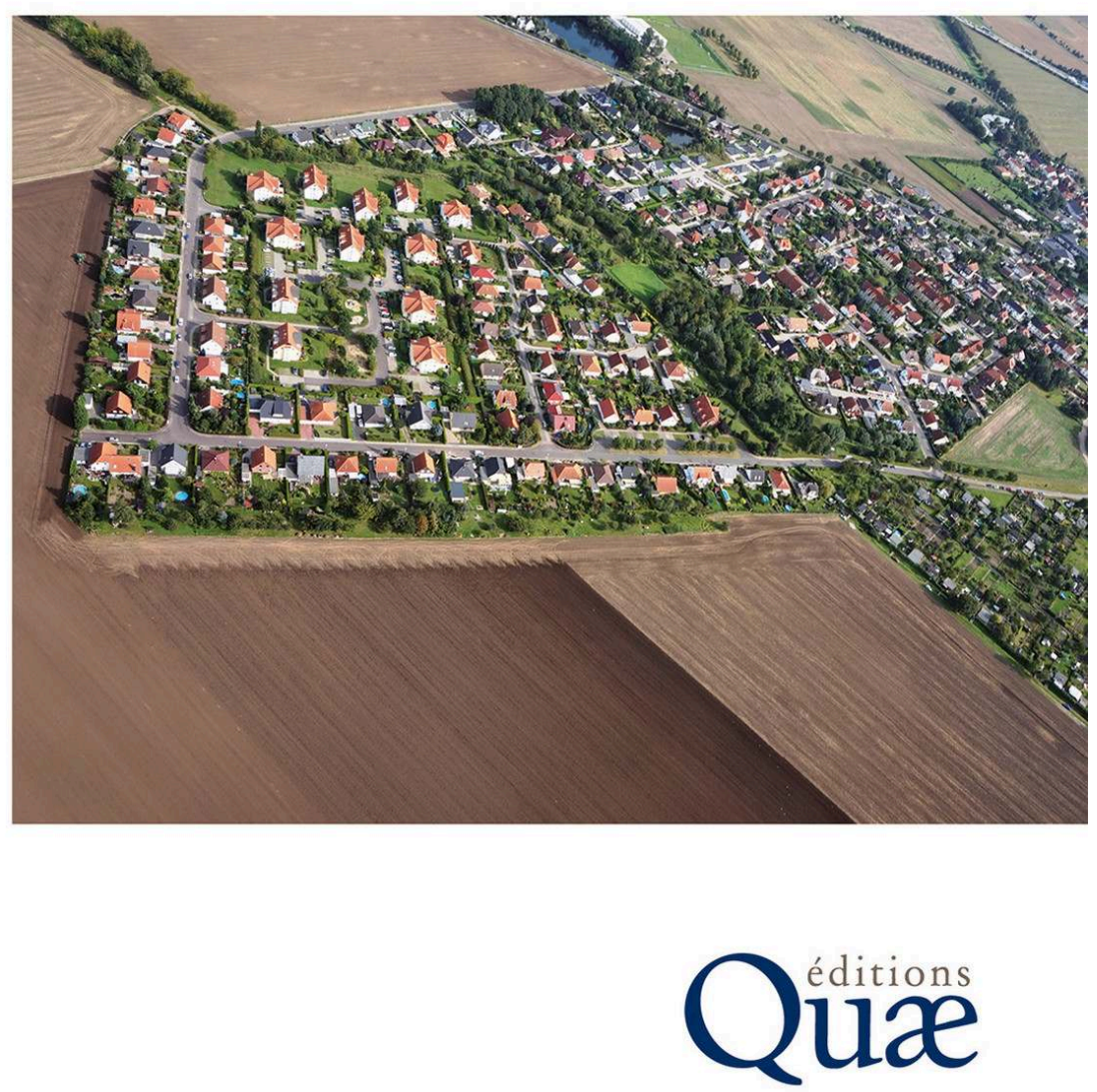




\section{Les terres agricoles face à l'urbanisation}

De la donnée à l'action, quels rôles pour l'information?

Roel Plant, Pierre Maurel, Éric Barbe et Jane Brennan (dir.)

Éditeur : Éditions Quæ

Année d'édition : 2018

Date de mise en ligne : 23 février 2021

Collection : Update Sciences \& Technologie

ISBN électronique : 9782759230297

\section{Sbook bors}

http://books.openedition.org

\section{Édition imprimée}

Nombre de pages : 270

\section{Référence électronique}

PLANT, Roel (dir.) ; et al. Les terres agricoles face à l'urbanisation : De la donnée à l'action, quels rôles pour l'information? Nouvelle édition [en ligne]. Versailles : Éditions Quæ, 2018 (généré le 24 février 2021). Disponible sur Internet : <http://books.openedition.org/quae/28250>. ISBN : 9782759230297. 


\section{LES TERRES AGRICOLES FACE À L'URBANISATION \\ De la donnée à l'action, quels rôles pour linformation?}

Roel Plant, Pierre Maurel, Éric Barbe, Jane Brennan, coordinateurs

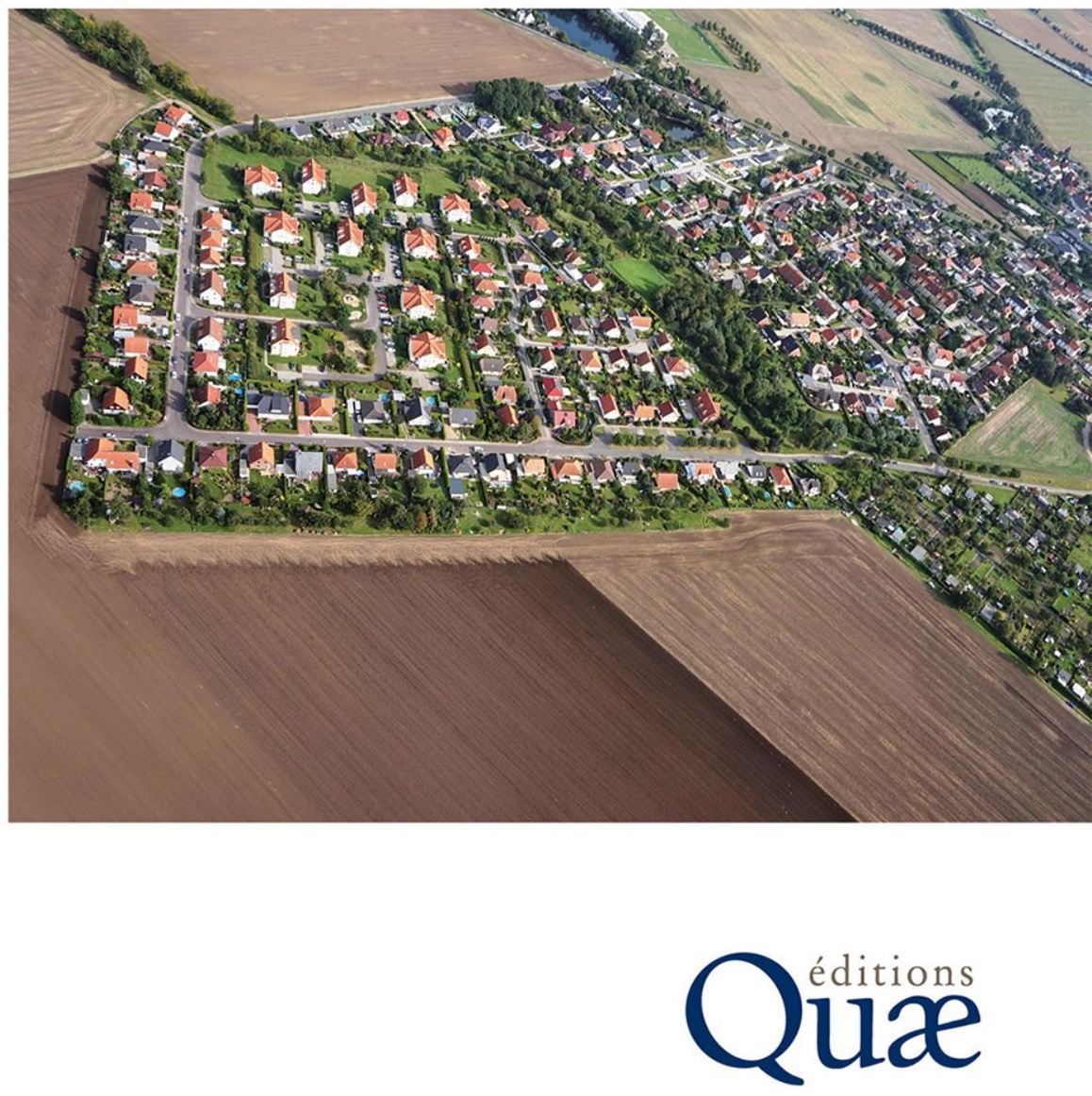




\section{Les terres agricoles face à I'urbanisation \\ De la donnée à l'action, quels rôles pour l'information?}

Roel Plant, Pierre Maurel, Éric Barbe, Jane Brennan, coordinateurs

(C) éditions Quæ, 2018

ISBN : 978-2-7592-2922-2

Éditions Quæ

RD 10

78026 Versailles Cedex

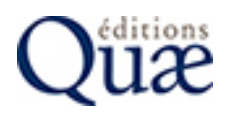

www.quae.com

\section{(c) $(1)(9)$}

Pour toutes questions, remarques ou suggestions : quae-numerique@quae.fr

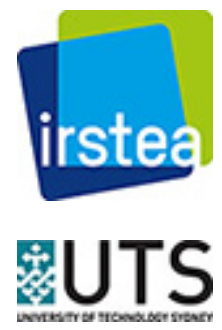





\section{Table des matières}

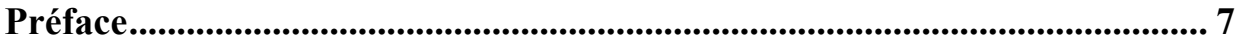

Introduction. Les terres agricoles face à l'urbanisation..................................... 9

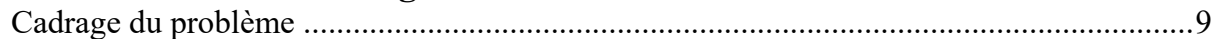

Genèse de l'ouvrage ............................................................................................. 10

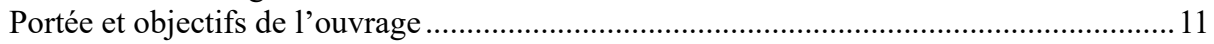

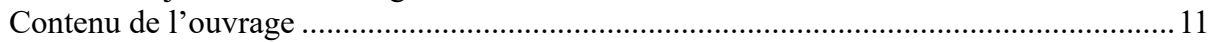

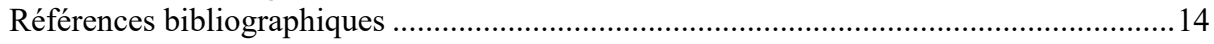

Chapitre 1 - Contexte : une étude comparée sur la planification spatiale de

l'artificialisation des sols en France et en Australie ...................................................... 17

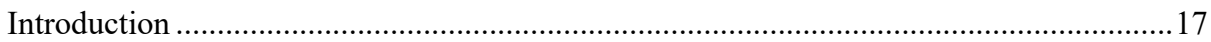

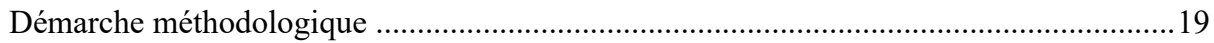

Logiques légales et administratives de la planification spatiale...........................................20

Instruments de planification spatiale, pratiques, participation et politique..........................25

Les approches de planification spatiale face à l'imperméabilisation des terres agricoles liée

à la périurbanisation ..............................................................................................

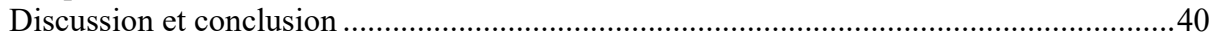

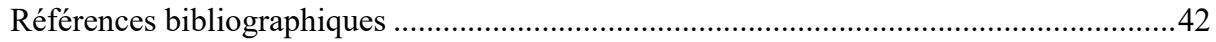

\section{Partie I \\ Base factuelle de la planification}

Chapitre 2 - Enjeux autour des terres agricoles et des données pédologiques : point de vue opérationnel d'un service de l'État en région......................................................51

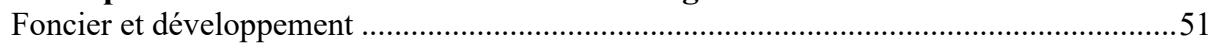

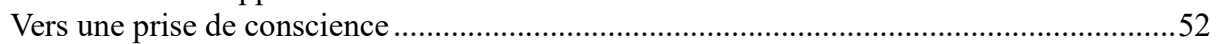

Éléments de stratégie en région : perspectives ..................................................................53

Origine et objectifs des travaux engagés ..........................................................................5

Développements méthodologiques régionaux à vocation nationale .....................................55

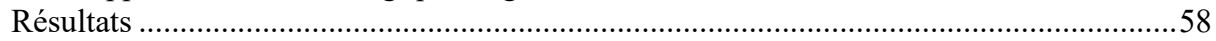

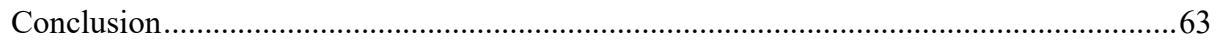

Références bibliographiques .........................................................................................63

Chapitre 3 - Consommation des terres agricoles en Languedoc-Roussillon : système d'indicateurs et démarche participative..........................................................65

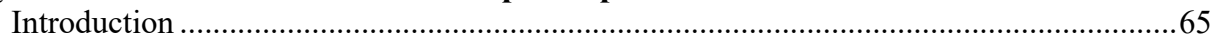

Une démarche modélisatrice et participative itérative pour aboutir à la production d'un

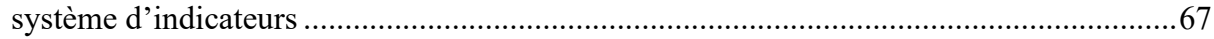

Mise en œuvre de la démarche ..................................................................................

Production d'un système d'indicateurs appliqué à l'artificialisation des sols en zone

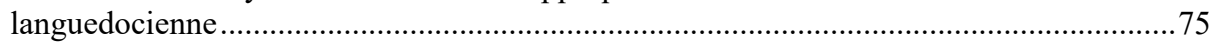

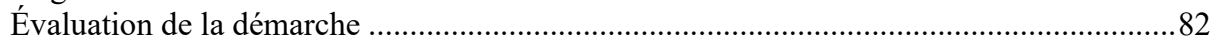

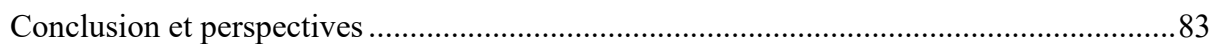

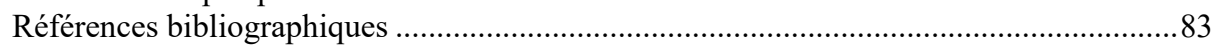


Chapitre 4 - Connaître les propriétés des sols pour éclairer les changements d'occupation du sol et l'aménagement du territoire. Une perspective

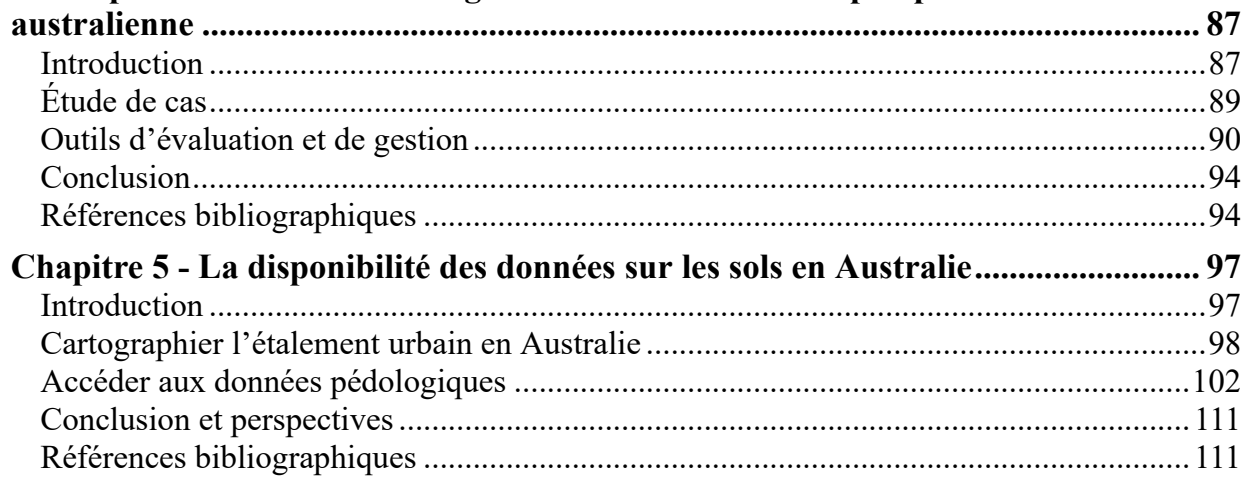

Chapitre 6 - Techniques géospatiales et de télédétection pour le suivi de l'état et des dynamiques des terres agricoles périurbaines .................................................. 113

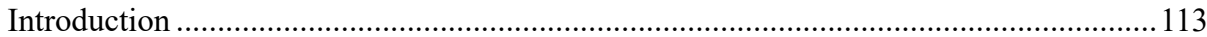

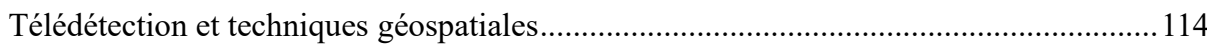

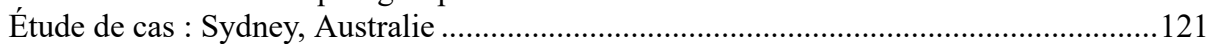

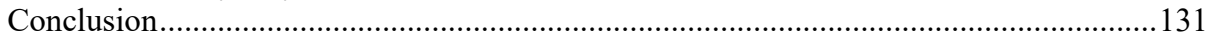

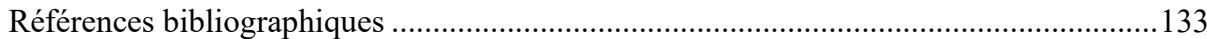

\section{Partie II \\ LA MESURE À L'ÉPREUVE DE L'HUMAIN}

Chapitre 7 - De la diffusion de l'information à la négociation du sens : l'exemple de la quantification et de la qualification de la consommation des terres agricoles dans l'ancienne région Languedoc-Roussillon ................................................................. 139

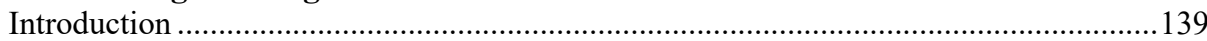

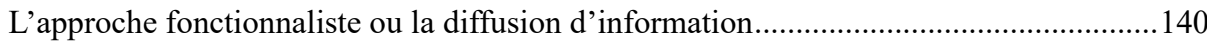

La sociologie de l'acteur-réseau ou la négociation du sens ............................................145

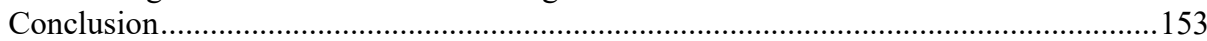

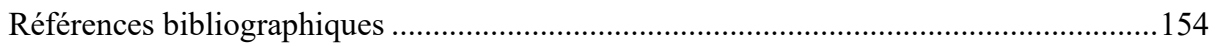

Chapitre 8 - Le concept de « politiques de l'identité de lieu » comme outil pour mieux comprendre la marginalisation des terres agricoles dans la commune périurbaine de Wollondilly, Nouvelle-Galles du Sud, Australie .............................. 157

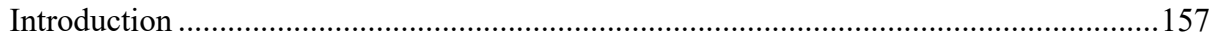

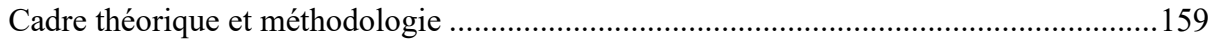

La marginalisation des terres agricoles dans les processus de planification en milieu

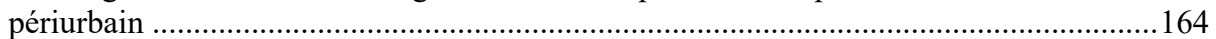

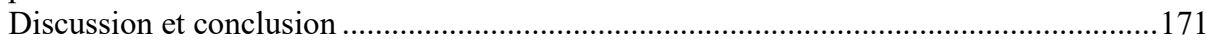

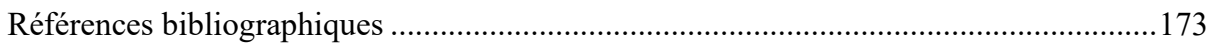

Chapitre 9 - Modèles et simulations spatio-temporels comme « objets intermédiaires $»$ : le cas de l'étalement urbain à la Réunion ................................... 177

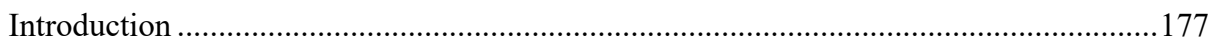

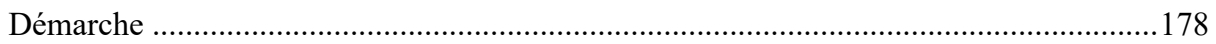

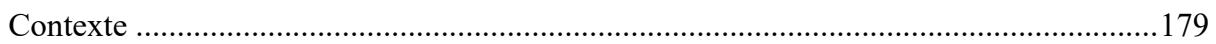

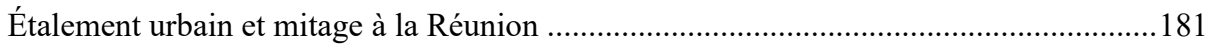

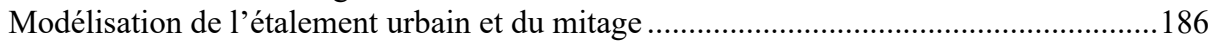




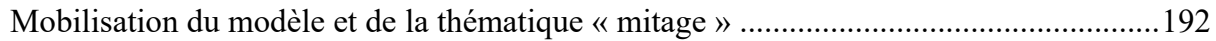

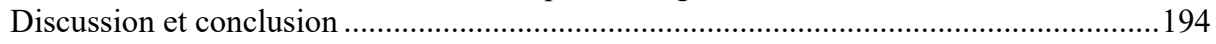

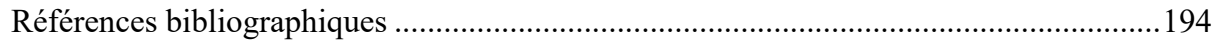

Chapitre 10 - La cocréation du futur alimentaire de Sydney : informer et transformer la gestion stratégique de l'agriculture périurbaine à travers un

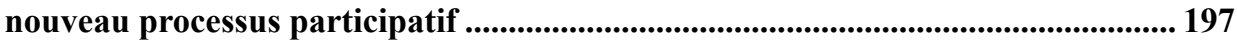

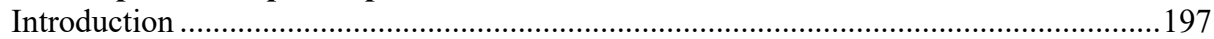

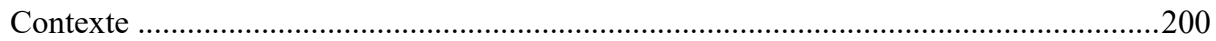

Modélisation de scénarios pour le futur de l'agriculture à Sydney .................................202

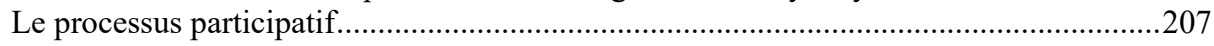

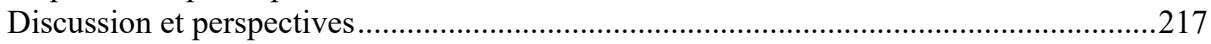

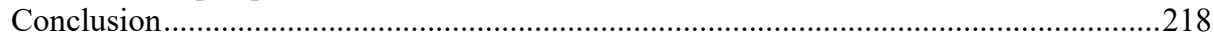

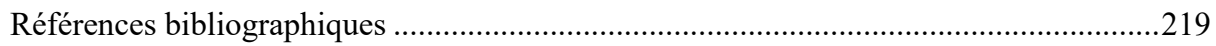

Chapitre 11 - Utilisation du concept de services écosystémiques pour une évaluation participative du rôle des terres agricoles périurbaines dans le sud de la

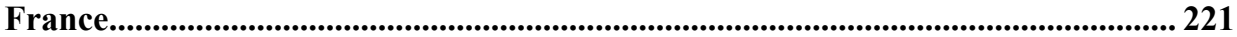

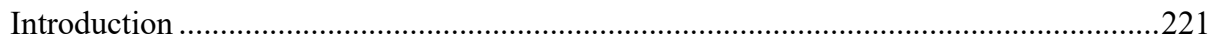

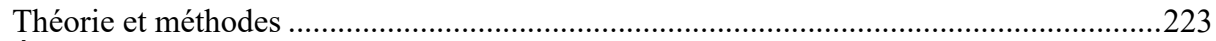

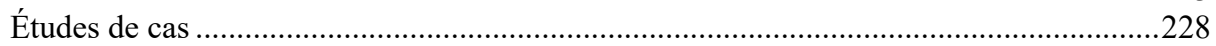

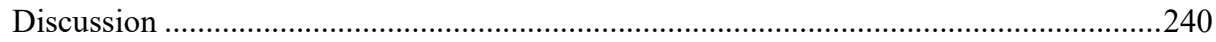

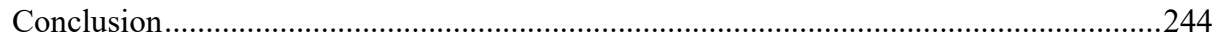

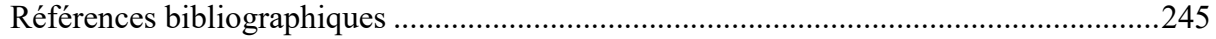

Synthèse. De la donnée à l'intelligence collective sur les terres agricoles périurbaines : quels rôles pour l'information, les savoirs et l'action ?..... 249

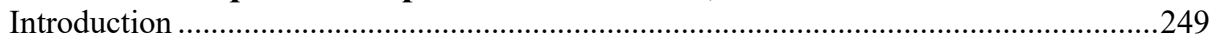

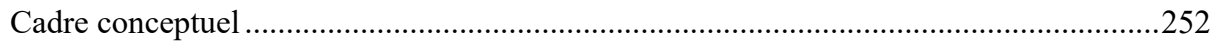

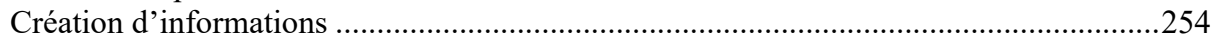

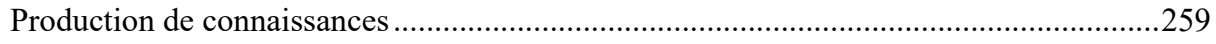

Créer de l'intelligence : vers une gouvernance durable des terres agricoles

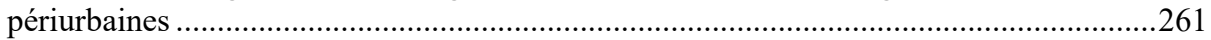

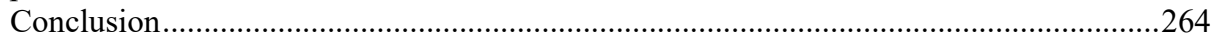

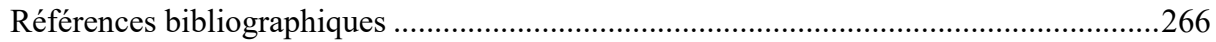

Liste des auteurs.................................................................................................. 269 



\section{Préface}

Pascal Augier

La préservation des terres agricoles constitue un enjeu stratégique majeur au regard de la production agricole en lien avec l'alimentation et l'aménagement du territoire, aux différentes échelles - locale, nationale et internationale; de nombreux pays dans le monde ont parfaitement identifié cet enjeu.

Au niveau mondial et à long terme, l'enjeu est de maintenir le potentiel de production agricole pour assurer la sécurité alimentaire des populations, alors que les décisions en matière d'aménagement du territoire relèvent essentiellement de l'échelon local, et s'inscrivent dans une perspective souvent à court et moyen terme. Ce double hiatus temporel et d'échelle de décision, renforcé par les intérêts financiers liés au changement de destination du foncier, contribue à une forte consommation, non durable, des terres agricoles au profit de l'artificialisation.

Ainsi, les infrastructures et l'étalement urbain observé depuis les années 1970 consomment d'importantes superficies de terres agricoles. En France, 800000 ha (dont $80 \%$ de terres agricoles) ont été artificialisés entre 1992 et 2004, et le rythme annuel d'artificialisation a progressivement augmenté jusqu'à atteindre un pic à $90000 \mathrm{ha} /$ an entre 2008 et 2009 (source: Teruti-Lucas) et une moyenne stabilisée d'environ 500000 ha consommés tous les 7 ans, soit l'équivalent de la superficie d'un département français.

Une expertise nationale réalisée en France en 2017 (ESCo - Sols artificialisés et processus d'artificialisation des sols : Déterminants, impacts et leviers d'action) a mis en exergue «que les dynamiques de l'artificialisation des sols, en particulier sur les terres agricoles, résistent en grande partie aux outils fiscaux et de planification élaborés par les pouvoirs publics depuis les années 1980. En dépit de lois et de règlements successifs, l'occupation économe du sol reste un objectif à atteindre à l'échelle nationale. »

Pour atteindre l'objectif d'une occupation économe des terres, il apparaît nécessaire de disposer de données fiables et répétables sur des temps longs, afin de mieux comprendre les ressorts des dynamiques à l'œuvre dans les territoires et de mettre en place collectivement des réponses adaptées.

Dès 2007, la DRAAF Languedoc-Roussillon (devenue DRAAF Occitanie depuis 2016), consciente de ce besoin, a sollicité des équipes de recherche d'Irstea et de l'Inra afin de produire de nouvelles données cartographiques sur les terres artificialisées et le potentiel agronomique des sols, ainsi qu'une série d'indicateurs spatiaux révélateurs des dyna- 
miques d'artificialisation du territoire régional. Des sessions de formation ont ensuite été menées auprès des services en charge de l'instruction des projets d'aménagement, afin de les sensibiliser à l'utilisation de ces informations pour répondre localement à l'enjeu de préservation des terres agricoles face à l'urbanisation.

Cet ouvrage rédigé par un collectif pluridisciplinaire de chercheurs français et australiens s'inscrit dans la continuité de ces travaux. S'appuyant sur des études de cas en France et en Nouvelle-Galles-du-Sud, en Australie, il interroge les rôles de l'information, depuis la fabrique de la donnée jusqu'à l'action collective, pour apporter des solutions concrètes face à la disparition des terres agricoles.

Les responsables politiques, ainsi que les services de l'État, partagent aujourd'hui le diagnostic qu'il s'agit d'un enjeu majeur de société et d'aménagement durable des territoires, à la fois pour garantir la sécurité alimentaire, mais aussi pour tisser de nouvelles solidarités entre les espaces ruraux et urbains. Ces objectifs sont ambitieux, mais ils sont à la hauteur de l'urgence à trouver des réponses aux mutations profondes que connaissent les territoires depuis quelques décennies. 


\title{
Introduction. Les terres agricoles face à l'urbanisation
}

\author{
Roel Plant, Pierre Maurel
}

\section{Cadrage du problème}

La perte de terres agricoles liée à l'urbanisation constitue une facette de la consommation des terres, ce que la Commission européenne dénomme "land take " (Decoville et Schneider, 2015). Elle la définit en termes généraux comme une perte d'espaces ouverts au profit d'espaces anthropisés et, de manière plus spécifique, comme la perte d'espaces agricoles, forestiers, naturels ou semi-naturels au bénéfice de l'urbain et d'autres espaces artificialisés. Cela inclut le bâti, les infrastructures ainsi que les espaces végétalisés en milieu urbain et les équipements de loisirs (EEA, 2006).

Même s'il a commencé dans les années 1950 (essentiellement du fait de l'étalement urbain), ce phénomène de consommation des terres agricoles prend aujourd'hui des proportions jusque-là inégalées. Au-delà de la simple conversion des terres d'usages non urbains à des usages urbains, l'étalement se caractérise aussi par une baisse de densité du bâti, une déconcentration des fonctions urbaines et le basculement de la ville compacte vers des formes irrégulières, discontinues et dispersées (Siedentrop et Fina, 2010).

Les constats et les chiffres pour illustrer ces processus à l'œuvre sont édifiants. À l'échelle européenne, plus d'un quart du territoire relève maintenant directement d'usages urbains et, d'ici 2020, plus de $80 \%$ de la population européenne vivra en ville, générant une intense pression foncière dans et autour des villes (EEA, 2006). Pour atteindre l'objectif que s'est fixé la Commission européenne d'arriver à une consommation nette de terres nulle d'ici 2050, un travail de modélisation a montré que la surface moyenne consommée par habitant et par an à partir de 2020 devrait être de l'ordre de $1,4 \mathrm{~m}^{2}$, alors qu'elle est de 2,2 $\mathrm{m}^{2}$ dans le scénario de référence basé sur les prévisions démographiques et économiques (Lavalle et al., 2013). En France, une étude publiée par le ministère de l'Agriculture en 2010 avait montré que l'artificialisation consommait en sept ans l'équivalent de la surface d'un département français (Bertrand, 2013). Ce rythme avait baissé les années suivantes pour atteindre l'équivalent d'un département tous les onze ans. Malgré l'absence d'une méthode incontestable pour quantifier le phénomène, les différentes sources de données amènent à une fourchette allant de 40000 à 90000 hectares par an en moyenne, surtout au détriment des terres agricoles, les surfaces forestières et naturelles restant stables, voire en légère augmentation (Oncea, 2014).

L'importante variabilité de ces chiffres s'explique principalement, d'une part, par l'absence d'une définition unique de la consommation des terres agricoles, couramment 
rapportée aux notions sensiblement différentes d'artificialisation, d'urbanisation ou d'imperméabilisation des sols, et, d'autre part, par la diversité des méthodes et techniques d'identification mises en œuvre (Béchet et al., 2017).

Les conséquences de ces processus d'artificialisation sont multiples et portent à la fois sur la production et sur la sécurité alimentaire (Gardi et al., 2015) ainsi que sur la perte de biodiversité (Colantoni et al., 2016). Une étude a par exemple estimé que la consommation des terres agricoles entre 1990 et 2006, mesurée à l'aide des cartes de la base de données Corine Land Cover sur 19 des 21 États membres, représentait l'équivalent d'une perte de production de plus de six millions de tonnes de blé (Gardi et al., 2015). Dans le champ plus large des écosystèmes, l'artificialisation génère la destruction d'habitats naturels et par conséquent une perte de biodiversité et d'un ensemble de services écosystémiques (Smiraglia et al., 2016), en plus du service de production alimentaire, tels que les aménités paysagères, l'approvisionnement en eau, la régulation de risques naturels (Maes et al., 2013). Alors que des politiques publiques sectorielles ont été mises en place en faveur de la conservation des habitats et des espèces, comme le réseau Natura 2000 en Europe ou la Trame verte et bleue (TVB) en France, les décideurs locaux restent livrés à eux-mêmes sur des questions d'aménagement de plus en plus sensibles et complexes, alors que ces dernières conditionnent la durabilité et la résilience des territoires. Comment arriver à maintenir des exploitations agricoles dans les espaces périurbains et à protéger de l'artificialisation les sols les plus productifs (Vandermeulen et al., 2009) ? Comment lutter contre le désintérêt vis-à-vis des dynamiques de changements d'occupation du sol évoquées ci-dessus ? Comment faire évoluer les représentations sociales de plus en plus marquées par une vision urbaine du monde (Sander et Polasky, 2009 ; Swanwick, 2009)?

D'un point de vue économique, les urbanistes ont pourtant montré depuis longtemps l'inefficacité de l'usage des ressources dans les villes étalées (Ciscel, 2001). Contrairement aux analyses relevant d'une approche orthodoxe de l'économie, celles portées par la vague du Nouvel Urbanisme privilégient la densification des zones urbaines et le développement des transports publics au lieu de laisser jouer les forces des marchés fonciers et de l'immobilier.

La préservation des terres agricoles périurbaines suscite désormais de nombreuses recherches, notamment en matière de gouvernance et de formes de coordination, sur les transformations en cours et les dispositifs innovants dans le domaine de l'action publique foncière (Bertrand et Duvillard, 2016). Cinq orientations majeures sont préconisées : raisonner la gestion des terres agricoles périurbaines de manière intégrée ; élargir les échelles de la planification; renouveler les relations sectorielles/territoriales par de nouvelles entrées : sécurité alimentaire, biodiversité ; renouveler les relations entre usages des terres et propriété foncière ; élargir les formes de régulation et de coordination entre acteurs.

\section{Genèse de l'ouvrage}

Le présent ouvrage s'inscrit dans la lignée de ces travaux qui visent à éclairer la décision territoriale pour mieux prendre en compte la question des terres agricoles dans les zones périurbaines. Il découle d'une collaboration scientifique franco-australienne initiée au début des années 2010 entre l'Université de technologie de Sydney (University of Technology Sydney, UTS) et l'Institut national de recherche en sciences et technologies pour l'environnement et l'agriculture (Irstea). Cette collaboration s'est progressivement structurée sous la forme du projet Knobimap (Knowledge-Based Biodiversity Mapping for Valuation of Ecosystem Services from Peri-Urban Agriculture). Elle s'est notamment 
traduite par les mobilités longues d'un chercheur français à l'Institute for Sustainable Futures (ISF) au sein de l'UTS, puis d'un chercheur australien au centre Irstea de Montpellier, à l'unité mixte de recherche Territoire, environnement, télédétection et information spatiale (UMR Tetis), et par le co-encadrement d'un stage de master en France puis d'une thèse en Australie. Deux séminaires d'échanges scientifiques ont également été organisés à Montpellier le 19 juin 2012 et à Sydney du 16 au 18 octobre 2013. Ils ont permis à des chercheurs et à des praticiens de la planification territoriale des deux pays de partager leur expertise ainsi que leurs retours d'expérience à partir de projets de recherche menés dans l'aire métropolitaine de Sydney en Australie et dans le sud de la France. Les équipes ont continué ensuite à mener des recherches sur la question de la planification territoriale de l'agriculture périurbaine.

Cet ouvrage dresse un panorama des résultats issus de cette collaboration entre la France et l'Australie. Il n'a pas la prétention d'offrir une analyse comparative approfondie de la planification des terres agricoles périurbaines entre les deux pays, mais plutôt de proposer une exploration des « boîtes à outils » de l'ingénierie territoriale développées et mobilisées pour faire face à cet enjeu.

Il offre également un "arrêt sur image " dans un panorama de champs de recherche en pleine évolution, autant du point de vue théorique (renouveau de la planification spatiale, approche par les services écosystémiques, systèmes alimentaires territoriaux...) que méthodologique (émergence du New Space en observation de la Terre, big data, sciences citoyennes...).

\section{Portée et objectifs de l'ouvrage}

L'ouvrage aborde des points clés de la problématique de la consommation des terres en se focalisant sur les terres agricoles en France et en Australie. Il est organisé autour de trois grandes questions :

Question 1 : Quels sont les enjeux ? Notre premier objectif est de clarifier la question de la perte de terres agricoles dans les zones périurbaines au moyen de cadres d'analyse de systèmes complexes pour comprendre les causes et les effets de ce phénomène.

Question 2 : Quels sont les outils disponibles pour faire face à cet enjeu? Il s'agit ici d'explorer les boîtes à outils actuelles et à venir, d'un point de vue théorique ou opérationnel, qui permettent de traiter la question de la planification territoriale de l'agriculture périurbaine et de limiter de nouvelles pertes de terres à haute valeur agricole.

Question 3 : Quelles sont les conditions d'adoption de nouvelles politiques et modalités de gouvernance et quels sont les bénéfices envisageables? Notre troisième objectif est de contribuer au défi de la réduction de la consommation des terres, à l'instar de l'Europe dont l'ambition est d'arriver à une consommation nette nulle d'ici 2050. Il s'agit ici d'étudier les conditions d'adoption d'outils et de méthodes développés par la communauté scientifique et d'analyser les évolutions nécessaires en matière de politiques publiques et de gouvernance. Il s'agit aussi de voir quels bénéfices (résilience, sécurité alimentaire, adaptation au changement climatique...) et quelles pertes évitées peuvent découler d'une telle ambition? Un dernier point plus prospectif porte sur les nouvelles recherches à développer par la communauté scientifique pour accompagner ces changements.

\section{Contenu de l'ouvrage}

Après une présentation générale et comparative de la planification territoriale en France et en Australie en lien avec la question des terres agricoles périurbaines l'ouvrage est 
organisé en deux grandes parties. La première traite des techniques d'observation, des données et des indicateurs pour représenter les dynamiques spatiales périurbaines (Base factuelle de la planification). La seconde aborde les modalités et techniques d'enrôlement des acteurs concernés par l'enjeu (La mesure à l'épreuve de l'humain). Une synthèse des résultats clôt l'ouvrage tout en proposant une ouverture sur des perspectives de recherche (Synthèse).

Le chapitre 1 traite, par une analyse comparative, de l'aménagement du territoire en France et en Australie, particulièrement en Nouvelle-Galles du Sud. À travers une analyse de documents et des entretiens individuels auprès de professionnels de l'aménagement, les logiques administratives et juridiques qui fondent l'aménagement du territoire dans ces deux pays sont présentées, ainsi que les instruments de planification qui en découlent. Malgré des différences significatives, la planification spatiale dans les deux pays repose sur des moteurs similaires, tels que la prise en considération d'un développement écologiquement durable et le renforcement de l'autonomie de la planification aux échelles locales avec la participation des communautés concernées. Ce chapitre met aussi l'accent sur l'utilisation récente d'outils de planification pour arbitrer les conflits d'usage autour de l'artificialisation des sols résultant du processus de périurbanisation.

\section{Partie I. Base factuelle de la planification}

Les chapitres 2 à 6 de la première partie traitent de la quantification des dynamiques spatiales périurbaines et de la qualité des sols, par des techniques d'observation (en particulier la télédétection satellitaire) et par la fabrique de données et d'indicateurs.

Le chapitre 2 introduit les enjeux de la préservation des terres agricoles du point de vue d'un service régional de l'État français relevant du ministère de l'Agriculture, la Draaf LR (Direction régionale de l'alimentation, de l'agriculture et de la forêt dans l'ancienne région Languedoc-Roussillon). Les auteurs mettent en avant un enjeu stratégique à long terme, celui de la sécurité et de l'autonomie alimentaires du pays. Il exige d'accorder plus d'importance à la qualité pédologique des sols, une dimension rarement prise en compte par les planificateurs et les aménageurs, par manque d'expertise et de données de référence dans ce domaine et faute d'une réglementation explicite.

Les auteurs du chapitre 3 présentent ensuite un système d'indicateurs spatiaux qui a été construit à partir des données décrites dans le chapitre 2. Ces indicateurs ont été développés à la demande de la Draaf LR pour surveiller et évaluer la dynamique de transformation des sols agricoles pour d'autres usages. L'originalité de l'approche a été de construire, de manière participative avec l'ensemble des parties prenantes, tout d'abord un modèle systémique traduisant une vision intégrée du développement urbain, puis un ensemble structuré d'indicateurs combinant des données pédologiques et des données sur les dynamiques d'artificialisation. L'objectif d'une telle approche était de garantir la qualité et la légitimité des indicateurs et de faciliter leur appropriation par les acteurs de la planification territoriale, afin d'étayer la prise de décision sur une base factuelle et objective.

Le chapitre 4 constitue un miroir australien des deux précédents chapitres. L'auteur montre comment la plupart des terres agricoles les plus productives en Australie, concentrées sur des zones littorales, ont été progressivement urbanisées durant les soixante dernières années. Elle met en exergue l'importance de disposer de données et de connaissances sur les propriétés des sols et d'adopter différentes stratégies, y compris réglementaires, pour arriver à les faire prendre en compte dans les projets d'aménagement périurbains. 
Pour aller plus loin sur la question des données en Australie, les auteurs du chapitre 5 détaillent l'avancement actuel de la cartographie de l'étalement urbain et de l'imperméabilisation des sols ainsi que de l'accessibilité des informations pédologiques en NouvelleGalles du Sud via le Système d'information sur les sols et le foncier (Soil and Land Information System, SALIS). Afin de rendre les informations sur les sols plus compréhensibles et exploitables par les aménageurs, elles sont incorporées dans des cartes de pédopaysages qui tiennent compte des contraintes et limitations imposées par différentes situations pédologiques et géologiques.

Dans le chapitre 6, les auteurs présentent les techniques géospatiales et de télédétection existantes pour caractériser l'état et suivre les dynamiques de l'artificialisation des terres agricoles périurbaines dans la région de Sydney. Ils montrent comment les images des satellites Landsat et Sentinel-2, qui sont gratuites et libres d'accès, peuvent être utilisées pour quantifier les changements paysagers et leur niveau de fragmentation, à une échelle synoptique et de manière répétée, à l'aide de techniques de classification, de suivi et de détection des changements. L'exploitation de la profondeur historique des images Landsat a aussi permis de retracer, sur les vingt-cinq dernières années, l'évolution des usages du sol aux interfaces entre l'urbain et le périurbain.

\section{Partie II. La mesure à l'épreuve de l'humain}

Cette partie de l'ouvrage (chapitres 7 à 11) s'intéresse à la dimension humaine dans les processus de planification territoriale qui traitent de la question des terres agricoles périurbaines.

Le chapitre 7 s'intéresse aux données et indicateurs sur la tache artificialisée et au potentiel agronomique des sols dans l'ancienne région Languedoc-Roussillon, en France. Ces données et indicateurs ont été présentés dans les chapitres 2 et 3 . Les auteurs ont ouvert la boîte noire des usages en étudiant le devenir de ces données, leur propagation et leur intégration dans les pratiques des acteurs de l'aménagement territorial, au moyen d'une analyse des processus communicationnels à l'œuvre tout au long de la «vie » de ces données. Ce chapitre interroge l'approche fonctionnaliste adoptée par les producteurs de ces données et montre que ce qu'ils pensent être une "mauvaise » utilisation des données par les destinataires qu'ils ciblaient est moins due à une « mauvaise communication » qu'à des questions de construction de réseaux d'intéressements, de traduction et de compromis.

À l'aide du concept de «politiques de l'identité de lieu», le chapitre 8 présente la manière dont les terres et activités agricoles sont marginalisées dans l'aménagement territorial de la commune périurbaine de Wollondilly, au sud-ouest de Sydney, en Australie. L'auteur montre comment le discours sur le développement de la commune, qui a pour objectif de combiner développement urbain et protection des terres agricoles, en maintenant une limite entre ville et campagne, mène, en pratique, à la marginalisation de ces terres dans l'aménagement communal. La priorité donnée aux projections du besoin en logement et l'utilisation de données à la validité discutable sur le potentiel agronomique des sols provoquent la marginalisation des terres agricoles. Selon l'auteur, ce problème ne pourra être résolu qu'en dépassant la dichotomie entre ville et campagne. Elle propose pour cela d'utiliser un modèle spatial où paysages urbains et agricoles seraient entremêlés, ce qui suppose de redéfinir le terme de viabilité de l'agriculture périurbaine dans la commune de Wollondilly, et de créer de l'information, qui fasse consensus, sur cette viabilité.

Dans le chapitre 9, un collectif de chercheurs présente les premiers résultats de la construction d'un dispositif partenarial sur le territoire d'une intercommunalité de l'île de 
la Réunion, un territoire français ultramarin. Ce dispositif mobilise un outil de simulation de dynamiques paysagères utilisé comme support d'un exercice participatif de prospective territoriale. L'exercice vise à explorer collectivement différents scénarios d'évolution de l'occupation du sol dans lesquels l'étalement urbain et ses impacts constituent un enjeu central. Les modèles sont ici utilisés comme des objets intermédiaires entre des acteurs hétérogènes pour faciliter les apprentissages collectifs.

Le chapitre 10 traite aussi de la manière dont les acteurs peuvent être accompagnés pour appréhender collectivement les processus qui mènent à la perte de terres agricoles et imaginer des solutions. Le territoire étudié ici est celui des zones périurbaines de Sydney. Pour faciliter les apprentissages du système territorial, les auteurs ont tout d'abord proposé aux participants un jeu de plateau sur l'aménagement du territoire. Ils se sont focalisés dans un deuxième temps sur un enjeu qui avait du sens pour tous les participants, celui du système alimentaire de Sydney. Ils ont identifié collectivement les vulnérabilités de ce système puis ils ont imaginé des trajectoires vers des futurs permettant de faire face à ces faiblesses. Ceci les a amenés indirectement à traiter la question de la durabilité des espaces agricoles dans les territoires périurbains de Sydney.

Dans le chapitre 11, les auteurs ont mené une recherche exploratoire pour évaluer l'intérêt du concept de services écosystémiques afin de décadrer la manière d'aborder le problème des terres agricoles périurbaines. Menée sur deux terrains dans le sud de la France, l'originalité de la démarche est de tenir compte des valeurs et des perceptions des acteurs locaux, basées sur leurs connaissances tacites ou d'experts de l'environnement local, pour arriver à une évaluation des services écosystémiques présents à l'échelle locale puis à leur traduction cartographique. Les auteurs étudient ensuite les conditions d'opérationnalisation de la méthode.

Enfin, une synthèse reprend les différentes approches théoriques, conceptuelles et méthodologiques ainsi que les cas d'application présentés dans les chapitres précédents. La synthèse s'organise pour cela autour d'un cadre conceptuel intégrateur adapté de la pyramide données-information-connaissance-sagesse (Data Information Knowledge Wisdom, DIKW) complété par deux notions supplémentaires relevant de l'anthropologie des connaissances (dimensions structurelles et humaines de la connaissance). En combinant les approches guidées par les données avec celles guidées par les usages pour créer des informations et inférer des connaissances, cette réflexion de synthèse arrive à la conclusion que les connaissances sur les terres agricoles périurbaines sont souvent mal structurées et/ou « mal » utilisées, du moins du point de vue de leurs producteurs. Les politiques publiques visant à préserver ces terres agricoles en sont d'autant moins efficaces. Les leçons tirées de l'analyse des études de cas en Australie et en France montrent toutefois que les dynamiques de production de connaissances dérivées à la fois de données factuelles et d'apprentissages collectifs sont particulièrement prometteuses. Une adhésion et des investissements dans ce type d'approches permettraient de les déployer dans les processus opérationnels de planification spatiale.

\section{Références bibliographiques}

Béchet B., Le Bissonnais Y., Ruas A., Aguilera A., Andrieu H., Barbe E., et al., 2017. Sols artificialisés et processus d'artificialisation des sols : déterminants, impacts et leviers d'action. Synthèse du rapport d'expertise scientifique collective, Ifsttar-Inra, France, 127.

Bertrand N., 2013. Terres agricoles périurbaines. Une gouvernance foncière en construction, éditions Quæ, 256 p.

Bertrand N., Duvillard S., 2016. Intégration des enjeux environnementaux dans la gestion du foncier agricole. Sciences Eaux et Territoires, 19 (2), 2-5. 
Ciscel D.H., 2001. The economics of urban sprawl: inefficiency as a core feature of metropolitan growth. Journal of Economic Issues, 35 (2).

Colantoni A., Grigoriadis E., Sateriano A., Venanzoni G., Salvati L., 2016. Cities as selective land predators? A lesson on urban growth, deregulated planning and sprawl containment. Science of the Total Environment, 545, 329-39.

Decoville A., Schneider M., 2015. Can the 2050 zero land take objective of the EU be reliably monitored? A comparative study. Journal of Land Use Science, 1-19.

EEA, 2006. Urban sprawl in Europe. The ignored challenge. European Environment Agency, Report No 10/2006.

Gardi C., Panagos P., Van Liedekerke M., Bosco C., De Brogniez D., 2015. Land take and food security: assessment of land take on the agricultural production in Europe. Journal of Environmental Planning and Management, 58 (5), 898-912.

Lavalle C., Barbosa A.L., Mubareka S., Jacobs-Crisioni C., Baranzelli C., Castillo C.P., 2013. Land use related indicators for resource efficiency: Part I Land Take Assessment. An analytical framework for the assessment of the land milestone proposed in the Roadmap for Resource Efficiency, JRC Scientific and Policy Reports. Publications Office of the European Union, Luxembourg.

Maes J., Teller A., Erhard M., Liquete C., Braat L., Berry P., et al., 2013. Mapping and Assessment of Ecosystems and their Services. An Analytical Framework for Ecosystem Assesments under Action 5 of the EU Biodiversity Strategy to 2020, Publications Office of the European Union, Luxembourg.

Oncea, 2014. Panorama de la quantification de l'évolution nationale des surfaces agricoles. Travail coordonné par le ministère de l'Agriculture, de l'Agroalimentaire et de la Forêt, direction générale des politiques agricole, agroalimentaire et des territoires, sous-direction de la biomasse et de l'environnement, bureau du foncier et de la biodiversité, mai 2014.

Sander H.A., Polasky S., 2009. The value of views and open space: Estimates from a hedonic pricing model for Ramsey County, Minnesota, USA. Land Use Policy, 26 (3), 837-45.

Siedentrop S., Fina S., 2010. Monitory urban sprawl in Germany: towards a GIS-based measurement and assessment approach. Journal of Land Use Science, 5 (2), 73-104.

Smiraglia D., Ceccarelli T., Bajocco S., Salvati L., Perini L., 2016. Linking trajectories of land change, land degradation processes and ecosystem services. Environmental Research, 147, 590-600.

Swanwick C., 2009. Society's attitudes to and preferences for land and landscape. Land Use Policy, 26 (0), suppl. 1, S62-S75.

Vandermeulen V., Gellynck X., Van Huylenbroeck G., Van Orshoven J., Bomans K., 2009. Farmland for tomorrow in densely populated areas. Land Use Policy, 26 (4), 859-868. 



\title{
Chapitre 1
}

\section{Contexte : une étude comparée sur la planification spatiale de l'artificialisation des sols en France et en Australie}

\author{
JASON Prior, Pierre MAURel
}

\section{Introduction}

«Les sols sont partout... sous nos villes et nos périphéries urbaines » (Hazelton et Murphy, 2011, p. 14). L'artificialisation anthropique des sols par des surfaces artificielles impénétrables, à travers des processus tels que l'urbanisation, est de plus en plus reconnue comme interférant avec les fonctions environnementales, économiques et sociales essentielles remplies par les sols (Scalenghe et Marsan, 2009 ; Salvati, 2013) comme la production alimentaire par l'agriculture (Nuissl et al., 2009 ; Munafo et al., 2010), y compris par des politiques alimentaires urbaines (Brand et al., 2017), la régulation des eaux (Hamdi et al., 2010 ; Yang et Li, 2011), l'énergie (Zhou et Wang, 2011) ou encore la fourniture d'habitats pour la biodiversité. Une attention croissante est portée à l'artificialisation anthropique des sols comme conséquence de l'expansion de l'urbanisation (Nuissl et al., 2009 ; Davis et al., 2010 ; Munafo et al., 2010 ; European Commission, 2012 ; Béchet et al., 2017). Comme l'ont constaté Scalenghe et Marsan (2009, p. 8), « l'avenir de la majorité [...] de l'humanité sera urbain et l'urbanisation s'accompagnera d'une artificialisation des sols à une échelle sans précédent dans l'histoire de l'homme ». En raison de ces préoccupations, de plus en plus de voix s'élèvent pour demander la création d'une gouvernance de l'artificialisation des sols, particulièrement dans les zones périurbaines où différents usages du sol sont en compétition avec l'agriculture.

Le terme « périurbain » est utilisé depuis longtemps en France pour décrire les zones aux usages du sol entremêlés, situées au-delà des banlieues (voir par exemple Pryor, 1968 ; Cadene, 1990). En Australie et au Royaume-Uni, les zones périurbaines sont aussi qualifiées de « franges rurales-urbaines » (rural-urban fringe) (voir par exemple Pryor, 1968), d' « arrière-pays urbain » (urban hinterland) (Furuseth et Lapping, 1999) ou encore de «terres périphériques» (edgelands) (Shoard, 2002). Tout au long du $\mathrm{xx}^{\mathrm{e}}$ siècle, des tentatives répétées ont été faites pour définir cette "zone complexe à la périphérie des zones urbaines en développement » (voir par exemple Pryor, 1968, p. 202 ; Bryant, 1982), dominée par la transition et la compétition entre un éventail d'usages du sol ruraux et urbains et d'activités fortement hétérogènes. Ce degré élevé d'hétérogénéité, tel qu'observé par Shoard (2002, p. 117), se caractérise par « des décharges et des entre- 
pôts, des hypermarchés et des usines à l'abandon, des parcs de bureaux et des campements de gens du voyage, des terrains de golf, des jardins ouvriers et des terres agricoles fragmentées et souvent mal entretenues ». La diversité des définitions et des nomenclatures utilisées pour définir ces zones n'a d'égal que celle des débats cherchant à identifier les principaux processus à l'origine de leur évolution tels que la « périurbanisation », l'« exurbanisation » ou la « rurbanisation » (voir par exemple Balchin et al., 1976 ; Bauer et Roux, 1976 ; Hoggart, 2005 ; Roux, 2017).

$\mathrm{Au}$ cours des dix dernières années, des chercheurs ont commencé à mettre l'accent sur le rôle que la planification spatiale pouvait jouer dans la gouvernance de l'artificialisation des sols induite par la périurbanisation (Huber, 2012 ; Bertrand, 2013). Ce présent chapitre s'ajoute à ces recherches récentes en présentant et en comparant la planification spatiale en Australie et en France, avec une attention particulière portée aux approches traitant de l'artificialisation anthropique des terres agricoles dans les zones périurbaines. En ce qui concerne l'Australie, nous nous concentrerons sur la planification spatiale dans l'État de Nouvelle-Galles du Sud. La planification spatiale, appelée aussi « planification urbaine et régionale » en Australie, peut-être décrite en termes généraux comme « des actions publiques délibérées qui impactent l'environnement bâti et naturel » (italique ajouté) (Seeley, 1962 ; Watson, 2002, p. 28), c'est-à-dire la manière dont l'homme façonne et gouverne l'environnement construit et naturel. La planification spatiale est formée par de nombreuses logiques (pragmatiques et idéologiques), légales, administratives, scientifiques et économiques entre autres. Ces logiques influencent à la fois les procédures et les contenus de la planification spatiale, comme par exemple les procédures de gestion de l'usage des terres au sein d'une zone périurbaine et les résultats attendus en termes d'usages du sol dans ces zones (Van Assche et Verschraegen, 2008 ; Allmendinger, 2009). Le réseau des organisations impliquées dans cet effort peut être qualifié de « système de planification spatiale ».

Les deux dernières décennies ont vu une augmentation des études comparatives en matière de planification spatiale (par exemple Getimis, 2012 ; Nadin, 2012 ; Reimer et Blotevogel, 2012). Un sujet d'intérêt majeur de ces recherches a été la comparaison systématique de la planification spatiale dans différents États de l'Union européenne (UE) (European Commission, 1997a ; 1997b ; 1997c ; ESPON, 2004). Une raison évidente du développement de ces études comparatives en Europe réside dans la volonté continue de comprendre les processus de planification spatiale à travers l'Union et de s'assurer de leur intégration (Healey et Williams, 1993 ; European Commission, 1997a ; 1997b ; 1997c ; Knieling et Othengrafen, 2009 ; Tosics et al., 2010). De plus, un certain nombre de ces études comparatives soutiennent que le contexte actuel de globalisation, d'européanisation ${ }^{1}$ et de dérégulation mène à un mélange des pratiques de planification spatiale entre des contextes différents, alors que traditionnellement, les systèmes de planification spatiale à l'échelle nationale dans un contexte tel que celui de l'UE ont été développés de manière relativement indépendante (European Commission, 1997a ; 1997b ; 1997c ; Nadin et Stead, 2008 ; Giannakourou, 2012). Si beaucoup d'études comparatives entre États de l'UE existent, peu de travaux finalement ont examiné les points communs et les différences entre les processus de planification spatiale au sein de l’UE et dans des pays en dehors de l'UE (voir par exemple Albrechts, 2006). L'étude de

1. L'« européanisation » fait référence ici au processus actuel qui cherche à intégrer les systèmes de planification spatiale à l'échelle européenne. L'usage de ce terme dans le cas présent est différent de l'usage qui en est fait dans d'autres contextes, comme en Australie, où il est utilisé pour décrire le processus par lequel le contexte australien a été transformé, dans les siècles derniers, par la colonisation européenne. 
la planification spatiale en Australie et en France présentée dans ce chapitre contribue à combler ce manque de travaux comparatifs entre Etats de l'UE et hors UE.

Les résultats de cette étude sont présentés en trois parties. Premièrement, nous décrivons les logiques politiques, légales et administratives qui ont façonné la planification spatiale en Australie et en France. Nous présentons ensuite les instruments et systèmes de planification qu'elles ont générés dans chaque pays. Dans la troisième partie, nous étudions les approches de planification spatiale ainsi que les instruments développés pour répondre aux enjeux spécifiques de l'artificialisation anthropique des terres agricoles dans les zones périurbaines en Australie et en France. Leur mise en œuvre concrète à l'échelle locale est ensuite décrite. La conclusion ouvre sur la question des données, des informations et de leurs usages pour accompagner ces politiques publiques dans les deux pays, question développée dans les chapitres suivants. Dans un premier temps toutefois, nous présentons la démarche et les données que nous avons utilisées pour réaliser cette étude comparative.

\section{Démarche méthodologique}

Pour comparer les processus de planification spatiale en Australie et en France, nous avons développé, dans les deux pays, des aperçus de cas d'étude tels qu'ils existaient en 2014, puis leur évolution depuis. Dans ce chapitre, nous présentons ces « arrêts sur image » et identifions leurs points communs et leurs différences. Le recueil de données et l'analyse sont présentés ci-dessous. Pour le cas australien, ils ont été faits conformément à l'approbation éthique requise par le Comité d'éthique pour la recherche en sciences humaines de l'Université de technologie Sydney.

Les données utilisées pour développer ces «arrêts sur images » dans les deux pays ont été recueillies à l'aide de différentes méthodes: des entretiens approfondis, un atelier de travail franco-australien et une analyse de documents (en matière par exemple de législation, de politiques publiques et d'instruments). Les documents recueillis ont porté sur 19 instruments internationaux (dont des chartes d'organisations internationales), 92 extraits de textes de loi et de politiques publiques de différents niveaux (sectoriel, transversal, national ou supranational) et 85 instruments de planification spatiale à des échelles plus fines (locales, communales, intercommunales et régionales). De plus, nous avons passé en revue les études comparatives sur la planification spatiale dans l'UE (voir par exemple European Commission, 1997a ; 1997b ; 1997c ; Knieling et Othengrafen, 2009 ; Prokop et al., 2011 ; European Commission, 2012).

Des entretiens ont été conduits avec 19 professionnels impliqués dans la gestion de l'artificialisation des sols en Australie et en France. Les participants ont été sélectionnés à l'aide d'un échantillonnage "boule de neige ${ }^{2}$ à partir de premiers contacts avec des universités et des instituts de recherche dans les deux pays. De plus, des données ont été collectées durant un atelier tenu à Montpellier en juillet 2012, qui a rassemblé des pédologues, des aménageurs et d'autres professionnels impliqués dans la planification urbaine et la gestion des ressources en France et en Australie (Plant, 2012). Les entretiens et l'atelier ont été menés de manière ouverte et les données recueillies ont permis de vérifier les résultats de l'analyse documentaire et de les replacer dans le contexte plus large des pratiques de planification spatiale dans les deux pays.

Nous avons aussi utilisé un codage thématique pour analyser les données recueillies dans les documents ainsi que durant l'atelier et les entretiens (Emerson et al., 1995). Nous

2. La méthode « boule de neige » consiste à sélectionner des personnes ayant les caractéristiques que l'on recherche puis de leur demander de suggérer d'autres personnes de profil similaire. 
avons commencé l'analyse en lisant les documents, les notes prises par les chercheurs durant les entretiens et l'atelier ainsi que les transcriptions des entretiens, pour évaluer, dans un premier temps, les logiques politiques, légales et administratives qui façonnent la planification territoriale et ses instruments dans les deux pays, et pour identifier, dans un second temps, la présence du concept d'imperméabilisation des sols dans les documents de planification, en notant les thèmes qui émergeaient. Les résultats de l'analyse sont présentés dans les sections suivantes.

\section{Logiques légales et administratives de la planification spatiale}

$\mathrm{Au}$ cours des dernières décennies, plusieurs chercheurs ont essayé de différencier les logiques légales qui façonnent la planification spatiale dans différentes juridictions (Newman et Thornley, 1996 ; Zewigert et Kotz, 1998). Des distinctions claires existent entre le système législatif australien, basé sur la tradition anglaise du droit commun, et le système légal français, basé sur la tradition napoléonienne. L'Australie, en tant que membre du Commonwealth, a plus de points communs avec le système de planification du Royaume-Uni que le Royaume-Uni n'en a avec son voisin français, bien qu'il y ait de plus en plus de similitudes entre les systèmes de planification de ces deux pays européens en raison des efforts d'intégration des États au sein de l'UE au cours des dernières décennies (European Commission, 1997a ; 1997b ; 1997c ; ESPON, 2004).

En Australie et au Royaume-Uni, le système juridique s'est développé à partir de la tradition anglaise du droit commun, un système de jurisprudence qui s'est construit progressivement, décision par décision. Cette approche favorise l'empirisme et accorde de l'importance à l'expérience passée et à la jurisprudence. L'application du droit en Australie respire le pragmatisme et le dédain pour l'uniformité. L'Angleterre a été à l'origine de ce système légal qui s'est étendu à l'Australie durant la colonisation. La France est quant à elle à l'origine du système judiciaire napoléonien. Ce système a tendance à utiliser des normes légales et des règles de droit abstraites. L'objectif est d'anticiper les problèmes et de préparer un système complet de règles basées sur la codification de principes abstraits.

Alors que certains soutiennent que les systèmes administratifs dérivent des systèmes juridiques, d'autres, comme Newman et Thornley (1996), établissent des distinctions claires entre les deux systèmes dans les États nations. Les systèmes administratifs peuvent être définis par des caractéristiques telles que le partage versus la séparation des pouvoirs, le fédéralisme versus l'unitarisme et la gouvernance territoriale versus la gouvernance sectorielle. Les systèmes administratifs en Australie et en France sont fondés sur des constitutions écrites, même si ces deux pays ont créé des échelons administratifs en plus de ceux listés dans leur constitution.

En Australie, la tradition administrative distingue clairement les pouvoirs et les responsabilités selon différents échelons de gouvernement. La Constitution australienne, signée en 1901 au moment de la fédération des États australiens, établit très clairement les pouvoirs de deux de ses trois niveaux de gouvernement - fédéral et étatique. N'importe quelle modification de leurs prérogatives nécessite un amendement de la Constitution. L'Australie compte un gouvernement fédéral et six États, plus dix territoires reconnus au niveau national comme des pseudo-États dotés d'un gouvernement propre. Il existe enfin un troisième échelon administratif, le gouvernement local, à qui les États délèguent des responsabilités. 
La Constitution australienne énonce clairement que la planification spatiale relève de la responsabilité des États. Le gouvernement fédéral n'a donc aucun pouvoir concernant la planification spatiale ou des sujets connexes, comme la gestion des ressources naturelles. Il est toutefois responsable des questions « environnementales d'importance nationale », comme la gestion de l'eau dans le bassin Murray-Darling. Bien que le gouvernement fédéral n'en ait pas le pouvoir, il a par moments cherché à s'impliquer dans la planification spatiale ${ }^{3}$. Du fait de la répartition des pouvoirs inscrite dans la Constitution, chaque État a développé son propre système de planification spatiale et de gestion de l'occupation du sol. Le présent chapitre se concentrera sur celui de l'État de Nouvelle-Galles du Sud. En Australie, les gouvernements des États ont souvent délégué aux gouvernements locaux les prérogatives en planification spatiale qui leur ont été conférées par la Constitution. La Nouvelle-Galles du Sud compte 152 gouvernements locaux qui sont en train de fusionner (figure 1.1).

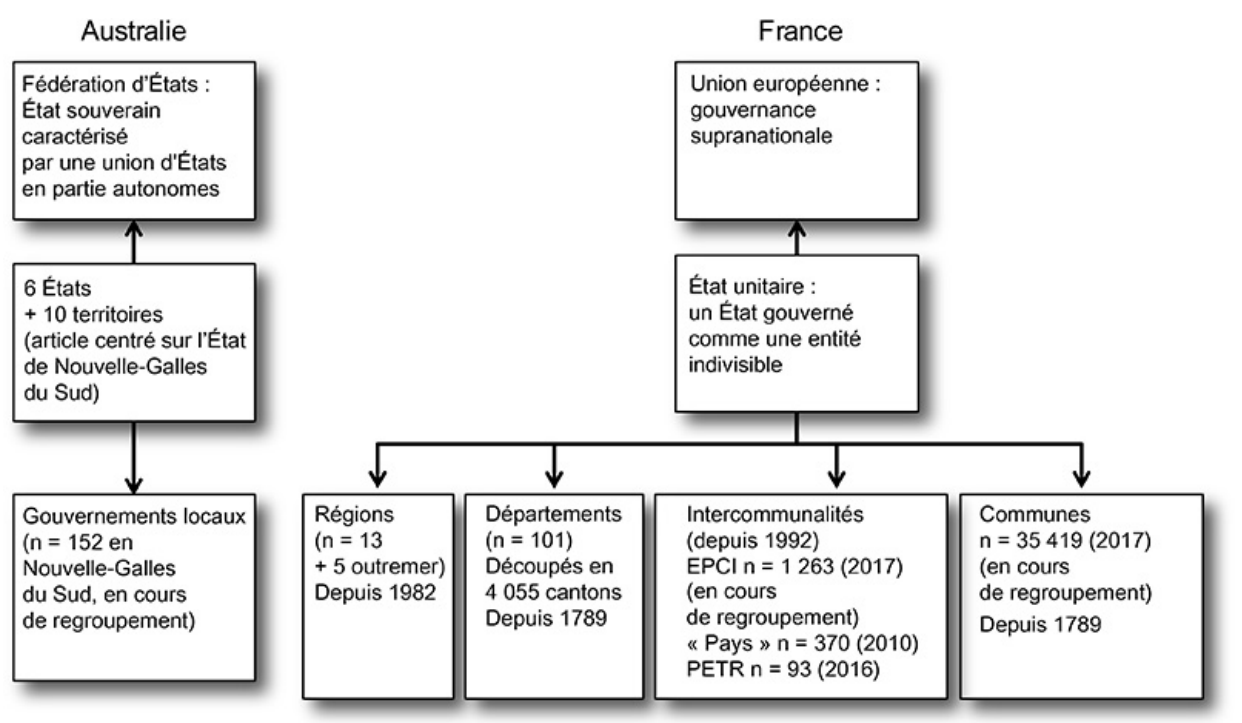

Figure 1.1. Logiques administratives pour la planification en Australie (Nouvelle-Galles du Sud) et en France.

EPCI : Établissement public de coopération intercommunale ; PETR : Pôle d'équilibre territorial et rural.

La République française, contrairement à la Fédération australienne, est un « État unitaire décentralisé », comme mentionné dans les changements constitutionnels de 2003. Conformément à sa Constitution, la France est une "République indivisible », qui garantit l'égalité de tous les citoyens devant la loi et définit le caractère unitaire de la République française. La Constitution garantit le principe de libre administration des autorités locales, selon les conditions stipulées par la loi ; cependant, le représentant de l'État (le préfet), assisté par l'administration locale, a la responsabilité de garantir l'intérêt national, le contrôle administratif et le respect de la loi, ainsi que de préparer des projets contractuels entre l'État et la région pour le développement socio-économique et

3. Durant l'après-guerre, la politique du Commonwealth sur les affaires urbaines et régionales n'a évolué qu'à deux reprises : durant le mandat de Whitlam (1972-1975) avec la création du Département du développement urbain et régional, et durant les gouvernements Hawke et Keating (1983-1996), en particulier avec le programme « Meilleures Villes » (Better Cities). 
l'aménagement régional (Contrats de plan État-région, CPER). Des distinctions existent donc entre les responsabilités de l'État et celles revenant à l'administration locale.

En France, les communes, les structures intercommunales, les départements et les régions sont des autorités locales autonomes ayant le statut de collectivités territoriales. Elles ont quatre caractéristiques : le pouvoir fiscal ; une personnalité juridique ; des champs de compétences spécifiques, mais pas complètement distincts, avec des ressources administratives propres ; un pouvoir de libre administration à travers un conseil d'élus, mais sans qu'aucune collectivité ne dispose d'une autorité sur une autre.

Avec le renforcement de la décentralisation, le nombre de structures intercommunales ayant un pouvoir fiscal, aussi appelées Établissements publics de coopération intercommunale (EPCI), a considérablement augmenté depuis le début des années 2000. Un large éventail de compétences obligatoires et optionnelles leur a été attribué, aux dépens des communes, même si leurs représentants politiques ne sont pas encore directement élus par les citoyens, mais choisis parmi les élus communaux. À la fin de 2013, 2456 EPCI avaient été créés dans $99,9 \%$ des communes. La promulgation de la loi de nouvelle organisation territoriale de la République (NOTRe), en 2015, a imposé de fusionner les plus petits EPCI pour atteindre un seuil minimum de 15000 habitants par EPCI. En janvier 2017, il y avait 35419 communes (35 885 début 2016, 36680 en 2000) et 1263 EPCI (2 062 au début de 2016).

Le renforcement des plus grosses conurbations françaises a aussi été dynamisé par la loi de réforme des collectivités territoriales, en 2010, et par la loi de modernisation de l'action publique territoriale et d'affirmation des métropoles, dite « loi Maptam », en 2014. Ces deux lois ont créé et renforcé le statut de métropole, qui est le niveau le plus intégré des EPCI, avec des compétences multiples, dont certaines ont été prises aux départements dans des domaines tels que les infrastructures routières, les transports publics, la culture. Seize conurbations françaises ont tout d'abord obtenu ce statut de métropole, puis cinq de plus en 2017 du fait de la fusion des régions.

Une nouvelle réforme territoriale conduite en 2015 et 2016, la loi NOTRe, a aussi réduit le nombre de régions métropolitaines de 22 à 13 et leur a transféré plus de compétences afin de renforcer leur visibilité et leur pouvoir à l'échelle européenne et internationale.

Des lois successives dans les années 1990 avaient aussi institué les « pays », une forme de coopération intercommunale volontaire (sans pouvoir fiscal) entre les communes d'un même bassin partageant des intérêts géographiques, culturels, économiques et sociaux communs. Un « pays » est guidé par un projet de développement durable rendu officiel à travers une charte. Au $1^{\text {er }}$ janvier 2010, il y avait 370 «pays » comprenant 29982 « communes » et couvrant 80 \% du territoire français. En 2010, la loi de réforme des collectivités territoriales a supprimé la possibilité de créer de nouveaux « pays ». En 2014, la loi Maptam a permis aux " pays » constitués de se transformer en une nouvelle forme de coopération intercommunale dans les zones rurales, appelée Pôle d'équilibre territorial et rural (PETR) pour contrebalancer la création de « métropoles » dans les conurbations les plus importantes. Il y avait 93 PETR au début de 2016, en incluant les anciens « pays » qui avaient choisi de se transformer en PETR.

La complexité du morcellement administratif français actuel, souvent qualifié de « millefeuille territorial », est présentée dans la figure 1.1.

Contrairement à la Constitution australienne, la Constitution française ne contient pas de dispositions détaillant quel échelon administratif est en charge de l'aménagement du territoire. La Constitution délègue cette responsabilité au législateur national, qui délègue 
à son tour les pouvoirs aux administrations de rangs inférieurs au gouvernement central à travers la promulgation de nouvelles lois plutôt que des changements constitutionnels.

Le cadre stratégique actuel pour la planification spatiale en France, dénommé « aménagement du territoire », a été développé au début des années 1950 par l'organe exécutif du gouvernement central pour accompagner la politique de planification économique. La notion même d'aménagement du territoire intègre une double dimension, la première, de nature économique, visant par des aides à un développement équilibré du territoire national, la deuxième, de nature aménagiste, visant à organiser et structurer l'espace géographique au moyen de grandes infrastructures (aéroports, ports, autoroutes...) ou de grandes opérations (villes nouvelles, stations littorales...). L'objectif global était de contrebalancer la domination de Paris, illustrée dans l'étude fondatrice de Jean-François Gravier Paris et le désert français (Gravier, 1947), en apportant une réponse à la fois en termes de justice spatiale et de rééquilibrage du développement économique. En 1963, le cadre pour la planification spatiale a été institutionnalisé avec la création de la Délégation à l'aménagement du territoire et à l'action régionale (Datar) ${ }^{4}$. Cette politique de planification stratégique peut opérer sans plan réglementaire et en l'absence d'un cadre juridique réel. La définition du rôle du législateur et des pouvoirs du Conseil constitutionnel dans la Constitution de 1958 a considérablement élargi les pouvoirs statutaires autonomes en matière de planification spatiale, l'État gardant toutefois la compétence juridique. Cependant, l'importance accordée par le Conseil constitutionnel au principe d'autonomie des autorités locales a réduit les pouvoirs d'intervention réglementaire par l'administration centrale française, ce qui a conduit, au cours des dernières décennies, à une implication significative de l'administration locale dans les questions de planification spatiale. À l'échelon régional, le cadre de la planification spatiale repose sur un partenariat fort entre l'État central et chaque région, qui partagent les coûts et les responsabilités du développement régional à travers un contrat pluriannuel, le CPER, aligné sur les politiques de l'UE et leur financement ${ }^{5}$. Jusqu'à présent, ce partenariat concernait essentiellement la dimension économique et peu la dimension de planification spatiale stratégique liée à la réalisation d'équipements structurants, dimension peu investie par les régions alors que la loi du 25 juin 1999 d'orientation pour l'aménagement et le développement durable du territoire (LOADDT) leur en donnait la possibilité (Jégouzo, 2015). La loi NOTRe de 2015 a renforcé les compétences des régions en matière de planification spatiale en supprimant la compétence générale pour les régions, les départements et les EPCI, et en désignant clairement les régions comme chefs de file de l'aménagement du territoire aux échelles régionales et infrarégionales. Elles sont depuis 2015 chargées d'élaborer un Schéma régional d'aménagement, de développement durable et d'égalité des territoires (Sraddet) qui a vocation à intégrer les précédents schémas régionaux sectoriels (transport, biodiversité, climat, énergie, biomasse...). Le Sraddet doit notamment fixer des objectifs de gestion économe de l'espace. Contrairement aux anciens schémas régionaux, le Sraddet est opposable aux documents d'urbanisme des collectivités territoriales (Schémas de cohérence territoriale, SCoT; Plans locaux d'urbanisme, PLU ; Plans locaux d'urbanisme intercommunal, PLUi ; chartes des

4. Ce qui sous-tendait ce cadre institutionnel était l'idée de performance économique basée sur une vision fordiste, où la division sociale du travail doit être alignée à une division spatiale des activités, menant à la spécialisation fonctionnelle des territoires, imbriqués comme des poupées russes, tous contribuant à la croissance nationale.

5. Deux financements européens s'occupent de l'aménagement du territoire : le Fonds européen pour le développement régional, Feder (European Regional Development Fund, ERDF) et le Fonds agricole européen pour le développement rural, Feader (European Agricultural Fund for Rural Development, EAFRD). 
parcs naturels...) pour certaines de ses composantes. De plus, la loi NOTRe a conforté la compétence des régions sur l'information géographique en désignant l'échelon régional comme pertinent pour assurer une mutualisation et une redistribution efficace de l'information géographique à des acteurs institutionnels au moyen d'infrastructures numériques de données géographiques pour une connaissance détaillée du territoire. Enfin, depuis 2014, les régions deviennent les seules autorités de gestion des fonds européens Feder et Feader, leur permettant ainsi de disposer des leviers financiers pour la mise en œuvre de leur stratégie de planification spatiale, dont la régulation des dynamiques foncières périurbaines, par les acteurs du territoire régional. Les nouvelles régions disposent donc aujourd'hui de nombreux pouvoirs pour jouer le rôle de chef de file de la planification territoriale parmi l'ensemble des collectivités locales (Desjardins et Béhar, 2017).

Parallèlement à ce mouvement de décentralisation au bénéfice des régions et des EPCI, l'État s'est réorganisé avec la mise en place d'une forme de " gouvernement à distance » (Epstein, 2009). Les services de l'État ne sont plus directement impliqués sur le terrain pour soutenir les municipalités à travers l'ingénierie publique, sauf dans les zones rurales. L'État opère désormais à travers des mécanismes à distance, en utilisant les outils et procédures de la «nouvelle gestion publique» tels que des appels d'offres concurrentiels, des primes, des indicateurs de performance pour suivre et contrôler le financement de projets afin d'encourager les gouvernements locaux à mettre en place des politiques de nature centralisée. Cette stratégie s'est appuyée sur une réorganisation de l'administration d'État, avec le redéploiement de son expertise à l'échelon régional, au détriment de l'échelon départemental. Les Directions départementales de l'équipement (DDE), responsables du développement urbain et des routes, et les Directions départementales de l'agriculture et de la forêt (DDAF), responsables des affaires rurales, ont fusionné à la fin des années 2000 pour former les nouvelles Directions départementales des territoires et de la mer (DDTM) ${ }^{6}$. Nous reviendrons sur cette réorganisation de l'État au niveau départemental dans la section suivante.

Il est difficile de faire une comparaison entre les différents échelons juridiques et administratifs en France et en Australie, étant donné qu'ils diffèrent considérablement en termes d'échelles, de nombre d'échelons et de structures administratives (beaucoup plus nombreux en France), de compétences. Si en Australie, les États sont clairement désignés par la Constitution comme les autorités responsables de la planification spatiale, la situation en France est moins nette, même si l'évolution de la législation ces deux dernières décennies a renforcé les pouvoirs des EPCI et surtout des régions en matière de planification spatiale. De plus, une des caractéristiques récentes de l'administration française qui la distingue de l'administration australienne est l'engagement de la nation envers l'UE, ce qui a mené à une forme d'administration supranationale. Bien que l'UE n'ait pas formellement d'autorité sur la planification spatiale, elle a mis en place plusieurs processus qui ont influencé les politiques de planification urbaine dans les États membres et les régions européennes et les ont poussés à adopter une approche polycentrique, fortement influencée par l'approche française de l'aménagement du territoire. Ces processus ont été conçus pour contrebalancer la domination économique et le déséquilibre résultant de la « banane bleue » (le pentagone Londres-Paris-MilanMunich-Hambourg), qui concentre $40 \%$ de la population de l'UE et $50 \%$ de son PIB. Ces processus incluent la Perspective européenne de développement spatial (European

6. À l'échelle locale, la diminution de l'expertise technique due au désengagement de l'État n'a été que partiellement contrebalancée par le recrutement d'ingénieurs par les autorités intercommunales, par la mobilisation d'acteurs intermédiaires (par exemple les chambres d'agriculture, les parcs naturels régionaux) et par l'utilisation de bureaux d'études privés. 
Spatial Development Perspective, ESDP) de 1999 ainsi que la logique de « cohésion territoriale », développée dans la stratégie de Lisbonne en 2000 et réaffirmée plus récemment dans l'Agenda territorial de l'Union européenne 2020 (Territorial Agenda of the European Union 2020) (Faludi, 2009a ; 2009b). Ces approches sont calquées sur les CPER français, afin de limiter les inégalités régionales au sein de l'UE, avec un intérêt particulier porté à la justice sociale et spatiale (Faludi, 2009a ; 2009b).

\section{Instruments de planification spatiale, pratiques, participation et politique}

Un aspect clé de la planification spatiale en Australie (qui a été adapté du contexte anglais) et en France est la planification de l'usage des sols. Celle-ci consiste à gérer l'espace à travers le développement d'un plan local déterminant l'utilisation future des terres au moyen de règles de zonage basées sur la régulation et le contrôle des terres, avec comme objectif de maîtriser le développement et la croissance du territoire. De manière générale, ce sont les autorités locales qui réalisent la majeure partie du travail de planification, même si l'administration centrale est en mesure d'exercer un certain pouvoir, soit en supervisant le système de planification, soit en établissant des objectifs politiques stratégiques, comme le fait la stratégie de 2014 pour la métropole de Sydney intitulée «Un plan pour la croissance de Sydney " (A Plan for Growing Sydney) (figure 1.2). De ce travail de planification découle en général un plan d'occupation du sol à l'échelle municipale ou un autre type de plan à l'échelon local. Ces plans font la distinction entre au moins trois catégories d'occupation du sol : les infrastructures, l'urbain et les espaces ouverts. Le système australien de planification spatiale est renforcé par un système juridique de jurisprudences en constante évolution, hérité de l'Empire britannique. Avant la Seconde Guerre mondiale, la planification de l'usage du sol en Australie relevait généralement de la législation de l'État et des gouvernements locaux, et constituait en grande partie une sous-catégorie des politiques de santé publique et de sécurité. À partir de la fin des années 1940, les gouvernements des différents États australiens ont commencé à développer des cadres stratégiques d'aménagement plus complets qui ont permis aux gouvernements des États de planifier l'occupation du sol sur des centres urbains plus étendus tels que Sydney. Le premier fut la loi de planification urbaine et rurale (Town and Country Planning Act), promulguée en 1945, à partir de laquelle a été établi le schéma de planification du comté de Cumberland (County of Cumberland Planning Scheme, CCPS) pour la métropole de Sydney. Les gouvernements locaux dans la métropole de Sydney préparèrent ensuite des schémas de planification locale exhaustifs alignés sur les objectifs stratégiques plus larges du CCPS. Cette évolution survenue en Australie après la guerre renvoie à des changements similaires apparus au Royaume-Uni à la même période : le CCPS a introduit les zonages, les zones d'emplois dans les banlieues, la planification du réseau de transport et le concept de « ceinture verte » dont l'objectif était de constituer des zones tampons entre les espaces urbains et de banlieues et les zones rurales. Cependant, au cours des décennies suivantes, la ceinture verte fut grignotée afin de répondre aux fortes pressions démographiques. Dans les années 1970, tous les États et territoires australiens ont développé une législation en matière de planification selon laquelle chaque gouvernement local devait disposer d'un schéma d'occupation du sol. Beaucoup d'États avaient aussi développé des plans stratégiques d'aménagement pour leurs principaux centres urbains (par exemple Melbourne en 1954, Perth en 1955 et Adelaïde en 1967).

Durant cette période, les systèmes de planification opérant en Australie étaient principalement contrôlés par des professionnels de la planification prenant des décisions au 
nom des membres de la communauté sans qu'aucun processus participatif soit organisé. En 1974, le gouvernement de la Nouvelle-Galles du Sud a remplacé l'Autorité de planification étatique (State Planning Authority) par la Commission pour la planification et l'environnement (Planning and Environment Commission, PEC), dont la tâche était de rendre compte, au ministre de la Planification, de la révision du système de planification. Cette révision a abouti à la promulgation en 1979 de la loi sur la planification et l'évaluation environnementale de Nouvelle-Galles du Sud (NSW Environmental Planning and Assessment Act, 1979, EPAA) (figure 1.2). L'EPAA a remplacé la réglementation précédente régissant la planification. Cette nouvelle loi a été saluée comme étant à la pointe de la législation sur la planification quant à sa structure, l'utilisation d'une terminologie compréhensible, sa relative simplicité et ses dispositions pour l'engagement du public à chaque étape de la planification stratégique et du développement. L'EPAA a profondément modifié la planification en Nouvelle-Galles du Sud et ceci de plusieurs manières. Un objectif clé de l'EPAA était de créer une distinction claire entre les enjeux de planification locale et ceux relevant de l'État, et de permettre leur résolution à l'échelon adéquat. L'État avait désormais la responsabilité des questions importantes à son échelle ou à celle de la région, alors que les gouvernements locaux gagnaient en autonomie en matière de planification à travers la création des Plans environnementaux locaux (Local Environmental Plans, LEP). Le ministre pour la Planification de l'époque avait ainsi souligné cette évolution :

« Le gouvernement se rend compte que le département [ndlr : de la Planification] devrait principalement s'occuper d'initier et de développer des politiques et des plans pour des questions d'importance à l'échelle de l'État ou de la région. Notre attention doit porter prioritairement sur ces questions. La capacité du département à endosser cette lourde tâche suppose qu'il soit déchargé de ses responsabilités en ce qui concerne les décisions et les détails de la planification locale. La décision de déléguer la responsabilité de la planification locale aux gouvernements locaux se justifie par la longue expérience pratique qu'ils ont acquise en matière de planification et de gestion. Aussi, l'État a pris la décision que le Département de l'environnement et de la planification devrait consacrer toutes ses ressources à la planification à l'échelle de l'État et de la région. » (Haigh, 1979.)

Une autre caractéristique clé de l'EPAA était l'intégration de la planification de l'occupation du sol avec la gestion de l'environnement, et l'utilisation du concept de Développement écologiquement durable (Ecologically Sustainable Development, ESD) comme principe directeur pour la planification et la gestion de l'environnement. Comme Hazelton et Clements (2011, p. 76) l'ont observé :

« L'EPAA a obligé les porteurs de projets de développement et les autorités compétentes à évaluer et à prendre en considération l'impact des développements envisagés sur la flore et la faune natives. Du fait de l'EPAA, des zones importantes de patrimoine naturel, dont des zones humides côtières, des friches en zones urbaines et [...] des forêts tropicales, ont pu être identifiées dans la loi sur la planification et l'évaluation environnementale de la Nouvelle-Galles du Sud. »

Au cours des vingt-cinq dernières années, l'ESD a été considéré comme la finalité principale, naturelle et rationnelle des systèmes contemporains de planification, et comme un concept fédérateur fixant un objectif de planification à long terme, celui d'un système équilibré d'un point de vue économique, social et environnemental (Campbell, 1996). Un tribunal spécial - le tribunal foncier et environnemental (The Land and Environment Court) - a aussi été mis en place en 1979 avec une compétence spécifique en matière de planification. 
Du fait de ces évolutions, et en accord avec la loi sur les gouvernements locaux (Local Government Act, 1993), la responsabilité de la planification stratégique, régulée par l'EPAA, a été déléguée aux gouvernements locaux en Nouvelle-Galles du Sud, et traite principalement de l'environnement bâti et de l'utilisation des terres. Dans ce contexte, les gouvernements locaux doivent préparer et gérer des instruments stratégiques de planification tels que les LEP, qui sont des plans de zonage, et les Plans de contrôle du développement (Development Control Plans, DCP), ainsi que des mécanismes de contrôle de conformité pour évaluer les projets de développement à la lumière de ces plans. Ces documents sont élaborés en consultant les communautés locales et doivent être en accord avec des objectifs stratégiques plus larges — par exemple le Plan de développement de Nouvelle-Galles du Sud (NSW State Plan 2021) —, les normes en vigueur ${ }^{7}$ ainsi que d'autres politiques déterminées par l'État.

Influencé par l'émergence de la démocratie participative, la volonté d'aller vers des formes de planification à plus long terme et plus intégrées, et une préoccupation croissante pour la durabilité et l'équité sociale, le gouvernement de Nouvelle-Galles du Sud a révisé durant la seconde moitié des années 2000 son cadre réglementaire relatif à la planification par les gouvernements locaux en y incluant un Plan stratégique communautaire obligatoire (Community Strategic Plan) d'une durée minimale de dix ans. Ce nouveau plan fait partie du «cadre de planification intégrée et de reporting pour les gouvernements locaux de Nouvelle-Galles du Sud », qui a été conçu pour que les gouvernements locaux « soient comptables de leurs actions » vis-à-vis des communautés locales et d'acteurs majeurs comme l'État (Local Government Act 1993, ch. 13). Ce plan intègre aussi «des principes d'équité sociale, d'accès, de participation et de droits » (Local Government Act 1993, s. 402(3)(b)). Comme ces plans stratégiques communautaires doivent être préparés et promulgués par tous les gouvernements locaux de NouvelleGalles du Sud, ils constituent le plus haut niveau de planification élaboré par ces gouvernements (Local Government Act 1993, ch. 13). Leurs objectifs stratégiques contribueront à façonner les instruments locaux de planification de l'occupation du sol, comme par exemple les Plans environnementaux locaux.

Alors qu'un renforcement de la participation du public dans la planification spatiale a pu être observé, certains affirment qu'il y a aussi eu, ces dernières années, des signes montrant que l'administration centrale de Nouvelle-Galles du Sud cherchait à limiter ces changements. Lipman et Stokes (2008) ont par exemple mené une analyse approfondie en Nouvelle-Galles du Sud, qui a mis en évidence un retour à la centralisation de la prise de décision en matière d'occupation du sol comme cela existait avant l'EPAA. Depuis 2005, des amendements de l'EPAA ont considérablement amoindri la distinction entre la prise de décision au niveau local et celle au niveau de l'État/région. La partie 3A de l'EPAA, introduite par des réformes en 2005, exige l'accord du ministre de la Planification pour tout projet d'importance majeure. Des amendements ultérieurs ont renforcé le processus de recentralisation du pouvoir entre les mains du ministre et de comités nommés par le ministère, en matière de planification. Les amendements introduits en 2008 semblent avoir été créés pour accélérer les procédures d'élaboration des plans et d'autorisation d'utilisation des terres, limitant d'autant les possibilités de participation du public dans la planification afin de réduire le temps de prise de décision. En centralisant ainsi la prise de décision, l'engagement du public tel qu'il figure en objet de la loi EPAA, et qui était un objectif clairement affiché au moment où la loi a été introduite,

7. Le gouvernement de la Nouvelle-Galles du Sud a introduit un modèle standard pour l'élaboration des Plans environnementaux locaux (structure commune, terminologie standardisée, types de zones) pour assurer une meilleure cohérence entre ses gouvernements locaux. 
perd de sa force, avec comme conséquence une moindre chance que les intérêts environnementaux et autres soient pris en compte. Des éléments tangibles amènent à penser que le gouvernement de la Nouvelle-Galles du Sud se recentre de plus en plus sur l'efficacité administrative et économique. Dans les récentes réformes du gouvernement en matière de planification, la prise en compte des dimensions sociales et environnementales apparaît largement en retrait par rapport à la rentabilité économique. De plus, les résultats attendus de la planification semblent se focaliser sur les effets immédiats plutôt que sur les effets à moyen — ou long terme — qui résulteraient d'une accumulation de décisions incrémentales.

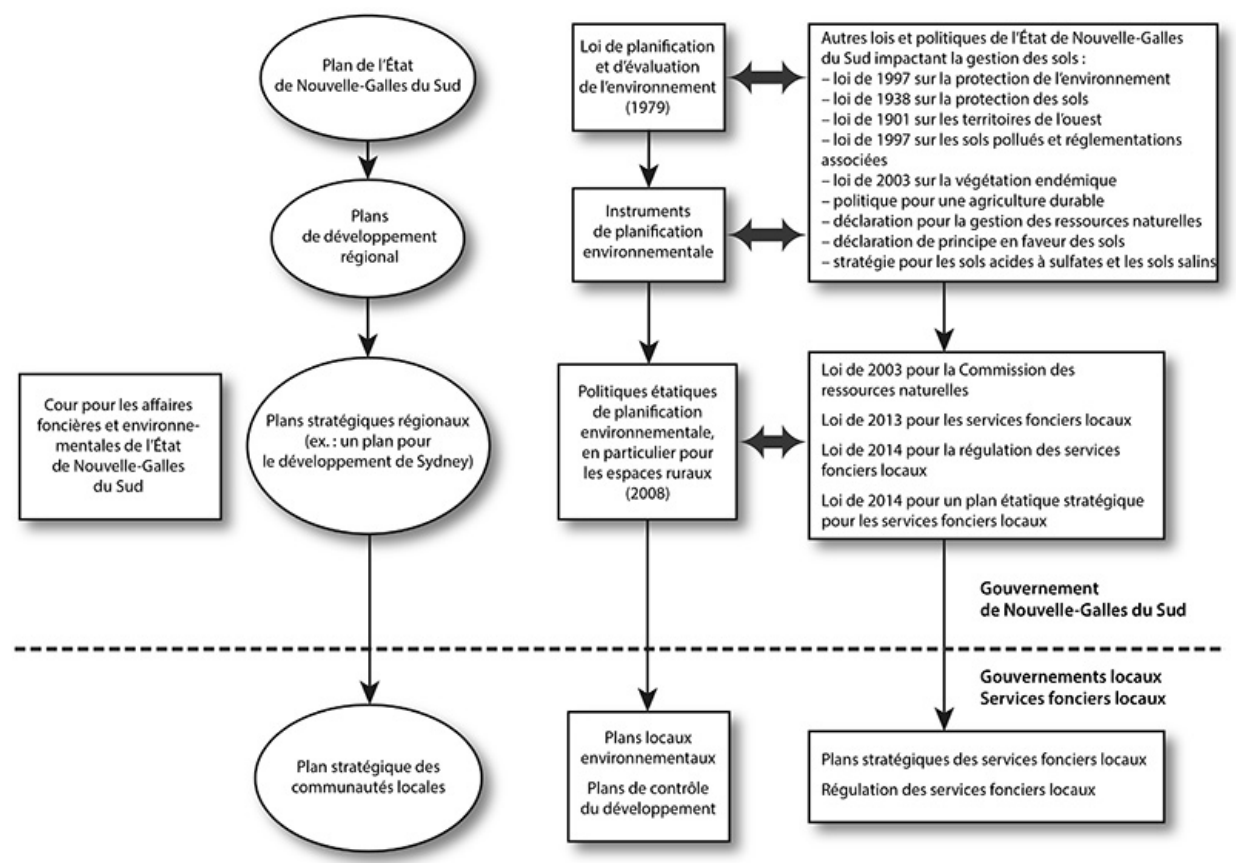

Figure 1.2. Structure du système de planification en Nouvelle-Galles du Sud montrant la législation, les politiques et les plans relatifs aux sols.

Alors que la culture de l'aménagement en Australie a traditionnellement été dominée par la planification de l'occupation du sol, où chaque État est doté de pouvoirs dans ce domaine, la planification spatiale française s'est construite quant à elle autour d'un code national de régulation de l'aménagement et d'une hiérarchie des plans. Les plus hauts niveaux se concentrent principalement sur l'expression de politiques de développement - économie, logements abordables, infrastructures. Aux niveaux les plus bas, l'accent est mis sur des plans plus spécifiques à une échelle plus détaillée et sur une approche de l'occupation du sol basée sur le zonage. Ceci est conforme au système légal français (napoléonien). La combinaison d'un contrôle centralisé et d'une réactivité aux pressions locales mène à l'établissement d'arrangements complexes. Au cours des dernières décennies, le mouvement de décentralisation et le renforcement de l'échelon régional ont modifié la culture de la planification en France, et l'aménagement prend désormais souvent place dans un éventail de coopérations verticales et horizontales. Les lois récentes Maptam de 2014 et NOTRe de 2015 offrent les moyens aux différentes couches du «mille-feuille territorial » français de faciliter le dialogue et la négociation 
pour arriver à une mise en cohérence des projets de développement et de leur contractualisation.

À partir des années 1990, les instruments de politiques publiques pour l'aménagement régional et urbain ont été de plus en plus influencés par le concept de développement durable. La Loi d'orientation pour l'aménagement et le développement durable du territoire (LOADDT), de 1999, a ainsi appelé au développement équilibré de l'intégralité du territoire national français, en combinant progrès social, efficacité économique et protection de l'environnement (figure 1.3). Cet objectif de développement durable a ensuite été renforcé par la Stratégie nationale de développement durable (SNDD) adoptée en juin 2003. Les instruments de planification spatiale ont alors évolué pour passer d'une logique de zonage réglementaire ou d'orientation générale non prescriptive, à une approche beaucoup plus stratégique et intégrée. La loi relative à la solidarité et au renouvellement urbains (SRU) de 2000 a ainsi introduit à l'échelon intercommunal le SCoT (en remplacement des Schémas directeurs d'aménagement) et, au niveau municipal, le PLU (en remplacement des Plans d'occupation du sol, POS). Ces deux documents doivent inclure un Projet d'aménagement et de développement durable (PADD). Le PLU inclut également des cartes de zonage réglementaires qui doivent être compatibles avec le SCoT (la « compatibilité » signifie que dans la hiérarchie des normes, une norme de rang inférieur n'empêche pas l'application d'une norme de rang supérieur) (figure 1.3). Afin de poursuivre le renforcement de la cohérence territoriale à l'échelle intercommunale, la loi Grenelle (2010) a tout d'abord donné aux municipalités volontaires la possibilité de mettre en place un PLUi. Puis la loi pour l'accès au logement et un urbanisme rénové (loi Alur) de 2014 a imposé à partir de mars 2017, sous certaines conditions, le transfert des compétences légales des communes aux EPCI pour élaborer un PLUi. En 2015, la loi NOTRe a renforcé le pouvoir des régions en matière de planification spatiale en leur confiant l'élaboration d'un Sraddet qui fixe de grandes orientations d'aménagement à moyen et long terme. Hiérarchiquement, il devient juridiquement opposable aux SCoT, PLU, PLUi, pour certaines de ses composantes, une fois qu'il a été approuvé par le préfet de région. Des documents locaux doivent donc prendre en compte les grandes orientations du Sraddet. La loi prévoit la possibilité pour les EPCI ou les PETR d'affiner conjointement avec la région la mise en œuvre du Sraddet sur leur territoire.

Un SCoT est un document de planification stratégique sur quinze à vingt ans utilisé au niveau intercommunal (un ou plusieurs EPCI) pour mettre en cohérence, à l'échelle locale, les politiques sectorielles ayant trait à l'urbanisme, au logement, à la mixité sociale, aux transports et aux équipements commerciaux. Les documents clés des politiques sectorielles doivent être compatibles avec le SCoT. Cela concerne le plan logement pour une conurbation (Plan local d'habitat, PLH), la Charte d'urbanisme commercial et le Plan de déplacement urbain (PDU) (figure 1.3). La loi Grenelle de 2010 puis la loi Alur de 2014 ont renforcé le pouvoir du SCoT en imposant aux PLU et aux PLUi des objectifs de densité urbaine et de consommation économe de l'espace. Les potentiels de densification et de mutation au sein des espaces urbains doivent être étudiés et complétés par des objectifs chiffrés par secteurs, argumentés sur la base d'enjeux. La loi Alur impose aussi d'inclure dans le rapport de présentation du PLU une analyse de la consommation d'espaces naturels, agricoles ou forestiers sur les dix dernières années. Elle permet le reclassement en zones naturelles d'anciennes zones à urbaniser. Les volets réglementaires des SCoT et des PLU doivent maintenir l'équilibre entre les différents types d'occupation du sol (zones bâties, de forêts, agricoles et naturelles) et préserver ou restaurer les corridors écologiques. 
Contrairement aux instruments précédents, le Sraddet, le SCoT et le PLU sont bien plus que de simples plans de zonage relevant du code de l'urbanisme. Ils doivent être basés sur des projets stratégiques de développement durable à moyen terme, dans le but de trouver un équilibre entre développement et conservation, et ils doivent aussi mettre en place un processus de participation du public. Ils peuvent ainsi porter de véritables visions politiques incarnées dans des projets de territoire et dotées d'une légitimité démocratique très forte dans le cas des communes et des régions, du fait des élections des conseils au suffrage universel direct. Ce n'est par contre pas encore le cas des représentants des EPCI, et encore moins des élus d'un syndicat mixte qui porterait le SCoT ou le PLUi de plusieurs intercommunalités. Ceci affaiblit leur légitimité politique et leur capacité à construire et à défendre des visions stratégiques intercommunales affranchies des petits arrangements avec les élus communaux. Au $1^{\mathrm{er}}$ janvier 2018, il y avait 469 SCoT (dont 316 déjà approuvés) représentant $80 \%$ des communes, $93 \%$ de la population française et près de $70 \%$ du territoire national. Ces chiffres reflètent un réel dynamisme, depuis 2000, de la planification spatiale stratégique locale en France.

Malgré le processus de décentralisation, le gouvernement national joue toujours un rôle majeur en s'assurant que les plans locaux respectent les exigences des politiques sectorielles ou des stratégies spatiales définies à des échelles de gouvernement plus élevées. Le rôle de l'administration centrale est ainsi passé d'une approche redistributive à un modèle endogène qui encourage les acteurs des territoires locaux à coconstruire des visions stratégiques de leur développement, en se reposant sur leurs caractéristiques et leur capital territorial. Cela a dynamisé les interactions orchestrées par l'administration centrale en impliquant les autorités régionales et locales. Ces interactions sont formalisées dans des «contrats » de territoire qui définissent les objectifs, les actions et les modalités de financement de leur stratégie (par exemple les CPER entre l'État et les régions ou les Contrats d'agglomération entre l'État, les régions et les EPCI). Ces contrats sont signés pour des périodes de six ans en adéquation avec les programmes des politiques régionales européennes, permettant ainsi des synergies financières avec les fonds structurels de l'UE. Les CPER comprennent une dimension infrarégionale qui permet par exemple de cofinancer un Contrat d'agglomération pour une conurbation de plus de 50000 habitants (figure 1.3).

Le gouvernement central a aussi établi des politiques spécifiques pour les zones soumises à de fortes pressions (loi Littoral de 1986, loi Montagne de 1985).

Même s'il existe de toute évidence des différences significatives dans la manière dont la France et l'Australie ont développé leurs instruments et leurs pratiques de planification spatiale, les deux pays ont néanmoins connu, sur certains aspects, des évolutions similaires. Premièrement, la planification spatiale dans ces deux pays a, au cours des dernières décennies, donné une voix à l'environnement dans un processus jusque-là dominé par l'anthropocentrisme, comme l'indique Joyce en référence au développement de l'EPAA :

« L'EPAA était l'une des premières lois en Nouvelle-Galles du Sud à traiter directement de questions environnementales et à reconnaître qu'un rôle crucial devrait être joué par le public pour faire appliquer la législation sur la protection de l'environnement [en lien avec la planification de l'occupation du sol]. Le fait que l'EPAA offre de plus en plus d'occasions d'impliquer le public dans la planification et l'évaluation environnementale est crucial pour la protection de l'environnement. Même si l'environnement est un sujet d'intérêt général, il reste en effet vulnérable à l'exploitation car les individus tirent plus de profit de son exploitation que de sa protection. » (Joyce, 1998.) 


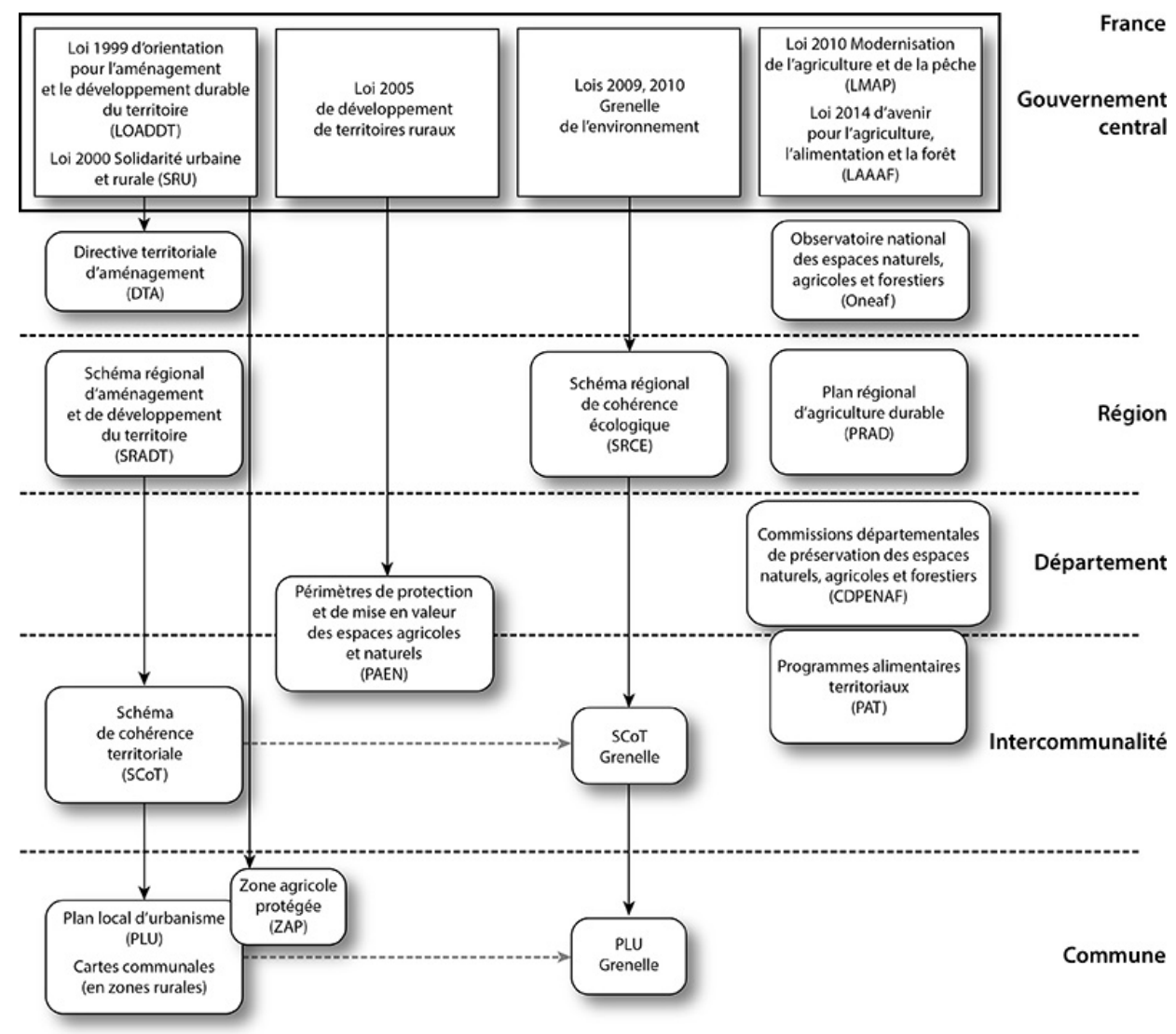

Figure 1.3. Structuration des instruments de planification utilisés en France.

Par ailleurs, à travers des approches quelque peu différentes, les systèmes de planification spatiale en France et en Australie ont cherché à renforcer l'autonomie des processus de planification locale, tout en s'assurant que le gouvernement central garde le contrôle sur des problématiques de planification d'importance nationale ou, en Australie, d'importance à l'échelle d'un État. Enfin, les deux systèmes de planification spatiale ont cherché à accroître le degré de participation des acteurs, dont le grand public, dans l'exercice de planification et dans la gestion.

\section{Les approches de planification spatiale face à l'imperméabilisation des terres agricoles liée à la périurbanisation}

\section{Législation, politiques et instruments de planification pour la régulation de l'imperméabilisation des sols en Nouvelle-Galles du Sud et en France}

Les gouvernements australiens et français disposent d'une grande variété de lois, de politiques et d'instruments de planification ayant trait à la gestion des sols. Au cours des dernières décennies, un intérêt pour le « développement accru d'approches stratégiques pour la gestion des sols » a pu être observé, avec pour objectif de lutter contre le prélè- 
vement des terres et des sols agricoles pour d'autres usages, du fait de la périurbanisation (National Committee on Soil and Terrain, 2009, p. 14). Les gouvernements des deux pays se sont de plus en plus mis à considérer la planification spatiale comme un cadre institutionnel pour « contraindre et décourager la gestion non durable des ressources en sols $[\ldots]$ en identifiant notamment $[. .$.$] des atouts tels que des terres de "haute qualité$ agricole" » (Department of Primary Industry, 2011), malgré la dimension relative de cette qualité. Il est important de noter que la récente prise de conscience des enjeux de l'artificialisation des sols en France (voir notamment l'expertise scientifique collective récente de Béchet et al., 2017), et dans une moindre mesure en Australie, a été renforcée par divers facteurs, dont: les récentes crises sanitaires dans l'UE qui ont contribué à mettre à l'agenda la question de la sécurité alimentaire en France (Tóth, 2012); le désir croissant de la population de consommer des aliments locaux dans les deux pays ; une meilleure compréhension de la contribution des zones agricoles à la préservation des habitats naturels et de la biodiversité dans les deux pays ; la compétition croissante pour les terres provoquée par une demande accrue en énergies renouvelables et en biocarburants dans les deux pays ; et les efforts de diverses organisations syndicales et professionnelles en France pour sensibiliser les élus, les parlementaires et les médias à l'importance de préserver les terres agricoles (comme par exemple la campagne de communication « Manger ou construire, il faut choisir!", réalisée par un syndicat agricole en 2011). Durant les années 1980, la recherche a principalement attribué, à tort, le déclin des sols agricoles à la déprise agricole qui mènerait à l'extension graduelle des friches et des forêts (Fottorino, 1990). Mais durant les quinze dernières années, des données et des statistiques plus fiables (même si les résultats varient selon les sources des données) ainsi que de nouvelles méthodologies cartographiques ont permis de mieux comprendre les liens entre l'étalement urbain, la qualité des sols et l'artificialisation des terres agricoles dans les zones périurbaines - voir, par exemple, le Centre européen de données sur le sol, et en France, le Groupement d'intérêt scientifique (GIS) sur les sols avec son premier rapport sur la situation des sols après dix ans de travaux intensifs (GIS Sol, 2011). Des compilations de méthodes pour mesurer la consommation des terres par l'artificialisation ont aussi été produites (Certu, 2010 ; Béchet et al., 2017, p. 27-56 ; ministère de l'Environnement et Virely, 2017). Ces aspects seront largement développés dans les chapitres suivants de cet ouvrage.

Selon la Constitution australienne, la responsabilité de la gestion de la ressource en sol revient principalement aux États, bien que le gouvernement fédéral ait cherché à jouer un rôle croissant dans ce domaine, en particulier dans les cas où les responsabilités ne sont pas clairement définies dans la Constitution australienne (par exemple en matière de séquestration du carbone). L'Australie dispose d'un large éventail de stratégies, de politiques, de cadres, de lois, d'initiatives et de programmes ayant trait à la gestion des sols, à l'échelle nationale, des États ou des territoires (Campbell, 2008 ; Hazelton et Clement, 2011). En Nouvelle-Galles du Sud, deux courants de législations et de politiques ayant à voir avec l'imperméabilisation des sols peuvent être identifiés : le premier est lié au système de planification de l'EPAA (présenté dans la section précédente) et le second à diverses lois pour la gestion des ressources naturelles, dont celles sur la Commission des ressources naturelles, 2003 (Natural Resources Commission Act 2003), la Conservation des Sols, 1938 (Soil Conservation Act 1938), la Gestion des terres contaminées, 1997 (Contaminated Land Management Act 1997), la Végétation primaire (Native Vegetation Act 2003 (NSW), et la Protection de l'environnement, 1997 (Protection of the Environment Act 1997) (figure 1.2). L'efficacité dans la mise en œuvre d'une grande partie de ces lois et politiques a été considérablement mise en doute et critiquée (Bradsen, 1988). Dans le contexte australien de ces dernières décennies, les sols ont souvent été perçus comme 
le « parent pauvre parmi les questions de [gestion des ressources naturelles]. [Contrairement à l'eau par exemple], ils ne profitent pas du parrainage de lobbies puissants et ils stimulent rarement l'imagination du public » (Campbell, 2008, p. 13). Néanmoins, une attention plus grande est maintenant portée aux implications de la gestion des sols, en raison de l'émergence de certaines questions liées à la séquestration du carbone, à la biodiversité des sols, à leur dégradation et à l'adaptation de l'agriculture au changement climatique.

Tout au long du $\mathrm{xx}^{\mathrm{e}}$ siècle, la Nouvelle-Galles du Sud, comme d'autres États en Australie, a développé des agences pour la préservation des sols matures ; ces dernières s'occupaient principalement des zones rurales, des organismes agricoles et des communautés agricoles. L'accent était principalement mis sur le contrôle de l'érosion des sols et, dans une moindre mesure, sur la dégradation de leur structure, la salinité et l'acidification, en utilisant une combinaison d'outils issus de l'agronomie et du génie civil afin de contrôler les mouvements des eaux de surface. Durant les dix dernières années, un changement d'orientation a vu le jour avec des politiques et des lois de plus en plus appliquées, dans les États, à tous les types d'espaces, que ce soit dans les zones urbaines, périurbaines ou rurales. Un facteur pouvant expliquer ce changement est la prise de conscience croissante de l'artificialisation ${ }^{8}$ de sols à haute valeur agricole au profit d'usages du sol « urbains » (zones résidentielles, commerciales...) dans ou autour de régions densément peuplées, soumises à une demande élevée pour d'autres usages comme l'habitat résidentiel ou l'industrie (voir Hulme et al., 2002, pour une discussion sur la perte des terres agricoles en Nouvelle-Galles du Sud). En Australie, des termes comme «étalement urbain » et " conservation de la biodiversité » sont de plus en plus utilisés dans les médias traditionnels et le débat public. Cela a contribué à sensibiliser plus fortement la population sur l'artificialisation des meilleurs sols agricoles dans les zones périurbaines, du fait du développement de la ville (Local Government and Shire Associations of NSW, 2009).

Les chercheurs de l'Organisation du Commonwealth pour la recherche scientifique et industrielle (Commonwealth Scientific and Industrial Research Organisation - CSIRO) ont observé que la périurbanisation ou l'expansion des zones urbaines pour loger une population croissante, incluant les besoins en nouvelles infrastructures, se produisait dans beaucoup de zones rurales australiennes, particulièrement les zones rurales littorales (CSIRO, 2008). Le gouvernement de Nouvelle-Galles du Sud a reconnu cette tension dans son dernier rapport sur l'état des sols (NSW-DPI, 2013, p. 2). Il cherche à adopter une approche plus globale dans sa gestion de ces derniers, et à les protéger et les améliorer en traitant la question des " conflits d'usage des terres et leurs impacts potentiels sur les sols» (figure 1.2). Depuis 2013, par exemple, une approche visant à conserver les terres agricoles de bonne qualité, dites « stratégiques » (Biophysical Strategic Agricultural Land, BSAL), est entrée en vigueur.

Pour traiter cet équilibre entre usages du sol concurrents, le système de planification de Nouvelle-Galles du Sud a mis en place, en 2008, une Politique de planification environnementale à l'échelle de l'État (State Environmental Planning Policy - Rural Land -, SEPP Rural). Cette politique prévoit des mesures pour aider à la bonne gestion, au développement et à la protection des terres rurales en limitant l'artificialisation des terres agricoles par des activités comme le développement résidentiel, ceci afin de promouvoir le bien-être social, économique et environnemental en Nouvelle-Galles du Sud (figure 1.2). Cette politique vient compléter des politiques plus anciennes comme celle pour l'agri-

8. Bien que les termes d'artificialisation ou de consommation des sols ne soient jamais utilisés explicitement dans les lois et politiques de la Nouvelle-Galles du Sud. 
culture durable en Nouvelle-Galles du Sud de 1998 (Policy for Sustainable Agriculture in NSW), qui intègre de la même manière des principes et objectifs du Développement écologiquement durable. Une disposition prévoit que le gouvernement identifie les terres agricoles présentant un enjeu à l'échelle de l'État pour garantir la viabilité à long terme de l'agriculture, et que le SEPP Rural soit appliqué dans les plans stratégiques régionaux. Une série de directives de l'État dans le domaine de l'aménagement a délégué aux gouvernements locaux le pouvoir d'appliquer eux aussi le SEPP Rural quand ils développent et mettent en œuvre des stratégies et des Plans environnementaux locaux (voir l'EPAA 1979, toutes les directions de planification se trouvent dans la section 117). Ces problématiques ont déjà été reconnues dans les plans stratégiques régionaux de NouvelleGalles du Sud, dont la région métropolitaine de Sydney (Un plan pour la croissance de Sydney) qui cherche à « protéger les terres agricoles pour maintenir la disponibilité d'aliments frais [et] minimiser les conflits » entre usages ruraux et urbains (Action 4.1.2, p. 98-99).

Parallèlement au développement d'outils de planification spatiale portant sur la gestion des sols agricoles dans les régions périurbaines, des lois et des politiques pour la gestion des ressources naturelles ont été instaurées (voir par exemple la loi sur la commission des ressources naturelles, Natural Resources Commission Act 2003, et celle sur les services fonciers locaux, Local Land Services Act 2013, LLS). Elles ont mené à la création de 11 services fonciers locaux qui couvrent l'ensemble de la Nouvelle-Galles du Sud (figure 1.2). Ces services permettent de traiter en même temps de la production agricole, de la biosécurité, de la gestion de crises et de la gestion des ressources naturelles qui inclut la gestion des sols et de l'eau. L'un des objectifs des LLS porte sur « la conservation des sols » (voir l'article 2(a)(i) de la loi sur les Services locaux de gestion des terres). Ce modèle des LLS cherche à rendre la gestion de l'environnement plus transparente et plus réactive aux besoins régionaux, à travers le développement d'une planification stratégique locale fondée sur une approche plus holistique de l'environnement. Ce changement reflète des tendances globales dans les pratiques de planification où il est question de prendre en compte, sur un pied d'égalité, les questions de gestion des ressources naturelles et de développement économique (Newman et Jennings, 2008). Certains ont fait valoir que cette connexion devrait être renforcée au moyen d'une réglementation (Robinson, 2009).

Le SEPP Rural et d'autres lois et politiques sur les sols constituent autant de moyens de prendre en compte la question de l'artificialisation des terres agricoles clés dans les processus de périurbanisation et permettent d'intégrer ces enjeux dans les plans d'occupation du sol à l'échelle de l'État, de la région ou au niveau local. Mais il est important de comprendre que ce n'est qu'un aspect d'un système de planification complexe qui comporte de multiples objectifs, alternatives, intérêts sociaux et préférences (Bantayan et Bishop, 1998 ; Duke et Aull-Hyde, 2002).

En France, bien que la préservation des terres agricoles ait été prise en compte indirectement dans diverses politiques publiques depuis des décennies, ce n'est qu'au début des années 2000 que de réels efforts ont été faits pour gérer les impacts de l'étalement urbain sur les terres agricoles à travers des politiques publiques et la planification spatiale.

Cette série de nouvelles approches a été confortée par l'engagement croissant de l'UE face au problème de l'artificialisation et de la perte de terres agricoles. Améliorer la planification spatiale est considéré comme l'une des meilleures solutions pour empêcher la progression de l'artificialisation des sols. De plus, en 2011, la Commission a développé une feuille de route pour l'Efficacité énergétique de l'Europe qui proposait que d'ici 2020, les politiques européennes prennent en compte leurs impacts sur l'occupation 
du sol afin d'arriver à une consommation d'espaces agricoles nulle d'ici 2050. L'UE a aussi publié un rapport sur les tendances de consommation des terres et d'artificialisation des sols dans l'UE et recommande l'adoption d'une séquence en trois étapes basée sur le principe « éviter, réduire, compenser » (Prokop et al., 2011).

En France, ces nouvelles orientations figurent dans les lois d'orientation agricole (LOA) de 1999 et de 2016 ainsi que dans la loi relative au développement des territoires ruraux (2005), suivies ensuite par les lois Grenelle $(2009,2010)$ et la loi d'avenir pour l'agriculture, l'alimentation et la forêt (LAAAF) de 2014. La LOA de 1999 avait déjà créé un instrument de zonage à l'échelle locale, la Zone agricole protégée (ZAP), qui est une servitude d'utilité publique prise par arrêté préfectoral à la demande des communes pour protéger des terres agricoles dont la préservation est d'intérêt général en raison de la qualité des productions ou de sa situation géographique. Tout changement d'occupation du sol qui altère durablement le potentiel agronomique, biologique ou économique doit être soumis à l'avis de la chambre d'agriculture et de la Commission départementale d'orientation agricole. En cas d'avis défavorable de l'une d'entre elles, le changement ne peut être autorisé que sur décision motivée du préfet. La LOA de 2016 a, quant à elle, instauré le « bail rural à clauses environnementales », dit « bail environnemental » entre un propriétaire foncier, qui peut être une collectivité publique, et un agriculteur, ceci afin d'encourager des pratiques culturales plus durables pour la protection de milieux, d'espèces et de ressources naturelles sur des parcelles situées dans des zones déjà soumises à une réglementation environnementale. La transformation des outils de planification à l'échelle communale (passage du POS au PLU) par la loi SRU (2000) a modifié les catégories de zonage de l'occupation du sol en supprimant celle correspondant aux «zones d'habitat diffus » afin de limiter l'étalement et d'encourager le renouvellement et la densification urbaine. La loi relative au développement des territoires ruraux (2005) a constitué une réponse aux profonds changements dans les zones rurales de plus en plus composites, et visait en partie la question de la préservation des terres agricoles face à l'urbanisation. Les articles 73 et 74 permettent aux départements de créer, en accord avec les communes concernées et sur conseil de la chambre d'agriculture, des Périmètres de protection et de mise en valeur des espaces agricoles et naturels périurbains (PAEN), périmètres qui doivent être compatibles avec les SCoT, les PLU et ne pas inclure de parcelles classées en zone urbaine ou à urbaniser dans les PLU ou situées dans une ZAP. Cet instrument de planification locale comprend des outils de gestion foncière pour permettre l'achat de terres par différentes structures : les départements, les municipalités, les Sociétés d'aménagement foncier et d'établissement rural (Safer) ainsi que les Établissements publics fonciers (EPF). Un PAEN inclut aussi un plan d'action qui détermine les règles en termes d'équipement et de gestion à l'intérieur du périmètre de protection (par exemple, renforcer la vocation maraîchère et vinicole d'une zone). Mais leur application reste toutefois anecdotique, comme dans le cas des ZAP créées par la LOA de 1999 (selon le ministère de l'Agriculture, 42 ZAP couvrant moins de 30000 ha étaient dénombrées en 2014). Une première étude sur les ZAP a montré que les quelques projets qui avaient été mis en œuvre ont surtout visé à maintenir une activité agricole élitiste, non généralisable, et à produire des paysages emblématiques (Vianey, 2012). Mais il serait nécessaire de disposer de plus de recul et de mener des études comparatives systématiques sur les PAEN et les ZAP mis en place pour pouvoir vraiment juger de leur efficacité. Le fait que la LAAAF de 2014 ait ignoré ces deux instruments au lieu de simplifier les textes ne va malheureusement pas contribuer à leur adoption par les territoires locaux. 
Les lois Grenelle $(2009,2010)$ ainsi que la Conférence environnementale tenue en 2012 ont abordé, quant à elles, indirectement les problèmes d'artificialisation des zones agricoles et boisées sous l'angle de la préservation de la biodiversité. Même s'il est nécessaire de développer des pratiques agricoles plus respectueuses de la biodiversité dans certaines zones, il est maintenant largement reconnu que maintenir de l'agriculture est un moyen de limiter l'imperméabilisation des sols et la fragmentation des espaces, ce qui a des bénéfices directs et indirects sur la production et la protection de la biodiversité. Les lois Grenelle ont abouti à une " écologisation » des politiques de planification urbaine aux échelles communales et intercommunales. Désormais, les outils de planification stratégique et de zonage de l'occupation du sol, comme la seconde génération de SCoT et de PLU connue sous les noms de « SCoT Grenelle » et « PLU Grenelle », doivent établir des objectifs quantitatifs et justifier la manière dont ces objectifs permettront une extension urbaine parcimonieuse. Des seuils minimaux de densité du bâti dans les zones urbanisables doivent être définis. Les efforts doivent porter en priorité sur la densification et la requalification des espaces déjà urbanisés. Les collectivités peuvent aussi imposer aux constructeurs de payer un versement pour sous-densité quand le seuil minimal de densité fixé pour un secteur donné n'est pas atteint. Mais ce type d'outil suppose une ingénierie technique peu accessible aux communes rurales et petites intercommunalités. Enfin, les lois Grenelle visent à la préservation ou à la restauration de la connectivité des habitats naturels (Trame verte et bleue, TVB) définie, à l'échelle régionale, au moyen du Schéma régional de cohérence écologique (SRCE) et, au niveau local, au moyen des SCoT et des PLU, via la prise en compte d'éléments naturels ou semi-naturels (prairies permanentes, haies, bois, arbres isolés, jachères et bandes enherbées). Les terres agricoles périurbaines peuvent ainsi être indirectement protégées du fait de leur contribution possible aux continuités écologiques.

La loi de modernisation de l'agriculture et de la pêche (2010) puis la loi d'avenir pour l'agriculture, l'alimentation et la forêt (2014) ont renforcé cette orientation en apportant de nouveaux outils, à différentes échelles, axés plus directement sur la consommation de terres agricoles et dans certains cas étendus aux espaces naturels et forestiers, avec comme objectif de réduire de moitié leur taux d'artificialisation d'ici 2020. Il s'agit :

- du Plan régional d'agriculture durable (PRAD) dans chaque région, qui détermine les tendances stratégiques régionales des politiques nationales dans le champ de l'agriculture durable, ainsi que des moyens de les accomplir. La LOA de 2010 avait confié la réalisation de ce plan au préfet de région en partenariat avec les acteurs du secteur agricole et de l'agroalimentaire. La LAAAF de 2014 a instauré une coresponsabilité de l'État et de la région, donnant la liberté aux régions de définir elles-mêmes leurs stratégies et priorités en matière d'agriculture durable, en échange de leurs contributions budgétaires. Par contre, le PRAD ne fait pas partie des plans régionaux intégrés dans le Sraddet ;

- de l'Observatoire national de la consommation des espaces agricoles (Oncea), qui a été créé pour mesurer, de manière fiable et à l'aide d'indicateurs de tendance standardisés, les changements d'occupation du sol dans les zones agricoles. Il a été étendu aux espaces forestiers et naturels par la LAAAF en 2014, pour devenir, en 2016, l'Observatoire des espaces naturels, agricoles et forestiers (Oenaf);

- des Commissions départementales de consommation des terres agricoles (CDCEA) mises en place dans chaque département français. Elles sont composées de représentants de l'État, des autorités locales (élus) et de la chambre d'agriculture. Elles se rencontrent régulièrement pour examiner chaque projet de développement (infrastructure urbaine ou de transport) qui se fera sur des terres agricoles et donnent un avis consultatif sur les problématiques agricoles sur le territoire concerné. La LAAAF de 2014 a étendu ces commissions aux espaces forestiers et naturels. Elles sont devenues les Commissions 
départementales de préservation des espaces naturels, agricoles et forestiers (CDPENAF) ;

- d'une nouvelle taxe créée à partir de la vente de terrains vacants reclassifiés dans le cadre de PLU en terrains constructibles. Le revenu de cette taxe est rattaché à un fonds pour l'installation de jeunes agriculteurs et le développement de projets agricoles périurbains innovants.

La LAAAF de 2014 a aussi transposé au monde agricole le principe de la compensation environnementale (séquence éviter, réduire, compenser), principe décrié par le monde agricole car la compensation se fait la plupart du temps au détriment de terres agricoles (Leras, 2016) et se heurte à des difficultés de mise en œuvre opérationnelle des mesures compensatoires par les agriculteurs (Roussel et al., 2018). L'objectif de la LAAAF est d'arriver à compenser de manière égale la perte de terres agricoles et les conséquences économiques qui en découlent dans les projets d'aménagement de grande ampleur. Enfin, la LAAAF s'est inscrite dans le mouvement général d'écologisation des politiques publiques en France en encourageant de nouveaux systèmes de production agricoles basés sur l'agroécologie et insérés dans une vision plus intégrée du développement territorial. Elle a introduit pour cela des instruments opérationnels destinés à mieux structurer l'économie agricole au sein des territoires locaux au moyen de circuits courts qui resserrent les liens entre production, transformation et consommation : il s'agit des Programmes alimentaires territoriaux (PAT), alignés sur les stratégies régionales établies dans les PRAD, et de la possibilité de créer des Groupements d'intérêt économique et environnemental (GIEE).

Malgré la multiplication de ces instruments réglementaires et opérationnels en France, force est de constater que dans la lutte contre l'artificialisation des terres agricoles périurbaines, la protection des sols, pris au sens agronomique du terme, occupe une place marginale. Les sols ne bénéficient en effet pas d'une protection juridique en tant que milieu naturel (Desrousseaux, 2014 ; Billet, 2017). Relevant du droit de la propriété privée, ils sont encore trop souvent perçus comme de simples supports au développement et non comme des ressources finies et des biens communs à protéger, au même titre que l'eau ou la biodiversité. Cette difficulté se retrouve au niveau européen. Malgré les efforts menés depuis une quinzaine d'années par l'Agence européenne pour l'environnement et le réseau du Bureau européen des sols (European Soil Bureau Network, ESBN) ${ }^{9}$ (Prokop et al., 2011 ; Tóth et Németh, 2011) pour faire aboutir une proposition de Directive-cadre sur les sols, celle-ci a été retirée en 2014 car, face au refus de certains États, elle ne revêtait plus un caractère d'actualité.

En France, les sols agricoles sont pour le moment pris en compte de manière indirecte dans différents codes juridiques (rural, urbanisme, environnement, santé). Il manque une cohérence d'ensemble, à travers une vision utilitariste et sélective de certains services écosystémiques qu'ils rendent à l'homme, quand par exemple leur dégradation a des impacts négatifs sur la qualité de l'environnement (ex. : périmètre de protection des captages d'eau potable prenant en compte les fonctions de filtration de l'eau par les sols), sur la production agricole ou sur la santé humaine (cas des sols pollués). Quelques avancées juridiques ont toutefois été obtenues ces dernières années. La loi Alur de 2014 intègre ainsi la préservation du potentiel agronomique des sols comme l'un des objectifs à prendre en compte dans les projets de développement durable des SCoT et des PLU et dans leur traduction juridique (cas par exemple des espaces classés en Agricole, A, dans les PLU). De son côté, la LAAAF de 2014 a fait évoluer la ZAP créée par la LOA de 1999 en rajoutant une servitude d'utilité publique qui s'impose au PLU et qui intègre un

9. L'Agence européenne pour l'environnement fédère les centres de recherche en sciences du sol. 
nouveau critère de qualité agronomique pour motiver la délimitation de la ZAP. Cette servitude est prise par arrêté préfectoral, précédée de l'avis des conseils municipaux des communes concernées. Enfin, la loi Biodiversité du 8 août 2016 a reconnu quant à elle que les sols concouraient à la constitution du patrimoine commun de la nation.

En termes d'ingénierie, d'innovation et d'apprentissage collectif, des groupes de travail hybrides combinant des expertises urbaines et rurales ont aussi émergé ces quinze dernières années. C'est par exemple le cas du Groupe de travail urbanisme et agriculture (GTUA) dans le département de l'Hérault, créé en 2002 pour réduire les tensions entre les agriculteurs et l'administration concernant les permis de construire. Composé de représentants de l'État, de la profession agricole et des autorités locales, le groupe a conduit de nombreuses expérimentations et analyses pour faciliter la prise de décision en matière d'agriculture périurbaine. Le résultat le plus remarquable est probablement le processus d'apprentissage social qui a découlé des interactions entre ces acteurs hétérogènes. En France, des groupes de travail de ce type se sont multipliés et ont été institutionnalisés dans tous les départements par le ministère de l'Agriculture, en 2008. La création plus récente par la LAAAF de 2010 des CDCEA, remplacées en 2016 par les CDPENAF pour intégrer également les espaces naturels et forestiers, influencera probablement les apprentissages collectifs dans les arènes locales dédiées aux arbitrages entre l'État, les collectivités et les acteurs économiques sur le devenir des terres périurbaines.

Un autre exemple d'innovation est le réseau « Terres en villes » (Villes, 2012), créé en 2000 par des élus, des autorités intercommunales et des représentants du monde agricole pour partager et développer des expériences en lien avec les politiques agricoles périurbaines. Cette association rassemble maintenant vingt-cinq aires urbaines majeures en France. Elle a collaboré avec des services de l'État pour coproduire un livre sur l'intégration de l'agriculture dans les SCoT (Villes, 2012). Un dernier exemple de mobilisation citoyenne est celui de l'association Terre de Liens, créée en 2003 et dont la fondation destinée à récolter des dons a été reconnue d'utilité publique en 2013. Cette structure s'est dotée d'un outil permettant d'émettre des actions pour recueillir de l'épargne citoyenne afin d'acquérir des exploitations destinées à des fermiers pratiquant une agriculture respectueuse de l'environnement au moyen de baux ruraux environnementaux.

\section{Interprétation locale et mise en œuvre d'instruments de planification pour la régulation de l'imperméabilisation des sols}

Toutes ces lois récentes et ces instruments de planification pour la régulation de l'étalement urbain et de la consommation des terres agricoles qui viennent d'être décrites montrent que la législation et le cadre politique national sont désormais bien établis en Australie et en France. Les politiques de planification spatiale dans les deux pays se veulent plus stratégiques et plus à même de contribuer aux objectifs du développement durable. La question est alors d'analyser la manière dont ces objectifs sont mobilisés à l'échelle locale dans les processus de prise de décision opérationnels pour la planification spatiale.

En Nouvelle-Galles du Sud, la législation sur les sols est ouverte à interprétation, ce qui signifie que leur mise en œuvre peut varier entre différentes juridictions locales et dépend souvent de la volonté des services de planification locale et des communautés de jouer sur place le rôle de porte-parole et de catalyseur (Jepson, 2004). Comme Gleeson et Low (2004) l'ont fait valoir, les défenseurs de la durabilité ou de l'environnementa- 
lisme cherchent généralement à réévaluer et à développer la planification en augmentant sa portée politique et en élargissant ses perspectives conceptuelles.

Des recherches récentes par Millar et Fyfe (2012) ont mis en lumière les diverses tensions qui sous-tendent la mise en œuvre de la législation et des politiques sur l'artificialisation des terres agricoles dans les zones périurbaines. Elles existent, selon eux, le long d'une « ligne de fracture entre ceux qui soutiennent le besoin de faire de l'agriculture et ceux qui soutiennent le droit de capitaliser sur les terres agricoles » (Millar et Fyfe, 2012, p. 1). Cette tension se retrouve à tous les niveaux du développement de la planification en Nouvelle-Galles du Sud, et par conséquent aussi au sein des communautés locales, en raison de leur implication croissante dans les processus d'aménagement (Farrier, 2007). Les aménageurs de Nouvelle-Galles du Sud que nous avons interviewés expliquaient que le droit des gouvernements de Nouvelle-Galles du Sud à limiter la consommation des terres agricoles périurbaines pour d'autres usages se heurtait souvent à leur propension à donner la priorité au développement économique (ce qui équivaut souvent à l'expansion pure et simple de l'habitat urbain et de zones commerciales) et à minimiser les coûts pour les promoteurs immobiliers.

En France, l'effort a été porté sur les échelles intercommunales et communales depuis que la décentralisation et les principes de gouvernance ont conforté leur position. Plusieurs études ont montré que les différents outils de planification décrits précédemment, censés favoriser le dialogue entre les politiques d'aménagement et les politiques agricoles aux échelles locales, restent encore peu utilisés. De plus, ils ne sont pas mobilisables partout à l'identique et doivent être adaptés selon la taille des collectivités territoriales et leurs capacités d'ingénierie, et, pour les communes, selon leur profil rural ou périurbain. La difficulté à les mettre en œuvre s'explique surtout par la complexité de gestion des systèmes fonciers locaux et des dilemmes auxquels sont confrontés les élus pour concilier les intérêts des propriétaires fonciers et les aspirations des nouveaux arrivants (Demazière et al., 2016 ; Melot et Bransiecq, 2016). Pour des raisons électorales, budgétaires et de proximité socio-spatiale entre les (inter)municipalités et certains acteurs locaux (propriétaires, promoteurs immobiliers), il est maintenant très difficile pour les élus de limiter l'artificialisation des sols cultivés (Serrano et Vianey, 2011), d'autant qu'une politique de développement de l'habitat et d'activités économiques dans des territoires ruraux soumis à la déprise agricole se justifie également. Enfin, les compromis auxquels sont confrontés les élus se jouent aussi entre des enjeux locaux, dominés par la priorité accordée au développement d'activités économiques et d'accueil de nouveaux habitants, sources de rentrées fiscales, et des enjeux globaux relevant de l'intérêt général (changement climatique, biodiversité, sécurité alimentaire...) portés par l'État et l'Europe. Le désengagement progressif de l'État dans l'ingénierie publique de proximité réduit d'autant ses capacités à aider les élus locaux à réguler l'artificialisation des terres au motif de ces enjeux globaux (Bombenger, 2011).

Une étude réalisée sur une ancienne communauté de communes située aujourd'hui dans la métropole de Tours a montré que les élus locaux responsables de la planification de l'occupation du sol privilégiaient encore le développement d'activités économiques et de l'habitat au détriment des terres agricoles (Serrano et Vianey, 2011). Une autre étude partiellement basée sur une approche qualitative (entretiens semi-directifs d'élus municipaux dans le sud-est de la France) a mis en évidence que la perception des zones non urbanisées différait selon le type de municipalité (Delattre et al., 2012).

Dans le cas des grandes collectivités, le renforcement récent du statut et des compétences des métropoles (loi Maptam de 2014) a permis à certaines d'entre elles de développer des stratégies novatrices pour prendre en compte l'agriculture périurbaine et sa compo- 
sante foncière dans le projet métropolitain. Grenoble a par exemple déployé une stratégie alimentaire de territoire (Vargas, 2016), Rennes a élaboré un SCoT dont le projet de développement s'appuie sur une agriculture multifonctionnelle (Demazière, 2016), Lille a créé une zone horticole et maraîchère et Nantes a abordé le traitement des friches agricoles (Schmitt et al., 2016).

En dépit des difficultés, il existe pourtant des facteurs favorables au maintien des terres agricoles périurbaines, comme par exemple : un niveau de rentabilité plutôt élevé pour les agriculteurs ; des dynamiques locales fortes (par exemple la présence de coopératives agricoles); des structures foncières et des équipements qui renforcent la viabilité des exploitations (remembrement des parcelles, habitation sur l'exploitation, réseau d'irrigation); le développement des systèmes alimentaires locaux (Perrin et Soulard, 2017) et le renouveau de l'agriculture en ville (Aubry et Consalès, 2014) ; ou encore une image positive de l'activité agricole constitutive d'une nouvelle identité territoriale (choix politique de promouvoir l'identité rurale, de développer des labels qualité pour des produits agricoles de terroir, d'acquérir une renommée touristique, d'être un village « biologique »).

Les solutions pour une meilleure préservation des terres agricoles périurbaines ne semblent plus passer par la création de nouvelles réglementations et de nouveaux instruments car ils sont déjà très (trop ?) nombreux, comme nous venons de le voir. Il s'agirait tout d'abord d'étudier comment rendre les dispositifs de gouvernance territoriale plus inclusifs vis-à-vis du monde agricole (Rey-Valette et al., 2011). Des études montrent que l'offre actuelle de participation correspond mal aux habitudes organisationnelles et sectorielles des agriculteurs (Houdart et al., 2012 ; Michel et al., 2018), même s'il existe des exemples réussis (Guiomar, 2014). La dimension foncière de la gouvernance territoriale est cruciale en France compte tenu de la multiplicité des acteurs et a déjà fait l'objet de nombreux travaux récents (Barthès et Bertrand, 2016 ; Gueringer et al., 2016 ; Perrin et al., 2016). Un autre aspect porte sur la capacité des territoires locaux à imaginer collectivement des visions de leur développement, sources d'identité territoriale, où les espaces agricoles ne seraient plus considérés comme des réserves foncières à l'urbanisation, mais comme le support d'une agriculture multifonctionnelle faisant partie intégrante du projet de développement (Perrin et al., 2016). Enfin, des données spécifiques peuvent être élaborées pour mieux caractériser spatialement l'agriculture périurbaine et aider les praticiens de l'aménagement à choisir les zones où intervenir préférentiellement. C'est le cas des cartes d'Unités agro-physionomiques (UAP) proposées par Deffontaines et Thinon (2008), utilisées pour le SCoT de Montpellier (Jarrige et al., 2006), et dont se sont inspirés récemment d'autres auteurs pour développer une méthodologie de caractérisation des Unités spatiales d'agriculture périurbaine (Usapu) (Sanz Sanz et al., 2017).

\section{Discussion et conclusion}

Ce chapitre a présenté en détail la planification spatiale en France et en Australie et a identifié leurs points communs et leurs divergences. À travers cette analyse comparative, nous avons identifié des mutations communes de la planification spatiale dans ces deux pays, en particulier : l'intégration de la planification de l'occupation du sol et de la gestion de l'environnement; les tentatives de renforcement de l'autonomie des processus de planification locale tout en assurant le contrôle de l'État sur les questions d'importance ; ainsi que le renforcement de la participation locale dans la prise de décision et la conception des instruments de planification. Chacune de ces transformations a sans doute eu un impact sur l'émergence en France et en Australie, au cours des quinze dernières années, de la question de l'artificialisation des terres agricoles dans la législation, les 
politiques et les instruments de planification. Il est probable qu'elles auront dans le futur des effets positifs tout en soulevant de nouveaux défis.

Le tournant vers une doctrine de développement écologiquement durable en France et en Australie a permis le rapprochement de deux domaines de la planification spatiale qui étaient traditionnellement séparés : la planification de l'occupation du sol, concentrée sur les zones urbaines, et celle des ressources naturelles, concentrée sur les zones rurales. Cette intégration a permis aux législateurs et aux aménageurs de développer des politiques plus efficaces pour traiter la question de l'artificialisation des sols agricoles dans les zones périurbaines, un problème jusqu'alors ignoré par ces deux approches de planification. Mais, si cette intégration représente une opportunité pour traiter cette question, elle est aussi source de tensions, la plus prégnante étant celle entre la prise en compte de facteurs sociaux et environnementaux d'un côté et la recherche de bénéfices économiques de l'autre.

Tenir compte de l'artificialisation des terres agricoles dans les zones périurbaines a été possible dans la planification spatiale en France et en Australie du fait de leur prise de distance par rapport à des systèmes de décision supervisés par des États centralisés et bureaucratiques (Scott, 1998) et de leur adoption d'un système de planification spatiale plus décentralisé et participatif (Healey et Williams, 1993 ; Healey, 1997 ; Innes et Booher, 2010). Comme cela a été présenté, cette mutation est bénéfique, puisqu'elle permet une approche plus contextualisée de l'artificialisation des sols en fonction des situations locales. Cependant, elle pose aussi des défis, le plus grand étant le possible échec des multiples politiques et instruments de planification. Il résulterait de la combinaison d'une bureaucratie planificatrice centralisée qui déléguerait la responsabilité politique aux autorités locales, et de la difficulté de ces dernières à les intégrer et à les mettre en œuvre de manière efficace, du fait d'intérêts multiples et divergents parmi les acteurs locaux ainsi que d'enjeux différents entre échelle locale et échelle globale. Pour surmonter ces obstacles, il conviendrait de mieux équilibrer les rôles et responsabilités entre une planification spatiale centralisée et bureaucratique et une planification décentralisée et participative, tournée vers l'intégration de l'agriculture périurbaine comme composante à part entière des projets de développement des territoires.

Une dimension rarement abordée dans les travaux académiques sur l'artificialisation des terres agricoles périurbaines est celle des dispositifs de mesure et des processus sociotechniques de production de données, d'information et d'apprentissage collectifs. Cette dimension se situe à la croisée de deux courants théoriques de la planification spatiale, celui de l'aménagement basé sur la preuve (Davoudi, 2006) et celui du tournant communicationnel (Innes, 1998). Quelles données, quels dispositifs d'observation permettent d'apporter des preuves tangibles, difficilement réfutables, des phénomènes et de leur dynamique ? Comment ces données et informations se construisent, circulent au sein des réseaux d'acteurs ? Quels apprentissages individuels et collectifs sont à l'œuvre ? Qu'estce que cela change dans les processus de décision en matière de planification spatiale et dans les pratiques concrètes sur le terrain ? Ce sont ces points qui seront abordés dans les autres chapitres de cet ouvrage.

Bien que les planifications spatiales australienne et française présentent des similitudes manifestes, notre analyse a mis en évidence que les approches de l'artificialisation des sols avaient émergé de systèmes d'aménagement très différents. Les systèmes de planification français et australien ont des géographies très distinctes (Hall, 1982) qui sont façonnées par des logiques légales, politiques et administratives qui n'existent pas $a$ priori et de manière définitive, mais qui résultent plutôt de constructions sociales qui évoluent au cours du temps (McGuirk, 2003). 


\section{Références bibliographiques}

Albrechts L., 2006. Shifts in strategic spatial planning? Some evidence from Europe and Australia. Environment and Planning A, 38 (6), 1149-1170.

Allmendinger P., 2009. Planning Theory, Basingstoke, New York, Palgrave Macmillan.

Aubry C., Consalès J.-N., 2014. L'agriculture urbaine en question: épiphénomène ou révolution lente ? Dialogue entre Christine Aubry et Jean-Noël Consalès. Espaces et sociétés, 158 (3), 119-131.

Balchin W.G.V., Green F.H.W., Burrows J.W., Perrin H.F.R., Bridgeman J., Hansford-Miller D., Munro N., Blake R.N.E., Coleman A., 1976. Is planning really necessary? Discussion. The Geographical Journal, 142 (3), 430-437.

Bantayan N., Bishop I., 1998. Linking objective and subjective modelling for land use decision making. Landscape and Urban Planning, 43, 35-48.

Barthès C., Bertrand N., 2016.

Bauer G., Roux J.M., 1976. La rubanisation ou la ville éparpillée, Paris, Seuil.

Béchet B., Le Bissonnais Y., Ruas A., Desrousseaux M., 2017. Sols artificialisés et processus d'artificialisation des sols : déterminants, impacts et leviers d'action, Inra, $622 \mathrm{p}$.

Bertrand N., 2013. Terres agricoles périurbaines. Une gouvernance foncière en construction, Canada, North America, Versailles, éditions Quæ.

Billet P., 2017. Aspects juridiques de l'artificialisation des sols. In : Sols artificialisés et processus d'artificialisation des sols : Déterminants, impacts et leviers d'action (B. Béchet, Y. Le Bissonais, A. Ruas, M. Desrousseaux, eds), chapitre 6, Ifsttar/Inra (France), 255-271.

Bombenger P.H., 2011. L'urbanisme en campagne. Pratiques de planification et d'aide à la décision dans des communes rurales françaises, université de Tours.

Bradsen J., 1988. Soil Conservation Legislation in Australia Report for the National Soil Conservation Program, University of Adelaide.

Brand C., Bricas N., D. Conaré, B. Daviron, J. Debru, Michel L., Soulard C.-T., 2017. Construire des politiques alimentaires urbaines : concepts et démarches, Versailles, éditions Quæ.

Bryant C.R., 1982. The City's Countryside: Land and Its Management in the Rural-Urban Fringe, London, Longman.

Cadene P., 1990. L'usage des espaces periurbains, une géographie régionale des conflits. Études rurales, 118-119, 225-267.

Campbell A., 2008. Managing Australia's Soils: A Policy. Discussion Paper Prepared for the National Committee on Soil and Terrain (NCST) through the Natural Resource Management Ministerial Council.

Campbell S., 1996. Green cities, growing cities, just cities? Urban planning and the contradictions of sustainable development. Journal of the American Planning Association, 62 (3), 296-312.

Certu, 2010. La consommation d'espace par l'urbanisation. Panorama des méthodes d'évaluation, Lyon, Éditions du Certu (Centre d'études sur les réseaux, les transports, l'urbanisme et les constructions publiques).

CSIRO, 2008. Landscapes in Transition. Improving the Sustainability of Peri-Urban Growth, Commonwealth Scientific and Industrial Research Organisation.

Davis A., Pijanowski B., Robinson K., Engel B., 2010. The environmental and economic costs of sprawling parking lost in the United States. Land Use Policy, 27,255-261.

Davoudi S., 2006. Evidence-based planning: rhetoric and reality. DisP: The Planning Review, 42 (165), 14-24. 
Deffontaines J.-P., Thinon P., 2008. Analyser la répartition et la dynamique des usages agricoles dans le territoire. La cartographie d'unités agro-physionomiques. FaçSADe, 27, 1-4. Delattre L., Chanel O., Napoléone C., 2012. Comment modéliser les déterminants locaux de préservation des espaces non artificialisés en France? L'apport d'une confrontation littérature-terrain. Revue d'économie régionale et urbaine, 5, 805-829.

Demazière C., 2016. Réformes de la planification spatiale et gestion " durable » des grandes agglomérations. Les cas de l'Angleterre et de la France. Revue d'économie régionale et urbaine, février (1), 81-100.

Demazière C., Douay N., Serrano J., 2016. Léviathan ou Picrochole ? La planification spatiale en France, entre vision stratégique et dépendance aux formes du pouvoir local. Un éclairage à partir des schémas de cohérence territoriale. Riurba, 2.

Department of Primary Industry, 2011. Soil Policy: Looking Forward, Acting Now. Draft NSW Soil Policy, New South Wales, Department of Primary Industry.

Desjardins X., Béhar D., 2017. Les régions françaises enfin aménageuses du territoire? Population et Avenir, 733 (3), 17-19.

Desrousseaux M., 2014. La protection juridique de la qualité des sols. Thèse de doctorat, Université Jean-Moulin (Lyon-III).

Duke J., Aull-Hyde R., 2002. Identifying public preferences for land preservation using the analytic hierarchy process. Ecological Economics, 42, 131-145.

Emerson R., Fretz R., Shaw L., 1995. Writing Ethnographic Fieldnotes, Chicago, University of Chicago Press.

Epstein R., 2009. Après la territorialisation : le gouvernement à distance. In : Territoires, territorialité, territorialisation. Controverses et perspectives (M. Vanier, ed.), Rennes, Presses universitaires de Rennes, 131-139.

ESPON, 2004. Governance of territorial and urban policies from EU to local Level. Final Report. Luxemburg, ESPON.

European Commission, 1997a. Regional development studies: The EU compendium of spatial planning systems and policies, Luxembourg, European Commission.

European Commission, 1997b. Regional development studies: The EU compendium of spatial planning systems and policies: France, Luxembourg, European Commission.

European Commission, 1997c. Regional development studies: The EU compendium of spatial planning systems and policies: Netherlands, Luxembourg, European Commission.

European Commission, 2012. Commission Staff Working Document: Guidelines on best practice to limit, mitigate or compensate soil sealing, Brussels, European Commission.

Faludi A., 2009a. Territorial cohesion under the looking glass: synthesis paper about the history of the concept and policy background to territorial cohesion, European Commission Regional Policy-Inforegio, 2.

Faludi A., 2009b. A turning point in the development of European spatial planning? The 'Territorial Agenda of the European Union' and the 'First Action Programme'. Progress in Planning, 71 (1), 1-42.

Farrier D.A., 2007. Biodiversity offsets and native vegetation clearance in New South Wales: The rural/urban divide in the pursuit of ecologically sustainable development. Environmental Planning and Law Journal, 24 (6), 427-449.

Fottorino E., 1990. La France en friche, Paris, Éditions Lieu Commun.

Furuseth O.J., Lapping M.B., 1999. Contested Countryside: The Rural Urban Fringe in North America, Aldershot, Brookfield, Vt., Ashgate.

Getimis P., 2012. Comparing spatial planning systems and planning cultures in Europe. The need for a multi-scalar approach. Planning Practice and Research, 27 (1), 25-40. 
Giannakourou G., 2012. The europeanization of National Planning: explaining the causes and the potentials of change. Planning Practice and Research, 27 (1), 117-135.

GIS Sol, 2011. L'état des sols de France, Groupement d'intérêt scientifique sur les Sols, $188 \mathrm{p}$.

Gleeson B., Low N., 2004. Revaluing, Planning. Rolling Back Neoliberalism in Australia. Progress in Planning, 22 (4), 345-366.

Gravier J.F., 1947. Paris and the French Desert, Paris, Portulan.

Gueringer A., Hamdouch A., Wallet F., 2016. Foncier et développement des territoires ruraux et périurbains en France. Une mise en perspective. Revue d'économie régionale et urbaine, octobre (4), 693-712.

Guiomar X., 2014. La mise en proximité de l'agriculture (péri)urbaine par les collectivités. Une (re)construction en trois temps, trois mouvements. Pour, 224 (4), 415-426.

Haigh W., 1979. Second Reading Speech on the Environmental Planning and Assessment Bill and Cognate Legislation, Legislative Assembly Hansard, New South Wales Parliament, November 14, 1979.

Hall J.M., 1982. The Geography of Planning Decisions, New York, Oxford University Press. Hamdi R., Termonia P., Baguis P., 2010. Effects of urbanization and climate change on surface runoff of Brussels Capital Region: a case study using an urban soil-vegetation-atmosphere model. International Journal of Climatology, 31, 1959-1974.

Hazelton P., Clement A., 2011. Historic and environmental significance of ecological communities in NSW, Australia. International Journal of Heritage and Sustainable Development, 1 (1), 72-83.

Hazelton P., Murphy B., 2011. Understanding Soils in Urban Environments, Melbourne, Earthscan.

Healey P., 1997. Collaborative Planning: Shaping Places in Fragmented Societies, London, Macmillan.

Healey P., Williams R., 1993. European Planning Systems: diversity and convergence. Urban Studies, 30 (4/5), 701-720.

Hoggart K., 2005. The City's Hinterland Dynamism and Divergence in Europe's Peri-Urban Territories, Aldershot, Hants, England, Ashgate.

Houdart M., Loudiyi S., Gueringer A., 2012. L'adaptation des agriculteurs au contexte périurbain. Une lecture des logiques agricoles à partir du cas de Billom-Saint-Dier (Auvergne). Norois, 224 (3), 35-48.

Huber S., 2012. Urban SMS Soil Management Strategy: Framework "Soils in spatial planning”, Brussels, European Union, European Regional Development Fund.

Hulme T., Grosskopf T., Hidle J., 2002. Agricultural Land Classification, Agfact AC.25, Wagga Wagga, NSW Agriculture.

Innes J., 1998. Information in communicative planning. Journal of the American Planning Association, 4, 52-63.

Innes J.E., Booher D.E., 2010. Planning with Complexity: An Introduction to Collaborative Rationality for Public Policy, Milton Park, Abingdon, Oxon, New York, NY, Routledge.

Jarrige F., Thinon P., Nougarèdes B., 2006. La prise en compte de l'agriculture dans les nouveaux projets de territoires urbains. Exemple d'une recherche en partenariat avec la Communauté d'Agglomération de Montpellier. Revue d'économie régionale et urbaine, août (3), 393-414.

Jégouzo Y., 2015. Les compétences "Aménagement du territoire et urbanisme »: Quelle décentralisation? Revue française d'administration, 4 (156), 1049-1054. 
Jepson E., 2004. The adoption of sustainable development policies and techniques: How wide, how deep, and what role for planners? Journal of Planning Education and Research, 23 (3), 229-241.

Joyce M., 1998. Privative Clauses under ss 35 \&104A of the Environmental Planning and Assessment Act 1979 (NSW) and the implications for public interest environmental litigation. Environmental and Planning Law Journal, 15 (5), 362-368.

Knieling J., Othengrafen F., 2009. Planning Cultures in Europe: Decoding Cultural Phenomena in Urban and Regional Planning, Farnham, England, Ashgate.

Leras G., 2016. C'est mieux que rien... Sciences Eaux et Territoires, 19 (2), 26-27.

Lipman Z., Stokes R., 2008. The technocrat is back: environmental land-use planning reform in New South Wales. Environmental and Planning Law Journal, 25 (5), 305-324.

Local Government and Shire Associations of NSW, 2009. Integrating Natural Resource Management into NSW Local Government Operations, Sydney, Local Government and Shire Associations of NSW.

McGuirk P.M., 2003. Producing the capacity to govern in global Sydney: A Multiscaled Account. Journal of Urban Affairs, 25 (2), 201-223.

Melot R., Bransiecq M., 2016. Règles d'urbanisme et choix politique : les observations de l'État sur les projets locaux. Revue d'économie régionale et urbaine, octobre (4), 767-798.

Michel L., Soulard C.-T., Chia E., 2018.

Millar R., Fyfe M., 2012. The Fertile Fringe, The Age, Melbourne, 15.

Ministère de l'Environnement, Virely B., 2017. Artificialisation. De la mesure à l'action. Théma. Paris, ministère de l'Environnement, CGDD, Service de l'économie, de l'évaluation et de l'intégration du développement durable, 46.

Munafo M., Norero C., Sabbi A., Salvati L., 2010. Soil sealing in the growing city: a survey in Rome, Italy. Scottish Geographical Journal, 126, 153-161.

Nadin V., 2012. International comparative planning methodology: introduction to the theme issue. Planning Practice and Research, 27 (1), 1-5.

Nadin V., Stead D., 2008. European spatial planning systems, social models and learning. DisP: The Planning Review, 172 (1), 35-47.

National Committee on Soil and Terrain, 2009. Managing Australia's Soils: A policy discussion paper. Draft summary of submissions from public consultation, National Committee on Soil and Terrain.

Newman P., Jennings I., 2008. Cities as Sustainable Ecosystems: Principles and Practices, Washington, DC, Island Press.

Newman P., Thornley A., 1996. Urban Planning in Europe: International Competition, National Systems and Planning Projects, London, Routledge.

New South Wales Department of Primary Industries (NSW-DPI), 2013. Position Statement on Soils.

Nuissl H., Haase D., Lanzendorf M., Wittmer H., 2009. Environmental impact assessment of urban land use transition: a context-sensitive approach. Land Use Policy, 26, 414-424.

Perrin C., Soulard C.-T., 2017. Introduction. L'agriculture dans le système alimentaire urbain : continuités et innovations. Natures Sciences Sociétés, 25 (1), 3-6.

Perrin C., Soulard C.-T., Chia E., 2016. La gouvernance du foncier agricole périurbain : entre planification urbaine et projets de développement. Revue d'économie régionale et urbaine, octobre (4), 713-736.

Plant R., 2012. Mapping soils, land use and urban sprawl in peri-urban France and Australia: French-Australian In: Collaborative Workshop, 20-22 juin 2012, Montpellier, Unpublished, Irstea/University of Technology Sydney. 
Prokop G., Jobstmann H., Schönbauer A., 2011. Report on best practices for limiting soil sealing or mitigating its effects in EU-27. Technical Report, 2011, 050.

Pryor R.J., 1968. Defining the rural-urban fringe. Social Forces, 47 (2), 202-215.

Reimer M., Blotevogel H.H., 2012. Comparing Spatial Planning Practice in Europe: A Plea for Cultural Sensitization. Planning Practice and Research, 27 (1), 7-24.

Rey-Valette H., Pinto M., Maurel P., Chia E., Guiheneuf P.Y., Michel L., Nougaredes B., Soulard C., Jarrige F., Guillemot S.C., Mate M., S., Ollagnon H., Delorme G., Prouheze N., Laurent A., Suita L.A., Bertrand J., Dieudonne C., Champorigaud A., Barbe E., 2011. Guide pour la mise en ouvre de la gouvernance en appui au développement durable des territoires, Montpellier, Inra Éditions.

Robinson D., 2009. Strategic Planning for Biodiversity in New South Wales. Environmental Planning and Law Journal, 26 (3), 213-235.

Roussel S., Tardieu L., Vaissière A.-C., 2018. Compensation écologique et agriculture : est-ce compatible? Revue économique, Prépublication (0), 183-198.

Roux J.-M., 2017. La naissance du périurbain. Tous urbains, 19-20 (3), 94-98.

Salvati L., 2013. The spatial pattern of soil sealing along the urban-rural gradient in a Mediterranean region. Journal of Environmental Planning and Management, 1-14.

Sanz Sanz E., Napoléone C., Hubert B., Mata R., Giorgis S., 2017. Repenser la planification urbaine à partir des espaces agricoles. Une méthodologie opérationnelle à l'échelle intercommunale. Revue d'économie régionale et urbaine, juin (3), 511-536.

Scalenghe R., Marsan F.A., 2009. The anthropogenic sealing of soils in urban areas. Landscape and Urban Planning, 90 (1-2), 1-10.

Schmitt G., Rouget N., Margetic C., 2016. Les métropoles lilloise et nantaise au prisme du foncier agricole. Sciences Eaux et Territoires, 19 (2), 32-37.

Scott J.C., 1998. Seeing like a state: how certain schemes to improve the human condition have failed, New Haven [Conn.], Yale University Press.

Seeley J.R., 1962. What is planning? Definition and strategy. Journal of the American Institute of Planners, 28 (2), 91-97.

Serrano J., Vianey G., 2011. Consommation d'espace agricole et relations entre acteurs privés et publics : un management en faveur de l'artificialisation. Norois, 221 (4), 111-124.

Shoard M., 2002. Edgelands. In: Remaking the Landscape: The Changing Face of Britain (J. Jenkins, ed.), London, Profile Books, 117-146.

Tosics I., Szemző H., Illés D., Gertheis A., 2010. National spatial planning policies and governance typology. PLUREL Deliverable Report 2.2.1 PLUREL.

Tóth G., 2012. Impact of land-take on the land resource base for crop production in the European Union. Science of the Total Environment, 435-436, 202-214.

Tóth G., Németh T. (eds), 2011. Land Quality and Land Use Information in the European Union, Luxembourg, Publications Office of the European Union.

Van Assche K., Verschraegen G., 2008. The limits of planning: Niklas Luhmann's systems theory and the analysis of planning and planning ambitions. Planning Theory, 7 (3), 263-283.

Vargas L., 2016. Définition de la stratégie agricole 2015-2020 de Grenoble-Alpes Métropole : le foncier au cœur des enjeux. Sciences Eaux \& Territoires, 19 (2), 28-31.

Vianey G., 2012. Les zones agricoles protégées (ZAP) : valoriser l'identité agricole locale, http://www.projetsdepaysage.fr/fr/

les_zones_agricoles_protegees_zap_valoriser_1_identite_agricole_locale.

Villes T.E., 2012. L'agriculture dans les SCoT, Lyon, Certu.

Watson V., 2002. The usefulness of normative planning theories in the context of Sub-Saharan Africa. Planning Theory, 1 (1), 27-52. 
Yang B., Li H., 2011. Assessing planning approaches by watershed flow modeling: case study of The Woodlands: Texas. Landscape and Urban Planning, 99, 9-22.

Zewigert K., Kotz H., 1998. Introduction to Comparative Law, Oxford, Clarendon Press.

Zhou X., Wang Y., 2011. Dynamics of land surface temperature in response to land-use/cover change. Geographical Research, 49, 23-36. 



\section{Partie I}

\section{Base factuelle de la planification}





\title{
Chapitre 2
}

\section{Enjeux autour des terres agricoles et des données pédologiques : point de vue opérationnel d'un service de l'État en région}

\author{
Bernard Clarimont, Éric Barbe, Philippe Lagacherie
}

\section{Foncier et développement}

En France, la planification de l'espace urbain relève du niveau local (communes et intercommunalités) dans le respect du cadre réglementaire qui impose la prise en considération d'enjeux nationaux tels que la préservation des populations (risques naturels en particulier) ou la protection de l'environnement.

Il n'existe pas aujourd'hui de prescriptions nationales imposant la préservation du potentiel agronomique. Or le foncier est au cœur des enjeux de développement. Tous les acteurs demandent un accès facile au foncier pour répondre à des besoins privés ou à des enjeux d'intérêt général (accueil de population, activités économiques, loisirs...).

Force est de constater que notre croissance effrénée a largement ignoré une réalité première : le sol, support de nos activités, est une ressource finie, non renouvelable à l'échelle humaine. Il assure de multiples fonctions essentielles telles que la production agricole, le support d'une importante biodiversité contribuant en particulier aux grands cycles biologiques (carbone, azote...), la contribution au cycle de l'eau, le stockage d'éléments divers (notamment toxiques) ou encore la régulation thermique. De ce fait, toute artificialisation, irréversible par définition (sauf à des coûts de remise en état prohibitifs), induit une atteinte à des équilibres globaux.

De plus, l'intensification des productions agricoles et la simplification des assolements ont été accompagnées d'une utilisation accrue d'intrants (fertilisants, phytosanitaires notamment), alors que les contraintes économiques et environnementales nécessiteront, à l'avenir, la recherche d'une efficience accrue (limitation de l'irrigation, réduction de la fertilisation...) et que les disponibilités naturelles en facteurs de production sont limitées (cas par exemple de la potasse et du phosphore, pivots de la fertilisation).

Dans tous les cas, les sols aux potentiels agronomiques les plus propices seront les plus aptes à répondre aux attentes sociétales et aux contraintes économiques.

La terre était d'ailleurs, historiquement, la source essentielle de richesses et revêtait de ce fait une importance particulière. Mais le lien à la terre a évolué. Dans nos sociétés urbaines, le sol est banalisé, réduit au rôle de support, objet de rente ou de spéculation. 
Dans ce contexte, les décideurs ont, durant des décennies, totalement méconnu l'enjeu de préservation des sols et a fortiori la notion de qualité des sols a été totalement absente de leurs réflexions. C'est ainsi que sur l'ensemble de la France, 800000 hectares ont été artificialisés entre 1992 et 2004, dont la moitié par de l'habitat individuel. $80 \%$ de ces surfaces artificialisées étaient des terres de production agricole. Ce phénomène s'est encore accéléré pour atteindre aujourd'hui le rythme de 500000 hectares artificialisés tous les sept ans.

\section{Vers une prise de conscience}

Depuis son entrée en vigueur en 1962, la Politique agricole commune de l'Union européenne a permis de relever le défi de l'autosuffisance alimentaire de l'Europe. Elle a ensuite été confrontée à des crises liées à la surproduction de nombreuses filières. C'est pourquoi, dans les années 1990, lors de l'élaboration des Plans d'occupation des sols (POS), les élus locaux n'étaient pas sensibles à la préservation des terres agricoles. Ils faisaient remarquer que les stocks de produits agricoles étaient importants et devaient faire épisodiquement l'objet de mesures de dégagement, et que l'Union européenne développait des politiques de maîtrise des productions (quotas...). L'État lui-même n'était que peu mobilisé sur ces questions, et les citoyens avaient le sentiment que l'approvisionnement alimentaire était assuré en abondance ad vitam aeternam.

La situation des marchés agricoles, les émeutes de la faim en 2008 ainsi que la perspective d'une population mondiale de 9 milliards d'individus en 2050 ont révélé l'importance de l'enjeu. En France, il y a eu à cette époque une prise de conscience de l'urgence à mettre en œuvre des mesures concrètes visant à limiter la consommation croissante d'espace par l'urbanisation.

La loi de modernisation agricole du 13 juillet 2010 a ainsi créé un Observatoire national de la consommation des espaces agricoles (Oncea), renforcé en 2014 dans le cadre de la loi d'avenir pour l'agriculture, l'alimentation et la forêt et élargi aux espaces naturels et forestiers, devenant ainsi l'Observatoire des espaces naturels, agricoles et forestiers (Oenaf). La loi de modernisation agricole a également institué, dès 2010, une Commission départementale de consommation des espaces agricoles (CDCEA), qui émet notamment un avis quant à l'opportunité de certaines procédures ou autorisations d'urbanisme au regard de l'objectif de préservation des terres agricoles. Il est à noter que la loi de juillet 2010 n'impose pas d'objectif de réduction du rythme de consommation des terres et n'évoque pas la question de la qualité des sols concernés par l'artificialisation.

C'est pourquoi, dans l'ancienne région Languedoc-Roussillon, les services de l'État ont donné une traduction réglementaire à ces objectifs de préservation en intégrant dans un arrêté préfectoral de mars 2012 relatif au Plan régional d'agriculture durable l'obligation de diviser par deux d'ici 2020, dans chacun des cinq départements régionaux, le rythme de consommation des sols, et de préserver particulièrement les sols présentant les meilleurs potentiels pour répondre aux enjeux alimentaires. Il s'agit des sols identifiés comme étant les plus aptes à porter des cultures diversifiées, et en particulier des grandes cultures telles que les céréales et les oléoprotéagineux.

Il faut souligner que dans cette région deux facteurs de vulnérabilité des sols sont particulièrement prégnants :

- l'importance des espaces protégés au titre de l'environnement (espaces dits «naturels »). Un des effets de cette logique vertueuse pour l'environnement est toutefois de reporter la pression sur l'espace agricole et de le transformer en gisement privilégié pour l'artificialisation ; 
- l'importance du faire-valoir direct comme mode d'exploitation agricole. Dans les départements méditerranéens, les deux tiers des agriculteurs sont pour l'essentiel propriétaires de leurs terres, qui font alors l'objet d'une gestion patrimoniale. Lors de la cessation d'activité, cette situation entraîne des réticences à consentir un bail rural à un repreneur et une tendance au gel du foncier dans l'attente d'une valorisation non agricole. De plus, le morcellement du parcellaire (la superficie moyenne des parcelles dans ces départements est de 0,5 hectare) facilite la cession progressive du foncier.

\section{Éléments de stratégie en région : perspectives}

Au-delà de l'indispensable évolution législative qui donnera le cadre sur lequel fonder une protection efficace des sols à vocation agricole, l'action locale demeure aujourd'hui déterminante car c'est à ce niveau que se décide, pour l'essentiel, le devenir de ces espaces.

En région, le propos n'est pas de préserver tous les sols pour l'agriculture d'aujourd'hui, d'autant plus qu'il n'existe pas toujours localement une demande suffisante pour mobiliser toutes ces terres. Mais si ce sont surtout des sols à potentiels limités qui sont laissés aujourd'hui aux jeunes agriculteurs (Balestat et al., 2011), les autres ayant été sacrifiés à l'extension urbaine, aux infrastructures et aux autres zones d'activités, les professionnels agricoles seront dans l'impossibilité de satisfaire aux attentes de citadins revendiquant une alimentation toujours plus écologique, et aux besoins de la société qui sera, dans quelques décennies, confrontée à des enjeux alimentaires majeurs. Il s'agit donc avant tout de préserver un potentiel productif indépendamment de son utilisation actuelle, et surtout de ne pas considérer qu'un sol non utilisé aujourd'hui pour l'agriculture est, de ce fait, disponible pour des aménagements. C'est pourquoi les services de l'État en région considèrent que les conséquences des aménagements doivent être examinées à deux niveaux en ce qui concerne l'enjeu agricole :

- tout d'abord, au niveau de l'entreprise agricole et des filières, comme c'est le cas actuellement. Il s'agit de s'intéresser aux unités économiques créatrices de richesses et d'emplois. Dans ce contexte, la préoccupation est avant tout la recherche de foncier pour compenser l'emprise des nouveaux aménagements (infrastructures, urbanisation...) afin de permettre à l'exploitant agricole qui le souhaite de poursuivre son activité. La contribution des Sociétés d'aménagement foncier et d'établissement rural (Safer) est à ce niveau essentielle, en particulier lors de la réalisation de grandes infrastructures ;

- le deuxième niveau, distinct du précédent et tout aussi important, est celui de la préservation des capacités (potentialités) productives des sols. Tout aménagement constitue une atteinte définitive à une ressource non renouvelable à l'échelle humaine. Aucune compensation physique à cette stérilisation ne peut exister. Il ne s'agit pas pour autant d'interdire par principe toute possibilité d'évolution, mais il est indispensable que les décideurs :

- aient tout d'abord connaissance des potentiels agronomiques des différents secteurs de leur territoire ; c'est aujourd'hui le cas dans les départements de l'ex-LanguedocRoussillon grâce au travail de traduction cartographique réalisé par l'Institut national de la recherche agronomique (Inra) à partir de ses bases de données,

- soient sensibilisés et puissent intégrer cette dimension dans leurs décisions et contribuent ainsi à la préservation des meilleurs potentiels.

Ainsi, à titre d'exemple, porter atteinte à des sols de bonne potentialité n'apparait pas légitime lorsqu'il s'agit de réaliser un habitat pavillonnaire sur grandes parcelles; par contre, implanter un habitat dense afin de répondre à des contraintes spécifiques (création d'habitat social pour répondre aux obligations liées à la loi relative à la solidarité 
et au renouvellement urbains, SRU, par exemple) ne peut être exclu en l'absence d'une autre alternative sur le territoire de la commune. Il s'agit donc de réaliser une analyse des valeurs en jeu avant toute décision afin de faire prévaloir l'option la plus satisfaisante dans une perspective d'intérêt général à long terme.

S'intéresser au potentiel des sols suppose de ne plus considérer un sol non utilisé aujourd'hui par l'agriculture comme disponible pour réaliser des aménagements. Dans le contexte des difficultés qu'ont connu les filières méditerranéennes, en particulier la crise de la viticulture, ce mode de pensée encore trop fréquent a conduit à légitimer de multiples artificialisations d'espace.

\section{Origine et objectifs des travaux engagés}

Dans l'ancienne région Languedoc-Roussillon, l'artificialisation des sols agricoles s'est considérablement accentuée avec l'arrivée massive et continue de nouveaux résidents permanents au cours des cinquante dernières années. Le développement de l'urbanisme et des infrastructures a été réalisé le plus souvent au détriment des terres agricoles des zones de plaine et de piémont.

Ce phénomène de consommation irréversible (stérilisation) que l'action de l'État n'a pas contribué à endiguer résulte d'un double hiatus :

- un hiatus d'échelle lié au niveau décisionnel en matière d'urbanisme. Au niveau local, le raisonnement des décideurs est de type marginal et n'intègre pas les conséquences de telles actions au niveau national, lorsque ces consommations sont multipliées par les milliers de centres de décision de l'Hexagone et reproduites dans le temps (révisions de PLU...). À ce niveau local sont traités des enjeux d'intérêt général mais de portée locale (équipements publics communaux, accueil de nouveaux habitants par exemple). Ces décideurs n'intègrent pas spontanément les enjeux, plus globaux, de niveau national concernant, en particulier, la préservation du potentiel agronomique des sols ;

- un hiatus temporel. La logique des décisions locales en matière de planification s'inscrit dans la durée d'une mandature ou deux. Les enjeux stratégiques à l'horizon 2050 et relevant de surcroît d'autres champs de préoccupation ne sont pas pris en considération.

Le rythme de consommation des terres confirme bien que la question foncière n'a pas encore reçu de réponse satisfaisante en France. Il apparaît clairement que la somme des actions de milliers de centres locaux de décision ne peut pas, en l'absence d'une politique nationale forte appuyée sur un cadrage réglementaire précis, répondre à ces enjeux stratégiques nationaux. Les quelques tentatives pour développer des outils de préservation, dont l'initiative de mise en œuvre dépend de la volonté locale, se sont soldées par un échec (Zones agricoles protégées, ZAP ; Périmètres de protection et de mise en valeur des espaces agricoles et naturels périurbains, PAEN). Il apparaît aujourd'hui nécessaire de développer de nouveaux instruments d'évaluation et de régulation, mais également de doter les services déconcentrés de l'État (Directions départementales des territoires) des moyens techniques permettant de faire valoir l'enjeu de préservation du potentiel agronomique qui nous préoccupe.

C'est pourquoi la Draaf Languedoc-Roussillon, désormais Draaf Occitanie, travaille depuis 2006 en partenariat avec Irstea (anciennement Cemagref) et l'Inra pour développer des instruments et des méthodologies généralisables (Balestrat et al., 2008) à l'Hexagone afin de :

- quantifier précisément l'atteinte aux sols agricoles à partir d'une maille communale agrégeable à différents niveaux d'analyse (supra-communal, départemental, régional...) 
non seulement en superficie, mais également en termes de potentiel agronomique. Des indicateurs doivent permettre de suivre l'évolution de ces atteintes ;

- mettre à disposition des directions départementales des territoires des outils, notamment cartographiques, et des indicateurs destinés à éclairer les options d'aménagement (en particulier les documents d'urbanisme) et à enrichir le « dire de l'État », notamment dans le cadre des « porter à connaissance».

\section{Développements méthodologiques régionaux à vocation nationale}

Pour apporter des éléments de réponse en matière de superficie et de qualité des terres consommées par l'artificialisation, les travaux ont été réalisés selon deux approches distinctes et complémentaires :

- une approche quantitative visant à développer une méthode de cartographie et de suivi des espaces artificialisés (Irstea, UMR Tetis) (Balestrat et al., 2011) ;

- une approche qualitative visant à développer une méthode de cartographie du potentiel agronomique des sols (traduit par l'aptitude à accueillir de grandes cultures) s'appuyant sur les données de sols existantes et en utilisant une typologie pertinente à l'échelle nationale (Inra, UMR Lisah).

Ces travaux ne se sont pas focalisés sur l'analyse de l'évolution des espaces à usage agricole en matière d'occupation du sol, mais se sont plus généralement intéressés à l'analyse de l'artificialisation des terres, étant entendu que le potentiel agricole d'une terre ne dépend pas de son usage actuel, mais de ses qualités agronomiques et de sa disponibilité.

\section{Une vocation nationale}

En tenant compte du fait que les enjeux de préservation d'un patrimoine de terres agricoles s'expriment à l'échelle nationale, les méthodes ont été élaborées dans un objectif de reproductibilité dans l'espace (c'est-à-dire sur tout le territoire métropolitain) et dans le temps (à plusieurs dates) afin de permettre un suivi homogène de l'artificialisation des terres et de sa caractérisation. Les méthodes et données mobilisées sont donc « génériques », afin d'être disponibles et applicables sur tout le territoire, et " performantes », afin d'envisager avec réalisme leur mise en œuvre à cette échelle.

\section{Cartographie des espaces artificialisés : méthode générale}

En France, plusieurs sources de données dédiées à l'occupation du sol (Corine Land Cover, Teruti-Lucas, fichiers fonciers...) et disponibles depuis plusieurs décennies permettent d'appréhender la notion d'artificialisation. Cependant, les données chiffrées issues de ces sources varient considérablement (rapport de 1 à 4 à l'échelle nationale). Les objectifs (connaissance générale de l'occupation du sol, suivi parcellaire...) et les méthodes (enquête statistique, photo-interprétation d'images satellitaires avec des seuils d'agrégation, déclaration fiscale...) mises en œuvre pour chacun de ces outils justifient ces écarts, sans remettre en question leur pertinence et leur fiabilité pour les usages ayant conditionné leur réalisation. Ce constat, opéré dès 2007 dans l'ancienne région Languedoc-Roussillon, a orienté le développement d'une méthode spécifiquement dédiée au suivi de l'artificialisation. Pour autant, la question de la mesure de l'artificialisation abordée en 2014 dans le rapport de l'Oncea (Oncea, 2014) reste toujours d'actualité en 2018, comme le souligne le chapitre « Des méthodes de mesure à l'estimation de l'artificialisation des sols en France » dans l'Expertise scientifique collective (ESCo) finalisée en décembre 2017 (Béchet et al., 2017). 
La méthode développée dès 2007 pour la quantification et le suivi des superficies de terres consommées par le développement de l'habitat en zones périurbaines et en zones rurales vise à produire une cartographie des espaces artificialisés. Elle est basée sur l'analyse d'images satellitaires, véritables «photographies»du territoire à un instant donné, garantissant l'exhaustivité et l'homogénéité des informations, et comporte deux étapes :

- l'extraction d'informations sur l'occupation du sol à partir d'images satellitaires selon une nomenclature adaptée, puis leur agrégation en deux classes (artificialisé/non artificialisé) ;

- la mise en œuvre d'un processus de dilatation/érosion appliqué à ces objets afin d'obtenir une « tache artificialisée » représentative de l'emprise au sol de l'artificialisation.

Tableau 2.1. Nomenclature retenue.

\begin{tabular}{|c|c|}
\hline Nom de classe & Méthode utilisée \\
\hline Zone artificialisée & \multirow{7}{*}{$\begin{array}{l}\text { Images obtenues par télédétection, puis corrigées par } \\
\text { photo-interprétation }\end{array}$} \\
\hline Végétation urbaine & \\
\hline Espace agricole & \\
\hline Zone naturelle & \\
\hline Zone en eau & \\
\hline Zone naturelle humide & \\
\hline Carrière, chantier, décharge & \\
\hline Route $10 \mathrm{~m}$ & \multirow{2}{*}{ Images obtenues à partir de la BD Carto® de l'IGN } \\
\hline Route $20 \mathrm{~m}$ & \\
\hline
\end{tabular}

En appliquant cette méthode à des images couvrant un même territoire prises à des dates différentes, il est possible de créer puis de comparer les taches artificialisées, afin de mesurer l'évolution de l'artificialisation de ce territoire. Cette méthode constitue un compromis entre la finalité opérationnelle locale d'évaluation et de localisation des terres artificialisées et la contrainte de reproductibilité dans le temps et dans l'espace en vue de sa mise en œuvre potentielle sur tout le territoire national. L'intérêt majeur de la méthode réside dans sa capacité à être appliquée sur des superficies importantes, tout en produisant des résultats exploitables à l'échelle locale (1/15 000). Cependant, les processus mis en œuvre relèvent d'une modélisation qu'il ne faut pas chercher à comparer à une analyse exhaustive, de type photo-interprétation par exemple, certes plus précise, mais économiquement impossible à réaliser, ne serait-ce qu'à l'échelle d'un département.

\section{Classification d'images}

Nous avons défini une nomenclature simple et discriminante pour l'identification des espaces artificialisés, constituée de neuf classes (tableau 2.1). Certaines classes difficilement identifiables par télédétection (routes, souvent masquées par les houppiers des arbres les bordant) proviennent de données externes de référence disponibles sur tout le territoire (IGN notamment). 


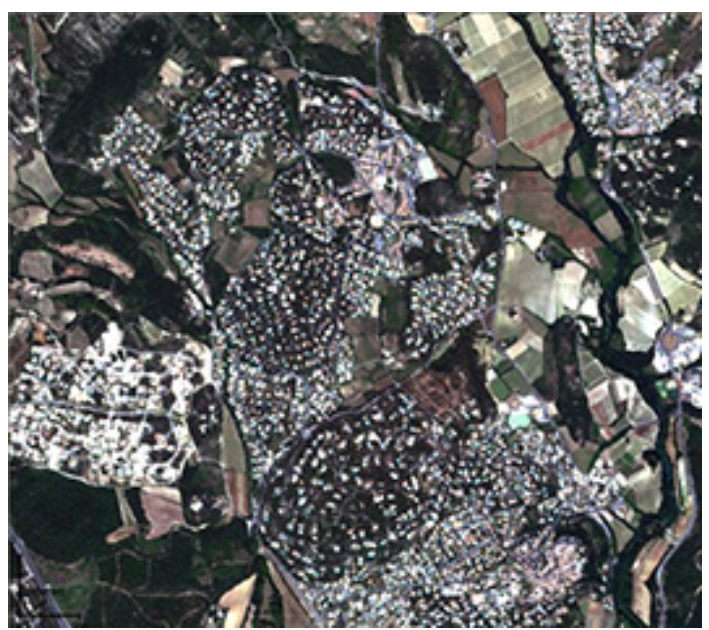

Figure 2.1. Extrait d'une image du satellite RapidEye.

La méthode générale de traitement d'image est constituée de trois étapes à partir d'une image satellitaire (figure 2.1).

1. Réalisation d'une classification orientée objet combinant des étapes de segmentation et de classification afin d'extraire les différentes classes d'occupation du sol définies dans la nomenclature, et intégration des routes et infrastructures de la BD Carto ${ }^{\circledR}$ IGN (figure 2.2).

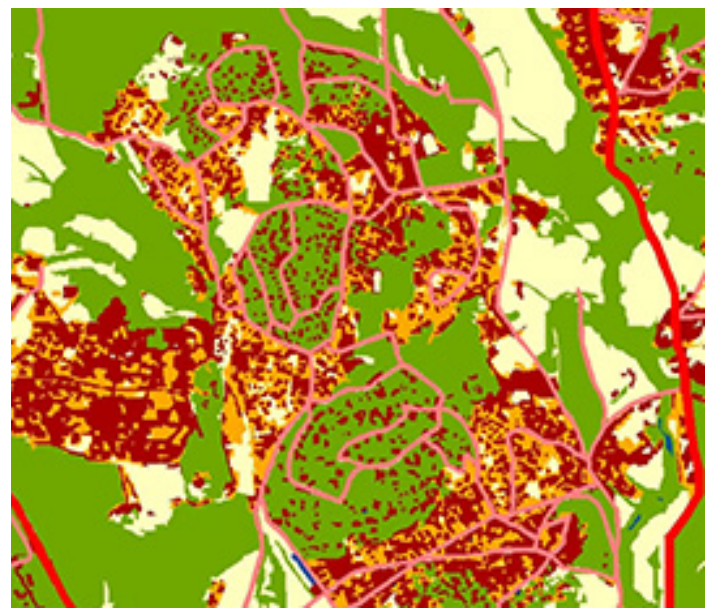

Figure 2.2. Exemple de classification.

2. Regroupement des classes obtenues en deux classes synthétiques (artificialisé/non artificialisé). La figure 2.3 représente un extrait du résultat de regroupement en deux classes : 


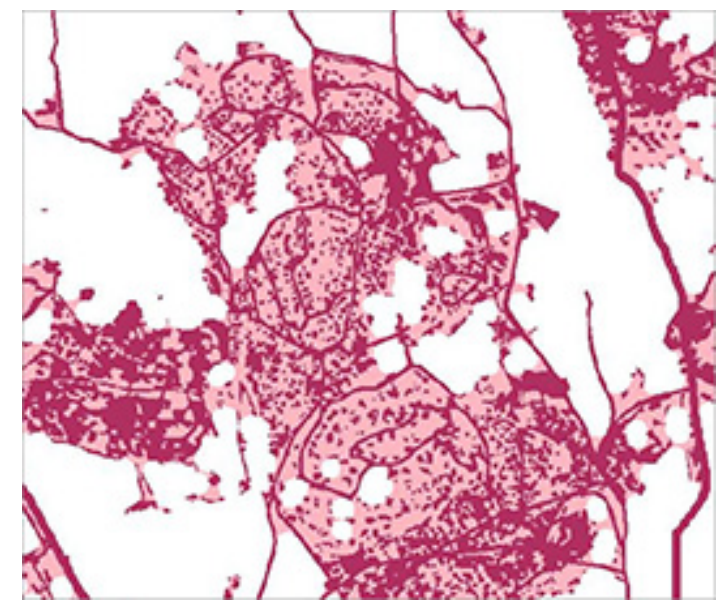

Figure 2.3. Classification simplifiée.

3. Application d'un processus de dilatation/érosion (seuil $50 \mathrm{~m}$ ) pour fusionner les objets artificialisés dans un périmètre de $100 \mathrm{~m}$ afin de produire la « tache artificialisée » représentative des espaces inaptes à une activité agricole (figure 2.4).

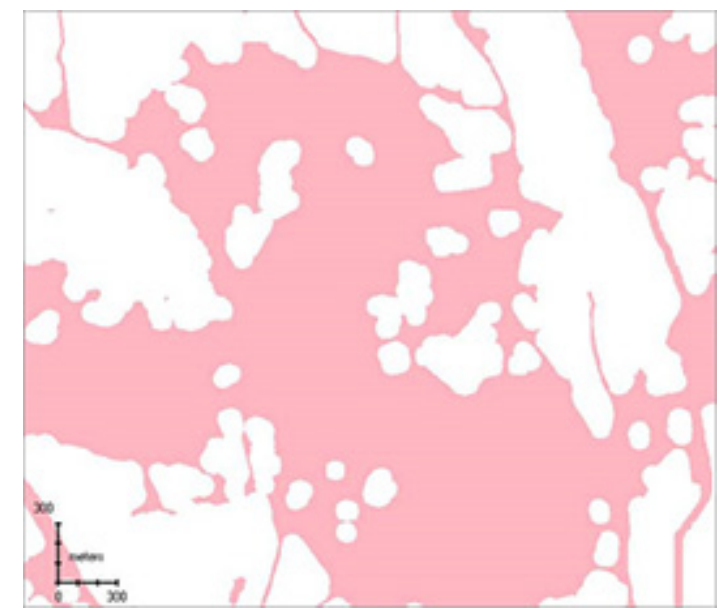

Figure 2.4. Tache artificialisée.

\section{Résultats}

Des images satellitaires RapidEye (2009) et IRS (1997) ont été acquises et utilisées pour la réalisation de ces travaux à l'échelle des quatre départements littoraux de l'ancienne région Languedoc-Roussillon (ainsi que le sud de la Lozère), où se concentrent les enjeux d'artificialisation et de pression foncière associée (Dupuy et al., 2012). La mise en œuvre des traitements sur ces images acquises à deux dates différentes a permis de caractériser l'évolution de l'artificialisation par son emprise et sa superficie sur une période de douze ans (figure 2.5). 

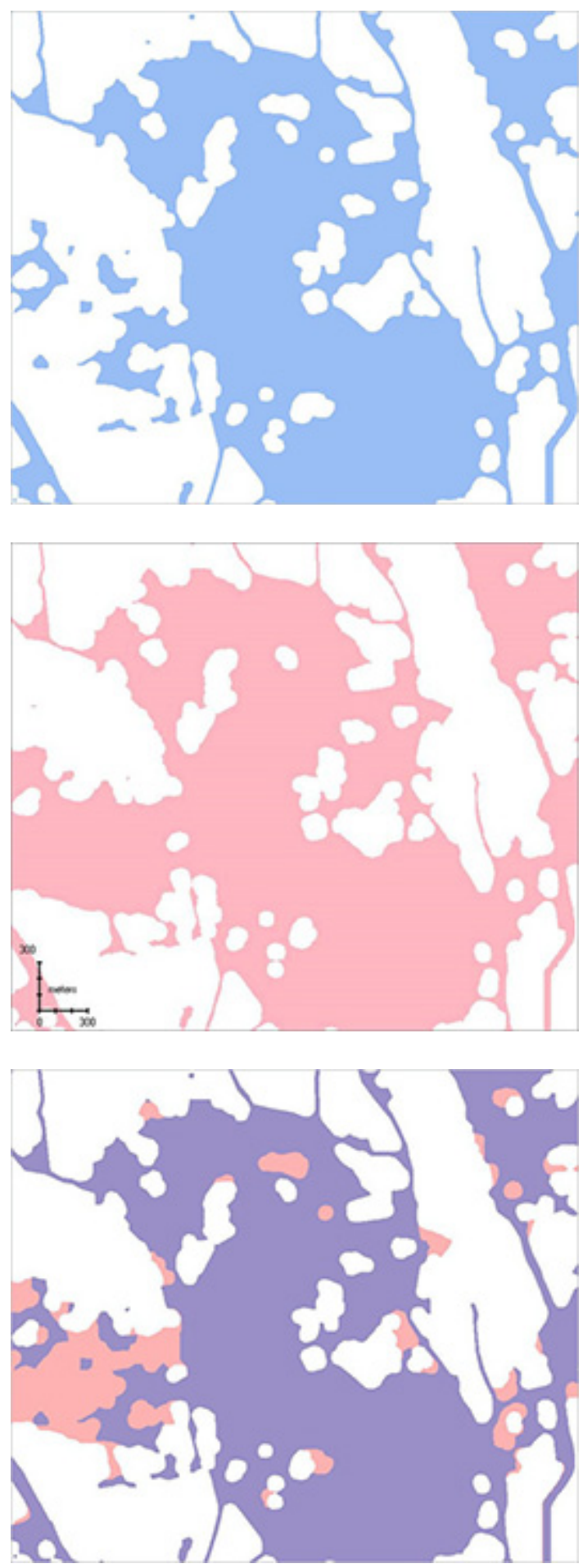

Figure 2.5. Suivi de l'évolution des taches artificialisées dans le temps (extrait à l'échelle d'une commune). 
Le taux moyen d'évolution de l'artificialisation entre 1997 et 2009 sur ces territoires déjà fortement urbanisés est de $8 \%$, mais il varie de $4 \%$ à près de $10 \%$ à l'échelle départementale (tableau 2.2).

Tableau 2.2. Évolution de la tache artificialisée intégrant les routes, de 1997 à 2009, pour chacun des départements littoraux.

\begin{tabular}{|l|l|l|l|l|l|l|l|}
\hline \multirow{2}{*}{$\begin{array}{c}\text { Départements } \\
\text { littoraux }\end{array}$} & \multicolumn{4}{|c|}{ Superficie (ha) } & \multicolumn{2}{c|}{$\begin{array}{c}\text { Part (\%)/super- } \\
\text { ficie de la zone } \\
\text { d'étude }\end{array}$} & \multirow{2}{*}{$\begin{array}{c}\text { Taux d'évolu- } \\
\text { tion 1997-2009 } \\
\text { (\%) }\end{array}$} \\
\cline { 2 - 9 } & $\begin{array}{c}\text { Superficie } \\
\text { totale }\end{array}$ & $\begin{array}{c}\text { Tâche } \\
\text { en } \\
\mathbf{1 9 9 7}\end{array}$ & $\begin{array}{c}\text { Tâche } \\
\text { en } \\
\mathbf{2 0 0 9}\end{array}$ & $\begin{array}{c}\text { Écart } \\
\mathbf{1 9 9 7 - 2 0 0 9}\end{array}$ & $\mathbf{1 9 9 7}$ & $\mathbf{2 0 0 9}$ & \\
\hline Pyrénées-Orientales & 416139 & 43174 & 46349 & 3175 & 10,4 & 11,1 & 7,4 \\
\hline Hérault & 624789 & 77475 & 84470 & 6995 & 12,4 & 13,5 & 9,0 \\
\hline Gard & 588826 & 68746 & 75583 & 6837 & 11,7 & 12,8 & 9,9 \\
\hline Aude & 634700 & 53000 & 55304 & 2304 & 8,4 & 8,7 & 4,3 \\
\hline Total & 2264454 & $\begin{array}{l}242 \\
396\end{array}$ & $\begin{array}{l}261 \\
707\end{array}$ & 19311 & 10,7 & 11,6 & 8,0 \\
\hline
\end{tabular}

Si l'on exclut les routes, le taux moyen de progression de la tache artificialisée atteint alors $17,5 \%$ et rend compte plus fidèlement de l'importante dynamique d'artificialisation, et de «l'inertie » engendrée par les routes dont l'emprise notable évolue peu dans le temps.

\section{Cartographie du potentiel agronomique des sols}

L'analyse uniquement quantitative des surfaces consommées par le développement de l'habitat ne saurait à elle seule constituer une base suffisante pour orienter les décisions en matière de patrimoine du sol. Les sols sont en effet extrêmement variables dans l'espace compte tenu de la multiplicité et de la variabilité des facteurs du paysage qui interagissent à leur formation (relief, géologie, occupation du sol, etc.). Chaque surface agricole perdue à cause de l'extension de l'urbanisation ou susceptible de l'être doit donc être caractérisée par un degré de qualité qui permettra au décideur de mieux apprécier le préjudice que cette perte fait subir à la collectivité.

Dans ces travaux réalisés par l'Inra (UMR Lisah), un indicateur de qualité spécifique au problème posé, adapté à l'agriculture régionale et aux données pédologiques disponibles, a été proposé en utilisant comme données sources le Référentiel régional pédologique du Languedoc-Roussillon.

\section{Construction d'un indicateur de qualité des sols du Languedoc-Roussillon}

L'indicateur de qualité des sols vise à évaluer une qualité « globale » du sol pour un usage futur (d'ici un siècle) en privilégiant le potentiel agronomique du sol et la capacité maximale de diversification des usages du sol. Il doit remplir les conditions suivantes :

- permettre d'apprécier le potentiel d'utilisation globale d'un sol à long ou très long terme. À ce titre, aucune aptitude particulière à une culture ou aucune fonction du sol n'était à privilégier par rapport à une autre. S'agissant d'un impact s'exerçant au-delà de plusieurs dizaines d'années, il est en effet impossible de se référer à tel ou tel agrosystème de référence ou usage agricole et/ou environnemental du sol. Il a cependant été 
retenu comme référence la production de grandes cultures en pluvial, qui correspond vraisemblablement à l'un des systèmes de culture les plus exigeants vis-à-vis des caractéristiques du sol ;

- proposer des modalités permettant de comparer un maximum de sols du LanguedocRoussillon entre eux tout en restant simple. Il s'agissait pour cela de proposer une classification avec un nombre limité de modalités, ces modalités ayant des effectifs comparables entre elles ;

- s'appliquer facilement dans un délai très court. Il s'agissait de privilégier un indicateur utilisant des données spatiales pédologiques déjà disponibles sur l'ensemble du Languedoc-Roussillon.

L'approche retenue est une approche par combinaison logique de classes de sol qui prend en compte les contraintes rencontrées en milieu méditerranéen. L'indicateur est présenté dans la figure 2.6 sous forme d'un arbre de décision avec trois niveaux hiérarchisés de classification :

- les contraintes « absolues », qui discréditent systématiquement et entièrement le potentiel du sol lorsqu'elles existent (classe 4). Il s'agit de la présence de salinité et de la pente lorsque celle-ci est supérieure à $15 \%$;

- la réserve utile $p$, qui constitue le paramètre principal de hiérarchisation, représentée par trois classes (classes 1, 2 et 3 ) ;

- les contraintes « secondaires » $n$ (battance, hydromorphie, pierrosité ou abondance des éléments grossiers et $\mathrm{pH}$ ), dont la prise en compte permet de moduler la qualité des sols au sein de chaque classe de réserve utile. Ainsi, la présence de $n(n=1$ à 5$)$ contraintes sur un sol de classe de réserve utile $p(p=1$ à 3$)$ permet de classer le sol en classe $n . p$, avec n.p d'autant plus défavorable que $n$ (le nombre de contraintes secondaires) est grand.

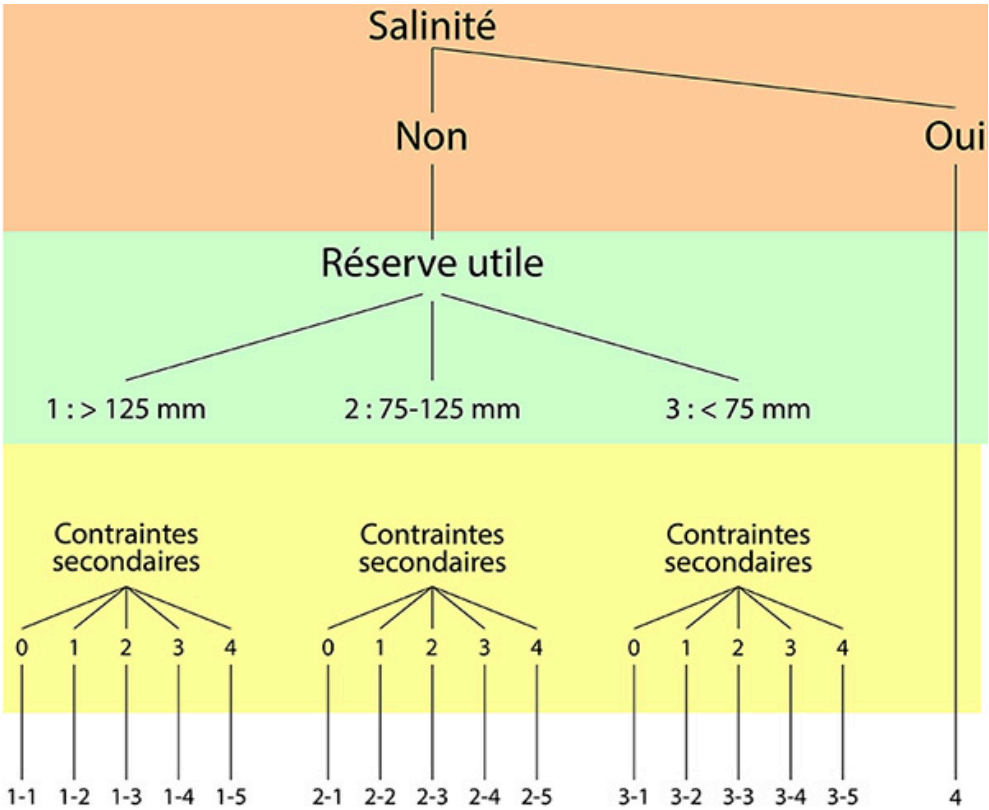

Figure 2.6. Arbre de décision de détermination du score « qualité des sols ». 


\section{Spatialisation de l'indicateur de qualité des sols : résultats}

L'indicateur de qualité des sols a été calculé en utilisant la base de données spatialisées Référentiel régional pédologique du Languedoc-Roussillon et représenté sous la forme d'une cartographie à l'échelle 1/250 000 issue des unités cartographiques de sols disponibles sur le territoire national. Le résultat peut être représenté sous forme d'une carte synthétique (figure 2.7) figurant six classes de sols exprimant les sols du plus fort (classe 1) au plus faible (classe 6) potentiel agronomique, la classe 7 représentant les sols salins impropres à la culture (Balestrat, 2011).

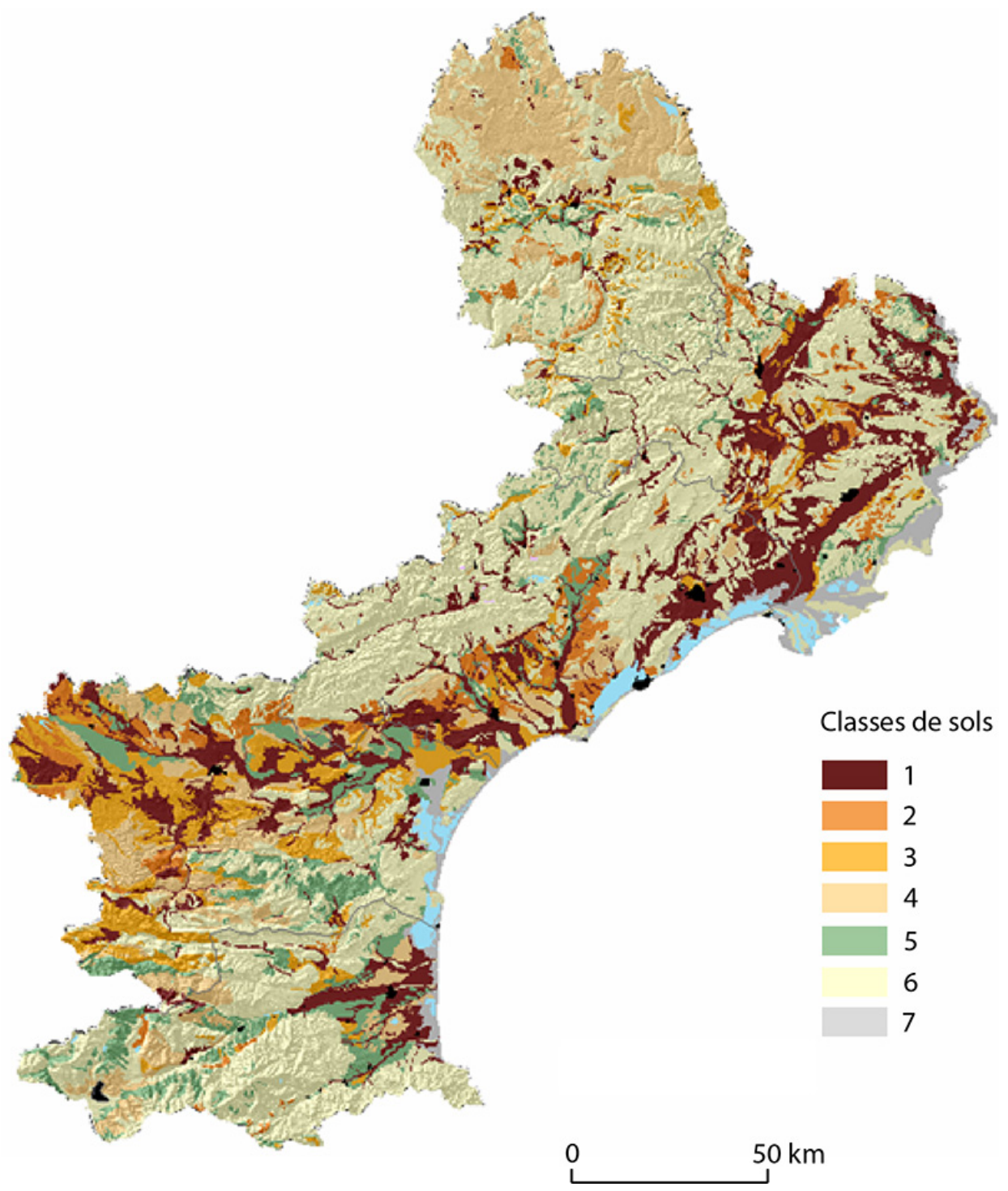

Figure 2.7. Carte du potentiel agronomique des sols du Languedoc-Roussillon. 


\section{Conclusion}

Désormais, les acteurs de l'aménagement du territoire du Languedoc-Roussillon disposent de cartes représentant non seulement l'emprise des espaces artificialisés entre 1997 et 2009, mais aussi le potentiel agronomique des sols. Ces informations permettent d'établir une cartographie des enjeux agricoles et participent à l'élaboration, à l'instruction et à la validation des projets d'aménagement. Le chapitre suivant (chapitre 3) présente la démarche mise en œuvre pour élaborer des indicateurs complémentaires issus du croisement de ces cartes.

Citons en conclusion de ce chapitre un indicateur issu du croisement entre les taches artificialisées et le potentiel agronomique des sols qui a permis de mettre en exergue la pression foncière exacerbée sur les sols à plus fort potentiel agronomique. Entre 1997 et 2009, près de 20000 hectares ont été artificialisés sur les quatre départements littoraux de l'ancienne région Languedoc-Roussillon (Aude, Hérault, Gard, Pyrénées-Orientales). Ils ont porté à $42 \%$ sur des sols de classes 1 et 2 (plus fort potentiel agronomique), qui ne couvrent pourtant que $22 \%$ du territoire.

Un nouveau projet à l'horizon 2019 vise à étendre ces travaux (artificialisation, potentiel agronomique, indicateurs) à l'échelle de la nouvelle région Occitanie, issue de la fusion des régions Languedoc-Roussillon et Midi-Pyrénées et désormais deuxième plus grande région de France, s'étalant sur une superficie de $72000 \mathrm{~km}^{2}$.

\section{Références bibliographiques}

Balestrat M., 2011. Système d'indicateurs spatialisés pour la gouvernance territoriale: application à l'occupation de sols en zone périurbaine languedocienne. Thèse de doctorat en géographie.

Balestrat M., Barbe E., Dupuy S., 2011. Analyse du potentiel agronomique des terres affectées par l'aménagement du territoire en Languedoc-Roussillon: Phase 2 2009-2010. Application des méthodologies de quantification des terres artificialisées et de caractérisation des dynamiques de consommation des terres sur les départements littoraux du Languedoc-Roussillon.

Balestrat M., Barbe E., Dupuy S., Lagacherie P., Meynard T., 2008. Analyse du potentiel des terres agricoles affectées par l'aménagement du territoire. Étude méthodologique sur une zone pilote (département de l'Hérault, 34). Rapport d'étude, Cemagref, Inra, Draaf LR.

Béchet B., Le Bissonnais Y., Ruas A., Aguilera A., Andrieu H., Barbe E., et al., 2017. Sols artificialisés et processus d'artificialisation des sols : déterminants, impacts et leviers d'action. Synthèse du rapport d'expertise scientifique collective, Ifsttar-Inra, 127.

Dupuy S., Barbe E., Balestrat M., 2012. An object-based image analysis method for monitoring land conversion by artificial sprawl use of RapidEye and IRS data. Remote sensing, 4, 404-423.

Oncea, 2014. Panorama de la quantification de l'évolution nationale des surfaces agricoles. Travail coordonné par le ministère de l'Agriculture, de l'Agroalimentaire et de la Forêt, Direction générale des politiques agricole, agroalimentaire et des territoires, sous-direction de la biomasse et de l'environnement, bureau du foncier et de la biodiversité. 



\title{
Chapitre 3
}

\section{Consommation des terres agricoles en Languedoc-Roussillon : système d'indicateurs et démarche participative}

\author{
Maud Balestrat, Éric Barbe, Jean-Pierre Chery, Jean-Philippe \\ TONNEAU
}

\section{Introduction}

Dans l'ancienne région Languedoc-Roussillon, comme dans toutes les zones à forte croissance urbaine, les acteurs du secteur agricole doivent convaincre les élus et l'ensemble de la société de l'intérêt de préserver les sols aux meilleures potentialités productives. En effet, ces potentialités sont peu prises en compte dans les politiques d'aménagement et les procédures d'urbanisme. Cela peut s'expliquer en partie par le manque d'informations disponibles. En conséquence, les acteurs agricoles souhaitent disposer d'outils de mesure spatialisés pour analyser les dynamiques de consommation des terres et sensibiliser à l'importance de la perte d'un patrimoine agronomique ${ }^{10}$.

Qui dit mesure dit indicateurs. Les expériences menées dans de nombreux pays et institutions (Pingault et Préaault, 2007 ; Roussel, 2007) montrent la difficulté de choisir des indicateurs qui rendent compte de la complexité des phénomènes. Dans une optique d'exhaustivité, une réponse est souvent de développer des listes présentant de nombreux indicateurs sectoriels qui s'avèrent, dans les faits, peu opérationnelles (Chamaret et al., 2006 ; Lavoux, 2006 ; Rey-Valette et al., 2008a,b ; Lyytimäki et al., 2013 ; Reinhard et Markus, 2013). Une représentation systémique est nécessaire pour identifier et caractériser les relations de cause à effet, expliquant la dynamique de l'étalement urbain et ses impacts sur les terres agricoles. C'est pourquoi nous avons choisi de développer un système d'indicateurs. Les indicateurs peuvent être en nombre, et ainsi prendre en compte la complexité des situations, mais ils sont structurés dans un ensemble cohérent (Joerin et al., 2005). Un système d'indicateurs découle d'un modèle. La modélisation des différents phénomènes qui interviennent dans l'évolution des surfaces artificialisées permet d'identifier les éléments et les relations déterminants, qui seront objets de la mesure.

Cependant, la production d'indicateurs ne suffit pas pour améliorer les choix d'aménagement. L'information produite par le système d'indicateurs s'insère dans des démarches,

10. Le patrimoine agronomique des sols dépend de la qualité intrinsèque des sols, liée à leurs caractéristiques pédologiques, qui détermine leur potentialité productive en fonction d'un type de culture spécifique. Il s'agit donc des sols «méritant d'être transmis du passé » (d'après Lazzarotti, 2003) pour trouver une valeur — dans le présent — de potentiel de production pour l'avenir. 
des méthodes, des procédures et des pratiques d'aménagement largement marquées par un implicite : l'artificialisation des espaces pour répondre aux besoins des activités industrielles et tertiaires, secteurs considérés comme garants du développement, a été longtemps encouragée. Ces dernières décennies, la prise de conscience de la dégradation des espaces naturels a conduit à leur protection. L'espace agricole est devenu la seule marge de manœuvre d'extension de l'urbain face à la nouvelle et forte valeur environnementale de certains espaces (Boutet et Serrano, 2013 ; Gueringer et al., 2016). Pour modifier cet état de fait, la préservation des terres agricoles nécessite d'impliquer les acteurs ayant a priori un rôle dans la gestion des terres agricoles. Cette implication passe par une approche participative. La participation constitue un des principes fondamentaux du développement durable, mais elle est d'intensité diverse et les résultats sont souvent limités. Des typologies existent (Reed, 2008 ; Barbier et Larrue, 2011), basées sur le degré d'engagement des parties prenantes (inspirées des travaux d'Arnstein, 1969) et sur les flux de communication entre parties prenantes (de la communication à la coconstruction en passant par la consultation). Les évaluations montrent que le niveau de participation reste généralement peu élevé et le plus souvent limité à certaines catégories sociales. La plupart des dispositifs connaissent des difficultés de mobilisation sur la durée. Ces limites sont souvent liées à la faible capacité d'influence ou à la difficulté de sortir des phases de diagnostic. Les procédures participatives sont trop souvent conçues par les élus comme un moyen de communication, de mobilisation, de contrôle et de légitimation à leur service, d'autant plus que ce sont souvent les mêmes qui participent, créant les conditions d'une cooptation par la sphère politique de « représentants légitimes ».

Au-delà de ces difficultés, l'intérêt des démarches participatives réside dans l'organisation d'un espace de dialogue qui permet l'expression des acteurs et qui interroge des représentations normées et figées. De nouvelles relations et de nouveaux supports pour la réflexion et l'action en découlent, en créant confiance et synergies. Les effets d'apprentissage sont importants : il en résulte des compétences nouvelles et des expériences à capitaliser.

La modélisation d'accompagnement, ou modélisation participative (Bousquet et al. 1999 ; Lynam, 2002 ; Voinov et Bousquet, 2010), tente de répondre à ce double enjeu : lecture de la complexité et implication des acteurs. Elle associe modélisation et espace de dialogue social. La modélisation vient en appui à une communauté d'acteurs - c'està-dire à un ensemble de personnes physiques ou morales - qui décident de s'organiser pour s' « emparer » et traiter d'un enjeu prioritaire.

À partir de l'analyse de nombreuses expériences, Voinov et Bousquet (2010) donnent un certain nombre d'orientations: favoriser les itérations, les adaptations; former l'ensemble des acteurs à l'utilisation des instruments, du point de vue à la fois conceptuel et opérationnel ; utiliser les outils les plus adaptatifs possible... Une dernière remarque est faite sur la nécessaire qualité de l'animation et de la facilitation en citant Creighton (2005), qui affirme que la "participation est un art et non une science» (p. 245). En résumé, c'est le processus de coconstruction du modèle qui va être déterminant. La qualité et la pertinence du modèle vont être jugées en fonction de sa capacité à porter la réflexion, les échanges, la planification, l'évaluation, etc.

Ce sont ces orientations que nous avons mises en œuvre dans une démarche modélisatrice et participative. Cette démarche a été élaborée dans le cadre d'une étude commanditée par la Draaf ${ }^{11}$ Languedoc-Roussillon (aujourd'hui Draaf Occitanie) à un collectif de 
recherche pour étudier la consommation des sols par l'artificialisation et qui fait l'objet du présent chapitre.

C'est cette démarche que nous souhaitons exposer ici en présentant successivement la méthode, le contexte de l'étude de cas, sa mise en œuvre et le système d'indicateurs produits.

\section{Une démarche modélisatrice et participative itérative pour aboutir à la production d'un système d'indicateurs}

\section{Modéliser le système territorial}

Le territoire est polysémique, mais les différentes définitions convergent (Ferrier, 2003). Le territoire est une entité, une portion de la surface terrestre aménagée et administrée par un groupe social pour répondre à ses besoins, en fonction d'un projet. C'est à la fois un espace de ressources, un espace de projet et un espace de gestion (territoire administratif). La diversité et la variété des éléments qui font un « territoire » et sa complexité justifient qu'il soit considéré comme un système, le système territorial ${ }^{12}$, qu'il convient de représenter grâce à la modélisation (Le Berre, 1992 ; Joerin et Rondier, 2007 ; Moine, 2008).

Un modèle est «une représentation schématique de la réalité élaborée en vue d'une démonstration » (Hagget, 1965, cité par Ferras, 1993). Il permet de mettre en relief une logique d'organisation et constitue une aide à la lecture de la complexité des processus qui produisent les territoires.

Modéliser implique plusieurs étapes. La première est consacrée à l'observation du fonctionnement du système territorial étudié. La seconde étape est celle de l'analyse systémique qui consiste ensuite à « définir les frontières du système à modéliser », à identifier les différents éléments le composant et les «types d'interactions entre ces éléments 》 et à « déterminer les liaisons qui les intègrent en un tout organisé » (De Rosnay, 1975, p. 122). La troisième étape est celle de la représentation du système réel étudié.

L'exercice de modélisation permet de construire une représentation intégrée et partagée qui aide à la compréhension du fonctionnement du système étudié. Le modèle constitue aussi un support pour mutualiser les savoirs locaux et confronter les représentations des acteurs qui participent à son élaboration. Enfin, la modélisation oriente l'élaboration d'un système d'indicateurs. Ce type de méthode permet de dépasser l'approche des tableaux de bord et des listes d'indicateurs sectoriels proposés par thématiques. L'élaboration d'un système peut aussi permettre d'éviter les redondances et les lacunes entre indicateurs.

\section{Adapter le modèle DPSIR}

Le choix d'un cadre d'analyse n'est pas neutre car il oriente ensuite l'approche modélisatrice. Il doit donc se faire en cohérence avec le système à modéliser et la problématique d'étude. Le modèle DPSIR (Driving forces, Pressures, State, Impacts, Responses; forces motrices-pressions-états-impacts-réponses) a été considéré comme pertinent pour aider à l'identification et à la compréhension des enjeux du territoire liés aux dynamiques d'artificialisation des sols, sous réserve de l'adapter (Balestrat et al., 2011 ; 2013). Proposé en

l'autorité du préfet de région, elles exercent leurs missions dans les domaines de compétence du ministère chargé de l'agriculture.

12. Eckert (1996) en propose la définition suivante : « Le système territorial est formé par l'arrangement des relations entre les lieux et les acteurs de l'espace considéré, pris dans leur environnement. » 
1998 par l'Agence européenne de l'environnement (Borja et al., 2006) comme extension du modèle PER (pression-état-réponse) de l'Organisation de coopération et de développement économiques (OCDE) (Maurizi et Verrel, 2002), le modèle DPSIR est une représentation schématique des interactions entre la société et l'environnement. Nous l'avons adapté en considérant l'état d'un système territorial, produit des activités humaines pour valoriser les ressources d'un territoire. Cela permettait notamment de dépasser une vision déterministe des mécanismes physiques, simplement accélérés ou atténués par des facteurs sociaux. En outre, le système n'était pas réduit à un écosystème ou à un milieu naturel, ceux-ci ne correspondant pas nécessairement à l'échelon de gestion des territoires, le système territorial étant aussi administré et approprié par un groupe humain.

Les indicateurs de forces motrices permettent d'exprimer les facteurs indirects à l'origine d'une évolution interne d'un système en termes d'évolutions structurelles (économiques et sociales). Ce sont, par exemple, les effets de la mondialisation sur le développement d'un territoire. Les indicateurs de pression (positive ou négative) décrivent les incidences d'un système (milieu physique et humain environnant) sous l'effet de phénomènes liés à une action. Ce peut être l'augmentation des besoins en logements, l'arrivée de migrants ou l'augmentation de la pollution des ressources naturelles par les activités agricoles, etc. Comme leur nom l'indique, les indicateurs d'état mesurent l'état du système (à un instant $\mathrm{T}$ ) pour le comparer à une ou plusieurs situations antérieures. Les indicateurs d'impacts expriment l'ensemble des conséquences (positives ou négatives) induites par la variation d'un état sous l'effet des pressions. Les indicateurs de réponse mettent en évidence le degré d'efficacité des actions mises en œuvre pour contrer les effets non désirés des pressions. Il s'agit de mesures publiques ou de comportements privés adoptés pour contrer ou renforcer les effets induits des variations du système (impacts), lorsque ceux-ci ne sont plus acceptés. Ces réponses se répercutent, comme rétroactions, de façon positive ou négative, en modifiant les éléments de pression, en réorganisant et en conditionnant (directement ou indirectement) l'état du système et, dans une moindre mesure, en contribuant à infléchir les forces motrices.

\section{Analyser les besoins des acteurs}

La pertinence d'un indicateur peut être évaluée selon deux critères (Roth, 2002). Le premier est celui de la validité scientifique (ou fiabilité), l'indicateur devant être scientifiquement fondé sur des données sûres et représentatives ; le second est celui de la légitimité politique (ou acceptabilité), le système d'indicateurs n'étant utile que s'il est accepté par l'ensemble des utilisateurs. Il doit d'abord être en adéquation avec la question que les acteurs se posent. Mais l'acceptation dépend d'un processus d'appropriation, fruit d'un travail de coconstruction (Offredi, 2005). Les acteurs doivent réfléchir ensemble aux différentes dimensions du champ que couvre la question, aux critères d'évaluation et donc au choix des indicateurs, au niveau d'agrégation requis et à la pérennité des indicateurs. La légitimité d'un système d'indicateurs se construit ainsi au fur et à mesure du processus de coconception avec l'ensemble des parties concernées. Il s'agit donc d'accompagner les acteurs à la fois dans la formulation des objectifs à atteindre et dans l'appropriation des connaissances nécessaires à la compréhension et à l'utilisation du système d'indicateurs.

L'implication des acteurs doit partir d'une démarche volontaire. L'« accompagnant », dans notre cas le chercheur, encourage et dynamise la participation en multipliant les phases d'interactions et en fournissant des supports graphiques et théoriques utiles pour enrichir les échanges (modèles, système d'indicateurs prototypes, cartes, graphiques, 
photographies aériennes, etc.). Ces échanges multiples ont pour principal objectif d'identifier les indicateurs les plus représentatifs et adaptés aux besoins des acteurs.

\section{Confronter le modèle à l'analyse des besoins pour guider le choix des indicateurs}

Pour orienter et organiser le processus de sélection des indicateurs, nous nous sommes inspirés des travaux de Rey-Valette (Rey-Valette et al., 2008a), formalisés dans la méthode « principes-critères-indicateurs ». Le travail conduit avec les acteurs permet de hiérarchiser les enjeux prioritaires (qui reflètent les principes défendus par les acteurs et leurs représentations du territoire). Ces enjeux sont traduits en actions concrètes à mettre en œuvre pour y répondre. Il s'agit alors d'identifier des critères pour mesurer ces enjeux, c'est-à-dire de choisir des «variables aptes à rendre compte de ces principes ». Ces critères ou variables peuvent ensuite être mesurés sous forme d'indicateurs, c'est-àdire sous forme d'indices et de valeurs seuils.

Desthieux (2005) a démontré, à partir des notions de convergence, de divergence et de redondance, comment la confrontation des représentations des acteurs peut guider le processus de sélection des indicateurs. Les acteurs se retrouvent généralement sur un certain nombre d'éléments stratégiques (convergence). En revanche, certains éléments, dont « la perception de l'amplitude ou la mise en relation», suscitent des désaccords, « révèlent des conflits d'interprétation sur les faits réels" (divergences) (Desthieux, 2005, p 145). Enfin, les critères pour traduire les enjeux retenus comme prioritaires peuvent se traduire, en termes de mesure, par des informations similaires (redondance). La figure 3.1 illustre le processus de sélection des indicateurs, confrontant les résultats de la modélisation et de l'analyse approfondie des besoins et guidé par ces trois conditions.

Groupe 1

Enjeu prioritaire

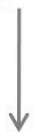

Critère de mesure
Groupe 2

Enjeu prioritaire

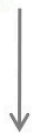

Critère de mesure
Groupe 3

Enjeu prioritaire

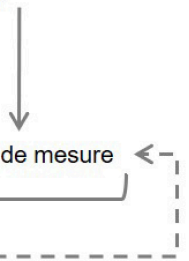

Contribue à

faire évolue

Critère de mesure
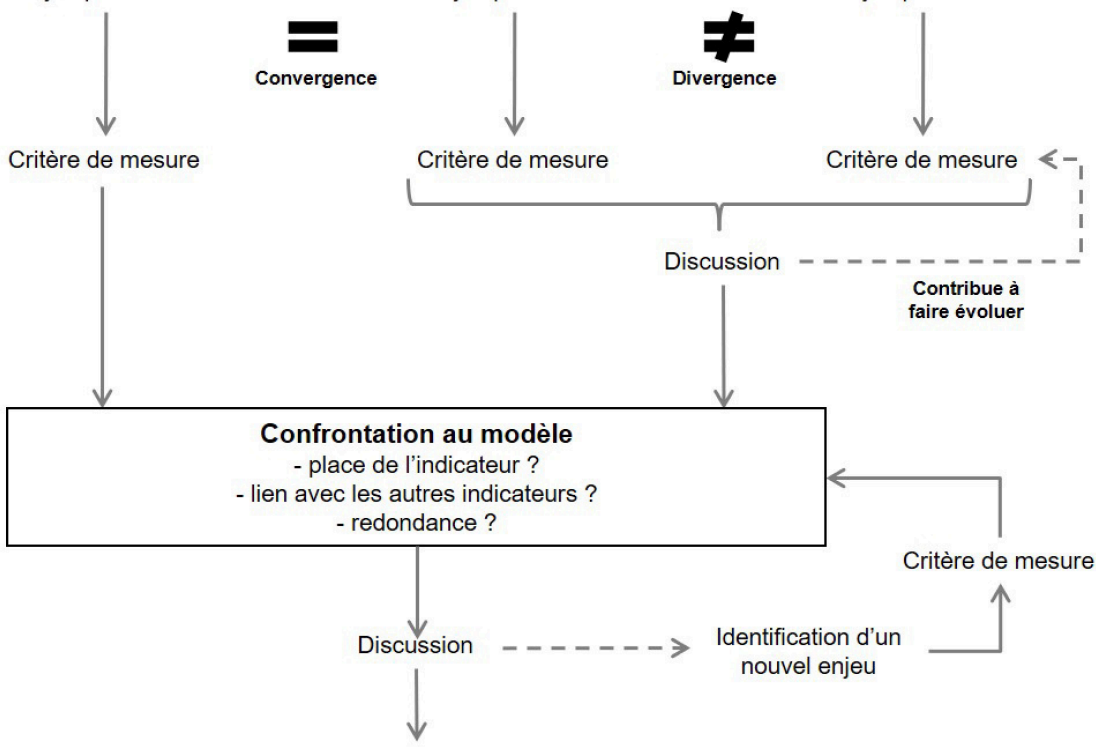

Choix des indicateurs les plus représentatifs

Figure 3.1. Processus de sélection des indicateurs composant le système d'indicateurs final (source : Balestrat, 2011). 
Le modèle, discuté avec les acteurs, permet d'identifier les indicateurs qui apparaissent les plus adaptés pour objectiver les décisions en matière de planification. Les relations causales entre indicateurs reposent sur celles identifiées entre les éléments du système. En reprenant chacune de ces interactions, les acteurs sélectionnent les indicateurs les plus représentatifs pour les qualifier (figure 3.2).

L'approche analytique et exploratoire, présentée ici pour trier les éléments et relations, n'est qu'un support pour effectuer une première proposition d'un ensemble d'indicateurs les plus pertinents. Ces indicateurs peuvent être des indices. Le terme « indice » désigne un indicateur synthétique construit en agrégeant d'autres indicateurs dits « de base ». La production d'indices nous a ainsi permis de proposer des indicateurs inter-reliés permettant de caractériser les relations causales qui établissent qu'un phénomène est la cause d'un autre. L'indice va ainsi exprimer la relation de causalité entre deux phénomènes (ex. : impacts ou réponses engendrés par la variation de l'état du système territorial).

Le choix définitif des indicateurs est ensuite inévitablement restreint par des contraintes de disponibilité des données. Il est donc également nécessaire d'identifier les sources d'information disponibles quant à leur exhaustivité, leur qualité et leur pertinence.

\section{Mise en œuvre de la démarche}

La démarche a été mise en œuvre pour l'analyse et le suivi de l'artificialisation des sols. Nous illustrons ses différentes étapes en présentant les résultats obtenus.

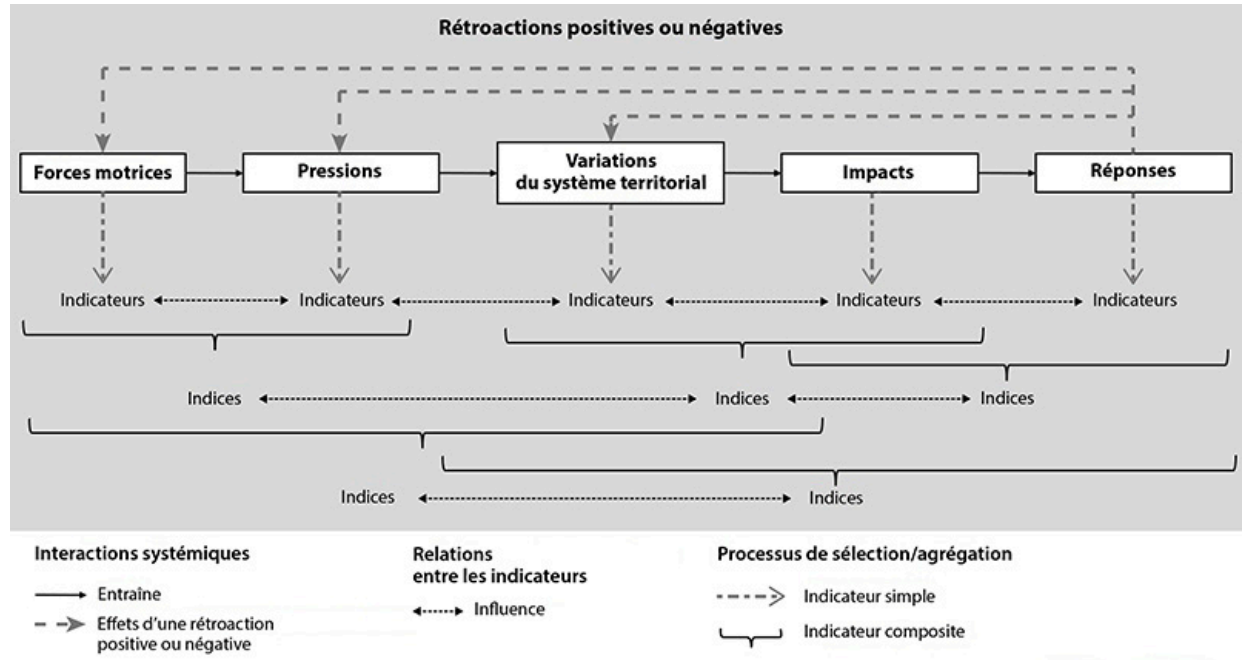

Figure 3.2. Processus de sélection des indicateurs sur la base du modèle DPSIR adapté (source : Balestrat, 2011).

\section{Modélisation des dynamiques d'artificialisation du capital foncier languedocien}

Le modèle DPSIR adapté a d'abord permis de caractériser les dynamiques de périurbanisation et d'identifier leurs relations de cause à effet dans le contexte languedocien d'artificialisation du capital foncier ${ }^{13}$ (figure 3.3). 


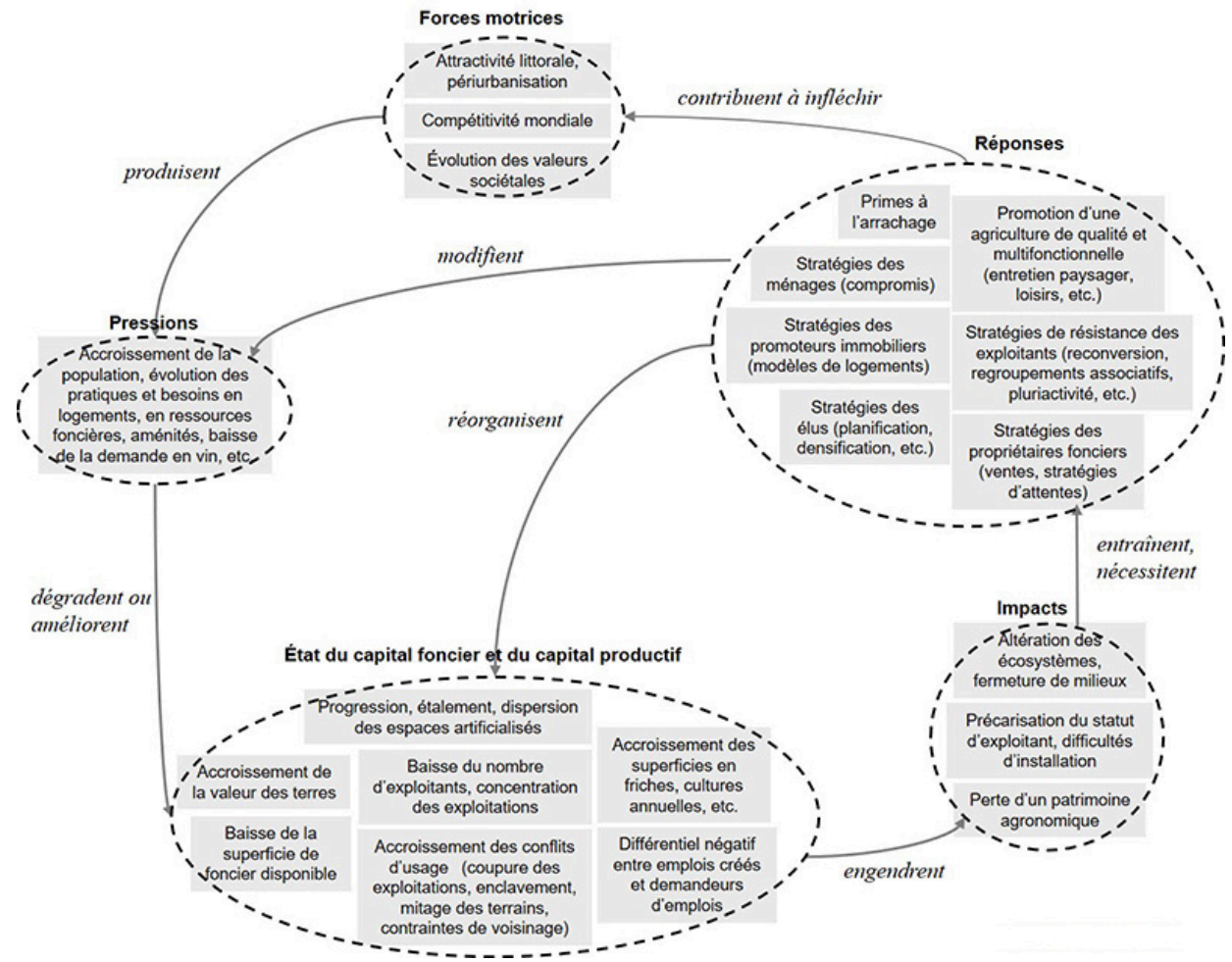

Figure 3.3. Modèle des dynamiques de périurbanisation du capital foncier languedocien, dans les années 2000 (figure réalisée par Maud Balestrat).

Dans l'ancienne région Languedoc-Roussillon, située sur la côte méditerranéenne (figure 3.4), l'attractivité littorale favorise une croissance démographique soutenue qui se manifeste par une forte dynamique de périurbanisation.

Les logiques de compétitivité mondiale entraînent une concurrence accrue sur la production agricole, en particulier viticole. L'évolution des valeurs sociétales se traduit par une homogénéisation et une généralisation des modes de vie urbains. La pression urbaine et périurbaine se manifeste par une diminution des superficies foncières disponibles et par un accroissement de la valeur des terres, qui favorisent une concurrence accrue sur l'usage des ressources foncières et se répercutent sur le système productif. Pour se maintenir, l'activité agricole, en zones périurbaine et rurale, doit désormais satisfaire une demande urbaine de qualité du cadre de vie (paysagère, environnementale, etc.) et de loisir. La pression urbaine se traduit par des difficultés de transmission et une concentration des exploitations qui conduisent à une précarisation du statut d'exploitant. Les stratégies de résistance s'expriment par des mécanismes de reconversion, les exploitations pour se maintenir se diversifient et se « labellisent». La labellisation consiste à faire reconnaître certaines caractéristiques d'un produit ou d'un processus de fabrication. La labellisation se matérialise généralement par la présence d'un logo. Les labels sont source de différenciation et garantes de qualité. La labellisation permet une meilleure rémunération des productions. 


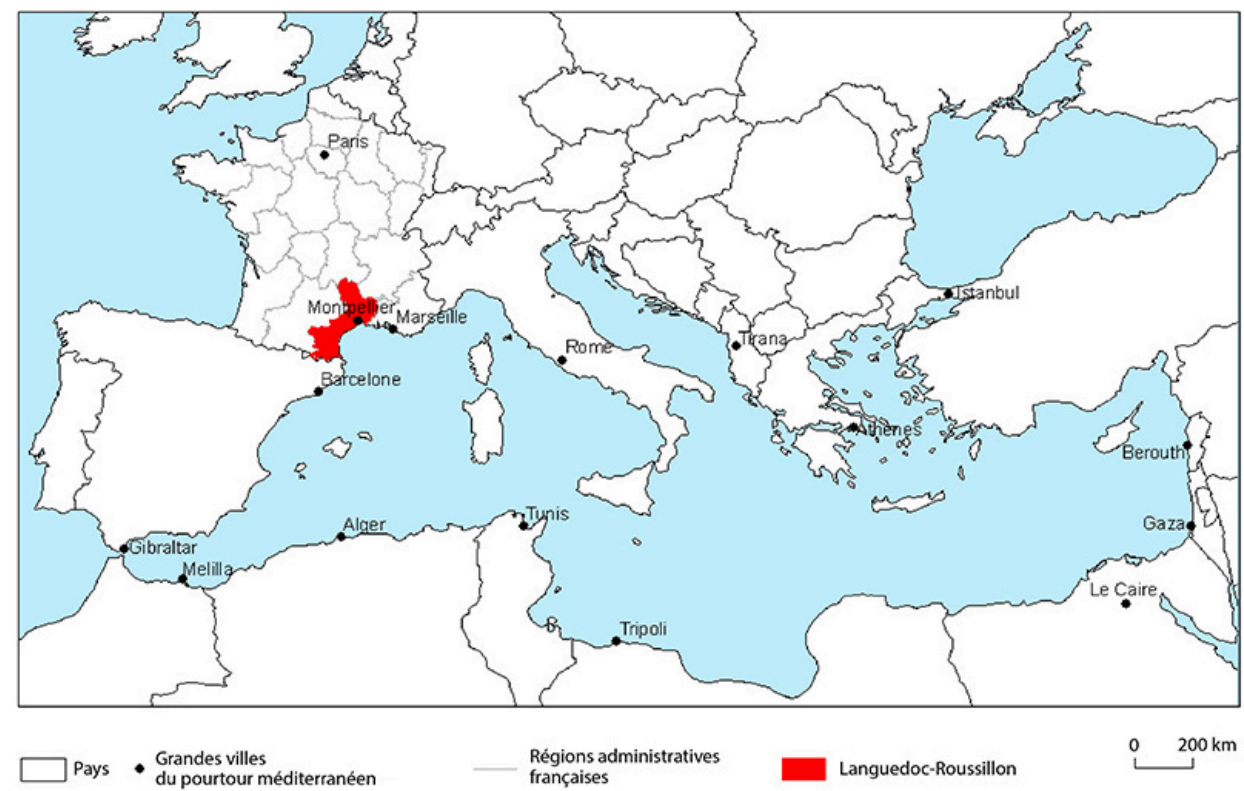

Figure 3.4. Localisation de l'ancienne région Languedoc-Roussillon dans le bassin méditerranéen (figure réalisée par les auteurs).

\section{Résultats de l'analyse approfondie des besoins}

\section{Contexte et organisation}

La volonté de participation a conduit à élargir le débat au-delà de la problématique soulevée par la Draaf Languedoc-Roussillon, principalement centrée sur des enjeux agricoles. Nous avons mobilisé d'autres acteurs publics œuvrant pour l'aménagement et la planification du territoire languedocien. Une analyse fine de la demande, des statuts et fonctions des acteurs mobilisés a été essentielle pour comprendre leurs différents degrés d'intervention (Balestrat et al., 2011). Une série d'entretiens en groupes restreints a été conduite de novembre 2008 à juillet 2009. Diverses tables rondes, ouvertes à un large panel d'acteurs, ont été organisées. Trois formations, portant sur la conduite de démarches de production d'indicateurs de diagnostic des territoires en matière de dynamiques d'artificialisation des sols, ont également été mises en place, mobilisant chacune une dizaine d'acteurs des services déconcentrés de l'État. Enfin, trois journées de restitution ont informé un panel plus large d'acteurs sur l'avancée du projet. Au total, une cinquantaine d'acteurs institutionnels ont été mobilisés et se sont investis, à des degrés divers, pendant la durée du projet.

\section{Une présélection des indicateurs adaptée aux besoins des acteurs}

À l'origine du projet, les besoins exprimés par la Draaf Languedoc-Roussillon étaient doubles : un besoin en outils pour quantifier et spatialiser la perte de terres à potentiel agricole, afin de contribuer à l'analyse globale des dynamiques à l'œuvre et de sensibiliser à l'importance de la perte du patrimoine agronomique ; un besoin en outils pour qualifier la potentialité agronomique des sols à accueillir les grandes cultures, afin d'appuyer l'expertise et l'argumentaire des représentants du monde agricole lors de l'élaboration des documents d'urbanisme. 
La confrontation des différents points de vue des acteurs mobilisés (agents des DDT(M), du CG34, de la Dreal, de plusieurs EPCI et collectivités) ${ }^{14}$ sur la convergence et la divergence des enjeux (figure 3.1) a ensuite permis un classement des enjeux identifiés comme prioritaires selon leurs critères de mesure et leurs indicateurs correspondants. Les acteurs ont identifié quatre grands enjeux :

- sensibiliser à la préservation du patrimoine agronomique des sols ;

- freiner les dynamiques d'artificialisation des sols (étalement, mitage, fragmentation) ;

- maintenir et favoriser le dynamisme de l'activité agricole ;

- maintenir et favoriser les capacités de développement durable du territoire.

Tableau 3.1. Structuration des critères de mesure pour répondre aux enjeux prioritaires.

\begin{tabular}{|c|c|c|c|c|}
\hline Critères & $\begin{array}{l}\text { Enjeu 1. Sensibi- } \\
\text { liser à la préser- } \\
\quad \text { vation du } \\
\text { patrimoine agro- } \\
\text { nomique des sols }\end{array}$ & $\begin{array}{l}\text { Enjeu 2. Freiner les } \\
\text { dynamiques d'artifi- } \\
\text { cialisation des sols }\end{array}$ & $\begin{array}{l}\text { Enjeu 3. Main- } \\
\text { tenir et favoriser } \\
\text { le dynamisme de } \\
\text { l'activité agricole }\end{array}$ & $\begin{array}{l}\text { Enjeu 4. Maintenir et } \\
\text { favoriser les capacités } \\
\text { de développement } \\
\text { durable du territoire }\end{array}$ \\
\hline 1 & $\begin{array}{l}\text { Caractéristiques et } \\
\text { variation du } \\
\text { patrimoine } \\
\text { agronomique initial } \\
\text { (avant } \\
\text { artificialisation) }\end{array}$ & $\begin{array}{l}\text { État et variation du degré } \\
\text { d'artificialisation des sols }\end{array}$ & $\begin{array}{l}\text { Caractéristiques et } \\
\text { dynamisme des } \\
\text { exploitations } \\
\text { (reconversion, } \\
\text { espaces de déprise, } \\
\text { etc.) }\end{array}$ & $\begin{array}{l}\text { Caractéristiques et } \\
\text { variations du système } \\
\text { productif } \\
\text { (complémentarité et } \\
\text { dynamisme des secteurs } \\
\text { d'activités, création } \\
\text { d'emplois, etc.) }\end{array}$ \\
\hline 2 & $\begin{array}{l}\text { Caractéristiques et } \\
\text { variation du } \\
\text { patrimoine } \\
\text { agronomique } \\
\text { artificialisé } \\
\text { (définitivement } \\
\text { perdu) }\end{array}$ & $\begin{array}{l}\text { État et variation de } \\
\text { l'occupation du sol } \\
\text { initiale (espaces agricoles, } \\
\text { naturels avant } \\
\text { artificialisation) }\end{array}$ & $\begin{array}{l}\text { Caractéristiques et } \\
\text { dynamisme des } \\
\text { exploitants agricoles } \\
\text { et agriculteurs } \\
\text { (capacités de reprise, } \\
\text { ancienneté des } \\
\text { exploitants, etc.) }\end{array}$ & \\
\hline 3 & & $\begin{array}{l}\text { État et variation du degré } \\
\text { d'étalement, de mitage et } \\
\text { de fragmentation des } \\
\text { terres }\end{array}$ & $\begin{array}{l}\text { État et variation des } \\
\text { espaces agricoles } \\
\text { (cultivés ou non) }\end{array}$ & \\
\hline 4 & & $\begin{array}{l}\text { État et variation de la } \\
\text { disponibilité foncière } \\
\text { (vocation des sols dans } \\
\text { les documents } \\
\text { d'urbanisme) }\end{array}$ & & \\
\hline 5 & & $\begin{array}{l}\text { Caractéristiques et } \\
\text { dynamiques du marché } \\
\text { foncier }\end{array}$ & & \\
\hline 6 & & $\begin{array}{l}\text { État et variation du niveau } \\
\text { d'attractivité du territoire } \\
\text { et de la répartition des } \\
\text { populations }\end{array}$ & & \\
\hline 7 & & $\begin{array}{l}\text { Caractéristiques et } \\
\text { variation du parc de } \\
\text { logements }\end{array}$ & & \\
\hline
\end{tabular}

14. DDT(M) : Direction départementale des territoires (et de la mer) ; CG : Conseil général ; Dreal : Direction régionale de l'environnement, de l'aménagement et du logement ; EPCI : Établissement public de coopération intercommunale. 
L'analyse des interactions entre chercheurs et acteurs révèle des préoccupations différentes et des enjeux communs. Les enjeux $n^{\circ} 1$ et $n^{\circ} 4$ ont fait débat, notamment entre la Draaf et la Dreal. Les positions reflètent les mandats et actions respectives en matière d'agriculture et d'équipement. L'enjeu $\mathrm{n}^{\circ} 2$ était plus consensuel et traduisait les préoccupations liées aux dynamiques d'artificialisation, difficiles à mesurer et à maîtriser. Nous rejoignons ici Rey-Valette et al. (2008b), qui défendent l'idée que la diversité des résultats ne doit pas être interprétée comme une faiblesse. Au contraire, elle exprime les divergences des points de vue des différents groupes d'acteurs mobilisés, résultats du processus d'apprentissage mis en œuvre. Ce processus laisse une large place au débat et s'en inspire pour enrichir la coconstruction du système d'indicateurs.

Pour chacun des quatre enjeux, des critères de mesure pertinents du point de vue des acteurs et traduisant les différents niveaux de préoccupation, ont été identifiés (tableau 3.1). Ces critères ont été formulés de façon volontairement synthétique pour favoriser les échanges et faciliter la compréhension de la démarche suivie. Cette relative simplicité favorise également l'implication volontaire du plus grand nombre tout au long du processus de coconstruction.

L'organisation des critères de mesure, présentée dans le tableau 3.1, constitue une base de référence qui a guidé le travail de sélection des indicateurs. Le tableau 3.2 présente les résultats pour l'enjeu 1 et le critère 1 (pour l'ensemble des résultats, voir Balestrat, 2011).

La mise en débat des critères de choix des indicateurs a été l'occasion de discuter non seulement la portée et l'intérêt de chaque indicateur, mais également les données à mobiliser pour leur calcul et leur mise en œuvre.

\section{Le choix définitif des indicateurs}

L'exercice de modélisation renvoie à la pertinence spatio-temporelle des indicateurs à retenir et donc à l'information spatiale à mobiliser. L'importance du phénomène de littoralisation a fait émerger le besoin de centrer spatialement le système d'indicateurs sur la plaine languedocienne. Les données spatiales exploitées devaient permettre de construire le système d'indicateurs en référence aux années 2000, période de profondes mutations spatiales (occupation et usage du sol) dans laquelle s'inscrit l'action des acteurs (à l'origine de l'étude sur l'analyse du potentiel agronomique affecté par l'aménagement du territoire languedocien) en matière de planification territoriale.

Les échanges sur la finalité du système d'indicateurs ont fait émerger la nécessité de disposer de données spatiales qui permettent, d'une part, de qualifier le potentiel agronomique des sols et, d'autre part, de mesurer et de suivre la consommation de ce potentiel par les espaces artificialisés. Les notions clés d'espaces artificialisés et de potentiel agronomique des sols ont été précisées et acceptées par tous (Balestrat et al., 2013). Les phénomènes ont été ainsi caractérisés sur une base commune qui a donné du sens et de la légitimité aux indicateurs produits. 
Tableau 3.2. Extrait des indicateurs retenus et classés par critères.

\begin{tabular}{|c|}
\hline Enjeu 1. Sensibiliser à la préservation du patrimoine agronomique des sols \\
\hline Critère 1. Caractéristiques et variation du patrimoine agronomique initial \\
\hline Superficies (ha) et répartition des classes de potentiel agronomique initial \\
\hline Part (\%) des classes de potentiel agronomique par rapport à la superficie de la zone d'étude \\
\hline Superficie (ha) et répartition des espaces non artificialisés par classe de potentiel agronomique des sols \\
\hline $\begin{array}{l}\text { Part }(\%) \text { des espaces non artificialisés par classe de potentiel agronomique des sols par rapport à la } \\
\text { superficie initiale de la classe de potentiel agronomique des sols }\end{array}$ \\
\hline $\begin{array}{l}\text { Part }(\%) \text { des espaces non artificialisés par classe de potentiel agronomique des sols par rapport à la } \\
\text { superficie totale des espaces non artificialisés }\end{array}$ \\
\hline $\begin{array}{l}\text { Part }(\%) \text { des espaces non artificialisés par classe de potentiel agronomique des sols par rapport à la } \\
\text { superficie de la zone d'étude }\end{array}$ \\
\hline Variation de la superficie (ha) des espaces non artificialisés par classe de potentiel agronomique des sols \\
\hline Évolution (\%) des espaces non artificialisés par classe de potentiel agronomique des sols \\
\hline $\begin{array}{l}\text { Superficies (ha) et répartition des espaces non artificialisés par classe de potentiel agronomique compris } \\
\text { dans un périmètre irrigable (présence d'équipements) }\end{array}$ \\
\hline $\begin{array}{l}\text { Part (\%) des espaces non artificialisés par classe de potentiel agronomique compris dans un périmètre } \\
\text { irrigable (présence d'équipements) par rapport à la superficie de la zone d'étude }\end{array}$ \\
\hline $\begin{array}{l}\text { Part }(\%) \text { des espaces non artificialisés par classe de potentiel agronomique compris dans un périmètre } \\
\text { irrigable (présence d'équipements) par rapport à la superficie non artificialisée de la classe de potentiel } \\
\text { agronomique correspondante }\end{array}$ \\
\hline $\begin{array}{l}\text { Variation de la superficie (ha) non artificialisée des classes de potentiel agronomique comprise dans un } \\
\text { périmètre irrigable (présence d'équipements) }\end{array}$ \\
\hline $\begin{array}{l}\text { Évolution de la superficie (\%) des espaces non artificialisés par classe de potentiel agronomique compris } \\
\text { dans un périmètre irrigable (présence d'équipements) }\end{array}$ \\
\hline
\end{tabular}

Pour produire les indicateurs spatialisés quantifiant les espaces consommés par l'artificialisation, nous avons opté pour la production de données continues et homogènes à l'échelle de l'ancienne région Languedoc-Roussillon. Deux méthodes de production de données spatiales ont été développées : une méthode pour mesurer et suivre l'emprise des espaces artificialisés qui s'appuie sur le traitement d'images satellitaires pour la production de taches artificialisées (Dupuy et al., 2012) ; une méthode de qualification du potentiel agronomique des sols qui s'appuie sur la construction et la spatialisation d'un indice de qualité des sols (chapitre 2).

\section{Production d'un système d'indicateurs appliqué à l'artificialisation des sols en zone languedocienne}

Le système d'indicateurs produit est composé de 141 indicateurs spatialisés pouvant être déclinés à différentes mailles d'analyse (départementale, intercommunale, etc.) et sur différents territoires d'étude (région, départements, groupes de communes, etc.). Le tableau 3.3 présente un extrait des indicateurs produits classés par grands objectifs (ex. : localiser et quantifier le potentiel agronomique des sols initial, les espaces artificialisés). 
Tableau 3.3. Extrait des indicateurs composant le système d'indicateurs final.

\begin{tabular}{|c|c|c|c|c|c|}
\hline $\begin{array}{l}\text { Objectif pour- } \\
\text { suivi }\end{array}$ & Description & $\begin{array}{c}\text { Maille } \\
\text { d'analyse }\end{array}$ & Étendue & Données & $\begin{array}{c}\text { Place dans le } \\
\text { système } \\
\text { d'indicateurs }\end{array}$ \\
\hline \multicolumn{6}{|c|}{ Potentiel agronomique des sols initial } \\
\hline \multirow{2}{*}{$\begin{array}{l}\text { Localiser et } \\
\text { quantifier le } \\
\text { potentiel } \\
\text { agronomique des } \\
\text { sols initial avant } \\
\text { toute } \\
\text { artificialisation }\end{array}$} & $\begin{array}{l}\text { Superficie (ha) } \\
\text { initiale des classes } \\
\text { de potentiel } \\
\text { agronomique des } \\
\text { sols (CPAS) }\end{array}$ & \multirow{2}{*}{$\begin{array}{l}\text { Départementale, } \\
\text { cantonale, } \\
\text { communale }\end{array}$} & \multirow{2}{*}{$\begin{array}{l}\text { Région } \\
\text { entière }\end{array}$} & \multirow{2}{*}{$\begin{array}{l}\text { Potentiel } \\
\text { agronomique des } \\
\text { sols }\end{array}$} & \multirow{2}{*}{$\begin{array}{l}\text { État du capital } \\
\text { foncier }\end{array}$} \\
\hline & $\begin{array}{l}\text { Part (\%) initiale } \\
\text { des CPAS par } \\
\text { rapport à la } \\
\text { superficie totale de } \\
\text { la zone d'étude }\end{array}$ & & & & \\
\hline \multicolumn{6}{|c|}{ Artificialisation des sols } \\
\hline \multirow{4}{*}{$\begin{array}{l}\text { Localiser et } \\
\text { quantifier les } \\
\text { espaces } \\
\text { artificialisés }\end{array}$} & $\begin{array}{l}\text { Superficie (ha) des } \\
\text { espaces } \\
\text { artificialisés en } \\
1997\end{array}$ & \multirow{4}{*}{$\begin{array}{l}\text { Départementale, } \\
\text { cantonale, } \\
\text { communale }\end{array}$} & \multirow[b]{2}{*}{$\begin{array}{l}\text { Départements } \\
\text { littoraux }\end{array}$} & \multirow{6}{*}{$\begin{array}{l}\text { Taches } \\
\text { artificialisées } \\
\text { (sans routes, } \\
\text { grandes routes et } \\
\text { toutes routes) }\end{array}$} & \multirow{4}{*}{$\begin{array}{l}\text { État du capital } \\
\text { foncier }\end{array}$} \\
\hline & \begin{tabular}{|l|} 
Part $(\%)$ des \\
espaces \\
artificialisés en \\
1997 par rapport à \\
la superficie de la \\
zone d'étude
\end{tabular} & & & & \\
\hline & $\begin{array}{l}\text { Superficie (ha) des } \\
\text { espaces } \\
\text { artificialisés en } \\
2009\end{array}$ & & \multirow[b]{2}{*}{$\begin{array}{l}\text { Région } \\
\text { entière }\end{array}$} & & \\
\hline & $\begin{array}{l}\text { Part (\%) des } \\
\text { espaces } \\
\text { artificialisés en } \\
2009 \text { par rapport à } \\
\text { la superficie de la } \\
\text { zone d'étude }\end{array}$ & & & & \\
\hline \multirow{2}{*}{$\begin{array}{l}\text { Évaluer le degré } \\
\text { d'artificialisation } \\
\text { des sols entre } \\
\text { deux dates }\end{array}$} & $\begin{array}{l}\text { Variation de la } \\
\text { superficie (ha) des } \\
\text { espaces } \\
\text { artificialisés entre } \\
1997 \text { et } 2009 \\
\end{array}$ & \multirow{2}{*}{$\begin{array}{l}\text { Départementale, } \\
\text { cantonale, } \\
\text { communale }\end{array}$} & \multirow{2}{*}{$\begin{array}{l}\text { Départements } \\
\text { littoraux }\end{array}$} & & \multirow{2}{*}{$\begin{array}{l}\text { Variation du } \\
\text { capital foncier }\end{array}$} \\
\hline & $\begin{array}{l}\text { Évolution (\%) de la } \\
\text { superficie des } \\
\text { espaces } \\
\text { artificialisés entre } \\
1997 \text { et } 2009\end{array}$ & & & & \\
\hline \multicolumn{6}{|c|}{ Potentiel agronomique des sols perdu } \\
\hline $\begin{array}{l}\text { Localiser et } \\
\text { quantifier les sols } \\
\text { artificialisés en } \\
\text { fonction de leur }\end{array}$ & $\begin{array}{l}\text { Superficie (ha) des } \\
\text { espaces } \\
\text { artificialisés par } \\
\text { CPAS en } 1997\end{array}$ & $\begin{array}{l}\text { Départementale, } \\
\text { cantonale }\end{array}$ & $\begin{array}{l}\text { Départements } \\
\text { littoraux }\end{array}$ & $\begin{array}{l}\text { Potentiel } \\
\text { agronomique des } \\
\text { sols }+ \text { taches } \\
\text { artificialisées }\end{array}$ & $\begin{array}{l}\text { État du capital } \\
\text { foncier }\end{array}$ \\
\hline
\end{tabular}




\begin{tabular}{|c|c|c|c|c|c|}
\hline $\begin{array}{l}\text { Objectif pour- } \\
\text { suivi }\end{array}$ & Description & $\begin{array}{c}\text { Maille } \\
\text { d'analyse }\end{array}$ & Étendue & Données & $\begin{array}{l}\text { Place dans le } \\
\text { système } \\
\text { d'indicateurs }\end{array}$ \\
\hline \multirow{7}{*}{$\begin{array}{l}\text { potentiel } \\
\text { agronomique }\end{array}$} & $\begin{array}{l}\text { Part }(\%) \text { des } \\
\text { espaces } \\
\text { artificialisés par } \\
\text { CPAS en 1997 par } \\
\text { rapport à la } \\
\text { superficie de la } \\
\text { CPAS initiale }\end{array}$ & & & \multirow{8}{*}{$\begin{array}{l}\text { (sans routes, } \\
\text { grandes routes et } \\
\text { toutes routes) }\end{array}$} & \multirow[b]{8}{*}{$\begin{array}{l}\text { Variation } \mathrm{du} \\
\text { capital foncier }\end{array}$} \\
\hline & \begin{tabular}{|l|} 
Part (\%) des \\
espaces \\
artificialisés par \\
CPAS par rapport à \\
la superficie totale \\
des espaces \\
artificialisés en \\
1997
\end{tabular} & & & & \\
\hline & $\begin{array}{l}\text { Part }(\%) \text { des } \\
\text { espaces } \\
\text { artificialisés par } \\
\text { CPAS en 1997 par } \\
\text { rapport à la } \\
\text { superficie de la } \\
\text { zone d'étude }\end{array}$ & & & & \\
\hline & $\begin{array}{l}\text { Superficie (ha) des } \\
\text { espaces } \\
\text { artificialisés par } \\
\text { CPAS en } 2009\end{array}$ & & \multirow{4}{*}{$\begin{array}{l}\text { Région } \\
\text { entière }\end{array}$} & & \\
\hline & $\begin{array}{l}\text { Part }(\%) \text { des } \\
\text { espaces } \\
\text { artificialisés par } \\
\text { CPAS en } 2009 \text { par } \\
\text { rapport à la } \\
\text { superficie de la } \\
\text { CPAS initiale }\end{array}$ & & & & \\
\hline & $\begin{array}{l}\text { Part (\%) des } \\
\text { espaces } \\
\text { artificialisés par } \\
\text { CPAS par rapport à } \\
\text { la superficie totale } \\
\text { des espaces } \\
\text { artificialisés en } \\
2009\end{array}$ & & & & \\
\hline & $\begin{array}{l}\text { Part }(\%) \text { des } \\
\text { espaces } \\
\text { artificialisés par } \\
\text { CPAS en } 2009 \text { par } \\
\text { rapport à la } \\
\text { superficie de la } \\
\text { zone d'étude }\end{array}$ & & & & \\
\hline $\begin{array}{l}\text { Évaluer la perte } \\
\text { de ressources } \\
\text { foncières, entre } \\
\text { deux dates, en } \\
\text { fonction de leur }\end{array}$ & $\begin{array}{l}\text { Variation de la } \\
\text { superficie (ha) de } \\
\text { sols non } \\
\text { artificialisés } \\
\text { (espaces agricoles }\end{array}$ & & $\begin{array}{l}\text { Départements } \\
\text { littoraux }\end{array}$ & & \\
\hline
\end{tabular}




\begin{tabular}{|c|c|c|c|c|c|}
\hline $\begin{array}{l}\text { Objectif pour- } \\
\text { suivi }\end{array}$ & Description & $\begin{array}{c}\text { Maille } \\
\text { d'analyse }\end{array}$ & Étendue & Données & $\begin{array}{l}\text { Place dans le } \\
\text { système } \\
\text { d'indicateurs }\end{array}$ \\
\hline \multirow{4}{*}{$\begin{array}{l}\text { potentiel } \\
\text { agronomique }\end{array}$} & $\begin{array}{l}\text { et naturels) par } \\
\text { CPAS }\end{array}$ & & & & \\
\hline & $\begin{array}{l}\text { Évolution de la } \\
\text { superficie (\%) de } \\
\text { sols non } \\
\text { artificialisés } \\
\text { (espaces agricoles } \\
\text { et naturels) par } \\
\text { CPAS }\end{array}$ & & & & \\
\hline & $\begin{array}{l}\text { Variation de la } \\
\text { superficie (ha) des } \\
\text { espaces } \\
\text { artificialisés par } \\
\text { CPAS entre } 1997 \text { et } \\
2009\end{array}$ & & & & \\
\hline & $\begin{array}{l}\text { Évolution (\%) des } \\
\text { espaces } \\
\text { artificialisés par } \\
\text { CPAS entre } 1997 \text { et } \\
2009\end{array}$ & & & & \\
\hline
\end{tabular}

La liste d'indicateurs retenus a été structurée sur la base du modèle conceptuel systémique. La figure 3.5, présentée et décrite ci-après, illustre l'une des nombreuses relations de causalité qui ont pu être mises en évidence et renseignées par les indicateurs retenus.

Variations de l'état

Impacts

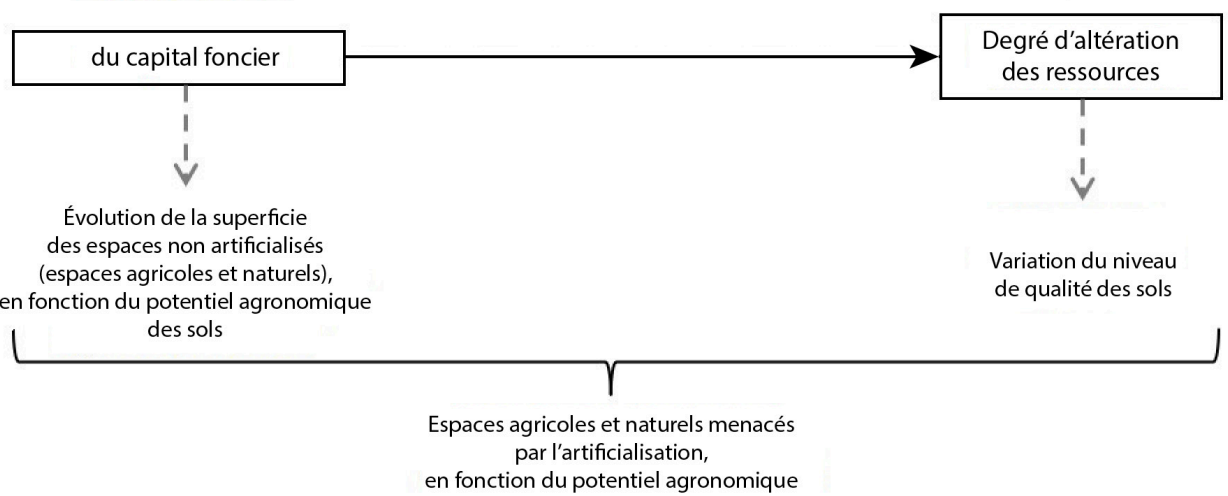

Figure 3.5. Exemple d'indicateurs représentatifs des interactions entre variations de l'état du capital foncier et impacts (source: Balestrat, 2011).

La variation de l'état du capital foncier disponible peut être évaluée en calculant l'évolution des superficies non artificialisées (espaces naturels et agricoles), en fonction du potentiel agronomique des sols. En termes d'impacts, il est possible de mesurer le degré d'altération des ressources environnementales en considérant la variation du niveau de qualité des sols. Il est alors envisageable d'extrapoler les superficies potentiellement menacées par l'extension des espaces construits, en fonction du potentiel agronomique des sols. En effet, les terres qui se trouvent à proximité directe d'espaces nouvellement 
artificialisés sont, a priori, davantage soumises à un risque d'artificialisation. Dans un objectif de prévention, pour déterminer les terres directement menacées, des critères de distance ont été définis avec les acteurs institutionnels, en fonction des tendances passées. Ce type d'indicateur doit cependant être complété pour pallier les risques liés à son interprétation qui pourraient conduire à surestimer les superficies ainsi mesurées. Une exploitation tournée vers la production d'une Appellation d'origine contrôlée $(\mathrm{AOC})^{15}$ ou un espace naturel classé devraient, a priori, mieux résister à la pression foncière que génère la construction d'une zone résidentielle ou commerciale, comparativement à un espace agricole faiblement rentable, qui se trouve par ce fait dans une situation nouvelle d'enclavement.

Un autre type de relation (forces motrices/pressions) peut être caractérisé par la densité nette, c'est-à-dire l'évolution du nombre d'habitants rapportée au nombre d'hectares artificialisés et non à la superficie. Cette donnée exprime " grossièrement » la tendance à la compacité (étalement) des espaces artificialisés (pression/variation du capital foncier) (figure 3.6).

La carte ci-dessus représente l'évolution de la densité nette à une maille communale. Une évolution positive du nombre d'habitants par hectare artificialisé est synonyme d'une densification ; une évolution négative indique une tendance à l'étalement. Les communes périphériques du pôle urbain montpelliérain subissent une tendance à l'étalement.

Les indicateurs produits sont accompagnés de fiches informatives détaillées (figure 3.7). Celles-ci fournissent, pour chaque indicateur, un ensemble d'informations techniques (mode de calcul, données mobilisées, maille d'analyse, etc.), des éléments d'aide à son interprétation (limites d'utilisation, comparabilité spatio-temporelle, etc.), et donnent sa position au sein du système (ex. : l'indicateur A exprime une réponse, l'indicateur B exprime le lien entre une force motrice et une pression). Une table de liaison permet de connaître les relations entre indicateurs (ex. : l'indicateur A influence les indicateurs B et E et est influencé par l'indicateur C).

L'ensemble organisé d'indicateurs a été mis à disposition des acteurs mobilisés dans la coconstruction du système d'indicateurs par l'intermédiaire d'un prototype d'interface de consultation en ligne. Celle-ci s'appuyait sur une automatisation du calcul des indicateurs et sur un mode de restitution dynamique des cartes et graphiques statistiques. L'outil a été spécifiquement adapté aux besoins des utilisateurs (acteurs de la Draaf et des DDTM en charge de la gestion des espaces agricoles) et structuré sur le modèle du système d'indicateurs afin de restituer les interactions entre indicateurs. La plateforme web développée spécifiquement pour accompagner la prise de décision a servi de support à l'évaluation des indicateurs par les utilisateurs du système d'indicateurs.

15. L'Appellation d'origine contrôlée (AOC) désigne « des produits répondant aux critères de l'AOP [Appellation d'origine protégée] et protège la dénomination sur le territoire français. Elle constitue une étape vers l'AOP, désormais (depuis 2009) signe européen. Elle peut aussi concerner des produits non couverts par la réglementation européenne (cas des produits de la forêt par exemple). C'est la notion de terroir qui fonde le concept des Appellations d'origine » (Inao, 2017). 


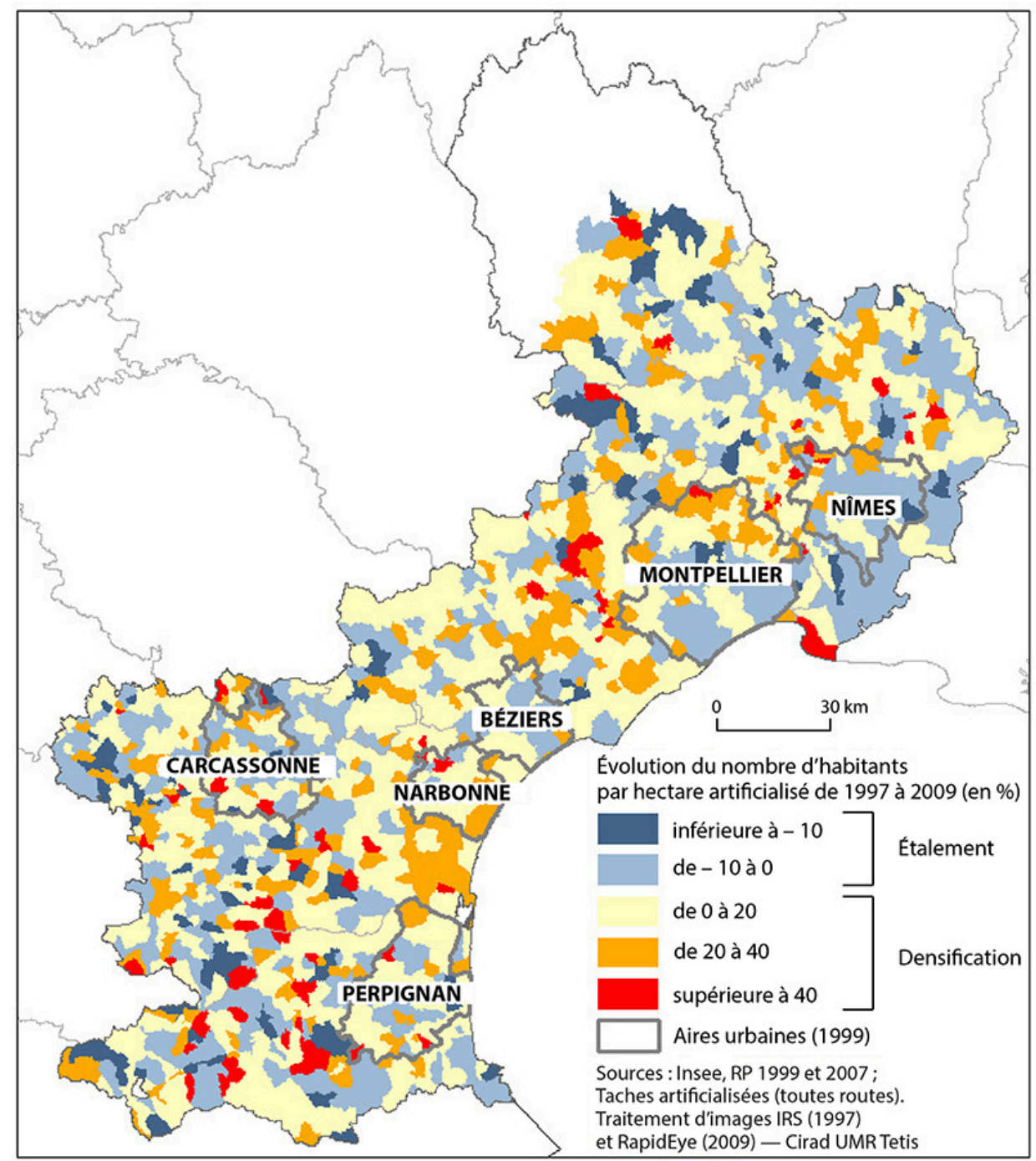

Figure 3.6. Évolution du nombre d'habitants par hectare artificialisé de 1997 à 2009 (source : Balestrat, 2011). 


\begin{tabular}{|c|c|}
\hline Thématique & Artificialisation des sols \\
\hline Sous-Thématique & Degré d'étalement urbain \\
\hline Identifiant & coeff_dispersion_gdes_routes_09 \\
\hline $\begin{array}{l}\text { Description de } \\
\text { l'indicateur }\end{array}$ & $\begin{array}{l}\text { Coefficient (\%) de dispersion, rapport (\%) entre la superficie occupée par les } \\
\text { taches artificialisées (grandes routes) inférieures à } 3 \text { ha et la superficie occupée } \\
\text { par les taches artificialisées (grandes routes) supérieures à } 3 \text { ha en } 2009\end{array}$ \\
\hline Nature & Etat \\
\hline \multicolumn{2}{|c|}{ Informations techniques } \\
\hline $\begin{array}{l}\text { Description de la } \\
\text { donnée source }\end{array}$ & $\begin{array}{l}\text { Tache artificialisée grandes routes issue d'une méthode de dilatation / érosion } \\
\text { (création de zones tampons positives puis négatives de } 50 \mathrm{~m} \text { de rayon) } \\
\text { appliquée aux "espaces artificialisés" (Tissu urbain, Zones industrielles et } \\
\text { commerciales, Décharges carrières et chantiers) extraits des images satellites } \\
\text { Rapide Eye 2009. Tache agrégée aux grandes routes issue de la BDCarto } \odot \text { ) } \\
\text { IGN 2000. }\end{array}$ \\
\hline $\begin{array}{l}\text { Précision de la } \\
\text { donnée source }\end{array}$ & Mosaïque d'images satellites Rapide Eye résolution 5 m couleur pour 2009. \\
\hline Etendue & Quatre départements littoraux du Languedoc-Roussillon + sud Lozère \\
\hline Maille d'analyse & Région, Départements, catégories ZAUER, Cantons, Communes \\
\hline Unité & Hectares \\
\hline Date & $1997-2009$ \\
\hline $\begin{array}{l}\text { Nomenclature de } \\
\text { restitution }\end{array}$ & espaces artificialisés grandes routes \\
\hline Méthode de calcul & $\begin{array}{l}\text { Calcul de la superficie de la superficie de la tache artificialisée (grandes routes) } \\
\text { inférieure à 3ha en } 2009 \text { par rapport à la superficie de la tache artificialisée } \\
\text { (grandes routes) supérieure à 3ha en } 2009 . \\
\text { CoefDisp } 09=\frac{T A G R 09 \operatorname{Inf} 3}{T A G R 09 \operatorname{Sipp} 3} \\
\text { TAGR09Inf3 = Superficie (ha) occupée par les taches artificialisées inférieures à } \\
3 \text { ha en } 2009 \\
\text { TAGR09Sup3 }==\text { Superficie (ha) occupée par les taches artificialisées } \\
\text { supérieures à } 3 \text { ha en } 2009\end{array}$ \\
\hline \multicolumn{2}{|c|}{ Evaluation de l'information } \\
\hline $\begin{array}{l}\text { Pertinence des } \\
\text { données par rapport } \\
\text { à l'objectif de } \\
\text { l'indicateur }\end{array}$ & $\begin{array}{l}\text { L'objectif de l'indicateur est d'offrir un suivi dans le temps de l'artificialisation des } \\
\text { sols dispersée et isolée. Les taches artificialisées produites ont une précision } \\
\text { relative à la résolution de l'image traitée, pour une échelle de restitution au } \\
1 / 15000 \text { e. }\end{array}$ \\
\hline $\begin{array}{l}\text { Comparabilité dans } \\
\text { le temps }\end{array}$ & $\begin{array}{l}\text { Le rapport entre la superficie des taches artificialisées inférieures et supérieures } \\
\text { à } 3 \text { ha est comparable d'une année à lautre à condition de tenir compte des } \\
\text { différences de résolution entre les données sources utilisées pour produire les } \\
\text { taches artificialisées. }\end{array}$ \\
\hline $\begin{array}{l}\text { Comparabilité dans } \\
\text { l'espace }\end{array}$ & $\begin{array}{l}\text { Le rapport entre la superficie des taches artificialisées inférieures et supérieures } \\
\text { à } 3 \text { ha est comparable entre les communes, les cantons et les départements. }\end{array}$ \\
\hline Limites d'utilisation & $\begin{array}{l}\text { L'indicateur offre un ordre de grandeur de la part que représente la superficie } \\
\text { des espaces artificialisés inférieures à } 3 \text { ha relatif à la résolution des images } \\
\text { traitées et à la méthode appliquée à l'échelle de l'espace régional. }\end{array}$ \\
\hline $\begin{array}{l}\text { Aide à } \\
\text { l'interprétation }\end{array}$ & $\begin{array}{l}\text { La part de la superficie des espaces artificialisés inférieure à 3ha indique } \\
\text { l'importance des espaces artificialisès (grandes routes) définitivement perdus } \\
\text { (non mobilisables pour la production agricole) dispersés et isolés. }\end{array}$ \\
\hline \multicolumn{2}{|c|}{ Position au sein du système d'indicateur } \\
\hline \multicolumn{2}{|c|}{$\begin{array}{l}\text { Indicateur qui exprime l'état du capital foncier en lien avec les pressions (variation des besoins en } \\
\text { logements) et les réponses apportées (stratégies des communes en termes de planification) }\end{array}$} \\
\hline
\end{tabular}

Figure 3.7. Fiche de l'indicateur d'état « Degré d'étalement urbain ». Exemple d'aide à l'interprétation fournie aux acteurs pour accompagner le système d'indicateurs final (source : Balestrat, 2011). 


\section{Évaluation de la démarche}

\section{Apports et limites de l'approche modélisatrice}

L'expérience nous a permis de confirmer les hypothèses avancées sur l'utilité du modèle comme support d'échanges et de réflexivité. Les principales difficultés de l'exercice de modélisation ont été de concilier la possibilité pour chaque acteur de partager ses connaissances de terrain, indispensables à l'enrichissement des savoirs de tout un chacun (acteurs et chercheurs), et la nécessité de rattacher les discussions à un cadre d'analyse rigoureux pour ne pas perdre des éléments essentiels à la compréhension du système. Nous avons dû, en particulier, répondre à des risques de démobilisation des acteurs liés aux décalages nombreux entre une approche théorique et les besoins opérationnels de l'action, auxquels doivent répondre, au quotidien, les acteurs institutionnels. La démarche modélisatrice devait être suffisamment souple, condition indispensable à l'implication volontaire de chacun dans la démarche. La capacité du modèle à répondre aux questionnements des acteurs garantissait leur intérêt et leur mobilisation. Il est difficile de dépasser l'approche sectorisée qui semble faciliter, de prime abord, l'intelligibilité des phénomènes, mais qui fait perdre les liens/interactions entre les différents critères analysés. La parole d'un acteur à l'occasion d'un atelier de travail portant sur le modèle est révélatrice : "Pourquoi ne s'intéresse-t-on pas à chaque thématique, une à une, pour ensuite les répartir sur le modèle? »Certains ont manifesté une relative frustration de devoir se restreindre à un cadre théorique. Sur ce point, l'itération est apparue comme une clé essentielle pour construire une vision partagée et assurer la légitimité du système d'indicateurs aux yeux du plus grand nombre.

\section{Des enjeux liés à l'interprétation et à l'opérationnalité des indicateurs}

Le choix d'un indicateur dépend des représentations que l'utilisateur se fait de la réalité. La mobilisation des acteurs, dès le stade de définition des objectifs, a été une condition indispensable pour fournir des éléments d'appui à l'interprétation. Outre l'importance d'utiliser des concepts et techniques partagés, supports à la définition d'objectifs « communs » et reconnus par tous, cette mobilisation a permis d'entamer un processus de vulgarisation de connaissances techniques indispensables à la compréhension et à l'exploitation du système d'indicateurs. Les discussions ont mis en avant la nécessité d'assortir la diffusion de la donnée d'une documentation précise et de formations adaptées aux publics concernés (services de l'État, collectivités et bureaux d'études). Les acteurs ont soulevé le besoin d'être formés afin d'extraire une information utile et utilisable.

En matière d'opérationnalité, les premiers retours d'expérience sur l'utilisation du système d'indicateurs ont mis en évidence la nécessité de vulgariser les notions et techniques d'analyse spatiale afin d'utiliser un langage commun. Malgré la simplicité d'utilisation que requiert un « bon indicateur », il paraît pourtant indispensable aux utilisateurs, sinon de maîtriser, du moins d'appréhender les langages et procédés qui ont conduit à son élaboration. La tâche est peu aisée car les indicateurs se fondent sur des connaissances scientifiques et des techniques parfois « complexes ». Les contraintes liées à la disponibilité des données ont également constitué un frein important pour produire un système d'indicateurs répondant aux ambitions de départ.

Cependant, on peut noter que nombre d'indicateurs ont éclairé, voire confirmé, la perception du phénomène d'artificialisation et la nécessité de prendre en compte le potentiel agronomique des sols dans les projets d'aménagement. Désormais, nombre d'utilisateurs 
mobilisent couramment une sélection d'indicateurs pour faire valoir les enjeux à l'œuvre sur le territoire.

\section{Conclusion et perspectives}

L'approche proposée ouvre des perspectives intéressantes pour améliorer l'usage de modèles dans les processus d'élaboration d'un système d'indicateurs. L'implication des acteurs, tout au long du processus de coconstruction, a permis de mettre en évidence les enjeux liés à l'interprétation et à l'opérationnalité du système d'indicateurs produit. Le choix de proposer une approche modélisatrice et participative itérative oblige à concevoir des méthodes et outils novateurs. Il faut envisager des méthodes qui permettent de prendre en compte la multiplicité des points de vue et le choix de supports adéquats pour favoriser la mobilisation à chaque étape. Ces interrogations, sur les options à retenir pour fournir des méthodes et outils adaptés aux négociations, soulèvent d'autres questions liées à la communication et à l'opérationnalité, qui passent, nous semble-t-il, nécessairement par un accompagnement des acteurs tout au long du processus de coconstruction du système d'indicateurs.

La mise à disposition de ces informations change la donne. Les porteurs des enjeux stratégiques de la production agricole à long terme peuvent désormais se saisir de ces indicateurs et des cartographies comme source d'argumentaires, de démonstrations et d'outils d'aide à la décision pour souligner leur intérêt et accompagner les procédures d'aménagement. Il reste à apprécier l'impact de ces informations auprès des nombreux acteurs de l'aménagement du territoire, et vérifier notamment si elles favorisent les consensus ou si elles constituent plutôt de nouvelles sources de tensions. Ces points ont fait l'objet d'une recherche supplémentaire présentée dans le chapitre 7.

\section{Références bibliographiques}

Arnstein S.R., 1969. A ladder of citizen participation. Journal of the American Institute of Planners, 35 (4), 216-224.

Balestrat M., 2011. Système d'indicateurs spatialisés pour la gouvernance territoriale: application à l'occupation des sols en zone périurbaine languedocienne. $\mathrm{PhD}$, univérsité Paul-Valéry, Montpellier-III.

Balestrat M., Barbe É., Chery J.-P., Lagacherie P., Tonneau J.-P., 2011. Reconnaissance du patrimoine agronomique des sols : une démarche novatrice en Languedoc-Roussillon. Norois, 221 (4), 83-96.

Balestrat M., Chéry J.-P., Tonneau J.P., 2013. Co-construction d'un système d'indicateurs spatialisés de la consommation des terres agricoles en Languedoc-Roussillon : une démarche d'implication des acteurs du territoire. In: Géogouvernance : utilité sociale de l'analyse spatiale (M. Masson-Vincent, N. Dubus, coord.), Versailles, éditions Quæ, 215.

Barbier R., Larrue C., 2011. Démocratie environnementale et territoires : un bilan d'étape. Participations, 1 (1), 67-104.

Borja Á., Galparsoro I., Solaun O., Muxika I., Tello E.M., Uriarte A., Valencia V., 2006. The European Water Framework Directive and the DPSIR, a methodological approach to assess the risk of failing to achieve good ecological status. Estuarine, Coastal and Shelf Science, 66 (1), 84-96.

Bousquet F., Barreteau O., Mullon C., Weber J., 1999. An environmental modelling approach. The use of multi-agents simulations. In: Advances in Environmental and Ecological Modelling (F. Blasco, A. Weill, eds), Paris, Elsevier, 113-122.

Boutet D., Serrano J., 2013. Les sols périurbains, diversification des activités et des valeurs. Quelques éléments de comparaison et d'analyse. Économie rurale, 338 (6), 5-23. 
Chamaret A., O’Connor M., Recoche G., 2006. Approche top-down/bottom-up pour l'élaboration d'indicateurs de développement durable applicables au secteur minier. L'exemple des mines d'uranium du Niger. In: Colloque international "Usages des indicateurs de développement durable. Mise en relation des pratiques et savoirs pour les ressources marines et les territoires littoraux avec les acquis d'autres domaines et espaces ", Montpellier, Réseau Indicateurs de développement durable et durabilité, 15.

Creighton M., 2005. The Public Participation Handbook: Making Better Decisions Through Citizen Involvement, Wiley.

De Rosnay J., 1975. Le macroscope. Vers une vision globale, Paris, Éditions du Seuil.

Desthieux G., 2005. Approche systémique et participative du diagnostic urbain : processus de représentation cognitive du système urbain en vue de l'élaboration d'indicateurs géographiques. Thèse.

Dupuy S., Barbe E., Balestrat M., 2012. An object-based image analysis method for monitoring land conversion by artificial sprawl use of RapidEye and IRS data. Remote Sensing, 4 (2), 404-423.

Eckert, D., 1996. Évaluation et prospective des territoires. Montpellier/Paris, GIP Reclus/La Documentation française, coll. Dynamiques du territoire, $\mathrm{n}^{\circ} 16$.

Ferras R., 1993. Les modèles graphiques en géographie, Paris, Economica/RECLUS.

Ferrier J.P., 2003. Territoires. In : Dictionnaire de la géographie et de l'espace des sociétés (J. Lévy, M. Lussault, coord.), Paris, Belin, 912-917.

Gueringer A., Hamdouch A., Wallet F., 2016. Foncier et développement des territoires ruraux et périurbains en France. Une mise en perspective. Revue d'économie régionale et urbaine, octobre (4), 693-712.

Hagget P., 1965. Locational Analysis in Human Geography, London, Arnold.

Inao, 2017. AOP-AOC, Institut national de l'origine et de la qualité, https://www.inao.gouv.fr/ Les-signes-officiels-de-la-qualite-et-de-1-origine-SIQO/

Appellation-d-origine-protegee-Appellation-d-origine-controlee .

Joerin F., Rondier P., 2007. Les indicateurs et la décision territoriale. Pourquoi ? Quand? Comment? In: Les indicateurs socioterritoriaux. Perspectives et renouvellement (G. Sénécal, ed.), chapitre 1, Laval, Presses de l'Université de Laval, 9-36.

Joerin F., Lebreton M., Desthieux G., 2005. Des systèmes d'indicateurs pour aider les acteurs à manipuler la complexité territoriale. In : "Développement urbain durable, gestion des ressources et gouvernance ", Colloque de l'Observatoire universitaire de la ville et du développement durable, Lausanne, 21-23 septembre 2005.

Lazzarotti O., 2003, Patrimoine. In : Dictionnaire de la géographie et de l'espace des sociétés (Levy J., Lussault M., eds), Paris, Belin, 692-693.

Lavoux T., 2006. L'offre et la demande d'indicateurs: les leçons des expériences internationales, nationales et locales. Colloque international Usages des indicateurs de développement durable. Mise en relation des pratiques et savoirs pour les ressources marines et les territoires littoraux avec les acquis d'autres domaines et espaces, organisé par le Réseau des indicateurs de développement durable et durabilité, 3-4 avril 2006, Montpellier.

Le Berre M., 1992. Le territoire. In : Encyclopédie de la géographie (R. Ferras, D. Pumain, A.S. Bailly, eds), Paris, Economica.

Lynam T., Bousquet F., Le Page C., D’Aquino P., Barreteau O., Chinembiri F., Mombeshora B., 2002. Adapting science to adaptive managers: Spidergrams, belief models, and multi-agent systems modeling. Conservation Ecology, 5 (2).

Lyytimäki J., Tapio P., Varho V., Söderman T., 2013. The use, non-use and misuse of indicators in sustainability assessment and communication. International Journal of Sustainable Development and World Ecology, 20 (5), 385-393. 
Maurizi B., Verrel J.L., 2002. Des indicateurs pour les actions de maîtrise des pollutions d'origine agricole. Ingénieries EAT, (30), 3-14.

Moine A., 2008. Le territoire : comment observer un système complexe, L'Harmattan.

Offredi C., 2005. La dynamique de l'évaluation face au développement durable, Paris, L'Harmattan/Société française d'évaluation.

Pingault N., Préaault B., 2007. Indicateurs de développement durable : un outil de diagnostic et d'aide à la décision. Notes et études économiques, 28, 43.

Reed M.S., 2008. Stakeholder participation for environmental management: A literature review. Biological Conservation, 141 (10), 2417-2431.

Reinhard S., Markus H., 2013. Objectives and indicators in sustainable development strategies: similarities and variances across Europe. Sustainable Development, 21 (4), 224-241.

Rey-Valette H., Clément O., Lazard J., 2008a. Les usages des indicateurs de développement durable. Compte rendu de colloque, Montpellier, 3-4 avril 2006, Natures Sciences Sociétés, 16 (1), 73-75.

Rey-Valette H., Clément O., Aubin J., Mathé S., Chia E., Legendre M., Caruso D., Mikolasek O., Blancheton J.P., Slembrouck J., Baruthio A., Rene F., Levang P., Morissens P., 2008b. Guide de co-construction d'indicateurs de développement durable en aquaculture, EVAD.

Roth I., 2002. Mesurer le développement durable. Bulletin SVU ASEP ASAP, 8, 8.

Roussel S., 2007. Efficacité d'une gestion intégrée de la zone côtière (GIZC). Thèse de doctorat, Montpellier.

Voinov A., Bousquet F., 2010. Modelling with Stakeholders. 



\title{
Chapitre 4
}

\section{Connaître les propriétés des sols pour éclairer les changements d'occupation du sol et l'aménagement du territoire. Une perspective australienne}

\author{
Pamela A. Hazelton
}

\section{Introduction}

Le développement de zones résidentielles, commerciales et industrielles a transformé l'utilisation des terres de l'ancienne frange périurbaine - l'espace en périphérie des zones urbaines. Ceci a entraîné la disparition de jardins maraîchers, de zones horticoles, ainsi que d'anciennes friches, qui pouvaient potentiellement abriter des espèces et des communautés écologiques en danger. Une fois les espaces naturels et agricoles urbanisés, la disponibilité de la ressource en sols change, puisque leurs conditions physicochimiques et biologiques sont altérées. Comme il n'existe pas de type de sol idéal pour remplir toutes les fonctions, il est nécessaire de comprendre l'importance des propriétés du sol propres à un site donné avant tout projet de développement urbain sur une zone agricole. Bénéficier d'informations sur la nature des sols assurera la préservation de zones propices à l'usage agricole ainsi que de certaines zones de paysages naturels.

\section{Changement d'usage du sol : du rural à l'urbain}

Dans les zones périurbaines, de larges portions de végétation primaire et de terres agricoles ont été converties en zones urbaines ou en banlieues à une vitesse supérieure au taux de croissance de la population urbaine (Hazelton et Clements, 2014). Cette conversion de végétation "primaire » ou de terres agricoles en zones urbaines, incluant les banlieues résidentielles, a connu une accélération dans les soixante dernières années avec l'expansion rapide de villes initialement compactes (Pickett et al., 2001). Ceci a eu pour conséquence la perte de terres à haute valeur agricole dans les zones périurbaines.

\section{Les zones périurbaines}

Des recherches ont montré que les zones périurbaines se développent en Europe et en Australie (Sinclair, 1996 ; Piorr et al., 2011) et que les sols sur lesquels elles se développent ne sont plus considérés comme une ressource utile (agricole ou naturelle), quel que soit leur usage passé (Pickett et al., 2001 ; Piorr et al., 2011). Le développement résidentiel est de loin le type de développement progressant le plus vite en Europe ainsi que dans les autres pays occidentaux. Par conséquent, la diversité d'utilisation des sols en zones périurbaines se trouve menacée. 
En France, par exemple, le taux de conversion des terres à un usage non agricole est équivalent à la surface d'un département français $(0,5 \mathrm{M}$ ha) tous les dix ans. Des tendances similaires peuvent être observées en Asie. En Chine, par exemple, la population de la ville de Nanjing est passée d'approximativement 700000 habitants en 1949 à 5,5 millions en 2009. Dans le même temps, la zone urbaine est passée de 42 à $577 \mathrm{~km}^{2}$ (Wei et al., 2013). Lorsque le développement urbain devient incontrôlé et entraîne la perte d'espaces ouverts, cela se traduit par une diminution en valeur absolue de terres agricoles et de zones naturelles tampons (EEA, 2006). Cependant, une part importante de l'alimentation des populations urbaines est produite sur les sols fertiles des zones périurbaines qui accueillent différents types d'habitats, des infrastructures ainsi que des activités agricoles (Mason et Knowd, 2010). Dès lors, le rôle des sols pour une agriculture durable et multifonctionnelle ainsi que pour la conservation de la nature devient vital dans une perspective globale de sécurité alimentaire (Hazelton et Murphy, 2011). De surcroît, l'environnement biophysique et le bien-être des populations locales se dégradent du fait de la disparition de zones récréatives et de friches, dont certaines contiennent les vestiges d'écosystèmes menacés (Hazelton et Clements, 2011).

Par conséquent, la diminution et finalement la disparition complète des zones périurbaines peuvent entraîner de nombreux problèmes sociétaux et environnementaux (Hazelton et Clements, 2014). De ce fait, l'impact des sociétés humaines sur les sols dépendra à la fois de l'ampleur des changements d'occupation du sol, des propriétés spécifiques des sols ainsi que du développement de futures stratégies de gestion durable (Hazelton et Clements, 2014).

\section{Comprendre et gérer les propriétés des sols}

Pour réduire l'impact humain, l'aménagement de l'espace requiert une approche plus holistique d'appréciation de la valeur des terres qui inclurait des compromis entre les dimensions sociales, économiques et environnementales (Dominati et al., 2010). Bien que traditionnellement la connaissance des propriétés des sols et de leur gestion technique ait considérablement influencé l'appréciation de leur capacité à soutenir une activité agricole, ceci est beaucoup moins le cas quand les sols sont convertis en habitat résidentiel. Étant donné qu'aucun type de sol ne peut remplir toutes les fonctions, il est nécessaire, avant de planifier un quelconque changement d'usage de l'espace, d'utiliser les données pédologiques locales pour s'assurer que les types de sols adaptés aux usages agricoles soient conservés. De la même façon, il est essentiel, pour que l'approche soit holistique, de conserver et de restaurer les zones boisées « primaires » (Tozer, 2003).

L'évaluation des propriétés des sols et des contraintes qui en découlent est nécessaire dans des contextes qui vont bien au-delà de l'agriculture et de l'horticulture. Il est donc capital qu'en matière de planification urbaine, l'importance et la pertinence de ces évaluations ne soient pas négligées. Cependant, dans les processus d'urbanisation, les propositions d'affectation d'usage du sol sur des sites donnés se fondent souvent sur des critères qui ne tiennent pas compte de facteurs pédologiques.

\section{Pourquoi l'évaluation des propriétés des sols est nécessaire}

Beaucoup d'exemples illustrent le besoin d'avoir à disposition des informations, aux échelles locale et parcellaire, sur les propriétés physiques, chimiques et biologiques des sols, lors de l'évaluation environnementale de projets de développement résidentiel. Un grand nombre de ces propriétés, telles que le $\mathrm{pH}$, la texture du sol, la teneur en argile et 
sa nature, la salinité, la teneur en sodium, les propriétés hydrauliques, la concentration en nutriments, sont connues et peuvent être gérées, in situ, dans les zones agricoles.

Si les aménageurs n'ont pas connaissance des contraintes posées par les propriétés des sols, ces dernières peuvent impacter de manière significative le futur du projet de développement et la gestion du site. Souvent, la conception, voire même la viabilité de nouveaux projets de développement peuvent être affectées (Pickett et al., 2001).

De surcroît, les projets de développement, une fois achevés, induisent un certain nombre de changements, tels que l'absorption de la chaleur par des surfaces imperméables, le nombre accru de sédiments contaminés et le ruissellement des eaux de pluie riches en nutriments alcalins. Ces changements impactent négativement l'activité microbiologique des sols, le cycle naturel des nutriments et la santé générale des sols. Une fois que le sol est imperméabilisé ou goudronné, les risques de réduction d'absorption des eaux de pluie sont significatifs (Burghardt, 2006 ; Kaye et al., 2006 ; Pouyet et al., 2007). Ces eaux de ruissellement peuvent devenir un problème majeur en provoquant des inondations à certains endroits, du fait de la saturation des réseaux pluviaux ou de cours d'eau. Elles peuvent aussi être contaminées par des fertilisants, entraînant une baisse de la qualité de l'eau et une dégradation des écosystèmes terrestres et aquatiques. Les nappes phréatiques peuvent, elles aussi, être contaminées (Hazelton et Murphy, 2011). De plus, les résidus de chantiers sur les sites des projets sont souvent enfouis sur place, ce qui affecte les propriétés physiques, chimiques et biologiques des sols (Hazelton et Murphy, 2011).

Le changement d'usage du sol n'impacte pas uniquement le site du projet, mais également les zones avoisinantes, ce qui se traduit par une perte des qualités paysagères et une diminution de la santé des sols. Par conséquent, la connaissance des contraintes posées par la nature des sols est essentielle pour déterminer si un changement d'affectation du sol sera un succès ou un échec. La section qui suit présente une étude de cas illustrant ce point.

\section{Étude de cas}

L'étude de cas présentée ici traite de l'installation de la première colonie européenne à Sydney, en Australie, à la fin du XVIII ${ }^{\mathrm{e}}$ siècle, et met en avant l'importance qu'a eue la connaissance des propriétés des sols. Pour que cette colonie puisse s'installer avec succès et durablement, ses membres ont dû en effet chercher à comprendre les propriétés des sols, en particulier le niveau de fertilité nécessaire à la sécurité alimentaire de la colonie. La compréhension du contexte australien a été particulièrement complexe pour les Anglais car ils n'avaient jusqu'alors jamais été confrontés à de tels types de sol, de végétation et de conditions climatiques.

\section{Les problèmes initiaux lors de l'implantation}

Dans les années 1790, la colonie pénitentiaire britannique s'installa tout d'abord autour de la baie de Sydney, où l'approvisionnement en eau était suffisant (Clark, 1963). Au premier abord, les terres de la baie ont paru fertiles avec la «perspective luxuriante de ses rivages couverts d'arbres jusqu'aux berges » (Tench, 1789). Cependant, lorsque les provisions de nourriture diminuèrent et que les tentatives de développement de l'agriculture échouèrent, les colons réalisèrent que le sol provenant du grès était peu profond, sableux et avait un faible potentiel agricole (Perry, 1963).

Finalement, les efforts pour développer la production agricole finirent par payer avec la découverte de zones argileuses et parfois même de poches de sols issus de résidus volcaniques à l'ouest de la colonie. Cette découverte permit à de petites communautés 
de se développer dans ces zones cultivables éparses (Spearritt, 1978 ; Benson et Howell, 1990 ; Hazelton et Clements, 2011). La répartition des terres arables détermina largement les schémas d'implantation et de défrichage des terres. En revanche, peu d'attention fut portée à la préservation de la végétation primaire, la principale préoccupation étant la sécurité alimentaire afin d'assurer la survie de la colonie. Durant le XIX siècle, l'élevage bovin et la production de produits agricoles devinrent la base de l'économie australienne.

\section{L'urbanisation}

Vers le milieu du $\mathrm{xx}^{\mathrm{e}}$ siècle, du fait des évolutions de l'économie, la population commença à converger des zones rurales vers les abords des villes, dont les périphéries commencèrent alors à s'étendre (Clark, 1963). Ainsi, en 2013, 85\% de la population australienne vivait en ville, avec des espaces agricoles transformés sous les effets de l'étalement urbain.

Les espaces agricoles ont progressivement été remplacés par des zones urbaines et périurbaines, avec un recouvrement des sols fertiles par du développement résidentiel. Une propriété in situ des sols telle que le retrait/gonflement (variable selon le type et la teneur en argile), qui a joué un rôle essentiel pour la production de cultures dans les zones agricoles fertiles de Sydney jusqu'à la fin du $\mathrm{Xx}^{\mathrm{e}}$ siècle, n'a plus été d'aucune utilité. Alors que cette propriété des sols argileux était bénéfique pour des terres agricoles productives, elle est devenue au contraire une contrainte coûteuse pour la construction de bâtiments et d'infrastructures.

Sur les terrains qui avaient été profondément modifiés, de sévères problèmes d'interface ont été observés, avec une végétation qui répondait mal aux aménagements paysagers (Handreck, 1994). Cela a souvent mené à une augmentation de l'érosion et du transport de sédiments dans les cours d'eau, au détriment des milieux terrestres et aquatiques.

La détérioration des milieux agricoles et naturels engendra des problèmes environnementaux, en plus de problèmes structurels. Une large proportion des endroits où des communautés écologiques avaient prospéré par le passé a d'abord été défrichée pour l'agriculture avant de se transformer, dans les cinquante dernières années, en zones de développement urbain. Les reliquats de végétation naturelle sont devenus les vestiges historiques de ce qu'étaient de vastes étendues de communautés végétales avant la colonisation de l'Australie et les implantations qui ont suivi. Ces vestiges de végétation, souvent situés dans des zones rurales en cours d'urbanisation ou en banlieues, étaient la plupart du temps de petite taille et épars (Charman, 2007).

\section{Outils d'évaluation et de gestion}

En Australie, dans les vingt-trente dernières années, des efforts ont été faits en termes de législation étatique et fédérale pour protéger et conserver les terres agricoles dans les zones périurbaines (cf. chapitre introductif).

\section{Protection des terres agricoles de bonne qualité}

Depuis 2013, par exemple, une approche visant à protéger et conserver les terres agricoles de bonne qualité est entrée en vigueur. Les terres agricoles dites « stratégiques » (Biophysical Strategic Agricultural Land, BSAL) sont des terres aux sols de bonne qualité et aux ressources en eau capables de supporter de hauts niveaux de productivité. Selon le Département de l'aménagement du territoire et de l'environnement de la Nouvelle-Galles du Sud (New South Wales State Government Department of Planning and Environment), ces terres jouent un rôle déterminant dans la production agricole de 
cet État, qui représente 12 milliards de dollars australiens ${ }^{16}$. Dans cet État, 2,8 millions d'hectares de terres agricoles stratégiques (BSAL) ont été identifiés et cartographiés à une échelle régionale ${ }^{17}$.

En octobre 2013, 1,74 million d'hectares de terres agricoles stratégiques ont été cartographiés dans les régions du Haut-Hunter et de la Nouvelle-Angleterre, situées dans la partie nord-ouest de la Nouvelle-Galles du Sud. Puis, en janvier 2014, le gouvernement a achevé la cartographie d'un million d'hectares additionnels dans le reste de l'État (voir les figures 4.1 et 4.2 pour des exemples de cartographie des terres agricoles stratégiques).

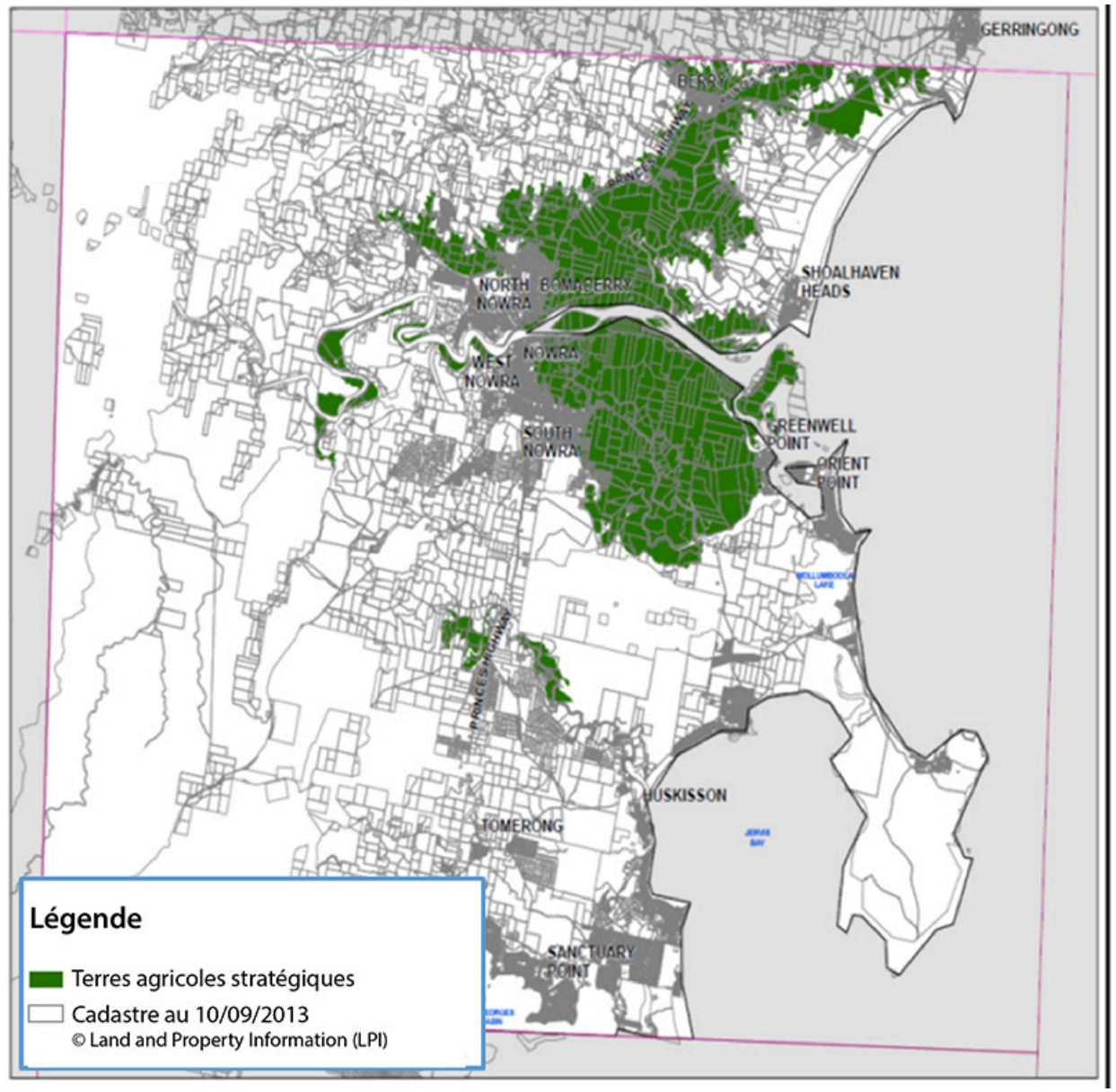

Figure 4.1. Exemple de cartographie de terres agricoles stratégiques pour la zone de Shoalhaven en Nouvelle-Galles du Sud.

16. https://www.planning.nsw.gov.au/Policy-and-Legislation/Mining-and-Resources/Safeguardingour-Agricultural-Land .

17. Ibid. 


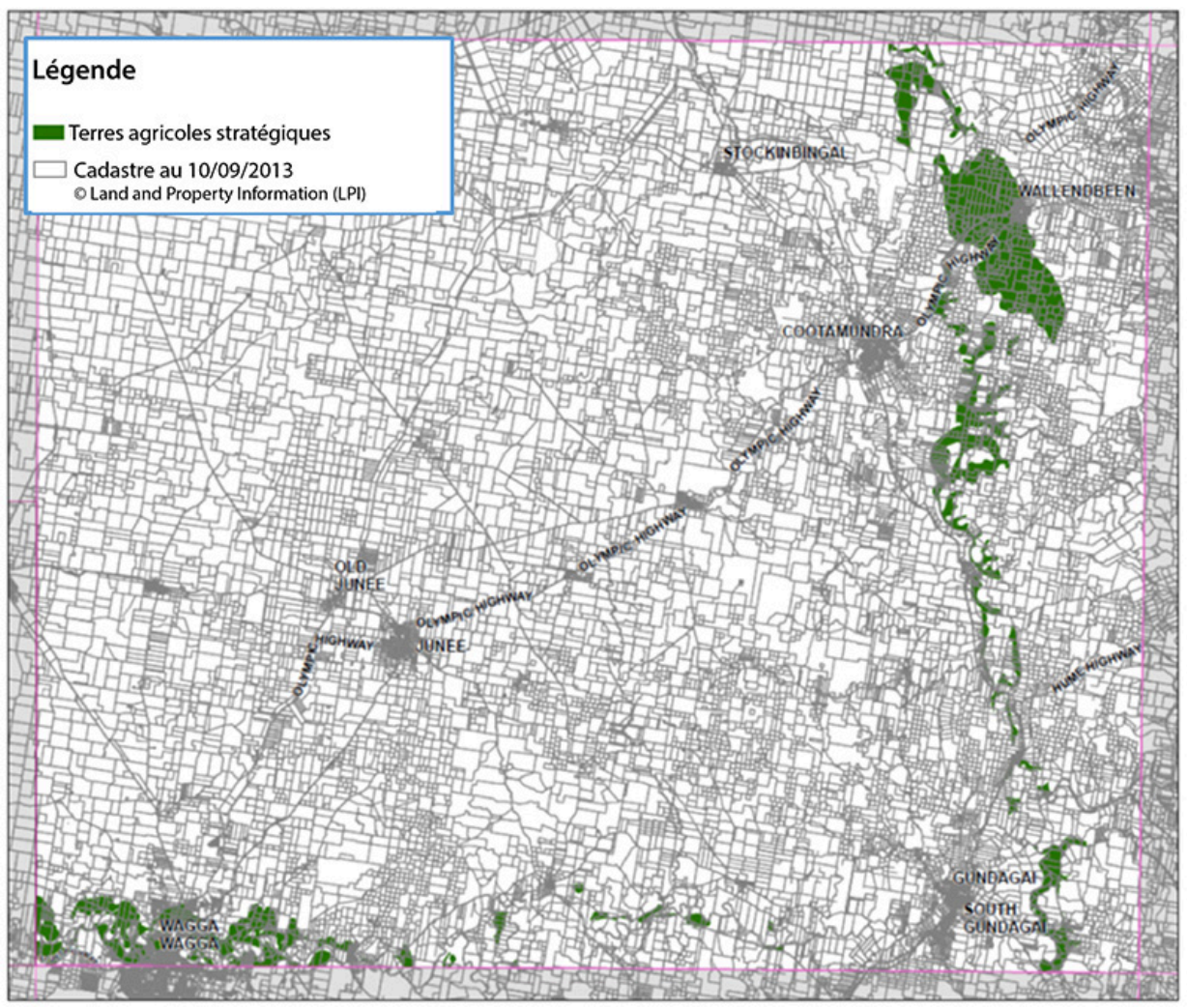

Figure 4.2. Exemple de cartographie de terres agricoles stratégiques pour la zone de Wagga Wagga en Nouvelle-Galles du Sud.

\section{Les communautés écologiques menacées d'extinction}

De la même façon, durant les vingt dernières années, l'Australie a instauré une série de lois visant à la conservation écologique en obligeant à maintenir, améliorer ou augmenter la connectivité entre les zones résiduelles de végétation primaire, ainsi qu'à prendre des mesures pour la compensation écologique. L'interprétation des propriétés des sols et des roches-mères associées est devenue l'une des composantes indiscutables de l'évaluation de la présence d'un grand nombre de ces communautés écologiques dans la réglementation environnementale, non seulement en Australie mais partout ailleurs dans le monde. Ceci contribue au regain d'intérêt plus général pour les sols en tant que milieux vivants et non de simples supports inertes.

Pour maîtriser la perte de biodiversité et l'extinction des espèces, la loi pour la conservation des espèces menacées (Threatened Species Conservation Act) a été adoptée en 1995 en Nouvelle-Galles du Sud. En 2004, un amendement de la loi sur les espèces menacées (Threatened Species Legislation Amendment Act) a été adopté, prévoyant la prise en compte des valeurs de la biodiversité dans l'aménagement stratégique du territoire, ainsi que des changements dans le processus d'évaluation du développement dans les zones urbaines et côtières.

En 2016, la loi pour la conservation des espèces menacées ainsi que la loi sur la végétation " primaire » (Native Vegetation Act 2003) ont été abrogées et remplacées par deux projets de loi sur la conservation de la biodiversité (Biodiversity Conservation Bill) et 
un amendement de la loi sur les services fonciers locaux (Local Land Services Amendment Bill). Ces deux nouveaux projets de loi avaient pour but de fournir un plan de compensation écologique ainsi que des programmes et recommandations pour aider les propriétaires fonciers à gérer leurs terres afin de mettre en place des techniques et des systèmes agricoles plus productifs. Ces recommandations fournissent aussi des informations précises concernant la gestion de la végétation.

L'Union internationale pour la conservation de la nature (International Union for Conservation of Nature, IUCN) (Keith et al., 2013) a développé des critères similaires pour conserver et restaurer des communautés écologiques résiduelles (Nicholson et al., 2009). Les récents changements de législation en Nouvelle-Galles du Sud en 2016 ont mis l'accent sur le besoin d'aider les propriétaires à gérer leur végétation ainsi qu'à cultiver leur terre de manière productive. Cependant, les défenseurs de l'environnement s'inquiètent $\mathrm{du}$ fait que la nouvelle législation ne change pas significativement le processus d'évaluation urbaine et rurale et d'approbation de projets de développement. Ces projets de loi leur apparaissent comme un pas en arrière en matière de protection environnementale.

Cependant, planifier un changement de l'usage du sol, avec des basculements entre vocations environnementales, agricoles ou urbaines, nécessite plus qu'une connaissance de la législation en vigueur. Il est nécessaire que tous les professionnels impliqués dans les politiques publiques d'aménagement aient une bonne compréhension des propriétés des sols, ainsi qu'un accès à diverses données spécifiques au site pour déterminer ses aptitudes à passer d'un usage agricole à un projet de conservation ou de développement urbain.

Dans les milieux urbains modernes, la capacité des sols à supporter le développement de la végétation est vitale et pourrait jouer un rôle croissant, particulièrement si l'on considère le défi du changement climatique. Cependant, plutôt que de continuer à construire et à défricher des terres mieux adaptées à un usage agricole ou de conservation, les futures stratégies de gestion pourraient se servir de sols et de matériaux reconstitués de manière innovante pour réhabiliter des sites contaminés ou dégradés tels que l'ancien site industriel de Barangaroo, à Sydney. Même si ces stratégies nécessitent de repousser les limites des connaissances sur les propriétés des sols, l'usage de l'eau et la nutrition végétale (Leake et Haege, 2014), l'utilisation de terrains réhabilités réduirait le besoin d'étendre les limites de la ville.

\section{Système d'information géographique}

L'évaluation et la gestion de l'information requièrent un certain nombre de stratégies. La législation ainsi que l'utilisation des systèmes d'information géographique (SIG) tels que WEB Soil Survey (WSS) et SRIC World Information sont d'excellents outils pour améliorer, afficher et manipuler les données spatiales relatives à l'usage du sol et la planification. Divers outils et techniques, traités dans le chapitre 5, tels que les cartes des pédopaysages, les cartes de contraintes et les cartes de risques relatifs aux sols, peuvent être utilisés à différentes échelles pour déterminer le potentiel des terres à partir de données sur les sols. À une grande échelle, la cartographie des pédopaysages utilisée pour les zones urbaines dans beaucoup de régions d'Australie, comme la Nouvelle-Galles du Sud et l'Australie-Méridionale, est tracée au 1:25 000 pour l'évaluation de terrain et publiée au 1:100 000. Elle sert à la planification territoriale et à l'identification des zones à risque. Les pédopaysages correspondent à des portions de territoires qui ont des topographies et des sols identifiables et spécifiques qu'il est possible de cartographier (Northcote, 1978). La cartographie des pédopaysages peut être utilisée pour différencier 
cartographiquement des sols sur la base de facteurs causaux similaires qui déterminent la formation des paysages et des sols. De la même manière, les cartes de contraintes relatives aux risques pour les projets de développement, tels que le retrait et le gonflement des sols, le risque d'érosion, sont disponibles à une échelle au 1:100 000 et sont liées aux limitations du paysage et du sol. Le concept de pédopaysage permet d'intégrer des contraintes relatives au sol et à la topographie sur une seule et même carte, qui peut alors être utilisée comme information de base dans les processus de planification précédant des changements d'usage du sol. Les informations qui en découlent doivent pouvoir être communiquées clairement à tous les acteurs pour faciliter la prise de décision concernant la poursuite ou non d'un projet. L'utilisation d'outils SIG combinée à la législation, tels les projets de loi sur la conservation de la biodiversité, l'amendement de la Loi sur les services locaux fonciers ainsi que la détermination de terres agricoles stratégiques, constituent autant de moyens pour contenir l'exploitation des zones périurbaines.

\section{Conclusion}

La compréhension des propriétés fondamentales des sols sur un site naturel ou agricole est indispensable pour déterminer si le site devrait être développé, conservé ou restauré. Dans l'étude de cas présentée, la survie de la colonie australienne était primordiale et la production agricole fut déterminante dans son succès, sur des terres dont le potentiel agronomique était inconnu. Aujourd'hui, pour aboutir à un modèle de développement urbain plus équilibré et durable, une politique publique éclairée, prenant en compte la dimension d'intérêt public des zones agricoles périurbaines en matière de sécurité alimentaire et de bien-être, doit être mise en place (Hazelton et Murphy, 2011 ; Vandermeulen et al., 2009). À la réflexion, il se peut que le succès de la conservation et la restauration des écosystèmes urbains dépendent du contexte propre à chaque ville (Heneghan et al., 2008), nécessitant alors des données environnementales aux échelles locales et régionales. Aujourd'hui, cependant, les planificateurs, qui font face à des enjeux majeurs tels que l'augmentation de la population et les aléas économiques, doivent choisir entre le potentiel agronomique des terres ou leur capacité à supporter des projets de développement. In fine, la prise en compte de facteurs liés à l'évaluation des propriétés physico-chimiques et biologiques des sols est souvent perdue de vue dans les processus de planification. La complexité des questions environnementales et d'ingénierie met l'accent sur la nécessité, pour les planificateurs, non seulement de développer des projets urbains qui soient conformes à la loi, mais aussi de travailler avec des équipes pluridisciplinaires incluant des pédologues, des écologues et des experts SIG pour une production cartographique intégrée et de qualité. C'est seulement à ces conditions qu'une approche holistique pourra être déployée pour identifier les caractéristiques des sols et leurs limitations, et permettre ainsi la mise en œuvre de stratégies de gestion durable pour la préservation de terres agricoles productives et d'espaces naturels jugés d'importance.

\section{Références bibliographiques}

Benson D.H., Howell J., 1990. Sydney’s vegetation 1788-1988: utilization, degradation and rehabilitation. Proceedings of the Ecological Society of Australia, 16, 115-127.

Burghardt W., 2006. Soil sealing and soil properties related to sealing. Geol. Soc. Lond. (Spec. Publ.), 266, 117-124.

Charman P.E.V., 2007. Soil chemical properties. In: Soils: Their Properties and Management (Charman P.E.V., Murphy B.W., eds), Melbourne, Oxford University Press.

Clark M., 1963. A Short History of Australia, New American Library. 
Dominati E., Patterson M., Mackay A., 2010. A framework for classifying and quantifying the natural capital and ecosystem services of soils. Ecological Economics, 69, 1858-68.

EEA, 2006. Urban Sprawl in Europe. The Ignored Challenge, European Environment Agency.

Handreck K., 1994. Home gardens and landscaping. In: Extended Abstracts of the Workshop "Soil in the City" (Peterson D. R., Weatherley A.J., White R.E., eds), Parkville, Victoria, University of Melbourne.

Hazelton P.A., Clements A., 2011. Historic and environmental significance of ecological communities in NSW, Australia. International Journal of Heritage and Sustainable Development, 1, 72-83.

Hazelton P.A., Clements A., 2014. Urban soil and the challenge of restoring natural ecosystems. In: Interactions Soil: Promoting Plant Growth (Dighton J., Krumins S., eds), Springer Press.

Hazelton P.A., Murphy B.W. (eds), 2011. Understanding Soils in an Urban Environment, CSIRO Publishing Melbourne.

Heneghan L., Miller S.P., Baer S., Callaham Jr M.A., Montgomery J., Pavao-Zuckerman M., Rhoades C.C., Richardson S., 2008. Integrating soil ecological into restoration management. Restoration Ecology, 16, 608-617.

Kaye J.P., Groffman P.M., Grimm N.B., Baker L.A., Pouyat R.V., 2006. A distinct urban biogeochemistry? Trends in Ecology and Evolution, 21, 192-

Keith D.A., Rodriguez J.P., Rodriguez-Clark K.M., Nicholson E., Aapala K., Alkonso A., et al., 2013. Scientific Foundations for an IUCN Red List of Ecosystems. PLOS One, 8, e62111. Leake S., Haege E., 2014. Soils for Landscape Development, Melbourne, Australia, CSIRO Publishing.

Mason D., Knowd I., 2010. The emergence of urban agriculture: Sydney, Australia. International Journal of Agricultural Sustainability, 8, 62-71.

Nicholson E., Keith D.A., Wilcove D.S., 2009. Assessing the conservation status of ecological communities. Conservation Biology, 23, 259-274.

Northcote K.H., 1978. Soils and land use. In: Atlas of Australian Resources, Canberra, Australia, Division of National Mapping.

Perry T.M., 1963. Australia's First Frontier. The Spread of Settlement in New South Wales 1788-1829, Melbourne, Melbourne University Press.

Pickett S.T.A., Cadenasso M.L., Grove J.M., Nilon C.H., Pouyat R.V., Zipperer W.C., Costanza R., 2001. Urban ecological systems: linking terrestrial ecological, physical, and socio-economic components, of Metropolitan Areas. Annual Review of Ecological Systems, 32, 127-157.

Piorr A., Ravetz J., Tosics I. (eds), 2011. Peri-urbanisation in Europe. Towards European policies to sustain urban-rural futures. Synthesis Report, Copenhagen, University of Copenhagen/Academic Books Life Sciences.

Pouyet R.V., Yesilonis I.D., Russell-Anelli J., Neerchal N.K., 2007. Soil chemical and physical properties that differentiate urban land-use and cover types. Soil Sci Soc Am J., 71, 1010-1019.

Sinclair I., 1996. "A view from the edge": issues in rural and metropolitan fringe planning. New Planner, 28.

Spearritt P., 1978. Sydney since the Twenties, Sydney, Hae and Iremonger Publishers.

Tench W., 1789. A Narrative of the Expedition to Botany Bay: With an Account of New South Wales, ch. 8, London, J. Debrett.

Tozer M., 2003. The native vegetation of the Cumberland plain, Western Sydney: systematic classification and field identification of communities. Cunninghamia, 8, 1-76. 
Vandermeulen V., Gellynck X., Van Huylenbroeck G., Van Orshoven J., Bomans K., 2009. Farmland for tomorrow in densely populated areas. Land Use Policy, 26, 859-868.

Wei Z., Wu S., Zhou S., Lin C., 2013. Installation of impervious surface in urban areas affects microbial biomass, activity (potential $\mathrm{C}$ mineralisation), and functional diversity of the fine earth. Australian Journal of Soil Research, 51, 59-67. 


\title{
Chapitre 5
}

\section{La disponibilité des données sur les sols en Australie}

\author{
Jane Brennan, Andrew Murrell
}

\section{Introduction}

Par le passé, la qualité des sols a souvent été négligée dans les processus d'aménagement $\mathrm{du}$ territoire. La diminution constante des zones périurbaines autour des grandes villes australiennes montre l'urgence de considérer la qualité des sols dans le processus d'aménagement du territoire, afin de préserver les terres propices à un usage autre que le développement urbain. Si l'on considère, par exemple, les activités agricoles, la qualité du sol combinée à ses caractéristiques topographiques devrait être prise en compte pour déterminer si une zone peut être imperméabilisée ou devrait être préservée pour un usage agricole. Cela semble être une tâche relativement simple à effectuer ; cependant, lorsque l'on explore plus en détail les informations sur les sols, il devient évident qu'une véritable expertise en pédologie est nécessaire pour comprendre ce qu'est un «bon » sol agricole, tant l'interprétation de la composition biochimique des sols est complexe. On pourrait même soutenir que la définition de la «qualité du sol» peut varier en fonction de la nature et de l'intensité de la production agricole envisagée ${ }^{18}$, voire qu'il n'existe pas de définition scientifique, objective de la qualité du sol. Enfin, il est parfois difficile de disposer de données relatives aux sols. Dans ce cas il est nécessaire de collecter et d'analyser les données, ce qui relève d'un processus onéreux.

L'étalement urbain et l'imperméabilisation des sols qui lui est associée sont des phénomènes existant à l'échelle mondiale, qui impactent considérablement la disponibilité de terres pour les activités agricoles, la préservation de l'environnement et les activités récréatives. Être en mesure de prendre des décisions éclairées est essentiel pour faire des choix pertinents en matière d'aménagement du territoire et assurer ainsi un futur durable. Cela inclut une bonne connaissance de l'étendue de l'étalement urbain pour évaluer la faisabilité de projets de développement futurs, mais aussi une bonne compréhension des propriétés des sols pour déterminer leur valeur et, par conséquent, la nécessité de les préserver. Pour évaluer la qualité des sols, il est nécessaire d'avoir accès à de l'information sur leurs propriétés. À cette fin, un large éventail de sources d'informations sur les sols est disponible en Australie. Avant de présenter ces sources en détail, nous nous pencherons brièvement sur les initiatives de cartographie de l'étalement urbain en Australie. Le chapitre 5 du présent volume offre une revue détaillée des méthodes géospatiales de cartographie de l'occupation des sols.

18. Agriculture intensive versus agriculture biologique. 


\section{Cartographier l'étalement urbain en Australie}

La plupart des projets qui portent sur l'étalement urbain utilisent l'imagerie satellitaire pour en identifier les limites géographiques ou décrire l'évolution de la croissance urbaine à des échelles de temps spécifiques. Généralement, l'imagerie satellitaire nocturne est utilisée pour analyser la croissance urbaine sur de plus longues échelles temporelles. Ces analyses sont disponibles dans des publications, telles que le rapport annuel Demographia sur les Zones urbaines mondiales (World Urban Areas) (Demographia, 2017). L'utilisation de l'imagerie satellitaire nocturne ne va pas sans poser de problèmes, particulièrement en ce qui concerne la définition de l'intensité lumineuse caractérisant une zone urbaine. Néanmoins, en complément des données de recensement, ce type d'imagerie permet de délimiter l'étendue des zones urbaines à l'échelle mondiale. Graetz et al. (1998), par exemple, ont cartographié les changements de couverture du sol entre 1972 et 1992 pour trois grands centres urbains en Australie. Sutton et al. (2010) et Smart Growth America (2014) ont aussi utilisé des images satellites nocturnes pour cartographier l'étendue de l'étalement urbain en Australie et développer un index d'étalement dans le cadre d'une étude comparative avec les États-Unis. Le rapport de Smart Growth America (2014) est une mise à jour de l'étude américaine « Measuring Sprawl and Its Impact » de 2002.

Alors qu'aucune donnée géographique exhaustive sur l'étalement urbain n'est disponible en Australie, une collection relativement large et complète d'images satellites relatives à l'expansion urbaine peut être acquise auprès de Géosciences Australie (Geoscience Australia), une agence du gouvernement fédéral australien qui fournit la majorité des données nécessaires pour la cartographie thématique en Australie. Cette agence gouvernementale gère un portail en ligne (Earth Observation Gallery ${ }^{19}$ ) qui permet d'accéder à des images satellites sur les catastrophes naturelles et les phénomènes extrêmes. Dernièrement, le projet de cube de données australiennes en géosciences (Australian Geoscience Data $\mathrm{Cube}^{20}$ ), appelé maintenant Terre numérique Australie (Digital Earth Australia $^{21}$, DEA), met à disposition non seulement des données satellitaires en ligne, mais également une plateforme d'analyse d'images satellites et autres données d'observation de la Terre. Elle comporte aussi des données sur l'Expansion urbaine australienne (Australian Urban Expansion) sur une période de plus de vingt ans, visualisables sous forme de séquences temporelles Landsat. Ces images à haute résolution en format JPEG sont disponibles gratuitement en couleurs naturelles et fausses couleurs pour des usages non commerciaux ${ }^{22}$. Comme il existe encore peu de cartes de l'étalement urbain, ces images pourraient, en principe, être utilisées pour cartographier la croissance des plus grandes agglomérations australiennes, puisqu'elles couvrent une large proportion du territoire national. Les figures 5.1 et 5.2 donnent un exemple du type d'images disponibles sur le site de Géosciences Australie. La figure 5.1 présente deux images en couleurs naturelles de la ville de Dubbo, acquises respectivement en 1987 et 2010, qui mettent en valeur la croissance urbaine. L'ampleur de ce phénomène ressort toutefois plus nettement sur les images en fausses couleurs présentées dans la figure 5.2. En utilisant ces images, les zones de développement urbain et, par conséquent, les zones où

19. http://www.ga.gov.au/scientific-topics/earth-obs/basics-archive/gallery (consulté le 28 septembre 2017).

20. http://www.datacube.org.au/ (consulté le 28 septembre 2017).

21. http://www.ga.gov.au/about/projects/geographic/digital-earth-australia (consulté le 28 septembre 2017).

22. (C) Commonwealth of Australia (Geoscience Australia), 2013. Ce produit est soumis à la Creative Commons Attribution 3.0 Australia Licence, http://creativecommons.org/licenses/by/3.0/au/deed.en 
les sols ont été imperméabilisés pourraient être délimitées, mais aussi cartographiées au moyen d'un système d'information géographique (SIG). Les cartes pourraient ensuite être utilisées pour d'autres analyses ainsi que pour de la modélisation.
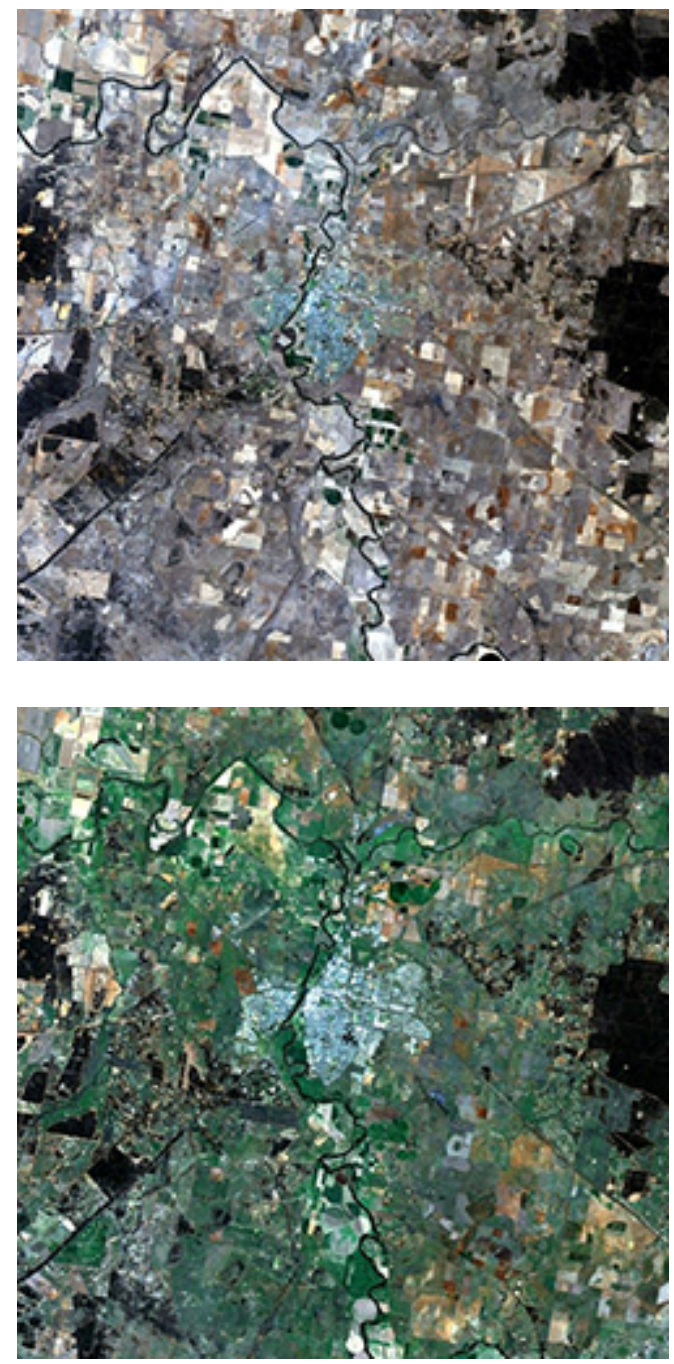

Figure 5.1. Images Landsat de Dubbo en couleurs naturelles en 2010 montrant l'étendue de l'étalement urbain (৫ Commonwealth of Australia, Geoscience Australia, 2013). 

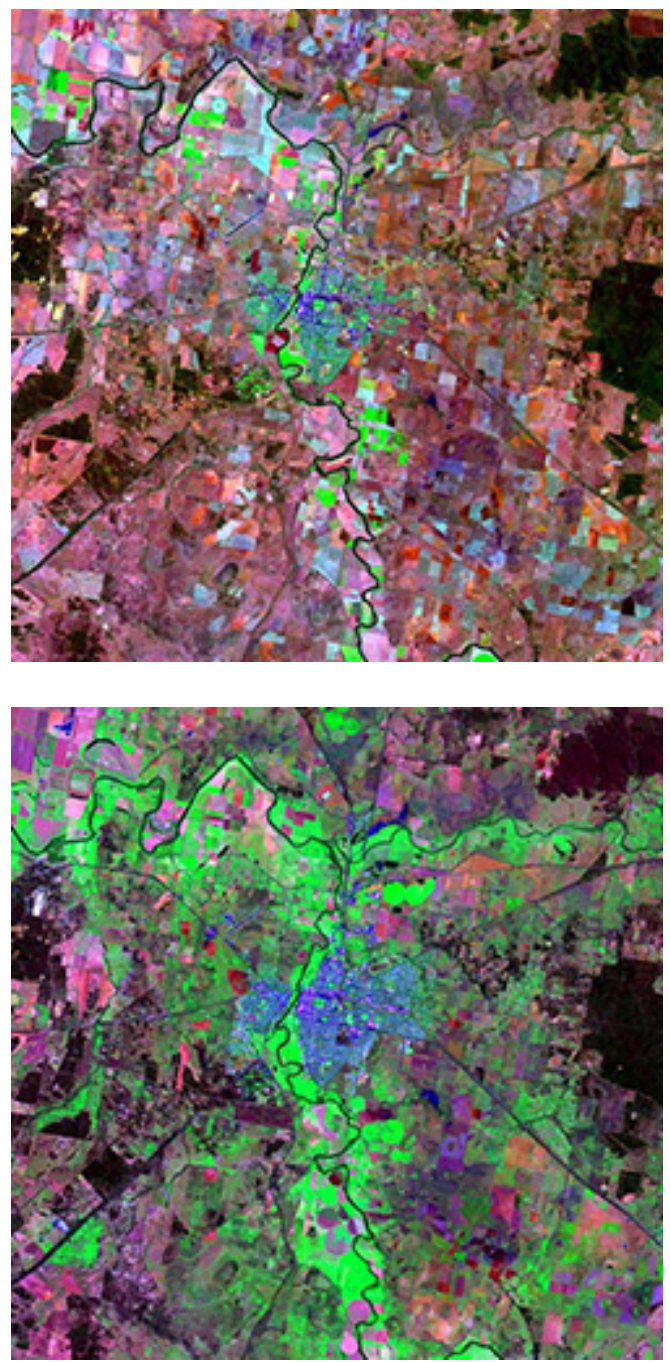

Figure 5.2. Images Landsat de Dubbo en fausses couleurs en 2010 montrant l'étendue de l'étalement urbain (๔ Commonwealth of Australia, Geoscience Australia, 2013).

Même si Graetz et al. (1998) n'ont pas spécifiquement produit de cartographies du phénomène d'étalement urbain, ils ont cependant utilisé des images satellites prises à différentes périodes pour déterminer les changements de l'occupation des sols. Ceci était une approche relativement novatrice à une époque où la croissance urbaine était déterminée à partir de données statistiques sur la population et de référentiels spatiaux comme les codes postaux ou les noms de quartiers. Graetz et al. (1998) ont constaté que la détermination de la croissance urbaine à partir des données démographiques du Bureau australien des statistiques (Australian Bureau of Statistics, ABS) était insuffisante parce qu'elles ne prenaient pas en compte l'effet d'étalement de cette croissance. Par exemple, la population peut augmenter dans des zones à forte densité sans toutefois engendrer (spatialement) d'étalement urbain. Graetz et al. (1998) ont utilisé des images satellites Landsat pour cartographier la croissance des grandes villes australiennes (Canberra, Sydney, Melbourne, Brisbane, la Gold Coast, Adelaïde, Perth, Hobart et Darwin). Bien 
que leurs méthodes d'analyse d'images aient été, de manière générale, relativement efficaces pour identifier la dynamique générale du développement urbain, ils ont observé que des changements marqués du taux d'humidité de la végétation affectaient leurs résultats. Dans le cas de Melbourne en particulier, la sécheresse présente en 1973 et la végétation plus verte et humide présente en 1988 ont rendu la comparaison plus difficile, car les contrastes étaient très marqués et rendaient les configurations spatiales moins visibles. Par exemple, les routes se distinguaient moins nettement des zones résidentielles contiguës quand elles avaient un terre-plein central végétalisé. Ceci a amené à une sous-estimation de l'accroissement de l'imperméabilisation des sols entre 1973 et 1988. Ce type d'effet du couvert végétal sur la cartographie de l'étalement urbain doit par conséquent être pris en compte pour ne pas biaiser l'estimation du phénomène.

Sutton et al. (2010) ont développé une méthode capable de remédier à ce type de problème en utilisant les émissions lumineuses captées par des images satellites prises de nuit dans le domaine spectral du visible pour détecter les limites des zones urbaines et leur configuration. Les auteurs n'étaient pas seulement intéressés par l'étendue globale de l'étalement urbain en Australie. Ils voulaient aussi différencier les zones urbaines des zones périurbaines pour identifier les développements à faible densité propices à l'usage de la voiture. En plus des images satellites, une grille de densité de la population a été développée à partir des données de 2001 du Bureau australien de statistiques et utilisée en conjonction avec les résultats de l'analyse d'image, pour déterminer les limites des zones urbaines et périurbaines en Australie. Il ressort de cette étude qu'approximativement $82 \%$ de la population australienne vit dans des zones urbaines, $15 \%$ dans des zones périurbaines et $3 \%$ dans des zones rurales. Sutton et al. (2010) ont compilé leurs résultats sur plus de 200 centres urbains australiens ${ }^{23}$, en enregistrant les données démographiques ainsi que les données sur les surfaces cartographiées. Ils ont pu ainsi développer un indicateur d'étalement urbain (Urban Sprawl Index) représentant « une mesure ajustée [par exemple, par rapport à la population] de la consommation d'espace par habitant, pour la population d'une aire urbaine donnée » (Sutton et al., 2010, p. 123). Par exemple, la population réelle de Sydney enregistrée était de 3760490 habitants, la superficie de $2258 \mathrm{~km}^{2}$ et l'indicateur d'étalement urbain de 32. Cet indicateur varie de manière inversement proportionnelle à l'étalement urbain. Plus il est élevé, moins l'étalement est important et inversement. Avec un indicateur d'étalement urbain de - 38, Brisbane est la ville australienne la plus étalée, alors que Sydney est la plus compacte (Sutton et al., 2010). Cet indicateur permet aussi de comparer le phénomène d'étalement urbain entre plusieurs aires métropolitaines dans le monde. Sutton et al. (2010) l'ont développé pour l'Australie dans le but de le comparer à un indicateur d'étalement urbain déjà utilisé aux États-Unis. Il en ressort par exemple que New York est la ville la mieux connectée et la plus compacte des États-Unis avec un indicateur de 203,4, alors que la ville de Hickory, en Caroline du Nord, n'a obtenu un score que de 24,9, étant, de ce fait, la métropole avec le taux d'étalement urbain le plus élevé du pays (Smart Growth America, 2014).

Le chapitre 4 de ce volume traite de l'importance d'une bonne connaissance des propriétés des sols et des besoins en outils pour faciliter l'accès aux informations sur les sols. Nous nous plaçons dans la continuité de cette démarche, et nous allons maintenant nous pencher sur la question de l'accessibilité des données sur les sols en Australie. Le Système d'information australien de ressources sur les sols (Australian Soil Resource Information System, ASRIS) fournit un cadre général pour différents systèmes d'informations sur les sols, comme le Système d'information sur les sols et les terres de la

23. Les centres urbains sont définis comme accueillant plus de 10000 habitants. 
Nouvelle-Galles du Sud (New South Wales Soil and Land Information System, SALIS). Nous présenterons d'abord ces systèmes. Puis nous traiterons des différentes manières d'interpréter les données relatives aux sols pour créer des cartes de pédopaysages ainsi que des cartes thématiques. Les outils et les principes de ces méthodes seront illustrés à travers l'exemple de la région de l'Hawkesbury-Nepean en Nouvelle-Galles du Sud, Australie.

\section{Accéder aux données pédologiques}

Les agences gouvernementales australiennes à l'échelon étatique et fédéral gèrent une série de bases de données pédologiques ainsi que d'autres systèmes d'informations qui leur sont associés. Le système ASRIS, développé par l'Organisation du Commonwealth pour la recherche scientifique et industrielle (Commonwealth Scientific and Industrial Research Organisation, CSIRO) dans le contexte d'un projet sur l'agriculture durable, « fut conçu pour construire une base de données cohérente à l'échelle nationale, à partir des nombreuses données pédologiques recueillies, au fil des ans, par les agences du Commonwealth, des États et des Territoires » (Johnston et al., 2003, p. 2). Cela fut fait dans le but de fournir des jeux de données homogènes et une multitude de collections de cartes sur les sols (Biggs et Grundy, 2010). Le système ASRIS est disponible en ligne ${ }^{24}$, et permet de visualiser des cartes et d'effectuer des recherches à l'aide de métadonnées et d'une interface SIG simplifiée. L'une des agences étatiques participant à l'archivage des données du système ASRIS est le Bureau de l'environnement et du patrimoine (Office of Environment and Heritage, $\mathrm{OEH}$ ) de Nouvelle-Galles du Sud. Il gère le système SALIS ainsi que les outils numériques associés, comme le portail web eSPADE pour l'accès aux cartes et aux profils pédologiques ainsi que l'application mobile eDIRT (Digital Infield Regolith Tool,) pour le recueil sur le terrain de données sur les profils pédologiques. Le système eSPADE tire la plupart de ses informations de SALIS. Auparavant, des fiches de données pédologiques (soil data cards) étaient fournies pour permettre l'enregistrement sur le terrain d'informations relatives aux sols. Même si ces fiches sont encore disponibles, elles ont été remplacées en pratique par l'application eDIRT. Il s'agit d'une application internet qui fonctionne sur PC ou appareil mobile et qui peut aussi être utilisée hors ligne, avec, dans ce cas, une synchronisation des données au serveur eDIRT dès que l'appareil est reconnecté. Les données enregistrées avec eDIRT sont ensuite utilisées pour mettre à jour la base SALIS. La fiche d'enregistrement des données dispose aussi de champs pour vérifier si les valeurs renseignées qualifient le sol mesuré comme terre agricole stratégique (Biophysical Strategic Agricultural Land, BSAL).

Des informations sur les sols sont recueillies depuis les années 1950 en Nouvelle-Galles du Sud au moyen, jusqu'à récemment, de fiches de données, comme mentionné plus haut. Ces fiches indiquent clairement le type d'informations sur les sols qui doivent être recueillies à chaque point de collecte. Elles s'appuient pour cela sur des attributs et une terminologie standard, fournissant ainsi une structuration de l'information qui a facilité sa numérisation. Les données pour chaque point de collecte sont souvent résumées dans un profil de sol (soil profile) propre à chaque point. Ces profils constituent les informations fondamentales sur les sols dans les bases de données utilisées depuis les années 1980 en Nouvelle-Galles du Sud. La base de données SALIS est elle-même une version améliorée du système originel, le Système de données du sol de NouvelleGalles du Sud (NSW Soil Data System, NSW SDS), mis en place à la fin des années 1990 (Milford et al., 2001). Un rapport détaillé des attributs descriptifs requis peut être 
consulté dans le guide de saisie des données sur les sols (Soil Data Entry Handbook) (Milford et al., 2001) et dans le guide de l'application eDIRT (NSW OEH, 2016).

\section{Le Système d'information sur les sols et les terres de Nouvelle-Galles du Sud (SALIS)}

Les données du système SALIS sont fournies principalement par des services gouvernementaux, et parfois par des géomètres indépendants. Les fiches de données pédologiques sont maintenant largement remplacées par l'application eDIRT pour la collecte de données sur le terrain et l'importation dans SALIS. Comme les relevés comprennent la localisation géographique du point de collecte, l'incorporation des données dans des bases de données SIG se fait directement. Ceci a permis de donner accès aux profils pédologiques au moyen d'outils en ligne. La visionneuse cartographique eSPADE est un système d'information basé sur Google Maps ${ }^{\mathrm{TM}}$, ce qui permet un accès gratuit et facile, sur ordinateur fixe et appareil mobile, à une multitude d'informations sur les sols et les terres en Nouvelle-Galles du Sud et dans le Territoire de la capitale australienne. Les données accessibles dans eSPADE comportent plus de 70000 profils pédologiques, en plus de cartes de paysages (figure 5.3). Comme nous le montre la figure 5.4, les cartes de paysages ne sont pas disponibles sur l'intégralité du territoire de Nouvelle-Galles du Sud, et les pédopaysages ne couvrent que les zones densément peuplées.

Sur eSPADE, la carte pédologique de Nouvelle-Galles du Sud affiche les points de collecte de données sur les sols sous la forme d'éléments SIG ponctuels aux coordonnées figurant dans les fiches pédologiques (remplacées depuis par des écrans de saisie dans l'application eDIRT). Chaque élément ponctuel est accompagné de trois rapports sur le sol : un rapport sur les informations essentielles (soil essentials) ; un rapport sur le profil pédologique ; et un rapport technique. Le rapport technique contient la description du paysage, les attributs du sol ainsi que les résultats des analyses de sol en laboratoire à différents niveaux de détail.

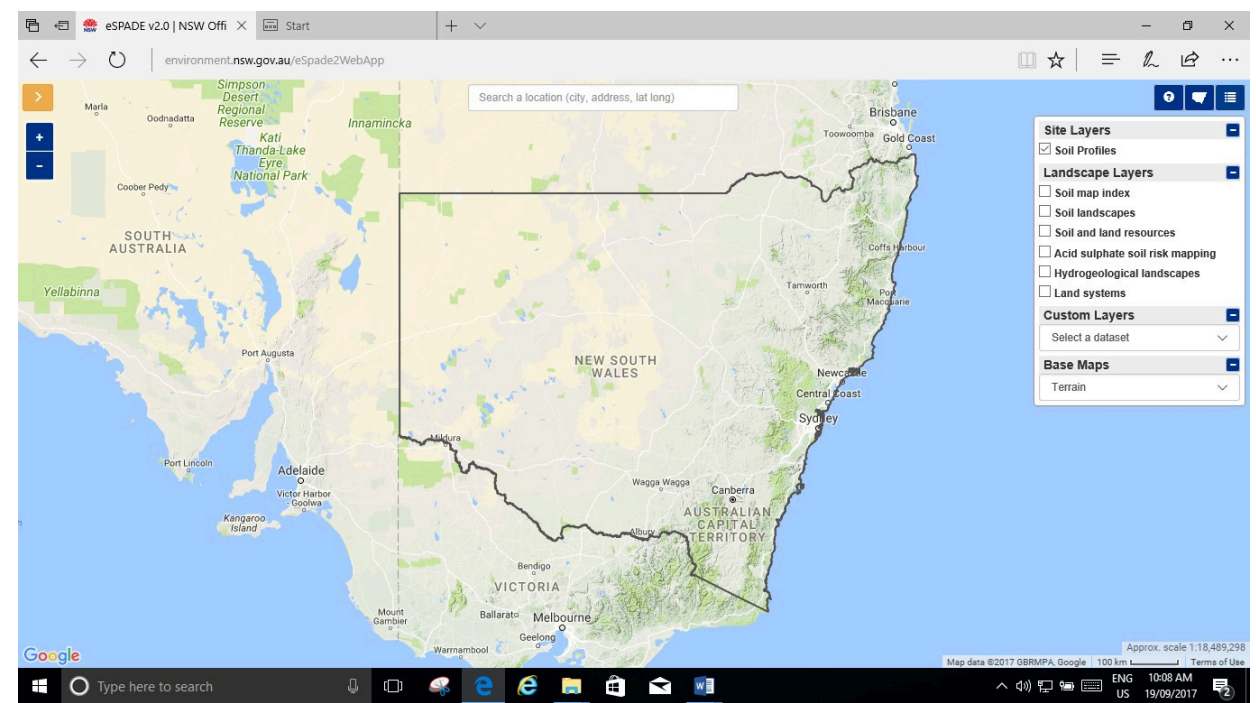

Figure 5.3. Écran d'accueil d'eSpade (๔ State of NSW and Office of Environment and Heritage 2017, capture d'écran du 23 septembre 2017, http://www.environment.nsw.gov.au/eSpade2Webapp , consulté le $1^{\text {er }}$ octobre 2017). 
Nous proposons d'examiner la région d'Hawkesbury-Nepean pour illustrer les concepts présentés dans ce chapitre. Les profils pédologiques disponibles sur cette zone sont présentés dans la figure 5.5 avec un zoom sur le résumé du profil 261, Baker's Lagoon. En cliquant sur un point de relevé, on accède à la localisation d'un profil pédologique spécifique, et les rapports qui lui sont associés peuvent alors être consultés. La figure 5.6 montre un exemple d'informations essentielles sur les sols pour le site de Baker's Lagoon, situé dans le pédopaysage de Penrith. Le rapport sur le profil pédologique (qui n'est pas présenté ici du fait de sa longueur) contient la liste complète de tous les tests de laboratoire menés sur les échantillons de sols prélevés sur le site.

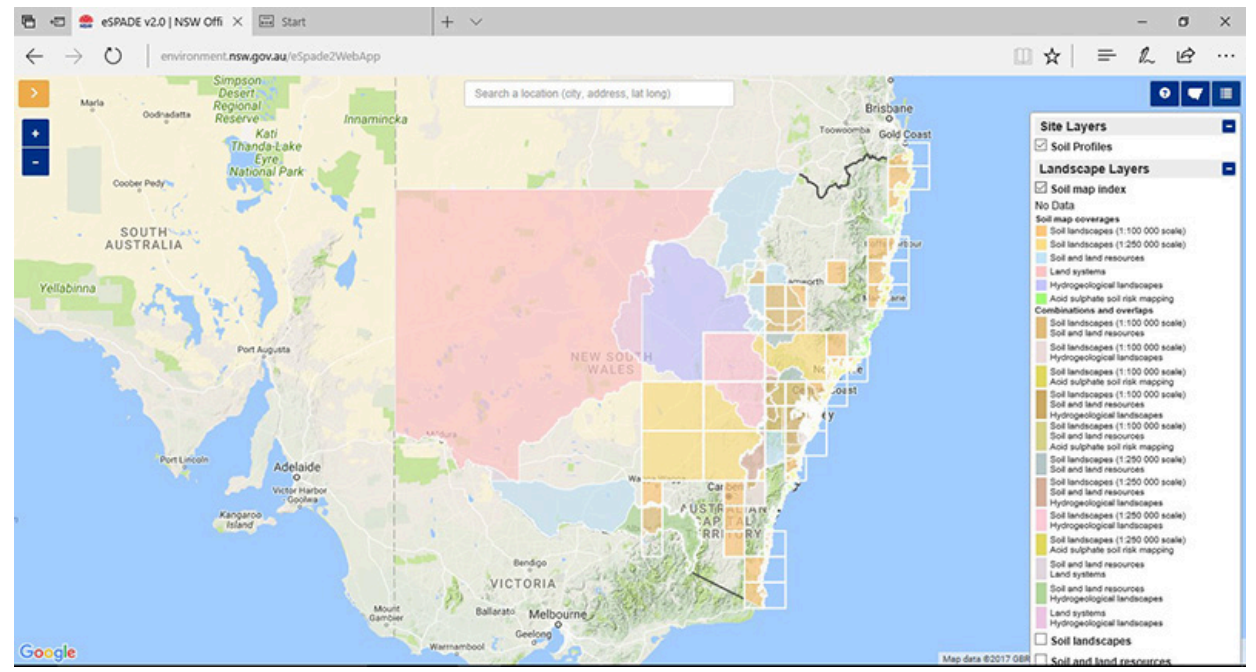

Figure 5.4. Copie d'écran d'eSpade montrant les couches disponibles (৫ State of NSW and Office of Environment and Heritage, 2017).

Cependant, pour les non-experts, l'utilité des données sur les sols est souvent assez limitée. Des compétences en pédologie sont nécessaires pour en extraire des informations utilisables pour évaluer, par exemple, l'aptitude des sols à supporter des activités agricoles. Afin de rendre les informations pédologiques accessibles aux décideurs n'ayant pas de formation en pédologie, des cartes thématiques de contraintes (constraint maps), parfois appelées aussi cartes de potentiel (capability maps), ont été développées. Ces types de cartes constituent depuis longtemps un outil de visualisation des contraintes liées au sol et au paysage, et font partie intégrante de SALIS.

Les cartes de contraintes ou de potentiel représentent « le potentiel, les limites ou les risques pour un usage du sol spécifique » (Yang et al., 2007, p. 569). Elles permettent l'identification de zones " pouvant être utilisées à des fins très variées » (Yang et al., 2007, p. 569). L'intérêt d'utiliser des cartes de contraintes est qu'elles montrent clairement les usages potentiels adaptés à chaque zone. Cela permet d'utiliser les terres « en fonction de leurs capacités, [et de ce fait] la probabilité de conflits d'usage diminue fortement » (Yang et al., 2007, p. 569). En Nouvelle-Galles du Sud, l'équipe de SALIS développe des cartes de potentiel qui font partie intégrante de leurs produits sur les ressources pédologiques et foncières (soil and land resource products). La figure 5.7 montre la disponibilité actuelle des cartes de sols en Nouvelle-Galles du Sud. La couche « ressources en sols et en terres » (soil and land resources) dans la légende correspond aux zones qui ont bénéficié d'une importante cartographie du potentiel des terres. Elle 
regroupe une série de cartes thématiques, telles que des cartes sur le potentiel agronomique des terres, ainsi que des cartes sur les risques comme, entre autres, les risques d'érosion, de salinisation ou d'inondation. Elles peuvent être obtenues sur DVD auprès du Bureau de l'environnement et du patrimoine de Nouvelle-Galles du Sud moyennant paiement. Les informations sur les pédopaysages peuvent être téléchargées sur eSPADE.

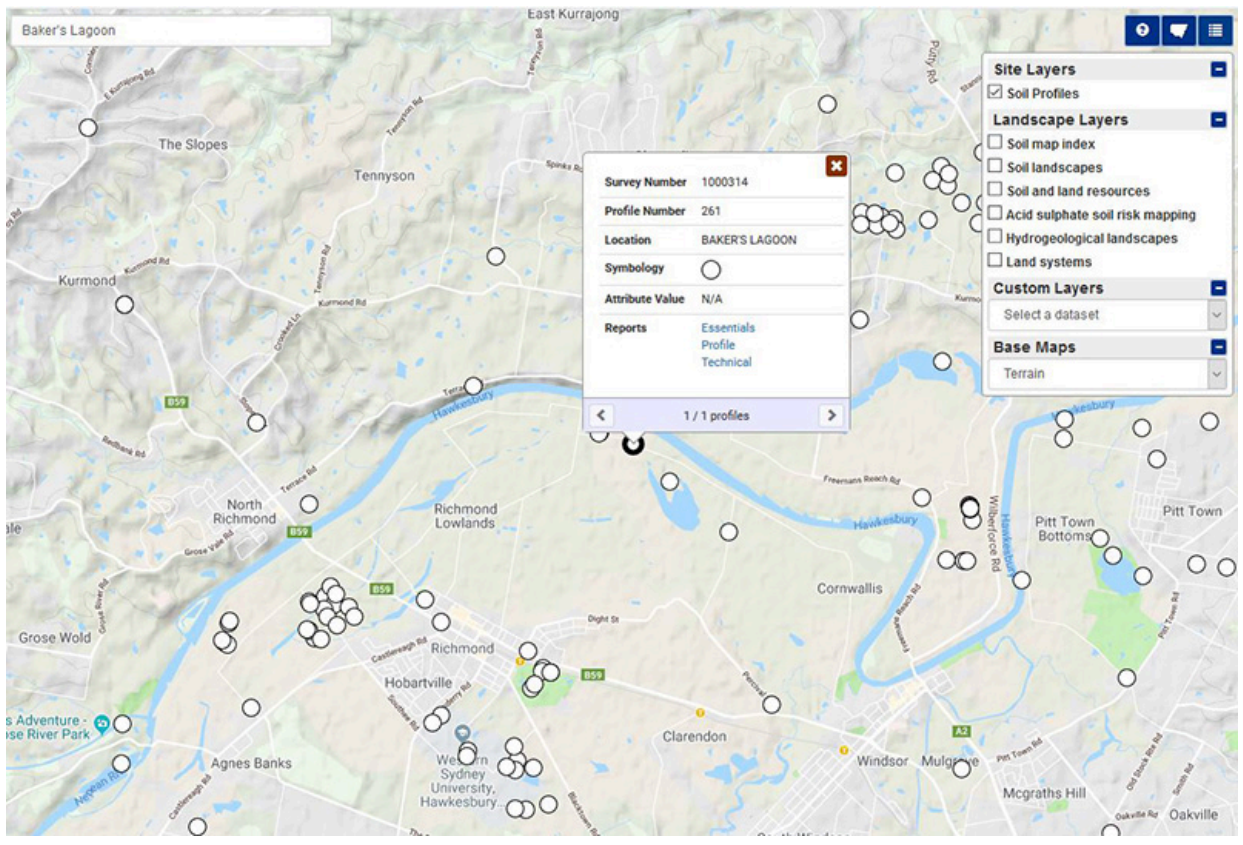

Figure 5.5. Carte des profils pédologiques et gros plan sur le profil 261, Baker's Lagoon (৫) State of NSW and Office of Environment and Heritage, 2017). 


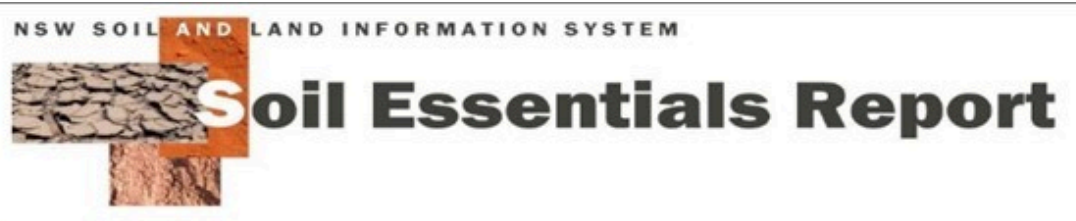

Site Location BAKER'S LAGOON

Map Reference: MG A G rid Reference : Easting 293705, Northing 6282990 PENRITH (9030) 1:100,000 sheet

Profile Details: Soil Landscapes of the Penrith 1:100 000 Sheet Survey, Profile 261, collected by Susan Abraham on December 01,1988

Physiography. swamp complex and used for volun.native pasture. Slope $1 \%$ (?). profile is imperfectly drained, erosion hazard is slight, and no salting evident

Soil Type: No suitable group (GSG), Dd4.52(PPF)

Soil Description:

Layer 0

Layer 1 field $\mathrm{pH}$ is 5 . Coarse fragments are not evident, pans are not evident. Segregations are not

00.00 -

$00.04 \mathrm{~m}$ evident, not evident Layer notes are: Horiz on given as 0 .; clear $(20-50 \mathrm{~mm})$ boundary to...

Layer 2

00.04 -

$00.30 \mathrm{~m}$

loam with massive structure field $\mathrm{pH}$ is 5 . Coarse fragments are not evident, pans are not evident. Segregations are not evident, not evident; clear $(20-50 \mathrm{~mm})$ boundary to...

A1 Horizon

Layer $310 \mathrm{~m}$ with massive structure field $\mathrm{pH}$ is 5 . Coarse fragments are not evident, pans are not 00.30 - evident. Segregations are not evident, not evident gradual $(50-100 \mathrm{~mm})$ boundary to...
$00.30 \mathrm{~m}$

B1 Horiz on

Layer 4 clay with massive structure field $\mathrm{pH}$ is 6.5 . Coarse fragments are not evident, pans are not

00.30 -

$00.75 \mathrm{~m}$ evident. Segregations are not evident, not evident gradual $(50-100 \mathrm{~mm})$ boundary to...

B21 Horizon

Layer 5 clay with massive structure (earthy), field $\mathrm{pH}$ is 8 . Coarse fragm ents are not evident, pans

00.75 -

$01.50 \mathrm{~m}$

B22 Horizon

Layer 6 (earthy). Coarse fragments are not evident, pans are not evident Segregations are not

01.50 -

$01.50 \mathrm{~m}$ are not evident. Segregations are not evident, not evident

Laboratory Test Data:

Figure 5.6. Exemple de fiche des informations essentielles sur les sols. Cas du profil 261, Baker's Lagoon (৫ State of NSW and Office of Environment and Heritage, 2017). 


\section{Cartographie des pédopaysages et cartes thématiques}

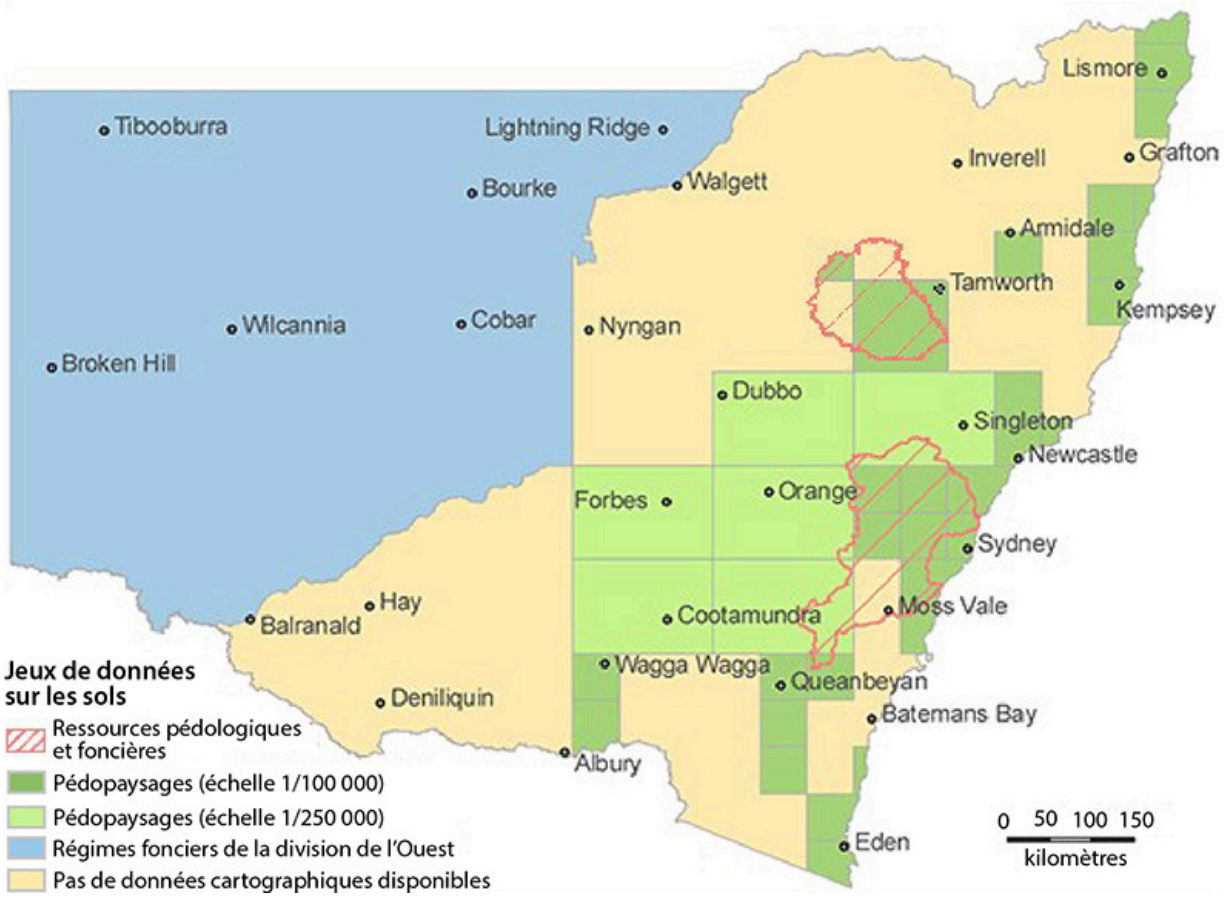

Figure 5.7. Carte des sols de la Nouvelle-Galles du Sud (৫) State of New South Wales through the Office of Environment and Heritage).

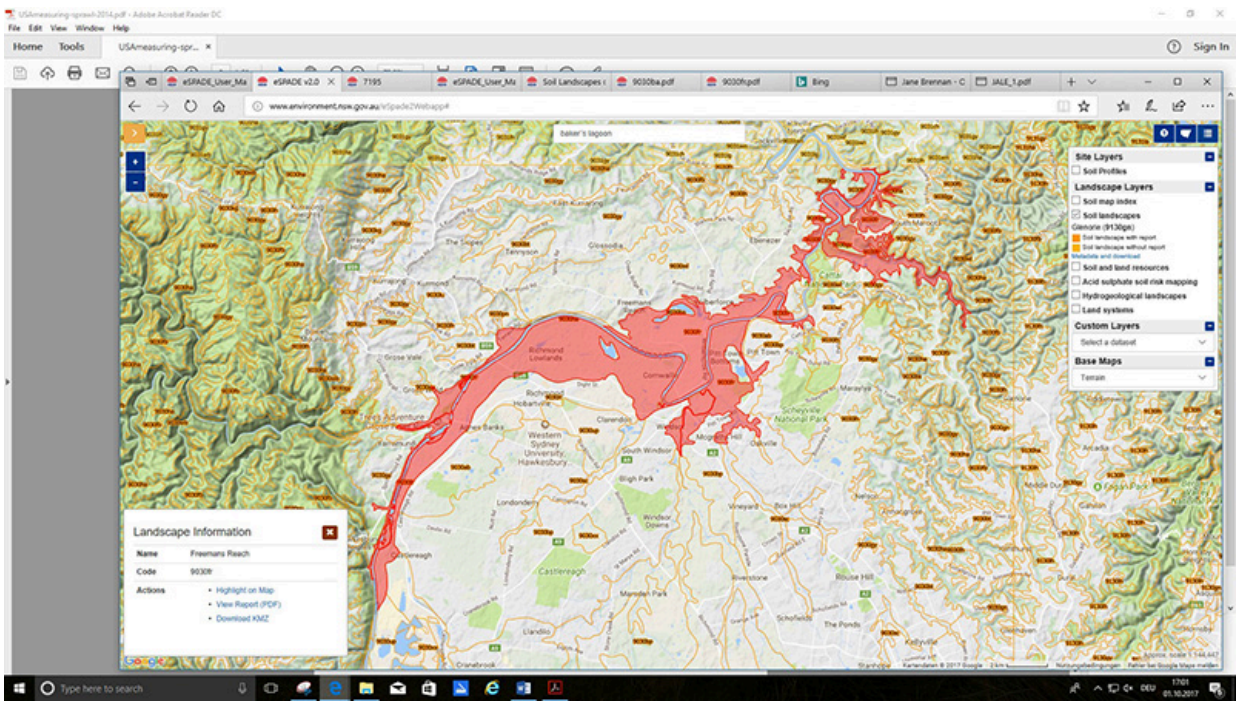

Figure 5.8. Zones de pédopaysages autour de Baker's Lagoon ( $\odot$ State of New South Wales through the Office of Environment and Heritage, capture d'écran du $1^{\text {er }}$ octobre 2017). 
Nous allons maintenant nous concentrer sur le potentiel des sols en milieu rural pour présenter plus en détail les concepts des cartes interprétatives et leurs applications. Les informations téléchargeables sur les pédopaysages comprennent pour certaines régions un résumé des informations sur les sols. La figure 5.8 représente les pédopaysages pour les zones situées autour de Baker's Lagoon dans la région de l'Hawkesbury-Nepean. Une fois la zone sélectionnée, le rapport sur le pédopaysage peut alors être consulté. Ce rapport est un extrait des pages pertinentes, pour le cas étudié, du guide de saisie des données sur les sols de Milford et al. (2001). Les cartes de contraintes ainsi que les cartes de potentiel agronomique des sols résultent des modalités d'interprétation décrites dans le guide. Le bassin versant de l'Hawkesbury-Nepean est l'une des régions pour lesquelles il existe un très grand nombre de cartes de potentialité. Les cartes de pédopaysages et de potentialité présentées ci-dessous (figures 5.9 et 5.10) ont été produites à partir des données du DVD mentionné plus haut (C) NSW Government, 2009) avec l'autorisation du Bureau de l'environnement et du patrimoine.

Pour créer des cartes de potentialité, « les cartes paysagères et les attributs des sols sont indispensables » (Yang et al., 2007, p. 569). Leur potentiel n'est pleinement exploité que lorsqu'elles sont intégrées dans des cartes de pédopaysages. Les pédopaysages sont, selon Northcote (1978), des zones clairement délimitées, identifiables du fait de leur topographie et de leurs sols particuliers. Ces zones peuvent être visualisées sur des cartes et décrites en termes concis. La figure 5.9 montre une carte des pédopaysages d'une zone autour de Baker's Lagoon, clairement identifiée comme comportant des sols alluviaux. Cette information n'est cependant pas d'une grande utilité pour une personne non formée à la pédologie. C'est pour cette raison qu'une fois les pédopaysages identifiés, ils sont utilisés, avec d'autres critères, comme données d'entrée pour produire des cartes de contraintes qui traduisent une interprétation finalisée, directement utilisable de ces pédopaysages. Concernant le potentiel agronomique des terres rurales, objet central de notre chapitre, il est défini en huit catégories selon un gradient allant des terres les plus propices aux cultures aux terres inaptes à toute production agricole.

En plus des pédopaysages, d'autres facteurs comme l'eau et l'érosion éolienne, la salinité et la dégradation de la structure des sols sont pris en compte pour cartographier le potentiel des terres et des sols (NSW OEH, 2012). La prise en compte de toutes ces contraintes permet alors de générer une carte de potentiel agronomique des sols ou une carte de contraintes, comme celle présentée figure 5.10. Sur cette carte thématique, la zone autour de Baker's Lagoon apparaît à nouveau comme homogène. Cette fois cependant, il est aisé pour un non-spécialiste d'évaluer le potentiel agronomique des sols pour cette zone classée dans la catégorie 1, à savoir le potentiel agronomique le plus élevé de la classification. Cette classe correspond à la définition suivante : « terre au potentiel agronomique extrêmement élevé, soumise à aucune contrainte. Aucune pratique de gestion particulière n'est requise. La terre peut supporter tous les usages agricoles et les types de pratiques » (NSW OEH, 2012, p. 10). En plus de cette catégorisation en termes de potentiel agronomique des sols, une carte du potentiel productif agricole des terres est fournie sur le DVD des Ressources en terres et en sols. Cette autre classification (potentiel productif agricole) combine les huit catégories de potentiel agronomique des sols en trois catégories. Elle figure également parmi les cartes des sols de l'Atlas des ressources naturelles de Nouvelle-Galles du Sud consultable en ligne. Sur la zone autour de Baker's Lagoon, la carte du potentiel productif agricole des terres montre à nouveau que cette zone dispose du plus haut potentiel. La simple observation visuelle de l'image satellite fournie avec le jeu de données montre qu'une large part des terres classifiées comme ayant un potentiel agronomique très élevé ne sont pas artificialisées et sont encore utilisées pour l'agricul- 
ture, à l'exception de quelques parties converties à des usages résidentiels ou industriels. Cependant, cela n'est pas toujours le cas et il est important que les décideurs aient une vue d'ensemble des zones déjà développées, c'est-à-dire où les sols pourraient déjà avoir été imperméabilisés et, de ce fait, être inutilisables pour l'agriculture ou d'autres activités. Par conséquent, il est nécessaire de préserver les zones aux potentiels agronomiques les plus élevés d'un point de vue strictement pédologique, mais également, dans un objectif de sécurité alimentaire, de maintenir dans les aires périurbaines et dans l'arrière-pays les zones disponibles ayant le meilleur potentiel agronomique.

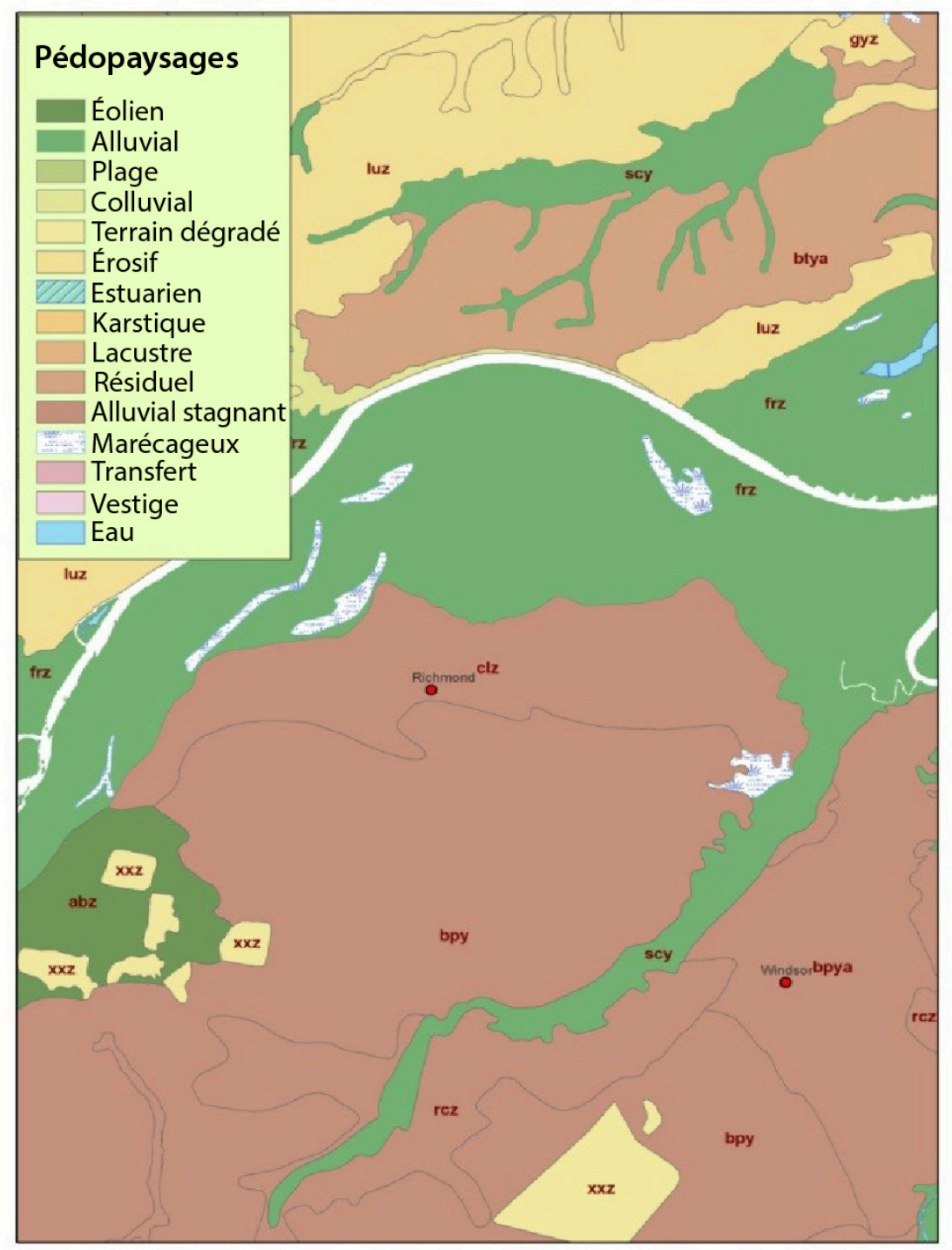

Figure 5.9. Carte de pédopaysage (zone autour de Baker’s Lagoon). 


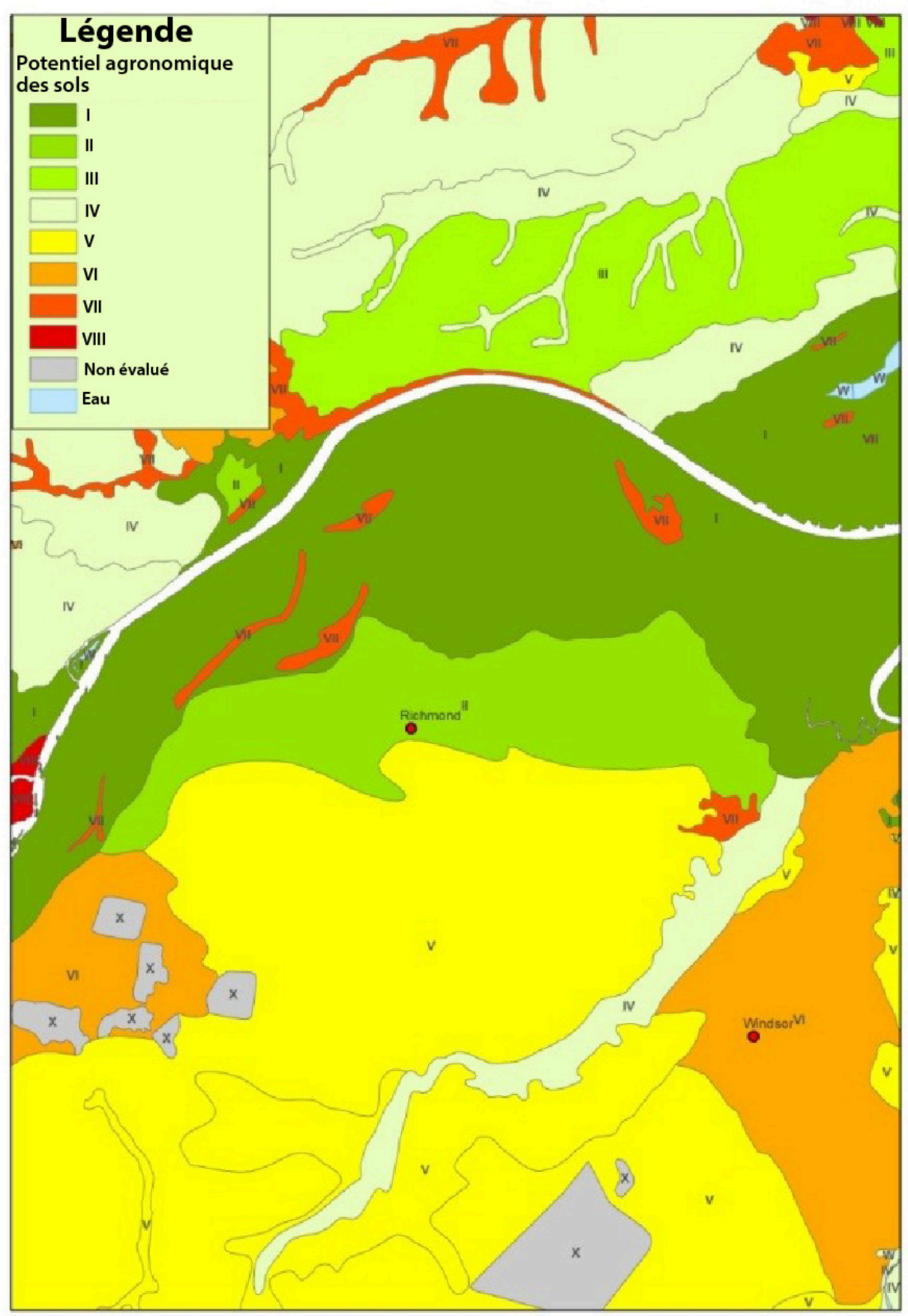

Figure 5.10. Carte du potentiel agronomique des sols (zone autour de Baker's Lagoon). 


\section{Conclusion et perspectives}

L'étalement urbain et l'imperméabilisation des sols qui lui est associée sont des phénomènes existant à l'échelle mondiale, qui affectent considérablement la disponibilité de terres pour les activités agricoles, la préservation de l'environnement et les activités récréatives. Pour assurer un usage durable des terres, les aménageurs doivent correctement appréhender ces phénomènes. Dans un premier temps, il s'agit de développer de manière proactive des fondements théoriques et des pratiques en matière d'aménagement du territoire afin d'éviter l'étalement urbain et l'imperméabilisation des sols. L'objectif d'aménagement durable des territoires ne peut être atteint qu'au moyen de processus de décision éclairés, basés sur des données factuelles. Ce chapitre s'est penché sur l'état d'avancement de la cartographie de l'étalement urbain et de l'imperméabilisation des sols en Australie, mais aussi sur l'accessibilité des données sur les sols afin d'améliorer encore le contenu informationnel véhiculé par la cartographie. Comme nous l'avons vu, ces données peuvent être utilisées pour créer des cartes de pédopaysages qui combinent les contraintes et les limites imposées par différents types de sols ainsi que différentes conditions géologiques. Les pédopaysages constituent la base des cartes de potentiel et de contraintes utilisées à des fins diverses, telles que l'aménagement urbain et rural, en permettant une appréhension facile des sols et d'autres types de contraintes.

La perte de bonnes terres agricoles rend nécessaire la préservation des terres agricoles restantes dans les zones périurbaines et dans l'arrière-pays pour la production alimentaire, même si elles n'ont pas les potentiels agronomiques les plus élevés. Les cartes de pédopaysages et les cartes thématiques, telles que celles représentant le potentiel agronomique des sols, sont des sources d'information facilement interprétables par les aménageurs pour identifier les zones à préserver pour assurer la sécurité alimentaire. Face à l'expansion continue des villes, il est important de fournir une couverture plus complète de ces cartes de potentiel ou de contraintes. Créer et mettre à jour ces cartes manuellement, comme cela se pratique actuellement, reste un processus très lent et onéreux. Il serait donc particulièrement utile de mener des travaux pour automatiser, au moins en partie, le processus de cartographie afin de fournir plus de données et de cartes interprétatives sur les sols à une plus large audience. Par exemple, en utilisant l'analyse d'images pour délimiter les limites paysagères et en les combinant ensuite avec les données sur les sols pour tous les points relevés sur une zone donnée, des pédopaysages prédéfinis pourraient être fournis aux experts et permettraient ainsi la production plus rapide de cartes. L'automatisation complète n'est pas envisageable, du moins à l'heure actuelle, étant donné que les cartes des paysages, et encore davantage les cartes de potentiel et de contraintes, sont toujours créées à des fins spécifiques et exigent un certain degré d'expertise.

\section{Références bibliographiques}

Biggs A.J.W., Grundy M.J., 2010. The need for better links between pedology and soil carbon research in Australia. Australian Journal of Soil Research, 48, 1-6.

Demographia, 2017. Demographia. World Urban Areas. In: World Urban Areas (Demographia, ed.), $13^{\mathrm{e}}$ ed.

Graetz R.D., Fisher R.P., Wilson M.A., Campbell S.K., 1998. Looking back. The changing face of the Australian continent: 1972-1992. In: Applications (C.O.O.S.S., ed.), COSSA Publication, CSIRO and the Commonwealth of Australia.

Johnston R.M., Barry S.J., Bleys E., Bui E.N., Moran C.J., Simon D.A.P., et al., 2003. ASRIS: the database. Australian Journal of Soil Research, 41, 1021-1036. 
Milford H.B., Mcgaw A.J.E., Nixon K.J., 2001. Soil Data Entry Handbook for the NSW Soil and Land Information System (SALIS), 3rd edition, Parramatta, Australia, NSW Department of Land and Water Conservation, Resource Information Systems Group.

Northcote K.H., 1978. Soils and land use. In: Atlas of Australian Resources, Canberra, Division of National Mapping.

NSW OEH, 2012. The Land and Soil Capability Assessment Scheme. Second Approximation, Sydney, Australia, State of NSW and Office of Environment and Heritage.

NSW OEH, 2016. eDIRT user manual for the Digital Infield Regolith Tool (eDIRT), version 1.1. Sydney, Australia, State of NSW and Office of Environment and Heritage, $113 \mathrm{p}$.

Smart Growth America, 2014. Measuring Sprawl 2014. Smart Growth America. Making Neighborhoods Great Together, www.smartgrowthamerica.org .

Sutton P.C., Goetz A.R., Fildes S., Forster C., Ghosh T., 2010. Darkness on the edge of town: mapping urban and peri-urban Australia using nighttime satellite imagery. The Professional Geographer, 62, 119-133.

Yang X., Chapman G.A., Gray J.M., Young M.A., 2007. Delineating soil landscape facets from digital elevation models using compound topographic index in a geographic information system. Australian Journal of Soil Research, 45, 569-576. 


\title{
Chapitre 6
}

\section{Techniques géospatiales et de télédétection pour le suivi de l'état et des dynamiques des terres agricoles périurbaines}

\author{
Paras Sidiqui, Rakhesh Devadas, Alfredo Huete
}

\section{Introduction}

Depuis toujours, les sociétés humaines modifient l'environnement dans lequel elles évoluent. Des villes de plus en plus étendues, à l'échelle mondiale, accueillent actuellement plus de $50 \%$ de la population mondiale, et ce pourcentage continue d'augmenter (DESA, 2012). L'étalement urbain rapide exerce des pressions sur les zones périurbaines et rurales environnantes, menant à la perte d'espaces et d'activités agricoles. De plus, l'urbanisation est un facteur majeur de perte de biodiversité et d'homogénéisation biologique.

Les zones périurbaines sont situées immédiatement au sortir des périphéries urbaines en expansion et sont caractérisées par une population moins dense et un nombre réduit d'infrastructures par rapport aux zones urbaines. Dans beaucoup de villes, les zones périurbaines accueillent des activités agricoles plus ou moins intensives, qui répondent aux besoins de production alimentaire locale et de sécurité alimentaire des villes avoisinantes. Ces zones peuvent même abriter, dans certains cas, l'essentiel des ressources en nourriture, en fibres et en énergie d'un pays (Verburg et al., 2009). Les zones périurbaines jouent aussi un rôle important dans la préservation de la biodiversité, du patrimoine culturel et de la beauté d'une région, et ont des caractéristiques socio-économiques, politiques et écologiques uniques (Pauchard et al., 2006).

Au-delà des zones périurbaines, les zones rurales comprennent des paysages naturels et des zones naturelles protégées qui constituent un réservoir de ressources pour des services et des fonctions écosystémiques vitales pour les populations périurbaines et urbaines. Ainsi, les zones périurbaines peuvent être définies comme des « zones de transition » entre les villes et les espaces ruraux, soumises à des changements rapides de leurs paysages (Pauchard et al., 2006).

L'urbanisation croissante exerce une pression grandissante sur les producteurs locaux (producteurs laitiers, arboriculteurs et maraîchers) ainsi que sur d'autres types d'activités agricoles. L'étalement urbain conduit au déplacement, à la perte et à la fragmentation des terres agricoles et des espaces naturels. À leur tour, ces phénomènes perturbent les fonctions des écosystèmes, les services écosystémiques ainsi que la production agricole. De 
plus, l'urbanisation croissante peut altérer les propriétés physiques d'un paysage, comme l'albédo, le bilan de rayonnement à la surface et le bilan énergétique, affectant ainsi le climat régional et global (Zhai et al., 2014). La magnitude du forçage radiatif induit par le changement de couverture du sol peut aggraver l'effet d'îlot de chaleur urbain dans les villes en modifiant les températures locales.

La cartographie et le suivi des activités agricoles ainsi que d'autres activités, dans les zones périurbaines, ont principalement reposé sur des approches conventionnelles exigeant une main-d'œuvre importante et impliquant du travail de terrain et le recueil d'échantillons de données pour la préparation des cartes. Ces approches sont onéreuses et ne peuvent être répétées que tous les cinq ou dix ans. À l'inverse, les données de télédétection permettent de faire des observations synoptiques, cohérentes et reproductibles avec de l'information spatiale précise. Ces jeux de données peuvent facilement produire des informations sur les configurations et les processus de transformation des paysages ainsi que des métriques paysagères pointues (Peng et al., 2007 ; Tang et al., 2006).

Quarante ans d'archives d'images satellites permettent de mener une analyse historique des données pour retracer les dynamiques passées des paysages périurbains et mieux comprendre les changements des trajectoires agricoles en réponse à l'urbanisation. La télédétection offre de nombreuses possibilités de suivi des dynamiques passées, présentes et en temps réel des activités agricoles afin de mieux appréhender les processus environnementaux dans les zones périurbaines, et ainsi d'améliorer la gestion et l'aménagement du territoire.

Dans ce chapitre, nous explorons comment la télédétection et les technologies géospatiales nous permettent de cartographier et de quantifier l'urbanisation ainsi que les changements paysagers au sein des espaces agricoles dans les zones périurbaines. Les mêmes outils et images sont utilisés pour déterminer les impacts de l'urbanisation sur les terres agricoles périurbaines. L'accent est mis sur les données satellitaires disponibles publiquement et gratuitement. Ces dernières sont utilisables pour offrir des solutions durables pour une gestion et une planification plus efficaces des zones urbaines et périurbaines. Après avoir introduit brièvement les techniques disponibles et les utilisations possibles de la télédétection et des systèmes d'information géographique (SIG) pour le suivi de ces zones, nous présentons l'étude de cas de la métropole de Sydney en Australie. Nous y décrivons les utilisations possibles de la télédétection et des SIG pour la classification des terres et l'analyse historique des changements du paysage durant les vingt dernières années.

\section{Télédétection et techniques géospatiales}

La télédétection et les SIG offrent la possibilité de cartographier et de faire un suivi régional à des échelles synoptiques. «Le format numérique peut apporter des bénéfices supplémentaires, tels que des changements d'échelles simples, l'agrégation et la désagrégation, un accès facile aux statistiques et aux valeurs attributaires, ainsi que l'intégration aisée d'autres données SIG numériques » (Ridd, 1995, p. 2175).

La télédétection consiste à détecter des objets sur des surfaces terrestres et aquatiques, sans contact physique, par des mesures à distance, et à enregistrer des données au moyen de capteurs qui ne sont pas en contact avec les surfaces observées. Si l'on s'en tient à cette définition générale, l'œil humain est un exemple de système imageur de détection à distance. Le signal détecté est transmis sous la forme d'énergie électromagnétique lorsqu'il est réfléchi et/ou émis par la surface, en direction du capteur. Le résultat final est souvent une image numérique représentant la scène observée. Plusieurs étapes 
de traitement et d'interprétation de l'image sont la plupart du temps nécessaires pour extraire de l'image des données et des informations de qualité suffisante. Prise au sens restreint du terme, la télédétection désigne la technologie d'acquisition d'informations à l'aide de capteurs embarqués sur des plateformes aéroportées (avions, ballons, drones) ou spatiales (satellites, navettes spatiales). Dans la section qui suit, nous présentons des capteurs de télédétection qui peuvent être utiles pour le suivi des zones périurbaines, ainsi que certaines de leurs applications.

Tableau 6.1. Exemples de satellites et de capteurs pouvant être utilisés pour l'analyse des espaces périurbains

\begin{tabular}{|c|c|c|c|c|}
\hline $\begin{array}{l}\text { Satellite/ } \\
\text { capteur }\end{array}$ & $\begin{array}{c}\text { Début de } \\
\text { disponibilité } \\
\text { des archives }\end{array}$ & $\begin{array}{l}\text { Résolution } \\
\text { spatiale au } \\
\text { nadir1 (en } \\
\text { mode multis- } \\
\text { pectral) }\end{array}$ & $\begin{array}{l}\text { Résolution } \\
\text { temporelle à } \\
\text { l'équateur }\end{array}$ & Applications \\
\hline \multicolumn{5}{|c|}{ Open Source Data } \\
\hline $\begin{array}{l}\text { Série satellites } \\
\text { NOAA/ } \\
\text { AVHRR }\end{array}$ & 1979 & $1,1 \mathrm{~km}$ & 1 jour & $\begin{array}{l}\text { Suivi de la température de surface } \\
\text { (Kidder et Wu, 1987; Streutker, 2002) }\end{array}$ \\
\hline $\begin{array}{l}\text { Série satellites } \\
\text { Landsat }\end{array}$ & 1987 & $30 \mathrm{~m}-60 \mathrm{~m}$ & 16 jours & $\begin{array}{l}\text { Suivi des changements de la } \\
\text { température de surface ; cartographie } \\
\text { de la couverture du sol ; cartographie } \\
\text { de l'albédo et de la sécheresse de la } \\
\text { végétation (Aniello et al., } 1995 \text {; } \\
\text { Odindi } \text { et al., 2015) }\end{array}$ \\
\hline ASTER & 1999 & $15 \mathrm{~m}-90 \mathrm{~m}$ & 16 jours & $\begin{array}{l}\text { Suivi des changements de la } \\
\text { température de surface ; cartographie } \\
\text { de l'occupation du sol (Chrysoulakis, } \\
2003 \text {; Zhu et Blumberg, 2002) }\end{array}$ \\
\hline $\begin{array}{l}\text { Satellites } \\
\text { Aqua/Terra } \\
\text { (MODIS) }\end{array}$ & 2000 & $1 \mathrm{~km}$ & 4 images/jour & $\begin{array}{l}\text { Suivi de la température de surface et de } \\
\text { la température de l'air ; cartographie de } \\
\text { l'occupation du sol ; cartographie des } \\
\text { caractéristiques des matériaux, de } \\
\text { l'albédo et de la végétation }\end{array}$ \\
\hline Sentinel-2 & 2015 & $10 \mathrm{~m}$ & 10 jours & $\begin{array}{l}\text { Détection de changements } \\
\text { d'occupation du sol (Wang } \text { et al., 2016) }\end{array}$ \\
\hline $\begin{array}{l}\text { Himawari } 8 \text { et } \\
9\end{array}$ & 2016 & $0,5-2 \mathrm{~km}$ & 15 minutes & $\begin{array}{l}\text { Suivi des précipitations, de la } \\
\text { température de surface de la mer, et de } \\
\text { l'atmosphère (Kurihara et al., 2016; } \\
\text { Yumimoto et al., 2016) }\end{array}$ \\
\hline \multicolumn{5}{|c|}{ Données commerciales } \\
\hline $\begin{array}{l}\text { Ikonos, } \\
\text { QuickBird, } \\
\text { GeoEye-1, } \\
\text { WorldView }(1, \\
2,3,4)\end{array}$ & 1999 & $0,4-1,24 \mathrm{~m}$ & Programmable & $\begin{array}{l}\text { Classification de l'occupation du sol } \\
\text { urbaine ; détection d'éléments urbains, } \\
\text { cartographie de la végétation en ville } \\
\text { (Davis et Wang, } 2002 \text {; Grau et al., } \\
\text { 2008) }\end{array}$ \\
\hline $\begin{array}{l}\text { Série satellites } \\
\text { SPOT }(5,6,7)\end{array}$ & 2002 & $6-10 \mathrm{~m}$ & Programmable & $\begin{array}{l}\text { Occupation du sol (Durieux et al., } \\
\text { 2008) }\end{array}$ \\
\hline
\end{tabular}

${ }^{1}$ Le point le plus bas. 


\section{Données satellitaires}

Les capteurs satellitaires enregistrent de l'information sur la surface terrestre, les océans et l'atmosphère dans différentes bandes spectrales pouvant aller, sur le spectre électromagnétique des longueurs d'onde, du domaine du visible jusqu'aux micro-ondes. La résolution spatiale, qui correspond à la taille du pixel, peut aller de quelques centimètres à plusieurs kilomètres, et la fréquence de revisite au-dessus d'un même point peut aller de quinze minutes à plusieurs semaines (Xie et al., 2008). Pour la cartographie de la couverture et de l'occupation du sol dans les zones périurbaines, il existe une série de capteurs satellitaires à haute résolution, comme QuickBird (Grau et al., 2008), Pléiades, SPOT, Sentinel-2 et Landsat. Ces capteurs permettent de faire des cartographies détaillées des états de surface. Le tableau 6.1 liste les satellites les plus utilisés pour l'étude des zones urbaines et périurbaines, ainsi que les caractéristiques de leurs capteurs, leur durée de vie, leurs résolutions, et des exemples d'applications. Cela comprend des satellites à haute résolution temporelle (journalière) tels que MODIS (Moderate Resolution Imaging Spectroradiometer) et AVHRR (Advanced Very High Resolution Radiometer), ainsi que des satellites à très haute résolution spatiale $(<1 \mathrm{~m})$ comme QuickBird, WorldView 1-4 ou Pléiades.

Bien que certaines données satellitaires soient uniquement commerciales et payantes, ces dix dernières années ont vu une augmentation importante du nombre de données satellitaires publiques et gratuites, comme les satellites Aqua/Terra, Landsat, ASTER, Himawari et Sentinel-2. Les capteurs MODIS embarqués sur les satellites Aqua/Terra offrent des données multispectrales à 36 bandes, depuis 2000 jusqu'à aujourd'hui. Le satellite Aqua passe à l'équateur à $1 \mathrm{~h} 30$ et $13 \mathrm{~h} 30$ heure locale, tandis que le satellite Terra

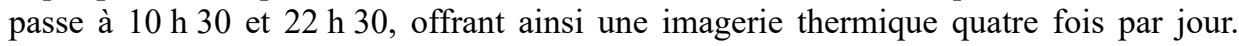
Ces données sont particulièrement adaptées à la cartographie à des échelles macroscopiques et pour des analyses régionales de température, d'albédo, ainsi que du niveau de sécheresse de la végétation. Les produits dérivés de MODIS sont disponibles sur le site internet de la NASA ${ }^{25}$. Les données Landsat, qui ont une résolution spatiale de $30 \mathrm{~m}$ pour les bandes optiques, de $60 \mathrm{~m}$ pour les bandes thermales et une résolution temporelle de 16 jours, sont disponibles sur le site internet de 1'USGS ${ }^{26}$. La mission Sentinel-2 est une constellation de deux satellites destinée au suivi des terres émergées, qui offre une imagerie optique à haute résolution, disponible sur le site internet de l'Agence spatiale européenne (European Space Agency, ESA) ${ }^{27}$. L'Instrument multispectral (MultiSpectral Instrument, MSI) embarqué sur les satellites Sentinel-2 offre une imagerie optique avec une résolution bien plus élevée $(10 \mathrm{~m})$ que les séries Landsat $(30 \mathrm{~m})$ et peut fournir des cartes de classification des paysages avec plus de détails et de classes, permettant potentiellement d'améliorer la représentation des impacts de l'urbanisation sur les aires périurbaines environnantes.

\section{Produits satellites et indices spectraux}

La télédétection fournit des informations diverses, qui sont utiles pour délimiter et classifier la végétation, le bâti, la surface terrestre et les surfaces en eau. Les indices spectraux sont des mesures biophysiques de l'état, de la structure ou de la composition de la surface d'éléments paysagers. Ces indices sont formés en additionnant, en divisant ou en multipliant des bandes spectrales pour détecter diverses caractéristiques d'absorption (ex. : pigments, minéraux, composés organiques) et faire apparaître les propriétés spécifiques

25. https://urs.earthdata.nasa.gov/ .

26. http://www.earthexplorer.usgs.gov/ .

27. https://scihub.copernicus.eu/ . 
de la surface terrestre, comme la végétation vivante, la neige, la végétation ligneuse ou l'eau. Le tableau 6.2 dresse la liste des indices spectraux les plus utilisés pour le suivi satellitaire des dynamiques des surfaces terrestres, et disponibles comme produits standard.

Tableau 6.2. Exemples de produits satellitaires adaptés au suivi des aires périurbaines. Les termes «Bleu », « Rouge», « Vert », « proche infrarouge » (PIR) et « infrarouge court » (IRC) indiquent les valeurs de réflectance de la surface telles que mesurées dans des bandes spectrales spécifiques, et $T_{B}$ représente les températures de brillance mesurées par le satellite.

\begin{tabular}{|c|c|c|}
\hline Indice spectral & Formule & Application \\
\hline $\begin{array}{l}\text { Indice de végétation } \\
\text { par différence } \\
\text { normalisée }\end{array}$ & $\begin{array}{l}\text { PIR - Rouge/PIR + Rouge } \\
\text { (Rouse Jr et al., 1974) }\end{array}$ & $\begin{array}{l}\text { Met en valeur la végétation verte et en } \\
\text { bonne santé }\end{array}$ \\
\hline $\begin{array}{l}\text { Indice de végétation } \\
\text { amélioré }\end{array}$ & $\begin{array}{l}\mathrm{G} \times \mathrm{PIR}-\text { Rouge } / \mathrm{PIR}+\mathrm{C} 1 \times \text { Rouge }+ \\
\mathrm{C} 2 \times \mathrm{Bleu}+\mathrm{L} \\
\text { Avec } \mathrm{G}=2,5, \mathrm{C} 1=6, \mathrm{C} 2=7,5 \text { et } \mathrm{L}=1 \\
(\text { Huete } \text { et al., } 2002)\end{array}$ & $\begin{array}{l}\text { Fait ressortir la végétation/la verdure. La } \\
\text { réflectance dans le bleu est utilisée pour } \\
\text { éliminer le signal du sol et réduire les } \\
\text { effets atmosphériques, y compris la } \\
\text { diffusion par les aérosols }\end{array}$ \\
\hline $\begin{array}{l}\text { Indice de différence } \\
\text { normalisée du bâti }\end{array}$ & $\begin{array}{l}\text { IRC }- \text { PIR/IRC }- \text { PIR } \\
\text { (Zha } \text { et al., 2003) }\end{array}$ & Met en valeur les caractéristiques urbaines \\
\hline $\begin{array}{l}\text { Indice modifié de } \\
\text { différence } \\
\text { normalisée de l'eau }\end{array}$ & $\begin{array}{l}\text { Vert }- \text { IRC/Vert }+ \text { IRC } \\
(\mathrm{Xu}, 2006)\end{array}$ & $\begin{array}{l}\text { Met en valeur les caractéristiques des } \\
\text { surfaces en eau tout en supprimant le bruit } \\
\text { du bâti, de la végétation et du sol }\end{array}$ \\
\hline $\begin{array}{l}\text { Température de } \\
\text { surface }\end{array}$ & $\begin{array}{l}\mathrm{T}_{\mathrm{B}} / 1+\left(\lambda \times \mathrm{T}_{\mathrm{B}} / \mathrm{c}_{2}\right) \times \ln (\mathrm{e}) \\
\text { Avec } \lambda=\text { longueur d'onde du } \\
\text { rayonnement émis, et } \mathrm{c}_{2}=0,014388 \mathrm{~m} \\
\mathrm{~K} \\
(\text { Weng et al., 2004) }\end{array}$ & $\begin{array}{l}\text { Permet de mesurer la température de la } \\
\text { surface terrestre }\end{array}$ \\
\hline Albédo & $\begin{array}{l}\text { Radiation sortante à ondes courtes/ } \\
\text { radiation entrante à ondes courtes }\end{array}$ & $\begin{array}{l}\text { Permet d'identifier les surfaces et leurs } \\
\text { propriétés }\end{array}$ \\
\hline
\end{tabular}

\section{Les indices de végétation}

Plusieurs indices de végétation fournissent des mesures quantitatives du niveau de sécheresse de la canopée. Ils portent sur la surface foliaire verte, la structure de la canopée et la teneur des feuilles en chlorophylle (Veroustraete et al., 1994). L'un des indices de végétation les plus largement utilisés est l'indice de végétation par différence normalisée (normalised difference vegetation index, NDVI) (Tucker, 1979), dont les valeurs vont de -1 à +1 . L'indice de végétation amélioré (enhanced vegetation index, EVI) est un indice de végétation « optimisé » pour améliorer le signal de la végétation grâce à une plus grande sensibilité dans les régions à forte biomasse, et pour mieux suivre l'état de la végétation en découplant le signal de fond de la canopée et en réduisant l'influence de l'atmosphère (Huete et al., 1997). Les indices de végétation sont souvent utilisés pour faire un suivi des écosystèmes dans le temps et pour évaluer les stades phénologiques de la végétation.

\section{L'albédo}

L'albédo de surface, défini comme le ratio du rayonnement réfléchi sur la surface hémisphérique et du rayonnement total incident, est une grandeur sans dimension décrivant les caractéristiques du rayonnement de surface et le bilan énergétique. Les changements 
de couverture du sol impactent directement l'albédo de surface, altérant ainsi le bilan énergétique et provoquant des effets climatiques à la surface (Zhai et al., 2014). Dans certains cas, la magnitude du forçage radiatif causée par l'albédo peut être plus importante, à l'échelle locale, que les émissions de gaz à effet de serre, et peut ainsi déterminer le climat d'une région (Zhai et al., 2014).

\section{La température de la surface terrestre}

Les bandes des capteurs satellitaires dans l'infrarouge thermique sont souvent utilisées pour évaluer les motifs spatiaux et temporels ainsi que les évolutions de la température de la surface terrestre. Les images thermiques qui représentent la température de brillance sont ensuite traitées pour obtenir des valeurs de température de la surface terrestre à partir d'estimations de l'émissivité (Kalma et al., 2008 ; Weng et al., 2004).

\section{L'indice modifié de différence normalisée de l'eau}

Les bandes sensibles à l'eau se situent dans la plage spectrale de l'infrarouge à courte longueur d'onde qui est absorbé par l'eau. Elles peuvent être utilisées pour identifier sur les images satellitaires les surfaces en eau et les surfaces inondées. L'indice modifié de différence normalisée de l'eau est souvent utilisé pour aider à la classification des pixels d'eau, mais il peut aussi servir à masquer les surfaces en eau avant une classification de l'occupation du sol (Xu, 2006).

\section{Analyse et cartographie de l'occupation du sol}

Dans cette partie, nous présentons des techniques géospatiales et de télédétection utilisées pour la classification des paysages. Les utilisations possibles de ces techniques pour cartographier, faire le suivi et mieux comprendre les dynamiques des surfaces terrestres dans les zones périurbaines, seront illustrées à partir de l'étude de cas de Sydney en Australie.

Les cartes de couverture du sol donnent des informations sur les surfaces couvertes en forêts, en zones humides, en zones imperméabilisées, en zones de végétation ou tout autre élément présent à la surface terrestre. Les cartes d'occupation du sol fournissent quant à elles des informations sur les activités (ou l'absence d'activités) humaines, comme les activités commerciales, résidentielles, agricoles et d'élevage ou les zones de friches dans les zones périurbaines. Les cartes des fonctions des terres intègrent quant à elles davantage d'informations locales fournies par les propriétaires, afin de développer des cartes détaillant l'état et les dynamiques des surfaces terrestres. Les cartes de couverture ou d'occupation du sol à différentes échelles peuvent être utilisées pour mettre et maintenir à jour les informations, et ainsi permettre une meilleure gestion et planification des activités agricoles dans les zones périurbaines. Diverses techniques ont été utilisées par les chercheurs, comme les classifications d'occupation du sol basées sur l'imagerie multispectrale (Atkinson, 2005 ; Dougherty et al., 2004), l'analyse sub-pixellaire des mélanges spectraux (Weng, 2001 ; 2002 ; Wu et al., 2005), ainsi que des méthodes plus perfectionnées reposant sur l'apprentissage machine, les réseaux neuronaux et les classifications orientées objet (Yang et al., 2003).

\section{La classification multispectrale}

Les outils de télédétection et les SIG peuvent être utilisés pour mieux comprendre et définir les limites des zones urbaines, périurbaines et rurales. Les données satellitaires peuvent servir à catégoriser les paysages en fonction du type de couverture ou d'occupation du sol, à l'aide de techniques de classification d'image. La classification multispectrale peut être décrite comme permettant la catégorisation d'images de la surface 
terrestre en différents groupes de couverture et d'occupation du sol, grâce à l'utilisation des propriétés de certaines bandes spectrales. Ce type de classification est la façon la plus simple d'utiliser la télédétection pour classer des surfaces n'ayant qu'une caractéristique : chaque pixel dans une image appartient à une classe particulière, afin de produire une carte finale avec un nombre donné de catégories. Ces méthodes de classification numérique présentent l'avantage d'être reproductibles dans le temps et dans l'espace et de pouvoir faire l'objet de vérifications de terrain.

Il existe deux principaux types de méthodes de classification d'image, l'une appelée « classification non supervisée » et l'autre « classification supervisée ». La classification non supervisée implique la sélection initiale de groupes de pixels sur la base des propriétés spectrales de l'image numérique pour ensuite affecter chaque groupe de pixels à différentes classes de couvertures du sol. Cette approche permet de classer les pixels sans qu'il soit nécessaire d'établir au préalable la liste et les intitulés des classes d'occupation du sol et leurs noms.

À l'inverse, la classification supervisée est basée sur la connaissance préalable des classes d'occupation du sol présentes sur une image. Par conséquent, la classification est dite " guidée » ou « supervisée ». Avec cette méthode, les pixels sont regroupés dans différentes classes d'occupation du sol sur la base d'échantillons. Ces échantillons sont générés en dessinant des zones d'intérêt sur l'image pour que chaque type d'occupation du sol étudié puisse être identifié. L'établissement de ces classes présélectionnées est basé sur des informations et des connaissances recueillies au préalable sur le terrain à l'aide, par exemple, de photographies aériennes, de cartes, d'images Google Earth ou encore d'une analyse spectrale des images à classer.

Les informations sur les surfaces végétales et non végétales obtenues en se servant de l'indice de végétation amélioré ou de l'indice de végétation par différence normalisée peuvent aussi être intégrées au processus de détermination des classes. D'autres caractéristiques de l'image comme le ton, la couleur, la texture et la forme peuvent également être prises en compte pour définir les attributs des différentes occupations du sol. Des couleurs et des intitulés différents sont alors attribués aux différentes classes durant l'analyse et constituent la légende de la carte. Les classes ainsi générées peuvent ensuite être regroupées dans des classes plus générales selon la nature des applications pour lesquelles les cartes ont été produites. Il existe différents algorithmes de classification, tels que le maximum de vraisemblance, la distance minimale ou l'approche par parallélépipède, le plus utilisé étant cependant le maximum de vraisemblance. Pour plus d'informations sur les méthodes de classification, il est possible de se référer à l'ouvrage Remote Sensing and Image Interpretation, de Lillesand et al. (2014).

\section{L'analyse linéaire des mélanges spectraux}

Une autre approche de classification repose sur l'hypothèse que la plupart des pixels sur une image satellite contiennent des signatures spectrales mélangées appartenant à deux catégories d'occupation du sol ou plus. Par exemple, un pixel peut contenir à la fois du sol nu et des plantes, ou un toit de maison avec des arbres. Cette méthode de classification d'occupation des sols sur des pixels mélangés est appelée " analyse linéaire de mélange spectral » (Garcia-Haro et al., 1996).

L'analyse linéaire de mélange spectral est une méthode de traitement d'image basée sur la physique du signal qui considère le spectre mesuré par un capteur d'image comme étant une combinaison linéaire des spectres de tous les composants à l'intérieur d'un pixel. Les résultats obtenus sont des images fractionnaires qui représentent la quantité de chaque composant dans l'image. Cette méthode modélise la réflectance de chaque pixel 
comme une combinaison linéaire de la réflectance de chaque composant présent dans le pixel. Dans cette approche, le calcul des images fractionnaires se fait en plusieurs étapes : traitement de l'image d'origine, choix des composants des pixels, opération de démélange linéaire et évaluation des images fractionnaires obtenues. De toutes ces étapes, le choix des composants les plus appropriés est la plus critique pour obtenir des images fractionnaires de bonne qualité.

Dans les études urbaines, cette technique est souvent utilisée pour classifier et quantifier les zones imperméabilisées qui empêchent l'infiltration de l'eau comme par exemple les rues, les routes, les parkings, les trottoirs et les bâtiments (Hu et Weng, 2009). Généralement, trois types de composants sont identifiés au départ sur une image multispectrale : la végétation chlorophyliennement active, les sols et les infrastructures urbaines (surfaces bétonnées, parkings, bâtiments/immeubles, maisons, aéroports, etc.) (Hu et Weng, 2009). La classe "végétation » peut être caractérisée à partir de zones d'herbe dense ou de pâture; la classe "sols » à partir de parcelles en sol nu parmi les terres agricoles; et les différents types de surfaces bâties (résidentielles et non résidentielles) à partir de toits de bâtiments, de pistes de décollage d'aéroports ou de croisements de routes principales. Le résultat du démélange est une série d'images montrant l'abondance fractionnaire dans chaque pixel d'un type d'occupation du sol donné (Garcia-Haro et al., 1996). Afin d'arriver à la meilleure qualité possible d'images fractionnaires pour l'estimation des surfaces bâties et des éléments de végétation, différentes combinaisons de composants sont souvent examinées et comparées.

\section{Autres méthodes}

Il existe d'autres approches de cartographie et de suivi de l'occupation du sol, telles que la classification par régression sous pixels (Song et al., 2005), la classification par réseau neuronal (Civco et Hurd, 1997), les régressions multiples (Bauer et Curran, 2005) ou encore les classifications orientées objet (Blaschke, 2010). Dans les classifications orientées objet, les pixels sont regroupés en objets homogènes résultant d'une segmentation des images. Cette méthode mène à la création d'objets à différentes échelles qui représentent différents éléments de l'image. Pour plus d'informations sur les classifications orientées objet, il est possible de se référer à l'ouvrage de Blaschke et al. (2008) intitulé Object-Based Image Analysis: Spatial Concepts for Knowledge-Driven Remote Sensing Applications.

\section{La fragmentation des paysages}

La fragmentation des paysages correspond au fractionnement et à la perte de connectivité de zones d'occupation du sol contiguës, liés soit à des processus perturbateurs, soit à certaines pratiques d'usage du sol (Saunders et al., 1991). La fragmentation de zones de végétation endémique a des impacts significatifs sur le fonctionnement des écosystèmes et sur la diversité biologique et paysagère (Fahrig, 2003). La caractérisation détaillée des formes de l'étalement urbain et de la fragmentation qui l'accompagne dans les zones périurbaines a progressé du fait de l'accessibilité croissante à des données spatiales et du développement de métriques spatiales adaptées de l'écologie du paysage (Kane et al., 2014 ; Turner et al., 1989). Les métriques paysagères sont dérivées de l'analyse de cartes thématiques-catégorielles qui, dans un premier temps, divisent le paysage étudié en taches de pixels contigus d'une même classe, puis utilisent cette information pour quantifier différents motifs paysagers (Kane et al., 2014). Les métriques spatiales communément utilisées incluent : le nombre de taches (densité des taches), la superficie totale des taches ; la densité des bordures (somme de toutes les longueurs des côtés des taches divisée par la superficie totale du paysage); la richesse des taches, des métriques, des 
formes et des bordures ; ainsi qu'un indice d'agrégation (He et al., 2000). Il a été prouvé que la surface totale des taches est étroitement liée aux questions de sécurité alimentaire et de durabilité (Su et al., 2011).

Pour quantifier l'étendue et le degré de fragmentation du paysage, la composition ainsi que la configuration des composants doivent toutes deux être prises en compte (Munroe et al., 2005; Neill et al., 1997). Le nombre de taches et leur densité sont un indice de l'hétérogénéité spatiale du paysage. La diminution au cours du temps de la densité des taches de végétation native révèle généralement une réaffectation de l'occupation du sol, tandis qu'une augmentation de la densité de ces taches traduit une fragmentation de l'occupation du sol au sein d'une même zone (Schneider et Woodcock, 2008). L'indice de diversité de Shannon est un exemple de métrique de diversité. Cet indice est basé sur les informations contenues dans chaque type de tache (Saunders et al., 1991).

L'indice d'agrégation constitue un indicateur global de l'étendue des modifications subies par un paysage du fait de perturbations telles que l'urbanisation ( $\mathrm{Su}$ et al., 2011). Cet indice est calculé sur la base d'une matrice de contiguïté des taches qui donne la fréquence avec laquelle des paires de taches de types différents se retrouvent côte à côte sur la carte, et quantifie ainsi le degré d'isolement ou de fragmentation d'un type d'occupation du sol (Ji et al., 2006). Le tableau 6.3 donne les formules des métriques paysagères les plus importantes. Lorsque l'on utilise des données satellites d'occupation du sol ou d'autres jeux de données géospatiales, l'indice d'agrégation, le nombre de taches (densité des taches) et la superficie totale peuvent être calculés facilement à l'aide du programme Fragstats dans le logiciel QGIS (McGarigal et Marks, 1995).

Tableau 6.3. Les métriques paysagères et leurs formules, telles que définies dans le programme Fragstats sur QGIS (McGarigal et Marks, 1995).

\begin{tabular}{|l|l|}
\hline Métriques paysagères & \multicolumn{1}{|c|}{ Formules } \\
\hline Nombre de taches & $\begin{array}{l}\mathrm{n}_{\mathrm{i}} \\
\mathrm{n}_{\mathrm{i}}=\text { nombre de taches de classe i dans le paysage }\end{array}$ \\
\hline Surface totale & $\begin{array}{l}\mathrm{A} \times(1 / 1000)^{2} \\
\text { Avec } \mathrm{A}=\text { surface totale du paysage }\left(\mathrm{m}^{2}\right)\end{array}$ \\
\hline Indice d'agrégation & $\begin{array}{l}100 \times\left(\mathrm{g}_{\mathrm{ii}} / \mathrm{max}-\mathrm{g}_{\mathrm{ii}}\right)^{2} \\
\text { Avec } \mathrm{g}_{\mathrm{ii}}=\text { nombre d'adjacences similaires entre pixels d'une classe de type } \mathrm{i} \text {, et } \\
\mathrm{max}-\mathrm{g}_{\mathrm{ii}}=\text { nombre d'adjacences similaires entre classes de type } \mathrm{i}\end{array}$ \\
\hline
\end{tabular}

\section{Étude de cas : Sydney, Australie}

En Australie, $89 \%$ de la population vit en zone urbaine, et il est prévu que cette part atteigne $93 \%$ en 2050. La population australienne est de plus en plus concentrée dans les cinq plus grandes villes du pays : Sydney, Melbourne, Brisbane, Perth et Adelaïde (Cleugh et al., 2011), Sydney totalisant à elle seule $20 \%$ de la population. Les zones périurbaines ou rurales dans la zone métropolitaine de Sydney sont d'une importance cruciale, car elles jouent un rôle vital dans l'agriculture australienne. D'après le Département des industries du secteur primaire de Nouvelle-Galles du Sud et le Bureau australien du recensement statistique agricole, $7 \%$ de la production agricole de l'État de Nouvelle-Galles du Sud provient de la région de Sydney. Les rapports montrent également qu'environ la moitié de la valeur de la production maraîchère de Nouvelle-Galles du Sud provient de la région de Sydney. 
Nous allons présenter ci-après une étude de cas des dynamiques des paysages périurbains qui entourent Sydney. Cette étude explore les changements d'usage et d'occupation du sol survenus durant les vingt dernières années, ainsi que les taux de fragmentation et les changements des propriétés de la surface des terres au cours du temps. Le but est de montrer comment plusieurs applications de télédétection et de SIG, telles que présentées plus haut, peuvent être utilisées pour le suivi et la cartographie des zones périurbaines.

\section{Données et zone d'étude}

Nous utilisons deux jeux de données, à savoir des images Landsat corrigées des effets atmosphériques et rectifiées géométriquement, et des images Sentinel-2 traitées avec le module d'extension de classification semi-automatique du logiciel QGIS. Des traitements supplémentaires ont été réalisés sur les logiciels ArcGIS, Microsoft Excel et RStudio.
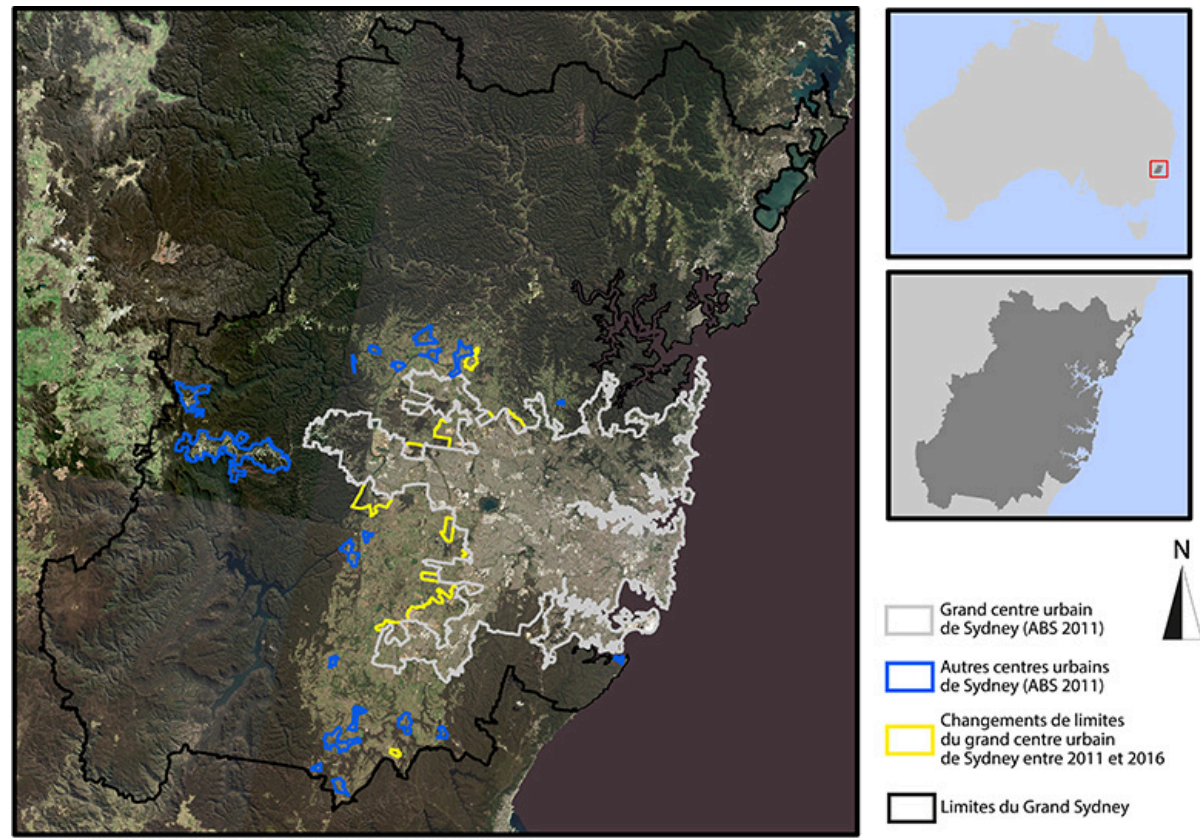

15 60 kilomètres

Figure 6.1. Région de Sydney couverte par deux images Landsat-8 respectivement acquises les 8 et 15 septembre 2017. Les polygones en noir et en gris désignent respectivement les limites de la région de Sydney et les limites de la partie urbaine de Sydney (ABS, 2011 ; 2016). Les polygones bleus représentent les zones urbaines à faible densité, tandis que les polygones jaunes représentent les zones urbaines créées entre 2011 et 2016.

La figure 6.1 montre la zone d'étude (région de Sydney) observée par le capteur OLI de Landsat-8 en septembre 2015 avec, en superposition, les limites administratives de la région de Sydney établies par le Bureau australien de statistiques, pour les recensements de 2011 et 2016. La couche correspondant aux limites urbaines a été créée en se basant sur le critère suivant : la densité de population doit être supérieure à 4 personnes par hectare pour qu'une zone soit considérée comme urbaine (ABS, 2016). Cette couche a été produite à partir des données du recensement de population le plus récent, datant de 2016, et est dérivée des données sur la population et le logement (ABS, 2016). Le 
Bureau australien des statistiques définit les centres urbains et les localités en fonction de la concentration des développements urbains et de la population. Cet organisme qualifie de « grands centres urbains » les localités de plus de 100000 habitants, d' " autres centres urbains », celles avec une population comprise entre 1000 et 100000 habitants, et de « rurales », celles de moins de 1000 habitants (ABS, 2016).

La figure 6.1 montre les limites des « grands centres urbains» et des « autres centres urbains ", respectivement en gris et bleu, établies à partir du recensement de la population et du logement de 2011. Les traits jaunes représentent les changements des limites des « grands centres urbains » entre 2011 et 2016. Une portion de cette image a été sélectionnée pour faire des analyses plus poussées sur une zone périurbaine de la région de Sydney (figure 6.2).

La figure 6.3 montre l'influence de la résolution spatiale des images sur la perception de l'occupation du sol. Des extraits d'images Sentinel-2 à $10 \mathrm{~m}$ de résolution sont comparés aux images Landsat- 8 à $30 \mathrm{~m}$ de résolution sur une zone de transition entre l'urbain (sous-zone II) et le périurbain (sous-zone I) dans l'ouest de Sydney.
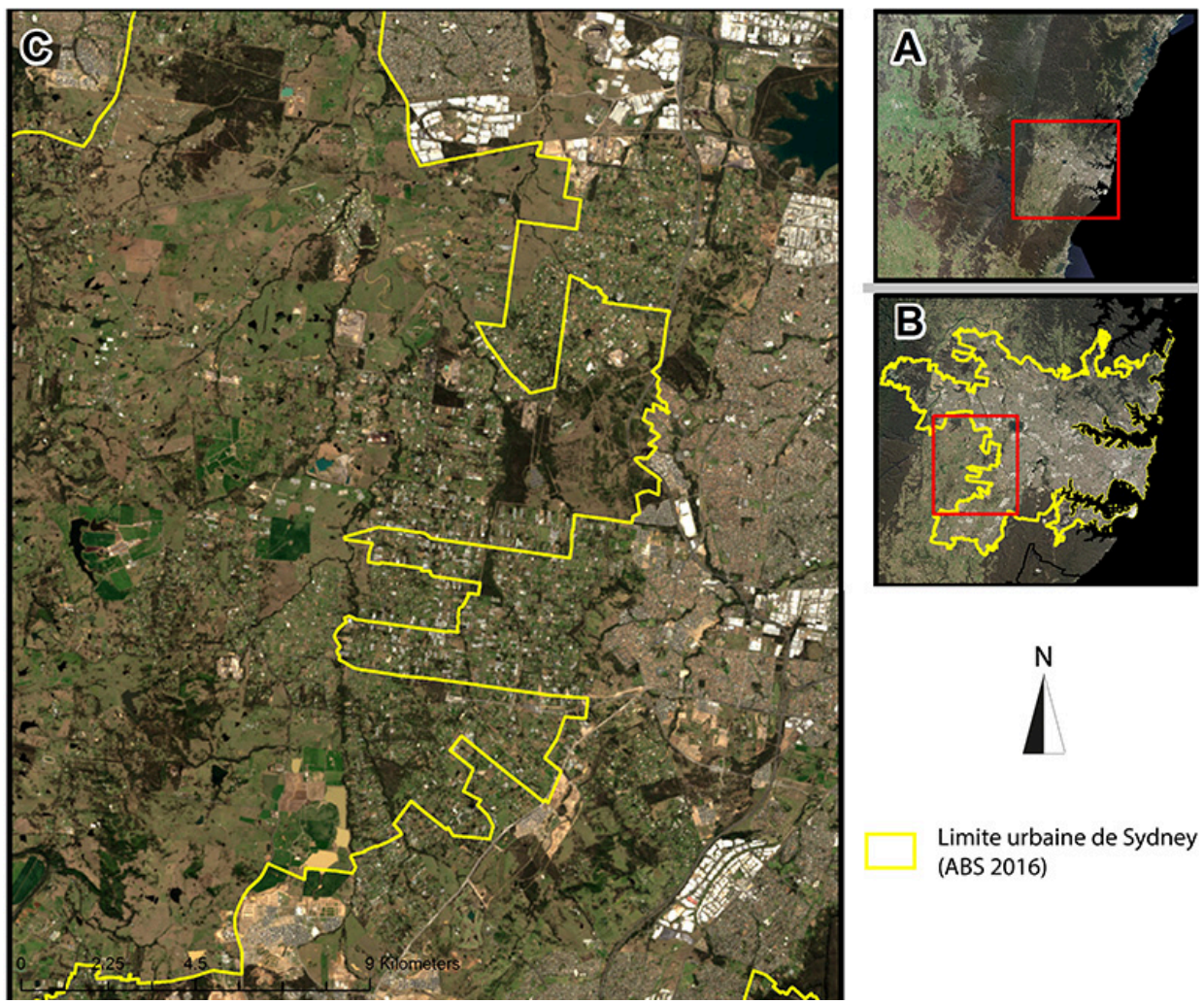

Limite urbaine de Sydney (ABS 2016)

Figure 6.2. Exemple de transition entre l'urbain et le périurbain sur une zone test. La partie A montre la mosaïque des deux images Landsat- 8 du 8 et du 15 septembre 2017. Le polygone rouge de la partie A correspond à la sous-zone urbaine et périurbaine de Sydney représentée dans la partie B ; celui de la partie B correspond à la zone périurbaine étudiée dans ce chapitre et agrandie dans la partie $\mathrm{C}$.

La résolution spatiale plus fine proposée par l'imagerie Sentinel-2 (figures 6.3C et 6.3E) fournit plus de détails que les images Landsat et donc plus de possibilités pour catégo- 
riser différents composants présents sur le terrain (figures 6.3B et 6.3D). Sur ces images, les structures urbaines apparaissent en blanc, avec un albédo élevé, alors que les prairies et les cultures ressortent en vert et prennent des formes géographiques variées. Les prairies ainsi que d'autres composants du paysage apparaissent plus nettement sur les images Sentinel-2 du fait de la meilleure résolution spatiale (figure 6.3C). Les deux types d'images sont disponibles en accès libre. Les images Landsat, quoique plus grossières, sont disponibles depuis les années 1980, alors que le premier satellite Sentinel-2, offrant des données plus fines, n'a été lancé qu'en 2016. Les deux constituent d'excellents moyens pour mieux comprendre les impacts sur l'agriculture des pressions exercées par une croissance urbaine très dynamique.
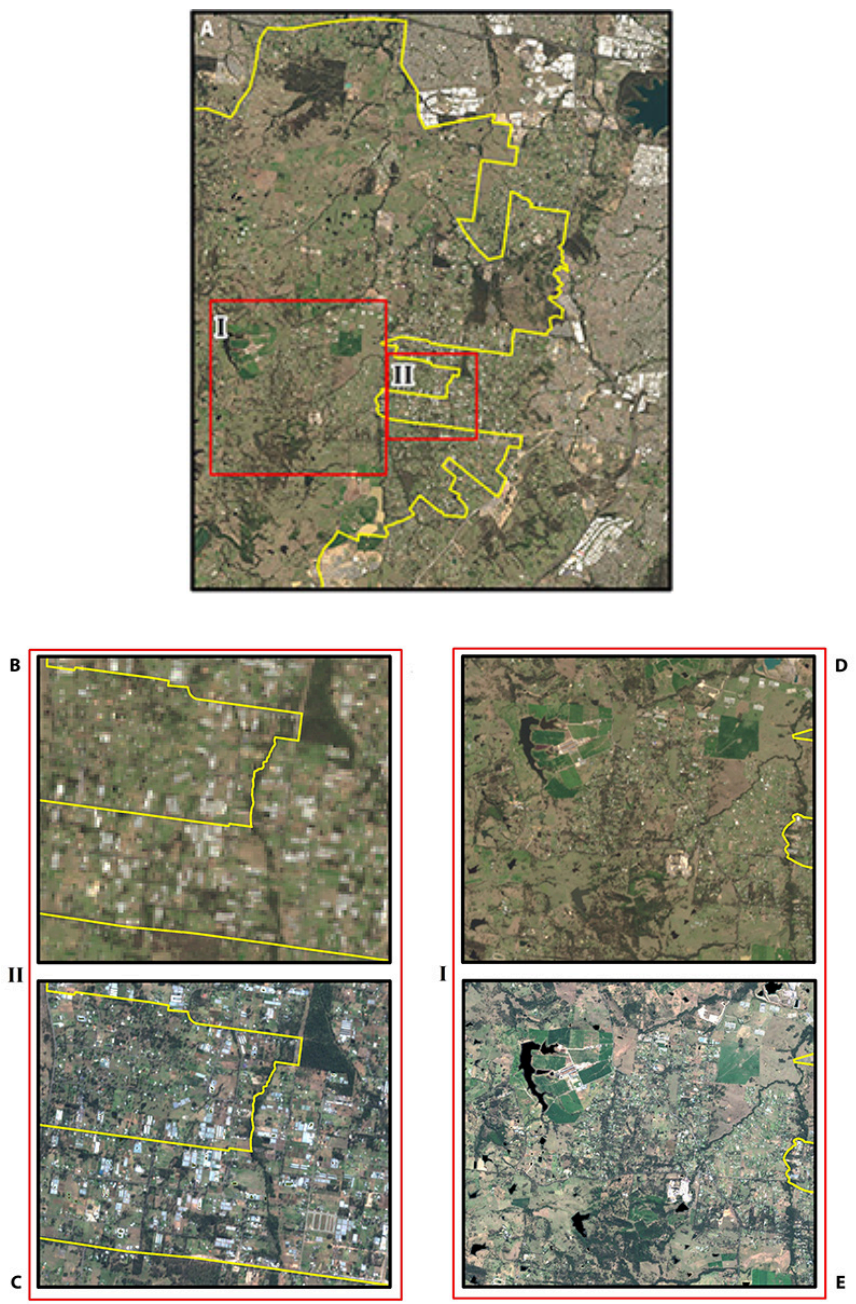

Figure 6.3. (A) Image Landsat-8 (8 septembre 2015) avec deux sous-zones en rouge (I : espace périurbain ; II : espace urbain) ; (B) image Landsat-8, sous-zone I ; (C) image Sentinel-2, sous-zone I ; (D) image Landsat-8, sous-zone II ; (E) image Sentinel-2, sous-zone II. 


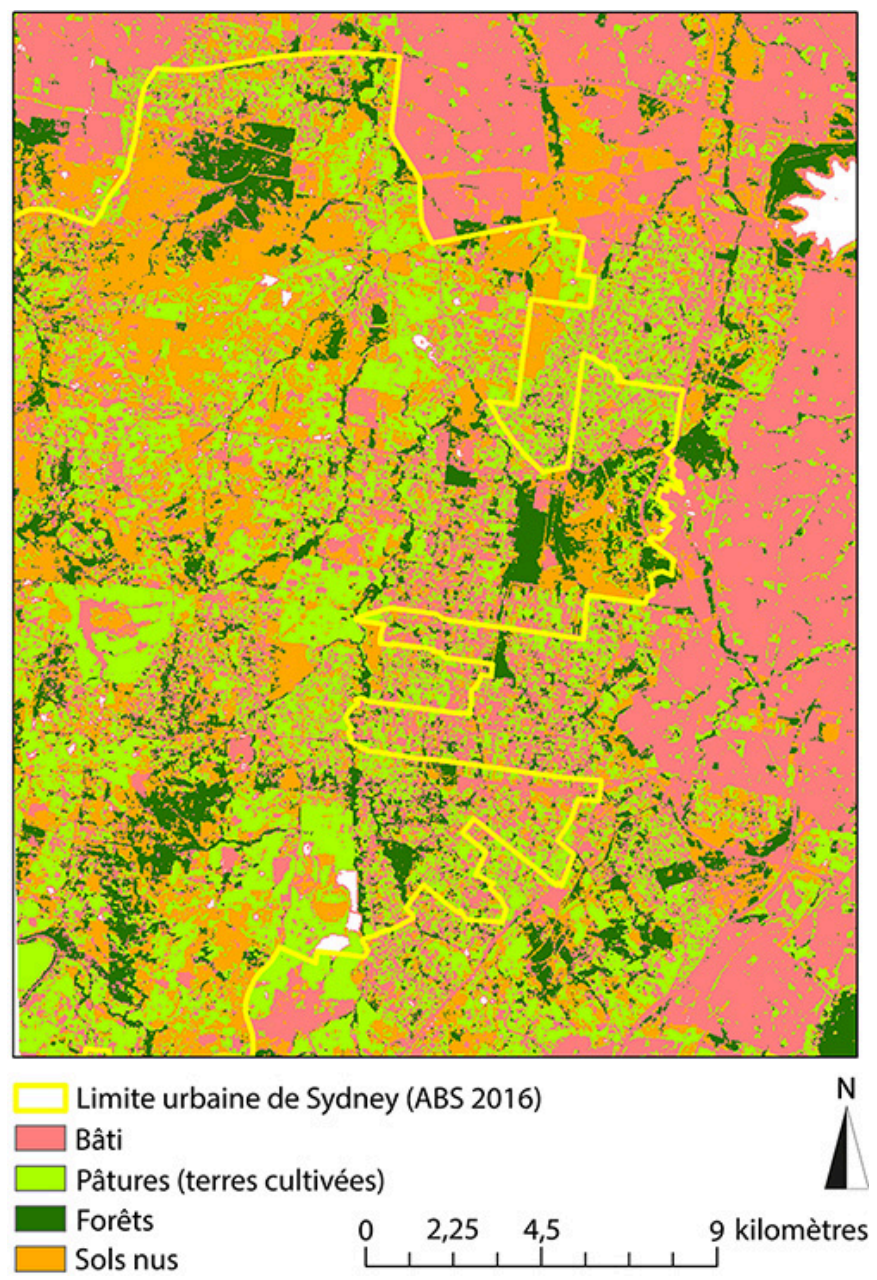

Figure 6.4. Classification multispectrale par maximum de vraisemblance d'une image Landsat-8 (8 septembre 2015) sur une zone de transition périurbaine (partie ouest de Sydney).

\section{La classification multispectrale des zones périurbaines}

Des classifications multispectrales par maximum de vraisemblance à partir des jeux de données Landsat-8 et Sentinel-2 ont été faites pour produire des cartes d'occupation du sol de la région de Sydney. La figure 6.4 montre la classification obtenue sur la zone de transition périurbaine sélectionnée à partir des images Landsat-8. Quatre classes principales ont été distinguées : bâtis, pâtures (terres cultivées), sols nus et forêts.

L'application aux images Sentinel-2 d'une classification similaire sur la même zone de transition périurbaine a permis de distinguer sept classes principales (figure 6.5) : pâtures, terres cultivées, sols nus, forêts, routes, zones résidentielles et zones commerciales. Ces résultats confirment l'intérêt de la meilleure résolution spatiale de Sentinel-2 pour distinguer plus de classes d'occupation du sol. 


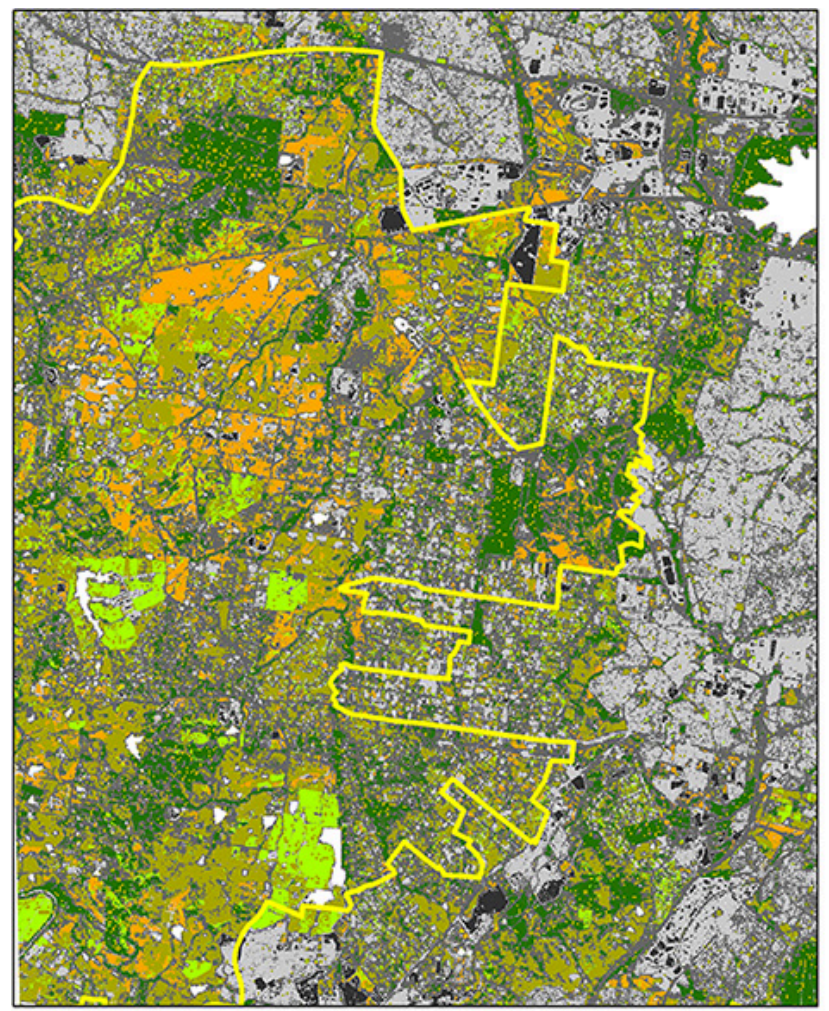

Limite urbaine de Sydney (ABS 2016)

Pâtures

Routes

Terres cultivées

Zones résidentielles

Forêts

i

Sols nus

Zones commerciales

Figure 6.5. Classification multispectrale par maximum de vraisemblance d'une image Sentinel-2 (23 août 2017) sur une zone de transition périurbaine (partie ouest de Sydney).

\section{La détection de changements entre 1989 et 2013 par analyse d'images Landsat}

\section{Les changements d'usage et d'occupation du sol}

Des images Landsat-5 et Landsat-8 acquises à différentes dates (27 septembre 1987, 10 octobre 1993, 9 octobre 1998, 9 octobre 2003, 18 septembre 2008 et 18 septembre 2013) ont été utilisées pour retracer les changements dynamiques et historiques d'occupation et d'usage du sol. Les principales classes identifiées ont été : forêts, sols nus, pâtures/terres cultivées et zones urbaines. 

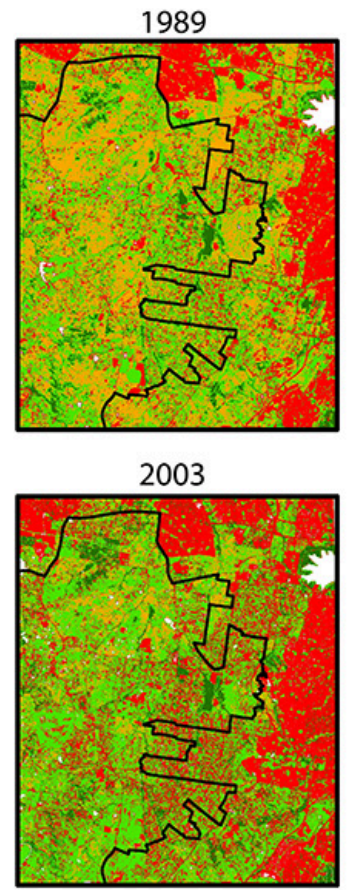

Sols
nus

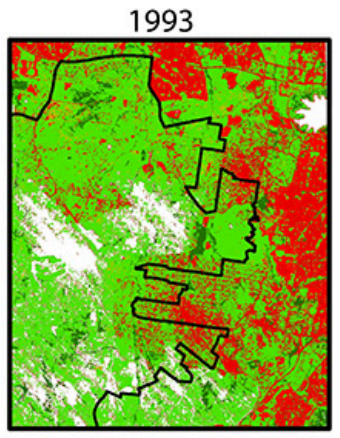

2008

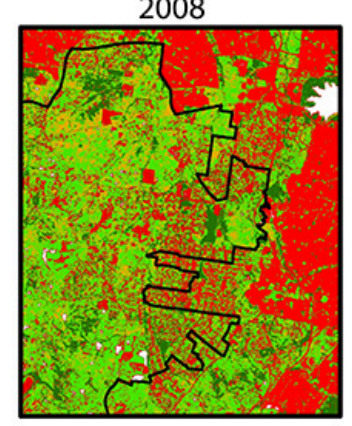

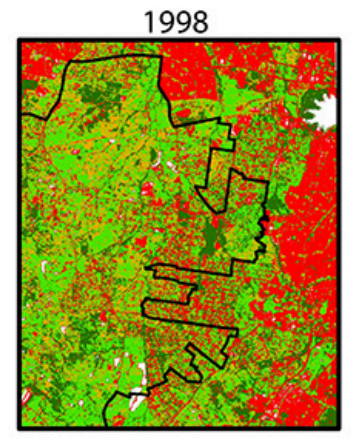
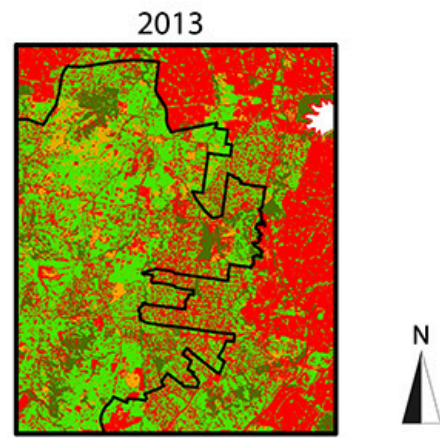

13 kilomètres

Figure 6.6. Série de classification d'images Landsat (5 et 8) sur vingt-cinq ans (1989 à 2013) montrant les changements d'occupation du sol dans la zone de transition périurbaine de Sydney.

La figure 6.6 montre que la zone périurbaine de Sydney Ouest a connu des changements très dynamiques de l'occupation du sol entre 1989 et 2013. L'étalement urbain est clairement visible dans l'expansion vers l'ouest de la zone urbaine de Sydney. La classe « sols nus » correspond aux terres arables ou à des zones ouvertes qui n'avaient pas de couvert végétal au moment où les images ont été acquises. L'analyse de cette séquence d'images révèle une augmentation générale des zones bâties et une diminution des terres agricoles (pâtures et terres cultivées) (figure 6.7). En surface, les zones urbaines ont progressé de $38 \%$ à $50 \%$ sur vingt-cinq ans, alors que les activités agricoles dans les zones périurbaines ont baissé de $35 \%$ à $25 \%$. En revanche, peu de changements ont été observés dans la classe « forêts ». La croissance des zones urbaines impacte donc surtout les zones périurbaines où se développent la plupart des activités agricoles. La poursuite de l'urbanisation amènera, in fine, à la disparition de zones périurbaines, à une pression accrue sur les forêts naturelles et les espaces ruraux, et à d'autres types de changements environnementaux.

\section{La fragmentation des paysages}

La fragmentation des paysages sur vingt-cinq ans a été analysée à l'aide de métriques paysagères appliquées à des cartes d'occupation du sol faites à partir d'images Landsat de 1989 et 2013. Un échantillonnage concentrique a été utilisé le long d'un transect urbain/ périurbain allant du centre-ville de Sydney jusqu'à l'extérieur de la ville. Cet échantillonnage est constitué de cercles allant de 10 à $60 \mathrm{~km}$ de rayon et espacés de $10 \mathrm{~km}$ (figure 6.8). Plusieurs métriques paysagères ont ensuite été calculées pour six zones 
échantillons, situées respectivement dans le cercle central et dans les cinq intervalles de $10 \mathrm{~km}$ de large (10 à $20 \mathrm{~km}, 20$ à $30 \mathrm{~km}, 30$ à $40 \mathrm{~km}, 40$ à $50 \mathrm{~km}$ et 50 à $60 \mathrm{~km}$ ).

A
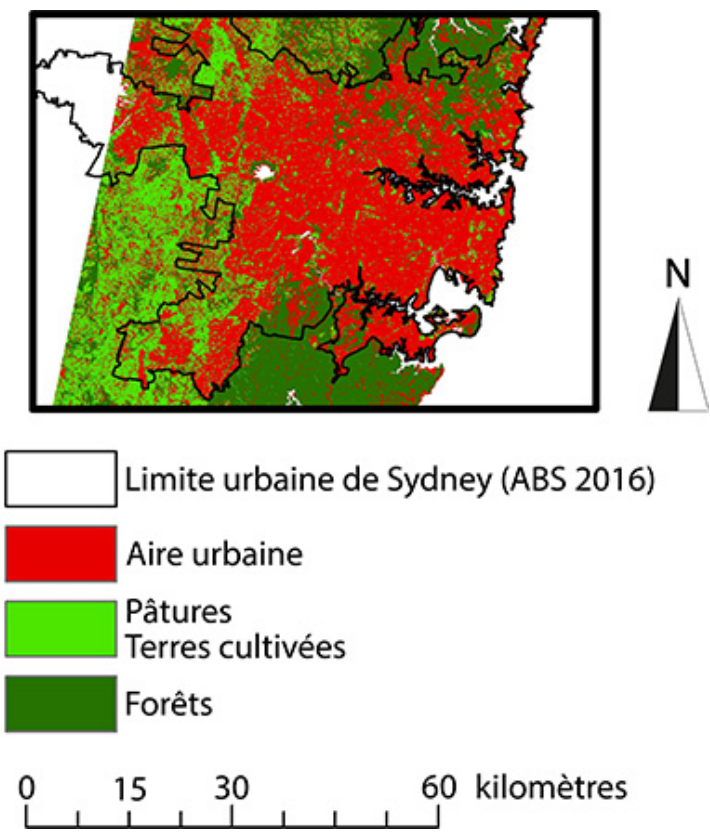

\section{Couverture (\%)}

B
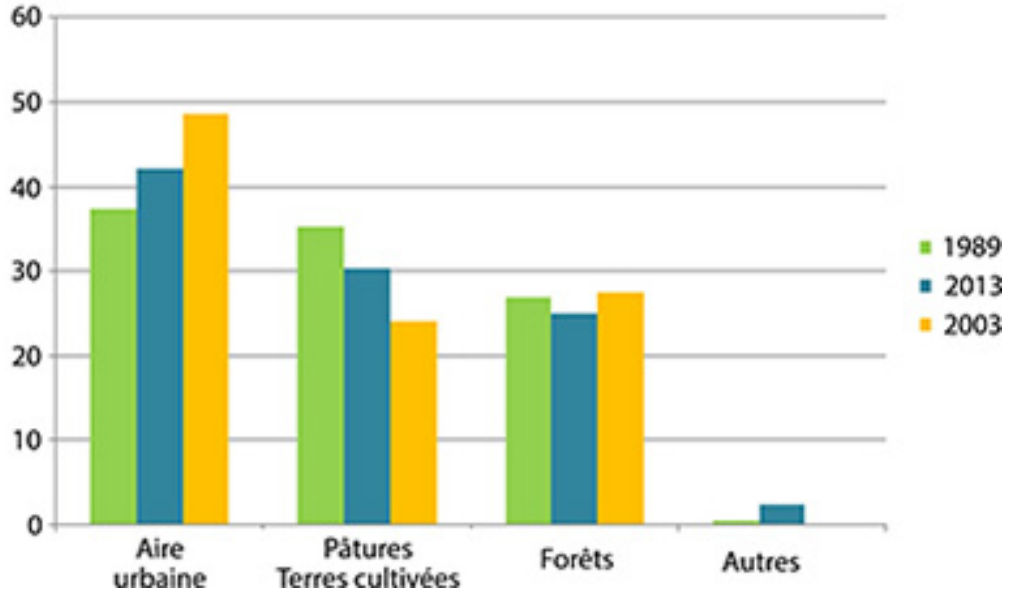

Figure 6.7. Dynamique des changements d'occupation du sol dans la région de Sydney entre 1989 et 2013. (A) Localisation de la zone d'étude ; (B) Évolution en \% des surfaces de quatre classes d'occupation du sol entre 1989, 2003 et 2013. 
1989

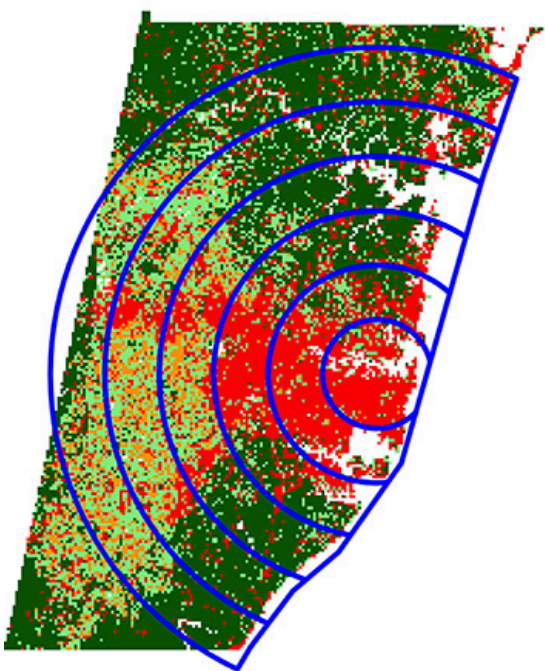

2013

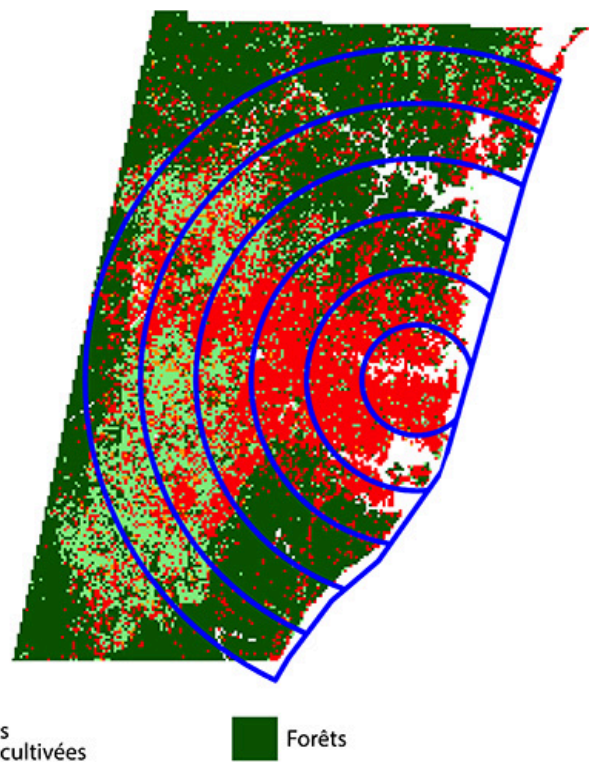

Sols nus

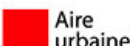

Terres cultivées

Figure 6.8. Échantillons en cercles concentriques sur une image de couverture du sol Landsat de 1989 (A) et 2013 (B). Les cercles bleus sont dessinés tous les $10 \mathrm{~km}$ à partir du centre-ville de Sydney.

L'analyse montre que sur l'ensemble des zones échantillons, le nombre de taches de pâtures/terres cultivées était plus élevé en 2013 qu'en 1989, alors que le nombre de taches urbaines a diminué sur la même période (figure 6.9). Le nombre de taches urbaines dans le cercle externe était plus élevé en 1989, indiquant l'agrégation de zones urbaines à l'extérieur de Sydney et la formation de taches urbaines plus grandes (figure 6.9A). L'augmentation de la densité des taches de pâtures/terres cultivées entre 1989 et 2013 indique la nature de plus en plus éparse et fragmentée du paysage. La plus faible densité des taches représentant les zones urbaines en 2013 traduit une diminution du caractère épars du développement urbain et suggère l'urbanisation des zones interstitielles au fil du temps. Ainsi, les zones périurbaines périphériques, autrefois utilisées pour des activités agricoles, ont été progressivement converties en espaces urbains (figure 6.9A). Les résultats montrent aussi que le caractère épars des zones urbaines est peu présent près du centre-ville et qu'elles deviennent moins denses et plus fragmentées lorsque l'on s'éloigne du centre-ville (figure 6.9A). Les surfaces urbanisées ont globalement augmenté dans tous les échantillons entre 1989 et 2013, alors que celles en pâtures/terres cultivées ont diminué (figure 6.9B). Ceci indique une augmentation de l'isolement et de la fragmentation de la classe pâtures/terres cultivées au fil du temps. Enfin, l'index d'agrégation démontre que la perte globale de pâtures/terres cultivées est déterminée par l'urbanisation progressive (figure 6.9C). Ces métriques paysagères fournissent des analyses précieuses concernant l'impact du développement urbain sur l'agriculture périurbaine et constituent autant d'opportunités pour leur gestion durable.

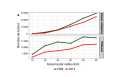



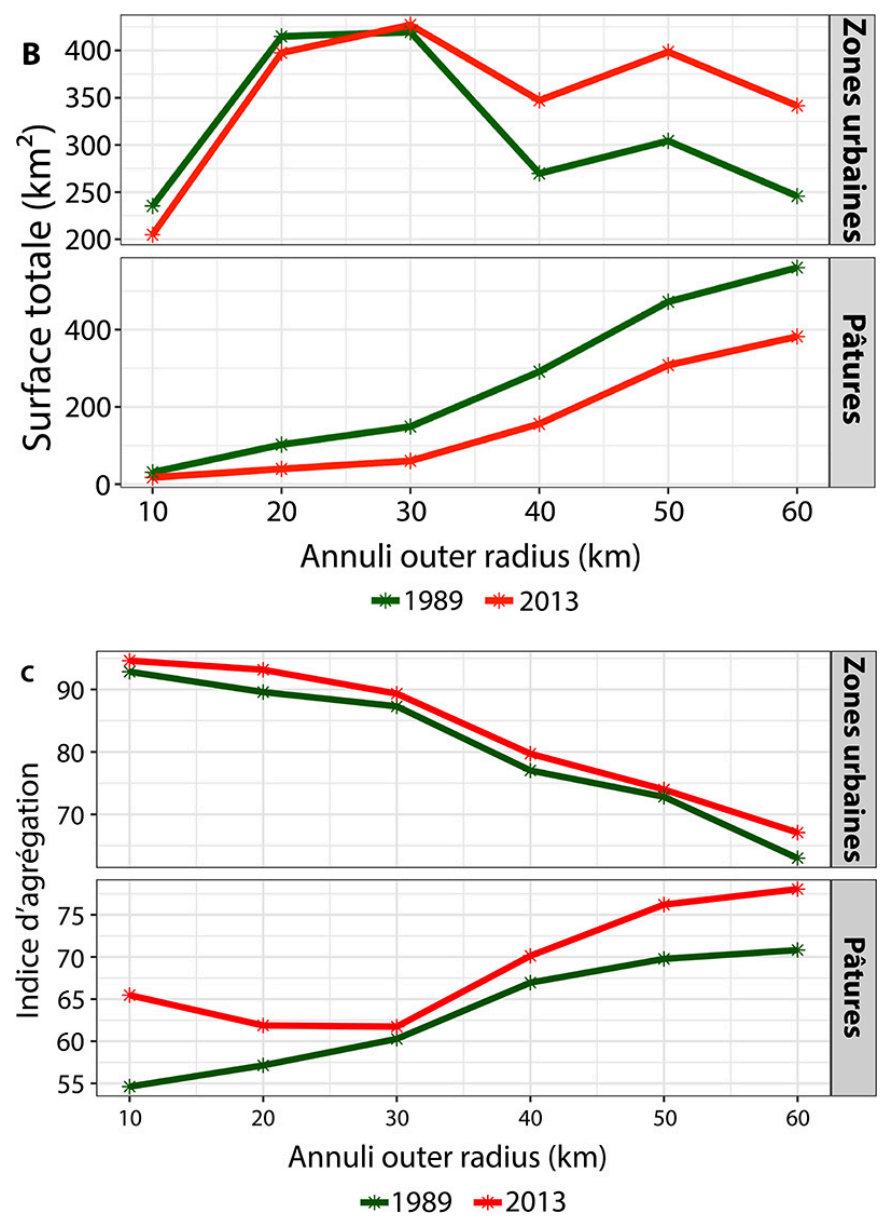

Figure 6.9. Changements dans la fragmentation du paysage entre 1989 et 2013 dans les différents cercles concentriques du transect d'échantillonnage. (A) nombre de taches en zones urbaines et en pâtures ; (B) surface totale des zones urbaines et de pâtures ; (C) indice d'agrégation mesurant la fragmentation.

\section{L’analyse linéaire de mélange spectral}

Les méthodes d'analyse linéaire de mélange spectral appliquées aux images multispectrales permettent de déterminer la proportion de chaque type d'occupation du sol à l'intérieur d'un pixel. Ces méthodes ont été appliquées à des données Landsat pour cartographier des indicateurs urbains, dont le pourcentage de surface en zones imperméables (\% ISA, impervious surface area), et mesurer leur évolution au fil du temps (Hu et Weng, 2009). L'indicateur \% ISA est particulièrement important pour la modélisation du ruissellement des eaux et des inondations en milieu urbain. Il peut aussi être utilisé pour distinguer les zones urbaines des zones périurbaines et rurales grâce à des mesures de l'étendue des infrastructures. Différents seuils ont été utilisés pour cartographier la limite entre l'urbain et le rural sur la base de l'indicateur \% ISA. Par exemple, Yuan et Bauer (2007) ont utilisé des seuils d'ISA $\geq 25 \%$ pour définir la classe « urbain », $<25 \%$ pour la classe « autre urbain » et $<5 \%$ pour la classe « rural ».

Nous avons appliqué cette technique d'analyse linéaire de mélange spectral à l'image Landsat- 8 pour produire une carte de l'indicateur \% ISA pour la région de Sydney 
(figure 6.10). Nous avons défini les zones urbaines et les banlieues comme ayant un ISA $>12 \%$, et les zones rurales comme ayant un ISA $<12 \%$. Nous avons trouvé des similarités entre nos résultats et les cartes des limites urbaines développées par le Bureau australien des statistiques (figure 6.10). Toutefois, les cartes basées sur l'ISA ont fourni des informations supplémentaires sur le développement urbain en lien avec la densité de population, et nous avons localisé plusieurs zones avec un ISA faible, alors qu'elles étaient considérées par le Bureau australien des statistiques comme des zones urbaines (figure 6.10). Ceci s'explique par le fait que les résultats des cartes des \% ISA sont basés sur une approche physique des infrastructures, alors que les limites établies par le Bureau australien des statistiques sont déterminées sur des critères statistiques et de population dans les localités.

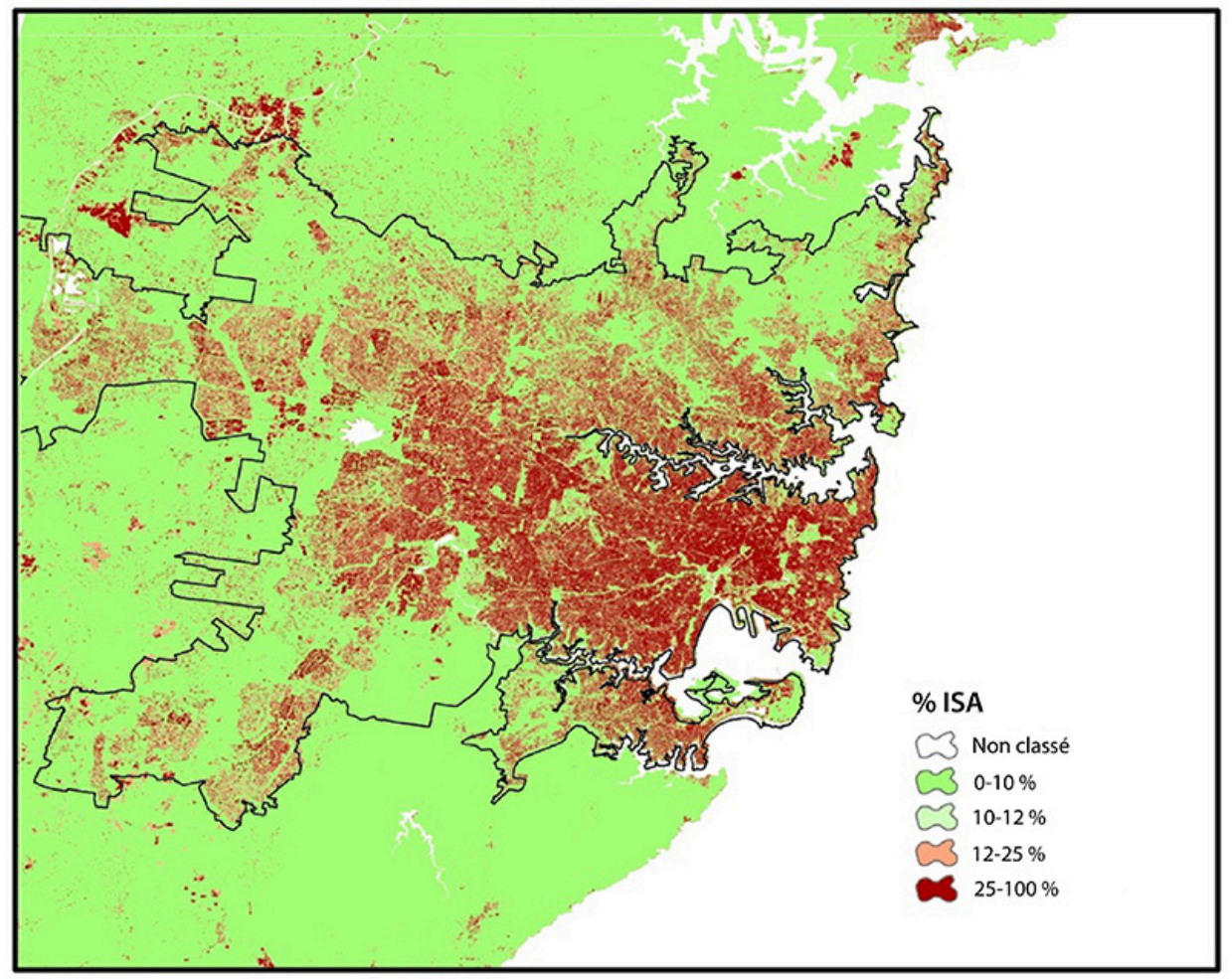

Figure 6.10. Carte des zones imperméables (impervious surface area, ISA) de la région de Sydney.

Cette carte a été produite au moyen d'une analyse linéaire de mélange spectral appliquée à une image Landsat-8 (17 septembre 2013) afin d'extraire une classe urbaine en niveaux d'ISA. Le polygone noir montre les limites du centre urbain principal de Sydney telles que définies par le Bureau australien des statistiques (ABS, 2016).

\section{Conclusion}

L'agriculture périurbaine apporte d'importants bénéfices aux zones métropolitaines en satisfaisant à des besoins commerciaux et récréatifs en matière d'alimentation. Elle constitue aussi une source d'emploi, améliore les moyens d'existence et fournit des services écosystémiques (Appeaning, 2010). Dans la région de Sydney, la périphérie urbaine et les zones périurbaines sont des zones de transition extrêmement dynamiques. 
Elles subissent des changements qui sont difficiles à quantifier par des relevés de terrain et des recensements, car la collecte de ces données demande beaucoup de main-d'œuvre, a un coût prohibitif et ne peut être mise à jour que tous les cinq ans ou plus. À l'inverse, les techniques satellitaires et géomatiques constituent des moyens économiques et efficaces pour le suivi et la cartographie à intervalles réguliers de l'évolution des pâtures et des terres cultivées dans les zones périurbaines. Les données satellitaires fournissent des informations spatiales cohérentes, une vue synoptique, un niveau de détail élevé, avec un accès gratuit aux images ayant des résolutions spatiales de 10 à 30 mètres. Quarante années d'imagerie Landsat sont disponibles pour retracer les changements d'occupation $\mathrm{du}$ sol et documenter les changements dans les zones cultivables et de pâtures. Cette source d'information potentiellement riche permet une meilleure planification et une gestion durable des activités agricoles en milieu périurbain, sur la base d'informations géospatiales précises (Appeaning, 2010).

Dans ce chapitre, nous avons présenté plusieurs utilisations géospatiales potentielles des données satellitaires ainsi que des algorithmes et des outils pour le suivi, l'évaluation et la gestion des terres agricoles périurbaines. Nous avons montré que les images des satellites Landsat et Sentinel-2 pouvaient être utilisées pour comprendre les dynamiques périurbaines au moyen de classifications et de suivis de l'occupation du sol ainsi que d'outils pour détecter les changements d'occupation du sol. Une série temporelle d'images sur vingt-cinq ans, extraite des archives des satellites Landsat, a permis de retracer et de mieux comprendre les changements et les tendances en matière d'occupation du sol.

Comme nous l'avons montré dans notre étude de cas sur Sydney en Australie, les zones périurbaines sont constamment soumises à la fragmentation induite par l'urbanisation. Cette fragmentation s'accompagne d'une perte de pâtures et de terres cultivables et d'un déplacement continu de la limite urbain/périurbain vers l'ouest de la métropole. Quelques années seulement après la création ou la mise à jour des tracés de la limite urbaine (ex. : ABS, 2011 ; 2016), l'imagerie satellite a permis de mettre en évidence la perte de nouvelles zones périurbaines récemment converties à l'usage urbain. Cela démontre que la mise à jour tous les cinq ans de la délimitation de la bordure urbaine est inadaptée. De plus, la méthodologie basée sur l'indicateur \% ISA apporte une mesure plus physique de la croissance urbaine, qui peut être particulièrement précise pour détecter et suivre l'étalement urbain ainsi que la perte de terres cultivables et de pâtures.

Malgré les nombreuses avancées faites dans les études sur l'occupation du sol dans les milieux urbains et périurbains, les recherches utilisant l'imagerie satellitaire et aéroportée en sont encore à leurs débuts et requièrent plus d'attention, particulièrement si l'on tient compte de l'intérêt croissant pour le devenir des paysages périurbains et des activités agricoles (Weng, 2009). Des avancées supplémentaires en télédétection et en analyse d'images seront nécessaires pour obtenir un meilleur suivi et une meilleure gestion des paysages dans les zones de transition entre l'urbain et le périurbain. Les données images acquises en mode maillé offrent la possibilité d'extraire des informations spatiales plus complexes, notamment des métriques paysagères sur les taches, les bordures, la diversité et la fragmentation des paysages. Comme illustré dans notre étude de cas sur Sydney, les mesures de fragmentation peuvent aider à représenter le caractère épars des zones périurbaines, avec la perte de pâtures et de terres cultivables, ainsi que la consolidation des zones urbaines résultant de l'aménagement des zones interstitielles.

Les mesures de diversité paysagère semblent prometteuses et pourraient permettre une meilleure compréhension des impacts des changements de composition et de configuration dans les zones périurbaines sur la productivité agricole et les services écosystémiques qui lui sont associés. Néanmoins, l'utilisation de métriques spatiales paysagères 
reste encore relativement peu exploitée en écologie des paysages dans les milieux urbains et périurbains. Les processus et les relations spatiales de fragmentation dus à des perturbations, l'occupation du sol et ses dynamiques, le fonctionnement de l'agriculture et la sécurité alimentaire restent encore mal compris et mériteraient que l'on s'y attarde plus longuement. D'autres avancées prometteuses sont à venir dans ce domaine, notamment grâce aux algorithmes d'apprentissage automatique et aux méthodes de classification orientées objet. Elles permettront, en effet, une distinction plus précise et détaillée des types d'occupation du sol. Cependant, à l'heure actuelle, elles reposent encore sur l'utilisation d'images satellitaires onéreuses et de logiciels commerciaux.

\section{Remerciements}

Ce chapitre est le résultat d'un travail d'équipe incluant tous les auteurs. Il est né des suites d'un atelier franco-australien qui s'est tenu à Sydney en octobre 2013.

\section{Références bibliographiques}

Aniello C., Morgan K., Busbey A., Newland L., 1995. Mapping micro-urban heat islands using Landsat TM and a GIS. Computers and Geosciences, 21 (8), 965-969.

Appeaning K., 2010. Urban and peri-urban agriculture in developing countries studied using remote sensing and in situ methods. Remote Sensing, 2 (2), 497-513.

Atkinson P.M., 2005. Sub-pixel target mapping from soft-classified, remotely sensed imagery. Photogrammetric Engineering and Remote Sensing, 71 (7), 839-846.

Australian Bureau of Statistics (ABS), 2011. Australian Statistical Geography Standards 2011 ABS Structures. http://stat.abs.gov.au/itt/r.jsp?ABSMaps (consulté le 8 décembre 2018).

Australian Bureau of Statistics (ABS), 2016. Australian Statistical Geography Structures 2016 ABS Structures. http://stat.abs.gov.au/itt/r.jsp?ABSMaps (consulté le 8 décembre 2018). Bauer D.J., Curran P.J., 2005. Probing interactions in fixed and multilevel regression: Inferential and graphical techniques. Multivariate Behavioral Research, 40 (3), 373-400.

Blaschke T., 2010. Object based image analysis for remote sensing. ISPRS Journal of Photogrammetry and Remote Sensing, 65 (1), 2-16.

Blaschke T., Lang S., Hay G., 2008. Object-Based Image Analysis: Spatial Concepts for Knowledge-Driven Remote Sensing Applications, Springer Science and Business Media.

Chrysoulakis N., 2003. Estimation of the all-wave urban surface radiation balance by use of ASTER multispectral imagery and in situ spatial data. Journal of Geophysical Research: Atmospheres (1984-2012), 108 (D18).

Civco D.L., Hurd J.D., 1997. Impervious surface mapping for the state of Connecticut. Proceedings of the 1997 ASPRS Annual Conference, 124-135.

Cleugh H., Smith M.S., Battaglia M., Graham P., 2011. Climate Change: Science and Solutions for Australia, CSIRO.

Davis C.H., Wang X., 2002. Urban land cover classification from high resolution multi-spectral IKONOS imagery. In: Geoscience and Remote Sensing Symposium, 2002, IGARSS'02. 2002 IEEE International, vol. 2, IEEE, 1204-6.

DESA, 2012. World urbanization prospects, the 2011 Revision, ST/ESA/SER.A/319, United Nations Department of Economic and Social Affairs, Population Division, viewed 05-06-2015, http://www.un.org/en/development/desa/population/publications/pdf/ urbanization/WUP2011_Report.pdf .

Dougherty M., Dymond R.L., Goetz S.J., Jantz C.A., Goulet N., 2004. Evaluation of impervious surface estimates in a rapidly urbanizing watershed. Photogrammetric Engineering and Remote Sensing, 70 (11), 1275-84. 
Durieux L., Lagabrielle E., Nelson A., 2008. A method for monitoring building construction in urban sprawl areas using object-based analysis of Spot 5 images and existing GIS data. ISPRS Journal of Photogrammetry and Remote Sensing, 63 (4), 399-408.

Fahrig L., 2003. Effects of habitat fragmentation on biodiversity. Annual Review of Ecology, Evolution, and Systematics, 34 (1), 487-515.

Garcia-Haro F., Gilabert M., Melia J., 1996. Linear spectral mixture modelling to estimate vegetation amount from optical spectral data. International Journal of Remote Sensing, 17 (17), 3373-400.

Grau H., Hernández M., Gutierrez J., Gasparri N., Casavecchia M., Flores-Ivaldi E., Paolini L., 2008. A peri-urban neotropical forest transition and its consequences for environmental services. Ecology and Society, 13 (1).

He H.S., Dezonia B.E., Mladenoff D.J., 2000. An aggregation index (AI) to quantify spatial patterns of landscapes. Landscape Ecology, 15 (7), 591-601.

$\mathrm{Hu}$ X., Weng Q., 2009. Estimating impervious surfaces from medium spatial resolution imagery using the self-organizing map and multi-layer perceptron neural networks. Remote Sensing of Environment, 113 (10), 2089-2102.

Huete A., Liu H., Batchily K., Van Leeuwen W., 1997. A comparison of vegetation indices over a global set of TM images for EOS-MODIS. Remote Sensing of Environment, 59 (3), 440-51.

Huete A., Didan K., Miura T., Rodriguez E.P., Gao X., Ferreira L.G., 2002. Overview of the radiometric and biophysical performance of the MODIS vegetation indices. Remote Sensing of Environment, 83 (1), 195-213.

Ji W., Ma J., Twibell R.W., Underhill K., 2006. Characterizing urban sprawl using multi-stage remote sensing images and landscape metrics. Computers, Environment and Urban Systems, 30 (6), 861-879.

Kalma J.D., McVicar T.R., McCabe M.F., 2008. Estimating land surface evaporation: a review of methods using remotely sensed surface temperature data. Surveys in Geophysics, 29 (4-5), 421-469.

Kane K., Connors J.P., Galletti C.S., 2014. Beyond fragmentation at the fringe: a path-dependent, high-resolution analysis of urban land cover in Phoenix, Arizona. Applied Geography, 52, 123-134.

Kidder S.Q., Wu H.-T., 1987. A multispectral study of the St. Louis area under snow-covered conditions using NOAA-7 AVHRR data. Remote Sensing of Environment, 22 (2), 159-172.

Kurihara Y., Murakami H., Kachi M., 2016. Sea surface temperature from the new Japanese geostationary meteorological Himawari-8 satellite. Geophysical Research Letters, 43 (3), 1234-1240.

Lillesand T., Kiefer R.W., Chipman J., 2014. Remote Sensing and Image Interpretation, John Wiley and Sons.

McGarigal K., Marks B.J., 1995. FRAGSTATS: Spatial pattern analysis program for quantifying landscape structure. Gen. Tech. Rep. PNW-GTR-351. US Department of Agriculture, Forest Service, Pacific Northwest Research Station.

Munroe D.K., Croissant C., York A.M., 2005. Land use policy and landscape fragmentation in an urbanizing region: assessing the impact of zoning. Applied Geography, 25 (2), 121-141. Neill R.V., Hunsaker C.T., Jones K.B., Riitters K.H., Wickham J.D., Schwartz P.M., Goodman I.A., Jackson B.L., Baillargeon W.S., 1997. Monitoring environmental quality at the landscape scale. BioScience, 47 (8), 513-519.

Odindi J., Bangamwabo V., Mutanga O., 2015. Assessing the value of urban green spaces in mitigating multi-seasonal urban heat using MODIS Land SurfaceTemperature (LST) and Landsat 8 data. International Journal of Environmental Research, 9 (1), 9-18. 
Pauchard A., Aguayo M., Peña E., Urrutia R., 2006. Multiple effects of urbanization on the biodiversity of developing countries: the case of a fast-growing metropolitan area (Concepción, Chile). Biological Conservation, 127 (3), 272-281.

Peng J., Wang Y., Ye M., Wu J., Zhang Y., 2007. Effects of land-use categorization on landscape metrics: a case study in urban landscape of Shenzhen, China. International Journal of Remote Sensing, 28 (21), 4877-4895.

Ridd M.K., 1995. Exploring a VIS (vegetation-impervious surface-soil) model for urban ecosystem analysis through remote sensing: comparative anatomy for cities. International Journal of Remote Sensing, 16 (12), 2165-85.

Rouse Jr J.W., Haas R., Schell J., Deering D., 1974. Monitoring vegetation systems in the Great Plains with ERTS. NASA special publication, 351, 309.

Saunders D., Hobbs R., Margules C., 1991. Biological Consequences of Ecosystem Fragmentation: A Review. Conservation Biology, 5 (1), 18-32.

Schneider A., Woodcock C.E., 2008.Compact, dispersed, fragmented, extensive? A comparison of urban growth in twenty-five global cities using remotely sensed data, pattern metrics and census information. Urban Studies, 45 (3), 659-692.

Song M., Civco D., Hurd J., 2005. A competitive pixel-object approach for land cover classification. International Journal of Remote Sensing, 26 (22), 4981-4997.

Streutker D.R., 2002. A remote sensing study of the urban heat island of Houston, Texas. International Journal of Remote Sensing, 23 (13), 2595-2608.

Su S., Jiang Z., Zhang Q., Zhang Y., 2011. Transformation of agricultural landscapes under rapid urbanization: a threat to sustainability in Hang-Jia-Hu region, China. Applied Geography, 31 (2), 439-449.

Tang J., Wang L., Yao Z., 2006. Analyzing urban sprawl spatial fragmentation using multi-temporal satellite images. GIScience and Remote Sensing, 43 (3), 218-232.

Tucker C.J., 1979. Red and photographic infrared linear combinations for monitoring vegetation. Remote Sensing of Environment, 8 (2), 127-150.

Turner M.G., O’Neill R.V., Gardner R.H., Milne B.T., 1989. Effects of changing spatial scale on the analysis of landscape pattern. Landscape Ecology, 3 (3-4), 153-162.

Verburg P.H., Van De Steeg J., Veldkamp A., Willemen L., 2009. From land cover change to land function dynamics: a major challenge to improve land characterization. Journal of Environmental Management, 90 (3), 1327-1335.

Veroustraete F., Patyn J., Myneni R., 1994. Application of remote sensing in regional scale estimates of vegetation carbon budgets: the Belfix project. Technical Report, Federal Office for Scientific, Technical and Cultural Affairs, Space Scientific Research in Belgium. Volume 3: Earth Observations, Part 2; pp. 191-21.

Wang Q., Shi W., Li Z., Atkinson P.M., 2016. Fusion of Sentinel-2 images. Remote Sensing of Environment, 187, 241-252.

Weng Q., 2001. A remote sensing? GIS evaluation of urban expansion and its impact on surface temperature in the Zhujiang Delta, China. International Journal of Remote Sensing, 22 (10), 1999-2014.

Weng Q., 2002. Land use change analysis in the Zhujiang Delta of China using satellite remote sensing, GIS and stochastic modelling. Journal of Environmental Management, 64 (3), 273-284.

Weng Q., 2009. Thermal infrared remote sensing for urban climate and environmental studies: methods, applications, and trends. ISPRS Journal of Photogrammetry and Remote Sensing, 64 (4), 335-344. 
Weng Q., Lu D., Schubring J., 2004. Estimation of land surface temperature-vegetation abundance relationship for urban heat island studies. Remote Sensing of Environment, 89 (4), 467-483.

Wu J., Xu J., Yue W., 2005. VIS model for cities that are experiencing rapid urbanization and development. IGARSS, 1503-1506.

Xie Y., Sha Z., Yu M., 2008. Remote sensing imagery in vegetation mapping: a review. Journal of Plant Ecology, 1 (1), 9-23.

$\mathrm{Xu}$ H., 2006. Modification of normalised difference water index (NDWI) to enhance open water features in remotely sensed imagery. International Journal of Remote Sensing, 27 (14), 3025-3033.

Yang Y., Titus S.J., Huang S., 2003. Modeling individual tree mortality for white spruce in Alberta. Ecological Modelling, 163 (3), 209-222.

Yuan, F., Bauer M.E., 2007. Comparison of Impervious Surface Area and Normalized Difference Vegetation Index as Indicators of Surface Urban Heat Island Effects in Landsat Imagery. Remote Sensing of Environment, 106, 375-386.

Yumimoto K., Nagao T., Kikuchi M., Sekiyama T., Murakami H., Tanaka T., Ogi A., Irie H., Khatri P., Okumura H., 2016. Aerosol data assimilation using data from Himawari-8, a next-generation geostationary meteorological satellite. Geophysical Research Letters, 43 (11), 5886-5894.

Zha Y., Gao J., Ni S., 2003. Use of normalized difference built-up index in automatically mapping urban areas from TM imagery. International Journal of Remote Sensing, 24 (3), 583-594.

Zhai J., Liu R., Liu J., Zhao G., Huang L., 2014. Radiative forcing over China due to albedo change caused by land cover change during 1990-2010. Journal of Geographical Sciences, 24 (5), 789-801.

Zhu G., Blumberg D.G., 2002. Classification using ASTER data and SVM algorithms: the case study of Beer Sheva, Israel. Remote Sensing of Environment, 80 (2), 233-240. 


\section{Partie II}

\section{La mesure à l'épreuve de l'humain}





\title{
Chapitre 7
}

\section{De la diffusion de l'information à la négociation du sens : l'exemple de la quantification et de la qualification de la consommation des terres agricoles dans l'ancienne région Languedoc-Roussillon}

\author{
Anja Martin-Scholz, Éric Barbe
}

\section{Introduction}

Les terres agricoles favorables à la diversification des cultures deviennent de plus en plus rares sur le littoral méditerranéen français, et plus particulièrement sur le territoire correspondant à l'ancienne région Languedoc-Roussillon ${ }^{28}$. Face à ce phénomène, la Direction régionale de l'alimentation, de l'agriculture et de la forêt (Draaf) de cette région a mandaté l'UMR Territoires, environnement, télédétection et informations spatiales (UMR Tetis) et le Laboratoire d'étude des interactions entre sol-agrosystème-hydrosystème (UMR Lisah) pour concevoir des données qui qualifient et quantifient la consommation des terres agricoles par l'urbanisation (chapitres 2 et 3). Suite à la construction de ces données, à savoir les taches artificialisées, les indices de qualité des sols (IQS) et les classes de potentiel agronomique des sols (CPAS), un système d'indicateurs a été conçu à partir du modèle forces motrices-pressions-états-impacts-réponses (Driving forces, Pressures, State, Impacts, Responses, DPSIR) afin de « favoriser l'appropriation de ces informations » par les acteurs de l'aménagement (chapitre 3). Dans la continuité de ces travaux présentés dans les chapitres précédents, nous nous intéressons ici à la propagation des données produites et à leur intégration dans les pratiques des acteurs de l'aménagement territorial.

Il s'agit ici de faire une analyse « rétrospective » permettant de reconstituer le processus de création et d'appropriation des données. L'objectif de ce chapitre est de prendre du recul par rapport à l'approche fonctionnaliste de l'information qui a présidé à la construction de ces données, c'est-à-dire d'interroger les limites de la conception et de l'utilisation des données comme outil d'aide à la décision. Nous montrerons que les données

28. L'entité administrative appelée «région Languedoc-Roussillon» n'existe plus depuis le $1^{\text {er }}$ janvier 2016, quand cette région a fusionné avec celle de Midi-Pyrénées pour former la région Occitanie-Pyrénées-Méditerranée. Cette fusion étant postérieure aux événements que nous décrivons dans ce chapitre, et du fait de la taille et donc de la diversité des problématiques agricoles en jeu dans cette nouvelle région, nous utiliserons la désignation de l'ancienne région Languedoc-Roussillon. 
ne sont pas porteuses d'une signification intrinsèque, mais que leur sens est le fruit de négociations et de compromis partagés par les utilisateurs. Cette approche permettra également de montrer que les changements dans les processus de décision et dans le réseau d'acteurs sont moins le résultat de l'impulsion d'événements extérieurs qu'issus de processus d'intéressement et de négociation de l'identité des acteurs.

Cette analyse se fonde sur les matériaux recueillis lors d'une enquête réalisée sur neuf mois, en 2011, au sein de l'UMR Tetis (Martin-Scholz, 2011 ; Martin-Scholz et al., 2013). Lors de l'enquête, une quinzaine d'entretiens ont été réalisés avec des agents des services déconcentrés de l'État, des chambres d'agriculture et des laboratoires de recherche. Au cours de cette étude, nous avons pu également observer plusieurs réunions, dont une de la Commission départementale de consommation des espaces agricoles (CDCEA), ainsi qu'accéder à un certain nombre de documents afférents au projet DraafUMR Tetis-UMR Lisah.

Ainsi, dans un premier temps, nous décrirons la diffusion des données, leurs impacts et leurs limites. Dans un second temps, nous analyserons comment ces données et leur sens ont été construits en interaction avec les acteurs. Enfin, nous interrogerons la pertinence de ce processus et les enseignements qu'on peut en tirer pour améliorer la conception d'outils d'aide à la prise de décision et leur diffusion.

\section{L'approche fonctionnaliste ou la diffusion d'information}

Les tenants de l'ingénierie territoriale s'inscrivent dans ce que Scardigli (2001) désigne par la «pensée ingénieur ». Ce système de pensée, tourné vers l'action (Vatin, 2008), est associé à une pensée calculatoire, visant à découper « le monde » en grandes fonctions mesurables, sur lesquelles il est possible d'agir et d'évaluer l'action (Vinck, 1999). Ce découpage en fonctions mesurables et appréhendables amène les acteurs de l'aménagement territorial à s'inscrire dans le paradigme communicationnel fondé sur le modèle émetteur/récepteur de Shannon et Weaver (1949) conçu à l'origine pour le fonctionnement du télégraphe, où les auteurs représentaient la communication comme un flux d'informations allant d'un émetteur à un récepteur, dont le sens était intrinsèque au message (Maurel, 2012). Mobilisant implicitement ce modèle, les acteurs du projet étudié visaient à réduire le « bruit », les « distorsions » qui empêchaient de véhiculer fidèlement le message des membres du projet Draaf-UMR Tetis-UMR Lisah vers les destinataires, et à évaluer l'efficacité de la communication.

\section{Le message porté par les données}

La production des taches artificialisées (chapitre 2) par la Draaf et l'UMR Tetis avait pour objectif de quantifier la consommation des espaces agricoles par l'urbanisation. Pour construire ces données, les agents de la Draaf se sont placés dans une optique à très long terme, et plus précisément dans la perspective d'une crise alimentaire mondiale en 2050, qui, selon l'hypothèse de ces agents, devrait encourager l'État français à préserver son indépendance alimentaire. Ainsi, l'objectif de ces données est d'identifier les espaces artificialisés, c'est-à-dire les espaces techniquement ou financièrement impossibles à remobiliser pour un usage agricole, ou à un coût environnemental et financier prohibitif. Le message que souhaitent faire passer la Draaf et ses partenaires à travers la diffusion de ces données est d'alerter sur la forte expansion des espaces artificialisés, au détriment des espaces agricoles, naturels et forestiers. 
Dans un même temps, la Draaf et l'UMR Lisah ont produit les IQS dans le but de qualifier le potentiel agronomique des sols en Languedoc-Roussillon (chapitre 3). Ici, le potentiel agronomique signifie la capacité des sols à accueillir des cultures mécanisées de céréales. En effet, les plantes cultivées ne présentent pas toutes les mêmes besoins ni les mêmes restrictions vis-à-vis des différentes caractéristiques du sol. Ne pouvant multiplier les indicateurs et considérant que la base de l'alimentation humaine est fondée sur les céréales, les acteurs ont désigné les sols de qualité comme étant favorables à la culture de céréales. Les IQS sont des indicateurs à deux critères, primaire et secondaire. Le critère primaire retenu pour déterminer le potentiel agronomique des sols en milieu méditerranéen est la réserve utile. Celle-ci correspond à la hauteur d'eau disponible pour les plantes que peut contenir un sol. Dans un climat soumis à des épisodes de sécheresse en alternance avec de fortes pluies, cette caractéristique est déterminante pour la culture des céréales. Les IQS intègrent également un critère secondaire influant sur la capacité des sols à produire des céréales $(\mathrm{pH}$, présence d'une croûte de battance, hydromorphie et pierrosité) (voir chapitre 3). Par la suite, l'UMR Tetis a produit les CPAS, qui sont issus d'une recombinaison des IQS uniquement sur la base de la réserve utile. Au travers de la construction des IQS et des CPAS, les agents de la Draaf et de l'UMR Lisah souhaitent alerter sur la forte hétérogénéité de la distribution des classes de sols à l'échelle régionale et sur la faible quantité de sols à "bon » potentiel agronomique, aptes à accueillir de grandes cultures.

Enfin, la combinaison des données d'artificialisation et de potentiel agronomique est supposée mettre en exergue l'artificialisation exacerbée des rares sols à « bon » potentiel agronomique. C'est ce message très fort, militant pour la prise en considération de la dimension «sol» dans les opérations d'aménagement, que les agents de la Draaf souhaitent véhiculer.

\section{La diffusion du message}

Suite à leur production, les données ont été diffusées. La phase de diffusion est une phase délicate qui nécessite l'attention des émetteurs pour que le message ne soit pas déformé. L'objectif de la Draaf est de faire prendre conscience de la consommation d'espace par l'artificialisation, aux acteurs de l'aménagement du territoire, et de la nécessité de tenir compte du potentiel agronomique des sols dans leurs projets. La diffusion poursuit donc un double objectif : d'une part les émetteurs veulent diffuser l'information au plus grand nombre d'acteurs de l'aménagement, d'autre part ils doivent s'assurer de l'efficacité de leur communication, à savoir veiller à ce que leur message atteigne sans encombre ses destinataires. Ils sont donc tiraillés entre deux objectifs : contrôler la communication et toucher une large audience.

La phase de diffusion a donc oscillé entre une volonté de limiter le nombre d'émetteurs qui mettent en place des conventions pour diffuser ces données et la mise en place d'émetteurs relais diffusant largement ces données.

La Draaf, commanditaire et copropriétaire des données produites, les a mises à disposition des Directions départementales des territoires (DDT) et de la Direction régionale de l'environnement, de l'aménagement et du logement (Dreal) via le service intranet des services de l'État. En complément, la Draaf a organisé des séminaires de formation et d'information auprès des collectivités et des DDT.

L'UMR Tetis, conceptrice et copropriétaire des données portant sur l'artificialisation, a diffusé marginalement ces données auprès d'acteurs issus de la recherche. 
L'UMR Lisah a confié au pôle géomatique régional SIG-LR la diffusion des IQS dans le cadre d'un partenariat. Les données ont été diffusées librement auprès de tous les adhérents du pôle via son portail de téléchargement.

Enfin, certaines DDT ont par la suite rediffusé ces données, notamment via leur site internet.

Pour tous ces différents canaux, la diffusion des données s'est accompagnée de métadonnées explicitant comment elles avaient été construites.

À l'issue de cette phase de diffusion, les acteurs ayant reçu ces données ont à leur tour interprété le message qu'elles contenaient et ont décidé pour certains de les intégrer dans leur activité, et pour d'autres de ne pas en tenir compte.

\section{La réception du message et l'utilisation des données}

Si la Draaf a diffusé les données vers l'ensemble des DDT de la région Languedoc-Roussillon, au moment de l'enquête, une seule Direction départementale des territoires et de la mer (DDTM) en avait fait usage et les avait rediffusées, notamment dans le cadre de son activité de «porter à connaissance ». Il s'agit des dires de l'État, réalisés lors du processus d'élaboration d'un projet d'aménagement du territoire (voir chapitre 3), qui à la fois présentent les informations dont dispose l'État sur le territoire concerné, et signalent les données dont il ne dispose pas mais dont il juge la prise en compte pertinente.

La diffusion des IQS via le site internet d'une des DDTM a permis à certains bureaux d'études de les intégrer lors de l'élaboration de documents d'urbanisme et de consolider leur argumentation, notamment lors du passage devant la CDCEA $^{29}$. Cette utilisation des IQS par les bureaux d'études prolonge leur diffusion auprès des personnes siégeant dans les Commissions départementales de consommation des espaces agricoles (CDCEA) qui doivent être convaincues du bien-fondé du projet d'aménagement défendu par ces bureaux d'études.

Ces personnes (élus, représentants de l'État et du monde agricole) peuvent à leur tour en tenir compte dans leur argumentation. Mais ce n'est pas forcément le cas, certains représentants du monde agricole considérant cette information comme « administrative » et déconnectée de la réalité, donc inintéressante.

Le message porté par ces données vise à faire prendre conscience aux acteurs de l'aménagement du territoire de la nécessité de diminuer le rythme de consommation des espaces agricoles, tout en identifiant les espaces à préserver en priorité. Or nous avons constaté un dévoiement de ces informations qui sont souvent utilisées à contre-emploi par ces mêmes acteurs pour justifier la consommation des espaces agricoles (tous les sols non classés à fort potentiel constituent une inépuisable ressource foncière) (Martin-Scholz et al., 2013).

Les acteurs locaux ne reprennent pas le message porté par la Draaf. La vision à long terme et l'orientation patrimoniale et alimentaire des données semblent relativement éloignées des préoccupations quotidiennes des acteurs locaux, qui ont tendance à privilégier l'expertise de terrain et une vision multidimensionnelle plus large de l'agriculture (Martin-Scholz et al., 2013). Cette différence dans les logiques à l'œuvre rend difficiles la compréhension et le partage de l'intérêt patrimonial de l'agriculture.

29. Voir la note 10 pour une explication des CDCEA. 


\section{Des problèmes de communication ?}

Nous avons pu constater que les acteurs du développement territorial ne se sont pas appropriés (ou très peu) le message émis par la Draaf. En s'insérant dans une approche fonctionnaliste, il est possible de parler de « problèmes de communication ».

L'approche fonctionnaliste considère « la communication comme une substance tangible qui voyage de manière ascendante, descendante et latérale dans l'organisation » (Laramée, 1989, p. 68, cité par Bonneville et Grosjean, 2011). Ainsi, elle est quelque chose de contrôlable qui existe en soi, indépendamment des individus et de l'organisation. Cette approche de la communication est fondée sur le modèle déjà évoqué plus haut de la cybernétique, élaboré par Shannon et Weaver (1949) et développé par Wiener (1950) (figure 7.1).

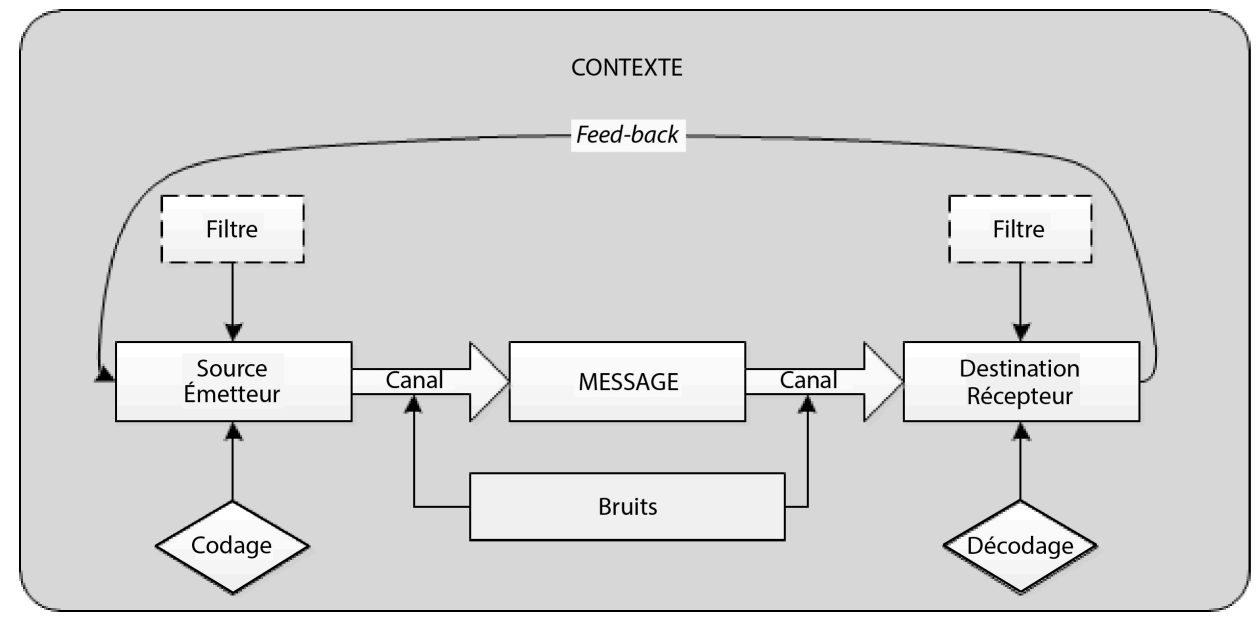

Figure 7.1. Schématisation du modèle émetteur-récepteur de Shannon et Weaver (1949).

La communication est, dans cette perspective, considérée selon le célèbre modèle du télégraphe, où l'émetteur code un message et le transmet via un canal à un destinataire qui le décode, le tout dans un contexte donné. Suite à la réception de ce message, le destinataire réalise un retour arrière (feed-back) vers l'émetteur. Dans ce second temps, le récepteur devient émetteur et inversement.

Selon l'approche fonctionnaliste, la signification du message est intrinsèque à celui-ci. Elle est considérée comme existante, comme réelle, indépendamment du contexte, de l'émetteur et du récepteur. Elle n'est donc pas construite et ne peut être, par conséquent, ni modifiable ni réinterprétable (Bonneville et Grosjean, 2011). L'enjeu de la communication est de transmettre une information de manière efficace, c'est-à-dire que le message émis atteigne le destinataire avec le moins de déformation possible, a contrario de ce qui s'est passé dans le cas de la diffusion des données d'artificialisation et de potentiel agronomique des sols. Selon Bonneville et Grosjean (2011), il est alors nécessaire d'identifier les sources d'interférences qui ont abouti à ces problèmes de communication, afin d'y remédier et de rendre la communication plus efficace. Nous avons déjà présenté quelques-unes de ces causes, notamment le fait que les destinataires, comme les DDTM ou les chambres d'agriculture, travaillent sur des projets à court terme (cinq ans) et locaux, d'une échelle qui varie de quelques hectares à celle de l'intercommunalité, alors que les données de la Draaf s'insèrent dans une perspective nationale à long terme 
(quarante ans), à une échelle régionale et selon une vision mondiale (les céréales comme « base » de l'alimentation humaine). Les destinataires du message peuvent donc rencontrer des difficultés pour interpréter le message initial, car le cadre de référence qui leur sert à décoder le message n'est pas le même que celui qui a servi à « l'encoder ». Enfin, l'utilisation des données par les bureaux d'études, différente de ce qu'avait imaginé la Draaf, peut être considérée comme ce que Dolan et al. (1996) nomment « l'écoute sélective ", c'est-à-dire qu'ils ont sélectionné les informations qui leur étaient utiles pour mener à bien leurs tâches.

$\mathrm{Au}$ vu de ces problèmes de communication, il semble que le processus de transmission d'information initié par la Draaf ait été peu efficace, puisqu'il n'a pas atteint ou très peu ses objectifs. Cependant, il est difficile de considérer que ce processus a été réellement inefficace, tant les objectifs initiaux étaient délicats à atteindre. Pour que les derniers destinataires puissent décoder le message et l'appréhender, il aurait fallu que le message ait une portée plus locale, tant au niveau spatial qu'au niveau temporel. Or, un tel message serait en contradiction avec les préoccupations de la Draaf.

Par ailleurs, l'investissement en temps pour décoder et appréhender ce message est conséquent. Puisque les données traitent d'un sujet auquel ses récepteurs ne sont pas familiers (la qualité des sols), ils doivent prendre le temps de comprendre ce qu'est la réserve utile et pourquoi elle permet de discriminer les différentes qualités de sol. Les récepteurs doivent aussi gérer des données dont la présentation ne leur est pas familière, puisqu'elles ont une échelle très globale alors qu'ils ont l'habitude de travailler à l'échelle de la parcelle cadastrale, ce qui nécessite des efforts et du temps de leur part afin d'appréhender ces changements d'échelle. Même si ce message est accompagné d'explications, sa réception reste coûteuse et nécessite toujours que les récepteurs identifient facilement les bénéfices qu'ils peuvent en attendre.

Ce manque de solutions pour améliorer «l'efficacité » du processus de diffusion du message nous amène à nous questionner sur les limites de cette approche fonctionnaliste de la communication, largement répandue dans l'ingénierie territoriale.

\section{Les limites de l'approche fonctionnaliste de la communication}

De l'analyse de ces problèmes de communication, il ressort que les « outils » techniques, comme les moyens de diffusion, sont relativement peu pris en compte dans l'approche fonctionnaliste, alors qu'ils participent à la coordination des acteurs et influent sur les actions (Vinck, 1999). En effet, cette approche considère les vecteurs du message comme des canaux, des tubes qui se contentent de transmettre le sens du message sans transformation. Cependant, Feenberg (2004) montre que ces vecteurs influent grandement sur la manière dont sont utilisées et interprétées les données. Il faut donc réintégrer la question des moyens de diffusion et de support des données dans l'analyse de la transmission du message de la Draaf.

L'étude de la diffusion suivant l'approche fonctionnaliste est également limitée par sa vision de la communication comme une relation duale entre émetteur et récepteur (Martin-Scholz, 2011). Or, dans le cas étudié ici, de nombreux acteurs interviennent soit au niveau de la production des données, où de nombreux échanges ont lieu pour spécifier les attentes de chacun et pour s'accorder sur les valeurs et les intérêts que doivent défendre ces données, soit lors de l'appropriation de ces données, où de nombreuses interactions et négociations ont lieu pour leur donner du sens. 
Ainsi, les limites de l'approche fonctionnaliste dans le cas présent ne nous permettent pas d'appréhender les compromis et les négociations à l'œuvre dans ces dispositifs, constitués à la fois d'interactions humaines et d'interactions avec les objets techniques, ni de comprendre les logiques et les intérêts représentés par les acteurs. Nous nous proposons donc d'apporter un éclairage différent sur la situation étudiée en adoptant une démarche issue d'une approche constructiviste : la sociologie de l'acteur-réseau (Callon, 1986 ; Latour, 2007).

\section{La sociologie de l'acteur-réseau ou la négociation du sens}

Face aux utilisations et surtout à la non-utilisation des IQS, des taches artificialisées et des CPAS, nous souhaitons mieux comprendre comment les différents acteurs impliqués dans la conception de ces données ont conçu ces dernières. Nous souhaitons observer comment leur conception et leur transmission influent sur le sens que leur attribuent les acteurs de l'aménagement du territoire. Cette compréhension passe par la prise en compte des interactions entre humains et non-humains et la multiplicité des «émetteurs » et des « récepteurs ».

En faisant appel au cadre d'analyse issu de la sociologie de l'acteur-réseau, nous tenterons de comprendre comment le réseau d'acteurs s'est constitué et comment les données ont été conçues et diffusées en son sein.

\section{Suivre les associations}

La sociologie de l'acteur-réseau est considérée comme une approche constructiviste, c'est-à-dire qu'elle ne se focalise pas sur les entités, leur identité et leurs fonctions. Au contraire, elle s'intéresse surtout aux relations qu'elles entretiennent entre elles (Latour, 2007). Il s'agit ainsi non pas de suivre comment des objets ou des personnes remplissent ou non les rôles, les fonctions qui leur seraient attribués, mais de se concentrer sur les associations entre ces objets et personnes ainsi que sur les compromis, les négociations et les traductions que supposent de telles associations. Selon cette approche, le réseau des acteurs ayant contribué à la création des données de la Draaf ne préexiste pas, mais est le fruit des négociations et des compromis qu'ils ont dû réaliser pour s'associer (Cooren et Robichaud, 2011).

Ainsi, la sociologie de l'acteur-réseau propose une focale pour analyser la conception des données et leur transmission en "suivant» les associations qui se nouent autour d'elles et avec elles (Latour, 2007). Cette perspective est particulièrement intéressante dans les situations inattendues, changeantes, innovantes, ou encore lorsque les catégories et les frontières sont en négociation (Latour, 2004). Elle ne se focalise pas sur les acteurs mais sur les relations qu'ils entretiennent entre eux. Pour cela, une étude suivant cette méthode se doit de ne rien considérer comme donné et ne pas suivre d'a priori, ni de préjugés. Nous devons donc analyser comment tous les acteurs, y compris non humains, s'associent, sans censurer les associations d'idées, de mots ou d'acteurs d'aucune sorte et sans faire de distinctions entre les acteurs qui ne seraient faites par les acteurs eux-mêmes.

L'analyse de la construction et de l'utilisation des données issues du projet initié par la Draaf a été présentée par ailleurs (Martin-Scholz et al., 2013). Nous en reprenons, ici, les principaux enseignements. 


\section{La construction des objets ou la formation d'un réseau}

Comme nous l'avons vu précédemment, la Draaf souhaite "faire prendre conscience » aux acteurs de l'aménagement du territoire de la nécessité de préserver ce qu'elle considère comme étant les terres à meilleur potentiel agronomique (chapitre 2). Afin d'atteindre cet objectif, elle a décidé d'avoir recours à des formes d'objectivation afin de légitimer son discours. Par formes d'objectivation, nous entendons les objets qui permettent de quantifier ou de qualifier un phénomène observé, dans le sens où leur finalité est de « fournir une "image objective" du monde » (Martin-Scholz et al., 2013). Étant conçus à partir de méthodes scientifiques et selon des techniques spécifiques, ces objets incarneraient une "rationalité supérieure » (Boussard, 2008, p. 116) donnant aux discours qu'ils accompagnent un « argument d'autorité » qui est légitime (Martin-Scholz et al., 2013). La Draaf n'a pas les ressources nécessaires en interne pour pouvoir répondre à sa préoccupation initiale, à savoir « faire prendre conscience » aux acteurs de l'aménagement du territoire de l'impératif de préserver les « meilleures terres agricoles ». Elle a donc dû avoir recours à une aide extérieure, et réussir à intéresser les parties prenantes de ce projet pour qu'elles y participent. En effet, la participation d'autres acteurs à ce projet n'est pas « donnée » d'avance, mais fait l'objet de négociations et de compromis afin de définir l'identité de ces acteurs et leurs intérêts, pour les convaincre de contribuer à la réalisation de ces formes d'objectivation (Callon, 1986). Ainsi, pour convaincre les acteurs de participer, les agents à l'initiative de ce projet doivent traduire les préoccupations et les motivations de chacun afin de construire ce que la sociologie de la traduction dénomme un «point de passage obligé » (PPO). Dans le cadre de notre étude et pour favoriser l'intéressement des acteurs, la Draaf a considéré que le PPO était la construction de données. Pour faciliter la construction de ces dernières, elle a divisé sa préoccupation initiale en deux questions : " Comment quantifier la consommation des terres agricoles par l'urbanisation ? » et « Comment qualifier les sols de la région au regard de leur capacité de diversification agricole ?» (Martin-Scholz et al., 2013).

La Draaf a donc dû négocier non seulement avec les «concepteurs » de ces données, mais aussi avec ceux qui, lors de la mise en place du projet, ont été identifiés comme étant les futurs utilisateurs des données. Par ailleurs, le projet ne regroupe pas que des acteurs humains, il comprend aussi des acteurs non humains, comme les terres agricoles et le référentiel désignant ce qu'est une «bonne terre agricole », avec lesquels la Draaf doit aussi négocier. Ces acteurs non humains sont considérés comme des acteurs à part entière dans la mesure où ils encadrent et influent très fortement sur les actions des autres acteurs. Ces négociations ont permis à la Draaf d'intéresser les acteurs à ce projet et de les convaincre d'y participer. En effet, en s'accordant sur les préoccupations, les objectifs et les valeurs des différents acteurs, la Draaf a pu favoriser l'inscription des acteurs dans le projet en les convaincant que, pour répondre à leurs attentes, il fallait participer au projet (Callon, 1986).

Parmi les premiers acteurs enrôlés se trouve Irstea, un organisme de recherche dont une des missions est l'appui aux politiques publiques via, notamment, la mise au point de méthodologies. Afin de le convaincre de participer au projet, la Draaf a mis en avant la portée mondiale de ses préoccupations et donc la possibilité pour Irstea de valoriser ses compétences, en lui donnant l'opportunité de mettre au point une méthode qui soit généralisable à l'échelle nationale.

Irstea n'a pas les ressources et les compétences en interne pour répondre à la deuxième question de la Draaf: "Comment qualifier les sols de la région au regard de leur capacité de diversification agricole ? » Pour répondre à cette question, un autre organisme de recherche a été approché : l'Inra. Ce dernier a été enrôlé via une de ses unités de 
recherche, l'UMR Lisah, qui a réalisé dans les années 1990 une base de données pédologique, la BD Sol. Les sols, et cette base de données en particulier, restent trop peu pris en compte, au goût de l'UMR Lisah, lors de l'élaboration de projets d'urbanisme. L'Inra a donc été intéressé par ce projet du fait de l'opportunité qui lui a été offerte de favoriser l'utilisation de cette base de données en simplifiant les données pour les rendre «plus opérationnelles », et d'améliorer la prise en compte des sols dans les politiques publiques territoriales.

Nous l'avons vu, la Draaf a, en outre, enrôlé des acteurs non humains dans ce projet, notamment le référentiel de « qualité » d'un sol agricole. Ce référentiel mondial peut être considéré comme un acteur à part entière, puisqu'il cadre le discours et influe sur les actions des autres acteurs. La Draaf l'a intégré dans le projet en estimant qu'il constituait une réponse à la crise alimentaire mondiale annoncée, puisqu'il visait à assurer la production alimentaire à cette échelle en postulant que c'étaient les terres favorables à la culture de céréales qui permettraient d'atteindre ce but.

Les terres agricoles ont également été recrutées dans le projet comme acteur non humain dans la mesure où elles sont le support physique des réflexions. De nombreux acteurs parlent en leur nom et, ce faisant, certains les défendent lors des nombreux conflits quant à l'usage qui doit être fait des sols. Ainsi, les terres agricoles « agissent», d'une certaine manière, sur d'autres acteurs en les incitant à faire quelque chose et à prolonger leur action. Les agents de la Draaf se sont faits les porte-parole des terres agricoles dans le projet en considérant que, face à la forte urbanisation qui les «consommait» dans l'ancienne région Languedoc-Roussillon, elles souhaitaient être maintenues dans leur capacité agricole.

Enfin, la Draaf a pris en compte, dans le projet, les Directions départementales de l'agriculture et de la forêt (DDAF), les services déconcentrés à l'échelon départemental du ministère en charge de l'Agriculture, en tant que futurs utilisateurs possibles des données conçues dans le cadre du projet. Les DDAF, qui ont fusionné en 2010 avec les Directions départementales de l'équipement (DDE) pour former les DDT, avaient pour mission de défendre les politiques de leur ministère de tutelle sur le territoire, notamment lors de la rédaction de documents d'urbanisme. Face à l'expertise et aux prérogatives des acteurs locaux, les DDAF n'ont à opposer que peu de « données objectives » sur les consommations des espaces agricoles. La Draaf les a intéressées au projet en mettant en avant la conception de formes d'objectivation qui leur permettraient d'asseoir leur discours.

Il est évident que ce n'est pas la Draaf dans son ensemble, avec tous ses agents, ses locaux, qui agit. Elle délègue son action à des agents qui parlent en son nom et qui deviennent ses représentants. De même, ce ne sont pas Irstea, les DDAF, l'Inra, les terres agricoles ou le référentiel qui interviennent directement dans le projet, mais chacun y est représenté par des porte-parole qui leur permettent de se déplacer vers des espaces-temps qui leur étaient inaccessibles ou difficilement atteignables (Latour, 2006). Ces différents acteurs ne sont pas appréhendables et déplaçables dans leur totalité. Pour qu'Irstea et la Draaf communiquent, il faut qu'ils soient représentés par des agents qui peuvent se rencontrer dans une même pièce et faire entendre la parole de leurs institutions respectives de vive voix. De même, ce n'est qu'une fois que les terres agricoles ont été représentées par des indicateurs et des cartes qu'elles peuvent entrer en contact avec les autres acteurs et « dialoguer » avec eux. Le projet s'est ainsi construit par porte-parole interposés, comme le montre la figure 7.2.

Cette construction par porte-parole interposés délègue à chaque représentant la charge de porter et de défendre les préoccupations et les objectifs de l'acteur qu'il représente. 
Ainsi, les cartes et les mesures conçues dans le cadre de ce projet deviennent les porteparole de l'ensemble des acteurs du projet et intègrent dans leur constitution l'ensemble des préoccupations et des objectifs exprimés.

Les cartes et les mesures forment ainsi des acteurs-réseau, c'est-à-dire qu'elles sont d'une part, des entités « ponctuelles » aux frontières bien définies, mais aussi des entités « distribuées » dans les différentes entités qui les composent. En effet, « [leur] forme, [leurs] propriétés, [leurs] caractéristiques [sont] les effets de toutes les interactions qui [ont eu] lieu, de tous les compromis sociotechniques qui [ont] été construits » (Callon et Law, 1997).

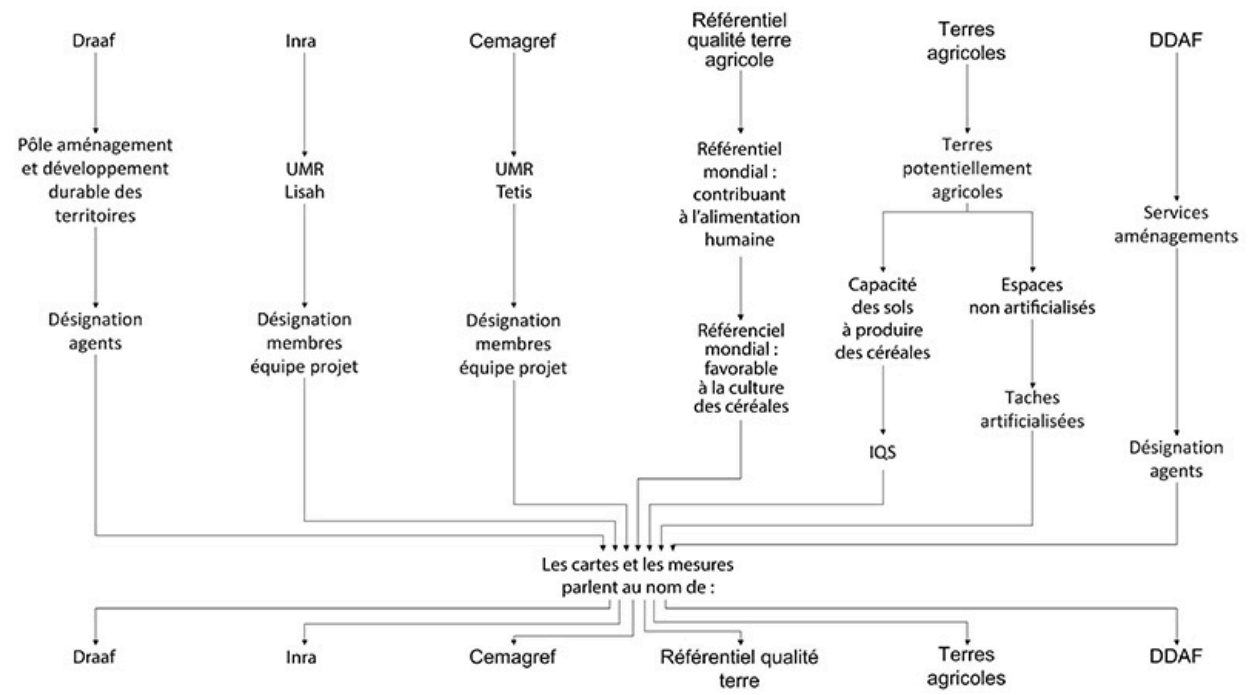

Figure 7.2. Construction des entités par porte-parole interposés.

Cependant, un compromis, une négociation peuvent être remis en cause, un porte-parole reconsidéré. À chaque instant, les acteurs impliqués dans la construction des cartes et des mesures peuvent se « désolidariser » et remettre en question la représentativité de ces formes d'objectivation, comme nous allons le voir par la suite.

\section{L'élargissement du réseau : avoir des mobiles immuables}

Une fois ces mesures et ces cartes constituées, l'ensemble des acteurs du projet va chercher à élargir le réseau d'acteurs afin de recruter de nouveaux alliés et faire adhérer de nouveaux acteurs aux préoccupations de la Draaf.

Recruter de nouvelles entités au sein du réseau pour l'élargir nécessite de se déplacer vers elles pour les toucher, les intéresser et les convaincre de s'inscrire. Comme il n'est pas aisé pour tous les acteurs de se déplacer, il est nécessaire d'avoir des « véhicules » qui puissent transporter l'énoncé, ici la préoccupation de la Draaf, d'un lieu vers un autre sans le déformer, ce que Latour appelle des « mobiles immuables » (Latour, 2006).

Nous l'avons vu, les cartes et les mesures sont le résultat de négociations et de compromis entre la Draaf, Irstea, l'Inra, les terres agricoles, le référentiel et les DDAF. Ce faisant, ces acteurs y inscrivent leurs objectifs, leurs valeurs, leurs stratégies, leurs préoccupations, leur permettant ainsi d'atteindre des lieux éloignés. En effet, pour 
atteindre les acteurs à convaincre, il est plus facile de déplacer des cartes et des mesures inscrites sur des feuilles de papier ou stockées sous forme de bits que l'ensemble des acteurs (les terres agricoles, la Draaf...) en un même lieu.

Ces objets ont ensuite été propagés selon les modalités présentées plus haut, c'est-à-dire en transférant aux DDTM ces données, celles-ci étant supposées les mettre à disposition des autres acteurs de l'aménagement du territoire, principalement dans le but d'atteindre les principales parties prenantes en matière d'aménagement territorial (Martin-Scholz et al., 2013).

Cependant, une fois les cartes et les mesures constituées, le processus qui a amené à leur construction n'est plus visible (Callon et Law, 1997). Une fois les compromis conclus et les négociations abouties, le processus commence à être occulté, et les données se présentent comme une « boîte noire » empêchant les acteurs qui pourraient s'y rallier de revenir aux postulats à la base de la constitution des objets. En effet, bien que ces données soient accompagnées de métadonnées, ces dernières ne disent rien des arbitrages et des négociations qui ont eu lieu. Les données sont présentées comme un « tout », dont on ne peut remettre en question les éléments qui le composent, ce qui a favorisé le développement de remises en cause lors de l'élargissement du réseau.

\section{Élargir le réseau, nécessaire mais déstabilisant}

Élargir le réseau est nécessaire. Une donnée, une technique fonctionnent peut-être parfaitement, sans être remises en cause, dans le cerveau de leurs concepteurs ou au sein de leur réseau stabilisé. Mais pour qu'elles cessent de n'être que des utopies, pour qu'elles fonctionnent réellement, il est nécessaire qu'elles élargissent le réseau des acteurs auxquels elles se confrontent (Latour, 1992), qu'elles se « frottent au réel ». Pour que la préoccupation de la Draaf devienne un enjeu reconnu en ce qui concerne l'aménagement territorial, il faut que cette préoccupation soit reprise par le plus grand nombre d'acteurs sans être déformée et serve de fondement aux réflexions concernant les décisions d'aménagement (Latour, 2006).

L'élargissement du réseau est nécessaire, mais ici il est aussi inévitable. Les acteurs du projet et les porte-parole du projet de la Draaf s'associent et se réorganisent en permanence dans différents collectifs. Chacun met en avant différentes identités en fonction du collectif dans lequel il s'insère. Un acteur peut être tour à tour collègue, ami, parent, patient d'un médecin, conducteur de voiture, passager dans les transports en commun, client d'un magasin... Lors de la construction du réseau et de la constitution des cartes et des mesures, l'intéressement des acteurs, les négociations quant à leurs objectifs, leurs préoccupations, bref, leur identité, avaient pour but de limiter l'influence des différentes " obédiences », des différents groupes dans lesquels les différents acteurs sont impliqués. Lors des réunions de l'équipe-projet, ses membres sont coupés des autres groupes avec lesquels ils interagissent pour ne s'intéresser qu'à la construction des données. Cependant, même s'il est éventuellement possible d'isoler les acteurs pendant un court laps de temps (le temps d'une réunion), cet isolement ne fait jamais totalement disparaître les autres groupes dans lesquels les différents acteurs sont impliqués. Ainsi, les DDAF ne sont pas isolées des autres parties prenantes de l'aménagement du territoire. Lorsqu'elles manient les cartes et les mesures et les prennent en compte dans leurs tâches quotidiennes, l'intégration de ces données leur permet d'atteindre d'autres acteurs avec lesquels elles interagissent. Que les différents actants (acteurs humains ou non humains) en aient conscience ou non, l'inscription de ces objets dans leurs activités permet à ces derniers de rallier d'autres acteurs, favorisant l'élargissement du réseau. 
Enrôler de nouveaux acteurs dans le réseau peut également le fragiliser (Martin-Scholz et al., 2013). Pour que de nouveaux acteurs intègrent le réseau, c'est-à-dire reprennent les préoccupations et les intérêts défendus par le dispositif initial sans les déformer, il faut que ceux-ci y voient un intérêt. Cela suppose que les actions spécifiquement conçues pour convaincre les actants du dispositif initial restent pertinentes pour d'autres acteurs. Ce qui est difficilement le cas, puisque chaque actant est porteur de son identité et de ses intérêts propres (Latour, 2006).

Dans le cas présent, avant même la propagation des données, le réseau était déstabilisé. Certains de ses acteurs, les DDAF, du fait de leurs liens avec d'autres collectifs, en l'occurrence ici l'État, ont été " désolidarisés » du réseau en raison de leur changement d'identité. Au $1^{\text {er }}$ janvier 2010, les DDAF ont fusionné avec les DDE pour former les DDT. Ainsi, elles se voient adjoindre la mission de faire appliquer et de défendre, au niveau départemental, les politiques des ministères en charge de l'Équipement et de l'Environnement, en plus de celles du ministère en charge de l'Agriculture. Le réseau des acteurs du projet s'est modifié, car d'organismes ayant une forte sensibilité agricole, ce sont devenus maintenant des institutions ayant certes toujours une certaine sensibilité agricole, mais surtout préoccupées par des questions relatives à l'aménagement et au développement durable. Le réseau a été d'autant plus déstabilisé qu'à la suite de la réorganisation des services consécutive à la fusion, ce sont majoritairement les agents issus de l'ex-DDE qui se sont retrouvés en charge de l'aménagement du territoire. Ainsi, les cartes et les mesures, construites pour des personnes ayant plutôt une sensibilité agricole, entrent désormais en contact avec des personnes plutôt concernées par des questions environnementales et d'urbanisme. Il était donc nécessaire de faire un effort pour les intéresser et les sensibiliser aux données réalisées dans le cadre du projet de la Draaf.

Des remises en cause ont également eu lieu en « interne » au sein du réseau initial, lors de la propagation des données. En effet, les acteurs étaient partagés entre deux injonctions contradictoires. D'un côté, il fallait convaincre et sensibiliser des acteurs aux préoccupations de la Draaf, afin qu'ils utilisent les données en poursuivant les différents objectifs définis par le réseau d'acteurs à l'origine de ces données, ce qui nécessite des efforts et du temps pour en maîtriser la propagation. D'un autre, l'objectif des acteurs était de faire partager rapidement ces préoccupations au plus grand nombre, exigeant de faciliter l'accès à ces données et de limiter les obstacles à leur propagation, en mettant en place des protocoles simples et rapides pour y accéder. Entre ces deux objectifs, certains ont privilégié l'idée de se concentrer sur la sensibilisation des acteurs et ont mis l'accent sur la maîtrise de la propagation des données en s'assurant de l'enrôlement des utilisateurs de ces données à la vision défendue par le réseau. Ils l'ont fait notamment en propageant différemment ces données en fonction des organismes visés (les acteurs de la recherche les transmettant aux organismes de la recherche, les services de l'État propageant vers les services de l'État). D'autres, plus nombreux, ont préféré propager les données le plus rapidement possible et auprès du plus grand nombre d'individus, en facilitant leur accès. L'intéressement de ces individus à la préoccupation portée par ces données et leur explicitation ont consisté ici à rédiger des fiches de métadonnées visualisables ou téléchargeables en même temps que les données, précisant comment ces données avaient été construites. Cependant, dans ces cas, l'intéressement est plus difficile car c'est un acteur seul face aux métadonnées et aux données qui doit négocier ses intérêts et ses motivations, favorisant les remises en cause ultérieures. 


\section{La remise en cause des données comme représentant du réseau des acteurs du projet}

La facilitation de l'accès aux données a permis de toucher une grande diversité d'actants comme les bureaux d'études en aménagement territorial. Pour que ces derniers « maîtrisent l'information » du point de vue des concepteurs, en d'autres termes, pour qu'ils partagent les préoccupations de la Draaf, il est nécessaire qu'ils soient intéressés, que les métadonnées soient compréhensibles, et qu'ils soient disposés et en capacité de les appréhender et de les comprendre. Les bureaux d'études sont mandatés par les élus locaux pour aider, préparer et/ou participer à l'élaboration de documents d'urbanisme. Ce sont pour la plupart des entreprises privées, mais ce peuvent être aussi des associations ou encore des chambres d'agriculture. Dans tous les cas, ils se doivent de satisfaire leurs clients, c'est-à-dire qu'ils doivent s'assurer que les projets soumis sont validés par les différentes instances et commissions en charge d'émettre un avis (Martin-Scholz et al., 2013). Pour ce faire, les bureaux d'études ont recours à de nombreuses formes d'objectivation qui légitiment, selon eux, leurs discours. Les données issues du projet de la Draaf ont été d'autant plus facilement utilisées qu'elles étaient aisément téléchargeables en ligne, et que les bureaux d'études considéraient qu'elles deviendraient un indicateur de référence (Boussard, 2001) pour les DDTM. Si les bureaux d'études utilisent les données issues du projet porté par la Draaf, ils n'en partagent pas pour autant les préoccupations, du fait que peu d'efforts ont été faits pour les intéresser et les enrôler dans cette vision. L'utilisation par les défenseurs de projets d'aménagement des cartes et des mesures pour justifier l'urbanisation de terres agricoles et légitimer leur projet puisque «les terres sont mauvaises » remet, en effet, en question leur fonction de porteparole du réseau des acteurs qui les ont construites, puisqu'elles peuvent être utilisées pour soutenir un discours à rebours de leurs préoccupations.

À l'inverse, les acteurs ayant été intégrés d'office dans le réseau, n'ayant pas pu négocier leurs intérêts et leur identité, contestent la capacité des données à les représenter. C'est le cas des agents issus de l'ex-DDE qui, suite à la création de la DDT, sont devenus les représentants de cette dernière au sein du projet. Ces agents n'ayant pas participé à la réalisation des données, ces dernières se présentent à eux sous la forme d'une «boîte noire », dont l'intérêt ne leur a pas été démontré. Les agents de l'ex-DDE ont surtout remis en cause l'utilité et la pertinence des taches artificialisées. Ces dernières se présentent comme des données utilisables à l'échelle du 1/15 000, alors que ces agents ont l'habitude de travailler avec des données ayant une échelle plus grande comme celle de la parcelle cadastrale. La pertinence de ces données est d'autant plus remise en cause qu'elles entrent en concurrence avec d'autres données portant sur des thématiques proches, notamment les taches urbaines réalisées à partir des fichiers fonciers, communément appelés Majic (Mise à jour des informations cadastrales), diffusées par la Direction générale des finances publiques (DGFiP). Ces taches présentent aussi une évolution du bâti mais à l'échelle cadastrale en utilisant des représentations familières aux personnes issues de l'ancien ministère de l'Équipement, amenant ces dernières à privilégier l'utilisation des taches urbaines au détriment des taches artificialisées, remettant ainsi en cause la capacité de ces dernières à représenter leurs préoccupations.

\section{La remise en cause de la définition d'une «bonne terre »}

La propagation facilitée des données et leur utilisation lors du processus d'élaboration de documents d'urbanisme les font entrer dans des arènes d'échanges, permettant de toucher 
de nouveaux acteurs. Par exemple, la mise en place de la CDCEA et les débats qui y ont lieu ont fait se rencontrer les données issues du projet initié par la Draaf et d'autres représentants des terres agricoles. Ces derniers vont contester aux cartes et aux mesures leur capacité à définir ce qu'est une « bonne terre agricole à préserver » (Martin-Scholz et al., 2013).

Les données de la Draaf représentent une vision qui considère l'agriculture pour sa fonction alimentaire et les terres agricoles comme un patrimoine public (Balestrat, 2011). À l'inverse, les agriculteurs de l'ancienne région Languedoc-Roussillon, majoritairement des viticulteurs très souvent propriétaires des terres qu'ils cultivent, considèrent les terres comme un bien privé. Ces derniers défendent une vision où les terres sont surtout considérées pour leur dimension économique, comme la qualité et la quantité de la production viticole qu'elles permettent ou leur valeur foncière. Cette divergence quant à la représentation des terres agricoles se manifeste surtout dans les CDCEA. Même si les agriculteurs n'y interviennent pas directement, ils y sont représentés par leurs syndicats professionnels et les chambres d'agriculture.

Dans les CDCEA, le représentant de la vision de l'État concernant les terres agricoles est la DDT. Si cette dernière prend en compte la vision de la Draaf, où les terres agricoles sont envisagées en termes de patrimoine agronomique, les DDT défendent surtout une vision multidimensionnelle de ces espaces. En effet, elles ont différentes missions portant sur différentes thématiques territoriales comme l'énergie, le logement, l'environnement, l'agriculture. Elles vont donc remettre en question l'utilisation des cartes et des mesures $\mathrm{du}$ fait de leur orientation uniquement alimentaire.

Ainsi, les DDT, les chambres d'agriculture et les syndicats agricoles défendent une vision locale à court et moyen terme, avec une agriculture multifonctionnelle, et privilégient le recours à l'expertise locale (Martin-Scholz et al., 2013), plus à même selon eux de répondre à des enjeux locaux, qu'un ensemble de cartes et de données qui s'inscrivent dans un contexte national, voire mondial. Ils remettent ainsi en cause la capacité des cartes et des mesures à être les porte-parole des terres agricoles.

\section{Retour sur la sociologie de l'acteur-réseau}

La Draaf est à l'origine de ce projet visant à modifier les pratiques des parties prenantes de l'aménagement territorial afin de préserver la capacité des terres de l'ancienne région Languedoc-Roussillon à accueillir différents types de cultures à visée alimentaire. Pour ce faire, elle s'est constituée porte-parole d'un référentiel mondial où l'agriculture est vue à travers sa capacité à subvenir aux besoins alimentaires des personnes. Cette dernière est donc appréciée pour sa capacité à accueillir des cultures céréalières, celles-ci fondant la base de l'alimentation humaine. La Draaf se fait aussi le porte-parole d'une vision à très long terme (quarante ans). Afin de faire partager ses préoccupations, elle a tenté de créer, à un échelon local, un système d'intéressement, en introduisant des références à une échelle globale et à long terme (Martin-Scholz et al., 2013). Pour ce faire, elle a réussi à enrôler différents acteurs et à négocier avec eux leurs intérêts afin qu'ils produisent ensemble un ensemble de cartes et de mesures qui deviennent les porte-parole de ce réseau d'acteurs. Cependant, lors de l'allongement du réseau, la Draaf a tenté d'y intégrer à moindre coût de nouveaux acteurs porteurs de logiques et de représentations différentes, qui ont donc remis en cause les cartes et les mesures.

Ces remises en cause, ou dissidences, montrent que penser la réalisation de formes d'objectivation sous l'angle de la « production d'information », du « destinataire » et des « données » présente des limites. Il n'y a pas ici de «production d'information », mais plutôt la formation d'un collectif qui tente de convaincre d'autres acteurs, pour faire 
partager une préoccupation commune en construisant des objets qui puissent transporter cette préoccupation vers des lieux que les membres de ce collectif peuvent difficilement atteindre. Les acteurs qui entrent en contact avec ces objets ne sont pas des destinataires passifs qui reçoivent une information, mais des acteurs à part entière avec lesquels il faut négocier leur intégration dans le réseau afin qu'ils puissent à leur tour porter les préoccupations de la Draaf. Enfin, le terme de données sous-entend qu'elles seraient " données » par la raison, alors que cet exemple le montre bien, rien n'est donné, rien n'est acquis et tout fait l'objet de négociation et de traduction (Martin-Scholz et al., 2013).

\section{Conclusion}

L'étude présentée dans ce chapitre visait initialement à déterminer les « effets » qu'avait eus la diffusion des données sur les processus d'aménagement du territoire, et plus précisément sur la prise en compte des « sols » dans l'élaboration des documents d'urbanisme (Martin-Scholz, 2011). Or, au-delà de l'impact des données, nous observons que leur diffusion et plus encore leur construction font intervenir des processus communicationnels afin de convaincre d'autres à agir.

Nous avons présenté dans ce chapitre deux approches communicationnelles différentes pour étudier la même situation. L'approche fonctionnaliste, fondée sur le modèle cybernétique qui semble relever du bon sens et de la raison, a montré que si elle permet d'apprécier la communication en termes d'efficacité, elle présente l'inconvénient de ne pas pouvoir prendre en compte la diversité des organisations concernées par la conception et l'utilisation de ces cartes et de ces mesures, ni de permettre d'appréhender les changements à l'œuvre dans le réseau formé par ces différentes organisations. La sociologie de l'acteur-réseau permet de sortir de cette vision, en proposant de ne plus se focaliser sur les individus qui « composent le système », mais de s'intéresser plutôt aux négociations, aux compromis qui ont lieu pour enrôler, inscrire les actants dans la défense d'une préoccupation commune. Les remises en cause qui semblent, dans un premier temps insurmontables, apparaissent comme une question d'intéressement.

Ce chapitre montre que pour les acteurs qui réalisent des formes d'objectivation, il est nécessaire de ne pas considérer ces formes comme porteuses d'un sens donné par avance et universel. Plutôt que de considérer la diffusion du message porté par la Draaf comme n'ayant pas été « efficace », ce changement d'approche nous permet d'observer que faire partager les valeurs, les préoccupations qui sont représentées par les données demande de prendre en considération le futur élargissement du réseau constitué autour de ces formes et de mettre en place des systèmes d'intéressement pour s'assurer de maintenir l'intérêt des acteurs inscrits et pour enrôler de nouveaux actants.

Enfin, il est important de rappeler que nous avons décrit un état des lieux à un instant donné sur une situation qui n'est pas figée mais évolue sans cesse. Nous avons insisté sur les actions d'intéressement à mettre en place pour favoriser l'inscription de nouveaux actants dans le réseau. D'une certaine manière, nous avons, nous aussi, inscrit les différents actants rencontrés, les avons traduits pour les réunir au sein de ce même chapitre afin de soutenir son énoncé, à savoir la nécessité de prendre en considération une approche communicationnelle fondée plus sur un réseau d'acteurs qui se constitue que sur une chaîne de processus de codage/décodage de messages.

À l'heure où nous écrivons ces lignes, de nouvelles actions de sensibilisation ont été réalisées. Les agents de la Draaf et de l'UMR Tetis ont enrôlé de nouveaux acteurs, dont des agents de la Dreal, afin de construire un module de formation, dont trois sessions ont été organisées en mars 2015, septembre 2016 et mai 2018. Le projet d'élaboration de ce 
module de formation avait pour objectif initial de convaincre les acteurs de l'aménagement territorial d'utiliser ces données issues du projet Draaf-UMR Tetis-UMR Lisah. Le suivi de l'élaboration de ce module a fait l'objet d'un travail de thèse (Martin-Scholz, 2017).

\section{Références bibliographiques}

Balestrat M., 2011. Système d'indicateurs spatialisés pour la gouvernance territoriale: application à l'occupation des sols en zone périurbaine languedocienne. Université Paul-Valéry Montpellier-III.

Bonneville L., Grosjean S., 2011. Les approches fonctionnalistes. In : La communication organisationnelle, Chenelière Éducation, 32-55.

Boussard V., 2001. Quand les règles s'incarnent: l'exemple des indicateurs prégnants. Sociologie du travail, 43 (4), 533-551.

Boussard V., 2008. Sociologie de la gestion. Les faiseurs de performance, Paris, Belin.

Callon M., 1986. Some elements of a sociology of translation: domestication of the scallops and the fishermen of St-Brieuc Bay. Power, action and belief: a new sociology of knowledge? London, Routledge.

Callon M., Law J., 1997. L'irruption des non-humains dans les sciences humaines : quelques leçons tirées de la sociologie des sciences et des techniques. In : Les limites de la rationalité. Tome 2. Rationalité, éthique et cognition, La Découverte, 99-118.

Cooren F., Robichaud D., 2011. Les approches constitutives. In : La communication organisationnelle, Chenelière Éducation, 140-175.

Dolan S.L., Lamoureux G., Gosselin E., 1996. Psychologie du travail et des organisations, Gaëtan Morin.

Feenberg A., 2004. (Re)penser la technique. Vers une technologie démocratique, Paris, La Découverte/MAUSS, 234 p.

Laramée A., 1989. La communication dans les organisations : une introduction théorique et pragmatique, Presses de l'Université du Québec.

Latour B., 1992. Aramis ou l'amour des techniques, Paris, La Découverte.

Latour B., 2004. Comment finir une thèse de sociologie. Petit dialogue entre un étudiant et un professeur (quelque peu socratique). Revue du MAUSS, 24 (2), 154-172.

Latour B., 2006. Les « vues » de l'esprit. Une introduction à l'anthropologie des sciences et des techniques. In : Sociologie de la traduction : textes fondateurs, Paris, Presses de l'École des Mines, 33-70.

Latour B., 2007. Changer de société, refaire de la sociologie, Paris, La Découverte.

Martin-Scholz A., 2011. Analyse de l'impact sur la gouvernance territoriale de la mise à disposition de nouveaux contenus informationnels. Projet Gouv. Innov. Mémoire d'école d'ingénieur, Ensat.

Martin-Scholz A., 2017. Communiquer et organiser en échafaudant un «big data » manuel. Le cas d'un projet de formation en aménagement du territoire. Thèse de doctorat en sciences de l'information et de la communication de l'Université Toulouse-III.

Martin-Scholz A., Mayère A., Barbe E., Valette E., Maurel P., 2013. Quand l'information échappe à ses créateurs. Le cas de l'artificialisation des terres agricoles en Languedoc-Roussillon. Études de communication, 40, 145-166.

Maurel P., 2012. Signes, données et représentations spatiales : des éléments de sens dans l'élaboration d'un projet de territoire intercommunal. Application au territoire de Thau. Thèse en Sciences de l'information et de la communication, université du Sud Toulon/Var.

Scardigli V., 2001. Un anthropologue chez les automates, Presses universitaires de France. 
Shannon C., Weaver W., 1949. The Mathematical Theory of Information, University of Illinois Press.

Vatin F., 2008. L'esprit d'ingénieur: pensée calculatoire et éthique économique. Revue française de socio-économie, 1, 131-152.

Vinck D., 1999. Ingénieurs au quotidien, PUG, coll. Génie industriel.

Wiener N., 1950. Cybernétique et société. L'usage humain des êtres humains, Éditions du Seuil. 



\title{
Chapitre 8
}

\section{Le concept de «politiques de l'identité de lieu » comme outil pour mieux comprendre la marginalisation des terres agricoles dans la commune périurbaine de Wollondilly, Nouvelle-Galles du Sud, Australie}

\author{
LAURE-ÉLISE RUOSO
}

\section{Introduction}

Les terres agricoles périurbaines fournissent divers types de services, qui contribuent à la résilience, à la durabilité et à la sécurité alimentaire des villes ainsi qu'à la santé et au bien-être de leur population (Agricultural Reference Group, 2013 ; Knowd, 2006 ; 2013 ; Olsson et al., 2016 ; Burton et al., 2013). Du point de vue de la durabilité et de la résilience, l'agriculture périurbaine peut participer, entre autres, à la mitigation du changement climatique et à la gestion des déchets urbains (déchets verts, eaux usées, etc.) (NSW Agriculture, 1998 ; De Zeeuw et al., 2011 ; Attwater et Derry, 2017). Du point de vue de la sécurité alimentaire, l'agriculture périurbaine constitue une source sûre de produits frais, et permet de réduire la dépendance au système alimentaire global qui est soumis à toute une variété de risques (perturbations du transport, pics pétroliers, changement climatique, etc.) (Olsson et al., 2016). Du point de vue social, l'agriculture périurbaine permet, entre autres, l'intégration de populations aux origines ethniques diverses, l'approvisionnement en denrées alimentaires culturellement appropriées ainsi que la recréation d'un lien entre producteurs et consommateurs (Parker, 2007 ; James, 2009 ; Knowd, 2013).

Cependant, les villes sont confrontées, à l'échelle mondiale, au phénomène de l'étalement urbain, qui engendre la perte de terres productives dans les zones périurbaines (Millar et Fyfe, 2012; Houston, 2005 ; Zasada, 2011 ; Pointereau et Coulon, 2009), et avec elles la disparition de nombreux services. L'Australie, pays auquel cet ouvrage s'intéresse, est l'un des pays les plus urbanisés au monde, et la ville de Sydney, sur laquelle nous nous concentrerons ici, rassemble $65 \%$ de la population de la NouvelleGalles du Sud (Australian Bureau of Statistics, 2012) et connait une croissance continue de sa population. Pour répondre à cette croissance, des logements sont construits sur des terres agricoles. 
Malgré la pression exercée par l'étalement urbain sur les terres agricoles périurbaines, la production agricole dans le bassin de Sydney continue. D’après le Département des industries primaires, le bassin a produit $6 \%$ de la valeur totale de la production agricole de la Nouvelle-Galles du Sud en 2016 (Department of Primary Industries, 2016). Ce chiffre s'élève à $65 \%$ lorsque l'on considère la production de légumes, à $36 \%$ pour la production de volailles, et à $37 \%$ pour la production d'œufs (Department of Primary Industries, 2016).

Cependant, trois phénomènes pourraient mettre en péril cette production agricole périurbaine : une planification qui favorise le développement urbain aux dépens de la protection des terres agricoles; une politique néolibérale en matière agricole; et des transformations sociales et culturelles dans les zones périurbaines, qui impactent les activités agricoles. En effet, même si le besoin de protéger les terres agricoles autour de Sydney a été reconnu dès le premier document de planification de Sydney publié en 1951 (County of Cumberland Planning Scheme), dans les faits l'accroissement du nombre de logements a toujours eu la priorité (James, 2009 ; Wilkinson, 2011). De plus, la domination d'une idéologie néolibérale dans l'aménagement urbain des vingt dernières années a mené le gouvernement à jouer progressivement un rôle de facilitateur du développement urbain, en créant des conditions favorables à l'investissement privé (Department of Planning and Environment, 2015 ; McFarland, 2014). La protection des terres agricoles et des services qu'elles fournissent ne constitue donc pas une priorité dans les orientations d'aménagement urbain de Sydney, et la question de la protection des terres agricoles périurbaines n'est que vaguement considérée. En outre, la politique néolibérale en matière agricole, poursuivie par l'Australie depuis les années 1960, a rendu les exploitations familiales, qui représentent une large part des exploitations australiennes (Alston, 2004), économiquement vulnérables du fait de l'ouverture et de la dérégulation du marché australien (Dibden et Cocklin, 2005 ; Lawrence, 1999 ; Pritchard, 2005a ; 2005b ; Vanclay, 2003). Pour ajouter à cela, les zones périurbaines sont souvent le lieu de profondes transformations sociales et culturelles. Majoritairement agricoles par le passé, elles accueillent aujourd'hui une diversité d'acteurs aux valeurs différentes, parfois contradictoires (Argent et al., 2011 ; Tonts et Greive, 2002 ; Willis, 2007). De ces modifications du tissu social émergent souvent des conflits liés aux représentations que les acteurs ont du paysage et des pratiques qui y sont jugées acceptables (Abrams et Gosnell, 2012 ; Foley et Scott, 2014 ; Walker et Fortmann, 2003). Ces conflits peuvent être considérés comme de nature identitaire, car ils reviennent souvent à définir qui est reconnu comme faisant partie des paysages périurbains et qui en est exclu (Trudeau, 2006).

Dans ce chapitre, qui présente certains des résultats développés dans notre thèse (Ruoso, 2018), nous essaierons, à l'aide du concept de «politiques de l'identité de lieu », de montrer comment certains acteurs parviennent à marginaliser l'agriculture dans les paysages périurbains en instrumentalisant les processus de planification, et plus particulièrement les demandes de rezonage des terres. Nous verrons aussi comment ce mécanisme se trouve renforcé, d'une part, par la priorité donnée, dans l'aménagement, aux prévisions démographiques et aux besoins en logements nouveaux qui en découlent, et, d'autre part, par l'utilisation d'informations, dont la validité peut être discutée, en ce qui concerne la viabilité des terres agricoles.

Nous présenterons tout d'abord notre cadre théorique (un modèle de la politique de l'identité de lieu) et notre méthodologie. Puis nous montrerons comment les processus de planification sont instrumentalisés par certains groupes sociaux pour marginaliser l'agriculture. Nous traiterons ensuite de la question de la domination des prévisions démogra- 
phiques et de l'utilisation d'informations peu pertinentes sur la viabilité des terres dans les processus de rezonage. Puis nous conclurons par quelques mots sur la manière dont le concept de " politiques de l'identité de lieu » permet de mieux comprendre la marginalisation des terres et activités agricoles en milieu périurbain, ainsi que sur les options possibles pour réintégrer les terres et usages agricoles dans la planification périurbaine.

\section{Cadre théorique et méthodologie}

\section{Cadre théorique}

Notre modèle de la politique de l'identité de lieu est basé sur une conceptualisation politique des notions de lieu et de paysage qui a été développée en géographie, mais aussi dans d'autres disciplines telles que la sociologie, la psychologie sociale ou encore les sciences du loisir (Cresswell, 1996 ; Hayden, 1995 ; Massey, 1994 ; Benson et Jackson, 2013 ; Stokowski, 2002 ; Dixon et Durrheim, 2000 ; Setten, 2006 ; Mitchell, 1996 ; 2003 ; Gailing et Leibenath, 2017). Cette conceptualisation politique de la notion de lieu ou de paysage est basée sur l'idée que lieux ou paysages sont structurés par des relations de pouvoir entre différents groupes sociaux. Larsen (2004), qui reprend et développe un modèle créé par Harner (2001), identifie trois moments de la politique de l'identité de lieu, dont chacun représente une articulation différente des relations de pouvoir. Il identifie un moment hégémonique, qui se produit lorsqu'un groupe social a le pouvoir de donner un sens au paysage ou au lieu, sens qui est accepté comme légitime par les autres groupes sociaux, même si cela se fait à leurs dépens. Une phase de résistance se produit lorsque certains groupes sociaux remettent en cause le sens donné au paysage par le groupe social dominant. Enfin, une phase d'émergence apparaît lorsque le sens donné à un lieu ou paysage et le contrôle exercé sur ce dernier sont renégociés entre différents groupes sociaux.

L'identité de lieu ou de paysage peut être considérée comme étant constituée de quatre dimensions : la dimension matérielle, les pratiques, les représentations et l'identité. La dimension matérielle renvoie aux caractéristiques physiques d'un lieu ou d'un paysage. Les pratiques font référence au fait qu'un groupe développe une relation à un lieu ou à un paysage à travers différentes activités qui modèlent l'environnement physique (Di Masso et Dixon, 2015 ; Benson et Jackson, 2013 ; Setten, 2006). La dimension des représentations (sociales) s'attache à montrer comment la manière dont des groupes sociaux se représentent un lieu ou un paysage contribue à la construction de leur relation à ce lieu ou à ce paysage, et définit la façon dont ils s'expriment à son sujet. En effet, dans plusieurs conceptualisations de la politique de l'identité ou de l'attachement au lieu, la dimension discursive est prédominante (Dixon et Durrheim, 2000 ; Stokowski, 2002). Plusieurs auteurs expliquent que c'est à travers des "actions discursives » (Dixon et Durrheim, 2000, p. 28) ou des «structures langagières cohérentes » (Stokowski, 2002, p. 372) que la relation d'un groupe à un lieu se crée. Ils expliquent aussi que ce sont ces mêmes structures langagières qui excluent certains groupes d'un lieu ou d'un paysage. Les représentations que des groupes sociaux ont des paysages qui les entourent sont donc utilisées pour inclure certains groupes et en exclure d'autres. Finalement, la dimension de l'identité peut être définie de deux manières pour comprendre l'identité de lieu ou de paysage. Premièrement, elle peut désigner le caractère unique d'un lieu ou d'un paysage, ou genius loci (Lewicka, 2008 ; Stobbelaar et Pedroli, 2011). Deuxièmement, elle peut être définie comme la manière dont un lieu ou un paysage contribue à la création de l'identité d'une personne ou d'un groupe (Lewicka, 2008 ; Hauge, 2007 ; Twigger-Ross et Uzzell, 1996 ; Stobbelaar et Pedroli, 2011). Quatre principes de l'identité ont été identifiés par Breakwell (1986) : la singularité, la continuité, l'estime de soi et l'efficacité. Lorsqu'appli- 
quées à l'identité de lieu, la singularité renvoie à la manière dont un lieu ou un paysage permettent à une personne de se distinguer des autres ; la continuité, à la manière dont un lieu ou un paysage permettent à une personne de maintenir un sentiment de continuité de son identité ; l'estime de soi, à la manière dont un lieu ou un paysage permettent à un individu ou un groupe de développer une vision positive de lui-même ; et l'efficacité personnelle, à la manière dont l'environnement permet à une personne de faire ce à quoi elle aspire et d'atteindre ses objectifs. L'identité de lieu peut donc être de deux natures. Soit elle fait référence aux caractéristiques spécifiques d'un lieu qui le rendent unique (genius loci), soit elle fait référence à la manière dont un lieu ou un paysage contribuent à créer l'identité d'une personne ou d'un groupe.

L'identité de lieu peut être considérée comme émergeant de l'interaction entre ces quatre dimensions. Ces interactions sont bidirectionnelles : un groupe social peut développer une représentation du paysage matériel et des pratiques qui y sont considérées comme acceptables, cette représentation venant renforcer l'identité du groupe, et par conséquent son identité de lieu. À l'inverse, des pressions externes peuvent mener des groupes à changer leurs pratiques pour s'adapter à ces nouvelles conditions, ce qui pourrait ensuite mener à un changement de représentations et d'identité de lieu. Ces quatre dimensions de l'identité de lieu sont intégrées dans les trois moments de la politique relative au lieu décrits précédemment (figure 8.1). Chaque moment (hégémonique, résistant, émergent) est donc constitué des quatre dimensions (paysage matériel, pratiques, représentations, identité). Ce modèle de la politique de l'identité de lieu est discuté plus en détail ailleurs (Ruoso, 2018 ; Ruoso et Plant, 2018).

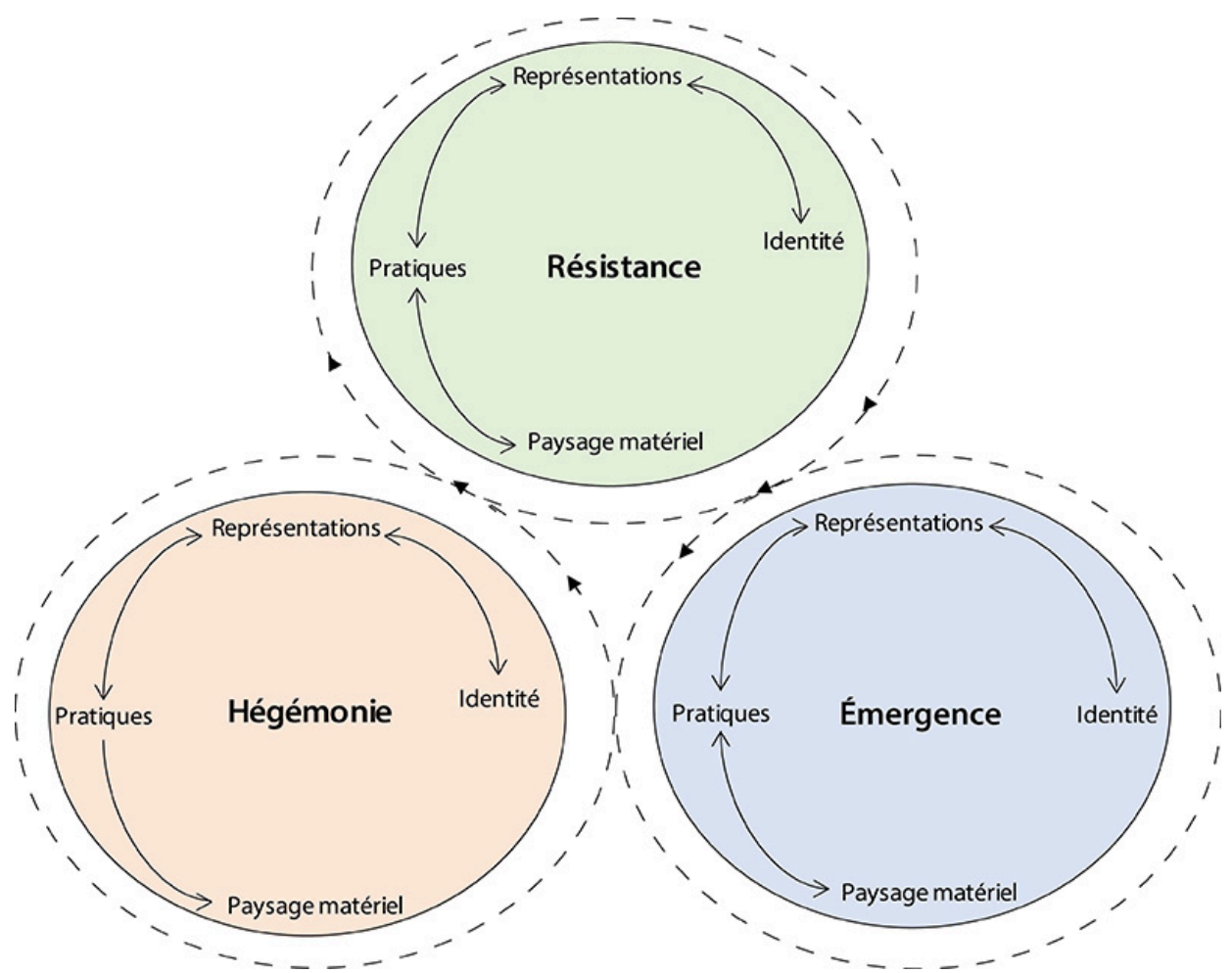

Figure 8.1. La politique d'identité de lieu (d'après Ruoso et Plant, 2018, et Ruoso, 2018). 
Dans ce chapitre, nous nous concentrerons sur la première dimension du modèle : l'hégémonie. Nous verrons comment les représentations dominantes du paysage sont reproduites à travers certaines pratiques de planification et influencent le développement du paysage et le rôle que l'agriculture y joue.

\section{Méthodologie : étude de cas}

Pour ce projet, nous avons adopté la méthodologie de l'étude de cas. Cette méthodologie nous est apparue comme pertinente, puisqu'elle nous permet d'étudier un phénomène dans un contexte réel (Yin, 2009) et d'explorer « la complexité d'une situation donnée » (Denscombe, 1998, p. 36), or notre but ici est d'explorer les formes que prend la politique liée à l'identité de lieu dans un contexte réel. Nous avons choisi comme zone d'étude la commune de Wollondilly (Wollondilly Shire), située dans le sud-ouest de l'aire métropolitaine de Sydney (figure 8.2). Une première raison de ce choix est que la commune de Wollondilly est impactée par la périurbanisation et les changements sociaux qu'elle engendre, mais qu'elle maintient une activité agricole relativement importante. En effet, l'agriculture dans cette commune représente $11 \%$ de la valeur agricole totale du bassin de Sydney (Macarthur Regional Organisation of Councils, 2016). L'activité agricole principale est l'élevage intensif de volaille (54,6\% de la valeur agricole de la commune), suivie du maraîchage $(20,1 \%)$, de l'élevage laitier $(7,2 \%)$, de la production d'œufs $(6,1 \%)$ et de la floriculture $(3,0 \%)$ (Macarthur Regional Organisation of Councils, 2016). D'autres productions plus marginales sont aussi présentes, telles que l'arboriculture, la culture de fruits à coque, la céréaliculture, la viticulture et la production de laine (Macarthur Regional Organisation of Councils, 2016). La deuxième raison du choix de la commune de Wollondilly est que la question du rôle de l'agriculture dans l'identité de Wollondilly est prise en compte dans les documents de planification de la commune. En effet, dans sa Stratégie de gestion de la croissance (Growth Management Strategy), Wollondilly se définit comme une commune caractérisée par son «Cadre de vie rural» (Rural Living) (Wollondilly Shire Council, 2011). L'idée de maintenir un mode de vie rural et de préserver les activités agricoles fait partie intégrante de la manière dont la commune se définit. Pour ces deux raisons, il nous a semblé pertinent de nous centrer sur cette commune.

\section{Méthodes}

Pour mener à bien notre recherche, nous avons analysé divers documents de planification à l'échelle locale : la Stratégie de gestion de la croissance de la commune de Wollondilly, qui définit les orientations de développement de la commune; le Plan local environnemental (Local Environmental Plan), qui détermine le zonage du sol à Wollondilly; ainsi que 24 propositions de rezonage de terrains, du rural vers le résidentiel, faites entre 2011 et 2016. Le processus de rezonage comporte plusieurs étapes décrites dans le tableau 8.1.

Durant l'analyse, la plupart des propositions de rezonage étudiées avaient été acceptées par le ministre de la Planification et de l'Environnement (à travers le processus de Gateway; voir définition dans le tableau 8.1), ce qui veut dire qu'elles avaient été acceptées par le gouvernement local, avaient fait l'objet d'une consultation initiale, et avaient ensuite été envoyées au ministère de la Planification et de l'Environnement. Les documents produits durant ces trois premières étapes font l'objet de notre analyse (Ruoso, 2018). Depuis notre analyse, le statut des propositions de rezonage étudiées a évolué (Wollondilly Shire Council, 2017) : neuf propositions ont été finalisées, treize sont dans les dernières phases (études supplémentaires, consultation publique, révisions), une proposition a été rejetée car des ressources minières ont été identifiées sur le terrain 
à rezoner, et une autre a été rétractée car les propriétaires n'avaient plus assez de fonds pour payer la continuation du projet.

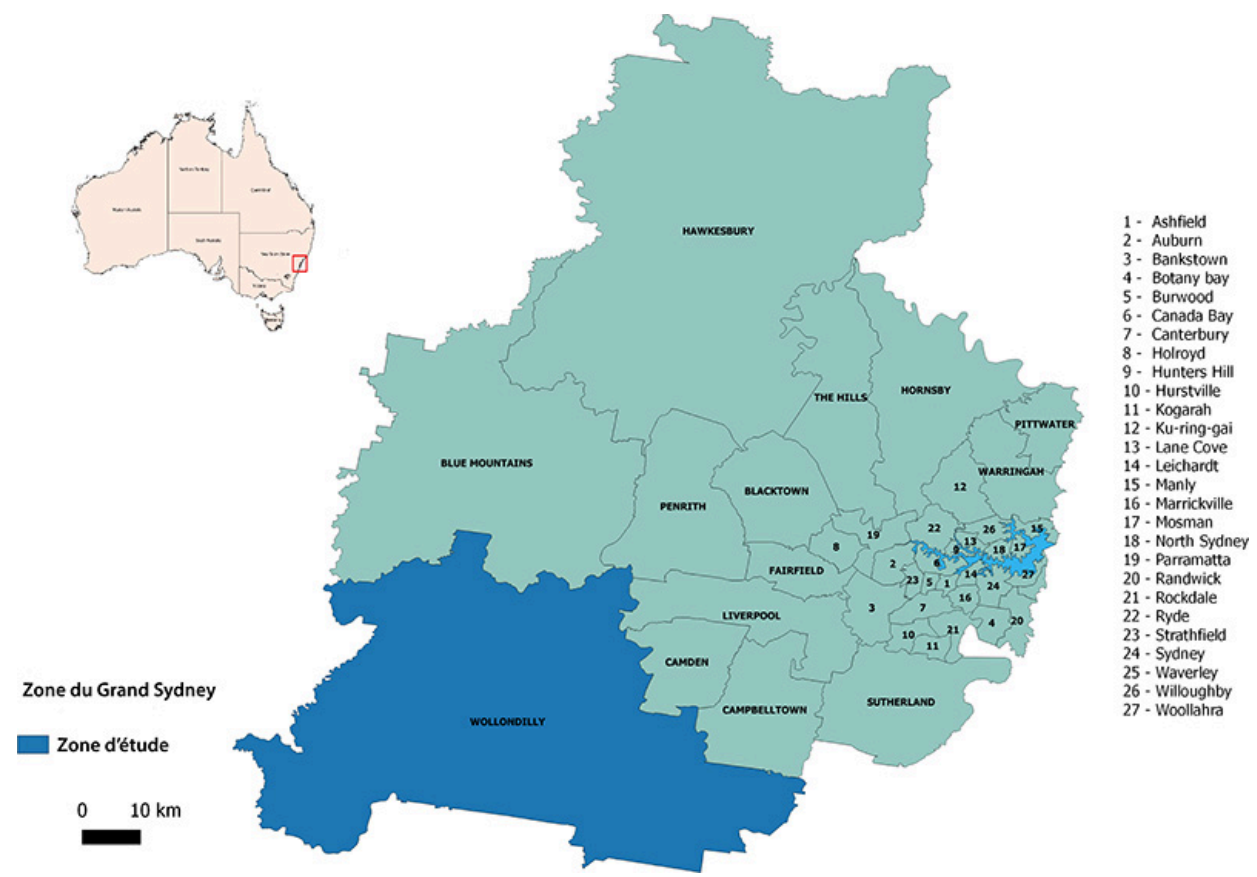

Figure 8.2. Localisation de la zone d'étude (d'après Ruoso et Plant, 2018).

La carte de la zone du Grand Sydney a été développée à partir des limites administratives produites par PSMA Australia Limited, agréée par le Commonwealth d'Australie et distribuée sous la licence Creative Commons Attribution 4.0 International licence - CC BY 4.0. La source pour la carte de l'Australie est Natural Earth.

Nous avons choisi d'analyser les documents d'aménagement parce qu'ils donnent des indications sur « les luttes de pouvoir entre différents intérêts » et sur la manière dont « certaines pensées, idées, connaissances et pratiques sont acceptées, marginalisées ou passées sous silence » (Richardson, 2000, p. 55). Ils permettent donc de comprendre quelles représentations d'un phénomène sont privilégiées par rapport à d'autres. Nous nous concentrons plus précisément sur les documents traitant des questions de zonage, d'une part parce que le zonage reste, à ce jour, le principal instrument de régulation du développement résidentiel en Australie (Sinclair et Bunker, 2007), et d'autre part parce que le zonage peut être considéré comme « des prescriptions normatives pour décider de la manière dont un morceau de terre peut être utilisé, qui peut y être présent, et à quoi il devrait ressembler» (Trudeau, 2006, p. 422). Analyser les processus de zonage nous permet donc de mieux comprendre les pratiques qui sont favorisées et celles qui sont découragées. Cela nous donne donc des indications sur le rôle attribué à l'agriculture dans la commune de Wollondilly. En plus de l'analyse de ces documents, nous avons conduit des entretiens avec des aménageurs, des gestionnaires et des agriculteurs, qui nous ont permis de voir émerger des discours concurrents sur ce qu'est le périurbain et sur le type de développement qu'il devrait connaître. Les entretiens ne seront pas au centre des résultats présentés ici, mais viendront éclairer notre analyse de documents lorsque cela sera nécessaire. Cette analyse avait deux buts principaux : d'une part, 
comprendre la conceptualisation du développement (spatial) périurbain promue dans les documents d'aménagement, et d'autre part, voir comment cette conceptualisation est contestée ou renforcée dans les pratiques d'aménagement, telles que les propositions de rezonage.

Tableau 8.1. Le processus de rezonage (d'après Ruoso, 2018).

\begin{tabular}{|l|l|}
\hline \multicolumn{1}{|c|}{ Étapes du rezonage } & \multicolumn{1}{c|}{ Description } \\
\hline $\begin{array}{l}\text { La proposition de } \\
\text { rezonage }\end{array}$ & $\begin{array}{l}\text { Une proposition est développée par la commune, sur la base de la proposition } \\
\text { initiale faite par les propriétaires. Chaque proposition doit prendre en compte } \\
\text { différents paramètres, tels que leur cohérence avec les plans locaux d'urbanisme } \\
\text { (Stratégie de gestion de la croissance) et les stratégies métropolitaines et } \\
\text { sous-régionales, mais aussi avec diverses directives ministérielles. }\end{array}$ \\
\hline $\begin{array}{l}\text { La consultation initiale } \\
\text { spécificité de la } \\
\text { commune de } \\
\text { Wollondilly) }\end{array}$ & $\begin{array}{l}\text { À Wollondilly, la commune organise un premier processus de consultation, } \\
\text { durant lequel les habitants peuvent contester ou exprimer leur soutien au projet. }\end{array}$ \\
\hline Le Gateway & $\begin{array}{l}\text { Le ministre de la Planification et de l'Environnement (ou un délégué) approuve } \\
\text { ou rejette la proposition de rezonage (Gateway) et établit les conditions de son } \\
\text { approbation (par exemple, des études additionnelles doivent être faites, des } \\
\text { agences gouvernementales doivent être consultées, etc.). }\end{array}$ \\
\hline La consultation publique & $\begin{array}{l}\text { Une consultation publique est organisée durant laquelle les habitants peuvent } \\
\text { contester le projet ou exprimer leur soutien, et demander une audition publique. }\end{array}$ \\
\hline L'évaluation & $\begin{array}{l}\text { Les contestations ou marques de soutien écrites des habitants sont passées en } \\
\text { revue, et le conseiller parlementaire prépare le Plan local environnemental. }\end{array}$ \\
\hline $\begin{array}{l}\text { La modification du Plan } \\
\text { local environnemental }\end{array}$ & \begin{tabular}{l} 
Après l'approbation du ministre, le Plan local environnemental est publié. \\
\hline
\end{tabular} \\
\hline
\end{tabular}

Pour la Stratégie de gestion de la croissance et le Plan local environnemental, nous avons procédé à une analyse de contenu basée sur une lecture et une annotation systématique de ces deux documents, qui nous a permis d'identifier et d'analyser les parties de texte pertinentes. En ce qui concerne les propositions de rezonage et les commentaires soumis par certains habitants au gouvernement local, nous avons créé un fichier Excel regroupant les différents thèmes qui nous intéressaient : comment les terres à rezoner étaient décrites dans les propositions; la manière dont la cohérence entre la proposition et différents textes d'aménagement au niveau local et métropolitain était présentée; les différents commentaires faits par les habitants sur le rezonage ; la manière dont le département d'État chargé d'approuver les demandes de rezonage évaluait chaque demande.

Deux discours dominants sur le rôle des terres et activités agricoles ont été identifiés dans la commune de Wollondilly. Un premier, défendu dans les documents d'aménagement de la commune, promeut une vision de la ruralité basée sur la séparation entre la ville et la campagne, alors que le second discours, défendu par certains habitants de la commune, promeut une « hybridation » du paysage rural, à travers le développement de logements résidentiels à faible densité au sein du paysage rural. Dans ce chapitre, nous nous concentrerons exclusivement sur le premier discours. Une présentation du second discours dominant peut être trouvée ailleurs (Ruoso, 2018). 


\section{La marginalisation des terres agricoles dans les processus de planification en milieu périurbain}

La Stratégie de gestion de la croissance de Wollondilly est un document de planification locale qui fixe les objectifs de la commune en matière de développement (Wollondilly Shire Council, 2011). La question de la gestion de la croissance urbaine y est présentée comme l'un des enjeux fondamentaux pour la commune, qui programme de construire 7500 nouveaux logements dans les vingt ou vingt-cinq prochaines années (Wollondilly Shire Council, 2011). Toujours dans ce même document, cette question apparaît intrinsèquement liée à d'autres questions, telles que l'environnement, l'économie, les infrastructures, etc., mais également l'identité et le caractère de la commune, qui y sont définis comme offrant un «Cadre de vie rural» (Rural Living) (Wollondilly Shire Council, 2011).

\section{Identité : genius loci et représentations}

Ce concept de «Cadre de vie rural » est essentiel, car il définit la manière dont le développement urbain devrait se faire dans la commune : «Toutes les propositions d'occupation du sol doivent être compatibles avec le concept et la vision de Cadre de vie rural. » (Wollondilly Shire Council, 2011, p. 9.) Le concept de « Cadre de vie rural » est traduit en neuf caractéristiques, dont trois définissent le type de développement urbain jugé souhaitable dans la commune, et une le rôle qui doit être attribué à l'agriculture. Les trois caractéristiques se concentrant sur le développement spatial de la commune insistent sur la nécessité de maintenir une délimitation claire (et visuellement harmonieuse) entre les villes et villages d'un côté et les espaces agricoles et naturels de l'autre. Une autre caractéristique de ce «Cadre de vie rural» est la volonté de maintenir de terres et d'usages agricoles dans la commune. L'agriculture est définie comme une activité appartenant au paysage culturel et à l'économie de la commune. Cependant, lorsque l'on regarde les mesures prises pour mettre en place ces éléments du «Cadre de vie rural », des mesures précises sont préconisées pour le développement urbain : le développement d'habitations à proximité des villes et des villages, l'identification de zones de croissance et des densités plus élevées à proximité des villes et des villages. À l'inverse, les deux mesures prises pour protéger les espaces agricoles restent relativement vagues. La première explique que les terres ayant une valeur économique, environnementale ou culturelle spécifique devraient être protégées, sans expliquer comment ces valeurs seront identifiées. La seconde explique que les terres agricoles seront protégées en empêchant le mitage qui contribue à la fragmentation de l'espace rural. En d'autres termes, il est considéré que les terres agricoles seront protégées par le simple fait de limiter le développement urbain aux zones situées à proximité des villes et des villages.

Le concept de «Cadre de vie rural » définit donc l'identité de la commune, identité qui semble être bâtie sur le concept de genius loci présenté plus tôt. La commune est en effet définie comme ayant des caractéristiques physiques spécifiques (la séparation entre la ville et la campagne) qui doivent être maintenues afin de continuer à offrir un « Cadre de vie rural ». Cette identité est supportée par une représentation de la commune selon laquelle la commune serait capable d'accueillir un développement urbain contrôlé tout en protégeant les terres agricoles, et ce grâce à un modèle de développement spatial basé sur le maintien d'une distinction entre ville et campagne.

Dans la prochaine section, nous verrons comment l'identité promue par la commune à travers une représentation de cette dernière comme pouvant accueillir de nouvelles habitations tout en protégeant son agriculture, est utilisée par des propriétaires voulant 
rezoner leur terrain du rural vers le résidentiel, pour promouvoir le développement urbain aux dépens de la préservation des terres agricoles. Nous verrons ensuite que l'une des raisons pouvant expliquer ceci est la domination des projections du besoin en logements dans les processus de décision en matière d'aménagement du territoire. Une autre raison tient au fait que les terres agricoles soient souvent considérées comme non viables, sur la base d'informations dont la validité semble discutable.

\section{Les pratiques de planification}

Dans les 24 propositions de rezonage examinées, l'impératif de maintenir une distinction (harmonieuse) entre ville et campagne, en ne développant des habitations qu'autour des villes et villages, est utilisé par les propriétaires pour légitimer le rezonage de leurs terres et plus généralement le développement urbain dans la commune.

Le premier argument utilisé dans la plupart des propositions de rezonage est que les terrains à rezoner sont situés à proximité d'une ville ou d'un village existant. De ce fait, construire des habitations sur ces terres permettrait de maintenir une démarcation claire entre ville et campagne. Beaucoup de propositions expliquent donc que le développement d'habitations se fera " près d'une ville existante » ou « dans la continuité de développements résidentiels ». D'autres affirment que le développement sera « en adéquation avec les usages [résidentiels] avoisinants » et favorisera la création « d'une aire urbaine consolidée » qui « ne contribue[ra] pas à la croissance dispersée de la population ». Étant donné que ces terres sont situées en périphérie des villes et villages, il est bien évident que leur utilisation pour le développement d'habitations ne serait en adéquation qu'avec une partie des terres avoisinantes, puisque d'autres terres situées à proximité sont des terres agricoles ou des espaces naturels. Ce type de raisonnement reflète le point de vue « urbano-centré » qui domine ces propositions : à partir du moment où une terre est située à proximité d'un village, il est considéré comme approprié de la développer. Cela pourrait mener à long terme à un développement urbain continu au détriment des terres agricoles, étant donné qu'au fur et à mesure que des terres sont urbanisées, de nouvelles terres agricoles se trouvent à proximité de terres urbanisées. Ceci est illustré de manière particulièrement flagrante par le fait que plusieurs propriétaires ont proposé le rezonage de terres situées à proximité de terrains dont l'urbanisation était programmée mais non commencée ou terminée : «Le site ne sera pas un développement résidentiel isolé quand le développement sur les terres situées à l'ouest sera terminé. » Ici, la légitimité du rezonage émane de la proximité des terres à rezoner avec des terres qui seront développées dans le futur.

Le second argument souvent invoqué par les propriétaires pour légitimer le rezonage d'une terre est que le développement résidentiel de leur terre marquera la limite de la ville en créant une démarcation nette ou une zone de transition entre les villes et villages et les terres agricoles ou espaces naturels. Trois des propositions étudiées expliquent que le développement résidentiel du terrain à rezoner permettra la création d'une limite claire entre la ville et les espaces environnants. Par exemple, une des propositions indique qu'à « l'ouest, le site est limité par des terres plus hautes qui descendent ensuite vers la zone de captage d'eau potable de Sydney [Sydney drinking water catchment] et au sud et à l'est, par des routes qui joueront le rôle de séparation physique entre l'urbain et le rural ». Une autre souligne que le site « est contraint géographiquement à l'est par la rivière Nepean qui constituerait ainsi une barrière physique à l'étalement urbain ». Ces propositions tentent donc de démontrer que les développements résidentiels qu'elles proposent viendront renforcer des limites naturelles (rivière, topographie) ou artificielles (route) déjà existantes, et créeront, de ce fait, une limite « naturelle » au développement urbain. 
Dans d'autres propositions, le développement résidentiel des terres à rezoner est présenté au contraire comme un moyen de créer une transition entre les villes et villages (zonage résidentiel) et les espaces agricoles ou naturels (zonage rural ou environnemental). Par exemple, il est écrit dans deux propositions que la faible densité des résidences proposées créera une transition entre des zones résidentielles plus denses, parfois décrites comme des zones de «banlieue », et des espaces agricoles ou naturels. La transition que pourrait offrir le développement urbain peu dense de ces terrains y est qualifiée de différentes manières. Elle est considérée comme « une transition visuelle appropriée à la limite de la ville », « une limite logique du point de vue visuel » ou encore « une zone tampon transitionnelle nécessaire ». Le vocabulaire choisi n'est pas anodin : en définissant le développement résidentiel comme « approprié », « logique » et « nécessaire », il contribue à l'essentialiser et le légitimer dans le paysage périurbain. De ce fait, le développement résidentiel est présenté non seulement comme la meilleure, mais aussi l'unique solution.

Le dernier argument souvent utilisé pour justifier le rezonage porte sur la manière dont le développement résidentiel sera harmonieusement intégré dans le paysage. Un développement est généralement considéré comme harmonieux dans les propositions de rezonage lorsqu'il est similaire à un développement déjà existant en termes de zonage et de densité. Il est alors considéré comme en adéquation avec « le caractère existant», « l'atmosphère » de la ville ou du village, " l'interface urbain-rural », le « caractère résidentiel avoisinant» ou le « cadre rural ». À nouveau, la cohérence de l'aménagement à venir avec l'atmosphère existante est souvent considérée comme un argument faisant de cet aménagement une « progression logique».

Dans cette section, nous avons vu comment l'idée de maintenir une distinction (harmonieuse) entre ville et campagne, pour permettre le développement urbain tout en protégeant les terres agricoles, est utilisée, en pratique, pour justifier le développement d'habitations à Wollondilly. Cela se traduit dans le paysage matériel par l'extension du résidentiel sur les terres agricoles se trouvant à proximité des villages de la commune. À long terme, cela pourrait mener à un développement continu du résidentiel, car de nouvelles terres agricoles se trouveront, sans cesse, à proximité des villes et villages. Dans la section suivante, nous montrerons comment, à l'inverse du développement résidentiel, les terres agricoles sont souvent présentées comme illégitimes dans le paysage périurbain.

\section{Les terres agricoles}

Plus de la moitié des propositions analysées font mention de la perte de terres agricoles engendrées par le rezonage de leurs terres de la catégorie "rural » vers la catégorie « résidentiel ». Les raisons invoquées pour justifier la perte de ces terres sont qu'elles ne permettent pas le maintien d'une agriculture viable parce qu'elles sont trop petites, que leur valeur agricole est limitée, ou qu'un usage agricole de ces terres créerait (ou crée déjà) des conflits. De plus, dans beaucoup de propositions, il est expliqué que ces terres agricoles sont sous-utilisées et devraient donc être rezonées.

Dans cinq des propositions analysées, les terres à rezoner sont présentées comme n'étant pas viables pour l'agriculture car trop petites. La taille des parcelles considérées va de 5 à 111 hectares. Dans cinq autres propositions, les terres sont considérées comme non viables du fait de leur mauvaise qualité. Pour justifier ceci, il est souvent fait référence à une classification du potentiel agronomique des sols (agricultural land capability) faite par le Département de l'industrie primaire (Hulme et al., 2002). Les terres à rezoner sont 
des terres de classe 3,3 et 4 ou 4 et $5^{30}$. Leur productivité serait modérée ou faible et il serait donc impossible d'y pratiquer une agriculture intensive. Les arguments avancés dans ces propositions restent toutefois discutables. En effet, lorsque l'on considère la taille des exploitations, une étude de Malcolm et Fahd (2009) sur le maraîchage dans la région de Sydney révèle que la taille moyenne des exploitations maraîchères (plein champ, sous serre ou hydroponiques) est de 1,9 hectare. Il ne semble donc pas inutile d'envisager d'engager un débat sur la taille minimale d'une exploitation agricole. En ce qui concerne la question de la productivité, lorsque l'on consulte la carte du potentiel agronomique des sols pour Wollondilly, nous pouvons voir que beaucoup des terres considérées comme rurales (RU1, RU2, et RU4 ${ }^{31}$ ) appartiennent pourtant aux classes 3, 4 ou 5 (figure 8.3). De plus, la commune de Wollondilly est un lieu de production alimentaire relativement important depuis l'établissement de la première colonie, et contribue à hauteur de $11 \%$ à la production agricole du bassin de Sydney (Macarthur Regional Organisation of Councils, 2016). Il est donc permis de penser que le fait de ne prendre en considération que cette carte du potentiel agronomique des sols pour juger si une terre peut ou non être utilisée pour l'agriculture est probablement peu pertinent et insuffisant. Docking et Sreekumar (2008) abondent dans ce sens lorsqu'ils expliquent que beaucoup des exploitations agricoles dans le bassin de Sydney sont situées sur des terres de classe 3 , et non de classes 1 ou 2 .

Un autre argument, utilisé dans dix propositions, pour délégitimer l'utilisation agricole des terres à rezoner est que les pratiques agricoles sur ces terres auraient des effets négatifs sur les habitations avoisinantes. Dans quatre propositions, il est expliqué qu'un usage agricole de ces terres est difficilement envisageable car elles se situent à proximité de zones résidentielles, ce qui pourrait mener à des «conflits d'usage rural/urbain typiques ». Pour cinq propositions, le terrain à rezoner est décrit comme ayant un « potentiel agricole limité » ou un « potentiel limité pour d'autres usages ruraux », sans qu'aucun argument lié à la taille, à la productivité ou au potentiel conflictuel d'un usage agricole soit avancé.

Enfin, un dernier argument fréquemment invoqué pour justifier le rezonage est celui de la sous-utilisation des terres. Dans plusieurs propositions, les terres concernées sont qualifiées de «parcelle[s] vide[s]», et sont décrites comme étant utilisées de facto pour du résidentiel, "pour de l'agriculture passive », ou encore comme accueillant une activité agricole minimale « à faible intensité » ou « limitée ». D'autres propositions développent des argumentaires sur le fait que le rezonage de ces terres sous-utilisées n'aurait pas d'impact sur la sécurité alimentaire. Une de ces propositions explique que $38 \%$ des terres de la commune étant inutilisées, rezoner une terre serait par conséquent sans impact. Une autre proposition avance que la terre à rezoner aurait pu être utilisée pour de l'agriculture mais ne l'a pas été et peut donc être rezonée. Une terre qui n'est pas utilisée pour un usage agricole commercial est donc souvent considérée comme pouvant être rezonée.

30. Les terres de classe 3 sont définies comme des terres non propices à la cultivation continue et qui doivent être mises en pâture, faire l'objet de travail de conservation du sol et/ou être mises en jachère. Les terres de classe 4 sont définies comme des terres propices au pâturage et non à la culture. Leur niveau de production est comparativement faible, du fait de contraintes environnementales majeures. Finalement, les terres de classe 5 sont définies comme des terres n'étant pas propices à l'usage agricole, ou seulement à du pâturage léger. Leur niveau de production agricole est faible (ou nul) du fait de contraintes environnementales majeures.

31. RU1 : production primaire; RU2: paysage rural; RU4: production primaire sur petites parcelles. 


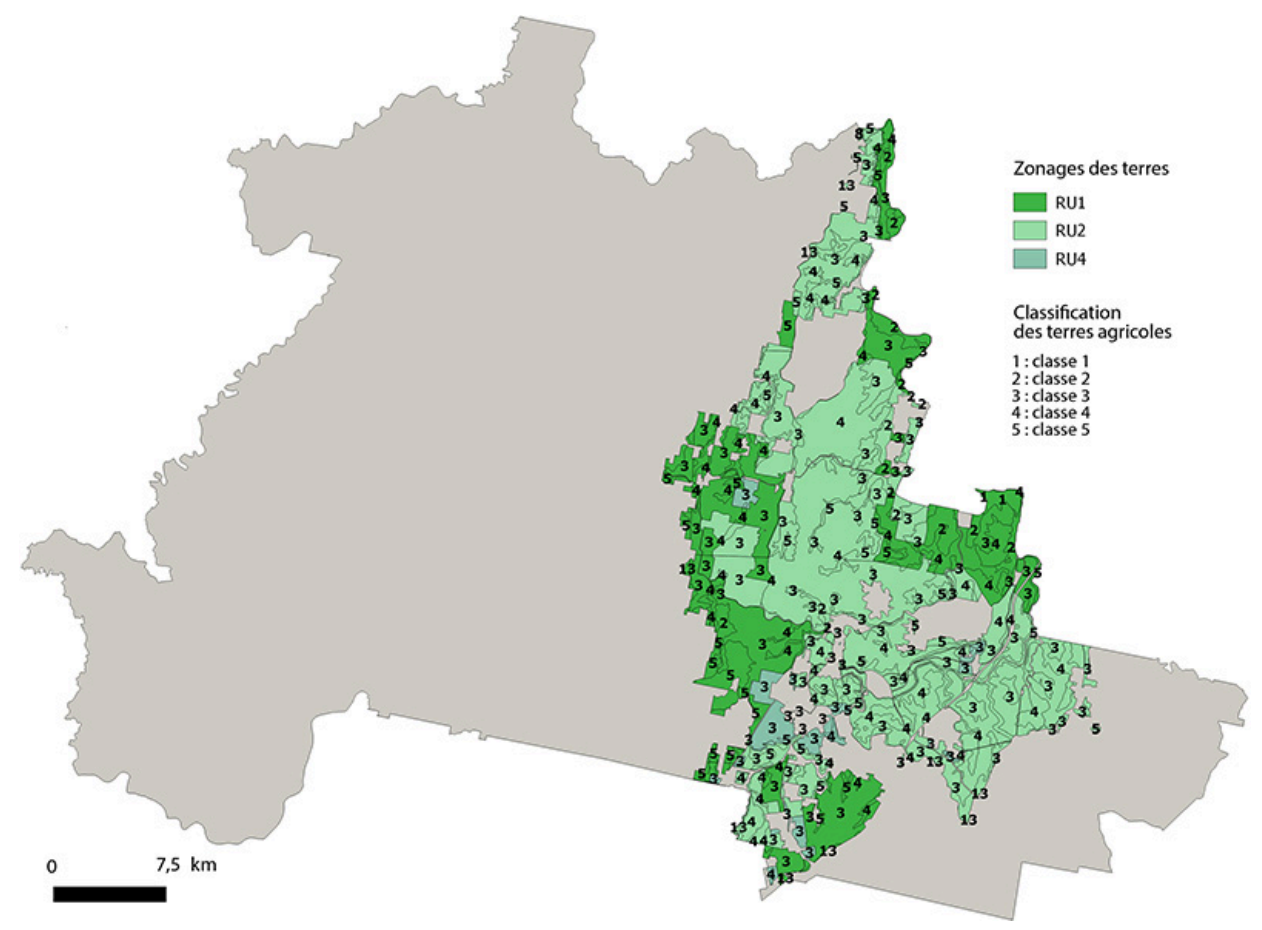

Figure 8.3. Potentiel agronomique des terres dans les zones rurales de la commune (RU1, RU2 et RU4) de Wollondilly (Ruoso, 2018).

Cette carte a été développée à partir du zonage (LZN) produit par le gouvernement de la Nouvelle-Galles du Sud et le Département de l'aménagement et de l'environnement et à partir des données sur la classification des terres agricoles de la Nouvelle-Galles du Sud produites par le Département des industries primaires.

Dans cette section, nous avons vu que les terres agricoles sont décrites comme non viables, non productives, sous-utilisées ou encore comme sources de conflits, tendant ainsi à faire de l'agriculture une activité illégitime dans la commune. Si nous appliquons notre modèle de l'identité de lieu à la situation décrite ici (figure 8.4), nous pouvons voir que l'idée de «Cadre de vie rural » qui constitue l'identité de la commune (en bleu) est basée sur une représentation de la capacité de la commune à accueillir du développement résidentiel tout en protégeant ses terres agricoles (en vert). Nous voyons aussi que cette représentation de la commune n'est utilisée que de manière partielle (flèche rouge en pointillés) dans les pratiques (propositions de rezonage), puisque ces dernières l'utilisent pour justifier le développement résidentiel, tout en remettant en cause les usages agricoles. Cela se traduit dans le paysage matériel par un développement résidentiel rapide en périphérie des villes et des villages de la commune (en gris).

Dans la section suivante, nous allons montrer comment le recours aux prévisions du besoin en logements constitue un trait dominant de la planification à l'échelle métropolitaine et locale, qui influence le processus de décision en ce qui concerne les propositions de rezonage. La domination des prévisions du besoin en logement dans la planification a déjà été observée ailleurs (Murdoch, 2000). 


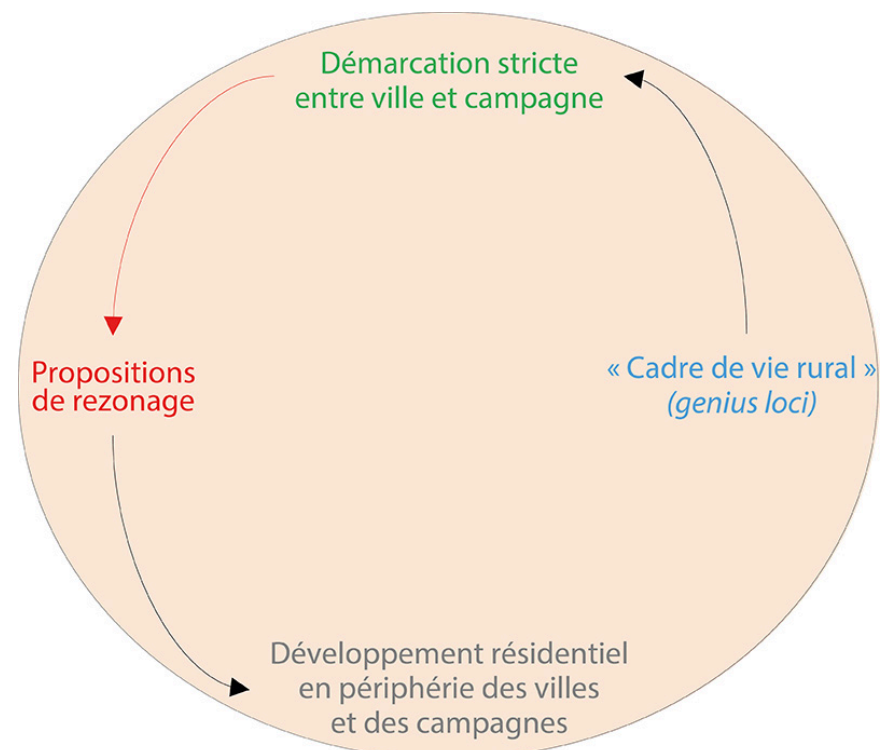

Figure 8.4. Description de l'identité de lieu hégémonique dans la commune de Wollondilly (d'après Ruoso, 2018).

\section{Les projections du besoin en logements}

Dans les documents de planification qui définissent les orientations d'aménagement pour la métropole de Sydney (stratégies métropolitaines), la nécessité de créer des habitations et des emplois pour accroître le rayonnement de Sydney comme «Ville globale» figure souvent comme une priorité, et fait l'objet d'études chiffrées. Par exemple, la stratégie Un plan pour Sydney 2036 ( A plan for Sydney 2036), publiée en 2011, préconise la construction de 770000 maisons d'ici 2036 (Department of Planning and Environment, 2010). Le rapport de la stratégie suivante publiée en 2015, Un plan pour la croissance de Sydney (A plan for Growing Sydney), chiffre à 664000 le nombre de nouvelles maisons à construire d'ici 2034 (Department of Planning and Environment, 2015). D'après un aménageur que nous avons interviewé, « les données sur la population sont un facteur clé pour comprendre et planifier la ville », confirmant ainsi l'importance de ces projections dans la planification de Sydney. D'autres objectifs peuvent toutefois être identifiés dans les stratégies métropolitaines, notamment celui du développement durable dans lequel pourrait s'inscrire la protection des terres agricoles périurbaines. Toutefois, les objectifs à atteindre concernant la durabilité ne sont pas clairement établis dans ces stratégies, et changent d'une stratégie à l'autre. Les mesures préconisées pour la protection des espaces agricoles périurbains ne sont pas cohérentes d'une stratégie à l'autre, et la seule que l'on retrouve dans plusieurs des stratégies les plus récentes se limite à contenir l'empreinte urbaine pour protéger les terres agricoles.

La priorité donnée aux projections du besoin en habitations dans la planification à l'échelle métropolitaine se retrouve à l'échelle locale. Le document de Stratégie de gestion de la croissance de Wollondilly établit à 7500 le nombre de maisons à construire à l'horizon 2035. À l'inverse, lorsqu'il s'agit de la protection des terres agricoles, aucun objectif de planification précis n'est établi, il est seulement fait référence aux mesures existantes (principalement le zonage) pour assurer la protection des terres agricoles de Wollondilly. Des mesures additionnelles concernant l'agriculture sont développées, mais 
leur objectif est souvent de promouvoir l'agriculture dans la commune et d'attirer de nouveaux agriculteurs, plutôt que d'influencer le processus de planification. La seule mesure qui pourrait avoir un impact sur ce processus et permettre le maintien de terres agricoles dans la commune est la création de parcs d'agrobusiness et de clusters pour l'industrie agricole, mais ces actions n'ont pas encore été mises en place. Un aménageur témoigne de l'absence de planification pour les zones agricoles de Wollondilly en expliquant que la Stratégie de gestion de la croissance identifie clairement les zones de développement urbain et ne définit les zones rurales que "par défaut», comme celles restantes.

L'analyse du processus de Gateway ${ }^{32}$, et plus particulièrement de la manière dont le Département de l'aménagement et de l'environnement (DAE) de la Nouvelle-Galles du Sud détermine l'impact d'un rezonage sur les terres agricoles durant le processus du Gateway, montre que le développement de logements additionnels est souvent considéré comme justifiant la perte de terres agricoles. Elle montre aussi que lorsque le DAE considère l'agriculture, il se base sur les informations fournies dans les propositions de rezonage. Or, la validité de ces informations semble, comme nous l'avons vu, discutable. Pour illustrer ce point, nous nous concentrerons sur la manière dont le DAE évalue la cohérence des propositions de rezonage avec la section 117 de la directive 1.2 relative aux zones rurales de la loi sur la planification et l'évaluation environnementale de 1979. Cette directive a pour but de protéger « la valeur productive des terres rurales » et doit être prise en compte avant tout rezonage. Pour cela, le DAE doit s'assurer que la proposition de rezonage «(a) ne reclassifie pas une terre rurale pour en faire des zones résidentielles, commerciales, industrielles, des villages ou des zones touristiques ; (b) ne contienne pas des clauses permettant d'augmenter la densité autorisée dans les zones rurales $[\ldots] »$. Théoriquement, il serait donc impossible de rezoner des terres rurales pour des usages résidentiels. Cependant, la directive prévoit que cela puisse avoir lieu sous certaines conditions : si cela est justifié par une Stratégie ; si une étude a été préparée dans la proposition de rezonage qui prend en considération les objectifs de cette directive ; si la proposition est en accord avec une stratégie régionale ou sous-régionale préparée par le Département qui prend en compte les objectifs de cette directive ; ou si le rezonage est d'importance mineure.

Dans presque la moitié des cas, le DEA se base sur les projections en besoins d'habitations pour justifier le manque de cohérence entre une proposition de rezonage et la section 117 de la directive 1.2 relative aux zones rurales. En effet, il est souvent expliqué que l'incohérence entre le rezonage et cette directive se justifie par le fait que le brouillon de la Stratégie sous-régionale pour le Sud-Ouest (Draft South West Sub-Regional Strategy) ${ }^{33}$ stipule que 5230 maisons devront être construites à Wollondilly d'ici 2031, dont 4000 sur des terrains non construits. Le deuxième argument auquel il est souvent fait appel est que le rezonage est d'importance mineure, sans que le terme «importance mineure » ne soit défini. Enfin, le DEA reprend souvent les arguments déjà avancés dans les propositions de rezonage, tels que la faible probabilité d'un usage agricole futur des terres, les conflits potentiels qui émergeraient d'un usage agricole, le faible potentiel agricole des terres, mais aussi la cohérence du développement avec les usages résidentiels avoisinants.

32. Le ministre de la Planification et de l'Environnement (ou un délégué) approuve ou rejette la proposition de rezonage (Gateway) et établit les conditions de son approbation (par exemple, des études additionnelles doivent être faites, des agences gouvernementales doivent être consultées etc.). 33. Cette stratégie vient compléter la stratégie métropolitaine en établissant des objectifs et priorités spécifiques pour la partie sud-ouest de Sydney. 
Nous pouvons voir ici que le DEA base souvent ses décisions concernant le rezonage de terres agricoles sur les projections du besoin en logements, et reprend les arguments présentés dans les propositions de rezonage sur la viabilité des terres agricoles, sans apporter de nouveaux éléments ou informations sur les caractéristiques des terres agricoles. Ce point est illustré par un aménageur interviewé, qui explique que «le gouvernement s'est toujours concentré sur l'emploi et le logement » et que peu d'informations sont disponibles en ce qui concerne la valeur des terres agricoles. D'autres agences gouvernementales qui pourraient disposer de plus d'expertise sur la valeur agricole des terres, comme par exemple le Département des industries primaires, sont rarement consultées durant ce processus de décision, ou le sont une fois que la proposition a été acceptée, pour donner des conseils sur des questions spécifiques, comme les conflits potentiels qui pourraient émerger entre l'usage résidentiel à venir et les exploitations agricoles environnantes.

Nous venons de voir ici que la marginalisation des terres agricoles dans les pratiques de planification, et plus particulièrement dans les propositions de rezonage, est rendue possible par la domination des projections du besoin en habitations dans les processus de décision et l'utilisation d'informations sur la viabilité agricole des terres à rezoner, dont la validité est discutable. Si nous reprenons notre modèle (figure 8.5) de l'identité de lieu, nous pouvons voir que la domination des projections du besoin en logements dans les processus de décision et le manque d'informations pertinentes relatives à l'agriculture contribuent à expliquer comment cette représentation inclusive de la commune (intégration du développement résidentiel et de la protection des terres agricoles) ne se traduit pas dans les pratiques de planification (propositions de rezonage).

\section{Discussion et conclusion}

L'utilisation de notre modèle sur les politiques de l'identité de lieu nous a permis d'identifier comment la représentation de la commune de Wollondilly développée dans la Stratégie de gestion de la croissance est instrumentalisée dans les pratiques (propositions de rezonage) pour promouvoir, dans le paysage matériel, le développement du résidentiel et la marginalisation des terres agricoles. Dans notre présentation du cadre théorique, nous avons vu comment la relation entre les quatre dimensions du modèle — dimension matérielle, pratiques, représentations et identité — était bidirectionnelle : l'identité et les représentations peuvent influencer les pratiques et vice versa. Ici, nous nous trouvons face à une situation hybride : l'identité promue, basée sur le genius loci et sur une représentation du périurbain qui maintient une séparation entre les villes et la campagne, ne se traduit pas complètement dans les pratiques de planification. Dans ces pratiques, la séparation entre villes et campagnes est utilisée comme un argument pour promouvoir le développement de logements, tout en présentant les terres agricoles comme non viables et comme des nuisances. Nous avons tenté ici d'expliquer pourquoi cette représentation promue par la commune ne se matérialisait pas dans les pratiques de planification. Il nous semble que dans le contexte de Sydney, la domination des projections du besoin en habitations dans les processus de planification — qui a déjà été observée ailleurs (Murdoch, 2000) — et l'utilisation d'informations dont la validité est discutable concernant les terres agricoles sont un élément de réponse. 


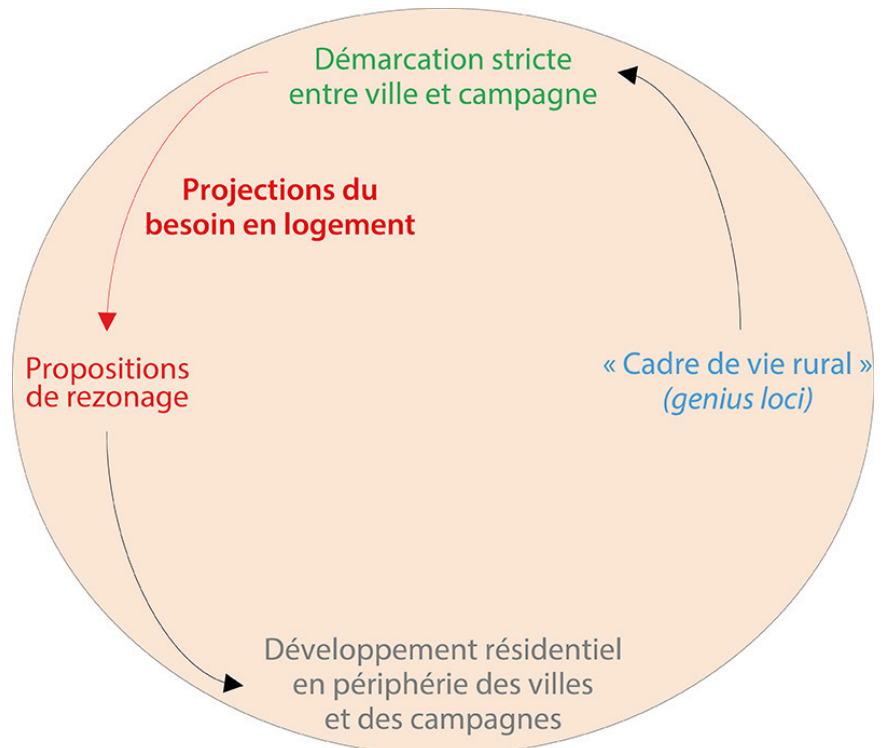

Figure 8.5. Le rôle des projections du besoin en logement dans les processus de décision concernant le rezonage (d'après Ruoso, 2018).

Dans le cas de Wollondilly, maintenir une séparation entre villes et campagnes ne semble donc pas mener à une protection efficace des terres agricoles périurbaines. Ce constat est corroboré par de nombreuses études qui ont montré que la création d'une séparation entre ville et campagne était inefficace pour protéger l'agriculture (Gant et al., 2011 ; Memon, 2003 ; Murdoch et Lowe, 2003 ; Paül et Tonts, 2005), notamment parce qu'une telle séparation crée une dichotomie artificielle entre ville et campagne qui est toujours transgressée (Murdoch et Lowe, 2003). Certains auteurs défendent l'idée que créer un mélange contrôlé de développements résidentiels et de terres à usage agricole pourrait potentiellement être plus efficace (Yokohari et al., 2000). Cependant, pour créer un paysage qui mélangerait développements résidentiels et espaces agricoles, il est nécessaire de redéfinir ce que sont les paysages agricoles de la commune de Wollondilly. En effet, nous avons vu dans ce chapitre que les terres agricoles de la commune sont souvent définies comme non viables, sur la base de leur taille, de leur potentiel agronomique ou des conflits potentiels qu'une activité agricole engendrerait. Or nous avons vu que la taille moyenne des exploitations dans le bassin de Sydney était moindre que celle des terres à rezoner, et que la carte du potentiel agronomique des sols utilisée pour définir la productivité des terres agricoles n'était pas nécessairement pertinente dans le bassin de Sydney. Il nous semble donc nécessaire, pour rendre possible ce mélange d'usages résidentiels et d'usages agricoles, de co-créer, avec les acteurs de la commune (planificateurs, agriculteurs, résidents) et des membres de la communauté scientifique (agronomes, pédologues, économistes), de l'information cartographique sur la viabilité des terres qui soit reconnue comme légitime par tous, et qui puisse être utilisée pour légitimer le maintien de certaines terres agricoles dans le bassin de Sydney.

Enfin, en ce qui concerne les conflits générés par l'activité agricole, plusieurs recherches ont démontré que l'existence de capital social entre agriculteurs et résidents pouvait mener à une réduction des conflits (Owen et al., 2000 ; Kelsey et Vaserstein, 2000 ; Sharp et Smith, 2003). Des agriculteurs interviewés ont expliqué qu'ils avaient mis en place un certain nombre de stratégies, qui ne peuvent pas être présentées en détail ici, pour 
développer du capital social entre les résidents et eux. Le gouvernement local pourrait jouer un rôle dans le développement du capital social de la commune en organisant des événements rassemblant agriculteurs et résidents. Nous ne développerons pas ce point plus avant car il dépasse le propos de ce chapitre.

\section{Remerciements}

Je tiens à remercier tous les participants à cette étude, ainsi que le gouvernement local de Wollondilly pour son soutien lors de mon projet de recherche.

Ce chapitre est issu de ma thèse faite à l'université de technologie de Sydney entre 2014 et 2018 (The politics of place identity in peri-urban environments. What role for productive farming landscapes? A case study of Wollondilly Shire, NSW, Australia - University of Technology Sydney).

\section{Références bibliographiques}

Abrams J.B., Gosnell H., 2012. The politics of marginality in Wallowa County, Oregon: Contesting the production of landscapes of consumption. Journal of Rural Studies, 28, 30-37. Agricultural Reference Group, 2013. Response to the draft Metropolitan Strategy - Review. Alston M., 2004. Who is down on the farm? Social aspects of Australian agriculture in the 21st century. Agriculture and Human Values, 21, 37-46.

Argent N., Tonts M., Jones R., Holmes J., 2011. Amenity-led migration in rural Australia: a new driver of local demographic and environmental change? In: Demographic Change in Australia's Rural Landscapes: Implications for Society and the Environment (Luck G.W., Race D., Black R., eds), Dordrecht, Springer.

Attwater R., Derry C., 2017. Achieving resilience through water recycling in peri-urban agriculture. Water, 9, 223.

Australian Bureau of Statistics, 2012. 4102.0 - Australian Social Trends, dec. 2012.

Benson M., Jackson E., 2013. Place-making and place-maintenance: performativity, place and belonging among the middle classes. Sociology, 47, 793-809.

Breakwell G.M., 1986. Coping With Threatened Identities, London, Methuen.

Burton P., Lyons K., Richards C., Amati M., Rose N., Fours L.D., Pires V., Barclay R., 2013. Urban Food Security, Urban Resilience and Climate Change, Gold Coast, National Climate Change Adaptation Research Facility.

Cresswell T., 1996. In Place/Out of Place: Geography, Ideology, and Transgression, Minneapolis, University of Minnesota Press.

De Zeeuw H., Van Veenhuizen R., Dubbeling M., 2011. The role of urban agriculture in building resilient cities in developing countries. The journal of Agricultural Sciences, 149, 153-163.

Denscombe M., 1998. The Good Research Guide: For Small-Scale Social Research Projects Buckingham, Open University Press.

Department of Planning and Environment, 2010. Metropolitan Plan for Sydney 2036.

Department of Planning and Environment, 2015. A plan for growing Sydney.

Department of Primary Industries, 2016. Status of Sydney's agriculture 2016.

Dibden J., Cocklin C., 2005. Sustainability and agri-environmental governance. In: Agricultural Governance: Globalization and the New Politics of Regulation (Higgins V., Lawrence G., eds), London, New York, Routledge.

Di Masso A., Dixon J., 2015. More than words: place, discourse and the struggle over public space in Barcelona. Qualitative Research in Psychology, 12, 45-60.

Dixon J., Durrheim K., 2000. Displacing place-identity: a discursive approach to locating self and other. The British Journal of Social Psychology, 39, 27-44. 
Docking A., Sreekumar C., 2008. Planning for food production within the Sydney Metropolitan region. In: International Cities, Town Centres and Communities Society Conference, Sydney Olympic Park, Sydney, Australia.

Foley K., Scott M., 2014. Accommodating new housing development in rural areas? Representations of landscape, land and rurality in Ireland. Landscape Research, 39, 359-386. Gailing L., Leibenath M., 2017. Political landscapes between manifestations and democracy, identities and power. Landscape Research, 42, 337-348.

Gant R., Robinson G.M., Shahab F., 2011. Land-use change in the 'edgelands': Policies and pressures in London's rural-urban fringe. Land Use Policy, 28, 266-279.

Harner J., 2001. Place identity and copper mining in Sonora, Mexico. Annals of the Association of American Geographers, 91, 660-680.

Hauge Å.L., 2007. Identity and place: a critical comparison of three identity theories. Architectural Science Review, 50, 44-51.

Hayden D., 1995. Urban landscape history: the sense of place and the politics of space. In: The Power of Place: Urban Landscapes as Public History, Cambridge, Massachusetts: MIT Press.

Houston P., 2005. Re-valuing the fringe: some findings on the value of agricultural production in Australia's peri-urban regions. Geographical Research, 43, 209-223.

Hulme T., Grosskopf T., John H., 2002. Agricultural Land Classification, NSW Agriculture. James S., 2009. Re-visioning Sydney from the fringe: productive diversities for a $21 \mathrm{st}$ Century City. Doctor of Philosophy, University of Western Sydney.

Kelsey T.W., Vaserstein G., 2000. Farming and non-farming neighbors: conflict, coexistence, and communication. Journal of Soil and Water Conservation, 55, 462-466.

Knowd I., 2006. Tourism as a mechanism for farm survival. Journal of Sustainable Tourism, $14,24-42$.

Knowd I., 2013. Hawkesbury Harvest: panacea, paradox and the spirit of capitalism in the rural hinterlands of Sydney, Australia. Doctor of Philosophy, Western Sydney University.

Larsen S.C., 2004. Place identity in a resource-dependent area of northern British Columbia. Annals of the Association of American Geographers, 94, 944-960.

Lawrence G., 1999. Agri-food restructuring: a synthesis of recent Australian research. Rural Sociology, 64, 186-202.

Lewicka M., 2008. Place attachment, place identity, and place memory: restoring the forgotten city past. Journal of Environmental Psychology, 28, 209-231.

Macarthur Regional Organisation of Councils, 2016. Wollondilly Shire. Employment by Industry (FTE), [en ligne], http://economy.id.com.au/macroc/ employment-by-industry-fte?WebID=120\&sEndYear=2001 (consulté le 18 septembre 2017).

Malcolm P., Fahd R., 2009. Ground Truthing of the Sydney Vegetable Industry in 2008, Sydney, Horticulture Australia Limited, NSW Depratment of Primary Industries.

Massey D., 1994. Space, Place and Gender, Cambridge, Polity Press.

McFarland P., 2014. The peri-urban land-use planning tangle: an Australian perspective. International Planning Studies, 1-19.

Memon P.A., 2003. Urban growth management in Christchurch. New Zealand Geographer, 59, 27-39.

Millar R., Fyfe M., 2012. The Fertile Fringe, The Age.

Mitchell D., 1996. Sticks and stones: the work of landscape (a reply to Judy Walton's "How real(ist) can you get?"). The Professional Geographer, 48, 94-96.

Mitchell D., 2003. Cultural landscapes: just landscapes or landscapes of justice? Progress in Human Geography, 27, 787-796. 
Murdoch J., 2000. Space against time: competing rationalities in planning for housing. Transactions of the Institute of British Geographers, 25, 503-519.

Murdoch J., Lowe P., 2003. The preservationist paradox: modernism, environmentalism and the politics of spatial division. Transactions of the Institute of British Geographers, 28, 318-332.

NSW Agriculture, 1998. Strategic Plan for Sustainable Agriculture. Sydney Region.

Olsson E.G.A., Kerselaers E., Søderkvist Kristensen L., Primdahl J., Rogge E., Wästfelt A., 2016. Peri-urban food production and its relation to urban resilience. Sustainability, 8, 1340.

Owen L., Howard W., Waldron M., 2000. Conflicts over farming practices in Canada: the role of interactive conflict resolution approaches. Journal of Rural Studies, 16, 475-483.

Parker F., 2007. Making periurban farmers on the fringe matter. State of Australian Cities Conference.

Paül V., Tonts M., 2005. Containing urban sprawl: trends in land use and spatial planning in the metropolitan region of Barcelona. Journal of Environmental Planning and Management, 48, 7-35.

Pointereau P., Coulon F., 2009. Abandon et artificialisation des terres agricoles. Le Courrier de l'environnement de l'Inra, 57, 109-120.

Pritchard B., 2005a. Constructing neoliberalism as a vision for agricultural policy. International Journal of Sociology of Agriculture and Food, 13, 1-12.

Pritchard B., 2005b. Strategies for securing neoliberalism. International Journal of Sociology of Agriculture and Food, 13, 1-14.

Richardson, T., 2000. Discourses of rurality in EU spatial policy: the European spatial development perspective. Sociologia Ruralis, 40 (1), 53-71

Ruoso L.-E., 2018. The politics of place identity in peri-urban environments. What role for productive farming landscapes? A case study of Wollondilly Shire, NSW, Australia. Doctor of Philosophy, University of Technology Sydney.

Ruoso L.-E., Plant R., 2018. A politics of place framework for unravelling periurban conflict: an example of periurban Sydney, Australia. Journal of Urban Management, OnlineFirst.

Setten G., 2006. Fusion or exclusion? Reflections on conceptual practices of landscape and place in human geography. Norsk Geografisk Tidsskrift - Norwegian Journal of Geography, $60,32-45$.

Sharp J.S., Smith M.B., 2003. Social capital and farming at the rural-urban interface: the importance of nonfarmer and farmer relations. Agricultural Systems, 76, 913-927.

Sinclair I., Bunker R., 2007. Planning for rural landscapes. In: Planning Australia: An Overview of Urban and Regional Planning (Thompson S., ed.), Cambridge, Port Melbourne, Vic, Cambridge University Press.

Stobbelaar D.J., Pedroli B., 2011. Perspectives on landscape identity: a conceptual challenge. Landscape Research, 36, 321-339.

Stokowski P.A., 2002. Languages of place and discourses of power: constructing new senses of place. Journal of Leisure Research, 34, 368-382.

Tonts M., Greive S., 2002. Commodification and creative destruction in the Australian rural landscape: the case of Bridgetown, Western Australia. Australian Geographical Studies, 40, 58-70.

Trudeau D., 2006. Politics of belonging in the construction of landscapes: place-making, boundary- drawing and exclusion. Cultural Geographies, 13, 421-443.

Twigger-Ross C.L., Uzzell D.L., 1996. Place and identity processes. Journal of Environmental Psychology, 16, 205-220.

Vanclay F., 2003. The impacts of deregulation and agricultural restructuring for rural Australia. Australian Journal of Social Issues, 38. 
Walker P., Fortmann L., 2003. Whose landscape? A political ecology of the 'exurban' Sierra. Cultural Geographies, 10, 469-491.

Wilkinson J., 2011. E-brief 2/2011 Agriculture in the Sydney region: historical and current perspectives. Sydney, NSW Parliamentary Library Research Service.

Willis A.-M., 2007. From peri-urban to unknown territory. Design Philosophy Papers, 5, 79-90.

Wollondilly Shire Council, 2011. Growth Management Strategy 2011.

Wollondilly Shire Council, 2017. Current status of planning proposals and projects. Detailed information as of 28 November 2017.

Yin R.K., 2009. Case Study Research: Design and Methods, Thousand Oaks, SAGE Publications.

Yokohari M., Takeuchi K., Watanabe T., Yokota S., 2000. Beyond greenbelts and zoning: a new planning concept for the environment of Asian mega-cities. Landscape and Urban Planning, 47, 159-171.

Zasada I., 2011. Multifunctional peri-urban agriculture. A review of societal demands and the provision of goods and services by farming. Land Use Policy, 28, 639-648. 


\title{
Chapitre 9
}

\section{Modèles et simulations spatio-temporels comme « objets intermédiaires" : le cas de l'étalement urbain à la Réunion}

\author{
Xavier Augusseau, Jeremy Bourgoin, Daniel David, Pascal \\ Degenne, Erwann Lagabrielle, Guillaume Lestrelin, Danny \\ LO SEEN
}

\section{Introduction}

Les processus de périurbanisation se traduisent par une forte tension sur le foncier agricole et par l'émergence d'une demande en agriculture "de services». De nouvelles fonctions sont attribuées à l'agriculture, comme la gestion des aménités rurales et environnementales (Beuret et Cadoret, 2009). L'espace agricole devient ainsi le support de multiples fonctions (production primaire et services environnementaux) et fait l'objet de controverses entre acteurs urbains, environnementalistes et acteurs du monde agricole (Bertrand et al., 2006).

Ainsi, l'étalement urbain, qui impacte majoritairement le foncier agricole en France ( $88 \%$ des espaces artificialisés sont des espaces agricoles (CGDD, 2012)), est devenu un enjeu prioritaire qui s'inscrit désormais dans différents agendas politiques. La loi de modernisation de l'agriculture et de la pêche (2010) et la loi d'avenir pour l'agriculture, l'alimentation et la forêt (2014) offrent différents instruments d'action publique pour mieux protéger le foncier agricole qui est considéré comme un facteur de production. Concomitamment, les différentes lois du Grenelle de l'environnement donnent de nouvelles prérogatives aux intercommunalités pour réguler la consommation par l'urbanisation des espaces agricoles, considérés comme supports d'aménités positives et contribuant au développement durable des territoires.

Les enjeux autour du foncier agricole dépassent donc le champ de la production et nécessitent de considérer les négociations réalisées par l'ensemble des acteurs et des instances de gouvernance (Jarrige et al., 2006). La diversification des questionnements autour de l'agriculture et la prise en compte d'une pluralité d'acteurs et de perspectives dans les négociations et dans la prise de décision nécessitent également des innovations sociales et organisationnelles. Ces nouvelles situations de gouvernance «partagée » rendent plus difficiles les choix et la recherche de consensus (Souchard, 2013).

Le recours à l'information géographique et "l'usage raisonné des représentations spatiales », notamment sous la forme de modèles spatialisés, semblent être une des voies 
privilégiées pour mettre en avant différents points de vue, en débattre et, potentiellement, dégager des consensus (Lardon et Piveteau, 2005 ; Maurel et al., 2007).

Dans des situations complexes où les facteurs d'incertitude sont importants, la prise de décision pour la gestion et l'aménagement des terres devient un véritable défi (McCarthy et Possingham, 2006). La modélisation rend possible l'adoption d'une posture de gestion adaptative (Schreiber et al., 2004) en permettant la manipulation du système et l'expérimentation de scénarios contrastés. Ces représentations artificielles sont de plus en plus utilisées pour explorer, apprendre de futurs possibles, et ainsi anticiper le potentiel impact d'instruments politiques avant leur implémentation (Geist et al., 2006). Les démarches de modélisation participative font état d'un besoin d'objectivité et de neutralité dans la gestion des ressources naturelles et soulignent le besoin d'impliquer un grand nombre de parties prenantes dans l'élaboration de scénarios prospectifs (Wollenberg et al., 2000 ; Alcamo et al., 2006 ; Lambin et Geist, 2006 ; Kok et al., 2007). Ces modèles sont ainsi perçus comme des supports de négociations plutôt que comme des outils de prise de décision (Verburg et al., 2006).

Si l'utilisation de modèles comme objets intermédiaires peut jouer un rôle important dans la médiation et les processus d'apprentissage (Vinck, 2000 ; Antona et al., 2005), il est donc nécessaire de promouvoir des démarches de coconstruction itératives pour assurer une bonne appropriation des modèles et leur mise en débat par les acteurs concernés (Van Ittersum et al., 1998 ; Volk et al., 2010).

Ce chapitre présente les premiers résultats de la construction d'un dispositif partenarial qui mobilise un outil de simulation de dynamiques paysagères utilisé comme support d'un exercice de prospective territoriale sur le territoire d'une intercommunalité de l'île de la Réunion. L'exercice vise à explorer différents scénarios d'évolution de l'occupation du sol dans lesquels l'étalement urbain et ses impacts constituent un enjeu central. Après avoir décrit les processus qui contribuent à l'étalement urbain à la Réunion, nous présenterons notre approche de modélisation de ces processus et de mobilisation des modèles comme supports de discussion.

Ce dispositif a été élaboré dans le cadre du projet Descartes de janvier 2013 à décembre 2014. Il a mobilisé un panel assez divers d'une quinzaine d'institutions locales : services de l'État, collectivités territoriales, filières agricoles, gestionnaires d'espaces naturels.

\section{Démarche}

Dans un contexte insulaire, le projet Descartes vise à construire une démarche d'élaboration participative d'un diagnostic sur la place et la contribution de l'agriculture à des projets de territoire sur l'île de la Réunion (quel espace agricole ? pour quelle production? pour quels services ?). Dans la perspective de développer des apprentissages, cette démarche intègre les partenaires institutionnels et la communauté scientifique sur l'ensemble de la mise en œuvre du projet. Le travail en partenariat s'appuie sur un outil de simulation cartographique visant à tester les " conséquences et cohérences », en matière de services (production et services environnementaux), de différents scénarios d'affectation de l'usage des sols dans un projet de territoire. Cet outil est construit comme interface entre les parties prenantes et les bases de données et modèles. Il est ainsi mobilisé comme support de débats et d'apprentissage collectif.

La mobilisation de modèles comme objets intermédiaires doit s'inscrire dans une démarche organisant les interactions entre scientifiques et acteurs concernés par le projet. 
Ces interactions sont considérées comme nécessaires à un processus d'apprentissage collectif, gage de l'utilisation des résultats du projet par les acteurs.

La première étape de notre démarche consiste à mettre en place et à consolider une arène qui garantisse la légitimité de l'exercice de prospective et en précise la finalité. Pour cela, nous avons choisi de développer un premier modèle démonstrateur qui représente les processus d'étalement urbain. Ce modèle est mobilisé pour mettre en débat la problématique de l'étalement urbain et plus spécifiquement du mitage agricole. Les interactions avec les partenaires se déroulent, selon une démarche itérative, au cours de forums - ateliers collectifs et groupes de travail plus restreints - durant lesquels le modèle et les simulations sont discutés puis améliorés.

\section{Contexte}

Le projet est mené à la Réunion, espace insulaire français de $2512 \mathrm{~km}^{2}$ situé dans l'océan Indien, qui se caractérise par des croissances démographique et économique importantes devant composer avec de fortes contraintes d'espace. La gestion du foncier est source de divergences. Depuis deux décennies, les acteurs du monde agricole tentent de protéger les terres arables, progressivement déclassées pour le développement urbain, tandis que les collectivités territoriales cherchent à assurer les besoins économiques et sociaux d'une population croissante (dans la partie « utile » de l'île, les densités sont proches de 600 hab./ha). L'espace agricole de l'île (environ 50000 ha) est le support de fonctions de production « primaire », mais devient aussi le support d'une production potentielle de services environnementaux. Les différentes filières agricoles doivent ainsi réaliser leurs propres objectifs (production et surfaces), potentiellement concurrentiels, tout en contribuant au développement durable de l'île. Or, les différentes politiques sectorielles se superposent sans qu'il y ait vraiment de lieux (forums) et d'outils pour diagnostiquer et débattre des interactions. Ainsi, les différents diagnostics territoriaux des projets de planification (comme les Plans locaux d'urbanisme, PLU, ou les Schémas de cohérence territoriale, SCoT) présentent généralement une vision segmentée des différents enjeux du territoire, bien loin de l'intégration nécessaire.

La création récente du Parc national de la Réunion (dont le « cœur de parc » couvre $40 \%$ de l'île) et d'une Réserve naturelle nationale marine sur les récifs coralliens du littoral ouest inscrivent dans l'espace géographique un projet de territoire insulaire orienté vers la conservation et la valorisation du patrimoine naturel (figure 9.1).

Le Territoire de la Côte Ouest (TCO), un Établissement public de coopération intercommunale (EPCI, voir chapitre 1) regroupant cinq communes, a été choisi comme territoire d'expérimentation. Il concentre d'importantes problématiques. Les élus et gestionnaires doivent trouver un équilibre et composer avec une démographie dynamique, des enjeux sociaux de taille (70\% des ménages sont éligibles au logement social) et des espaces agricoles qui ont été, en partie, sanctuarisés (la sole cannière) par des périmètres irrigués $^{34}$. Le TCO se caractérise également par la présence en aval d'un lagon, situé au nord-ouest du territoire, dont les ressources sont directement impactées par les activités anthropiques en amont, et par le cœur du Parc national dans les Hauts (figure 9.1). Ainsi, d'importants enjeux environnementaux, notamment sur la qualité de l'eau et les risques liés au ruissellement et à l'érosion, viennent se superposer aux conflits d'usage « historiques » sur le foncier. Enfin, pour mettre en œuvre son projet de territoire (c'est-à-dire

34. Un périmètre réglementaire protège la majorité de la sole cannière et des espaces concernés par un vaste projet d'irrigation. 
le SCoT), le TCO doit intégrer des politiques élaborées aux échelles régionales et nationales (comme les politiques agricoles) (figure 9.2).

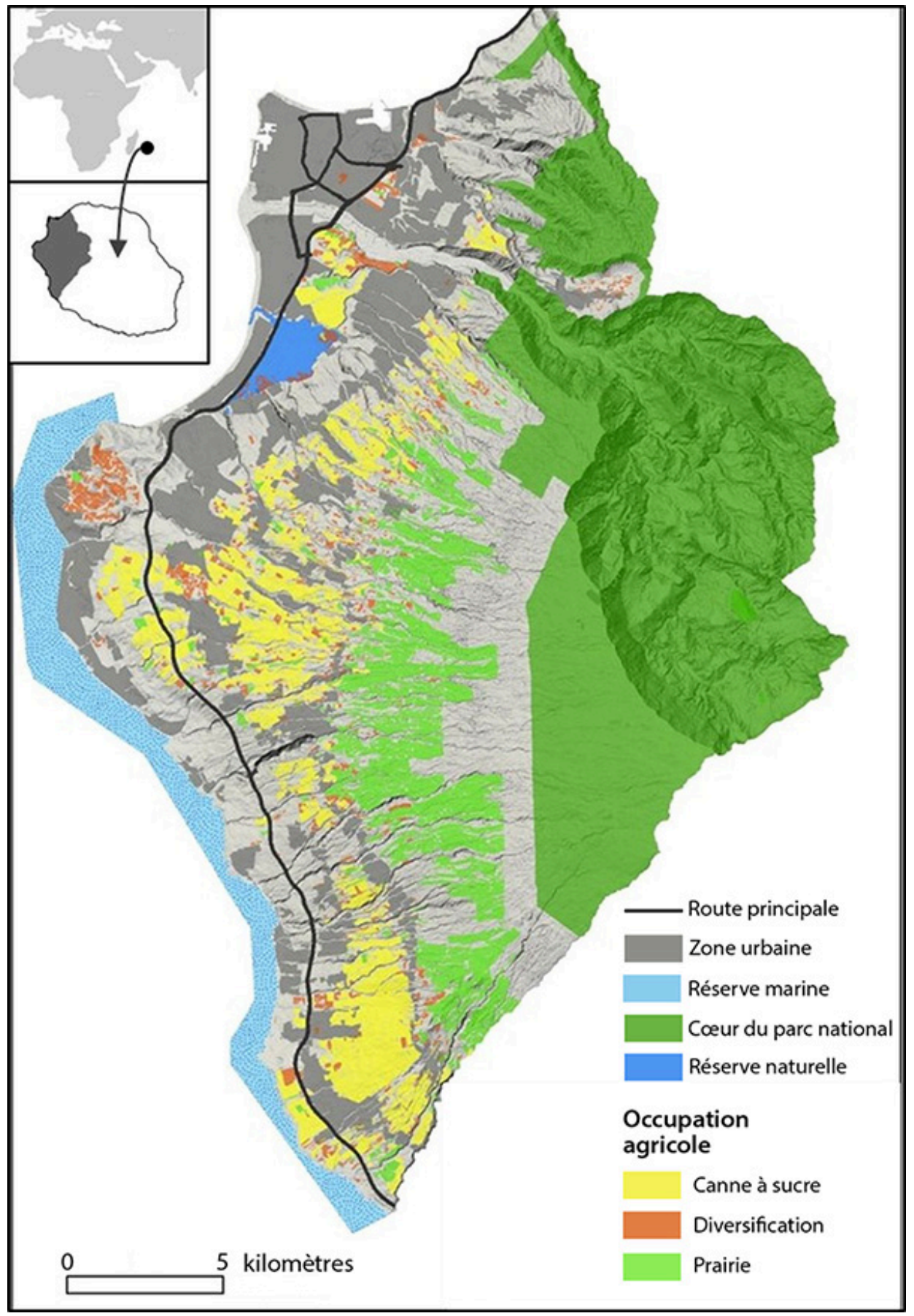

Figure 9.1. L'occupation du sol sur le Territoire de la Côte Ouest. 


\section{Réunion}

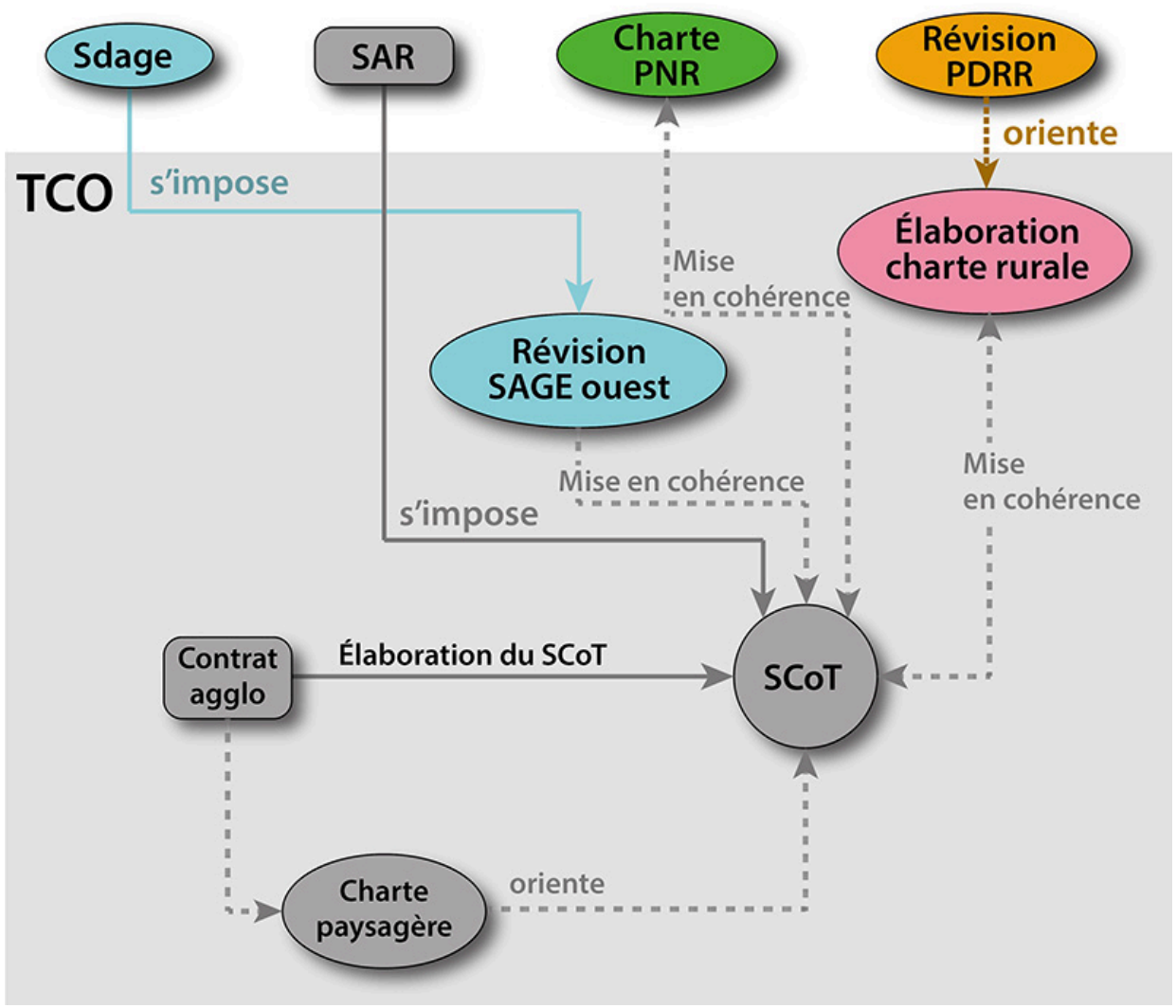

Figure 9.2. Interactions entre les principaux documents de planification territoriale et sectorielle, élaborés à différentes échelles.

Sdage : Schéma directeur d'aménagement et de gestion des eaux; SAR : Schéma d'aménagement régional ; PNR : Parc national de la Réunion ; PDRR : Plan de développement rural réunionnais ; SAGE : Schéma d'aménagement et de gestion des eaux ; SCoT : Schéma de cohérence territoriale.

\section{Étalement urbain et mitage à la Réunion \\ L'étalement urbain, une composante principale de l'urbanisation}

L'étalement urbain, dans des petits territoires insulaires comme la Réunion, relève de processus d'urbanisation très spécifiques liés à l'histoire de ces îles. Les impacts y sont également beaucoup plus marqués qu'en métropole en raison d'importantes contraintes d'espace. En conséquence, l'équilibre entre les espaces urbains et agricoles représente un des enjeux majeurs pour l'aménagement du territoire et la planification.

L'urbanisation à la Réunion est relativement récente et ne s'est vraiment développée qu'à partir des années 1960, après la départementalisation initiée en 1946. La forte croissance démographique et la transformation rapide d'une société rurale vers une économie tertiarisée ont induit une urbanisation très dynamique dans une logique de 
rattrapage en matière de logements et d'infrastructures (Jauze, 1998). Cette dynamique s'est traduite par une expansion spatiale importante (figure 9.3), produisant des agglomérations urbaines (au sens de l'Insee) éloignées du modèle métropolitain. Elles peuvent être assimilées à des « villes jardins », caractérisées par une faible densité d'une moyenne de 10 à 12 logements/ha (Lajoie et Actif, 2001). Dans les années 1990, selon les statistiques de l'Insee, l'urbanisation consommait de 500 à 600 ha de terres agricoles par an, soit près de $1 \%$ des terres agricoles (Insee, 2011). Depuis, l'étalement urbain tend à diminuer sous les effets conjugués de la planification territoriale, qui promeut la densification de projets urbains privilégiant le logement collectif, et du coût financier de l'aménagement (réseaux, etc.) induit par les faibles densités urbaines.

L'étalement urbain fait l'objet d'un suivi régulier, notamment avec la construction d'une tache urbaine réactualisée tous les cinq ans, à partir de la base de données topographique de l'IGN (BD TOPO $\left.{ }^{\circledR}\right)$ qui est mise à jour tous les cinq ans (Agence d'urbanisme à la Réunion, Agorah). La tache urbaine réunionnaise est essentiellement résidentielle, avec une densité de 11 logements par hectare en moyenne, et sa surface a augmenté entre 1997 et 2008 de 2,2 \% en moyenne par an. Elle occupait environ $10 \%$ du territoire en 2008 . L'analyse de l'évolution de la tache urbaine (Agorah, 2008a ; 2008b) montre que l'étalement urbain se produit pour moitié sur des espaces agricoles. Outre la consommation de terres cultivées, l'étalement urbain induit une fragmentation des espaces agricoles, au travers du mitage, qui fragilise les filières. Si cette tendance actuelle se poursuivait, près de 5000 ha de terres agricoles seraient consommés d'ici 2020. Les études prospectives sur l'étalement urbain estiment, en partant d'un besoin de logements évalué à 106000 résidences en 2020, que la moitié des nouveaux logements sera construite en zone urbaine et que les surfaces d'extensions, qui seront nécessaires, varieront de 4200 ha pour un scénario tendanciel à 1200 ha pour un scénario optimal (Coteba et Deal Réunion, 2010).

\section{Le mitage agricole, une composante autonome de l'étalement urbain}

Une des caractéristiques de l'urbanisation à la Réunion est qu'elle produit du mitage agricole. Le mitage désigne tout le bâti construit illégalement en zones réglementairement reconnues à vocation agricole et naturelle par les documents d'urbanisme (Plans d'occupation du sol, POS, et PLU). Il représente un phénomène constitutif de l'urbanisation à la Réunion qui peut se rattacher aux dynamiques historiques de peuplement vers les parties hautes de l'île. Mal connu, c'est un processus complexe qui est mû par des stratégies individuelles et se déploie différemment sur le territoire en fonction de facteurs historiques, sociaux et fonciers. Il représente un enjeu territorial important qui a des impacts sur la planification urbaine et le foncier agricole. Il est perçu négativement par les gestionnaires des collectivités et les services de l'État. L'analyse des facteurs à l'origine du mitage et les moyens de le réguler sont source de controverses entre les tenants d'une réglementation plus contraignante et les défenseurs d'une politique urbaine plus ambitieuse pour répondre aux besoins en logements.

L'analyse du mitage et de son impact est réalisée à partir de la mesure de son emprise spatiale, appelée «surface urbanisée » (figure 9.4). Elle prend en compte le voisinage du bâti construit selon la méthode tampon-érosion (Deal Réunion, 2011). 

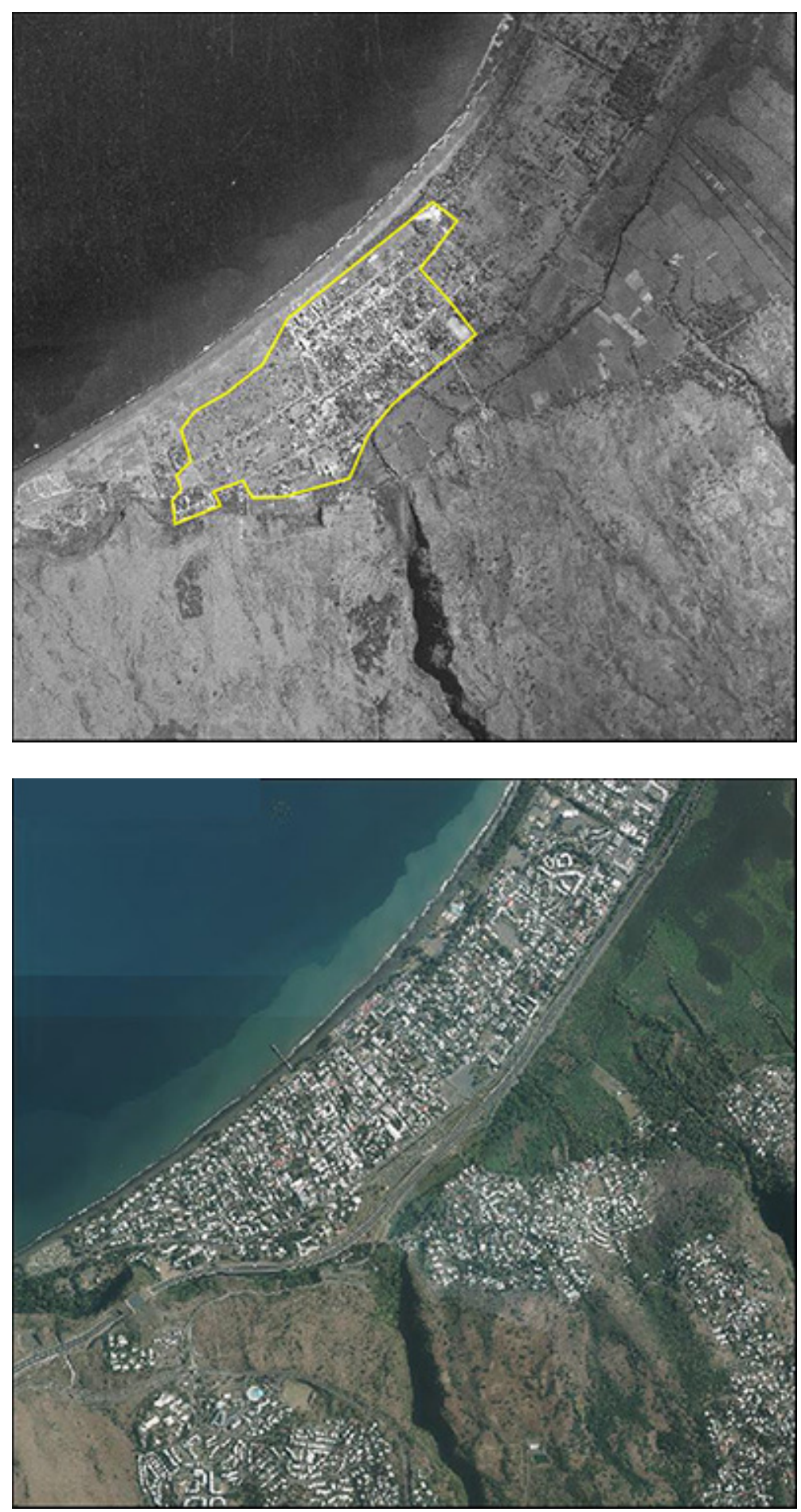

Figure 9.3. L'urbanisation de Saint-Paul (vieux centre-ville) et des planèzes périphériques en 2011 (source : IGN). 


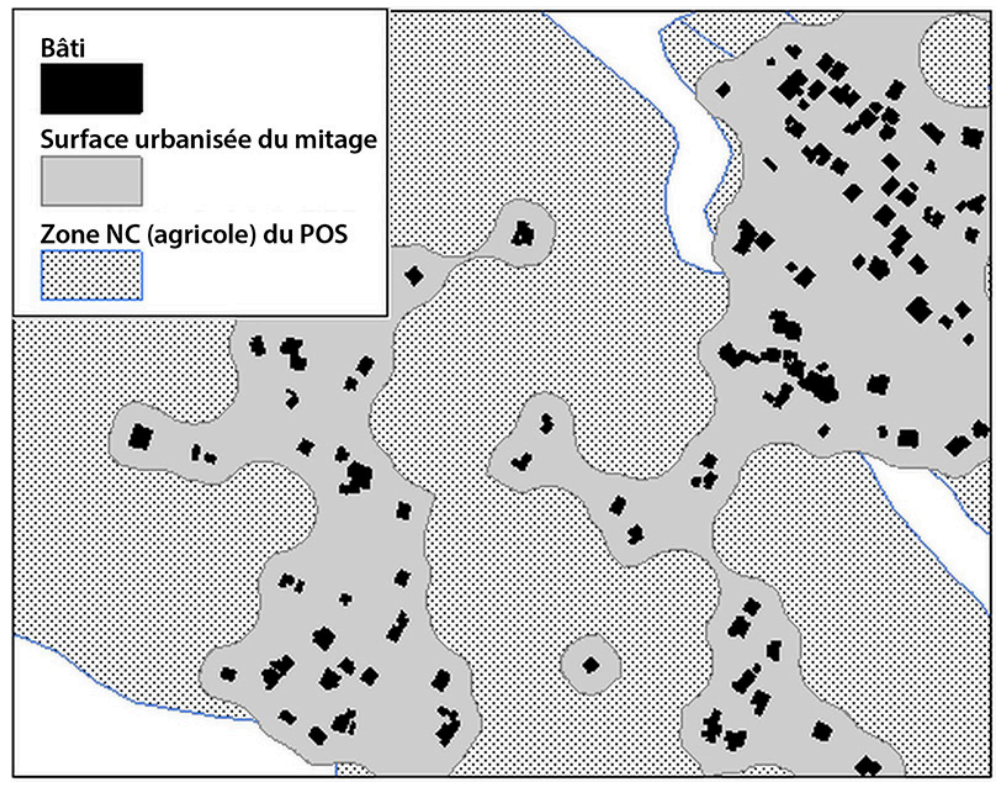

Figure 9.4. Emprise spatiale du mitage sur une planèze de la commune de Saint-Paul en 2009 (NC : zones non constructibles).

À la Réunion, $17 \%$ des surfaces urbanisées mitent les espaces agricoles. Après une forte croissance jusqu'en 2003, le phénomène de mitage a eu tendance à diminuer, voire à se stabiliser dans certaines communes. Différents facteurs favorisant le mitage ont été identifiés, même s'il est difficile de les hiérarchiser :

- la structure parcellaire : taille, forme, processus de division parcellaire ;

- la proximité du réseau routier, de centres urbains ou de zones de mitage préexistantes ;

- la topographie, qui conditionne en partie la structure parcellaire ;

- le potentiel et la dynamique agricole (souvent en contradiction avec la vocation réglementaire des usages des sols);

- les documents de planification urbaine (Schéma d'aménagement régional, POS), qui peuvent avoir un impact local sur la dynamique du mitage (pression foncière, conflit avec un projet agricole, etc.).

Les acteurs agricoles, et en particulier la filière de la canne à sucre, ont élaboré un zonage du potentiel agronomique en combinant différents critères agronomiques (type de sol, pente, altitude, pierrosité, etc.). Si sa finalité première est d'orienter les priorités d'aménagement agricole, il est souvent mobilisé lors des révisions des documents d'urbanisme pour remettre en cause certaines décisions de changement d'usage des sols au détriment de l'agriculture.

Le TCO est la région la plus marquée par l'étalement urbain, qui se développe à égalité sur les espaces à urbaniser réglementaires et sur les espaces agricoles et naturels. C'est aussi là que le mitage agricole est le plus important, notamment sur la commune de Saint-Paul, où l'urbanisation occupe près de $20 \%$ des espaces agricoles et naturels (Deal Réunion, 2011). L'analyse de différentes zones mitées sur le TCO montre que le mitage se déploie sur des espaces où l'agriculture est marginale et en déclin. Le potentiel agricole y est souvent faible. Les zones historiques de mitage se caractérisent par des densités de bâti qui sont relativement importantes, au minimum de 2 bat./ha. En zone 2NC (non constructible), à vocation agricole où seul l'habitat agricole est autorisé, ces densités sont 
proches de 8 bat./ha (Deal Réunion, 2011). Ces zones constituent de fait de petits bourgs ruraux. Après avoir culminé dans les années 1990, la dynamique de mitage s'est significativement atténuée depuis 2003 sur la majeure partie du territoire du TCO. Malgré tout, il existe une grande disparité en termes de progression, avec des zones de mitage historique stabilisées et des zones à forte dynamique, de +30 à $+60 \%$ depuis 1997 (figure 9.5).

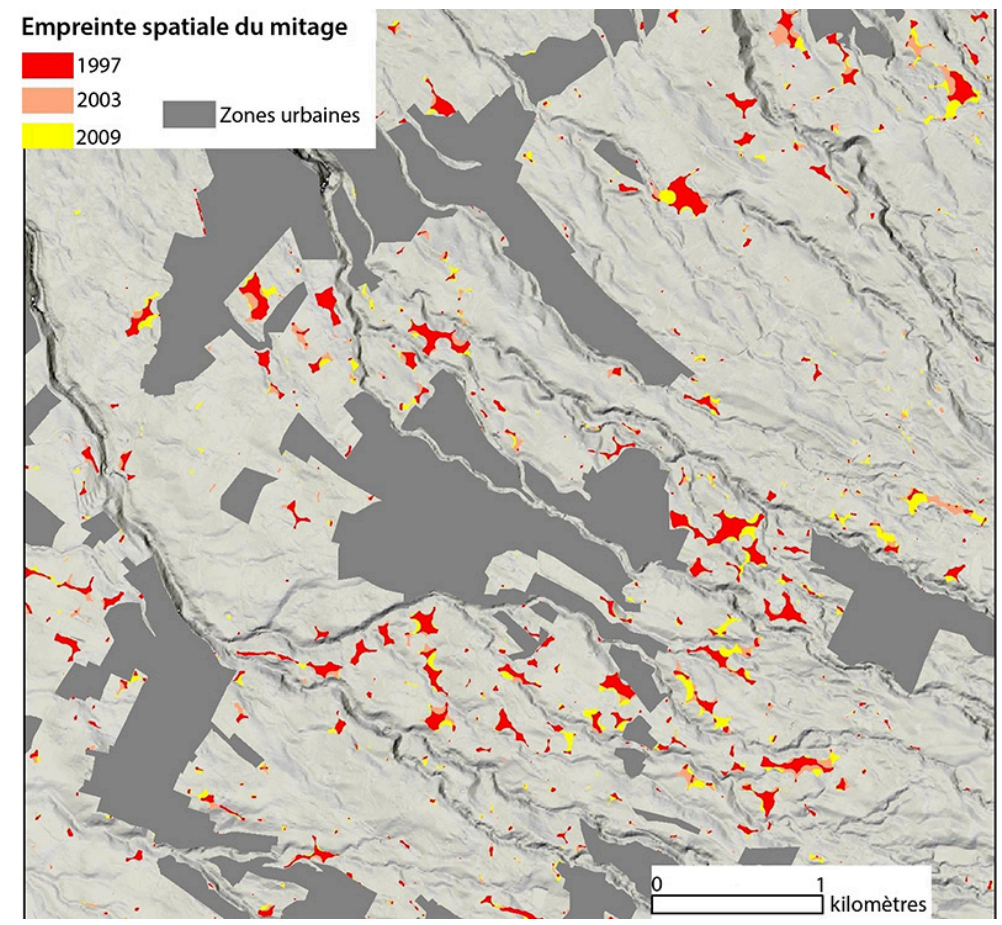

Figure 9.5. Évolution du mitage dans les hauts de la commune de Saint-Paul.

\section{Un mitage agricole pas ou peu pris en compte dans les politiques d'aménagement}

Depuis les années 1990, différents projets de planification territoriale tentent de réguler et limiter l'étalement urbain. Dès 1995, le premier Schéma d'aménagement régional $(\mathrm{SAR})^{35}$ fixe comme objectif prioritaire la préservation des espaces naturels et agricoles (Metzger, 2004). Dans le dernier SAR, révisé en 2011, les espaces urbains, organisés selon une hiérarchie, sont soumis à des objectifs de densification en matière de logement. Les espaces agricoles sont sanctuarisés et l'étalement urbain est strictement encadré par des quotas de surface, alloués aux communes, sur quelques zones agricoles en périphérie des pôles urbains qui ont fait l'objet d'âpres négociations avec la profession agricole.

Néanmoins, l'analyse du SAR de 2011 montre que la plupart des zones mitées du TCO sont situées dans des espaces classés comme agricoles mais où l'activité agricole est en fait relativement marginale. Le classement réglementaire de ces espaces, situés dans l'emprise du Projet d'irrigation du littoral ouest, apparaît ainsi moins guidé par la prise en compte de la réalité de l'usage des terres à l'échelle locale que par l'hypothèse, défendue ment qui s'imposent aux documents de planification des communes (POS, PLU). 
par le monde agricole, que l'irrigation pourrait améliorer le potentiel agricole et, à terme, induire une dynamique agricole. À l'échelle communale cependant, on commence à observer une prise en compte du mitage historique. Dans le cadre de révisions de PLU, certaines de ces zones bénéficient d'une réglementation spécifique autorisant le bâti sur un périmètre restreint - l'objectif étant de concentrer le mitage sur ces zones.

À l'instar de la planification territoriale, aux échelles intercommunale et communale, les documents de programmation comme le Plan local d'habitat (PLH) en matière de logement sont également censés jouer un rôle dans la régulation du mitage en précisant les moyens de répondre aux besoins en logement de la population. Dans le cas du TCO cependant, les résultats des PLH successifs depuis 2005 se sont avérés assez mitigés. En particulier, l'impossibilité d'atteindre les objectifs de construction de logements sociaux — des objectifs particulièrement élevés sur le territoire du TCO — a probablement eu un impact sur la poursuite du mitage et de l'étalement urbain.

En fin de compte, malgré les moyens mis en œuvre et l'existence de différents outils réglementaires pour réguler la consommation des espaces agricoles et naturels, de fortes incertitudes demeurent sur la capacité des collectivités à répondre à l'ensemble des besoins en logement et à réaliser les objectifs de densification inscrits dans les documents de planification.

\section{Modélisation de l'étalement urbain et du mitage}

\section{Le modèle conceptuel}

La consommation d'espaces agricoles (et/ou naturels) par l'urbanisation est modélisée comme résultant d'un double processus : d'une part le déclassement de terres agricoles par la planification urbaine, et d'autre part le mitage, qui obéit à des décisions individuelles. Nous avons fait le choix de représenter ces processus en nous basant sur des éléments de planification du SAR qui vont fortement influencer et réguler les dynamiques d'urbanisation à venir. En outre, les objectifs qui y sont assignés (en matière de densification, d'extension spatiale et de structuration des pôles urbains) donnent d'emblée un « scénario » de référence.

La planification urbaine se décline en trois sous-processus qui sont : la densification — le remplissage de "dents creuses » en zone urbaine; l'extension-déclassement de terres agricoles dans les zones AU (à urbaniser) selon un quota défini par le SAR; et la construction d'infrastructures — selon des projets planifiés à moyen terme (figure 9.6). Enfin, nous faisons l'hypothèse que le processus de mitage est lié à la non-satisfaction des besoins en logements par la planification urbaine. Autrement dit, le mitage est une réponse individuelle à un manque de logements non pourvus par les politiques publiques qui ont, notamment, en charge de développer le logement social.

\section{La modélisation avec Ocelet}

Modéliser un processus selon une approche systémique consiste essentiellement à identifier et à décrire les entités (éléments du système) qui interviennent dans le processus, à décrire les relations (interactions dynamiques) entre ces entités, et enfin, à simuler leurs évolutions selon plusieurs scénarios. Pour réaliser ce modèle, nous utilisons Ocelet, un langage « métier » de modélisation de dynamiques spatiales et paysagères (Degenne et Lo Seen, 2016). Celui-ci est conçu autour d'un ensemble restreint de concepts clés, dont celui central de graphe (objet mathématique constitué d'un ensemble de nœuds dont certains sont connectés par des arcs) d'interaction (la sémantique des interactions est portée par les arcs) pour exprimer les différents types de relations possibles (spatiales, 
fonctionnelles, sociales, hiérarchiques) entre les entités du modèle. Ocelet est associé à un environnement logiciel qui permet de construire les modèles et les scénarios, de lancer les simulations, et de sauver les résultats sous diverses formes, dont celle de cartes animées visualisables sous Google Earth.

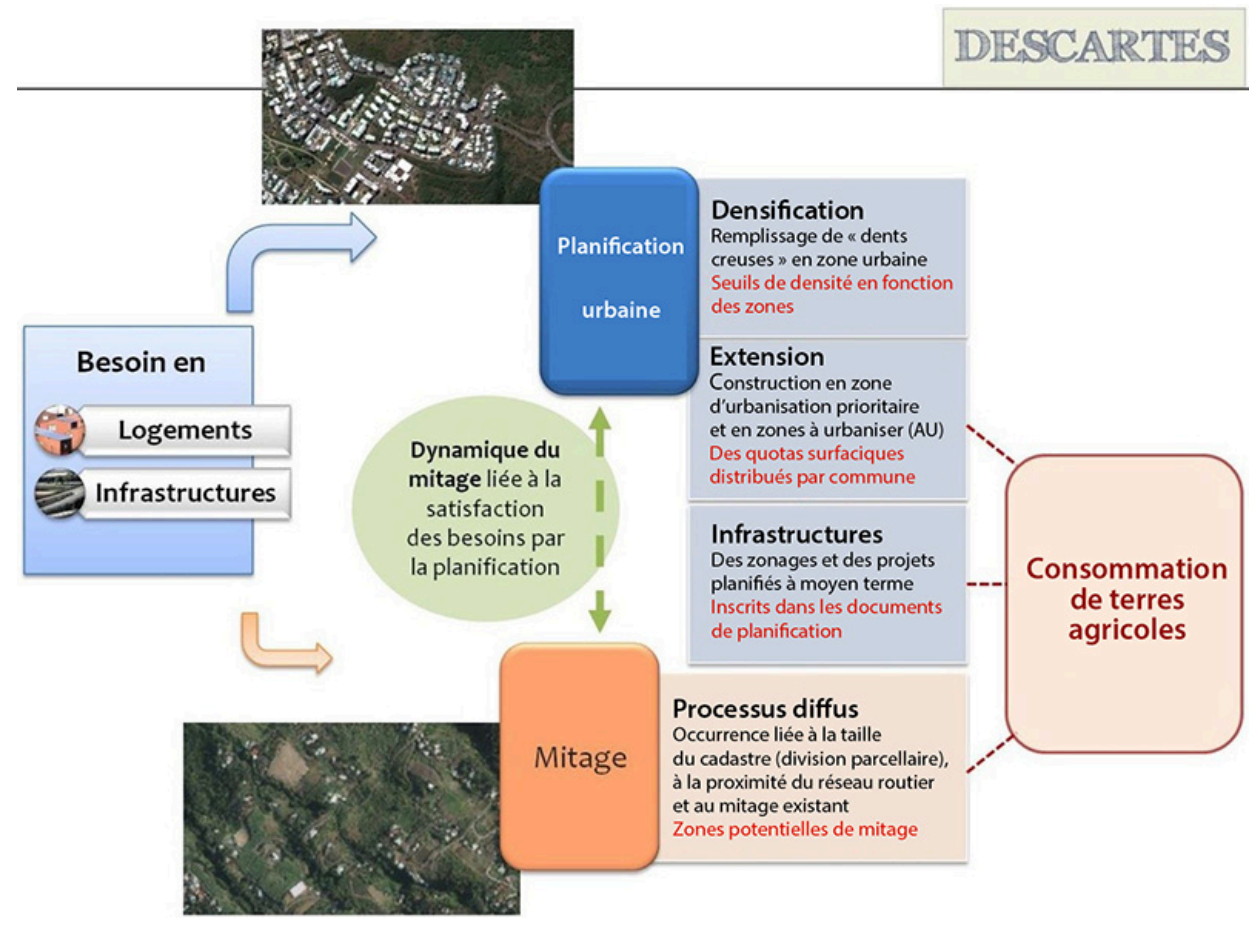

Figure 9.6. Représentation des dynamiques urbaines.

L'intérêt de ce langage est qu'il permet de manipuler aisément de l'information géographique, aussi bien en entrée qu'en sortie des modèles, en créant et en initialisant des entités Ocelet de façon quasi automatique à partir de données géographiques natives.

Un autre avantage que procure le choix d'Ocelet pour implémenter nos modèles découle du positionnement de ce langage au carrefour des approches de modélisation top-down (descendantes) et bottom-up (ascendantes). Ainsi, si la représentation de l'espace et des entités dans Ocelet est comparable, notamment en termes de granularité, à ce que l'on pourrait obtenir avec des approches de modélisation ascendantes comme les automates cellulaires (White et Engelen, 1993) ou les systèmes multi-agents (David, 2010), la description de son évolution au cours du temps se fait de façon plus globale, à la manière d'approches descendantes héritées du concept de dynamique des systèmes (Forrester, 1968). Ceci permet aux thématiciens d'avoir une plus grande compréhension du fonctionnement des modèles qu'ils ont contribué à coconstruire, dans la mesure où les blocs dictant l'évolution de ces modèles en langage Ocelet peuvent se lire à la manière d'un scénario narratif.

Enfin, afin de faciliter la visualisation des évolutions au cours du temps de notre territoire d'expérimentation (Sheppard et Cizek, 2009), Ocelet permet d'exporter les résultats des modèles dans les formats kml (pour Google Earth) ou shp (pour les systèmes d'information géographique) ayant des attributs temporels et de produire des cartes dynamiques en 
3D qui donnent aux utilisateurs une meilleure contextualisation des résultats des simulations sur leur territoire.

\section{La construction itérative du modèle}

Un premier prototype - simplifié et volontairement non calibré — a été développé entre chercheurs dans le but explicite d'exposer au TCO notre savoir-faire en matière de modélisation et de simulation cartographique sur une zone test (d'échelle communale). Pour mieux focaliser l'attention de nos interlocuteurs sur les possibilités offertes par notre approche, nous avons choisi de tester ce prototype sur une commune située en dehors du territoire du TCO.

Suite à ce prototype, nous avons enclenché avec les équipes du TCO la démarche de modélisation qui a permis d'aboutir au modèle plus complet dans lequel nous avons conceptualisé, implémenté et couplé deux dynamiques : l'urbanisation planifiée et le mitage. Les échanges avec le TCO et les autres partenaires ont alors permis d'enrichir et d'améliorer le modèle, notamment sur le mitage en distinguant deux processus distincts : le mitage « traditionnel », qui relève de stratégies individuelles et qui est autonome vis-àvis des dynamiques urbaines, et le mitage qui vise à répondre à des besoins en logements qui ne sont pas résolus par l'urbanisation planifiée et qui correspond à notre première hypothèse. Enfin, parmi les facteurs pouvant favoriser le mitage, nos partenaires ont proposé d'intégrer la notion d'attractivité qui peut être induite par la mise en service de nouveaux équipements ou la recherche de meilleures conditions d'habitat (recherche d'un « effet balcon» sur des hauteurs offrant un panorama exceptionnel).

\section{Le modèle implémenté : entités et processus à l'œuvre}

Plusieurs entités spatiales multi-échelles sont représentées et manipulées dans ce modèle, en particulier le TCO lui-même, ses pôles urbains, auxquels sont associés des zones d'extension urbaine, et ses parcelles cadastrales.

Chaque année, dans la dynamique d'urbanisation planifiée, une partie des parcelles cadastrales qui se trouvent au sein des zones d'extension urbaine définies dans les documents de planification (notamment le SCoT) s'urbanise en fonction des objectifs de nombre et de densité de logements associés à chacun des pôles urbains (à l'horizon 2020). En fonction des contraintes et des scénarios de simulation, la part des besoins en logement qui n'est alors pas satisfaite vient enrichir pour partie la dynamique de mitage. Dans cette dernière, des maisons sont construites illégalement sur des parcelles cadastrales se situant en dehors des zones urbaines réglementaires mais qui possèdent un fort potentiel en raison de leur attractivité et de leur proximité au réseau routier, aux pôles urbains et/ou aux zones historiques de mitage des terres agricoles.

La construction du modèle dans Ocelet passe par la définition des entités, des relations et des scénarios exprimant les deux processus présentés en figure 9.7.

Les entités de chaque type sont initialisées à partir d'un fichier SIG (shapefile) et sont renseignées par plusieurs propriétés (ex. : identifiant, surface, statut, géométrie, nombre de logements) dont les valeurs pour certaines vont évoluer durant la simulation. Ainsi, par exemple, chaque entité " pôle urbain » est dotée d'objectifs en termes de nombre de logements à bâtir, les entités "zone d'extension urbaine » sont renseignées par une date de mise en service supposée de réseaux d'assainissement, et chaque parcelle cadastrale située hors des zones urbaines se voit affecter des pondérations reflétant sa proximité au réseau routier. 


\section{Modèle de consommation de terres agricoles par l'urbain}

\section{DESCARTES}
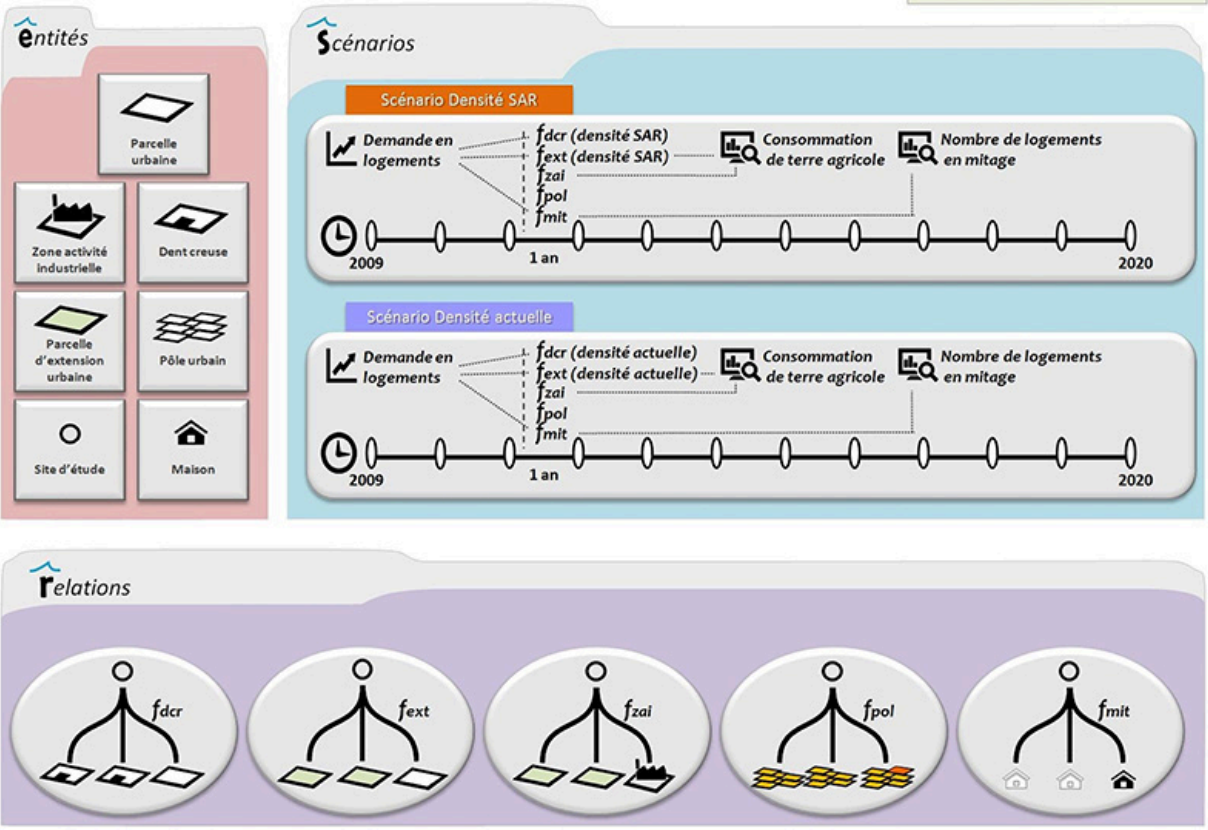

Figure 9.7. Les primitives du modèle de consommation de terres agricoles par l'urbain.

Les relations utilisées sont de type hiérarchique, reliant toutes les entités d'un même type à l'entité "site d'étude ». Ainsi, chaque entité " pôle urbain » est reliée à l'entité «TCO » par une relation d'appartenance, alors qu'une entité «zone d'extension urbaine » est reliée à une entité " pôle urbain » particulière par une relation de dépendance. Chaque relation est un graphe d'interaction, c'est-à-dire un ensemble de nœuds (les entités) reliés par des arcs. Les arcs portent les fonctions d'interaction; elles décrivent ce qui se passe entre les entités connectées par les arcs. Un tel graphe permet d'accéder individuellement à chaque entité et à chaque pas de temps pour y exécuter des fonctions telles que transformer une entité non construite (ex. : dent creuse ou parcelle d'urbanisation prioritaire) en entité construite à un moment donné de la simulation. Ces fonctions font intervenir des probabilités de changements qui sont aussi des paramètres du modèle.

Les scénarios servent à décrire l'évolution du système à partir d'un état initial, avec une durée et un pas de temps prédéterminés. Différents scénarios expriment alors différentes hypothèses d'évolution. Dans le cas présent, ils prennent en compte l'évolution de la demande en logements, un pourcentage de nouveaux logements sur le bâti existant, la construction de nouveaux logements dans les dents creuses en respectant une densité préconisée, un quota de surface agricole par pôle urbain à urbaniser en priorité, dont un pourcentage à utiliser d'ici 2020, et enfin le mitage pour absorber le décalage entre l'offre et la demande en logements.

\section{Simulation et exemples de sorties}

Les résultats des simulations issues de cette version du modèle ont été exportés sous forme de fichiers $\mathrm{kml}$ temporels pour comparer les évolutions spatiales associées à des 
scénarios tests. Les figures suivantes illustrent trois situations types que nous avons simulées sur une période entre 2010 et 2020, avec un pas de temps annuel.

Sur la figure 9.8, on observe la différence entre un scénario dans lequel nous avons maximisé l'importance du critère de proximité aux zones déjà mitées, ce qui explique que les cercles jaunes (les zones mitées au cours de la simulation) soient concentrés autour des zones rouges (les zones de mitage historique), et un scénario dans lequel nous avons favorisé le critère de proximité au réseau routier. La figure 9.9 illustre les effets sur le mitage de l'attractivité renforcée autour d'un échangeur mis en service sur l'axe routier principal du territoire. Et la figure 9.10 met en évidence le lien entre l'urbanisation planifiée et le mitage, puisque dans un cas l'urbanisation planifiée a su se faire selon de forts objectifs de densification de logements et d'urbanisation dans les zones d'extension urbaine, ce qui a engendré un mitage assez faible (qui correspond uniquement à la part relevant du mitage « traditionnel »), tandis que dans l'autre cas l'urbanisation planifiée n'a pas su tenir les hypothèses de densification ou d'extension qui étaient prévues, et cela s'est traduit par une augmentation du mitage dit « vases communicants » dont la part est venue s'ajouter au mitage « traditionnel ».

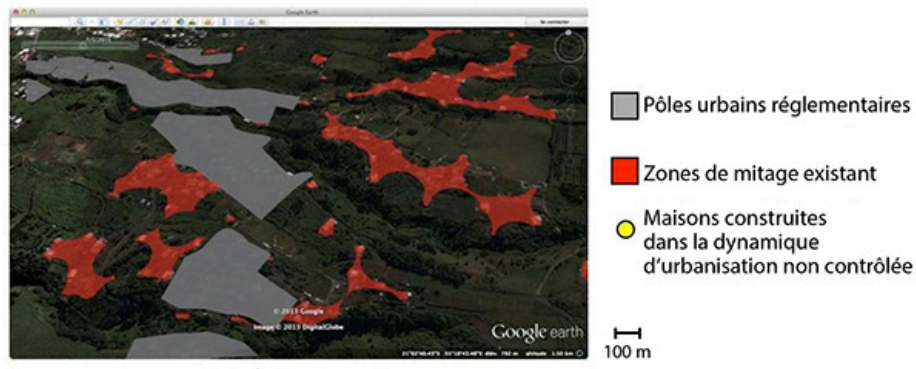

État final (2020) Scénario de simulation $A$ (proximité aux zones déjà mitées)

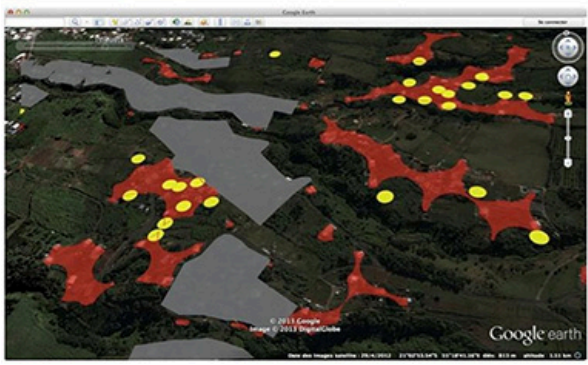

État initia
(2013)

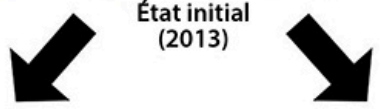

État final (2020)

Scénario de simulation B (proximité au réseau routier)

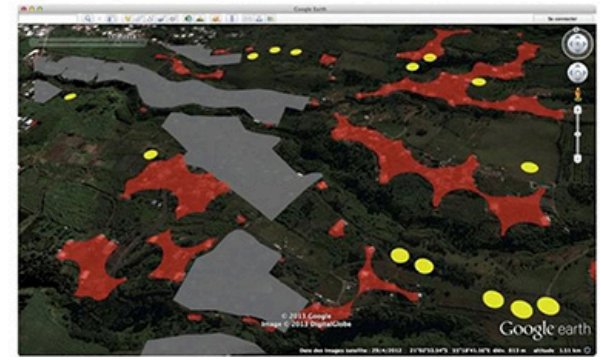

Figure 9.8. Impact de la proximité des zones de mitage existantes et de la proximité au réseau routier sur le mitage. 


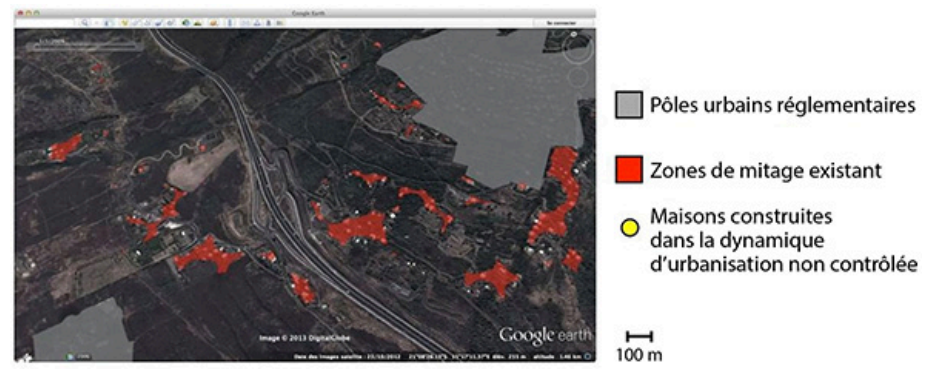

\section{État final (2020) \\ Scénario de simulation $\mathrm{A}$ (pas d'attractivité de l'échangeur)
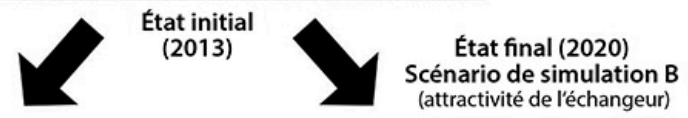
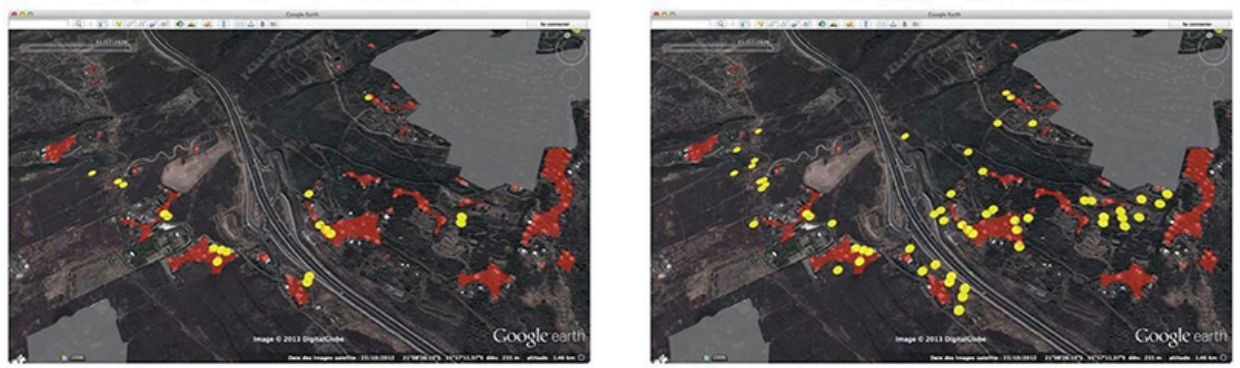

Figure 9.9. Impact de l'attractivité d'une zone particulière (ici un échangeur sur un axe routier majeur) sur le mitage.

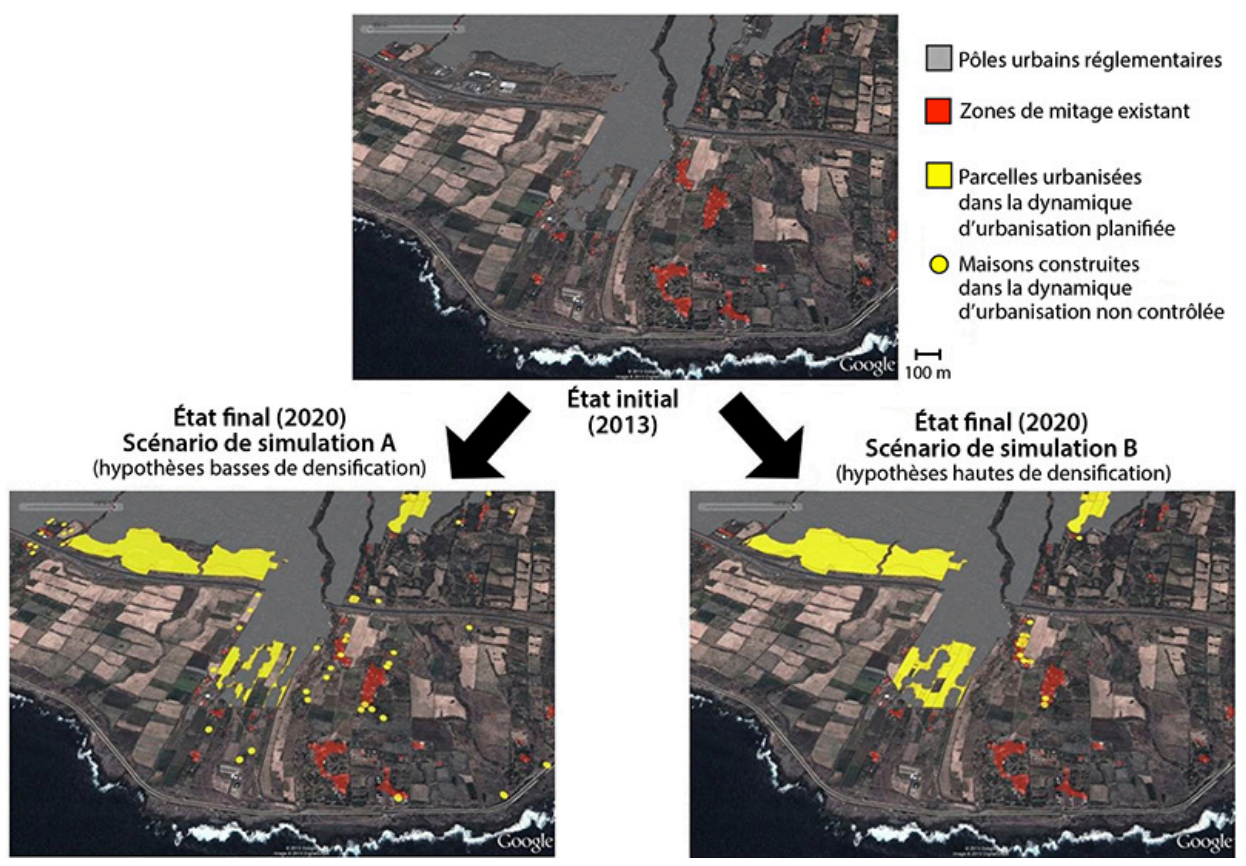

Figure 9.10. Impact des évolutions de l'urbanisation planifiée sur le mitage. 


\section{Mobilisation du modèle et de la thématique « mitage »}

Au cours de l'atelier de démarrage du projet organisé en mars 2012, il a été demandé aux représentants d'une dizaine d'organisations partenaires (ex. : chambre d'agriculture, services de l'État, filières agricoles, Parc national) de présenter leur vision des principaux enjeux liés à la planification et à l'aménagement du territoire à la Réunion. Les présentations ont été suivies d'une discussion sur les notions de "paysage » et de "services écosystémiques » et les interactions et conflits entre usages des terres. Les participants ont ainsi pu identifier un certain nombre de "propositions de recherche » visant à combler des manques d'informations et/ou organisationnels ayant trait à différents types d'interactions entre usages des terres. Concernant les interactions entre agriculture et développement urbain, il a été proposé d'analyser les dynamiques historiques de mitage, de mettre en évidence leurs moteurs, d'identifier des scénarios d'évolution politiques et socio-économiques réalistes et d'évaluer leurs impacts sur le mitage et la consommation des terres agricoles. Concernant les interactions entre écosystèmes naturels et usages anthropiques, il a été proposé d'identifier des indicateurs et d'évaluer les impacts écologiques des dynamiques agricoles et d'urbanisation (notamment, évaluer le potentiel agricole des zones de continuité écologique du SAR).

Bien que l'atelier ait permis de positionner le projet Descartes relativement aux principales problématiques auxquelles sont confrontés les acteurs de l'aménagement du territoire à la Réunion, la mise au point d'une démarche et de méthodes pour répondre à ces problématiques s'est avérée plus difficile. En cause, la réaction des participants aux propositions de l'équipe de recherche de développer des activités de prospective territoriale qui s'est finalement révélée très variable : certains ont fait montre d'une bonne compréhension et d'un intérêt marqué pour une démarche de modélisation spatiale et d'exploration de scénarios; d'autres étaient moins convaincus ou avaient une vision plus confuse de la démarche ; d'autres enfin étaient franchement rétifs à toute initiative d'ordre collectif et/ou à visée intégrative. La décision fut donc prise par l'équipe scientifique de se focaliser sur le développement d'un modèle permettant de simuler des dynamiques urbaines, au détriment, dans un premier temps, de la structuration d'une arène de travail collaboratif. Ce faisant, l'équipe faisait l'hypothèse que le développement d'un modèle " démonstrateur » permettrait d'illustrer le potentiel d'intégration et d'investigation de la démarche et, partant, d'accroître l'intérêt et le soutien des acteurs de l'aménagement du territoire.

Le mitage constitue un important sujet de controverse à la Réunion, générant souvent de vifs débats entre conseils municipaux et acteurs du secteur agricole et de la planification urbaine. En effet, les services de l'État et les filières agricoles privilégient une vision « aménagiste » de l'urbanisme et militent pour renforcer la réglementation afin de contraindre les candidats au mitage. À l'inverse, les collectivités et leurs services d'urbanisme argumentent que c'est en faisant de la « bonne ville » qui répond aux besoins des populations que l'on pourra limiter le mitage. Ils mettent donc en avant le projet urbain.

Partant de cette observation, l'équipe de recherche a fait le choix de concentrer le travail de modélisation sur les dynamiques de consommation des espaces agricoles et naturels en lien avec l'urbanisation planifiée et le mitage (cf. partie « Modélisation »). Après avoir passé en revue différents rapports et documents de planification, participé à des ateliers et collecté des données géographiques et statistiques en lien avec ces questions, un premier modèle a été développé et appliqué à la municipalité de Saint-Pierre. Des simulations dérivées de ce modèle et présentées sous forme de cartes dynamiques avec 
Google Earth ont ensuite été présentées et discutées au cours de réunions avec différents acteurs (ex. : filières agricoles, services de l'État, mairies et EPCI). Ce processus a permis d'attirer l'attention d'un certain nombre d'acteurs et de les engager dans un processus itératif de débat-amélioration progressive du modèle (ex. : choix des échelles de modélisation et des entités, définition des interactions entre variables). Il a également permis d'identifier l'intercommunalité du TCO comme une organisation partenaire clé, légitime et volontaire pour soutenir l'effort de structuration d'une arène de travail collaboratif.

Cette dernière étape a été grandement facilitée par l'actualité interne de l'équipe technique du TCO, en phase stratégique de redéfinition des grandes orientations de son territoire (ex. : validation du projet de SCoT et révision du SAGE). Des groupes de travail sur la modélisation des dynamiques d'urbanisation et la restitution de simulations sous Google Earth ont notamment permis d'alimenter des discussions entre l'équipe scientifique et le TCO autour du projet de SCoT et des différentes orientations possibles de l'urbanisation, en ciblant localement des incohérences ou des compromis potentiels. Suite à ces échanges, la collaboration a été formalisée par une charte de partenariat, signée par le président de l'EPCI en mars 2013, précisant la finalité du travail de prospective, les modalités d'interaction entre les acteurs du projet, et positionnant l'intercommunalité du TCO au centre d'un dispositif de réflexion et de débats sur l'évolution du territoire.

Dans le même temps, un premier atelier de travail collectif a été organisé, rassemblant des représentants de 15 organisations, pour discuter du modèle appliqué à la zone du TCO et des premières simulations, débattre des impacts sociaux et environnementaux du mitage ainsi que des moyens de régulation et de réduction du processus. Les simulations ont donné lieu à des débats sur les éléments déterminant la progression du mitage (ex. : accès aux réseaux « eau » et « électricité », proximité à la route, aux pôles urbains et aux poches historiques de mitage) et leur importance relative. L'existence et la portée des liens entre besoin en logements, urbanisation planifiée et mitage ont également été questionnées. Enfin, les participants ont insisté sur la nécessité de mettre à l'épreuve et de valider le modèle au travers d'une analyse des dynamiques historiques de mitage, et d'engager une réflexion spécifique sur les indicateurs permettant d'évaluer et de simuler les impacts socio-écologiques du mitage.

L'atelier s'est également révélé une bonne opportunité pour l'équipe technique du TCO, en défaut de communication récurrent avec le secteur agricole, d'exposer ses objectifs en lien avec le projet de recherche : à savoir, répondre à une demande des élus du TCO et développer des moyens techniques et institutionnels permettant d'explorer de manière non sectorielle les futurs possibles du territoire. Enfin, concernant le positionnement des différents acteurs, l'atelier a permis de mettre en évidence l'existence de perspectives divergentes concernant les moyens de gérer la problématique du mitage : un premier groupe de participants mettant l'accent sur la nécessité d'améliorer la réponse aux besoins en logements (c'est-à-dire plus de constructions et une meilleure prise en compte des différenciations socio-économiques); un second groupe favorisant plutôt le contrôle et la protection stricte des espaces agricoles et naturels.

Cette réflexion collective autour de la problématique du mitage a servi de fondation pour structurer et établir une arène de travail collaboratif portée par l'intercommunalité du TCO et animée par l'équipe scientifique au travers de forums (ateliers et groupes de travail) sur les dynamiques urbaines, agricoles et environnementales à venir au sein du TCO. Ainsi, entre avril et octobre 2013, une série de consultations et deux ateliers ont été organisés afin d'élaborer collectivement des scénarios contrastés et d'en débattre. Ces 
scénarios décrivent à moyen terme (jusqu'en 2025) les interactions possibles entre différentes politiques et dynamiques urbaines et agricoles planifiées ou déjà à l'œuvre sur le territoire du TCO. À terme, la traduction de ces scénarios en simulations dans différents modèles implémentés en Ocelet et la construction d'indicateurs de suivi permettront d'évaluer les impacts socio-économiques et environnementaux possibles.

\section{Discussion et conclusion}

Le choix de mobiliser des modèles contextualisés et le développement incrémental du modèle, passant d'une version simple, servant de démonstrateur, à une version plus élaborée pour représenter les processus complexes contribuant à l'étalement urbain, ont largement concouru à faciliter l'émergence d'une arène de travail collaboratif. Nous avons ainsi pu mobiliser un réseau d'acteurs et organiser différents ateliers. En ce sens, les modèles développés ont joué le rôle d'objet intermédiaire, facilitant, notamment, l'échange de points de vue sur les facteurs à l'origine de l'étalement urbain.

La capacité de la plateforme de modélisation Ocelet à produire des cartes dynamiques a aussi permis de donner à voir des résultats tangibles et concrets pour nos partenaires. Ces cartes ont été particulièrement efficaces, au début du projet, pour démontrer l'aptitude de notre équipe à représenter des processus de dynamiques territoriales complexes et, en conséquence, à intéresser nos partenaires. Néanmoins, la représentation cartographique des simulations des différents scénarios a fait l'objet de débats au sein du réseau d'acteurs. En effet, une bonne partie d'entre eux était réticente sur le choix d'une granularité fine, jugée trop précise et susceptible d'être mal interprétée, par les élus notamment.

Le point de vue du réseau d'acteurs sur la démarche a été globalement positif. Néanmoins, deux groupes s'opposent sur l'utilisation de ce type de modélisation. L'un privilégie une fonction de communication mobilisant des modèles très simples au service d'un plaidoyer. L'autre vise clairement à mobiliser ces outils en appui à la décision, ce qui requiert le développement de modèles complexes ayant une portée heuristique.

Les acteurs et gestionnaires du territoire d'expérimentation du projet Descartes ont trouvé un intérêt à la démarche du projet et à l'utilisation de ces outils de simulation. En effet, les gestionnaires de ce même territoire ont initié un projet de Gestion intégrée de la mer et du littoral (GIML). Ce projet, à l'échelle d'un bassin versant, remobilise certains des modèles développés dans le cadre du projet Descartes, dans la perspective d'objectiver les différents scénarios qui auront été construits et mis en débat.

\section{Remerciements}

Cette recherche est en partie financée par l'Agence nationale de la recherche à travers le projet Descartes (ANR 11-AGRO-002-01).

\section{Références bibliographiques}

Agorah, 2008a. Tache urbaine et espaces agricoles à la Réunion. Note de travail du groupe étalement urbain, nov. 2006, Agorah, 13 p.

Agorah, 2008b. Densification de la tache urbaine réunionnaise, tendances actuelles et perspectives 2020. Travaux du groupe « Étalement Urbain », Agorah, 46 p.

Alcamo J., Kok K., et al., 2006. Searching for the future of land: scenarios from the local to global scale. In: Land-Use and Land-Cover Change (E.E.F. Lambin, H.J. Geist), Springer Berlin Heildelberg.

Antona M., D’Aquino P., Aubert S., Barreteau O., Boissau S., Bousquet F., Daré W., Étienne M., Le Page C., Mathevet R., Trébuil G., Weber J., 2005. La modélisation comme outil d'accompagnement. Natures Sciences Sociétés, (13), 165-168. 
Bertrand N., Souchard N., Rousier N., Martin S., Micheels M.C., 2006. Quelle contribution de l'agriculture périurbaine à la construction de nouveaux territoires : consensus ou tensions ? Revue d'économie régionale et urbaine, (3), 329-353.

Beuret J.-E., Cadoret A., 2009. Une gouvernance territoriale endogène de l'environnement : contours et enjeux. In : $3^{e s}$ Journées de recherches en sciences sociales, Montpellier.

Coteba, Deal Réunion, 2010. Éléments de méthode pour une politique de densification à la Réunion. Rapport d'étude, juillet 2010, 255 p.

Commissariat Général au Développement Durable (CGDD), 2012. Urbanisation et consommation de l'espace, une question de mesure. Mars 2012.

David D., 2010. Prospective territoriale par simulation orientée agent. Phd Thesis, université de la Réunion.

Deal Réunion, 2011. Étude du mitage à la Réunion. Habitat et société, Fondation des villes. Rapport d'étude, juillet 2011, 166 p.

Degenne P., Lo Seen D., 2016. Ocelet: Simulating processes of landscape changes using interaction graphs. SoftwareX, 5, 89-95.

Forrester J.W., 1968. Principles of Systems, Wright-Allen Press Cambridge, Mass.

Geist H.J., McConnell W., et al., 2006. Causes and trajectories of Land-Use/Cover Change. In: Land-Use and Land-Cover Change (E.E.F. Lambin, H.J. Geist), Springer Berlin Heildelberg.

Insee, 2011. Le Tableau économique de La Réunion 2011, 92 p.

Jarrige F., Thinon P., Nougaredes B., 2006. La prise en compte de l'agriculture dans les nouveaux projets de territoires urbains. Exemple d'une recherche en partenariat avec la Communauté d'agglomération de Montpellier. Revue d'économie régionale et urbaine, (3), 393-414.

Jauze J.M., 1998. L'urbanisation de l'île de la Réunion : évolution et modèles de villes. Cahiers de géographie du Québec, 42 (116), 195-221.

Kok K., Verburg P.H., et al., 2007. Integrated assessment of the land system: the future of land use. Land Use Policy, 24 (3), 517-520.

Lajoie G., Actif N., 2001. Des villes-jardins qui s'étalent depuis dix ans. Économie de la Réunion, (108), Dossier : « La ville s'étale », 16-19.

Lambin E.F., Geist H.J., et al., 2006. Introduction: local processes with global impacts. In: Land-Use and Land-Cover Change, Local Processes and Global Impacts (E.E.F. Lambin, H.J. Geist), Springer Berlin Heidelberg.

Lardon S., Piveteau V., 2005. Méthodologie de diagnostic pour le projet de territoire : une approche par les modèles spatiaux. Géocarrefour, 80 (2), 75-90.

Maurel P., Craps M., Cernesson F., Raymond R., Valkering P., Ferrand N., 2007. Concepts and methods for analysing the role of Information and Communication tools (IC-tools) in Social Learning processes for River Basin Management. Environmental Modelling and Software, 22 (5), 630-639.

McCarthy M.A., H.P. Possingham, 2006. Active adaptive management for conservation. Conservation Biology, 21 (4), 956-963.

Metzger P., 2004. Les enjeux de la question urbaine dans l'aménagement régional à l'île de La Réunion. Espace populations sociétés, [en ligne], 2004/2.

Sheppard S.R., Cizek P., 2009. The ethics of Google Earth: Crossing thresholds from spatial data to landscape visualization. Journal of Environmental Management, 90 (6), 2102211.

Schreiber E.S.G., Bearlin A.R., Nicol S.J., Todd C.R., 2004. Adaptive management: a synthesis of current understanding and effective application. Ecological Management and Restoration, 5 (3), 177-182. 
Souchard N., 2013. Sortie de planification : les voies incertaines de la gouvernance des terres périurbaines. In : Terres agricoles périurbaines, une gouvernance foncière en construction (Bertrand N., ed.), éditions Quæ, p. 81-99.

Van Ittersum M.K., Rabbinge R., van Latesteijn H.C., 1998. Land use studies and their role in strategic policy making. Agricultural Systems, 58 (3), 309-330.

Verburg P.H., Kok K., et al., 2006. Modeling Land-Use and Land-Cover Change. In: Land-Use and Land-Cover Change, Springer Berlin Heildelberg.

Vinck D., 2000. Pratiques de l'interdisciplinarité. Mutation des sciences, de l'industrie et de l'enseignement, PUG, Grenoble.

Volk M., Lautenbach S., van Delden E., Newham L., Seppelt R., 2010. How can we make progress with decision support systems in landscape and river basin management? Lessons learned from a comparative analysis of four different decision support systems. Environmental Management, (46), 834-849.

White R., Engelen G., 1993. Cellular automata as the basis of integrated dynamic regional modelling. Environment and Planning A, 25, 1175-1199.

Wollenberg E., Edmunds D., et al., 2000. Using scenarios to make decisions about the future: anticipatory learning for the adaptive co-management of community forests. Landscape and Urban Planning, 47, 65-77. 


\title{
Chapitre 10
}

\section{La cocréation du futur alimentaire de Sydney : informer et transformer la gestion stratégique de l'agriculture périurbaine à travers un nouveau processus participatif}

\author{
Dana Cordell, Laura Wynne, Brent Jacobs
}

\section{Introduction}

La sécurité alimentaire est une priorité globale qui constitue le second objectif de l'Organisation des Nations unies en matière de développement durable ${ }^{36}$. Étant donné qu'aujourd'hui, plus de la moitié de la population mondiale vit dans les villes, la question de savoir comment nourrir la population urbaine de la planète est de la plus haute importance. Cependant les zones périurbaines - le grenier alimentaire traditionnel de nos villes - sont menacées. À mesure que les villes s'étendent, du fait de l'installation croissante de la population en zones urbaines (Rydin et al., 2012 ; Seto et al., 2012), les sols nourriciers se trouvant à leurs péripheries sont progressivement artificialisés. Ce processus met en péril l'ensemble des bénéfices fournis par l'agriculture périurbaine, qui vont de la sécurité alimentaire à l'habitabilité urbaine, en passant par la réduction des kilomètres alimentaires et des déchets. La conversion croissante de terres agricoles pour des usages commerciaux et résidentiels, la fragmentation des terres, les conflits d'usage ainsi que les défis globaux comme le changement climatique sont des menaces pour la viabilité de la production alimentaire dans les zones périurbaines. Malgré cela, les risques liés à la perte des terres agricoles périurbaines et les bénéfices liés à leur conservation ne sont pas pris en considération dans les processus d'aménagement urbain des métropoles du monde entier.

Répondre aux défis complexes de la durabilité nécessite des changements profonds et non des ajustements progressifs du système (Hackman et St Clair, 2012). Pour créer ces changements profonds, il est nécessaire, entre autres choses, d'encourager l'apprentissage collectif et la coproduction de savoirs entre acteurs et chercheurs (Mitchell et al., 2015). Dans le cadre d'un projet de recherche récent, le Futur alimentaire de Sydney (Sydney's Food Futures), qui vise à encourager la transformation de la planification périurbaine à Sydney, en Australie (ISF, 2016a), les acteurs ont été invités à participer à plusieurs étapes du projet de recherche. Leur participation avait deux buts clés : premiè-

36. http://www.undp.org/content/undp/en/home/sustainable-development-goals.html . 
rement, permettre l'apprentissage collectif et la coproduction de savoirs entre divers acteurs ayant des difficultés à communiquer, du fait de valeurs, perceptions et objectifs différents (situation proche de celle décrite par Grove et al., 2016, p. 5) ; et deuxièmement, créer une stratégie basée sur la collaboration pour définir les actions et les trajectoires à adopter afin de parvenir à un futur alimentaire résilient à Sydney.

Dans ce chapitre, nous discutons des nouvelles méthodes d'engagement employées pour étudier les défis et les opportunités de planification en matière d'agriculture périurbaine à Sydney. La situation dans les zones périurbaines de Sydney est semblable à n'importe quelle autre périphérie urbaine à travers le monde. Ainsi, les idées développées et les leçons tirées du cas de Sydney seront probablement pertinentes pour les chercheurs, les décideurs et les aménageurs dans d'autres contextes. Pour ancrer ce processus participatif dans son contexte, nous proposons d'abord de donner une vue d'ensemble des bénéfices tirés de l'agriculture périurbaine et des défis rencontrés par le secteur agricole à Sydney. Nous décrirons aussi le type de modélisation mis en œuvre pour comprendre les futurs possibles pour la production alimentaire dans la plus grande ville d'Australie.

Sydney compte approximativement 4,5 millions d'habitants et se situe dans un large bassin versant (le bassin de Sydney) sur la côte est de l'Australie, dans l'État de Nouvelle-Galles du Sud. La ville se caractérise par une absence historique de planification urbaine qui a conduit à un développement quasi organique, passant ainsi d'un petit village de colons européens sur la baie de Sydney, dans les années 1800, à une ville tentaculaire de plusieurs millions d'habitants où les banlieues se développent dans toutes les directions.

Traditionnellement, les zones périurbaines de Sydney étaient des zones de production alimentaire. La pluviométrie relativement régulière et le climat doux du bassin avaient permis le développement d'une ceinture verte agricole partant du sud de la ville, passant à l'ouest, et remontant au nord, vers la Central Coast et le bassin de l'Hawkesbury. Aujourd'hui, une part significative des terres productives de Sydney a été convertie à d'autres usages - principalement résidentiels — du fait d'une mauvaise planification, d'un développement désordonné et d'un manque de planification environnementale prenant en compte l'occupation du sol (Low Choy et al., 2008).

La conversion constante de terres agricoles pour des usages résidentiels a alimenté la perception, largement partagée par beaucoup d'Australiens et de décideurs dans les zones périurbaines, que les zones périurbaines étaient des «banlieues en devenir » (Bunker et Holloway, 2001, p. 13), et que les terres agricoles étaient simplement en sursis, en attendant d'être converties en logements par des investisseurs (Rothwell et al., 2015). En effet, en Australie, la définition qui est donnée des zones périurbaines englobe « les périphéries urbanisées des villes ainsi que les espaces sur lesquels elles se développent, physiquement et fonctionnellement » (italique ajouté) (Burnley et Murphy, 2004, p. 245). Cette conceptualisation des régions périurbaines, combinée à une augmentation continue de la population, a fait de Sydney une ville qui « absorbe constamment sa périphérie et crée une "nouvelle" périphérie toujours plus éloignée du centre-ville » (Golledge, 1960, p. 243). 


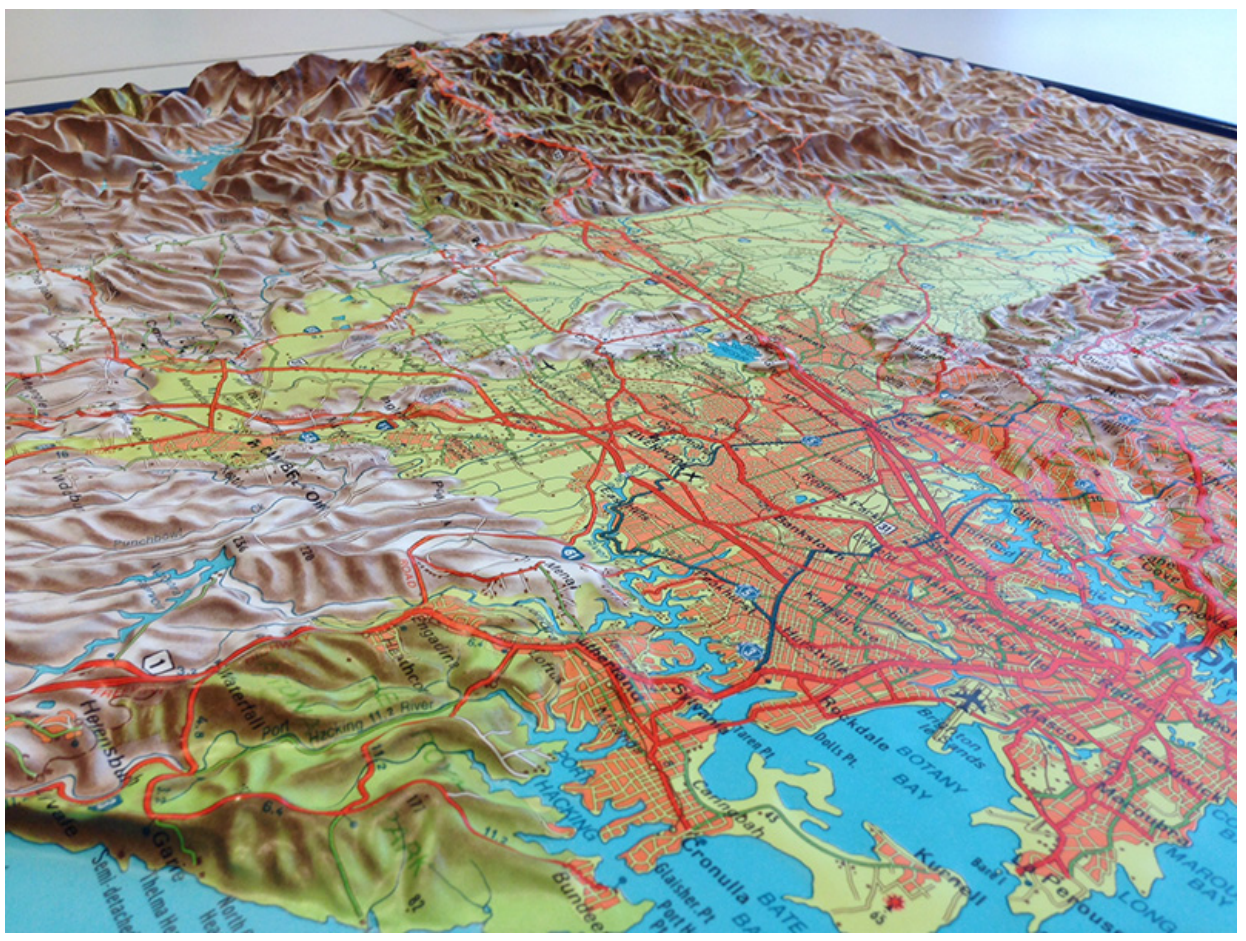

Figure 10.1. Un bassin aux contraintes géographiques importantes : la région de Sydney (the Greater Sydney region) (12300 km²) se situe dans un large bassin hydrographique délimité par des réserves naturelles, des bassins versants clos et la zone montagneuse de la Cordillère australienne (Great Dividing Range) (source : site web du projet Sydney's Food Future, www.sydneyfoodfutures.net/interactive-maps/ ).

La zone métropolitaine de Sydney est pourtant soumise à de nombreuses contraintes topographiques (figure 10.1). Avec des parcs nationaux au nord et au sud, l'océan à l'est et les Montagnes Bleues (Blue Mountains) à l'ouest, la ville ne peut pas s'étaler indéfiniment. Aujourd'hui, les terres agricoles se retrouvent prises en étau entre les banlieues périphériques qui se développent et les contraintes topographiques. Par conséquent, si Sydney veut conserver son grenier alimentaire, des mesures doivent être prises pour faire face au développement actuel de la ville dans les zones périurbaines.

Les zones périurbaines constituent une interface dynamique entre les villes et les espaces ruraux. Dans les pays développés, ces régions sont souvent composées d'un mélange d'usages urbains et ruraux. On y retrouve typiquement de l'habitat résidentiel (souvent sur de larges propriétés), des exploitations agricoles de petite et moyenne tailles avec des productions comme l'horticulture, des activités semi-rurales comme l'élevage de chevaux, ainsi que quelques villes, souvent concentrées autour de plateformes de transport. Même si les régions périurbaines présentent de nombreux points communs avec les régions urbaines, comme par exemple la législation en matière de planification ou les services d'approvisionnement en eau et en énergie, elles remplissent aussi d'autres fonctions, en appui aux zones urbaines qu'elles entourent.

Dans la section suivante, nous expliquons pourquoi les zones périurbaines méritent d'être préservées et nous discutons des principaux défis auxquels sont confrontées les périphéries des villes du monde entier. 


\section{Contexte}

\section{L'importance du système alimentaire périurbain}

En raison de leur proximité au marché et la population, les zones périurbaines jouent un rôle important dans l'alimentation des populations urbaines - particulièrement en ce qui concerne les denrées périssables ou inadaptées au transport sur de longues distances, comme les légumes frais ou les œufs. Cependant, les zones périurbaines sont aussi des endroits dans lesquels nos villes ont tendance à s'étendre au cours du temps. De ce fait, elles risquent d'être remplacées par des logements ou des infrastructures.

Innovations et opportunités émergent souvent en périphérie ou à l'intersection de systèmes. Il en va de même pour les zones périurbaines. Étant à l'interface entre l'urbain et le rural, elles offrent la possibilité de capitaliser sur les caractéristiques uniques des deux types d'occupation du sol - comme, par exemple, le recyclage des déchets alimentaires et des déchets verts pour en faire du compost de bonne qualité pour les exploitations agricoles.

L'agriculture périurbaine fournit d'importants bénéfices à nos villes, notamment (voir ISF, 2016a et ISF, 2016b pour une description complète de ces bénéfices) :

- la réduction des gaz à effet de serre et de la production de déchets, puisque les aliments produits localement sont transportés sur de plus courtes distances et stockés moins longtemps. Ils génèrent par conséquent moins de gaspillage alimentaire que les aliments produits plus loin du point de vente ;

- des bénéfices socio-économiques associés à l'emploi et au développement économique — l'agriculture périurbaine à Sydney contribue à hauteur de 4,5 milliards de dollars australiens à l'économie locale, avec un rendement à l'hectare plus élevé que les cultures à grande échelle (Houston, 2005), si l'on inclut les activités qui lui sont associées comme la transformation des aliments et l'agrotourisme ;

- des bénéfices liés à la sécurité alimentaire : une plus grande fiabilité de l'approvisionnement, une plus grande fraîcheur des produits, des prix plus abordables du fait des courts trajets, moins sensibles à des aléas et à des chocs pétroliers futurs (qui pourraient conduire à l'augmentation des prix de la nourriture importée) ;

- des bénéfices liés aux écosystèmes, tels que l'apport d'espaces verts et le maintien de la biodiversité ;

- des bénéfices liés à l'habitabilité urbaine, tels que le refroidissement de la température en ville et la réduction des inondations.

Cependant, les défis auxquels les zones périurbaines sont actuellement confrontées menacent leur capacité à produire ces bénéfices.

\section{Les risques et les défis}

Un des défis clés pour l'agriculture périurbaine dans le bassin de Sydney — et dans la plupart des régions périurbaines du monde - est la conversion de terres agricoles vers des usages résidentiels, commerciaux ou d'autres types d'usages non agricoles. L'urbanisation croissante des terres est un phénomène global. En Europe, au moins 2,8 \% des terres ont subi un changement d'occupation du sol entre 1990 et 2000, et la majorité de ces changements d'occupation du sol correspond à une augmentation des zones urbaines (La Rosa et al., 2014). À Sydney, comme dans d'autres villes australiennes, une part importante des terres utilisées pour la production alimentaire a été convertie à d'autres usages — principalement résidentiels. 
À l'heure actuelle, l'agriculture périurbaine à Sydney se trouve confrontée à plusieurs défis interdépendants (d'après ISF, 2016b) :

- de multiples acteurs aux intérêts divers. De nombreux acteurs sont impactés par l'utilisation des terres en milieu périurbain et influencent ce processus. Il s'agit des agriculteurs, des résidents, des promoteurs immobiliers, des gestionnaires de l'environnement, des aménageurs, des gestionnaires de l'eau et des prestataires de services de santé. Chacun a des priorités multiples qui entrent souvent en concurrence ou en conflit avec celles des autres;

- l'aménagement du territoire est dicté par le marché. La consolidation urbaine repose sur les usages générant le plus de profits, ce qui revient à condamner toute perspective d'un redéveloppement de la production alimentaire dans les zones urbaines (Gleeson, 2013). La valeur élevée de l'immobilier résidentiel à Sydney mène ainsi à une conversion continue de terres agricoles en logements ;

- les agriculteurs en zones périurbaines, qui sont les acteurs ayant le plus à gagner de la préservation des terres agricoles, font face à un dilemme qui se pose en des termes différents en fonction de leur âge (Cook et Harder, 2013). Certains agriculteurs préfèrent que leurs terres restent agricoles pour les générations futures, d'autres - forcés par l'âge, le handicap et les pressions financières liées à un déménagement de l'exploitation ou à un départ en retraite - voient la vente de leur ferme à des promoteurs comme une opportunité financière leur permettant de partir en retraite ou de développer une nouvelle exploitation agricole hors du bassin de Sydney;

- l'agriculture dans le bassin de Sydney n'a pas été considérée comme une priorité depuis plusieurs dizaines d'années. En raison de l'accent mis sur l'objectif de faire de Sydney une « ville globale» (global city), avec des emplois en centre-ville et le développement de logements, la situation très dégradée dans laquelle se trouve la production agricole passe inaperçue. Les plans d'aménagement actuels, comme par exemple le Plan pour la Croissance de Sydney (Plan for Growing Sydney) (NSW Department of Planning and Environment, 2015), évoquent peu l'agriculture et son rôle pour la résilience du bassin de Sydney ;

- l'intensification des conflits résultant de l'augmentation du nombre d'habitations résidentielles dans les zones périurbaines. Du fait du morcellement des terres dans les zones périurbaines, des habitations résidentielles se trouvent désormais à proximité d'exploitations agricoles, pouvant amener à des conflits entre agriculteurs et résidents (Ruoso, 2018). Dans beaucoup de cas, la résolution de ces conflits se fait au détriment des agriculteurs, qui connaissent ensuite des baisses de productivité dues aux contraintes qui s'exercent sur leurs exploitations. À Sydney, beaucoup de ces agriculteurs sont obligés de s'installer en dehors du bassin ou, plus radicalement, de vendre leurs terres et de prendre leur retraite ;

- les pressions globales comme le changement climatique ou la pénurie de phosphore à l'échelle mondiale sapent la résilience du système alimentaire urbain. En raison de la menace posée par un climat plus extrême et instable, sur le rendement des récoltes, il devient nécessaire de protéger les terres les plus fertiles des régions côtières pour approvisionner la population en nourriture. De plus, les systèmes alimentaires locaux peuvent mieux répondre à la menace globale grandissante de pénurie de phosphore ${ }^{37}$, qui pourrait mener à des envolées du prix des engrais et à des perturbations dans l'approvisionnement (Cordell et White, 2014);

- la dégradation des sols et la désertification, qui conduisent au déclin de la fertilité des sols à l'échelle mondiale et menacent la capacité productive des terres agricoles sur tous

37. http://phosphorusfutures.net/the-phosphorus-challenge/the-story-of-phosphorus-8-reasons-whywe-need-to-rethink-the-management-of-phosphorus-resources-in-the-global-food-system/ . 
les continents ;

- les risques pour la biosécurité, dus à l'augmentation du transport d'aliments, de personnes, d'intrants et de produits à travers les frontières et entre les continents. Ces mouvements croissants de biens et de personnes introduisent des risques potentiels pour la biodiversité, en raison du possible transport de maladies et de nuisibles ;

- des menaces économiques et financières dues à la consolidation grandissante du secteur agroalimentaire en un petit nombre de multinationales, plutôt qu'en une diversité de petites structures de production. Cela engendre une vulnérabilité aux chocs et aux crises économiques ;

- la moyenne d'âge des agriculteurs en Nouvelle-Galles du Sud est élevée, et peu de jeunes agriculteurs s'engagent dans le secteur agricole. Du fait du vieillissement des agriculteurs et de leur départ à la retraite, Sydney pourrait bientôt connaître une pénurie de main-d'œuvre pour la production de son alimentation (Henry et al., 2013) ;

- le duopole constitué par les supermarchés Coles et Woolworth en Australie exerce des pressions à la baisse sur les prix des produits agricoles. Ces pressions affectent négativement la viabilité des exploitations agricoles dans le bassin de Sydney et en Australie plus généralement ${ }^{38}$.

\section{Modélisation de scénarios pour le futur de l'agriculture à Sydney}

Pour développer une bonne compréhension des implications de la stratégie métropolitaine actuelle (Un plan pour la croissance de Sydney) et d'autres scénarios possibles pour le futur de l'agriculture à Sydney, nous avons créé un modèle géospatial basé sur la modélisation du « bassin alimentaire » (Peters et al., 2009 ; 2012). Le concept de bassin alimentaire est comparable à celui de bassin versant et est utilisé à la fois comme un outil pour comprendre comment les aliments circulent dans le système alimentaire, et comme un modèle pour concevoir des systèmes alimentaires locaux alternatifs. Notre modèle évalue la production et la demande actuelle et future en aliments dans le bassin de Sydney, grâce à des projections soumises à un certain nombre de changements d'occupation du sol et de scénarios de croissance urbaine. Cela implique l'utilisation de projections de croissance démographique, des objectifs de croissance des communes, de données sur la production alimentaire actuelle et de données géospatiales d'occupation du sol, pour identifier la production actuelle, les différentes formes possibles de la croissance urbaine à venir et les impacts de ces différentes formes de croissance sur la production alimentaire. Le gouvernement de Nouvelle-Galles du Sud a fixé des projections de croissance pour les gouvernements locaux dans son récent Plan pour la croissance de Sydney. Cela inclut le développement résidentiel de zones périurbaines. La demande alimentaire a été calculée en utilisant des données sur le régime alimentaire des Australiens fournies par le CSIRO et des données démographiques fournies par le Bureau australien des statistiques (ABS, Australian Bureau of Statistics).

Les cartes produites représentent la production alimentaire actuelle ainsi que quatre scénarios futurs :

- production actuelle ${ }^{39}$ : cette carte utilise des données existantes pour cartographier la production en 2011 ;

- scénario d'étalement urbain à l'horizon 2031 : cette carte part du principe que le

38. https://www.themonthly.com.au/issue/2014/august/1406815200/malcolm-knox/supermarketmonsters .

39. Les données disponibles les plus récentes datent de 2011. Un travail à venir mettra à jour les scénarios pour qu'ils reflètent la production et la demande en 2016-2017. 
Plan pour la croissance de Sydney est mis en œuvre selon les prévisions de croissance actuelles ;

- scénario d'étalement urbain limité à l'horizon 2031 : cette carte part du principe que le Plan pour la croissance de Sydney est mis en œuvre selon les projections de croissance actuelles, mais que l'étalement urbain sur des terres agricoles de classe 1 est interdit; - scénario donnant la priorité à l'agriculture à l'horizon 2031 : ce scénario interdit tout étalement urbain sur des terres agricoles. Les aires urbaines existantes doivent donc être densifiées pour absorber la croissance de la population ;

- scénario d'agriculture intensive à l'horizon 2031 : ce scénario modélise les gains en productivité qui pourraient être obtenus par le biais de la préservation et de l'intensification des terres agricoles de Sydney.

La base de connaissances résultant de cette recherche a été un point de départ important pour la consultation des acteurs sur la faisabilité et la désirabilité de différents scénarios et pour discuter des mesures potentielles à mettre en place pour s'engager sur la voie souhaitée. Chacun de ces scénarios et leurs implications pour la production alimentaire sont discutés plus en détail ci-dessous.

\section{Production actuelle (2011)}

L'analyse a montré qu'en 2011, le bassin de Sydney a produit un demi-million de tonnes d'aliments - suffisamment pour satisfaire $20 \%$ de la demande de Sydney. Le bassin fournit une proportion significative de certains types d'aliments pour répondre à cette demande, notamment $40 \%$ des œufs, $38 \%$ des produits laitiers et $55 \%$ de la viande. À l'inverse, le bassin ne produit que $10 \%$ de sa demande en légumes et $2 \%$ de sa demande en fruits, soit moins que Melbourne, qui fournit actuellement $40 \%$ des produits frais locaux consommés par sa population (VEIL, 2015).

Le but de ce modèle était de montrer quelle était la production alimentaire « actuelle », mais les données disponibles les plus récentes remontaient à 2011. Compte tenu de l'étalement significatif de l'aire urbaine de Sydney ces cinq dernières années, particulièrement sur des zones auparavant agricoles autour de Leppington, où une nouvelle gare a ouvert pour soutenir la croissance de la population, nous nous attendons à ce que des pertes significatives du niveau de production se soient déjà produites depuis 2011 .

\section{Scénario d'étalement urbain au fil de l'eau}

Dans le cas du scénario d'étalement urbain au fil de l'eau, qui repose sur les objectifs actuels de croissance de Sydney, le modèle montre que la ville risque de perdre environ $60 \%$ de sa capacité de production alimentaire totale d'ici à 2031 par rapport à 2011 (la configuration spatiale de cette perte est présentée en figure 10.2). Les légumes, la viande et les œufs seraient les plus touchés : $92 \%$ de la production actuelle de légumes frais pourrait être perdue, ainsi que $91 \%$ de la production de viande et $89 \%$ de la production d'œufs (figure 10.3). Ceci est dû à l'étalement urbain dans les zones périurbaines.

Ce scénario d'étalement urbain au fil de l'eau était formulé de la manière suivante : «Et si l'agriculture de Sydney n'était pas protégée et si la croissance de la population telle que présentée dans le Plan pour la croissance de Sydney se produisait sans contraintes particulières ? » Ceci signifie que l'accroissement démographique d'ici à 2031 se produirait conformément à ce qui est prévu dans le Plan pour la croissance de Sydney, avec une répartition entre chacune des municipalités locales et une concentration dans les pôles de développement au nord-ouest et au sud-ouest. Les résultats montrent que les pertes prévues affecteraient de manière disproportionnée un petit nombre de municipa- 
lités (figure 10.4), dont celle de Wollondilly où ont été réalisés les exercices participatifs décrits dans la section suivante.
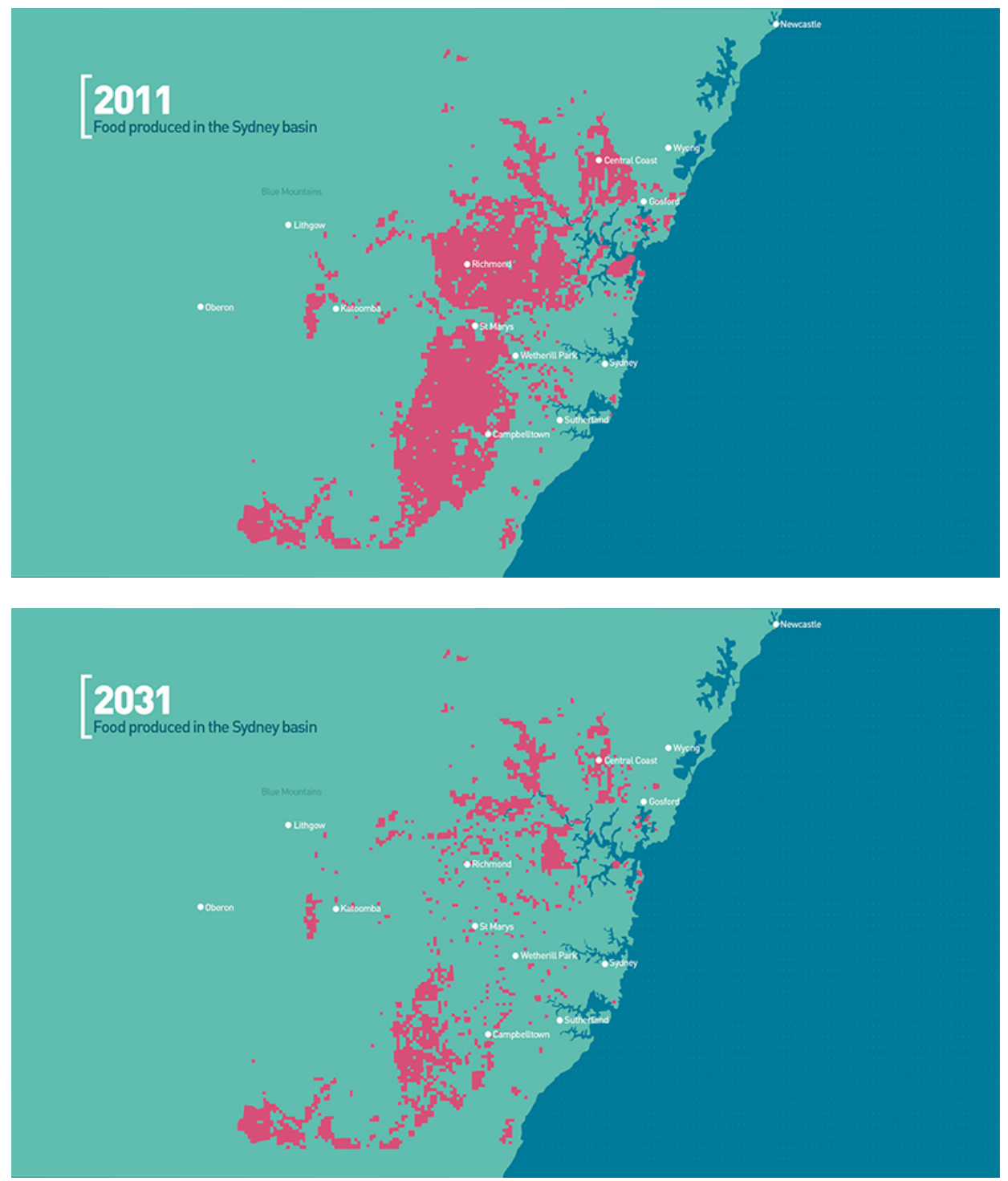

Figure 10.2. Représentation spatiale de la production alimentaire prévue entre 2011 et 2031 pour le scénario d'étalement urbain au fil de l'eau, qui illustre la perte potentielle pour l'agriculture si la croissance continue telle que prévue dans la planification stratégique urbaine (sydneyfoodfutures.net, 2016). 


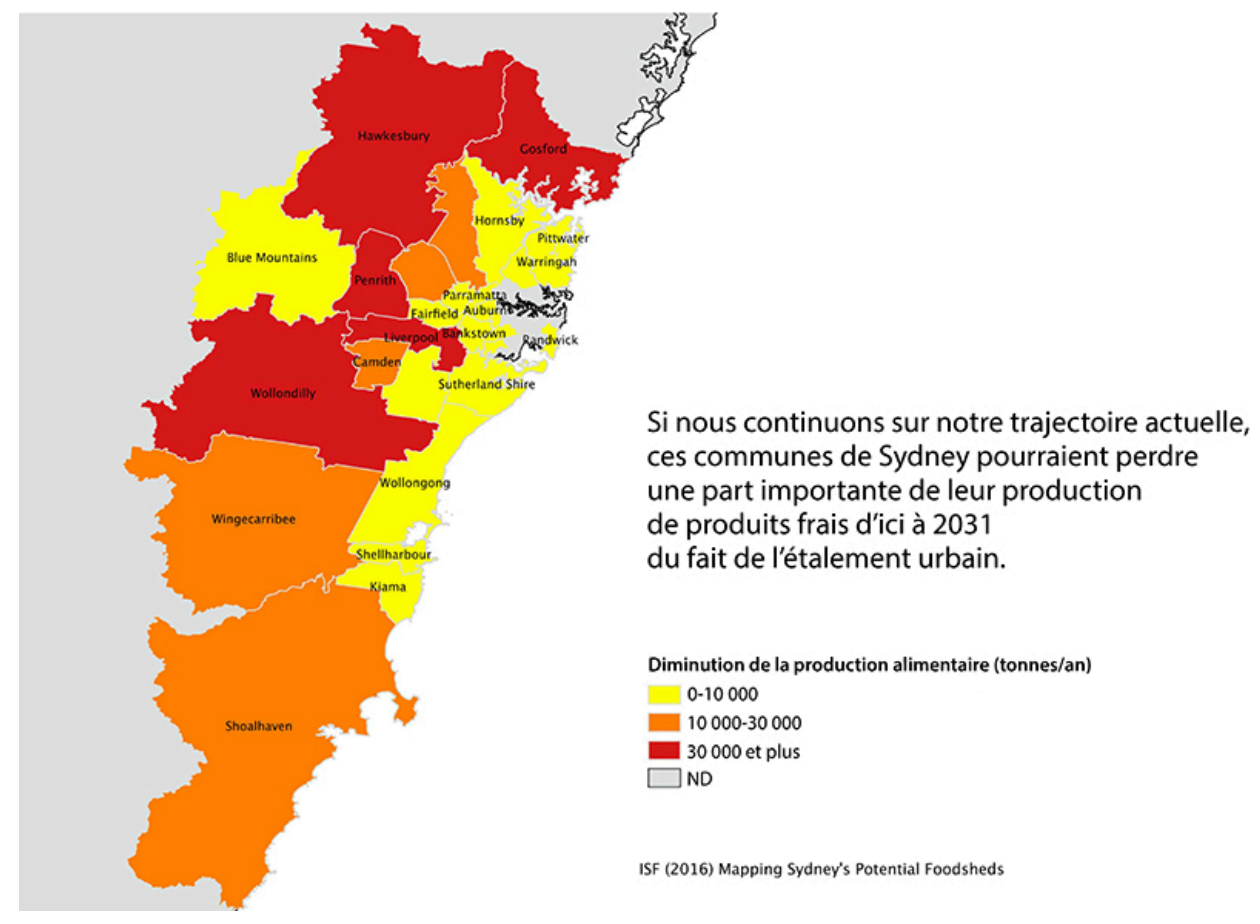

Figure 10.3. Perte en nourriture, par type d'aliment (en tonnes/an), dans le scénario d'étalement urbain.

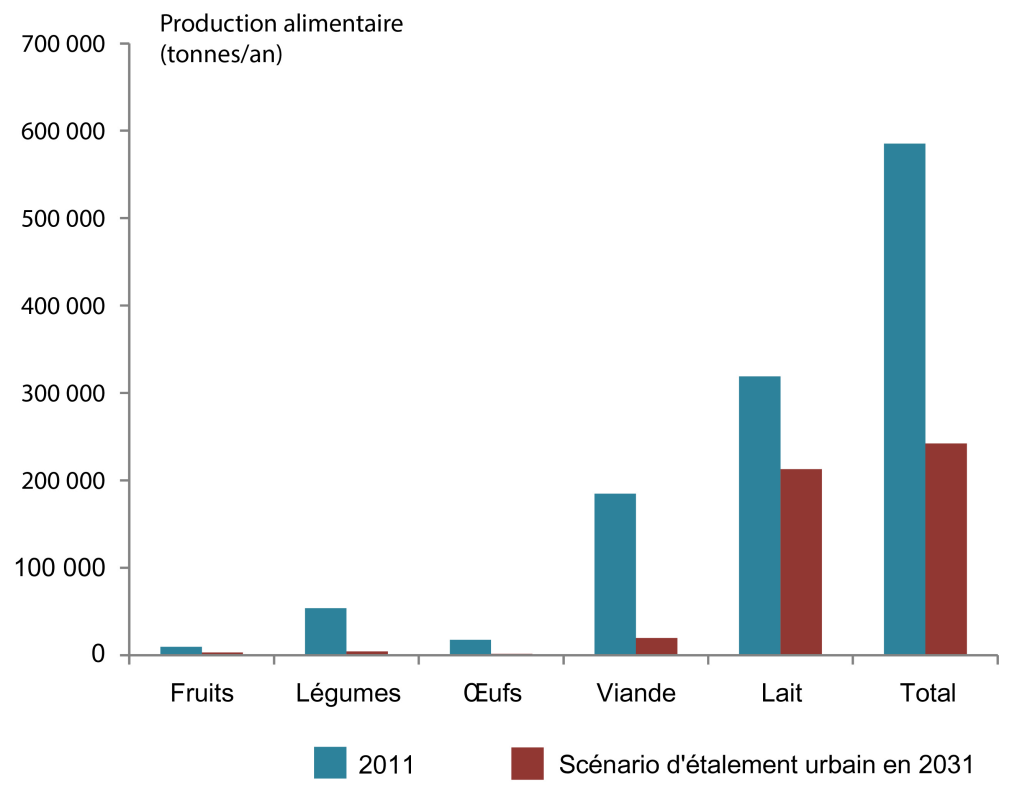

Figure 10.4. Baisse de la production alimentaire de 2011 à 2031 (en tonnes/an) pour chaque municipalité du bassin de Sydney. 


\section{Scénario d'étalement urbain limité}

Ce scénario posait la question suivante : «Et si le développement urbain se faisait dans des zones urbaines existantes ou des zones agricoles moins prioritaires ? » $\mathrm{Ce}$ scénario empêcherait l'étalement urbain sur des terres agricoles périurbaines de très bonne qualité (calculée sur la base de données sur la fertilité des sols et leur potentiel agronomique), mais autoriserait la croissance sur des terres agricoles de moins bonne qualité. Ce scénario a été développé en prenant en compte de nombreuses méthodologies d'évaluation des sols qui essaient de les classifier et de cataloguer pour ensuite faire des recommandations afin d'encourager la protection des terres considérées comme les plus précieuses pour l'agriculture périurbaine.

Les résultats montrent que ce scénario ne permettrait pas de protéger beaucoup plus de terres agricoles sur Sydney que le scénario d'étalement au fil de l'eau, l'écart entre les deux restant marginal. Ceci s'explique par le fait que seul un petit nombre de terres agricoles dans le bassin de Sydney sont considérées comme étant de " très bonne qualité ", ce qui signifie que ce scénario n'entraînerait qu'une protection limitée de la production alimentaire existante. La production alimentaire à l'horizon 2031 déclinerait presque aussi fortement que dans le cas du scénario d'étalement urbain au fil de l'eau. Dans le scénario d'étalement urbain limité, la capacité de Sydney à satisfaire ses besoins alimentaires avec des produits locaux passerait de $20 \%$ à $7 \%$ entre 2011 et 2031 . Comme pour le scénario d'étalement urbain au fil de l'eau, cela conduirait à une diminution de la production en légumes qui ne satisferait plus que $1 \%$ des besoins en 2031 .

\section{Scénario donnant la priorité à l'agriculture}

Ce scénario avait pour but d'étudier le cas où Sydney arriverait à préserver son agriculture périurbaine telle qu'elle est actuellement. La question était formulée ainsi : « Et si la croissance de la population prévue par le Plan pour la croissance de Sydney se produisait de manière contrainte, pour que les développements urbains prévus se fassent dans des zones résidentielles existantes, et non sur des terres agricoles ? »

Ce scénario suppose que Sydney protège ses capacités de production agricole actuelle (autour d'un demi-million de tonnes de nourriture par an) en optant pour un mode de développement urbain basé sur la densification des zones urbaines existantes plutôt que sur l'étalement urbain.

Néanmoins, même si, dans ce scénario, le tonnage reste le même qu'en 2011 en valeur absolue, le taux d'autosuffisance alimentaire de Sydney baisse en proportion, du fait de l'accroissement de la population et de la demande alimentaire accrue qui l'accompagne. Ce taux ne serait plus que de $14 \%$ en 2031 (figure 10.5). Nous avons estimé que les besoins alimentaires de Sydney passeraient d'environ 2,9 millions de tonnes par an en 2011 à 4,2 millions par an en 2031, du fait d'une augmentation de population de 1,6 million d'habitants.

\section{Scénario d'agriculture intensive}

Contrairement aux autres scénarios, ce scénario demandait : «Et si Sydney maximisait sa production agricole en termes de rendements (tonnes de nourriture produites par $\mathrm{km}^{2}$ ) ? » Dans ce scénario, les terres agricoles existantes sont converties en systèmes de productions agricoles maximisant les rendements, comme l'horticulture intensive (serres), l'élevage intensif de volailles et les parcs d'engraissement. Bien que ce scénario ne prenne pas en compte les questions de durabilité, comme la pollution de l'environnement, la réduction des aménagements urbains, le bien-être animal, et les questions de 
régime alimentaire équilibré, il indique qu'hypothétiquement la production alimentaire de Sydney pourrait être multipliée par huit et passer de 580000 tonnes par an actuellement à 5280000 tonnes. Sydney pourrait même devenir exportateur de légumes, d'œufs et de viande si la production agricole était maximisée et protégée. Le gain hypothétique dans ce scénario est largement associé à la conversion de pâturages en élevages et maraîchages intensifs à Wingecarribee, et, dans une moindre mesure, à l'intensification de l'agriculture dans les municipalités de Hawkesbury et Shoalhaven.

En réalité, l'intégralité de ces terres ne pourrait pas être utilisée pour de l'agriculture intensive, et beaucoup de limites existent quant à la plausibilité et la faisabilité de ce scénario, étant donné les risques en matière de rentabilité, d'investissement en capital, et d'autres questions comme la biosécurité. Ce scénario hypothétique a pour seul but de donner une idée du potentiel maximum de production alimentaire des terres agricoles, et n'a pas pour objectif d'indiquer la voie pour le futur.

Les figures 10.5 et 10.6 permettent de comparer ces quatre scénarios en montrant les écarts de production.

Étalement urbain à l'horizon 2031

A

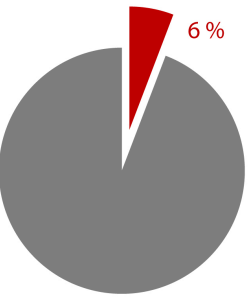

Étalement urbain limité à I'horizon 2031

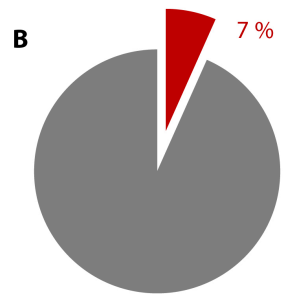

Priorité à l'agriculture à l'horizon 2031

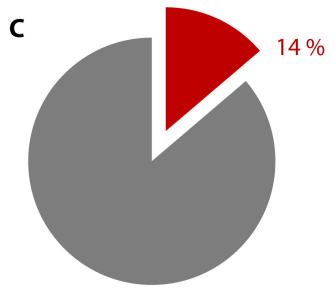

Production locale

Importation

Figure 10.5. Proportion de la demande alimentaire satisfaite par la production locale à Sydney, selon trois scénarios.

Note : le scénario d'intensification n'est pas représenté ici car la production locale de nourriture dépasse la demande (mais uniquement pour certaines productions dont les légumes, la viande et les œufs).

\section{Le processus participatif}

La modélisation géospatiale a montré les résultats de quatre scénarios possibles en matière de production alimentaire. Cependant, comprendre les implications sociales, financières, politiques et de santé ainsi que la désirabilité et la faisabilité de ces scénarios, nécessite de consulter les décideurs et les acteurs affectés par l'adoption de l'un ou l'autre de ces scénarios. 


\section{Production (t/an)}

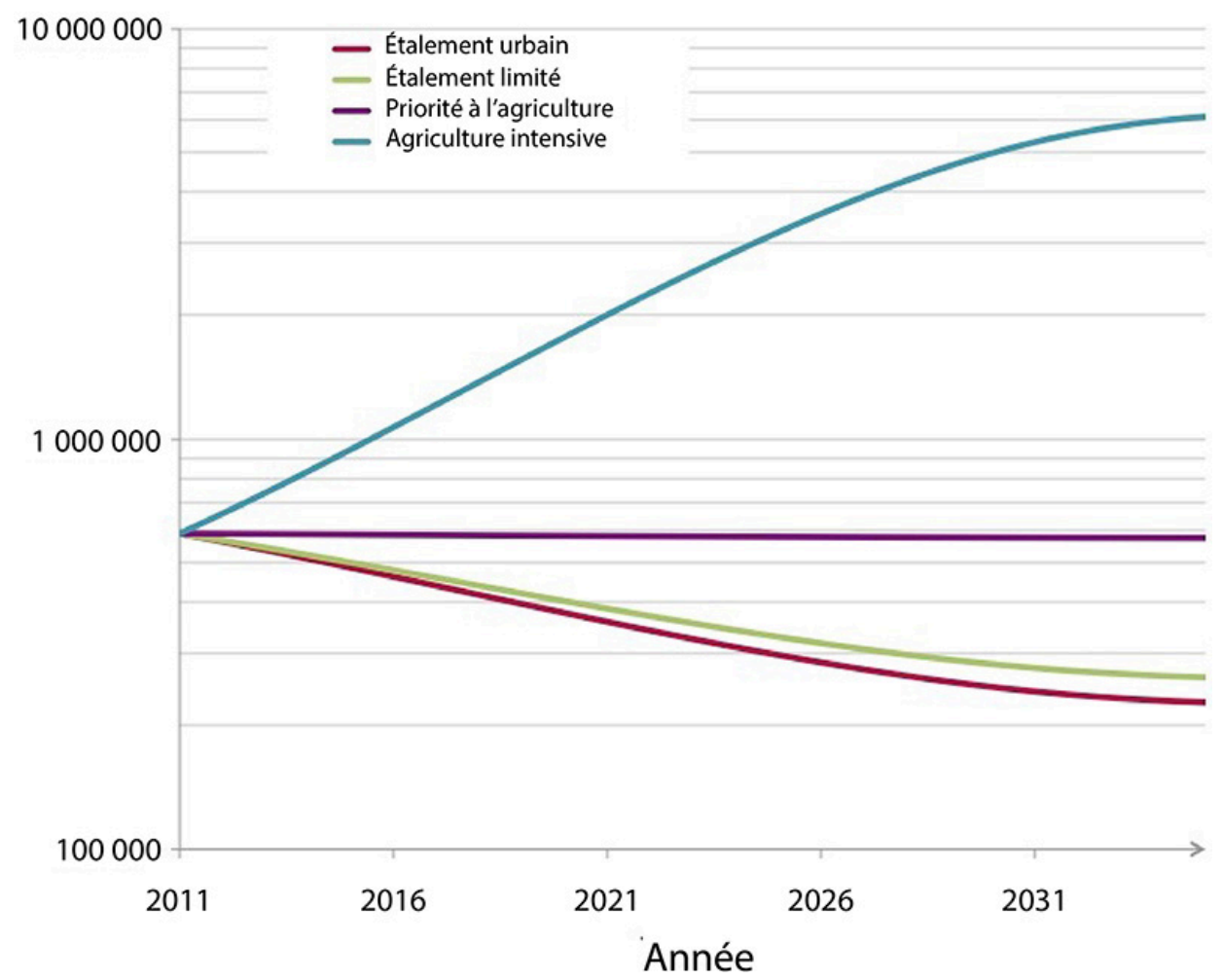

Figure 10.6. Quatre trajectoires possibles pour la production alimentaire future à Sydney.

En outre, la gestion des zones périurbaines reste complexe, du fait de la diversité des acteurs présents et de la variété de leurs motivations. Dans le bassin de Sydney, le manque de consensus et de vision commune entre les acteurs persiste en ce qui concerne les arrangements socio-institutionnels et la manière dont les zones périurbaines devraient être gérées. Par exemple, alors que les agriculteurs dans le bassin recherchent des conditions économiques favorables, un juste prix pour leurs produits, la sécurité foncière et l'acceptation sociale de leur activité, d'autres acteurs pensent que les exploitations agricoles devraient s'implanter en dehors du bassin. Les résidents estiment de leur côté qu'ils ont droit à un logement à des prix abordables, des emplois, des infrastructures pour le transport et l'accès à l'eau et à des prestations sociales. Les nutritionnistes et les professionnels de la santé défendent de plus en plus le droit des consommateurs à de la nourriture nutritive et abordable - particulièrement pour les individus les plus vulnérables et les habitants de Sydney Ouest - pour inverser les taux importants d'obésité et de diabète, et éviter les « déserts alimentaires $»^{40}$ (zones où l'accès à des épiceries ou supermarchés n'est ni facile ni équitable). Bien que ces priorités différentes puissent être perçues comme contradictoires, ceci n'est toutefois pas une fatalité.

Le but de nos deux processus participatifs était d'amorcer un dialogue et des débats constructifs sur les points de vue, les valeurs et les préférences de ces différents acteurs 
pour aller vers une meilleure compréhension mutuelle. Nous avons cherché, en particulier, à comprendre comment ces différences pouvaient être explorées et utilisées comme des pistes pour optimiser le système alimentaire, plutôt que d'être gérées comme des conflits directs ou des compromis où le gain pour un secteur constitue une perte pour un autre.

\section{Processus participatif. Première partie : apport du jeu dans la planification stratégique pour l'agriculture périurbaine}

Pour encourager la compréhension et l'apprentissage collectif, cet atelier participatif s'est concentré sur les idées, les réactions et les préférences des acteurs concernant les conséquences des scénarios préliminaires élaborés (tels que décrits dans la section sur la modélisation de scénarios pour le futur de l'agriculture à Sydney). Ensuite, pour renforcer l'apprentissage collectif, nous avons demandé aux acteurs d'élaborer ensemble un plan pour l'intégration de nouvelles habitations et, de manière plus générale, pour le développement de la commune de Wollondilly.

Cette commune située au sud-ouest de Sydney a traditionnellement été une région agricole. Cependant, elle fait face, à l'heure actuelle, à des pressions du gouvernement de Nouvelle-Galles du Sud et des marchés fonciers pour accueillir une part de la croissance urbaine prévue dans le Plan pour la croissance de Sydney. La figure 10.4 montre une perte importante de production alimentaire prévue pour la commune de Wollondilly. Les changements d'occupation du sol résultant de ces pressions génèrent des conflits qui ont été évoqués à plusieurs reprises par les participants au projet.

À travers ce processus participatif, nous avons cherché à mettre en lumière les perspectives et les priorités concurrentes et contradictoires des acteurs. Le but était de développer une compréhension collective au sein des différents groupes d'acteurs de la commune de Wollondilly. De plus, nous avons observé que, bien que les acteurs aient été conscients des problèmes existants, ils ne paraissaient pas savoir comment et pourquoi leurs intérêts concurrents créaient ces problèmes, ce qui a nécessité de se pencher plus avant sur la situation. Pour ce faire, un jeu basé sur la coproduction de savoirs a été conçu pour que les acteurs fassent l'expérience des difficultés inhérentes à la planification lorsqu'il s'agit de réconcilier des intérêts contradictoires.

La « ludification » est l'application de concepts utilisés dans les jeux vidéo à d'autres domaines comme l'éducation de la petite enfance, l'enseignement supérieur, le commerce, le marketing (Domínguez et al., 2013), dans le but de créer des « expériences ludiques » dans des contextes qui ne le sont pas (Hamari et al., 2014). Les jeux, activités interactives qui posent continuellement des défis et définissent des objectifs pour les utilisateurs, se sont avérés être un cadre porteur pour susciter la curiosité sur un grand nombre de sujets (Domínguez et al., 2013). Des recherches suggèrent que les jeux utilisés dans des contextes d'apprentissage peuvent renforcer la collaboration entre pairs (Rosas et al., 2003), développer l'envie d'apprendre (Domínguez et al., 2013), améliorer l'attention portée à une activité par les participants, et encourager l'acquisition fortuite de connaissances (Rosas et al., 2003).

Nous avons conçu un jeu de société comparable au processus de prise de décision dans une situation réelle, ce qui a permis aux acteurs de s'immerger dans les défis de la planification spatiale et de mettre en lumière la complexité des problèmes dans une zone géographique qui leur était familière. Le but du jeu était d'encourager les acteurs à partager leurs savoirs d'experts et leurs perspectives afin de générer une compréhen- 
sion collective, tout en résolvant des problèmes concrets de planification. Pour atteindre cet objectif, des acteurs de différents secteurs ont collaboré par équipes de dix environ, dans le cadre d'un atelier. Chaque équipe était constituée de représentants de différents secteurs — planification (représentants du gouvernement et des communes), agences gouvernementales (industries primaires et développement régional), organisations agricoles (par exemple, NSW Farmers), agriculteurs (par exemple, éleveurs de volaille) et associations de résidents. Chaque équipe comprenait des participants locaux et non locaux, certains amenant une connaissance de l'environnement intime et détaillée, alors que d'autres apportaient à la discussion des connaissances plus génériques sur les dynamiques dans le bassin de Sydney.

Tableau 10.1. Le processus participatif : ludification de la planification stratégique pour l'agriculture périurbaine.

\begin{tabular}{|c|c|}
\hline Étapes & Tâches \\
\hline Étape 1 & $\begin{array}{l}\text { Une carte de la commune de Wollondilly en format A0 a été fournie à chaque table, avec des } \\
\text { pièces représentant la croissance résidentielle prévue d'ici } 2031 \text { ( } 5000 \text { maisons), où chaque pièce } \\
\text { représente un groupe de } 50 \text { maisons pouvant avoir trois types de densités résidentielles : densité } \\
\text { rurale, densité de bourg rural, et densité de zone de croissance, basée sur la moyenne des zones } \\
\text { avoisinantes. Il a été demandé aux participants d'identifier sur la carte des zones où pourrait être } \\
\text { construit un nombre donné de maisons. Les participants ont été encouragés à prendre en } \\
\text { considération des facteurs comme les infrastructures, les services, les types de développements } \\
\text { existants, les espaces ouverts et l'occupation du sol existante. Cependant, aucune instruction } \\
\text { détaillée n'a été donnée au sujet de la localisation des maisons. À la place, ils étaient encouragés } \\
\text { à se baser sur les savoirs d'experts locaux du groupe. }\end{array}$ \\
\hline Étape 2 & $\begin{array}{l}\text { Une nouvelle couche cartographique a été introduite, indiquant la qualité des terres agricoles. La } \\
\text { couche était une feuille transparente qui indiquait l'index du potentiel agronomique des sols de la } \\
\text { région (NSW OEH, 2016), qui prend en compte la fertilité des sols, le drainage, la pente ainsi que } \\
\text { d'autres données pertinentes. Il était alors demandé aux participants de réévaluer leur décision } \\
\text { concernant la localisation de développements résidentiels à la lumière de cette nouvelle } \\
\text { information. }\end{array}$ \\
\hline Étape 3 & $\begin{array}{l}\text { Il a été demandé aux participants d'ajouter les services et les infrastructures — par exemple, les } \\
\text { écoles, les hôpitaux, les centres commerciaux — qui seraient nécessaires pour les nouveaux } \\
\text { résidents. }\end{array}$ \\
\hline Étape 4 & $\begin{array}{l}\text { Les participants ont dû localiser de nouveaux espaces pour développer plus de logements ( } 2000 \\
\text { de plus que ce qui était prévu par le plan actuel), en faisant l'hypothèse que la modélisation de la } \\
\text { population sous-estimait les demandes futures en logement (comme cela a été le cas dans le } \\
\text { passé). }\end{array}$ \\
\hline Étape 5 & $\begin{array}{l}\text { Un facteur de complexité supplémentaire a été introduit sous la forme d'un risque d'inondation } \\
\text { (une inondation tous les cent ans), un scénario qui pourrait se réaliser d'ici à } 2031 \text { compte tenu } \\
\text { des changements dans le profil des précipitations dans le bassin de Sydney (NSW OEH, 2016). } \\
\text { Ceci inonderait de vastes zones résidentielles à Wollondilly, dont la ville principale, Picton. Il a } \\
\text { été demandé aux participants de reconsidérer les sites sélectionnés pour le développement } \\
\text { résidentiel en prenant en compte la variable inondation. }\end{array}$ \\
\hline Étape 6 & $\begin{array}{l}\text { Pour la dernière étape, la période pour la planification du développement a été étendue jusqu'en } \\
2050 \text {, et il a été demandé aux participants de trouver des sites pour des logements, des services et } \\
\text { des infrastructures intégrés dans les zones de croissances urbaines prévues par le gouvernement } \\
\text { de la Nouvelle-Galles du Sud. Ceci impliquait de trouver des sites pour } 4000 \text { nouveaux } \\
\text { logements et les services associés. }\end{array}$ \\
\hline
\end{tabular}

\section{Le processus}

Il a été demandé aux participants d'entreprendre des tâches de planification stratégique, comme par exemple trouver des sites adaptés pour répondre aux prévisions de demandes 
en logements et en services associés aux logements. La complexité du défi augmentait à chaque nouvelle étape du jeu (tableau 10.1).

Les participants ont identifié collectivement les problèmes et solutions potentiels (figure 10.7) et se sont servis des connaissances des membres du groupe pour résoudre les problèmes. Par exemple, il a été observé que les participants utilisaient à la fois leurs connaissances de l'environnement local («Nous ne pouvons pas mettre des maisons là, cet élevage laitier est ici depuis plus longtemps que nous »), leurs connaissances de la situation globale («Ils vont amener la ligne de chemin de fer jusqu'à Leppington, peut être qu'un jour ils voudront l'étendre jusqu'ici, cela pourrait être un bon endroit pour des habitations ») et leur expertise sur des sujets spécifiques — par exemple, le potentiel agronomique des sols, la planification, les infrastructures.

Les principaux défis, idées et leçons auxquels le jeu a exposé les participants incluent : - la complexité : la difficulté de gestion de ce qui semble à première vue être un simple exercice de résolution de problème est devenue évidente lorsque chaque groupe a essayé de prendre en compte les intérêts, les priorités et les connaissances concurrentes des différents membres du groupe ;

- les informations et données nécessaires: le besoin et l'importance d'informations critiques lorsque des décisions sont prises sur le futur de l'occupation du sol ont été soulignés dans ce processus. Les participants ont observé que lorsqu'une nouvelle couche d'information était ajoutée (par exemple, les informations spatiales sur le potentiel agronomique des sols), une nouvelle série de questions émergeait concernant les décisions à prendre pour l'occupation du sol future. Il a été suggéré qu'intégrer ce type d'informations dans le processus de planification de l'occupation du sol pourrait améliorer le processus de prise de décision ;

- considérer de manière explicite les compromis : les participants ont observé que les décisions prenaient la forme d'une série de compromis entre des éléments considérés comme importants par différents secteurs, tels que développer des habitations à proximité de services essentiels versus protéger des terres agricoles de très bonne qualité. Le besoin d'une plus grande transparence concernant ces compromis a été considéré comme critique pour des acteurs approchant cette question sous des angles différents ;

- des perspectives concurrentes : malgré la faible diversité des parties prenantes dans chaque groupe de joueurs, les participants ont eu des difficultés à réconcilier des perspectives divergentes et ont reconnu qu'il serait nécessaire de mieux coordonner les activités et de communiquer de manière plus efficace sur les approches adoptées lors des processus de planification réels. 


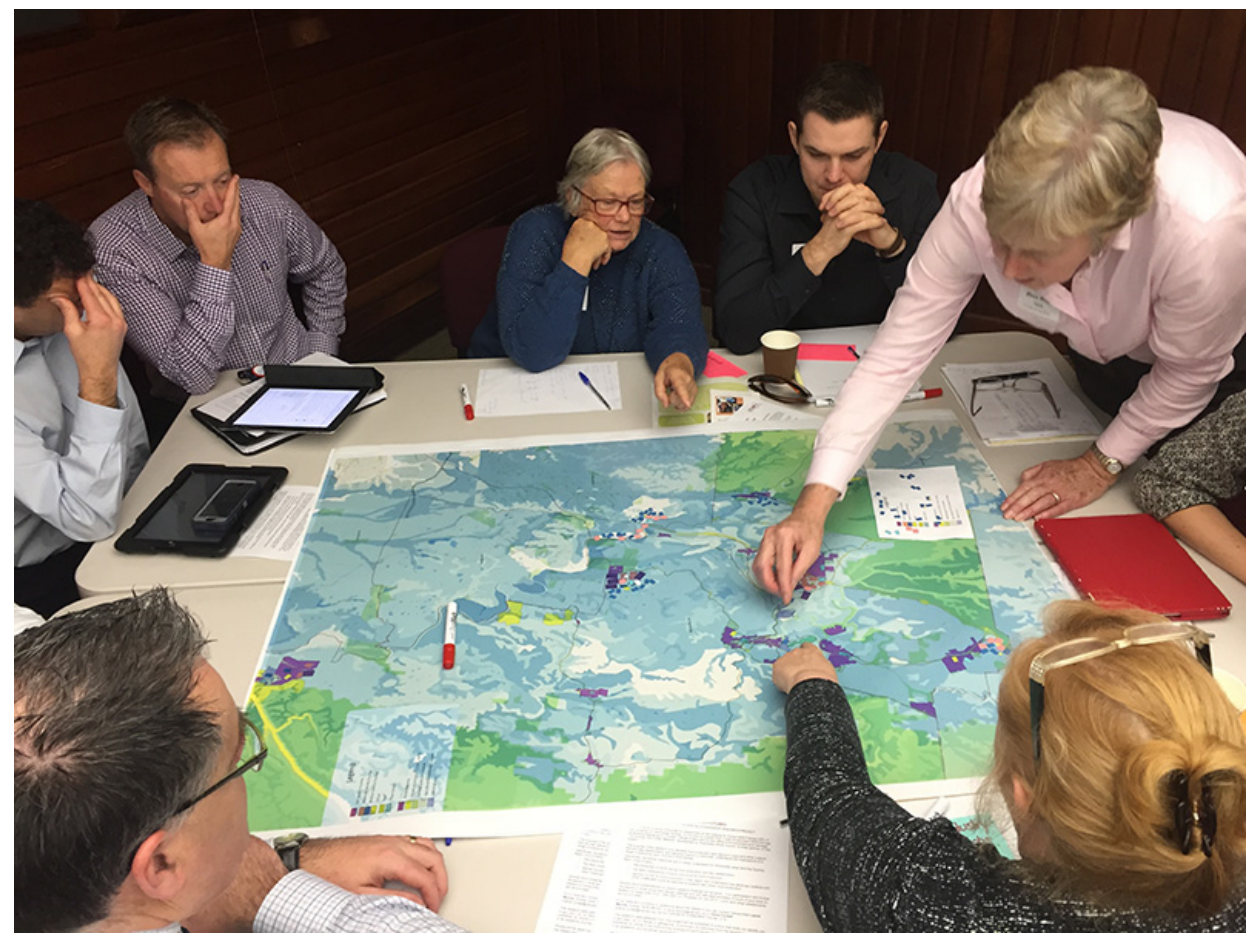

Figure 10.7. Acteurs participant à la renégociation de la localisation de nouvelles habitations dans une zone périurbaine de Sydney, après l'introduction d'une carte sur les inondations (période de retour centennale) comprenant des informations sur la fertilité des sols et leur potentiel agronomique.

\section{Processus participatif. Deuxième partie : synthèse et codéveloppement de trajectoires prioritaires}

Le deuxième processus participatif a pris la forme d'un atelier participatif avec des acteurs issus de divers secteurs du gouvernement, de l'industrie et du monde associatif, dont la santé, l'aménagement, le développement, l'environnement et l'agriculture. Le but de cet atelier était de consolider, de synthétiser et de construire une compréhension collective des vulnérabilités et des trajectoires possibles du système alimentaire de Sydney en explicitant et en synthétisant les savoirs tacites des acteurs présents (Grafton, 2010). Les activités principales de l'atelier ont été développées sur la base d'un modèle sur les trajectoires d'adaptation (figure 10.8). Elles visaient à :

- cartographier les impacts des facteurs de changements globaux et locaux sur le système alimentaire pour visualiser sa vulnérabilité (Loria, 2008 ; Shachter, 1986) ;

- identifier les étapes clés et les trajectoires nécessaires pour accomplir des changements dans le système (Haasnoot et al., 2013);

- développer une déclaration commune sur les attributs d'un système résilient, qui servirait de programme pour de futures actions collectives (Ostrom, 2014). 


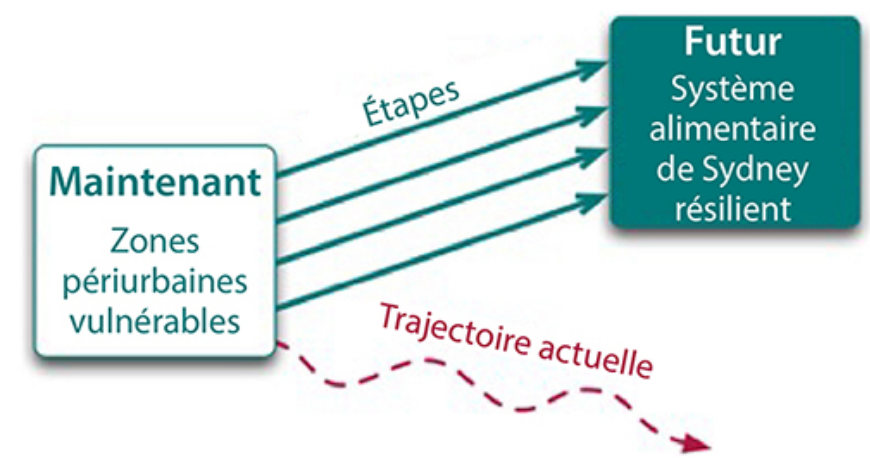

Figure 10.8. Modèle conceptuel du changement dans le système alimentaire de Sydney, utilisé pour guider la discussion lors de l'atelier.

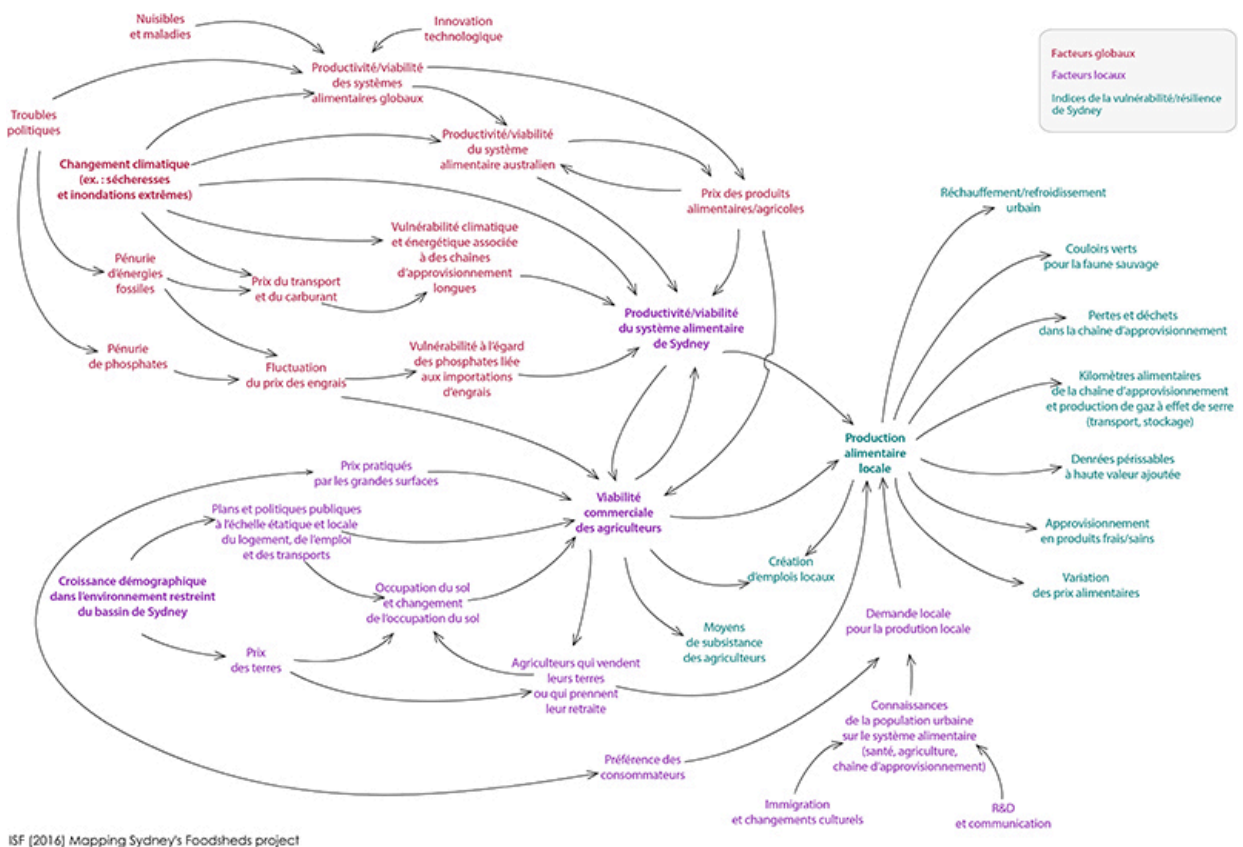

Figure 10.9. Les liens de causalité dynamiques qui influencent la vulnérabilité ou la résilience du système alimentaire de Sydney (d’après ISF, 2016a).

\section{Cartographier les liens de causalité et les vulnérabilités dans le système alimentaire}

Le diagramme sur les influences et les impacts (figure 10.9) a été développé entre la première et la deuxième partie du processus participatif. Il fait la synthèse des connaissances qui ont émergé du premier atelier participatif, de la littérature et de la modélisation. La partie en rouge du diagramme indique l'influence de changements globaux sur la productivité et la viabilité du système alimentaire de Sydney, comme le changement climatique ou la pénurie de phosphore. La partie en violet indique l'influence des changements locaux, tels que l'augmentation du prix des terres et les préférences des consommateurs sur la viabilité économique des exploitations agricoles et la demande pour les produits agricoles. Le côté en vert indique les impacts en bout de chaînes de causalité, 
allant de la température en milieu urbain aux kilomètres alimentaires, en passant par la génération de gaz à effet de serre et l'approvisionnement du bassin de Sydney en denrées périssables à haute valeur ajoutée.

En petits groupes, les participants - des gestionnaires de bassin versant, des agriculteurs, des consommateurs de denrées alimentaires, des aménageurs au sein des gouvernements locaux et des professionnels de la santé - ont annoté les trajectoires de vulnérabilité sur le diagramme pour des secteurs spécifiques. Il leur était notamment demandé de souligner les trajectoires pour le scénario «Production actuelle à l'horizon $2031 »$ pour leur secteur, en incluant les tendances des impacts (par exemple, $X$ mène à l'augmentation/diminution de la viabilité commerciale des exploitations agricoles). Ils devaient aussi ajouter les étapes ou les trajectoires manquantes dans le diagramme, et identifier les trois nœuds ou points d'influence les plus importants pour leur secteur.

\section{Définir les étapes et les trajectoires prioritaires}

Pour identifier les étapes et trajectoires destinées à réduire la vulnérabilité et à améliorer la résilience du système alimentaire de Sydney, il a été demandé aux participants de se baser sur les initiatives existantes ou en cours de développement. Ceci a été fait par petits groupes en complétant une matrice comprenant les questions suivantes : «Qu'estil nécessaire de faire? Quelles initiatives sont déjà en cours? Qui doit agir ? Quel est votre rôle ? Quelles sont les actions et les zones prioritaires ?»

À l'issue de cet exercice, les participants ont identifié plusieurs trajectoires prioritaires pour arriver à un système résilient à Sydney :

- élaborer une planification stratégique. La région de Sydney a besoin d'un plan stratégique qui reconnaît le rôle crucial que l'agriculture périurbaine joue dans l'économie, la résilience et la sécurité alimentaire de la ville. Par exemple, comme le Réseau périurbain de Sydney (Sydney Peri-Urban Network, SPUN) joue un rôle important à l'échelle des communes, certains participants ont suggéré que la position particulière de ces communes de la frange urbaine soit renforcée institutionnellement par le biais d'une loi sur le périurbain ;

- freiner la perte de terres agricoles. Les plans métropolitains stratégiques pour Sydney doivent explicitement identifier, préserver et protéger les zones agricoles, et empêcher l'étalement urbain dans ces zones. De tels plans doivent aussi reconnaître et empêcher la fragmentation des terres agricoles, qui crée des perturbations et contribue au développement de conflits d'usage. Pour cela, les participants ont suggéré de redéfinir le problème positivement, en montrant que la préservation des terres agricoles n'était pas incompatible avec le développement local ;

- concevoir de nouvelles formes de gouvernance. Un schéma métropolitain-rural (MetroRural Framework) élaboré de manière collaborative est nécessaire pour : développer une approche cohérente de la prise en considération de l'agriculture dans le bassin ; identifier les aires prioritaires pour l'agriculture, les principales pressions et l'impact cumulé sur la production agricole future ; et fournir un schéma de prise de décision clair pour les exécutifs locaux. Certains participants ont souligné l'inefficacité avérée d'une approche basée sur le zonage des terres et ont suggéré de tester d'autres mécanismes comme les crédits aux exploitations agricoles ou le développement de zones d'agriculture durable (ISF, 2016b) ;

- proposer un processus de planification holistique. Les processus de planification pour évaluer les changements d'occupation du sol proposés dans les zones périurbaines doivent pleinement prendre en compte les nombreux bénéfices que l'agriculture fournit. Beaucoup de participants ont estimé que, pour dépasser le principe du « meilleur usage » 
(highest and best use) appliqué par défaut, il était nécessaire de développer un schéma efficace qui prenne en compte les nombreux bénéfices apportés par l'agriculture périurbaine à l'économie, à l'environnement, au tissu social et plus généralement à la résilience de la ville (ISF, 2016b);

- mettre en place une méthode efficace de résolution des conflits. Des processus de résolution de conflits doivent être mis en place pour résoudre les tensions entre agriculteurs et résidents. Ces processus doivent garantir que les agriculteurs puissent exploiter leurs terres sans être affectés par des plaintes pour nuisance. S'appuyer sur le document développé par le Département des industries primaires de la Nouvelle-Galles du Sud sur le droit à pratiquer l'agriculture (right to farm policy) a été identifié comme la marche à suivre ;

- soutenir les agriculteurs. La protection, les incitations financières et d'autres formes de soutien sont nécessaires pour garantir aux agriculteurs l'égalité des chances sur le marché. Certains participants ont suggéré qu'un partenariat entre agriculteurs et promoteurs immobiliers pourrait être un mécanisme à explorer ;

- mettre en place une politique efficace de réutilisation des déchets. Des politiques et des initiatives de la part des industriels doivent être mises en place pour traiter sérieusement le fléau du gaspillage alimentaire. Le programme " Aime la nourriture, déteste le gaspillage » (Love Food, Hate Waste) de l'Autorité de protection de l'environnement (Environment Protection Authority, EPA) de la Nouvelle-Galles du Sud ainsi que des projets menés par des associations comme OzHarvest et Green Connect ont été identifiés comme des initiatives à succès. Certains participants ont toutefois estimé nécessaire d'accroître la réglementation, par exemple en interdisant le gaspillage alimentaire dans les supermarchés, comme cela a été récemment institué en France ;

- améliorer l'équité alimentaire. Garantir que toutes les régions dans le bassin de Sydney — et particulièrement l'ouest - aient accès à des points de vente d'aliments frais et sains, pour éviter les déserts alimentaires (food deserts) et renforcer les résultats en matière de santé publique. Certains participants ont suggéré de soutenir et de développer des initiatives comme les banques alimentaires (food pantries), et de prendre en compte les impacts sur la santé dans l'évaluation des projets d'aménagement ;

- éduquer le consommateur. Il s'agit de renforcer la prise de conscience chez les consommateurs et d'autres groupes de l'importance de la production alimentaire dans le bassin de Sydney, et des menaces qui pèsent sur elle. Cette question a récemment suscité l'intérêt des médias à la suite du lancement du Projet sur le futur alimentaire de Sydney et des stratégies actuelles de planification en Nouvelle-Galles du Sud. En outre, sensibiliser le public nécessite une stratégie à plus long terme à travers, par exemple, la création de matériel pédagogique pour les écoles ;

- soutenir le local. En tant que consommateurs, si nous achetons de la nourriture produite localement dans les marchés d'agriculteurs ou d'autres types de points de vente, nous pouvons soutenir les producteurs locaux et réduire l'empreinte écologique liée à l'alimentation. Beaucoup d'exemples de marchés d'agriculteurs prospères ont été identifiés dans le bassin, ainsi que des initiatives comme Hawkesbury Harvest ${ }^{41}$, un projet d'agrotourisme local qui a pour but de connecter les consommateurs avec les producteurs locaux par le biais par exemple du « sentier des fermes » (farm gate trails). Un moyen de faciliter le soutien aux productions locales pourrait être d'étiqueter les produits pour identifier leur origine ;

- développer des connaissances solides. Il s'agit de poursuivre des recherches et des expérimentations pour accroître les bases factuelles sur l'agriculture périurbaine. Les participants ont reconnu l'importance de la recherche, à l'instar du Projet sur le futur 
alimentaire de Sydney. Des recherches additionnelles sont nécessaires, par exemple sur le développement de scénarios pour les zones de production alimentaire durable, ou sur l'évaluation des bénéfices économiques et non économiques de la nourriture produite localement ;

- définir les mécanismes pour la redistribution spatiale de l'occupation du sol. L'objectif ici serait de développer des approches stratégiques pour relocaliser les agriculteurs du sud du bassin de Sydney, en s'appuyant par exemple sur un modèle d'incitations financières ;

- déterminer des objectifs pour le commerce agricole. Identifier des objectifs stratégiques pour Sydney en matière d'agriculture, comme par exemple: qu'avons-nous besoin de produire localement, et dans quelles quantités ? Que pouvons-nous relocaliser dans d'autres zones de productions ou importer?

\section{Codévelopper une vision pour un futur alimentaire résilient à Sydney}

La liste revisitée des composants d'un futur alimentaire résilient à Sydney qui a été codéveloppée par les participants inclut les points suivants :

- une compréhension collective de la valeur de l'agriculture périurbaine. Les planificateurs et les décideurs comprennent l'importance de protéger les terres et exploitations agricoles ainsi que les emplois liés à l'agriculture, pour la résilience de la ville. Les participants ont jugé indispensable que l'importance de l'agriculture périurbaine soit retranscrite de manière cohérente et stratégique dans les schémas de gouvernance à l'échelon étatique et local et dans les documents de planification;

- une protection par le biais de la planification. L'agriculture périurbaine, les jardins partagés/collectifs et l'aquaculture (y compris les petites fermes) à Sydney sont protégés, soutenus, promus et élargis afin de produire des produits frais, de haute qualité, et sont inclus de manière explicite et coordonnée dans les principaux documents de planification de l'État et des communes ;

- une réduction de l'empreinte écologique. Sydney se procure une part importante de ses produits frais auprès des fermes périurbaines et des jardins partagés. Le reste des produits sera importé d'Australie orientale pour réduire les importations de l'étranger ;

- un circuit fermé de nutriments. Sydney récupère et réutilise les déchets organiques urbains locaux (comme la nourriture compostée et les déchets verts, les eaux usées et le fumier des poulaillers) comme intrants pour l'agriculture périurbaine (plutôt que de les jeter dans les décharges) et pour la production d'énergie ;

- l'éducation alimentaire des consommateurs. Les consommateurs ont une meilleure compréhension du système alimentaire. Ils savent notamment d'où provient leur nourriture (par exemple, grâce à l'étiquetage des produits), quelles sont les ressources nécessaires pour produire cette nourriture, les déchets générés, et les implications de leurs choix alimentaires pour la santé. Ils sont aussi conscients de l'importance considérable de l'agriculture périurbaine et des jardins urbains. De plus, la population de Sydney est consciente et éduquée sur les bénéfices environnementaux et sanitaires liés à la consommation de légumes et de fruits frais et à la baisse de consommation d'aliments transformés et de viande rouge ;

- l'accès des consommateurs. Les consommateurs ont physiquement et financièrement accès à des produits locaux abordables et sains et ont les compétences pour préparer des repas et les conserver ;

- les infrastructures de soutien. Les infrastructures logistiques et de transports (par exemple, le train à grande vitesse, les tunnels routiers) sont en place pour étendre les opportunités en dehors du bassin de Sydney, au-delà de 2031. 


\section{Discussion et perspectives}

Les scénarios prospectifs de production alimentaire qui ont été développés dans cette recherche basée sur la modélisation des « bassins alimentaires » ${ }^{42}$ allaient du plus réaliste au plus hypothétique. Ils démontrent que la voie que prend Sydney à l'heure actuelle — l'étalement urbain ininterrompu — n'est pas " gravée dans le marbre ». Au contraire, nous pouvons - et nous avons besoin de - mettre en place des schémas de gouvernance et des stratégies efficaces visant à trouver un équilibre entre le développement d'habitations et la production alimentaire, les moyens de subsistance des agriculteurs, la protection de l'environnement et la disponibilité d'aliments sains.

La première étape de notre processus participatif a révélé que les défis posés à l'agriculture périurbaine sont aussi bien — si ce n'est plus - liés aux interactions sociales qu'à l'occupation du sol. Par exemple, la participation au jeu a permis aux agriculteurs ainsi qu'aux aménageurs, aux professionnels de la santé, aux fonctionnaires du gouvernement, de se mettre à la place de l'autre et de comprendre les défis, de leur point de vue. Les agriculteurs, par exemple, ont pu faire l'expérience de la complexité du processus de planification urbaine : le problème ne vient pas simplement du fait que les aménageurs ne prennent pas en compte les agriculteurs et les terres agricoles, mais plutôt d'un ensemble complexe de facteurs qui doivent être identifiés et évalués. Les aménageurs ont pu reconnaître qu'en matière de planification, ils ont rarement accès à des informations pertinentes, telles que des données géospatiales sur la fertilité des sols et leur potentiel agronomique. Avoir accès à de meilleures informations leur permettrait de prendre des décisions mieux fondées pour positionner de nouveaux espaces résidentiels et d'autres types de projets.

Le jeu a en plus permis aux participants de voir que les décisions sur l'occupation du sol future nécessitaient que des compromis complexes soient faits entre la construction de logements, la production alimentaire, le maintien d'espaces verts/ouverts, les infrastructures et toute une variété de facteurs sociaux, politiques, environnementaux et économiques inter-reliés. Reconnaître la complexité d'un tel système et les compromis nécessaires durant le processus de planification est une étape essentielle vers un futur plus résilient. Comme cela a été suggéré dans des recherches précédentes, l'usage de jeux durant l'atelier a contribué aux résultats obtenus, en faisant participer les acteurs à un processus interactif et en encourageant l'apprentissage collectif et le travail d'équipe. Les participants ont indiqué, une fois le jeu terminé, que des activités similaires pourraient être utilisées dans des processus participatifs impliquant la communauté sur d'autres aspects du processus de planification stratégique - particulièrement ceux qui impliquent des intérêts concurrents et conflictuels et nécessitent une série de compromis complexes.

La seconde étape du processus participatif a permis aux acteurs de dépasser le stade du débat ou de la dispute entre personnes ayant des points de vue différents, pour identifier ensemble des trajectoires et des étapes ambitieuses mais concrètes pour aller vers un système alimentaire résilient à Sydney, dans un contexte de croissance urbaine et d'autres pressions. Ce processus participatif a été particulièrement fructueux, car il a explicitement reconnu les points de vue de beaucoup d'acteurs sur ce défi vieux de plus de dix ans. Plutôt que de créer un énième lieu de débat, ce processus participatif a cherché à développer des actions collectives constructives. Cela a donné aux participants un sentiment de satisfaction et d'optimisme concernant la possibilité d'avancer de manière fédératrice plutôt que conflictuelle.

42. http://www.sydneyfoodfutures.net/interactive-maps/ . 
Cependant, l'atelier final a aussi soulevé de nouvelles questions importantes qui restent encore sans réponse et nécessitent d'être étudiées plus en profondeur. Il s'agissait notamment de la volonté politique de créer du changement, de l'évaluation et de la protection de l'agriculture, des agriculteurs et des consommateurs les plus vulnérables, et de la nécessité d'intégrer explicitement la gestion de l'eau dans la planification alimentaire et agricole. Dans ce dernier cas, les défis concernent les risques d'inondation, les besoins en eau pour de nouveaux logements, l'adaptation ou l'intensification de l'agriculture, ainsi que les possibilités de réutilisation des eaux usées pour la ferti-irrigation ${ }^{43}$. Ces sujets et d'autres questions d'importance devront être traités dans de prochains échanges entre les parties concernées.

\section{Conclusion}

Créer un futur alimentaire résilient à Sydney, et dans d'autres villes en pleine croissance, nécessite que la planification métropolitaine stratégique évalue et protège mieux l'agriculture de l'étalement urbain. Les planificateurs ont besoin de prendre des décisions basées sur des connaissances solides pour trouver un équilibre entre les occupations $\mathrm{du}$ sol et les besoins concurrents des acteurs. Ces décisions doivent prendre en compte l'ensemble des valeurs et des bénéfices fournis par l'agriculture périurbaine - et pas uniquement les gains économiques résultant de la conversion de terres vers un usage de «plus haute valeur». Bien que, si l'on considère l'histoire et la complexité des développements périurbains, de tels bouleversements dans les systèmes de planification urbaine aient peu de chance de se produire naturellement, il apparaît toutefois essentiel de rassembler les acteurs clés pour partager, faciliter l'apprentissage collectif et la construction critique d'une vision commune, et pour codéfinir activement les étapes clés et les trajectoires du changement. La mobilisation des parties prenantes dans des processus créatifs et interactifs est cruciale pour arriver à surmonter un problème faiblement structuré ou en apparence insoluble.

\section{Remerciements}

Ce projet a bénéficié du soutien du gouvernement de Nouvelle-Galles du Sud et a été appuyé par le programme Construire la résilience au changement climatique (Building Resilience to Climate Change), financé par le Bureau pour l'environnement et le patrimoine (Office of Environment and Heritage) de Nouvelle-Galles du Sud, et le crédit environnemental (Environmental Trust) de Nouvelle-Galles du Sud. Il a été géré par le gouvernement local de Nouvelle-Galles du Sud.

Les auteurs tiennent à remercier Ally Dench, du gouvernement local de Wollondilly (Wollondilly Shire Council), ainsi que les représentants du Département des industries primaires (Department of Primary Industries) de Nouvelle-Galles du Sud, du comité de Développement régional australien de Sydney (RDA Sydney), de l'Agence de santé de Nouvelle-Galles du Sud (NSW Health) et de l'Association des agriculteurs de NouvelleGalles du Sud (NSW Farmers).

Nous remercions également nos collègues de l'Institute for Sustainable Futures, Dr Steve Mohr, Kris Maras et Ben Madden, pour leur contribution à la modélisation des « bassins alimentaires » décrits dans ce chapitre ; Joanne Chong pour sa contribution concernant l'évaluation des bénéfices de l'agriculture périurbaine ; et Roel Plant pour avoir proposé et conçu le projet initial « Cartographier les bassins alimentaires potentiels de Sydney » (Mapping Sydney's Potential Foodsheds project).

43. La ferti-irrigation est une technique qui combine des engrais et d'autres types d'amendements avec l'irrigation, distribuant ainsi aux plantes des nutriments pour le sol en plus de l'eau. 


\section{Références bibliographiques}

Bunker R., Holloway D., 2001. Fringe city and contested countryside: population trends and policy developments around Sydney. Issues Paper No. 6 Urban Frontiers Program, University of Western Sydney.

Burnley I., Murphy P., 2004. Sea Change: Movement from Metropolitan to Arcadian Australia, Sydney, University of New South Wales Press.

Cook N., Harder S., 2013. By accident or design? Peri-urban planning and the protection of productive land on the urban fringe. In: Food Security in Australia (Farmar-Bowers Q., Higgins V., Millar J., eds), New York, Springer.

Cordell D., White S., 2014. Life's bottleneck: sustaining the world's phosphorus for a food secure future. Annual Review of Environment and Resources, 39, 161-188.

Domínguez A., Saenz-De-Navarrete J., De-Marcos L., Fernández-Sanz L., Pagés C., Martínez-Herráiz J.J., 2013. Gamifying learning experiences: practical implications and outcomes. Computers and Education, 63.

Gleeson, B. 2013. A New Harvest of the Suburds. In: Food Security in Australia (Farmar-Bowers Q., Higgins V., Millar J., eds), New York, Springer.

Golledge R.G., 1960. Sydney's metropolitan fringe: a study in urban-rural relations. Australian Geographer, 7, 243-255.

Grafton R.Q., 2010. Adaptation to climate change in marine capture fisheries. Marine Policy, 34, 606-615.

Grove J.M., Childers D.L., Galvin M., Hines S., Muñoz-Erickson T., Svendsen E.S., 2016. Linking science and decision making to promote an ecology for the city: practices and opportunities. Ecosystem Health and Sustainability, 2.

Haasnoot M., Kwakkel J.H., Walker W.E., Ter Maat J., 2013. Dynamic adaptive policy pathways: a method for crafting robust decisions for a deeply uncertain world. Global Environmental Change, 23, 485-498.

Hackman H., St Clair A.L., 2012. Transformative cornerstones of social science research for global change. Mundo Amazónico, 4, 117-152.

Hamari J., Koivisto J., Sarsa H., 2014. Does gamification work? A literature review of empirical studies on gamification. In: Proceedings of the Annual Hawaii International Conference on System Sciences, 3025-3034.

Henry B., Conant R., Carter J., Droulez V., Grace P., 2013. Increasing food production sustainably in a changing climate: understanding the pressures and potential Beverley. In: Food Security in Australia (Farmar-Bowers Q., Higgins V., Millar J., eds), New York, Springer.

Houston P., 2005. Re-valuing the fringe: some findings on the value of agricultural production in Australia's peri-urban regions. Geographical Research, 43, 209-223.

ISF, 2016a. Mapping Sydney's Potential Foodsheds, Sydney's Food Futures. Institute for Sustainable Futures, University of Technology Sydney.

ISF, 2016b. Planning tools for strategic management of peri-urban food production. Report for the Royal Institution of Chartered Surveyors.

La Rosa D., Barbarossa L., Privitera R., Martinico F., 2014. Agriculture and the city: a method for sustainable planning of new forms of agriculture in urban contexts. Land Use Policy, 41, 290-303.

Loria M., 2008. A review of the main approaches to knowledge management. Knowledge Management Research and Practice, 6, 77-89.

Low Choy D., Sutherland C., Gleeson B.J., Dodson J., Sipe N., 2008. Peri-Urban Futures and Sustainable Development, Research Monograph 4, Urban Research Program, Griffith University. 
Mitchell C., Cordell D, Fam D., 2015. Beginning at the end: the outcome spaces framework to guide purposive transdisciplinary research. Futures, 65, 86-96.

NSW Department of Planning and Environment, 2015. A plan for growing Sydney. Technical report.

NSW OEH, 2016. The land and soil capability assessment scheme, Second approximation. A general rural land evaluation system for New South Wales. Technical report.

Ostrom E., 2014. Collective action and the evolution of social norms. Journal of Natural Resources Policy Research, 6, 235-252.

Peters C.J., Bills N.L., Lembo A.J., Wilkins J.L., Fick G.W., 2009. Mapping potential foodsheds in New York State: a spatial model for evaluating the capacity to localize food production. Renewable Agriculture and Food Systems, 24, 72-84.

Peters C.J., Bills N.L., Lembo A.J., Wilkins J.L., Fick G.W., 2012. Mapping potential foodsheds in New York State by food group: an approach for prioritizing which foods to grow locally. Renewable Agriculture and Food Systems, 27, 125-137.

Rosas R., Nussbaum M., Cumsille P., Marianov V., Correa M., Flores P., Grau V., Lagos F., López X., López V., Rodriguez P., Salinas M., 2003. Beyond Nintendo: design and assessment of educational video games for first and second grade students. Computers and Education, 40, 71-94.

Rothwell A., Ridoutt B., Page G., Bellotti W., 2015. Feeding and housing the urban population: environmental impacts at the peri-urban interface under different land-use scenarios. Land Use Policy, 48, 377-388.

Ruoso L.-E., 2018. The politics of place identity in peri-urban environments. What role for productive farming landscapes? A case study of Wollondilly Shire, NSW, Australia. PhD, University of Technology Sydney.

Rydin Y., Bleahu A., Davies M., Dávila J.D., Friel S., De Grandis G., Groce N., Hallal P.C., Hamilton I., Howden-Chapman P., Lai K.M., 2012. Shaping cities for health: complexity and the planning of urban environments in the 21st century. Lancet, 379, 2079.

Seto K., Güneralp B., Hutyra L., 2012. Global forecasts of urban expansion to 2030 and direct impacts on biodiversity and carbon pools. PNAS, 109, 16083-16088.

Shachter R., 1986. Evaluating influence diagrams. Operations Research, 34, 871-882.

VEIL, 2015. Infographic: Melbourne's Urban Sprawl. https://veil.msd.unimelb.edu.au/ (consulté le 8 décembre 2018). 


\title{
Chapitre 11
}

\section{Utilisation du concept de services écosystémiques pour une évaluation participative du rôle des terres agricoles périurbaines dans le sud de la France}

\author{
Roel Plant, Pierre Maurel, Laure-Élise Ruoso
}

\section{Introduction}

Le phénomène d'étalement urbain, vu comme un développement urbain incontrôlé et irréfléchi, menace les zones agricoles périurbaines et représente une forme particulière d'emprise foncière. L'étalement urbain est considéré comme l'une des causes principales de changement d'occupation du sol en Europe (Smiraglia et al., 2016 ; Colantoni et al., 2016 ; Bio Intelligence Service et al., 2015). Les terres agricoles périurbaines sont généralement situées sur des terres fertiles et renferment, avec les zones naturelles, des éléments clés de réseaux socio-écologiques complexes (Billeter et al., 2008). Ainsi, des valeurs sociétales - et économiques - souvent méconnues ou non reconnues résident dans ces terres agricoles (Gardi et al., 2015). Dans le contexte d'un développement urbain dominé par les logiques de marché, où les régulations sont limitées et où le prix des terres détermine le sort des terres agricoles périurbaines, ces valeurs ne sont pas reconnues (Ciscel, 2001). Une façon d'empêcher cette situation est de proposer aux aménageurs et aux décideurs des approches innovantes prenant en compte les valeurs sociétales plus larges associées aux terres agricoles périurbaines, afin qu'elles puissent être intégrées de manière plus transparente, systématique et explicite dans les processus d'aménagement local du territoire.

La réflexion et la prise de décision basées sur les «valeurs » ont une longue et riche histoire dans la littérature scientifique (Keeney, 1970) et ont souvent été utilisées dans le contexte de la prise de décision environnementale (ex. : Plant et al., 2016 ; Kerselaers et al., 2011). Par exemple, Kerselaers et al. (2011) ont développé une méthode basée sur les processus d'analyse hiérarchique (Analythic Hierarchy Process, AHP ; Saaty, 1996) pour identifier les priorités en matière de préservation des terres agricoles dans les Flandres. Leur méthode s'appuie sur une approche participative menant à l'élaboration d'un « arbre de valeurs ». Ceci a permis de constituer une liste de critères afin de définir la valeur des terres agricoles, et de les lier de manière explicite aux objectifs de préservation de ces terres. Ce type de processus participatif aide à développer une vision partagée de cet enjeu. 
À l'heure actuelle, l'expression des valeurs fait l'objet de beaucoup d'attention dans le contexte de l'étude des services écosystémiques. Les services écosystémiques peuvent être définis comme la contribution de la nature au bien-être humain (MEA, 2005). Ceci inclut, par exemple, les services d'approvisionnement, comme la production alimentaire et l'eau ; les services de régulation, comme la régulation du climat et la pollinisation ; les services de support, comme le maintien du cycle de vie et des nutriments et la provision d'habitats pour la biodiversité ; et les services culturels comme les loisirs, les aménités paysagères et l'apprentissage. Alors que le concept de service écosystémique est souvent critiqué car considéré comme anthropocentré, il faut rappeler qu'il vise à mettre en valeur l'importance de l'ensemble du système socio-écologique. Comme Costanza et al. (2017, p. 3) l'expliquent dans leur réflexion sur vingt années d'utilisation de ce concept, " le concept de services écosystémiques est une vue "consciente de l'ensemble du système" qui considère les humains comme faisant partie d'une société et plus largement de la nature $»$.

Un des postulats initiaux du concept de service écosystémique, développé à la fin des années 1990, est qu'afin de susciter de l'intérêt pour la notion de «capital naturel » et ainsi de garantir une meilleure conservation, les services écosystémiques devraient être considérés comme des marchandises et mis sur le marché (Daily, 1997 ; Westman, 1977 ; Sukhdev et al., 2010). À cette époque - l'âge d'or de la politique de la troisième voie, où libéralisme économique et démocratie sociale convergeaient - , ceci était vu comme un moyen stratégique de faire entrer la question de la conservation de la biodiversité dans l'arène politique traditionnelle. Ces dernières années, la notion de capital naturel et l'idée de donner un prix à la nature et de la considérer comme une marchandise ont fait l'objet de nombreuses critiques dans la littérature scientifique (McCauley, 2006 ; Schröter et al., 2014 ; Hejnowicz et Rudd, 2017). Par conséquent, d'autres manières d'exprimer les valeurs, s'inspirant de traditions en économie, en théorie de la décision, en psychologie cognitive, en philosophie et en anthropologie, sont maintenant étudiées (Jacobs et al., 2016). Ce débat est loin d'être clos, et pourtant, comme Costanza et al. (2017) le constatent, " aussi longtemps que nous sommes forcés à faire des choix, nous procédons à un processus d'évaluation ». La définition du terme « évaluation » proposée par l'Oxford English Dictionary est « une estimation de la valeur de quelque chose ». Estimer la valeur revient en fin de compte à un processus mental, conduit individuellement ou collectivement, dans lequel les situations sont évaluées et comparées à une valeur de référence. Les résultats obtenus durant ce processus mental permettent de décider s'il faut agir (en cas de certitude raisonnable) ou s'abstenir (en cas de doute raisonnable).

Le concept de services écosystémiques a pour objectif de permettre une évaluation holistique des conséquences d'un changement majeur sur le bien-être humain. Par exemple, les écosystèmes et les services écosystémiques pourraient être sévèrement impactés par un changement d'occupation du sol planifié (par ex. un développement résidentiel à haute valeur ajoutée sur des terrains jamais construits, comme des villages de retraite) ou non planifié (par ex. l'étalement urbain sur des terres agricoles marginalisées). Dans de telles situations, l'évaluation structurée et transparente des services écosystémiques est appropriée pour envisager un futur désirable. Elle permet d'explorer comment des changements (planifiés ou non) conduiront à une modification des types et des quantités de services écosystémiques fournis par une zone particulière à un moment précis, dans le futur.

Pour conclure leur bref état des lieux du débat autour de l'évaluation des services écosystémiques, Costanza et al. (2017) soulignent que de telles évaluations devraient être faites « au niveau sociétal plutôt qu'au niveau individuel, et dans des contextes temporels et 
spatiaux appropriés ». Ce chapitre vise à explorer l'évaluation individuelle et collective des services écosystémiques. Il présente une méthode d'évaluation des services écosystémiques fournis par les terres agricoles périurbaines menacées par l'étalement urbain, en faisant le postulat que l'intégration des connaissances — tacites ou expertes - que les acteurs ont sur leur environnement local sont essentielles pour obtenir une évaluation signifiante dans un contexte local précis (Blackmore, 2007 ; Moller et al., 2004).

En premier lieu, nous présenterons la conceptualisation holistique des services écosystémiques adoptée dans ce chapitre ainsi que notre méthodologie. Puis nous nous concentrerons sur les résultats empiriques issus de deux études de cas dans le sud de la France où une évaluation sociale des services écosystémiques a été conduite. Notre réflexion se portera ensuite sur les approches et processus testés qui pourraient être codifiés pour être intégrés dans la planification locale (participative) de l'occupation du sol, afin de mieux prendre en compte, d'une façon holistique, les valeurs moins tangibles associées aux terres agricoles périurbaines et aux zones naturelles menacées par le développement urbain.

\section{Théorie et méthodes}

Dans cette section, nous développons les bases conceptuelles pour une approche holistique de l'évaluation des services écosystémiques en posant trois questions: Quelles valeurs doivent être incluses? Les valeurs de qui doit-on prendre en compte ? Comment ces valeurs sont-elles le mieux appréhendées et intégrées dans les processus de planification territoriale ? Nous présentons ensuite notre méthodologie.

\section{Quelles valeurs ? Les cadrages des services écosystémiques}

La représentation socio-spatiale du phénomène d'étalement urbain et ses effets sur les services écosystémiques constituent un défi scientifique majeur. Avec l'engagement pris par la Commission européenne de mettre un terme à l'augmentation nette des surfaces artificialisées d'ici 2050, une part importante de l'activité scientifique se concentre sur le développement d'indicateurs (Barbosa et al., 2017). Nous vivons à une époque où les nombres façonnent les décisions sociétales plus que toute autre chose (Van Dijk et Van der Valk, 2010). L'économie de l'environnement et des ressources offre de riches outils quantitatifs pour mesurer et évaluer les ressources environnementales (Hanley et Spash, 1993 ; Helliwell, 1969). Bien que les décideurs s'appuient fortement sur cette gamme d'approches économiques, ces dernières ne représentent qu'une manière d'exprimer les valeurs (Vatn, 2005), ou " cadrages » — un cadrage qui s'aligne sur la rationalisation économique de l'utilisation des terres, centrée sur le marché. Comme nous l'avons expliqué dans la section précédente, ce cadrage particulier pourrait mener à la perte de valeurs écologiques et sociétales plus larges, qui pourraient ne pas être reconnues, et n'être regrettées que lorsqu'il sera trop tard. Afin de favoriser la prise en compte de ces valeurs écologiques et sociétales plus larges dans la planification de l'occupation des sols, il est nécessaire d'être conscient du cadre à travers lequel nous déchiffrons nos relations à l'environnement.

Le concept de cadrage est issu de traditions de recherches en sociologie (Goffman, 1974), en psychologie (Kahneman et Tversky, 1983) et en linguistique cognitive (Lakoff, 2004). C'est Lakoff (2004) qui a importé le cadrage des choix dans le lexique des politiques publiques. Dans le contexte des politiques publiques, le cadrage fait référence, selon Thompson (2008), à la manière dont un domaine de gouvernement ou une activité sociale sont présumés tourner autour d'un certain enjeu politique. Il affirme que comprendre 
comment les choix individuels et publics sont limités et façonnés par le cadrage peut contribuer à améliorer les processus décisionnels.

La notion de cadrage peut être utilisée dans le domaine de l'évaluation des services écosystémiques, pour tenter de répondre, de manière holistique, à la perte fortuite de services écosystémiques due à l'urbanisation. Adopter un cadrage alternatif qui ne serait pas basé sur une logique économique, mais seulement sur des perspectives socioculturelles, pourrait être tout autant partiel et limité que le cadrage que nous avons critiqué précédemment. Plutôt que de choisir un cadrage en particulier, il nous paraît plus important d'être conscient des différents cadres de référence existants. La notion de « cadres » appliquée aux services écosystémiques a été récemment explorée dans une recherche sur la planification collaborative du paysage (Opdam et al., 2015). Les cadres utilisés dans cette étude étaient : le cadre socioculturel (accent mis sur les services socioculturels), le cadre économique (accent mis sur les services de production) et le cadre de la durabilité (accent mis sur les services de régulation). Ces trois cadres sont alignés sur les domaines de valeurs des services écosystémiques qui ont été établis dans la littérature (Martín-López et al., 2013). Opdam et al. (2015) reconnaissent aussi que le concept de service écosystémique lui-même est un cadre. Nous sommes en accord avec cette position, constatant que beaucoup d'autres cadres d'évaluation existent et qu'ils permettraient eux aussi d'aboutir à l'expression de valeurs et de bénéfices dans une région donnée et de comprendre comment ces valeurs et ces bénéfices pourraient influencer le comportement des acteurs. Nous pouvons citer comme autres cadres la théorie de l'acteur réseau (Callon, 1986), le modèle d'analyse IAD (Institutional Analysis and Development) (Ostrom, 2009), ou encore l'analyse du cycle de vie à l'échelle territoriale (Loiseau, 2014). De plus, il a été démontré que le concept de service écosystémique restait complexe sur le plan scientifique et sémantique et qu'il ne faisait pas écho de la même manière chez tout le monde (Plant et Ryan, 2013 ; Baker et al., 2013).

Pour ces raisons nous avons décidé, dans notre étude, d'adopter un cadrage implicite du concept de service écosystémique qui ne se limite pas à l'un des trois domaines de valeurs généraux (monétaire, biophysique et socioculturel). Un cadrage implicite signifie que notre méthodologie intègre le concept de service écosystémique, mais ne présente ni le concept, ni ses principes aux participants (voir la méthodologie de l'étude de cas pour les détails). En laissant ouverte la possibilité de multiples cadres de valeurs, notre approche n'adopte pas, a priori, de cadrage particulier. Nous considérons, cependant, qu'une approche sociale des valeurs n'est jamais très éloignée des services écosystémiques culturels, ou, tout du moins, amène à adopter une perspective « culturelle » sur les services écosystémiques.

\section{Les valeurs de quels acteurs ? Les bénéficiaires des services écosystémiques}

Lorsque nous examinons la valeur des terres agricoles périurbaines face à l'urbanisation, il est essentiel de prendre en considération ce qui pourrait être affecté, positivement ou négativement, par l'étalement urbain. L'urbanisation et l'étalement urbain sont des processus de changements dynamiques qui impactent les systèmes socio-écologiques autour des villes. Généralement, les citadins sont les « gagnants » — plus d'espaces résidentiels sont disponibles — et les habitants des espaces périurbains, dont les agriculteurs, sont les « perdants » - moins de terres agricoles seront disponibles, des conflits d'usage vont probablement émerger, et les agriculteurs ne sont plus en mesure de conserver ou de développer des exploitations agricoles viables. Une autre catégorie de " gagnants » est les nouveaux résidents, par exemple des retraités, qui veulent acheter un bien immobilier 
dans des villages seniors en milieu rural ou périurbain, ou des promoteurs immobiliers qui saisissent des opportunités commerciales et d'investissement.

Ainsi, le processus de changement de l'occupation du sol pose la question de savoir les valeurs de qui vont être affectées que ce soit positivement ou négativement. Dans les évaluations scientifiques, c'est souvent l'analyste, le chercheur, qui décide quelles valeurs (bénéfices) et coûts doivent être inclus dans l'analyse, et dans le cas où des valeurs non marchandes sont recherchées, comment un échantillon socialement représentatif peut être enquêté. Cependant, lorsqu'il s'agit d'aménagement du territoire (par exemple, planifier le futur des terres agricoles), les acteurs en présence ont souvent des perspectives et des intérêts différents, parfois même contradictoires. Par exemple, les agriculteurs dans une région donnée afficheront probablement des intérêts différents de ceux des citadins. D'autres personnes ne résidant pas dans la zone pourront lui trouver de la valeur simplement du fait de son existence (possibilité de la visiter un jour, région où l'on a grandi, etc.). Il est donc essentiel de prendre en compte les perspectives des différents acteurs à travers une évaluation participative des services écosystémiques.

La figure 11.1 présente une typologie ouverte des bénéficiaires des écosystèmes. Sur l'axe du temps, en abscisse, figurent les bénéficiaires actuels et futurs. Il se peut que les bénéficiaires actuels aient vécu dans la zone concernée par la planification depuis des décennies ou des générations - auquel cas il est probable qu'ils possèdent une connaissance profonde et détaillée de leur environnement local. Les bénéficiaires présents peuvent aussi être des gens de l'extérieur qui veulent s'installer pour des raisons personnelles (par exemple des retraités) ou pour développer des activités commerciales (par exemple des promoteurs immobiliers). Les bénéficiaires futurs représentent les personnes qui ne sont pas encore nées (les descendants des résidents locaux) ou les personnes qui pourraient s'installer plus tard dans la zone. La notion de bénéficiaires futurs est en accord avec la définition canonique du développement durable « qui répond aux besoins du présent sans compromettre la capacité des générations futures de répondre aux leurs » (italique ajouté) (WCED, 1987). L'axe des ordonnées, dans la figure 11.1, représente l'éloignement ou la proximité d'un bénéficiaire de services écosystémiques avec la zone fournissant ces services. Alors que le potentiel de bénéfices (actuels ou futurs) est évident pour les résidents locaux, la notion de bénéfice «à distance » désigne les valeurs d'option et les valeurs d'existence. Par exemple, des personnes vivant ailleurs pourraient accorder de la valeur à la présence de certains bénéfices dans cette zone spécifique - la zone pourrait leur rappeler leur enfance, ou contenir des zones écologiques emblématiques ou des paysages qui n'existent nulle part ailleurs. En utilisant ces deux dimensions (le temps et l'espace), une autre distinction peut être faite entre les bénéficiaires réels et potentiels. En effet, il est important de noter que les bénéficiaires potentiels peuvent être des personnes qui résident déjà dans la zone — ou qui vivent ailleurs - , mais qui ne sont pas conscientes des bénéfices fournis par les écosystèmes dans cette zone. Ceci montre le potentiel qu'a l'évaluation des services écosystémiques d'expliciter les bénéfices associés à des services écosystémiques et de communiquer sur ce sujet à un public plus large durant le processus de planification.

\section{Comment accéder à des valeurs holistiques?}

Après avoir montré le lien entre les valeurs rattachées aux services écosystémiques et les bénéficiaires concernés, nous allons maintenant voir comment ces bénéfices et bénéficiaires peuvent être pris en compte dans une étude empirique aux moyens limités. Beaucoup de chercheurs ont développé des procédures systématiques pour l'évaluation des services écosystémiques, à des fins variées et à des échelles spatio-temporelles 
différentes (TEEB, 2010 ; Watson et Albon, 2011). Ces dernières années, de nombreux travaux méthodologiques ont été menés pour produire des représentations cartographiques des services écosystémiques (Crossman et al., 2012 ; Burkhard et al., 2012b ; Martínez-Harms et Balvanera, 2012 ; Kareiva et al., 2011). Des inventaires à grande échelle des impacts de l'étalement urbain sur les services écosystémiques ont été développés en se basant sur des typologies formelles, telles que la classification MEA (MEA, 2003) ou des classifications de l'occupation du sol dérivées d'images satellites comme Corine Land Cover (Burkhard et al., 2012a). Les services de régulation sont les plus communément cartographiés, suivis des services de production, des services culturels et des services de support (Martínez-Harms et Balvanera, 2012). Des efforts importants ont aussi été faits pour intégrer les points de vue des acteurs dans la cartographie des services. Par exemple, Sheate et al. (2012) présentent une méthodologie de cartographie des services écosystémiques au moyen de systèmes d'information géographique (SIG) et de jeux de données d'occupation et d'usage du sol facilement disponibles. Un élément essentiel de leur méthodologie consiste à impliquer les acteurs dans le développement des typologies de services écosystémiques et dans l'identification des relations entre les services écosystémiques et les différentes classes d'occupation et d'usage du sol, afin qu'elles soient pertinentes pour les acteurs.

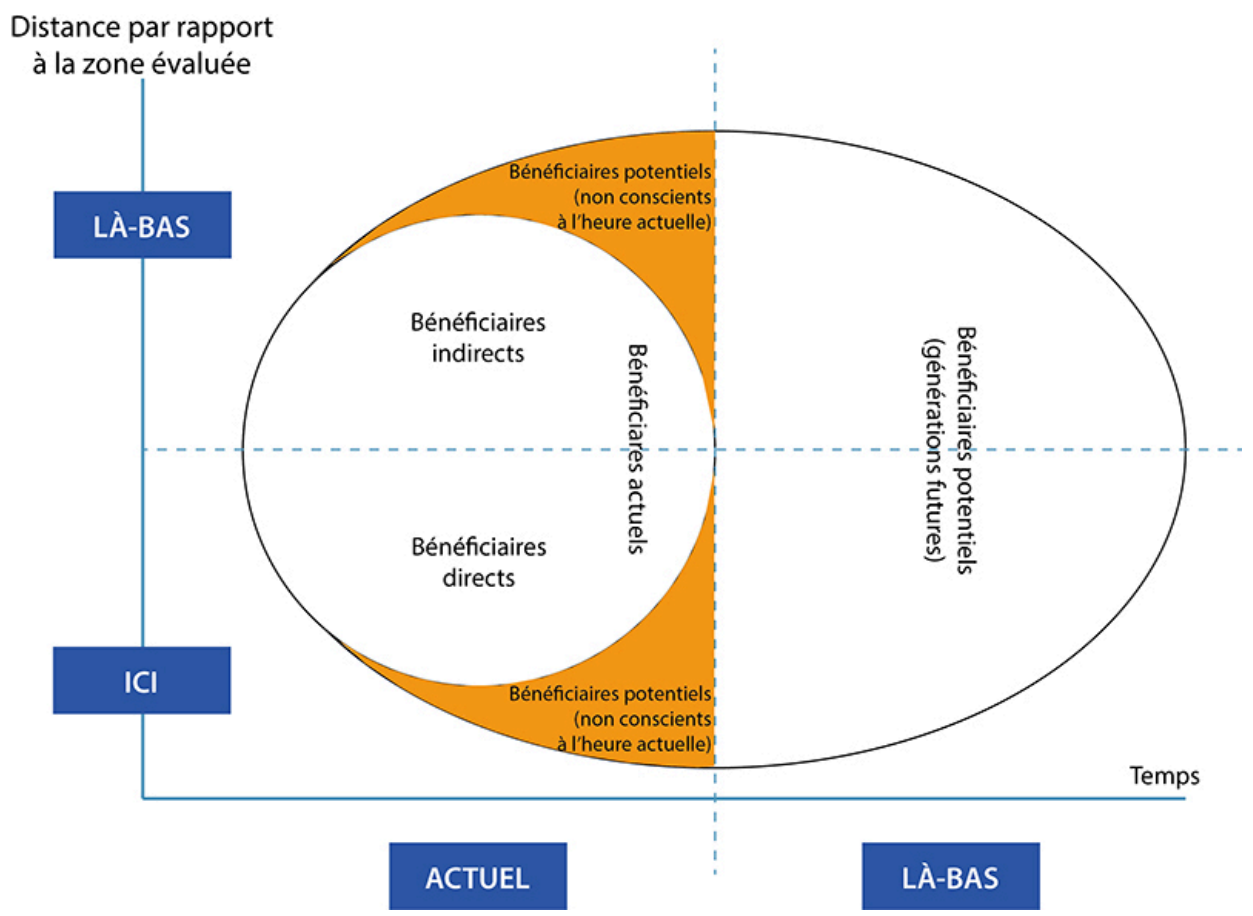

Figure 11.1. Typologie des bénéficiaires des services écosystémiques.

À ce jour, la plupart des études cartographiques de services écosystémiques ont été menées à l'échelle régionale ou nationale. Cependant, avec les résolutions toujours plus fines des images satellites et la baisse de leur prix d'acquisition, les possibilités de cartographie détaillée à l'échelle locale sont de plus en plus envisageables. Ces cartographies fines de services écosystémiques constituent des solutions prometteuses pour traiter les 
questions de l'étalement urbain, et plus largement de la consommation, voire de l'accaparement des terres agricoles (Bonifazi et al., 2016 ; Di Palma et al., 2016).

Beaucoup des procédures et des protocoles utilisés à ce jour sont marqués implicitement par un certain positivisme scientifique et mettent l'accent sur la quantification, affirmant que «nous ne pouvons pas gérer ce que nous ne pouvons pas mesurer». Par exemple, Primmer et Furman (2012, p. 86) suggèrent que « des fonctions écosystémiques importantes nécessitent d'identifier les éléments clés des écosystèmes. Une fois identifiés, ils peuvent alors être mesurés et des analyses supplémentaires peuvent être développées ». L'accent est mis ici sur la quantification des services écosystémiques relevant du domaine biophysique (services de régulation) ou sur l'identification des approximations quantifiables pour des services relevant du domaine humain. Or, il est nécessaire d'arriver à une compréhension plus fine des valeurs et des bénéfices pour pouvoir éclairer l'action publique et la prise de décision. Nous défendons l'idée que les valeurs et les bénéfices fournis par les services écosystémiques sont des constructions sociales et «n'existent pas » dans le sens positiviste du terme. Ils ne peuvent donc pas être appréhendés à l'aide de méthodes purement quantitatives. Le modèle en cascade des écosystèmes proposé par Haines-Young et Potschin (2010), bien que critiqué pour sa simplification d'une réalité complexe et dans le même temps pour sa complexification, jugée inutile, d'une définition claire des services écosystémiques (Costanza et al., 2017), propose une manière élégante de représenter visuellement ce problème complexe. Le modèle en "cascade » fait la distinction entre les structures et les processus biophysiques ; les fonctions écologiques; les services écologiques; et - franchissant la limite peu claire entre les domaines du biophysique et de l'humain — les bénéfices fournis par les écosystèmes, les valeurs qui leur sont accordées et leurs bénéficiaires. La « cascade » de services, depuis leurs origines biophysiques jusqu'à leurs contributions finales au bien-être humain, peut aussi être comprise comme un pont entre les domaines naturels et humains, et ce pont peut être emprunté des deux côtés - en adoptant une épistémologie positiviste ou, comme nous l'avons fait dans notre étude, une épistémologie constructiviste. Pour appréhender les services écosystémiques et arriver à une évaluation significative à l'échelle locale, nous avons fait appel à des méthodes participatives qui intègrent les savoirs locaux - tacites ou d'experts. Les savoirs locaux tacites sont les savoirs que les acteurs développent à partir de leur expérience personnelle dans la zone d'étude. Les savoirs locaux d'experts sont les savoirs que les experts locaux développent à partir d'études scientifiques passées ou présentes. L'information scientifique joue un rôle important dans la planification de l'occupation du sol ainsi que dans la planification du paysage (Opdam et al., 2015 ; Arts et al., 2017). Bien que nous adoptions une épistémologie constructiviste, notre étude utilise le modèle en cascade comme moyen de réconcilier les savoirs tacites et d'experts sur les services écosystémiques pour la planification locale (Plant et Prior, 2014). Dans notre première étude de cas (le bassin de Thau, voir description plus bas), une combinaison de récits faits par les acteurs (individuellement et collectivement), d'exercices de cartographie participative et de coconstruction de matrices combinant l'occupation du sol et les services écosystémiques a été utilisée pour mettre en lumière les connaissances, les perceptions et les valeurs des participants. Les participants étaient composés d'experts et de non-experts, d'où la dénomination « à dire d'acteurs » de cette méthode participative. Cette méthode peut être positionnée dans la partie « humaine» du modèle en cascade (figure 11.2), car l'accent est mis sur les connaissances scientifiques ou profanes que les acteurs ont développées sur leur environnement local, mais aussi sur leurs perceptions des services écosystémiques et les valeurs qu'ils leur accordent. Dans la seconde étude de cas (les Costières de Nîmes, voir description plus bas), notre méthode participative a impliqué un petit groupe de professionnels 
locaux représentant différents domaines de politiques publiques et s'est principalement centrée sur le développement d'une matrice combinant l'occupation du sol et les services écosystémiques. Nous avons nommé cette approche « à dire d'experts ». Elle peut être positionnée au croisement des domaines biophysiques et humains décrits dans le modèle en cascade, car l'accent est mis principalement sur les connaissances scientifiques des experts locaux.

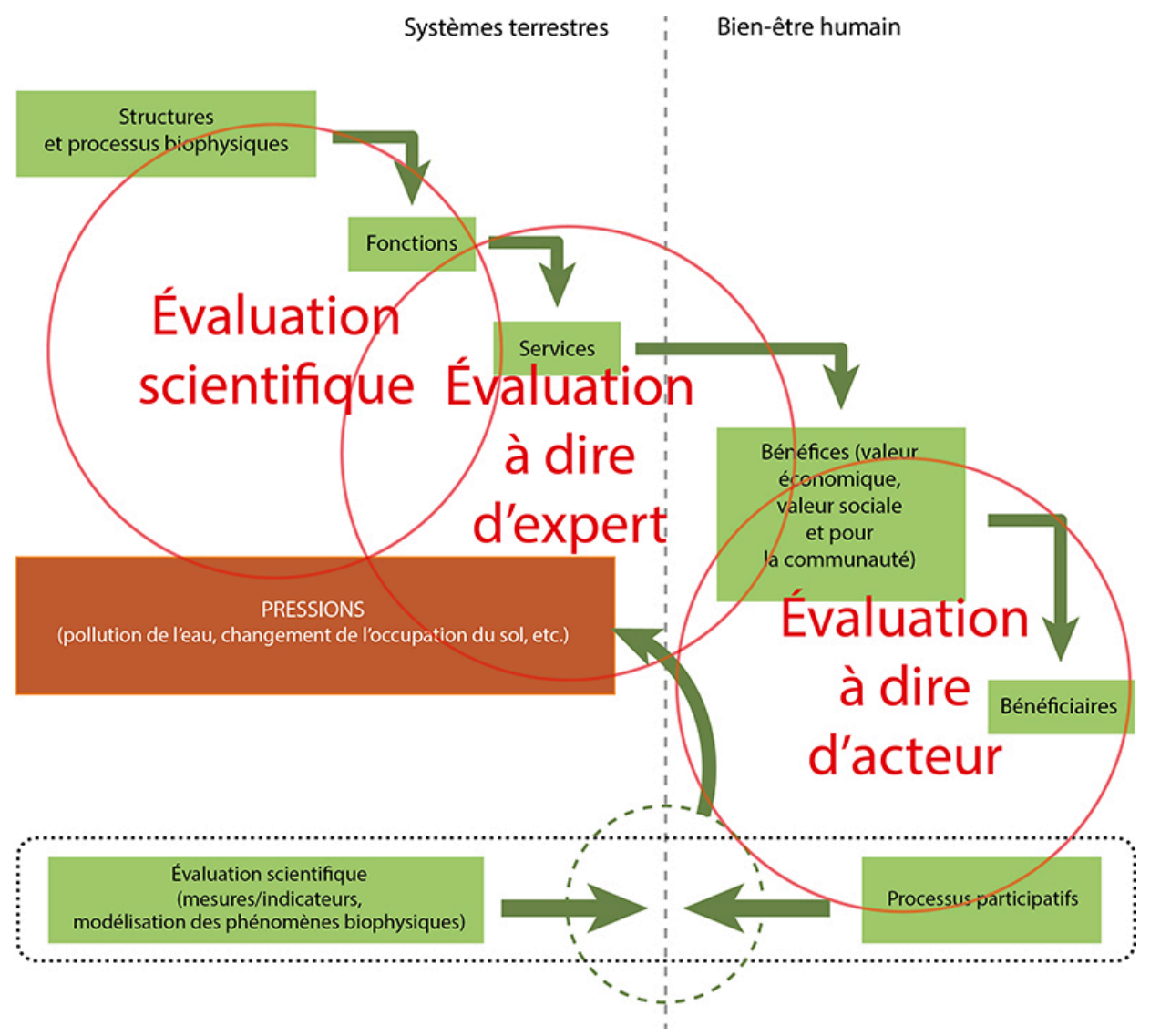

Figure 11.2. Trois manières de saisir les valeurs accordées aux services écosystémiques.

\section{Études de cas}

Dans cette section, nous présentons brièvement notre évaluation holistique et constructiviste des services écosystémiques à l'aide de deux études pilotes menées dans le sud de la France en 2012-2013 ${ }^{44}$ (figure 11.3). La première étude de cas, le bassin de Thau, situé sur le littoral méditerranéen près de l'aire métropolitaine de Montpellier (figure 11.3), est une zone où l'agriculture est perçue comme de moins en moins viable, du fait de l'incertitude autour de la demande en produits de la zone, mais aussi des pressions importantes pour le développement urbain. La zone attire de nouveaux résidents à un

44. Cette étude pilote a été entreprise dans le cadre du projet Knobimap (Knowledge-Based Biodiversity Mapping, and Ecosystem Services Research Project), mené conjointement par Irstea (France) et l'Université de technologie de Sydney (Australie). Ce projet a testé l'évaluation participative des terres agricoles périurbaines en se basant sur le concept de services écosystémiques. Voir aussi http://knobimap.blogspot.fr/ . 
rythme sans précédent et cela devrait continuer dans les années à venir. Par conséquent, une quantité considérable de terres agricoles situées sur des sols fertiles risque d'être convertie à des usages non agricoles. La deuxième étude de cas, les Costières de Nîmes situées dans l'aire métropolitaine de Nîmes (figure 11.3), est une zone où l'agriculture a été affectée par le développement d'infrastructures. Avec l'annonce de l'extension de la ligne de TGV Nîmes-Montpellier il y a une quinzaine d'années, les agriculteurs ont commencé à abandonner leurs terres et à spéculer sur les indemnisations de rachat. Étant donné que la planification et la mise en œuvre des infrastructures ont pris longtemps, la zone a progressivement subi une déprise agricole et un " ré-ensauvagement » des terres agricoles. Une conséquence inattendue de ce ré-ensauvagement a été le développement imprévu d'un habitat pour plusieurs espèces, dont l'outarde canepetière (Tetrax tetrax).

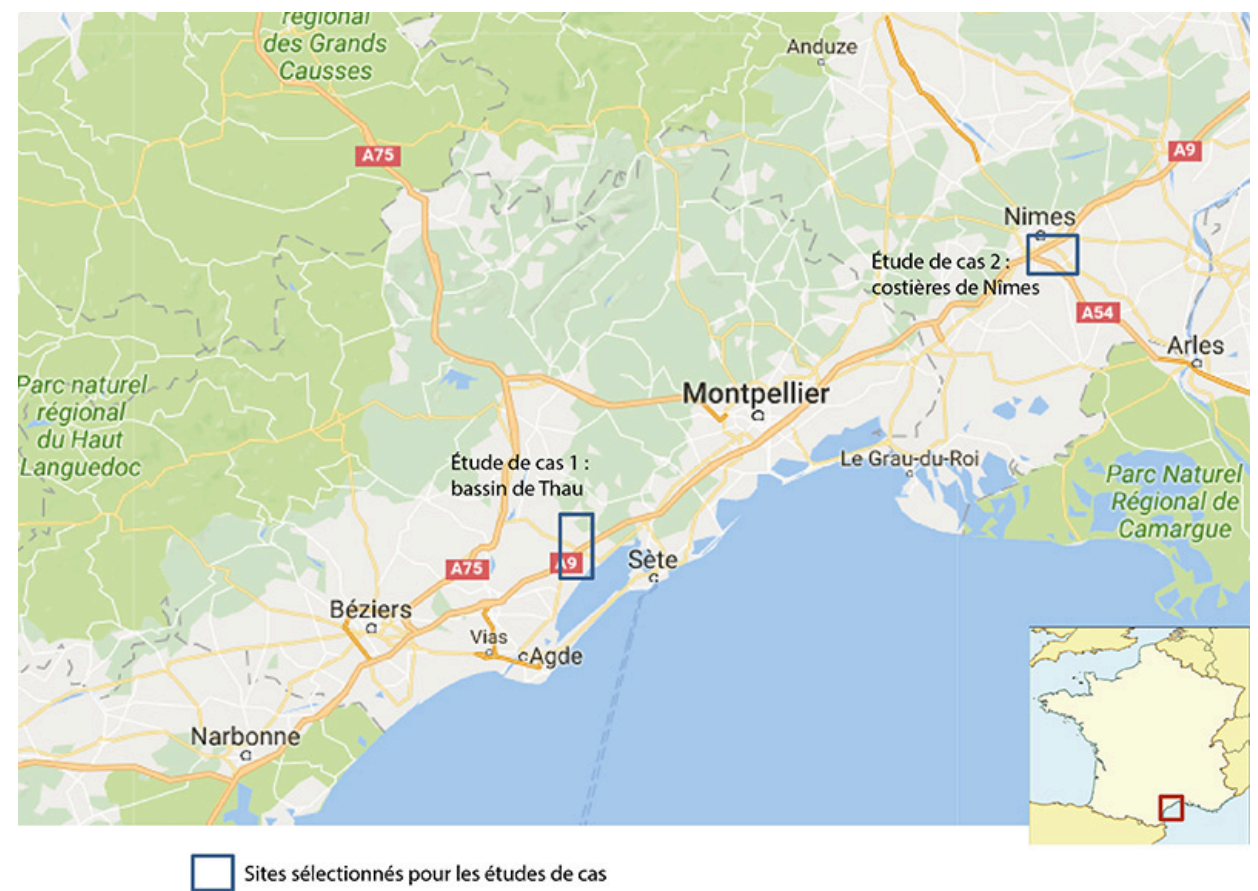

Figure 11.3. Localisation des zones d'études.

Les deux études de cas avaient pour but de mettre en lumière les impacts du développement - le développement urbain dans le cas du bassin de Thau et le développement d'infrastructures dans le cas des Costières de Nîmes - sur les services écosystémiques (figure 11.4). Le processus d'évaluation participative des services écosystémiques a été conçu pour capturer les connaissances et les perceptions que les acteurs ont des services écosystémiques en utilisant la perspective la plus large (holistique) possible, pour ensuite les structurer dans des matrices liant les classes d'occupation du sol à certains services écosystémiques.

La méthode pour l'étude de cas du bassin de Thau a inclus une phase préliminaire d'entretiens individuels avec les acteurs centrés sur les rôles qu'ils accordaient aux terres agricoles, suivie de deux ateliers participatifs. Ces ateliers ont consisté à : identifier des services écosystémiques présents sur le territoire; discuter collectivement des valeurs accordées à ces services; les localiser sur des images satellites; et enfin développer des 
matrices liant ces services aux classes d'occupation du sol (figure 11.4). Certains des résultats de cette étude de cas ont été présentés plus en détail dans Ruoso et al. (2015).

La méthodologie pour Nîmes a été quelque peu différente. Nous avons tout d'abord organisé deux ateliers participatifs centrés sur le renseignement structuré d'une matrice combinant services écosystémiques et classes d'occupation du sol afin de mettre en lumière les connaissances des participants sur les impacts de la construction d'une infrastructure sur les services écosystémiques rendus par les terres agricoles. Par la suite, un travail en laboratoire a été entrepris pour cartographier les résultats des deux ateliers au moyen d'un SIG.

Alors que l'étude de cas du bassin de Thau s'est concentrée à la fois sur la manière dont les acteurs exprimaient les valeurs qu'ils accordaient aux services écosystémiques et sur la création de matrices liant services écosystémiques et occupation du sol, l'étude de cas des Costières de Nîmes s'est focalisée uniquement sur la création de matrices. Deux raisons expliquent ce choix. La première est que pour le bassin de Thau, nous avons privilégié les « dires d'acteurs », ce qui englobe aussi bien les connaissances que les perceptions et valeurs des acteurs, alors que pour la seconde étude de cas, nous nous sommes concentrés sur les « dires d'experts » et donc plus sur leurs connaissances scientifiques que sur leurs perceptions et valeurs. Deuxièmement, alors que le cas de Thau ne présentait pas de contraintes méthodologiques particulières, notre travail sur Nîmes est venu se greffer sur un projet d'aménagement en cours, et seul l'emploi des matrices s'est révélé envisageable.

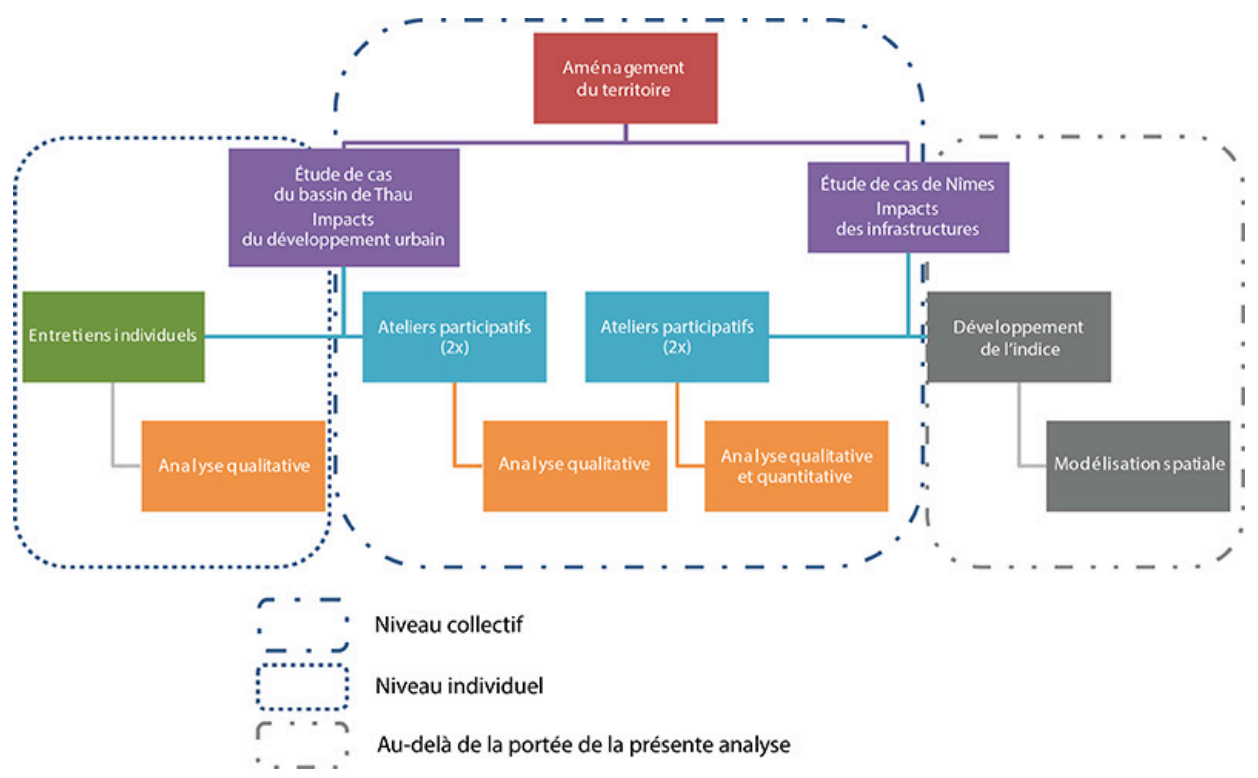

Figure 11.4. Méthodologie. 
Tableau 11.1. Liste des services écosystémiques présentée aux participants dans les deux études de cas (Thau et Nîmes).

\begin{tabular}{|c|c|c|c|}
\hline $\mathbf{N}^{\circ}$ & Service & $\mathbf{N}^{\circ}$ & Service \\
\hline 1 & Assurer le cycle des nutriments & 17 & Emploi, viabilité économique \\
\hline 2 & Maintenir ou améliorer la qualité des sols & 18 & Inspiration (artistique ou autre) \\
\hline 3 & Produire de la biomasse & 19 & Spiritualité et religion \\
\hline 4 & Séquestrer et stocker du carbone & 20 & Éducation, apprentissage et savoirs \\
\hline 5 & $\begin{array}{l}\text { Détoxifier et décomposer des déchets } \\
\text { « chimiques » et organiques }\end{array}$ & 21 & $\begin{array}{l}\text { Loisirs et tourisme (chasse, sports et loisirs de } \\
\text { nature) }\end{array}$ \\
\hline 6 & Réguler le climat & 22 & Historique et patrimoine \\
\hline 7 & $\begin{array}{l}\text { Réguler les aléas naturels (ex. : érosion, } \\
\text { inondations) }\end{array}$ & 23 & $\begin{array}{l}\text { Paysages (esthétiques et thérapeutiques - } \\
\text { paysages qui apaisent) }\end{array}$ \\
\hline 8 & Réguler le cycle de l'eau & 24 & Sentiment d'appartenance à un lieu \\
\hline 9 & Purifier l'eau & 25 & Importance « en soi » des espaces agricoles \\
\hline 10 & Réguler les maladies et les ravageurs & 26 & $\begin{array}{l}\text { Transmission des terres aux générations } \\
\text { suivantes }\end{array}$ \\
\hline 11 & Polliniser & 27 & Relations sociales \\
\hline 12 & Fournir un habitat (faune, flore) & 28 & Ressources pharmaceutiques et médicinales \\
\hline 13 & Produire de l'énergie & 29 & Qualité de l'environnement olfactif et sonore \\
\hline 14 & Produire des aliments & 30 & Santé (santé physique et mentale, force...) \\
\hline 15 & Cueillette & 31 & Fourniture d'ombre et d'abris \\
\hline 16 & Ressources ornementales & & \\
\hline
\end{tabular}

\section{Le bassin de Thau}

\section{Contexte}

Le territoire de Thau se situe dans l'ancienne région Languedoc-Roussillon, dans le sud de la France (figure 11.3), qui fait partie de la nouvelle région Occitanie depuis le $1^{\text {er }}$ janvier 2016. Il se caractérise par la présence d'une lagune principale de 7500 hectares sur un bassin versant de 44000 hectares (Blezat Consulting, 2011). Ce territoire est marqué par une diversité de paysages composés de plaines agricoles, de garrigues, de forêts, de zones humides et de lagunes, et par les activités qui leur sont associées comme l'agriculture, la pêche, l'ostréiculture et le tourisme. La présence de la lagune de Thau a fortement façonné l'activité économique autour de la conchyliculture et de la pêche. Malgré de fortes pressions urbaines, les terres et activités agricoles jouent toujours un rôle important et couvrent à l'heure actuelle environ $50 \%$ du territoire de Thau. La principale activité agricole est la viticulture $(33,5 \%$ des terres agricoles du bassin et $18 \%$ du territoire total) (Blezat Consulting, 2011). La céréaliculture, le maraîchage, l'arboriculture et l'élevage laitier (un élevage de chèvres) occupent au total $5 \%$ $\mathrm{du}$ territoire. Les surfaces en friches agricoles sont très importantes ( $24 \%$ du territoire). Au moment de notre étude, le territoire de Thau était constitué de deux intercommunalités : la Communauté d'agglomération du bassin de Thau (CABT) et la Communauté de communes du nord du bassin de Thau (CCNBT). Elles ont été regroupées en une 
seule intercommunalité le $1^{\text {er }}$ janvier 2017 : Sète Agglopôle Méditerranée. Une structure d'ingénierie territoriale créée en 2005, le Syndicat mixte du bassin de Thau (SMBT), a mis en place un Schéma de cohérence territoriale (SCoT), un document de planification spatiale qui intègre des politiques sectorielles pour ces deux intercommunalités. Dans ce document, les décideurs ont fait le choix d'orienter l'intensification du développement urbain dans le triangle formé par les villes de Sète, de Frontignan et de Balarucles-Bains (figure 11.5), et de préserver les terres agricoles dans la partie nord du bassin, principalement autour de Villeveyrac, Loupian et Montbazin. C'est pour cette raison que nous avons choisi de situer notre zone d'étude dans cette partie nord (figure 11.5). Elle couvre trois communes aux caractéristiques géographiques différentes, allant de la lagune à l'arrière-pays : Bouzigues, Loupian et Villeveyrac.

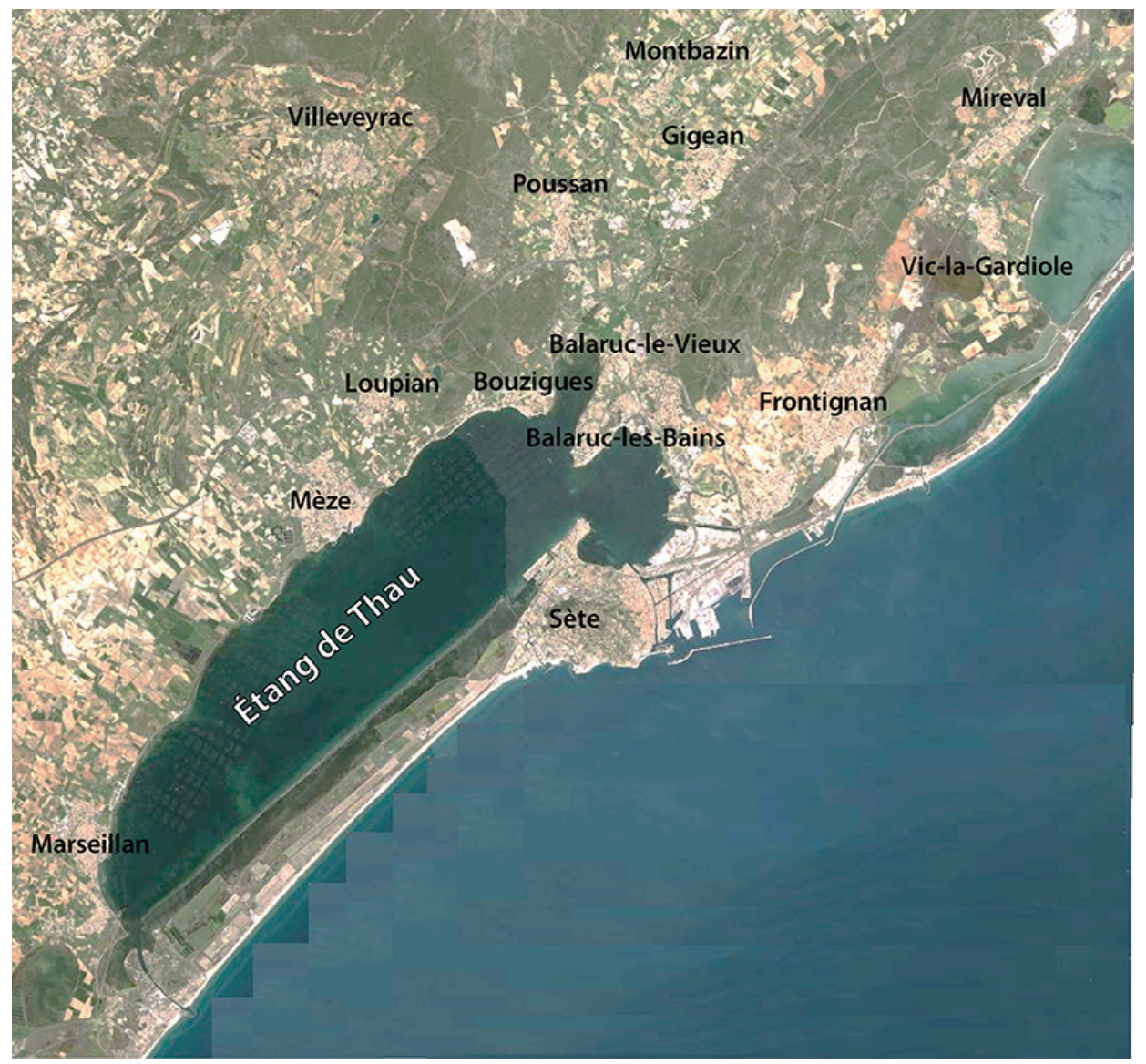

Figure 11.5. Bassin de Thau (contient des informations $\odot$ Airbus DS/Spot Image 2017, ๔ Production IRD, Irstea, IGN, tous droits réservés).

\section{Entretiens et ateliers participatifs}

Le but de l'étude était de comprendre les valeurs que les acteurs accordaient aux terres agricoles périurbaines, et de les capturer. Pour cela, nous avons conduit des entretiens individuels auprès de divers acteurs, ainsi que deux ateliers participatifs. Les entretiens portaient sur l'usage que les acteurs faisaient des terres agricoles, le rôle qu'ils leur donnaient et les défis qu'ils identifiaient dans ces espaces (identification des cadres), sans 
qu'il soit fait référence au concept de services écosystémiques et à ses principes. Dans le premier atelier, nous avons donné aux participants une liste de services écosystémiques (appelés « rôles ») en leur demandant de désigner ceux qui étaient fournis par les terres agricoles du bassin. Le but de cet exercice était de compléter les informations obtenues sur les services écosystémiques durant les entretiens individuels. Nous nous sommes basés pour cela sur 13 typologies des services écosystémiques issues de la littérature anglo-saxonne et française. S'ils estimaient que la liste n'était pas exhaustive, les acteurs pouvaient aussi ajouter des services, comme la capacité des terres agricoles à contrôler l'urbanisation et à produire de la diversité paysagère, mais aussi des disservices comme la pollution de la lagune par l'agriculture. À la fin de cet atelier, nous avons présenté de manière plus formelle, mais toujours très générale, la notion de services écosystémiques aux participants. Durant le second atelier, qui est celui sur lequel nous nous concentrerons ici, notre but était de spatialiser certains des services écosystémiques identifiés dans la zone d'étude (comment capturer les services ?). Pour des raisons de temps, seul un nombre limité de services a été abordé. Le programme de l'atelier s'est déroulé en deux phases avec les 16 participants répartis en quatre groupes de quatre. Durant la première phase, les participants devaient identifier la présence de certains services écosystémiques sur une image satellite multispectrale Pléiades (50 centimètres de résolution), où figuraient aussi le réseau hydrologique et les limites des communes (figure 11.6). Chaque groupe était invité à tracer sur les cartes les endroits (sous la forme de points, de lignes ou de polygones) où ils identifiaient des services. Les cartes ont été utilisées ici dans un rôle de référentiel partagé pour permettre aux acteurs d'objectiver et de spatialiser collectivement les services écosystémiques (Maurel, 2012).

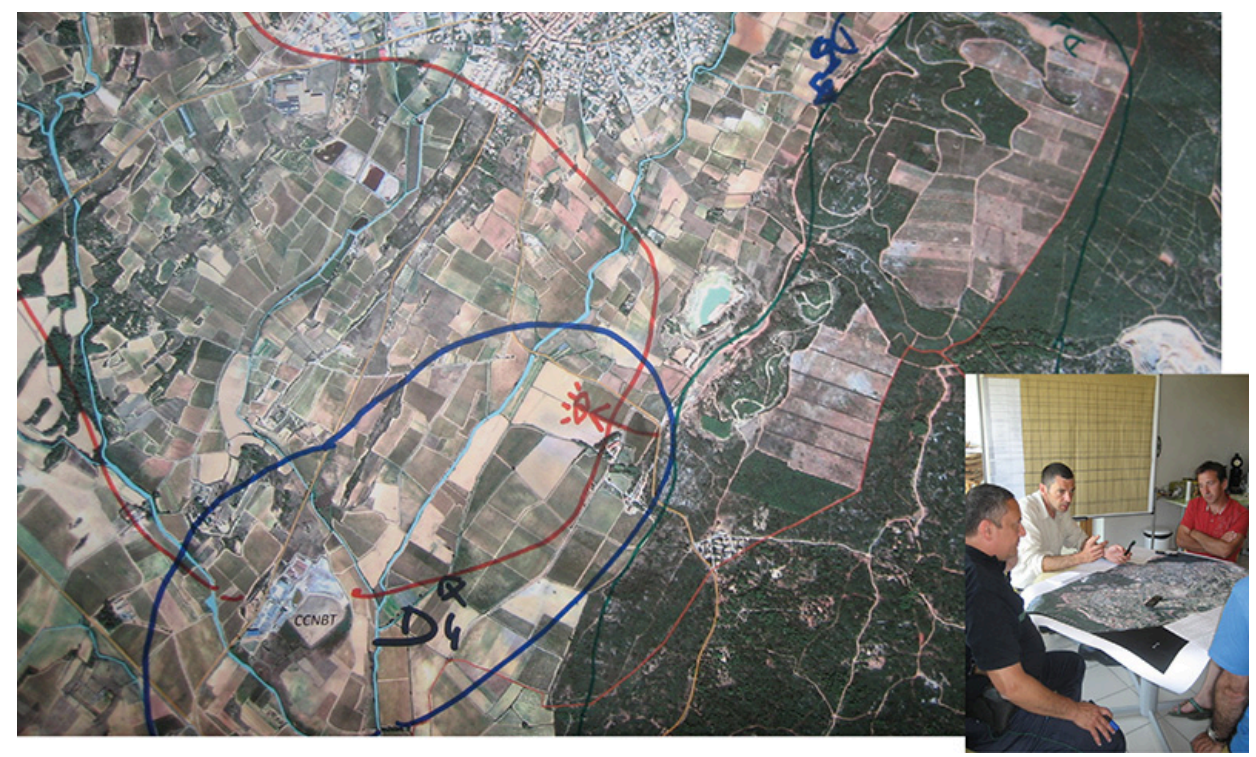

Figure 11.6. Identification de services écosystémiques sur une image Pléiades par un groupe de participants.

Durant la deuxième phase de l'atelier, le but était plus spécifiquement de décrire les « conditions d'existence » ou de production des services écosystémiques identifiés précédemment. Nous avons demandé pour cela aux acteurs de relier certains services — nous avons ici utilisé explicitement le terme de "services écosystémiques » - aux éléments 
du paysage qui leur permettaient d'exister. Ce travail a été fait au moyen d'une matrice créée à partir d'éléments considérés a priori comme pertinents et d'éléments physiques identifiés lors des entretiens individuels comme jouant un rôle dans la présence des services écosystémiques dans la zone d'étude. La matrice était constituée des éléments suivants : l'occupation du sol (vignes, maraîchage, oliviers, voierie, bâti, etc.) ; l'association d'éléments paysagers divers (par exemple, association d'éléments naturels et cultivés) ; le bassin versant ; des paramètres biophysiques (par exemple, climat, relief, exposition et ressource en eau) ; l'accessibilité des espaces agricoles (clôtures, manque de routes, droit d'accès, manque d'entretien des espaces, etc.) et la structure des exploitations agricoles (taille, distribution des parcelles, type d'agriculture : conventionnelle, raisonnée, biologique, etc.). Chaque groupe devait associer les services qu'il plaçait sur la carte à des éléments présents dans la matrice (figure 11.7). Les acteurs pouvaient rajouter, s'ils le souhaitaient, de nouveaux éléments, ce qu'ils ont fait en ajoutant par exemple dans la catégorie " bâti » les capitelles et les murs en pierre sèche. Le but était ici de créer, de manière participative, une matrice liant certains services écosystémiques à des éléments physiques pour produire ensuite une cartographie collective.

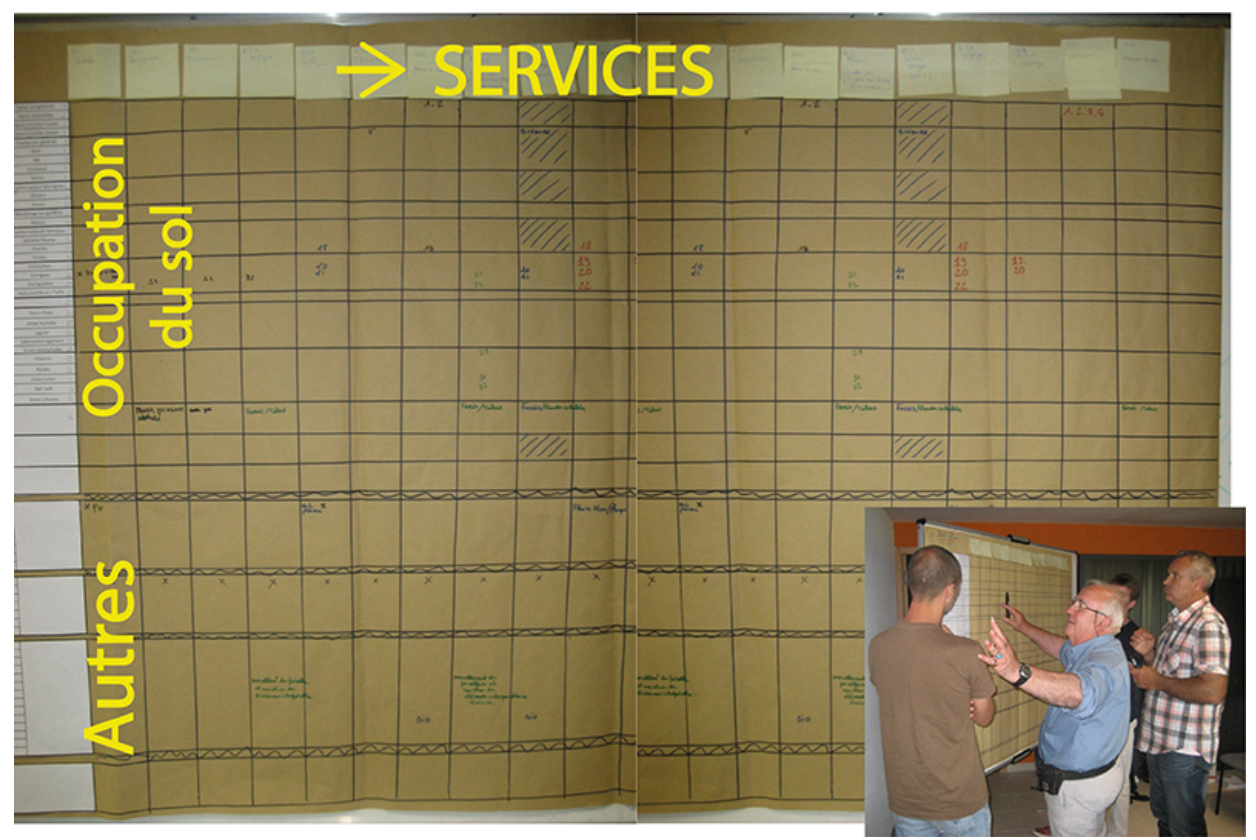

Figure 11.7. Remplissage d'une matrice (services $\times$ occupation du sol) par un groupe de participants.

\section{Informations obtenues}

Quelles valeurs? Tout d'abord, le cadrage initialement implicite du concept de service écosystémique, puis l'utilisation d'une simple liste de services ont permis aux acteurs d'adopter, durant les entretiens et les ateliers, une variété de «cadres » pour décrire les relations entre environnement et société. Cela les a aidés à aller au-delà d'un cadrage purement économique où les relations entre l'environnement et la nature ne sont considérées que sous l'angle de bénéfices à quantifier. En effet, l'usage implicite du langage et du concept de services écosystémiques a permis aux acteurs d'exprimer différentes manières d'envisager les relations entre société et environnement. Raymond et al (2013) 
expliquent que diverses métaphores peuvent illustrer ces relations, comme la métaphore du stewardship (considérer la Terre comme un foyer dont les sociétés humaines doivent prendre soin) ou celle du web of life (considérer les sociétés humaines comme faisant partie d'un système écologique plus large qu'elles doivent comprendre). Dans notre étude de cas, les acteurs ont exprimé différents points de vue sur ces liens entre nature et société, en phase avec les métaphores du stewardship et du web of life. Par exemple, ils ont souvent décrit le bassin de Thau comme étant un paysage complètement anthropisé et géré par l'homme. Selon eux, aucun espace ne pouvait être qualifié de naturel, y compris les garrigues qui portent encore les traces d'une activité agricole passée. Par conséquent, ils se considéraient souvent (les agriculteurs particulièrement) comme ayant le devoir de prendre soin de l'environnement et de le gérer afin de préserver le service de «beauté paysagère ». Ceci démontre une vision des relations entre sociétés humaines et environnement en accord avec la métaphore du stewardship. Certains acteurs étaient aussi en accord avec la métaphore du web of life, car ils avaient développé une vision systémique de leur environnement local. Ils voyaient certains services, tel celui de production alimentaire, comme ayant un rôle à jouer dans le système territorial local, en maintenant les paysages ouverts, en créant de l'emploi et de la solidarité entre producteurs et consommateurs locaux. Ils ont donc vu un composant du système - la production alimentaire - comme permettant de maintenir et de développer d'autres composants. Pour une description plus détaillée des relations sociétés-nature telles qu'exprimées par les acteurs, voir Ruoso et al. (2015).

Les valeurs de quels acteurs? En ce qui concerne les bénéficiaires, notre étude s'est principalement centrée sur les acteurs habitant ou travaillant actuellement dans la zone d'étude, et n'a pas pris en compte les bénéficiaires plus éloignés, ni les bénéficiaires futurs. Toutefois, les acteurs ont indiqué des éléments de changements en cours dans la zone d'étude qui allaient mener à la diminution, l'accentuation ou la mise en compétition de certains services écosystémiques. Cela nous a permis d'identifier certains services qui pourraient un jour disparaître de la zone d'étude, au détriment des générations à venir. Par exemple, pour certains participants, l'accroissement des friches mène à la perte de l'attachement aux espaces agricoles (service " sentiment d'appartenance à un lieu »), et est le signe du manque de viabilité économique des exploitations agricoles (perte du service " emplois et viabilité économique »). L'enfrichement est aussi considéré comme entraînant une diminution des services de régulation des feux et de beauté des paysages. D'autres acteurs considèrent que les friches profitent à la biodiversité (service de provision d'habitats), car elles permettent à certaines espèces de revenir sur la zone d'étude (par exemple l'outarde canepetière, Tetrax tetrax) (voir aussi l'étude de cas sur Nîmes ci-dessous). Nous pouvons voir ici que l'augmentation du nombre de friches entraîne la diminution de la présence de certains services, et l'augmentation de la présence d'autres services. Un autre exemple est celui des clôtures, dont le nombre croissant dans la zone d'étude a été perçu comme le signe d'une augmentation du nombre de propriétaires de chevaux avec leurs zones de pâturage (services récréatifs). Ce type de service entre en compétition directe avec un autre service récréatif, la chasse. En effet, les activités de chasse ne peuvent se dérouler que dans des paysages ouverts et accessibles fournis par les paysages agricoles traditionnels dans la zone d'étude.

Même si cette étude nous a permis d'entrevoir les conséquences possibles de certains changements en cours, elle n'a pas permis d'avoir une vision d'ensemble de la manière dont les changements qui affectent le territoire pourraient avoir un impact sur le territoire et priver les générations futures de certains services. De plus, notre étude ne nous a pas 
permis de développer une vision systématique de la compétition entre services du fait d'intérêts contradictoires parmi les acteurs.

Comment capturer les services écosystémiques? Finalement, utiliser une méthode participative à dire d'acteurs nous a permis de saisir les valeurs et perceptions que les acteurs avaient des services écosystémiques dans le bassin de Thau, ainsi que les éléments paysagers qui rendent leur existence possible. Par exemple, lors des entretiens individuels et des discussions autour des cartes et des matrices, il a été possible d'identifier certains éléments agricoles ayant une valeur patrimoniale pour les acteurs (service historique et patrimonial), tels que certains types de couverture du sol comme les vignes, les garrigues, les oliveraies, les champs de blé, mais aussi des éléments ponctuels comme les capitelles, les murs en pierre sèche, la voie Domitienne, etc. La signification de ces différents éléments du paysage a pu ensuite être discutée. Par exemple, les espaces de garrigue, leurs capitelles et murs en pierre sèche sont considérés par certains acteurs comme étant les témoins d'une présence humaine ancienne, dans la garrigue, de pratiques agricoles très différentes de celles d'aujourd'hui, ainsi que de l'histoire sociale du territoire, puisque les ouvriers agricoles cultivaient ces terres de garrigues le soir venu afin d'avoir une source d'alimentation supplémentaire. Un autre exemple est celui du mélange de vignes, de blé et d'oliviers autour de Villeveyrac qui évoque, pour certains, les vestiges des pratiques culturales romaines. La conjonction de discussions individuelles et collectives ainsi que les exercices de cartographie et de remplissage des matrices nous ont donc permis de commencer à identifier les éléments physiques qui conditionnent la présence sur le territoire de certains services liés à l'agriculture, et les valeurs ou significations que les acteurs accordent à ces éléments.

\section{Les Costières de Nîmes}

\section{Contexte}

Le territoire des Costières de Nîmes est situé au sud-est de la ville de Nîmes, dans le département du Gard, dans la même région que le territoire de Thau. Il couvre une surface de 25000 hectares répartis sur 24 communes. Le moteur principal du changement de l'occupation du sol est un projet d'infrastructure de transport terrestre (ITT) : la ligne TGV entre Nîmes et Montpellier. L'annonce de ce projet il y a plusieurs années a entraîné de la spéculation foncière et l'abandon de terres agricoles qui se sont progressivement transformées en habitats pour diverses espèces, dont un grand oiseau de la famille des outardes, l'outarde canepetière (Tetrax tetrax), le seul membre du genre Tetrax. Cette boucle de rétroaction inattendue pose un défi quant aux rôles et valeurs des services écosystémiques sur les terres agricoles abandonnées en raison d'un projet d'ITT. L'objectif de notre étude de cas était de développer et de tester une méthodologie pour évaluer l'ensemble des impacts (positifs et négatifs) de la réalisation d'une ligne TGV sur les services écosystémiques, en se basant sur le savoir expert de professionnels locaux représentant différents secteurs.

\section{Ateliers participatifs}

La méthodologie s'est centrée sur la création d'une matrice bidimensionnelle liant les services écosystémiques prioritaires aux occupations du sol générant ces services. Deux ateliers de trois heures avec des experts ont été conduits à Nîmes. Six participants représentaient les organisations listées dans le tableau 11.2. 


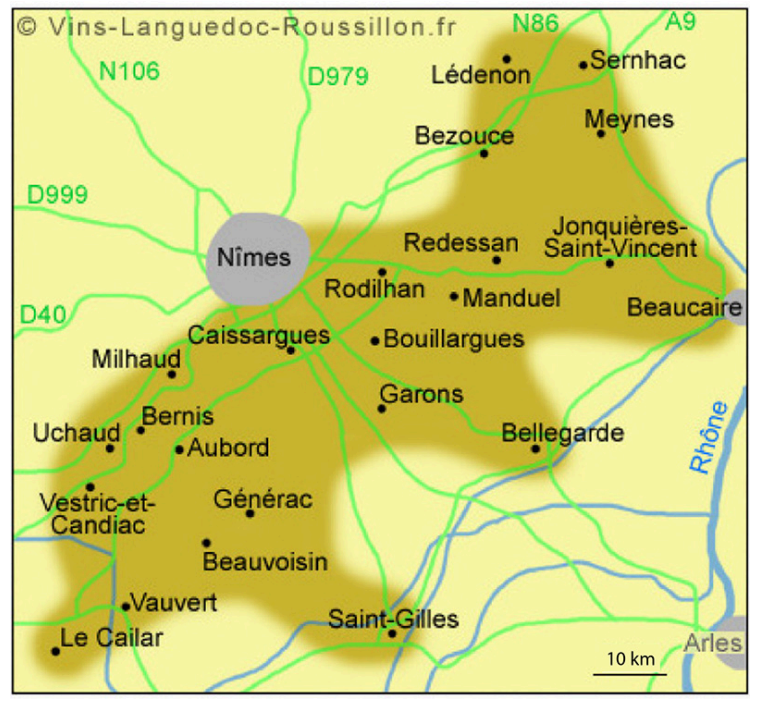

Tableau 11.2. Organisations représentées par les participants à l'étude de cas de Nîmes.

\begin{tabular}{|l|l|}
\hline \multicolumn{1}{|c|}{ Nom de l'organisation } & \multicolumn{1}{c|}{ Description } \\
\hline Nîmes Métropole & $\begin{array}{l}\text { Structure intercommunale, établie en 2002, centrée sur la ville } \\
\text { de Nîmes. }\end{array}$ \\
\hline Dreal Languedoc-Roussillon & $\begin{array}{l}\text { Direction régionale de l'environnement, de l'aménagement et } \\
\text { du logement pour l'ancienne région Languedoc-Roussillon }\end{array}$ \\
\hline Chambre d'agriculture du Gard & $\begin{array}{l}\text { Chambre d'agriculture du département du Gard, structure de } \\
\text { formation et de prestation de services pour les organisations } \\
\text { agricoles professionnelles. }\end{array}$ \\
\hline $\begin{array}{l}\text { Conservatoire d'espaces naturels } \\
\text { Languedoc-Roussillon (CEN L-R) }\end{array}$ & $\begin{array}{l}\text { Association pour la conservation et la protection du patrimoine } \\
\text { naturel dans le département du Gard (créée en 1990). }\end{array}$ \\
\hline Centre ornithologique du Gard (COGard) & $\begin{array}{l}\text { Centre pour l'étude, la protection et la promotion des } \\
\text { connaissances sur l'avifaune du département du Gard (créé en } \\
\text { 1980). }\end{array}$ \\
\hline $\begin{array}{l}\text { Agence d'urbanisme et de développement } \\
\text { des régions nîmoise et alésienne (A'U) }\end{array}$ & $\begin{array}{l}\text { Structure technique à but non lucratif en appui à l'État et aux } \\
\text { collectivités locales pour l'aménagement urbain et territorial } \\
\text { (créée en 1989). }\end{array}$ \\
\hline
\end{tabular}

Avant le premier atelier, une matrice initiale avait été développée, représentant les combinaisons possibles entre l'occupation du sol et les services écosystémiques. En colonne figuraient les 31 services dérivés de la littérature (tableau 11.1). Comme exercice introductif, les six experts ont examiné individuellement ces 31 services pour indiquer ceux qui devraient être conservés, d'après leur expertise, leurs connaissances du projet ITT et leur compréhension des bénéficiaires des services listés. Ils pouvaient également ajouter des services en utilisant leurs propres termes. En ligne figuraient les catégories d'occupation du sol basées sur Corine-Biotopes (tableau 11.3). Les acteurs devaient définir collectivement un niveau d'impact allant de 0 (pas d'impact) à 4 (impact fort ${ }^{45}$ ) pour chaque combinaison entre les classes d'occupation du sol et les services écosystémiques. En 
raison des contraintes de temps, il n'a pas été possible durant l'atelier de couvrir toutes les combinaisons. Il a donc été demandé aux experts de compléter la matrice individuellement chez eux et d'envoyer les résultats aux chercheurs avant le second atelier, ce qu'ont fait cinq des six experts. En utilisant l'index de variation qualitative - une mesure de dispersion statistique d'une distribution nominale - les matrices complétées individuellement ont été harmonisées pour obtenir des valeurs uniques (de 0 à 4 ) pour chaque combinaison d'occupation du sol et de services écosystémiques, valeurs considérées comme une représentation du consensus des acteurs. Les scores, pour chaque catégorie d'occupation du sol, ont ensuite été indexés au moyen d'un index composite pour conserver la variation des scores. Dans une telle approche, le score composite pour la combinaison 1-2-1-4 =8, par exemple, est différent de la combinaison 2-3-1-2=8. Le second atelier a donné l'opportunité aux participants de réfléchir sur les matrices complétées à partir du travail additionnel réalisé par les chercheurs.

Tableau 11.3. Classification de l'occupation du sol.

\begin{tabular}{|c|c|}
\hline Catégories utilisées dans la matrice & Classification Corine-Biotopes \\
\hline Vigne sur sol nu & Vignobles intensifs \\
\hline Vigne enherbée & Vignobles traditionnels \\
\hline Vigne récemment plantée & Vignobles \\
\hline Vigne récemment arrachée & Terrains en friche \\
\hline Friche herbacée & Terrains en friche \\
\hline Friche mixte & Terrains en friche \\
\hline Friche arbustive & Terrains en friche $\times$ fourrés décidus \\
\hline Friche viticole & Terrains en friche \\
\hline \multirow{2}{*}{ Pâture } & Pâtures mésophiles (si mésophile) \\
\hline & Terrains en friche (si non mésophile) \\
\hline \multirow{2}{*}{ Prairie } & Prairies fourragères des plaines (si mésophile) \\
\hline & Terrains en friche (si mésoxérophile) \\
\hline Luzerne (parcelles en légumineuse) & Grandes cultures \\
\hline Céréale & Grandes cultures \\
\hline \multirow{4}{*}{ Arboriculture } & Oliveraies traditionnelles \\
\hline & Vergers à amandiers \\
\hline & Rosacées \\
\hline & Agrumes \\
\hline Terre labourée & Cultures \\
\hline Maraîchage & Cultures et maraîchage \\
\hline \multirow{4}{*}{ Canal, rivière } & Lagunes \\
\hline & Plans d'eau douce \\
\hline & Eaux saumâtres sans végétation \\
\hline & Lit des rivières \\
\hline
\end{tabular}




\begin{tabular}{|l|l|}
\hline Catégories utilisées dans la matrice & \multicolumn{1}{|c|}{ Classification Corine-Biotopes } \\
\hline \multirow{5}{*}{ Bâti } & Salines \\
\cline { 2 - 3 } & Canaux navigables \\
\cline { 2 - 3 } & Fossés et petits canaux \\
\cline { 2 - 3 } & Lagunes industrielles et bassins ornementaux \\
\hline \multirow{5}{*}{ Boisement } & Villes \\
\cline { 2 - 3 } & Villages \\
\cline { 2 - 3 } & Sites industriels en activité \\
\cline { 2 - 3 } & Sites industriels anciens \\
\hline \multirow{5}{*}{} & Matorral arborescent de chênes sempervirents \\
\cline { 2 - 3 } & Galeries méditerranéennes de grands saules \\
\cline { 2 - 3 } & Chênaies blanches \\
\cline { 2 - 2 } & Forêts de pins d'Alep \\
\cline { 2 - 2 } & Bois de frênes riverains \\
\cline { 2 - 2 } & Chênaies vertes \\
\hline
\end{tabular}

\section{Informations recueillies}

Durant le premier atelier, les six experts ont collectivement observé tous les services écosystémiques qui leur étaient présentés sous la forme d'une simple liste (c'est-à-dire sans expliquer le concept sous-jacent de services écosystémiques et les différentes catégories). Seul un expert a choisi d'ajouter un service (« innovation d'infrastructure »). La liste contextualisée découlant de la réflexion individuelle des experts comprenait donc au final 32 services. La figure 11.9 fournit un échantillon de la diversité de ces services dans la sélection initiale des acteurs.

L'harmonisation des réponses divergentes des participants a été faite au moyen d'un index de variation qualitative. Alors que notre objectif premier était d'obtenir une valeur unique (0-4), nécessaire pour calculer un index composite des services écosystémiques, nous nous sommes aperçus que l'analyse de l'hétérogénéité des réponses des experts et le développement d'un outil informatique pour rendre ces divergences visibles (exemple sur la figure 11.10) offraient des informations additionnelles utiles.

Cette tentative d'évaluation participative «à dire experts » des services fournis par les terres agricoles à Nîmes a montré qu'il s'agissait d'un processus chronophage demandant beaucoup d'efforts aux participants. La simple taille de la matrice (débattre sur 352 cellules !) s'est avérée être un défi majeur. Travailler avec moins de combinaisons, en limitant par exemple les catégories d'occupation du sol à celles qui sont directement liées à l'agriculture, aurait minimisé le problème. Le remplissage de matrices individuelles et l'agrégation par les chercheurs constituent une alternative viable, notamment si les résultats de l'analyse peuvent être partagés et débattus avec le même groupe d'experts, ce que nous avons fait durant notre second atelier. 
Choix des services : 32 services écosystémiques retenus

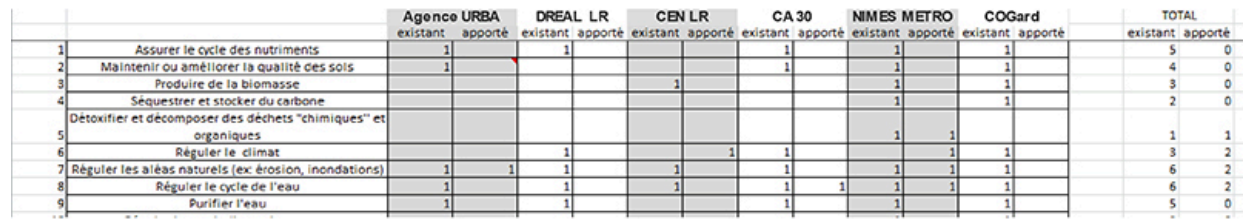

Capacité des classes d'occupation du sol pour fournir des services écosystémiques (de $4=$ fort à vide $/ 0=$ nulle)

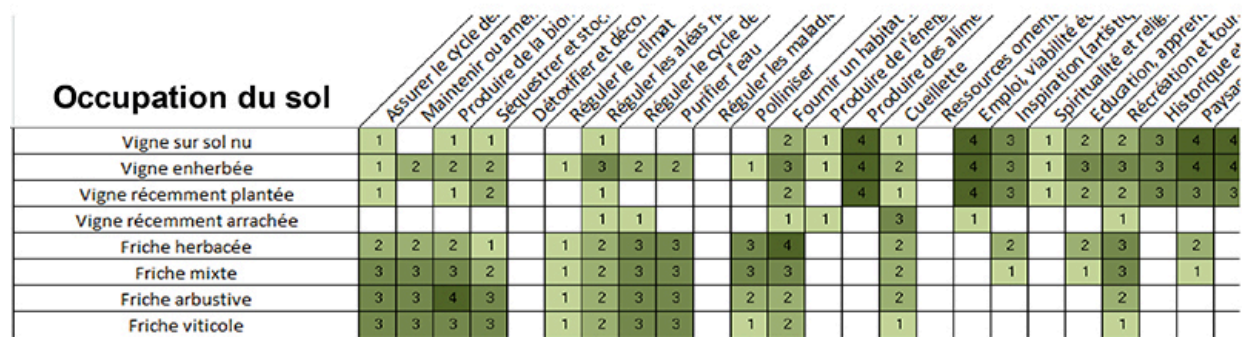

Figure 11.9. Sélection des services écosystémiques par les experts et évaluation des impacts sur les services écosystémiques.

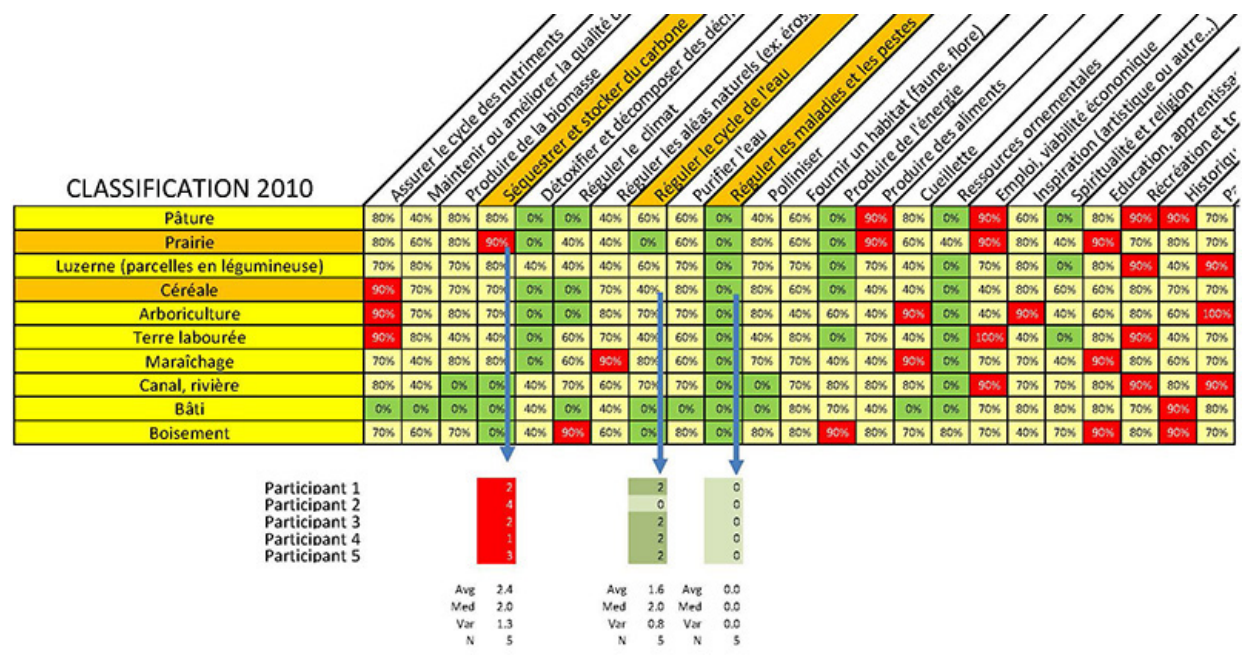

Figure 11.10. Intégration des différences entre les savoirs d'experts.

\section{Discussion}

\section{Réflexion critique sur l'approche adoptée}

Lorsque nous comparons les résultats de notre étude aux dernières avancées de la recherche sur les services écosystémiques, des éléments clés émergent.

Quelles valeurs ? Prendre du recul par rapport à la notion scientifique de service écosystémique en abandonnant le jargon et la complexité qui lui sont associés est une approche qui a bien fonctionné dans notre contexte. Notre cadrage implicite, qui consistait à poser des questions générales sur les rôles présents et futurs des terres agricoles périurbaines face à des changements rapides, a permis aux acteurs du bassin de Thau d'exprimer une 
grande variété de valeurs, de perceptions et de types de connaissances au cours des entretiens individuels et des discussions de groupe. Cela nous a permis de saisir les différents types de relations que les acteurs entretiennent avec leur environnement.

Les valeurs de quels acteurs? Afin d'obtenir la meilleure représentativité possible des acteurs concernés, dans la limite des ressources de notre projet de recherche, nous avons opté pour une approche pragmatique d'échantillonnage boule de neige pour recruter les participants. Une évaluation participative plus formelle nécessiterait sans doute une représentation plus exhaustive des bénéficiaires, à partir, par exemple, d'une cartographie préalable des acteurs avant les ateliers participatifs. De plus, bien que très importante pour la planification spatiale, l'intégration des valeurs, des perspectives et des connaissances des bénéficiaires futurs constitue un vrai défi. Cela pourrait être accompli dans une certaine mesure en présentant lors des ateliers participatifs des projections des changements sociodémographiques (et de leurs impacts) et en demandant aux participants leurs points de vue sur les bénéfices escomptés pour les générations à venir. Ceci pourrait faire l'objet d'un atelier participatif additionnel où seraient discutés les principes communs permettant d'assurer à la fois la protection des terres agricoles et la prise en compte des intérêts des générations futures.

Une approche ciblée sur les bénéficiaires correspond bien à la tendance actuelle où les responsabilités concernant la planification spatiale incombent désormais aux échelons politico-administratifs locaux et s'appuient de plus en plus sur l'intégration de perspectives, connaissances et valeurs locales (voir chapitre 1).

Comment capturer ces valeurs? Alors que les approches participatives sont fréquemment mises en avant comme un outil essentiel pour la gestion de l'environnement, dans notre cas, la transposition de nos résultats en représentations (spatiales) compatibles avec les exigences réglementaires en matière d'aménagement du territoire a constitué un véritable défi, et ce pour plusieurs raisons. Il a tout d'abord été difficile de produire des matrices qui prennent en compte l'intégralité des services et des bénéficiaires sur un territoire. Deuxièmement, même si les deux études de cas ont été suivies de près par des aménageurs et des décideurs légitimes sur ces territoires, notre travail était motivé par des questions de recherche plus que par l'ambition de répondre à des défis politiques et d'aménagement immédiats. Par conséquent, les résultats de ce projet de recherche n'ont pas trouvé d'application immédiate dans les processus de planification opérationnels sur ces deux territoires.

D'un point de vue méthodologique, nous avons rencontré un certain nombre de difficultés. Premièrement, certains acteurs, particulièrement dans le cas de Thau, n'ont pas adhéré à l'exercice de renseignement des matrices, et n'ont donc que peu participé, contrairement à l'exercice de cartographie qui avait suscité beaucoup de discussions. Des recherches futures pourraient se concentrer principalement sur la cartographie comme représentation plus intuitive, particulièrement pour les non-experts, plutôt que de préciser et de détailler la myriade de liens abstraits entre les catégories d'occupation du sol et la fourniture de services écosystémiques (Crossman et al., 2013). Deuxièmement, développer une compréhension profonde des perceptions des acteurs pour chaque service écosystémique prend un temps considérable, ce qui nous a obligés, sur Thau, à nous restreindre à quelques services. Nous n'avons ainsi pas pu explorer en détail les synergies et les contradictions potentielles entre services écosystémiques en matière d'aménagement du territoire. Dans le cas de Nîmes, le renseignement complet de la matrice n'a pu s'élaborer qu'en prolongeant le travail de groupe par un travail de remplissage individuel après l'atelier. Ces deux retours d'expérience montrent qu'il n'est pas aisé d'obtenir des matrices pour l'intégralité des services sur un territoire. Nous savons aussi que 
nos ateliers n'ont rassemblé qu'une petite partie des bénéficiaires, pas nécessairement représentatifs de la population du territoire. Pour éclairer véritablement la prise de décision, il faudrait reproduire ces exercices participatifs avec différents groupes sociaux et comprendre les relations de pouvoir qui existent dans la zone d'étude afin d'exploiter au mieux cette approche basée sur les services écosystémiques. Troisièmement, nous avons noté, dans le cas de Thau, que certains participants aux ateliers craignaient que les cartes de services écosystémiques créées puissent être utilisées comme des outils prescriptifs de zonage, synonymes de contraintes réglementaires supplémentaires, comme cela a été vu par ailleurs (Couderchet et Amelot, 2010). Ceci pourrait décourager certains acteurs de partager leurs connaissances. Enfin, dans le cas de certains services, comme celui de production alimentaire qui nécessiterait de cartographier les exploitations maraîchères et arboricoles, la production de cartes explicites et réalistes pourrait mener à accroître les vols ou les dégâts faits aux cultures, particulièrement si ce type de cartes devenait accessible au grand public. Dans le même ordre d'idée, la cartographie des services basés sur la pratique de cueillette (baies, champignons, etc.) pourrait mener à une surexploitation ou même à la disparition de ressources. Ces exemples soulignent la nécessité d'une réflexion approfondie en matière de cartographie des services écosystémiques.

\section{Perspective d'intégration des services écosystémiques dans l'aménagement des paysages et du territoire}

L'inclusion d'approches basées explicitement ou implicitement sur le concept de services écosystémiques dans les processus opérationnels de planification territoriale soulève un certain nombre de difficultés identifiées il y a plusieurs années (De Groot et al., 2010). Cependant, des progrès importants ont été faits depuis, en France, à travers le leadership du projet d'Évaluation française des écosystèmes et des services écosystémiques (Efese), le volet français du programme européen, Cartographie et évaluation des écosystèmes et de leurs services (Mapping and Assessment of Ecosystems and their Services; MAES), qui a mené à la production de cadres conceptuels désormais reconnus et diffusés comme nouveaux cadres de référence (Efese, 2016). L'un des composants essentiels concerne les valeurs (d'usage, patrimoniales, morales) de nos relations à la nature (Efese, 2016 ; Maris et al., 2016). Au niveau national, l'inventaire des services écosystémiques reste encore largement incomplet, particulièrement pour les services culturels liés aux dimensions patrimoniales de la nature. En ce qui concerne les zones agricoles, une étude récente conduite dans le cadre du projet Efese par l'Institut national de recherche agronomique pour caractériser les services fournis par ces zones s'est principalement concentrée sur les services de production et de soutien, sans intégrer les services de régulation des risques (inondations, incendies), ni les services culturels apportés par les paysages (Tibi et Therond, 2017). Pour la planification à l'échelle locale, il existe encore moins de données prêtes à l'emploi suffisamment précises et exhaustives, particulièrement sur la couverture et l'occupation du sol, pour quantifier et cartographier les services écosystémiques. Ceci est d'autant plus vrai pour les services culturels qui reposent sur les jugements de valeur d'individus ou de groupes sociaux, et qui impliquent, pour être identifiés, de faire des entretiens et des exercices participatifs.

Pour ces différentes raisons, utiliser le concept de services écosystémiques dans l'aménagement du territoire à l'échelle locale ne peut pas se faire sans une forte mobilisation des acteurs, afin de s'appuyer sur leurs savoirs, leurs représentations de la réalité et les valeurs qu'ils attachent à la nature et à ses services. Ceci nécessite une forte volonté politique, une culture démocratique de la participation pour mobiliser des ressources ainsi que de solides compétences en ingénierie territoriale et en ingénierie de la participa- 
tion. Ceci implique aussi que les élus et les équipes techniques de l'administration et des bureaux d'études se soient appropriés le concept de services écosystémiques ou bénéficient de soutiens scientifiques. Dans la plupart des communes, ces conditions sont encore loin d'être réunies.

Cependant, des avancées prometteuses ont été faites ces dernières années. En France, beaucoup d'expérimentations innovantes ont été conduites (voir, par exemple, la compilation d'études de cas dans le rapport provisoire d'Efese, 2016, ou Bertrand, 2016). Des cadres théoriques (Luque et al., 2017), des méthodologies et des outils concrets sont progressivement venus enrichir la "boîte à outils » de l'ingénierie territoriale afin d'intégrer les services écosystémiques dans la planification spatiale. La politique d'open data et la compilation de données participatives ou l'utilisation de données sur les réseaux sociaux offrent aussi de nouvelles perspectives pour inventorier et estimer les valeurs accordées aux services écosystémiques. Les réglementations à l'échelle européenne et nationale pour la protection de la nature, comme par exemple Natura 2000, et son intégration dans des politiques sectorielles (biodiversité, eau, agriculture, forêt, urbanisme, etc.) constituent des éléments favorables, même si le terme de "services écosystémiques » reste plus rarement employé que celui de «fonctions » (Mongruel et $a l ., 2016$ ), à l'exception de la protection des habitats naturels (politique de la Trame verte et bleue, séquence ERC, éviter, réduire, compenser). L'évaluation environnementale et les processus participatifs sont en effet devenus obligatoires dans les projets de développement, y compris à l'échelle locale, tout en laissant une marge suffisante dans la mise en œuvre opérationnelle pour pouvoir mobiliser des concepts et des approches innovantes.

Concernant l'approche exploratoire que nous avons développée dans les deux études de cas présentées ici, les perspectives de mise en œuvre opérationnelle sont très variées du fait d'évolutions différentes du contexte dans les deux cas.

À Nîmes, la ligne de TGV est maintenant installée. L'obligation légale de protéger la biodiversité a mené les acteurs à se concentrer uniquement sur les services de protection de l'habitat de certaines espèces protégées en suivant le principe ERC (éviter, réduire, compenser). Des efforts et des moyens significatifs ont été nécessaires pour calculer la valeur des habitats, mobiliser les acteurs et rendre le processus compréhensible et transparent (Quetier, 2015). Des compensations ont été réalisées pour contrebalancer les impacts sur les habitats et les populations d'outarde (Tetrax tetrax). Un observatoire environnemental, composé des autorités administratives et de l'autorité de gestion de l'infrastructure ferroviaire, a été mis en place pour faire le suivi de la mise en œuvre des compensations et mesurer leur efficacité en matière de protection des habitats naturels, y compris après le début du fonctionnement de la nouvelle infrastructure ferroviaire. Une option serait d'étendre le périmètre de l'observatoire à d'autres services écosystémiques en utilisant, comme cela a été fait dans notre étude exploratoire, une matrice entre services écosystémiques et occupation du sol.

Sur le territoire de Thau, le projet politique de développement à long terme basé sur la qualité des ressources environnementales se poursuit sur la base d'une approche intégrée et adaptative et d'un système de gouvernance pluri-acteurs (Plant et al., 2014). Concernant les instruments de planification, le SCoT (développement urbain, zonage, transports) a été approuvé en 2014, et le SAGE (Schéma d'aménagement et de gestion des eaux) devrait être approuvé à l'échelle du bassin versant courant 2018. Le Syndicat mixte du bassin de Thau (SMBT) assiste maintenant les communes dans le développement de leurs Plans locaux d'urbanisme (PLU) pour mieux prendre en compte la préservation des écosystèmes et de leurs services dans leur projet de développement. Des actions ont aussi été réalisées pour identifier et cartographier les continuités écologiques vertes 
et bleues qui sont à préserver ou à recréer, et pour les inclure dans le SCoT et dans les PLU des communes (Chaurand, 2017). En matière d'outils de gestion opérationnels, le $4^{\mathrm{e}}$ Programme d'action territorial (2013-2018) a pour but d'assurer la protection des zones agricoles et naturelles et le développement de pratiques agro-environnementales. Un observatoire du territoire a aussi été mis en place pour mesurer régulièrement et cartographier l'évolution du territoire afin d'évaluer les impacts du programme d'action et permettre à cet organisme de gouvernance multi-acteurs d'ajuster ses actions en conséquence (Lemoisson et al., 2016). L'observatoire est alimenté par des cartes et des indicateurs dérivés notamment du suivi, grâce à la télédétection, des changements dans la couverture et l'occupation du sol à une échelle très précise (Dupaquier et al., 2014 ; Tonneau et Maurel, 2016). Le bassin de Thau est aussi l'une des zones choisies pour le projet scientifique Imagine (programme européen Biodiversa, 2017-2020), qui vise à développer des stratégies pour un développement spatial durable et adaptatif des systèmes socio-économiques. Dans le cadre de ce projet, de nouveaux travaux ont été menés en 2017 pour l'évaluation quantitative et la cartographie de services écosystémiques sur le territoire de Thau (Billaud, 2017).

\section{Conclusion}

Ce chapitre a présenté les fondements pour une évaluation participative des services écosystémiques appliquée aux terres agricoles périurbaines dans le sud de la France. Nos résultats suggèrent que dans un contexte de planification de l'occupation du sol et des paysages, une approche basée sur les services écosystémiques peut favoriser l'émergence d'une représentation holistique des valeurs sociétales relatives aux zones agricoles et naturelles. Nous avons en effet montré qu'il était possible de mobiliser des acteurs - des experts ayant des connaissances scientifiques et/ou des acteurs locaux avec des connaissances tacites - pour évaluer les rôles multiples et complexes que jouent les terres agricoles en zones périurbaines, pour les bénéficiaires. Nous avons vu que les recherches actuelles qui visent à appliquer les avancées scientifiques sur les services écosystémiques à la planification spatiale et paysagère se sont fixé des objectifs ambitieux en ce qui concerne le cadrage des valeurs, l'inclusion des acteurs et le processus d'évaluation participative des services. Le fait d'avoir mené des expérimentations concrètes a mis en évidence la nécessité de faire des compromis entre la théorie et la pratique. Notre recherche a toutefois abouti à une grande variété de résultats : récits à dires d'acteurs ; remplissage par les acteurs de matrices d'interactions entre services et occupations du sol ; cartographie participative des services. Même si ces résultats n'ont pas été repris dans les processus de planification, quelques perspectives pour l'aménagement du territoire peuvent toutefois être dégagées de cette étude pilote. Un domaine de recherche original à explorer dans le futur porte sur l'intégration des évaluations et des perceptions locales des services écosystémiques dans les géo-observatoires soutenant les processus de planification. De plus, la rédaction d'un catalogue des bonnes pratiques pour l'évaluation des services apportés par les terres agricoles périurbaines aiderait à codifier ces pratiques et à les rendre plus facilement accessibles pour de futures évaluations.

\section{Remerciements}

Les auteurs remercient toutes les personnes qui ont participé aux entretiens individuels et aux ateliers pour leur disponibilité et les informations qu'ils ont partagées. Nous remercions aussi le Syndicat mixte du bassin de Thau pour son aide dans l'étude de cas du bassin de Thau, Claire Dupaquier pour l'appui lors des ateliers et pour son travail, avec Annie Debrosse, de classification de l'occupation du sol du bassin de Thau. Nous remercions aussi le Centre national d'études spatiales pour la mise à disposition gratuite 
des images Pléiades au titre du Programme de la RTU (Recette thématique utilisateurs) Pléiades. Ce projet de recherche a été financé par l'Institut national de recherche en sciences et technologies pour l'environnement et l'agriculture.

\section{Références bibliographiques}

Arts B., Buizer M., Horlings L., Ingram V., Van Oosten C., Opdam P., 2017. Landscape Approaches. Annual Review of Environment and Resources, 42, 439-463.

Baker J., Sheate W.R., Phillips P., Eales R., 2013. Ecosystem Services in Environmental Assessment. Help or Hindrance? Journal of Environmental Management, 90, 1692-1702.

Barbosa A., Vallecillo S., Baranzelli C., Jacobs-Crisioni C., Batista E., Silva F., Perpina-Castillo C., Lavalle C., Maes J., 2017. Modelling built-up land take in Europe to 2020: an assessment of the Resource Efficiency Roadmap measure on land. Journal of Environmental Planning and Management, 60, 1439-1463.

Bertrand N., 2016. Intégration des enjeux environnementaux dans la gestion du foncier agricole. Sciences Eaux et Territoires, (19).

Billaud O., 2017. Intégration des services écosystémiques dans la planification territoriale. Exemple $d u$ bassin de Thau. Rapport de stage de master 2 EEET Économie de l'environnement, de l'énergie et des transports, $94 \mathrm{p}$.

Billeter R., Liira J., Bailey D., Bugter R., Arens P., Augenstein I., et al., 2008. Indicators for biodiversity in agricultural landscapes: a pan-European study. Journal of Applied Ecology, 45, 141-150.

Bio Intelligence Service, IVM, IEEP, 2015. Land as a Resource. Problem definition and possible areas for EU action. Prepared for the European Commission (DG ENV).

Blackmore C., 2007. What kinds of knowledge, knowing and learning are required for addressing resource dilemmas? A theoretical overview. Environmental Science \& Policy, 10, 512-525.

Blezat Consulting, 2011. Étude action sur le développement de la filière agricole sur le territoire de Thau Agglomération. Rapport d'étude, $71 \mathrm{p}$.

Bonifazi A., Sannicandro V., Attardi R., Di Cugno G., Torre C.M., 2016. Countryside vs city: a user-centered approach to open spatial indicators of urban sprawl. In: Computational Science and Its Applications (Gervasi O., Murgante B., Misra S., Rocha A., Torre C.M., Tanier D., Apduhan B.O., Stankova E., Wang S., eds), ICCSA 2016, Pt Iv.

Burkhard B., Kroll F., Müller F., 2012a. Mapping ecosystem service supply, demand, and budgets. Ecological Indicators, 21, 17-29.

Burkhard B., Kroll F., Nedkov S., Müller F., 2012b. Mapping ecosystem service supply, demand and budgets. Ecological Indicators, 21.

Callon M., 1986. Some elements of a sociology of translation: domestication of the scallops and the fishermen of St-Brieuc Bay. In: Power, Action and Belief: A New Sociology of Knowledge? (Law J., ed.), London, Routledge.

Chaurand J., 2017. La cohérence interterritoriale des projets de continuités écologiques. L'exemple de la politique Trame verte et bleue en France. Thèse de doctorat en Science de l'environnement, Montpellier, AgroParisTech, 470 p.

Ciscel D.H., 2001. The economics of urban sprawl: inefficiency as a core feature of metropolitan growth. Journal of Economic Issues, 35.

Colantoni A., Grigoriadis E., Sateriano A., Venanzoni G., Salvati L., 2016. Cities as selective land predators? A lesson on urban growth, deregulated planning and sprawl containment. Science of the Total Environment, 545, 329-339. 
Costanza R., De Groot R., Braat L., Kubiszewski I., Fioramonti L., Sutton P., Farber S., Grasso M., 2017. Twenty years of ecosystem services: how far have we come and how far do we still need to go? Ecosystem Services, 28, 1-16.

Couderchet, L., Amelot, X., Faut-il brûler les Znieff ?, Cybergeo.

Crossman N.D., Burkhard B., Nedkov S., 2012. Quantifying and mapping ecosystem services. International Journal of Biodiversity Science, Ecosystem Services and Management, $8,1-4$.

Crossman N.D., Burkhard B., Nedkov S., Willemen L., Petz K., Palomo I., et al., 2013. A blueprint for mapping and modelling ecosystem services. Ecosystem Services, 4, 4-14.

Daily G.C. (ed.), 1997. Nature's Services: Societal Dependence on Natural Ecosystems, New York, Island Press.

De Groot R.S., Alkemade R., Braat L., Hein L., Willemen L., 2010. Challenges in integrating the concept of ecosystem services and values in landscape planning, management and decision making. Ecological Complexity, 7, 260-272.

Di Palma F., Amato F., Nole G., Martellozzo F., Murgante B., 2016. A SMAP supervised classification of Landsat images for urban sprawl evaluation. ISPRS International Journal of Geo-Information, 5.

Dupaquier C., Desbrosse A., Maurel P., Plant R., Ruoso L.-E., Roussillon J.-P., 2014. Apports de l'imagerie Pléiades à la gestion intégrée des zones côtières. Application au territoire de Thau. Revue française de photogrammétrie et de télédétection, 208, 45-50.

Efese, 2016. Rapport intermédiaire sur l'évaluation française des écosystèmes et des services écosystémiques. Rapport MTES, FRB, 162 p.

Gardi C., Panagos P., Van Liedekerke M., Bosco C., De Brogniez D., 2015. Land take and food security: assessment of land take on the agricultural production in Europe. Journal of Environmental Planning and Management, 58, 898-912.

Goffman E., 1974. Frame Analysis. An Essay on the Organization of Experience, Boston, Northeastern University Press.

Haines-Young R., Potschin M., 2010. The links between biodiversity, ecosystem services and human well-being. In: Ecosystem Ecology: A New Synthesis (Raffaelli D.G., Frid C.L.J., eds), Cambridge, Cambridge University Press/British Ecological Society.

Hanley N., Spash C.L., 1993. Cost-Benefit Analysis and the Environment, Edward Elgar.

Hejnowicz A.P., Rudd M.A., 2017. The value landscape in ecosystem services: value, value wherefore art thou value? Sustainability, 9, 850.

Helliwell D.R., 1969. Valuation of wildlife resources. Regional Studies, 3, 41-47.

Jacobs S., Dendoncker N., Martín-López N., Barton D.N., Al E., 2016. A new valuation school: integrating diverse values of nature in resource and land use decisions. Ecosystem Services, 22, Part B, 213-220.

Kahneman D., Tversky A., 1983. Choices, values and frames. American Psychologist, 39, 341-350.

Kareiva P., Tallis H., Ricketts T.H., Daily G.C., Polasky S. (eds), 2011. Natural Capital Theory and Practice of Mapping Ecosystem Services, Oxford, Oxford University Press.

Keeney B.C., 1970. Bridge of values. Science, 169, 26-28.

Kerselaers E., Rogge E., Lauwers L., Van Huylenbroeck G., 2011. Prioritising land to be preserved for agriculture: a context-specific value tree. Land Use Policy, 20, 219-226.

Lakoff G., 2004. Don't Think of an Elephant! Know Your Values and Frame the Debate. The Essential Guide for Progressives, White River Junction, VT, Chelsea Green Pub.

Lemoisson P., Tonneau J.P., Maurel P., 2016. L'intelligence territoriale dans le bassin de Thau : un observatoire pour penser et piloter l'action. In : Partenariats pour le développement territorial (Torre A., Vollet D., eds), Versailles, éditions Quæ, 59-73. 
Loiseau E., 2014. Élaboration d'une démarche d'évaluation environnementale d'un territoire basée sur le cadre méthodologique de l'Analyse du cycle de vie (ACV). Application au territoire du bassin de Thau. Thèse, Montpellier, SupAgro.

Luque S., Fürst C., Geneletti D., 2017. Nexus Thinking. How ecosystem services concepts and practice can contribute balancing integrative resource management through facilitating cross-scale and cross-sectoral planning. International Journal of Biodiversity Science, Ecosystem Services and Management, 13 (2), 1-3.

Maris V., Devictor V., Doussan I., Béchet A., 2016. Les valeurs en question. In : Biodiversité et services écosystémiques : regards croisés entre les sciences de la nature et les sciences humaines (Geijzendorffer I., Levrel H., Roche P., Maris V., eds), éditions Quæ.

Martín-López B., Gómez-Baggethun E., García-Llorente M., Montesa C., 2013. Trade-offs across value-domains in ecosystem services assessment. Ecological Indicators.

Martínez-Harms M.J., Balvanera P., 2012. Methods for mapping ecosystem service supply: a review. International Journal of Biodiversity Science, Ecosystem Services and Management, $8,17-25$.

McCauley D.J., 2006. Selling out on nature. Nature, 443, 27-28.

MEA, 2003. Ecosystems and Human Well-being: A Framework for Assessment, Washington, Millennium Ecosystem Assessment/Island Press.

MEA, 2005. Ecosystems and Human Well-being: Synthesis, Millennium Ecosystem Assessment, World Resources Institute/Island Press, Washington, 137 p.

Moller H., Berkes F., Lyver P., Kislalioglu M., 2004. Combining science and traditional ecological knowledge: monitoring populations for co-management. Ecology and Society, 9.

Mongruel R., Meral P., Doussan I., Levrel H., 2016. L'institutionnalisation de l'approche par les services écosystémiques : dimensions scientifiques, politiques et juridiques. In : Valeurs de la biodiversité et services écosystémiques: perspectives interdisciplinaires (Roche P., Geijzendorffer I., Levrel H., Maris V., coord.), Versailles, Quæ, 191-216.

Opdam P., Coninx I., Dewulf A., Steingröver E., Vos C., Van Der Wal M., 2015. Framing ecosystem services: affecting behaviour of actors in collaborative landscape planning? Land Use Policy, 46, 223-231.

Ostrom E., 2009. A general framework for analyzing sustainability of social-ecological systems. Science, 325, 419-422.

Plant R., Prior T., 2014. An ecosystem services framework to support statutory water allocation planning in Australia. International Journal of River Basin Management, 12 (3), 219-230.

Plant R., Ryan P., 2013. Ecosystem services as a practicable concept for natural resource management: some lessons from Australia. International Journal of Biodiversity Science, Ecosystem Services and Management, 9, 44-53.

Plant R., Maurel P., Barreteau O., Bertacchini Y., 2014. The role of territorial intelligence: the case of the Thau Territory, Southern France. In: River Basin Management in the Twenty-first Century: People and Place (Squires V., Milner H., Daniell K., eds), Boca Raton, London-New York, CRC Press/Taylor \& Francis Group.

Plant R., Boydell S., Prior J., Chong J., Lederwasch A., 2016. From liability to opportunity: an institutional approach towards value-based land remediation. Environment and Planning C: Government and Policy, Online First.

Primmer E., Furman E., 2012. Operationalising ecosystem service approaches for governance: do measuring, mapping and valuing integrate sector-specific knowledge systems? Ecosystem Services, 1, 85-92.

Quetier F., Van Teeffelen A.J.A., Pilgrim J.D., Von Hase A., Kate K.T., 2015. Biodiversity offsets are one solution to widespread poorly compensated biodiversity loss: a response to Curran et al. Ecol. Appl., 25, 1739-1741. 
Raymond, C.M.S, Singh G., Benassaiah, K., Bernhardt, J.R., Levine, J., Nelson, H., Turner, N.J., Norton, B., Tam, J., KaiK.M.A., 2015. Ecosystem services and beyond: using multiple metaphors to understand human-environment relationships, BioScience, 63 (7), 536-546.

Ruoso L.-E., Plant R., Maurel P., Dupaquier C., Roche P., Bonin M., 2015. Reading ecosystem services at the local scale through a territorial approach: the case of peri-urban agriculture in the Thau Lagoon, Southern France. Ecology and Society, 20, 11.

Saaty T., 1996. The Analytic Hierarchy Process, New York, McGraw Hill.

Schröter M., Van Der Zanden E.H., Van Oudenhoven A.P.E., Remme R.P., Serna-Chavez H.M., De Groot R.S., Opdam P., 2014. Ecosystem services as a contested concept: a synthesis of critique and counter-arguments. Conservation Letters, 7 (6), 514-523.

Sheate W.R., Eales R.P., Daly E., Baker J., Murdoch A., Hill C., Ojike U., Karpouzoglou T., 2012. Spatial representation and specification of ecosystem services: a methodology using land use/land cover data and stakeholder engagement. Journal of Environmental Assessment Policy and Management, 14.

Smiraglia D., Ceccarelli T., Bajocco S., Salvati L., Perini L., 2016. Linking trajectories of land change, land degradation processes and ecosystem services. Environmental Research, 147, 590-600.

Sukhdev P., Wittmer H., Schröter-Schlaack C., Nesshöver C., Bishop J., Brink P.T., Gundimeda H., Kumar P., Simmons B., 2010. The Economics of Ecosystems and Biodiversity: Mainstreaming the Economics of Nature: A synthesis of the approach, conclusions and recommendations of TEEB, European Communities.

TEEB, 2010. The Economics of Ecosystems and Biodiversity Ecological and Economic Foundations, Pushpam Kumar, Earthscan, London/Washington.

Thompson P.B., 2008. Agrarian Philosophy and Ecological Ethics. Science and Engineering Ethics, 14 (4), 527-44.

Tibi A., Therond O., 2017. Évaluation des services écosystémiques rendus par les écosystèmes agricoles. Une contribution au programme EFESE. Synthèse du rapport d'étude, Inra, $108 \mathrm{p}$.

Tonneau J.P., Maurel P., 2016. Satellite imagery, a tool for territorial Development 2016. In: Land Surface Remote Sensing in Urban and Coastal Areas (Baghdadi N., Zribi M., eds), Londres, ISTE-Elsevier, 101-140.

Van Dijk T., Van Der Valk A., 2010. Harde Cijfers En Ruimtelijk Beleid. In: Economische waardering van omgevingskwaliteit. Casestudies en toepassingen in de MKBA (Koetse M.J., Rietveld P., eds), Den Haag, Sdu.

Vatn A., 2005. Institutions and the Environment, Cheltenham, Edward Elgar.

Watson R., Albon S., 2011. UK National Ecosystem Assessment. Understanding Nature's Value to Society. Synthesis of Key Findings, Cambridge, UK National Ecosystem Assessment. Westman W.E., 1977. How much are nature's services worth? Science, 197, 960-964.

World Commission on Environment and Development (WCED), 1987. Our Common Future. Oxford: Oxford University Press. 


\section{Synthèse. De la donnée à l'intelligence collective sur les terres agricoles périurbaines : quels rôles pour l'information, les savoirs et l'action?}

Roel Plant, Pierre Maurel, Laure-Élise Ruoso, Éric Barbe, Jane Brennan

\section{Introduction}

\section{Les terres agricoles périurbaines face à l'étalement urbain}

Maintenir les terres agricoles périurbaines et les activités qui leur sont associées à proximité de centres urbains en expansion est une préoccupation à l'échelle mondiale. Face à une demande toujours croissante en terres à construire, le défi de conserver les terres agricoles périurbaines - notre préoccupation principale dans cet ouvrage - continue de faire débat.

La littérature récente présente différentes initiatives qui ont été adoptées, ou pourraient l'être, pour protéger et conserver ces terres. Par exemple, Akimowicz et al. (2016) ont évalué, de manière critique, les mérites de la loi sur la ceinture verte (Greenbelt Act) en Ontario (États-Unis) concernant l'adaptation des agriculteurs et leurs stratégies d'investissement. Inwood et Sharp (2012) ont rendu compte de la résilience des exploitations agricoles dans les zones périurbaines en étudiant les phénomènes de succession et l'adaptation des fermes aux États-Unis. En tant que telle, cette étude ne propose pas d'initiatives qui pourraient être mises en place pour protéger et conserver les terres agricoles périurbaines, mais identifie des facteurs contribuant à expliquer le maintien de certaines exploitations agricoles en milieu périurbain. À partir de l'étude des conflits autour des usages agricoles dans la région du projet de Grand Paris, Darly et Torre (2013) analysent les expériences vécues par les agriculteurs ainsi que la couverture médiatique du projet, et présentent des mécanismes innovants de résolution de conflit.

Les réseaux alimentaires alternatifs, comme initiatives contribuant à protéger les terres agricoles périurbaines, font aussi l'objet de recherches (Deverre et Lamine, 2010 ; Soulard et al., 2018; Brand et al., 2017). À travers l'étude de la région métropolitaine de 
Barcelone en Espagne, Paül et McKenzie (2013) concluent que ces réseaux alimentaires dans les zones périurbaines ne sont possibles que si la préservation des terres agricoles est garantie, mais qu'ils ne doivent pas en être qu'une simple conséquence car ils font partie de la solution. En Australie, Knowd (2013) a étudié le cas d'Hawkesbury Harvest, une organisation communautaire à but non lucratif créée dans les années 2000 dans le but de répondre aux menaces systémiques impactant l'agriculture dans le bassin de Sydney.

Les onze chapitres de cet ouvrage se sont concentrés sur les terres agricoles périurbaines, leurs sols et les activités agricoles qui leur sont associées en Europe, plus particulièrement en France, ainsi qu'en Australie, plus particulièrement dans l'État de la NouvelleGalles du Sud où se situe Sydney. Comme plusieurs chapitres l'ont illustré, la question de la protection des sols est complexe et le développement d'une gouvernance des sols durable est urgent étant donné que la qualité et la disponibilité des sols sont intrinsèquement liées à la fourniture de services écosystémiques, dont la production d'aliments locaux et la sécurité alimentaire. Alors que le monde s'urbanise de plus en plus, la sécurité alimentaire émerge progressivement comme un défi dans les pays développés et en développement (Guillou et Matheron, 2011). Ainsi, il est permis de penser que les notions de « sécurité des sols » et de "sécurité alimentaire » sont intrinsèquement liées. Face à la menace grandissante de l'imperméabilisation des terres agricoles périurbaines et de leurs sols, leur protection devient un enjeu majeur pour des raisons socio-économiques et environnementales.

Au-delà de la production alimentaire, les terres agricoles périurbaines remplissent un éventail plus vaste de services tels que les aménités paysagères, l'atténuation des îlots de chaleur en milieu urbain, les activités récréatives et le tourisme. Les agriculteurs contribuent également à la fabrique et l'entretien des paysages, services qui font partie intégrante de leur activité professionnelle. Des études montrent que certains agriculteurs commencent à considérer la protection de l'environnement comme faisant partie de leur métier et ne pratiquent plus uniquement l'agriculture à des fins de production alimentaire (Greiner et Gregg, 2011). L'idée de « cultiver les paysages » émerge progressivement comme une alternative à l'intensification et à l'industrialisation de l'agriculture : si l'agriculture productiviste traditionnelle ne s'avérait plus (complètement) viable économiquement, les agriculteurs pourraient aussi être rémunérés pour leur activité de gestion du paysage (Serrano, 2011). D'autres exemples concernent l'agriculture à faible intensité de carbone et la gestion du risque d'inondation par les agriculteurs. Ces activités consistent à préserver les paysages agricoles pour qu'ils remplissent des services allant au-delà de la production d'aliments et de fibres végétales, comme la régulation du climat, la réduction du risque d'inondation, la provision d'habitats, les aménités paysagères. Tout au long d'un continuum allant des sols aux paysages, les activités agricoles ont donc un rôle fondamental à jouer dans le maintien d'une variété de services écosystémiques.

Cependant, comme l'ont montré plusieurs chapitres dans cet ouvrage, dans la pratique, ni les sols, ni les activités productives et de gestion des paysages ne sont vraiment pris en compte dans les politiques publiques traitant des zones périurbaines en France et en Australie. Les pressions liées au développement urbain et économique sur des terres peu abondantes génèrent inévitablement des coûts d'opportunité pour les propriétaires, invitant à la maximisation de la rente et à la spéculation foncière (Reckien et Karecha, 2007 ; Bertrand et al., 2006). De plus, les politiques publiques ont trop longtemps considéré les espaces périurbains comme des «vides », des «banlieues en devenir » (Bunker et Holloway, 2001, p. 13). Les rôles multiples remplis par les terres agricoles périurbaines et leurs bénéfices socio-économiques et environnementaux passent encore trop souvent inaperçus et restent sous-évalués. 


\section{Retour sur les objectifs de l'ouvrage}

Le débat public sur l'agriculture périurbaine ne peut pas avoir lieu sans tenir compte de la production agricole, des sols et de la dimension paysagère. Cela soulève nécessairement la question de la nature et du rôle des données et informations sur les sols, les paysages et les dynamiques de l'étalement urbain dans les processus de décision traitant des terres agricoles périurbaines. Cet ouvrage a pour but de comprendre comment l'Australie (plus particulièrement l'État de Nouvelle-Galles du Sud) et l'Europe (plus particulièrement la France) tentent de répondre aux défis complexes posés par la protection des terres agricoles périurbaines, avec un intérêt particulier pour le rôle de l'information dans la prise de décision et l'action.

À ce stade, il est utile de rappeler au lecteur les questions posées dans l'avant-propos :

Question 1 : Quels sont les enjeux ? Notre premier objectif était de clarifier la question de la perte de terres agricoles dans les zones périurbaines.

Question 2 : Quels sont les outils disponibles pour faire face à cet enjeu? Notre second but était d'évaluer, de manière critique, les théories et méthodes qui pourraient permettre de traiter la question de la perte de ces terres.

Question 3 : Quelles sont les conditions d'adoption de nouvelles politiques et modalités de gouvernance et quels sont les bénéfices envisageables? Notre troisième objectif était d'établir une feuille de route pour le futur. Que peut-on dire du développement, de l'adoption et de l'utilisation d'outils et de méthodes scientifiques pour générer des données, de l'information, des connaissances et de nouvelles pratiques sur les terres agricoles périurbaines? En outre, qu'est-ce que cela supposerait comme changements en termes de gouvernance?

Les types d'informations présentés dans les divers chapitres de cet ouvrage incluent de l'information créée à l'aide d'une approche descendante, comme les cartes de potentiel agronomique des sols, de l'information créée à travers une approche ascendante, comme les services écosystémiques identifiés par les acteurs, ainsi que des informations créées par une approche hybride (ascendante et descendante), comme la coconstruction d'indicateurs. D'autres chapitres ont mis en évidence des cas d'utilisation d'informations peu adaptées, ou des usages à mauvais escient de données de qualité. Dans ce dernier chapitre, nous proposons une synthèse des divers enseignements et approches présentés dans les onze chapitres précédents, et cherchons à apporter des réponses à la question de savoir comment des données et des informations sur les sols et les paysages pourraient contribuer à une gouvernance durable des terres agricoles périurbaines et de leurs rôles et bénéfices.

\section{Structure du chapitre}

Afin de structurer cette synthèse de manière cohérente, nous nous sommes appuyés sur un cadre conceptuel, présenté dans la deuxième section, qui permet de relier donnée, information, connaissance, action et intelligence collective. Dans la troisième section, nous abordons la question de la fabrique de l'information et du passage de la donnée à la connaissance. Dans la quatrième section, nous adoptons une perspective anthropologique sur la question des connaissances, et mettons en exergue des exemples de sousutilisation, ou d'utilisation détournée, inattendue de l'information sur les terres agricoles périurbaines qui ouvrent des voies possibles d'innovation. La cinquième section explore la manière dont des données et des informations ad hoc, adaptées aux besoins, pourraient conforter des actions contribuant à une gouvernance durable des terres agricoles périurbaines et, en retour, bénéficier de moyens pour leur production. La coproduction 
d'informations ainsi que la notion d'observatoire sont abordées dans cette section. Dans la sixième et dernière section, nous concluons par une brève réflexion sur les leçons tirées de ces échanges franco-australiens, en nous interrogeant sur ce qu'apporterait l'adoption de bonnes pratiques en matière d'informations sur les terres agricoles périurbaines, avec quels résultats en fin de compte.

\section{Cadre conceptuel}

Notre cadre conceptuel pour la synthèse (figure 1) est une adaptation de la pyramide donnée-information-connaissance-intelligence (Data Information Knowledge Wisdom, $D I K W$ ) d'Adler (1986). Elle offre une conceptualisation de la manière dont les humains passent de la donnée à l'action et aux décisions. Elle a été revisitée par plusieurs auteurs, certains proposant de rajouter des boucles de rétroaction pour rendre le processus itératif et non plus linéaire (Rowley, 2007 ; Kitchin, 2014 ; Adler, 1986).

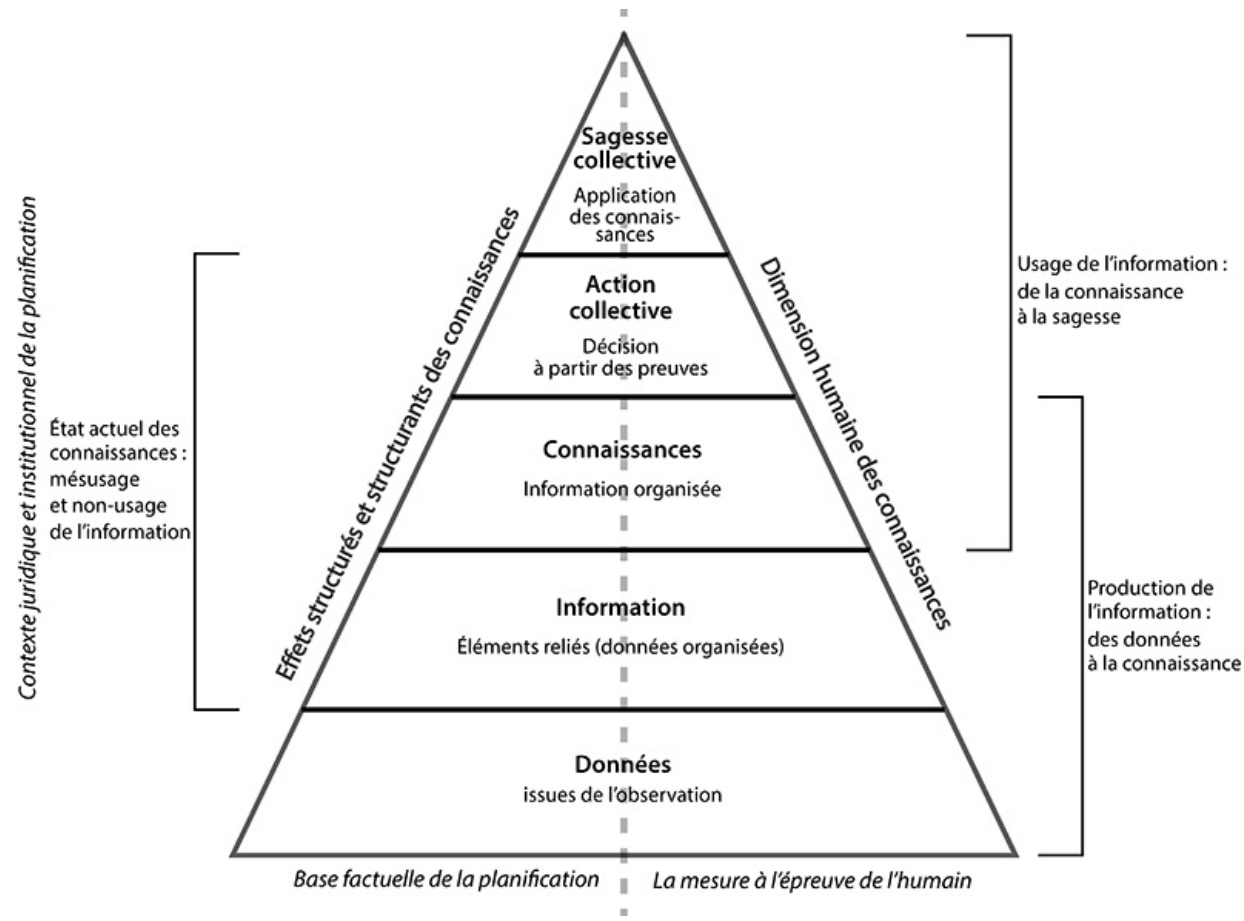

Figure 1. De la donnée à l'intelligence : conceptualiser le rôle de l'information, de la connaissance et de l'action. D'après Adler (1986) et McCandles (2000), in Kitchin (2014) et Graham et al. (2000).

Nous avons adapté cette représentation en insérant tout d'abord un niveau supplémentaire, celui de l'action (collective), entre la connaissance et l'intelligence (collective), pour insister sur le fait que la «preuve» de l'intelligence réside dans les conséquences pratiques de l'action et non dans la simple connaissance a priori. Cela revient à adopter la perspective épistémique pragmatique de Peirce (Peirce, 1905), que nous considérons comme une méthode de pensée réflexive sur les concepts et les hypothèses employés : nous clarifions une hypothèse en identifiant ses conséquences pratiques.

Dans notre cadre conceptuel modifié, la donnée fait référence à une observation abstraite de la réalité, considérée dans une perspective sémiotique peircéenne (Deledalle, 2001) 
comme un système de signes obtenus à travers la perception humaine ou celle d'une machine. L'information fait référence à des données organisées, reliant des éléments pour leur donner sens (du latin informare, "mettre en forme »). Ensuite, la connaissance, qu'elle soit tacite (dans la tête des individus) ou explicite (codée), est le résultat de processus d'apprentissage. Il s'agit d'informations organisées en vue d'une prise de décision sur la base de faits avérés à des fins d'actions individuelles ou collectives. L'intelligence est une forme de connaissance appliquée. Enfin, l'action correspond aux actes entrepris par un groupe (directement ou en son nom par le biais d'une organisation) pour atteindre des intérêts communs perçus par ses membres (Marshall G., 1998). Au niveau collectif, cela correspond à une compréhension profonde des personnes, des choses, des événements et des situations qui permet de prendre des décisions et de passer à l'action de manière optimale et coordonnée pour atteindre un objectif donné et une vision partagée du futur. Comme nous nous intéressons à des actions et des décisions pour générer des résultats relevant de biens collectifs, nous adoptons donc une perspective centrée sur cet objectif.

Nous avons apporté une deuxième modification à la pyramide en développant la composante connaissance. Le modèle de la connaissance à l'action développé par Graham et al. (2006) offre une conceptualisation où la production et l'application des connaissances sont intégrées, afin de clarifier les notions de traduction et de transfert de connaissance. Ces auteurs proposent une carte conceptuelle du processus allant de la connaissance à l'action pour aider ceux qui produisent et ceux qui utilisent les connaissances à comprendre la nature du terrain et à se frayer un chemin dans le processus complexe, itératif et organique de la traduction des connaissances.

Cette deuxième modification repose aussi sur une approche anthropologique de la connaissance. Selon Barth (2002), la connaissance comprend toujours trois dimensions : un corpus d'assertions sur le réel, un éventail de moyens de représentations, et une organisation sociale. Dans la logique de notre pyramide, en prolongeant le raisonnement de Barth, nous pouvons voir que les humains créent de la donnée, de l'information et de l'action sur la base de leurs connaissances (et vivent et agissent en fonction). Par conséquent, nous devrions nous demander comment les diverses données, informations et actions sont produites, représentées, transmises et mises en application. Une perspective anthropologique du savoir nous paraît donc essentielle pour faire avancer le débat public et entre experts sur les terres agricoles périurbaines.

Ceci nous amène à rajouter deux dimensions à notre pyramide, comme l'avait déjà fait Laplantine (2001) : la dimension de l'effet de structure et de structuration de la connaissance (à travers les systèmes d'informations et modèles qui accompagnent et cadrent les politiques publiques), présentée sur la gauche de la pyramide, et la dimension humaine de la connaissance sur la droite (ce que les acteurs décident en matière de connaissances nécessaires et de données sous-jacentes et ce qu'ils en font concrètement).

Ces deux modifications amènent à un spectre dialectique qui englobe la création, l'utilisation et l'interprétation de la donnée, juxtaposées à la dimension structurelle d'un côté et à la dimension humaine de l'autre, les deux étant intimement liées. Les deux parties de cet ouvrage traitent des deux dimensions, structurelles et humaines, de la connaissance (Maury et al., 2014).

La section ci-dessous ( «Création d'informations ») s'appuie sur des études de cas français et australiens où l'information devient de la connaissance à travers le traitement et l'organisation des données. Elle se concentre sur les parties : donnée, information et connaissance de la pyramide. Elle traite aussi de la question de l'acquisition des données, 
des processus de réduction et d'abstraction du monde « réel ». La section suivante se concentre sur l'interprétation et l'utilisation concrète de l'information, y compris ce qui peut être perçu comme de mauvais usages ou des interprétations erronées. Cette section propose une analyse des raisons pour lesquelles la connaissance sur les terres agricoles périurbaines ne se traduit pas encore en action « intelligente ». Dans la dernière section, nous dressons un bilan, de manière spéculative, de la manière dont une meilleure organisation et une meilleure interprétation de l'information pourraient mener à une gouvernance durable des terres agricoles périurbaines.

\section{Création d'informations}

\section{Création d'informations et production de connaissances guidées par les données}

La question des informations qui ont été produites, ou qui pourraient l'être d'un point de vue technique, pour mesurer la perte et la dégradation des terres agricoles périurbaines a fait l'objet de la partie I de l'ouvrage, Base factuelle de la planification (chapitres 2 à 6). De ces chapitres ressortent deux catégories d'information pertinentes : celles permettant le suivi des taux d'urbanisation (imperméabilisation des sols) et celles sur les sols et leur potentiel agronomique. Ces mesures doivent permettre aux décideurs de comprendre les dynamiques de changement, mais aussi d'identifier les terres dont la " bonne qualité » justifie de les protéger. Cependant, le choix des métriques détermine les types de connaissances qui peuvent être produites, en organisant l'information. Par exemple, mesurer l'imperméabilisation des sols ne renseigne pas sur leur qualité et leur potentiel agronomique, ceci nécessitant une analyse morpho-paysagère et des mesures de leurs propriétés bio-physico-chimiques. La métrique d'imperméabilisation des sols ne permet que d'identifier les terres ayant été construites ou qui sont en passe de l'être. Le chapitre 2 (par Bernard Clarimont, Éric Barbe et Philippe Lagacherie) présente un exemple de l'effet structurant de la connaissance qui peut émerger de la cartographie de l'imperméabilisation des sols et de leur potentiel agronomique. Dans le cas de l'ancienne région du Languedoc-Roussillon en France, des cartes représentant l'étendue de l'imperméabilisation des sols entre 1997 et 2009 et le potentiel agronomique des sols ont été produites à destination des acteurs de l'aménagement du territoire. C'est leur combinaison sous forme cartographique et d'indicateurs spatialisés qui a permis d'apporter des informations tangibles sur les dynamiques d'artificialisation et les types de sols concernés dans les commissions chargées d'évaluer les projets de développement urbain proposés par les collectivités locales.

En Australie et en France, plusieurs projets visant à produire des informations pour éclairer les processus de prise de décision concernant les terres agricoles périurbaines ont vu le jour. En France, en plus des bases de données statistiques existantes sur les milieux urbains et ruraux, des centres de recherche ont commencé à créer de nouvelles informations (étalement urbain, propriétés des sols) sur des régions pilotes, à la demande d'acteurs publics. La question de leur production régulière à l'échelle nationale reste toutefois encore en suspens. En Australie, des agences gouvernementales fournissent de l'information directement exploitable sur les terres agricoles périurbaines (par exemple le Système d'information sur les sols et le foncier ; Soil and Land Information System, SALIS). À part cela, le reste de la production de connaissances sur ce sujet reste du ressort de la communauté scientifique. En embrassant une perspective historique, le chapitre 4 (par Pamela Hazelton) a montré que la productivité agricole avait été à l'origine de succès ou d'échecs dans la conquête de nouvelles terres à une époque où 
les ressources pédologiques de l'Australie étaient encore méconnues. Mais aujourd'hui, malgré la disponibilité des informations sur les propriétés pédologiques des sols, les aménageurs australiens continuent de privilégier, dans leur prise de décision, les questions de croissance démographique et de fluctuations économiques plutôt que de potentiel agronomique des sols.

Le chapitre 5 (par Jane Brennan et Andrew Murrell) repose sur les hypothèses que des théories et des pratiques de planification proactives pourraient éviter l'étalement et l'imperméabilisation des terres, et qu'une prise de décision éclairée et basée sur des faits avérés est essentielle pour un aménagement du territoire durable. Il comprend un état de l'art sur la cartographie de l'étalement urbain et de l'imperméabilisation des sols en Australie ainsi qu'une analyse sur l'accès à des informations sur les sols. Les cartes de pédopaysages et de potentiel agronomique des sols constituent une source d'informations facilement interprétables par les aménageurs afin d'identifier des zones à préserver. Face au développement urbain rapide de l'Australie, la connaissance du potentiel agronomique sur l'intégralité du territoire sera essentielle. En France, il a été démontré que la cartographie de l'étalement urbain par télédétection et celle des propriétés des sols étaient réalisables à une échelle régionale (l'ancienne région Languedoc-Roussillon). En Australie, de telles cartes ne sont disponibles que sur certaines zones et ne couvrent pas de manière systématique les aires métropolitaines où les terres agricoles périurbaines sont les plus menacées (Sydney, Melbourne et Brisbane). De plus, elles nécessitent une évaluation par des experts, ce qui empêche une automatisation complète.

En ce qui concerne le suivi automatisé de l'étalement urbain, l'image satellite offre un potentiel important du fait des avancées récentes en télédétection et de l'existence d'archives d'images qui permettent de mesurer les tendances passées et les taux de perte/ préservation des terres agricoles périurbaines. Ces informations sont utiles aux décideurs, car elles leur offrent des bases objectives pour quantifier les dynamiques passées et caler des modèles prédictifs pour éclairer des scénarios de planification. Le chapitre 6 (par Paras Sidiqui, Rakhesh Devadas et Alfredo Huete) montre en quoi la télédétection et les SIG constituent des outils efficaces et à des coûts supportables pour suivre et cartographier, de manière régulière, les activités dans les zones périurbaines. Les images satellites, gratuites à partir de 10 mètres de résolution, fournissent des informations spatiales cohérentes ainsi que des vues à la fois synoptiques et détaillées. Quarante ans d'archives Landsat sont disponibles pour retracer les changements d'occupation du sol, notamment dans les zones de cultures et de pâtures. Plusieurs approches géospatiales pour le suivi, l'évaluation et la gestion des terres agricoles périurbaines sont présentées. L'étude de cas de Sydney (Australie) décrite dans ce chapitre démontre tout l'intérêt de passer par la production de paramètres biophysiques dérivés des images pour suivre avec précision la croissance urbaine et la consommation d'espaces agricoles. De nouvelles recherches combinant télédétection et écologie du paysage commencent aussi à être menées par la communauté scientifique pour aider à la gestion des dynamiques paysagères en milieu périurbain. Les métriques spatiales de diversité paysagère qui sont obtenues semblent prometteuses pour comprendre les impacts des changements de composition et de configuration paysagère sur la productivité agricole et les services écosystémiques qui lui sont associés. D'autres avancées prometteuses sont obtenues à partir de méthodes de classification orientée objet et d'algorithmes à base d'apprentissage machine. Elles permettent une distinction plus précise et détaillée de la couverture du sol. Jusqu'à présent, elles nécessitaient de disposer d'images satellites et de logiciels coûteux. Des programmes récents en observation de la Terre, tels que Copernicus en Europe, Geosud/Theia en France ou DataCube en Australie, diminuent ces limitations en fournissant un accès 
gratuit à des images satellites à haute et très haute résolution ainsi que des services à haute performance de traitements numériques en ligne des images.

Le chapitre 3 (par Maud Balestrat, Éric Barbe, Jean-Pierre Chery et Jean-Philippe Tonneau) traite la question des indicateurs spatiaux pertinents pour le suivi et l'évaluation des dynamiques d'artificialisation des sols en milieu urbain et périurbain à partir d'une étude de cas couvrant l'ancienne région Languedoc-Roussillon en France. Il existe encore peu de méthodes reproductibles conçues spécifiquement pour mesurer quantitativement et qualitativement l'expansion urbaine sur les terres agricoles. Les auteurs explorent un processus de "coconstruction » d'un système d'indicateurs, basé sur un modèle conceptuel dynamique de socio-écosystème, et de consultation itérative de ces indicateurs. Cette analyse éclaire la manière dont différents acteurs choisissent d'interpréter, de communiquer et d'opérationnaliser des indicateurs d'imperméabilisation du sol, d'étalement urbain et de potentiel agronomique. L'adoption d'une démarche itérative est déjà en soi une innovation, car elle permet de prendre en compte les perspectives multiples des participants. Ce travail de coconstruction d'indicateurs est une bonne illustration de l'effet structurant d'un apprentissage collectif de nouvelles connaissances pour permettre aux acteurs de converger vers un but commun - ici la gouvernance durable des terres agricoles périurbaines. Les auteurs soulignent le pouvoir transformateur d'une telle approche et le potentiel qu'elle offre pour aboutir à un consensus. Elle permet par exemple aux agriculteurs de faire connaître leurs intérêts et de les légitimer durant le processus de coconstruction d'indicateurs. En Australie, il n'existe pas encore à ce jour de cartes et d'indicateurs coconstruits de l'étalement urbain (chapitre 8 par Laure-Élise Ruoso ; chapitre 10 par Dana Cordell, Laura Wynne et Brent Jacobs), l'adoption d'une telle démarche supposant chez les acteurs de l'aménagement du territoire une volonté politique de mettre à l'agenda la question des terres agricoles.

Les processus de création d'information qui viennent d'être présentés ont comme point commun de reposer sur des approches soutenues par la technologie et axées sur la traduction d'informations scientifiques sur la qualité des sols en connaissances appropriables par les décideurs pour tenir compte de la dimension productive des terres agricoles périurbaines. Cependant, nous avons vu que ces espaces agricoles fournissaient une variété d'autres services qui ne peuvent être identifiés qu'à travers l'étude des perceptions et représentations que les acteurs ont de ces services.

\section{Collection de données et création d'informations guidées par les connaissances}

La création d'informations basées sur des données n'est pas toujours suffisante dans les processus d'apprentissage. Il est parfois nécessaire d'adopter des approches participatives pour découvrir les valeurs que les acteurs attribuent aux terres agricoles périurbaines, puis ensuite pour formaliser ces connaissances subjectives en les traduisant sous la forme de produits et d'outils communicables, tels que des tableaux renseignés ou des cartes. Il ne s'agit pas ici de traduire de l'information scientifique en produits directement utilisables par les décideurs, mais plutôt de collecter des données et de produire sur mesure de l'information appropriable. Nous reprenons ci-dessous les points essentiels qui relèvent d'une telle approche dans la seconde partie de l'ouvrage (La mesure à l'épreuve de l'humain).

Dans la partie II de l'ouvrage (La mesure à l'épreuve de l'humain), deux chapitres (chapitre 7 par Anja Martin-Scholz et Éric Barbe ; chapitre 8 par Laure-Élise Ruoso) illustrent ce qu'il peut se produire quand des données ne sont pas alignées sur les systèmes de valeurs des utilisateurs, ou ne sont pas adaptées à une situation. Les trois 
derniers chapitres (chapitre 9 par Xavier Augusseau, Jeremy Bourgoin, Daniel David, Pascal Degenne, Erwann Lagabrielle, Guillaume Lestrelin et Danny Lo Seen; chapitre 10 par Dana Cordell, Laura Wynne et Brent Jacobs ; et chapitre 11 par Roel Plant, Pierre Maurel et Laure-Élise Ruoso) présentent diverses manières de surmonter ce problème.

Le chapitre 7 (par Anja Martin-Scholz et Éric Barbe) traite d'un projet de recherche qui avait initialement pour but de déterminer les effets de la dissémination d'informations sur les terres agricoles périurbaines, en particulier sur les propriétés pédologiques des sols, auprès d'acteurs locaux de la planification spatiale en France. Cependant, il devint rapidement évident que les processus de communication associés à la construction et à la dissémination de l'information avaient joué un rôle critique. Ce chapitre fait appel à deux modèles théoriques de la communication - celui de Shannon et Weaver, fonctionnaliste et cybernétique, et celui de l'acteur-réseau - , pour étudier une seule et même situation dans laquelle une direction régionale du ministère de l'Agriculture (Draaf) a joué un rôle central (chapitre 2 par Bernard Clarimont, Éric Barbe et Philippe Lagacherie). La Draaf a adopté le premier modèle pour fonder une stratégie de dissémination des données supposée " efficace », c'est-à-dire permettant une appropriation parfaite du contenu et le bon usage des données tels qu'imaginés par leurs producteurs. Ce modèle s'est révélé incapable de représenter la complexité des processus communicationnels à l'œuvre parmi la diversité des acteurs et des organisations impliqués dans la cartographie de l'étalement urbain et de la qualité des sols et le développement de métriques. La théorie de l'acteur-réseau a permis une autre lecture de la situation en s'écartant de l'idée que les individus constituaient le système (de communication), pour s'intéresser aux relations qui se tissaient parmi l'ensemble des acteurs concernés lors des négociations et des intéressements successifs autour des données. L'analyse a mis en lumière la place centrale des valeurs des organisations et des individus à différentes échelles (du national au local) et la nécessité de les comprendre et de les partager. Une deuxième étude a été menée ultérieurement pour analyser les processus de communication à l'œuvre au sein d'un collectif d'acteurs publics et de scientifiques engagés dans la conception d'un module de formation à l'usage des données de la Draaf sur l'artificialisation et sur les sols (Martin-Scholz, 2017).

Le chapitre 8 (par Laure-Élise Ruoso) adopte une perspective « anthropo-politique » sur les questions de conservation des terres agricoles périurbaines. En utilisant la littérature sur les notions de lieu et de paysage comme point de départ, le chapitre présente un modèle de la politique de l'identité de lieu, offrant une heuristique pour analyser l'identité de lieu dans les milieux périurbains soumis à un développement urbain rapide. L'application de ce modèle au gouvernement local de Wollondilly, situé dans le sud-ouest du bassin de Sydney, montre que l'identité de lieu défendue par le gouvernement local de Wollondilly est basée sur le maintien du «cadre de vie rural » à travers une séparation entre ville et campagne, mais que cette identité n'est pas traduite dans les pratiques de planification, où la séparation ville/campagne est utilisée pour justifier le développement urbain et marginaliser les terres agricoles en les définissant comme non viables. Ce chapitre analyse pourquoi l'image de la ruralité qui domine dans les documents de planification ne se matérialise pas dans les pratiques de planification. Il ressort de cette analyse que cela pourrait être lié au fait que les projections sur le besoin en logements dominent le processus de planification, et que les informations qui sont utilisées pour déterminer la viabilité des terres agricoles ne sont pas pertinentes. En effet, sur ce territoire, l'agriculture est souvent menée, en pratique, sur des terres considérées comme " non viables » en matière de taille et de potentiel agronomique des sols. Une solution identifiée dans 
ce chapitre consisterait à définir collectivement les critères déterminant la viabilité des terres, au-delà de la taille et du potentiel agronomique des sols.

Le chapitre 9 (par Xavier Augusseau, Jeremy Bourgoin, Daniel David, Pascal Degenne, Erwann Lagabrielle, Guillaume Lestrelin et Danny Lo Seen) présente un exemple de planification spatiale sur l'île de la Réunion, dans l'océan Indien, à l'est de Madagascar et au sud-ouest de l'île Maurice, où la maîtrise de l'urbanisation constitue un défi majeur. $\mathrm{Au}$ cours d'un processus de modélisation participative, les participants ont identifié le problème de l'étalement urbain, et plus particulièrement du mitage, comme un enjeu à prendre en compte dans la planification spatiale, mais source de divisions. Pour l'équipe scientifique, l'un des principaux défis a été d'arriver à impliquer un grand nombre d'acteurs en leur donnant les moyens de débattre sur des questions transversales et de développer une approche intégrée. Pour cela, il a été fait appel à un langage de modélisation spatiale, Ocelet, déclinable sur des domaines spécifiques. Ce travail de modélisation participative et ses résultats en matière de connaissances spatiales ont permis d'évaluer rapidement un modèle, rendu ensuite plus pertinent, crédible et légitime. La légitimité du processus, du modèle et de ses résultats cartographiques a été démontrée à travers la capacité du projet à dépasser les divisions politiques sur les questions d'étalement urbain, et à stimuler des débats tournés vers la recherche de solutions. Les modèles développés ici ont agi comme des objets intermédiaires (Vinck, 2009).

Un autre exemple d'interaction fructueuse entre les connaissances des acteurs et celles des scientifiques est présenté dans le chapitre 10 (par Dana Cordell, Laura Wynne et Brent Jacobs). Ce chapitre se concentre sur un projet de recherche ayant pour but la création d'un futur alimentaire résilient pour Sydney, à travers la cartographie — et donc l'objectivation — du potentiel des terres agricoles périurbaines de Sydney à fournir des aliments pour ses résidents. Des villes avec une croissance aussi rapide ont besoin de développer une planification stratégique à l'échelle métropolitaine pour mieux évaluer et protéger l'agriculture de l'étalement urbain. Dans le même temps, les aménageurs doivent prendre des décisions basées sur des informations qui prennent en considération la diversité des valeurs et bénéfices fournis par les terres agricoles périurbaines. Le processus participatif créatif et interactif présenté dans ce chapitre constitue un exemple de la manière dont le défi a priori insoluble de la préservation des terres agricoles périurbaines de Sydney pourrait être relevé.

Guidé par des objectifs similaires, mais avec une méthodologie et dans un contexte sociospatial différents, le chapitre 11 (par Roel Plant, Pierre Maurel et Laure-Élise Ruoso) présente une méthode d'évaluation participative des services écosystémiques dans le sud de la France. L'hypothèse de base de ce projet est que l'incorporation des valeurs et perceptions fondées sur l'explicitation de connaissances, perceptions et représentations d'acteurs locaux et d'experts, est essentielle pour aboutir à une évaluation ayant du sens à l'échelle locale des services écosystémiques rendus par les espaces agricoles périurbains.

Ces chapitres montrent qu'il est possible de mobiliser des acteurs - experts avec des connaissances scientifiques et/ou acteurs et leurs savoirs locaux tacites - pour évaluer, de manière exhaustive, les rôles multiples que les terres agricoles périurbaines jouent pour leurs bénéficiaires, à l'échelle individuelle et collective. Ces projets de recherche ont abouti au développement d'une riche série d'artefacts allant de récits à des supports tangibles de connaissances tels que des matrices ou des cartes. Ces résultats constituent autant de points d'entrée légitimes pour envisager un «tournant scientifique » qui conduirait à une production accrue de données et d'informations fiables (par exemple, des cartes de services écosystémiques réalisées par les acteurs locaux à partir de cartes d'occupation du sol et d'images satellites). 


\section{Conclusion : vers des approches hybrides?}

La synthèse de ces différentes contributions nous amène à conclure que les données conduisent bien au développement de connaissances sur les défis posés par la préservation des terres agricoles périurbaines. Cependant, ces mêmes connaissances — qu'elles soient tacites, basées sur l'expérience, ou explicites, basées sur la science - interrogent elles-mêmes en retour ce travail de recueil de données et de création d'informations « sur mesure ». De ce fait, il n'existe pas de solution « miracle », qu'elle soit ascendante (guidée par les données) ou descendante (guidée par les connaissances). Une approche hybride, bien que souvent plus élaborée et adaptée au contexte, semble constituer une voie plus prometteuse. Les différents chapitres proposent diverses approches : la coconstruction de données et d'indicateurs, la modélisation spatiale des phénomènes conduisant à l'artificialisation des terres, l'évaluation participative des services écosystémiques et la modélisation du potentiel de « bassins alimentaires » locaux.

Cependant, la simple création d'informations et la production de connaissances, que ce soit par des approches descendantes, ascendantes ou hybrides, ne garantissent aucunement l'utilisation " appropriée » de ces connaissances dans les processus de planification. C'est ici que les politiques publiques peuvent entrer en jeu, en imposant réglementairement l'utilisation de certaines informations et connaissances, par exemple en rendant obligatoire la prise en compte de la qualité des sols dans les procédures d'approbation d'extensions urbaines. Avant de traiter plus en profondeur l'enjeu de la gouvernance dans la planification spatiale, nous allons développer, dans la prochaine section, la question de ce qui pourrait être considéré comme des "mauvais usages » ou des « non-usages » de l'information dans la création de connaissances.

\section{Production de connaissances}

\section{Un manque de consensus autour de l'information sur les terres agricoles périurbaines}

En adoptant une posture naïve vis-à-vis de la question de la protection des terres agricoles périurbaines, nous pourrions penser qu'il suffirait de produire de nouvelles informations pour qu'elles soient automatiquement prises en compte dans le processus de planification spatiale. Cependant, comme cela est ressorti des divers chapitres de cet ouvrage, la planification périurbaine concerne une grande diversité d'acteurs, avec des valeurs, des perceptions, des intérêts et des objectifs différents. Le sens qu'ils donnent à l'information reçue peut largement différer de celui que lui avaient donné ses producteurs. Cela a pu être observé dans l'étude de cas française présentée au chapitre 7, où les informations sur le potentiel agronomique des sols ont été utilisées par des bureaux d'études pour justifier de projets urbains sur des terres agricoles périurbaines au motif qu'elles n'avaient pas une productivité élevée. Ceci illustre bien le fait que la simple création d'information ne suffit pas et n'empêche pas des usages détournés de l'intention initiale des producteurs. Pour que l'information se transforme en connaissance, il est nécessaire qu'une diversité d'acteurs interrogent et négocient, de manière collective, le sens qu'ils attribuent à ces informations, afin qu'elles soient utilisées dans un but commun codéfini par les divers groupes d'acteurs. 


\section{Accorder trop de crédits aux informations sur les terres agricoles périurbaines}

La synthèse des connaissances, les outils et les produits créés (cartes, matrices, ensemble d'indicateurs, récits, etc., à l'aide d'approches ascendantes ou descendantes) devraient être utilisés comme des outils de négociation pour la prise de décision plutôt que comme des outils mécanistes d'aide au zonage de l'espace. Ceci se justifie par le fait que coexistent dans les zones périurbaines des acteurs avec des intérêts, des représentations et des objectifs différents, et que le sens attribué aux données et aux informations a toujours besoin d'être construit collectivement pour se transformer en connaissances partagées. Plus particulièrement dans le cas des approches descendantes (exemple du chapitre 11), il existe souvent de bonnes raisons qui permettent de justifier que l'information joue un rôle — même modeste — comme outil de négociation plutôt que comme une réponse définitive à un problème d'aménagement. Les raisons sont que des produits vecteurs de connaissances, tels que des cartes ou d'autres types de données codifiées, ne prennent pas en compte l'intégralité des valeurs et préoccupations des différentes parties prenantes, et, de ce fait, ne sont qu'une représentation partielle des valeurs de certains acteurs dans un lieu et à un moment donné. De telles informations ne pourront jamais être automatiquement extrapolées. Il existe toutefois des cas où des cartes développées à des fins d'information ont finalement été utilisées comme outil de zonage. C'est le cas des Znieff (Zones naturelles d'intérêt écologique, faunistique et floristique), où des zones qui avaient été identifiées pour leur richesse écologique ont été transformées en zonages prescriptifs dans le cadre du programme Natura 2000 (Couderchet et Amelot, 2010).

\section{Les informations sur les terres agricoles périurbaines ne sont pas obligatoires dans les procédures de planification et ne sont donc pas utilisées}

Il existe souvent un fossé entre les concepts utilisés en recherche (par exemple l'intérêt du monde académique pour le concept de services écosystémiques (chapitre 11), ou la création d'indices de qualité des sols par l'Inra (chapitre 2) et les besoins et/ou la capacité des décideurs à intégrer de tels concepts dans le processus de prise de décision. De ce fait, si des chercheurs créent de l'information pertinente d'un point de vue scientifique, mais qui ne répond pas au besoin immédiat en termes de politiques publiques et de planification, il est fort probable que cette information reste sous-utilisée ou soit laissée de côté.

\section{Conclusion : l'information n'est pas la connaissance}

Pour résumer, l'utilisation d'une démarche scientifique classique pour transformer des données sur les terres agricoles périurbaines en informations ciblées ne mène pas automatiquement à des connaissances actionnables. De plus, lorsque des initiatives lancées par la recherche mobilisent des acteurs territoriaux autour de concepts sans aucune référence à leurs préoccupations professionnelles ou personnelles, il est peu probable que la synthèse des connaissances qui en découle mène en retour à des recueils de données et à la génération d'informations plus pertinentes. Il apparaît nécessaire d'interroger continuellement les besoins des acteurs et les méthodes scientifiques requises pour favoriser des processus itératifs pouvant déboucher sur des connaissances actionnables pour arriver à réellement préserver des terres agricoles périurbaines. 


\section{Créer de l'intelligence : vers une gouvernance durable des terres agricoles périurbaines}

Jusqu'ici, notre synthèse s'est intéressée aux premiers niveaux de la pyramide donnéeintelligence présentée au début de ce chapitre. Qu'en est-il des niveaux de l'action et de l'intelligence? Il est tentant de penser que la solution pour une gouvernance durable des terres agricoles périurbaines réside dans les cadres législatifs et les régulations dont dispose un pays (France), un État fédéral (État australien) ou encore une région métropolitaine (le bassin de Sydney). Le chapitre 1 (par Jason Prior et Pierre Maurel) a présenté une vue d'ensemble comparative détaillée de la planification spatiale en France et en Australie. S'il existe des similarités évidentes entre les deux pays, ce chapitre met en lumière la manière dont l'approche en matière d'imperméabilisation des sols a émergé dans des contextes géographiques très éloignés et avec des logiques légales, politiques et administratives différentes. Trois points communs et trois différences concernant les terres agricoles périurbaines ressortent de l'analyse comparative : l'intégration progressive entre aménagement du territoire et gestion de l'environnement ; l'autonomie croissante des processus de planification aux échelles locales combinée au maintien du contrôle de l'État sur les affaires d'importance ; et la participation croissante des acteurs locaux et du citoyen dans la prise de décision et la conception d'instruments de planification. La capacité de répondre aux défis rencontrés par les terres agricoles périurbaines a changé, dans la planification spatiale française comme australienne, à travers le glissement progressif de systèmes de planification spatiale supervisés par des États centralisés et bureaucratiques vers une planification spatiale décentralisée et participative. En Australie, le terme d'aménagement axé sur les territoires (place-based planning) (Untaru, 2010) est de plus en plus fréquemment utilisé et peut être considéré comme un moyen pour les agences gouvernementales responsables de la planification régionale de se désengager, tout en encourageant les gouvernements locaux à gérer les problèmes qui se posent à leur échelle et qui peuvent profiter d'informations recueillies auprès des acteurs locaux. Avec l'émergence de la planification spatiale décentralisée et participative, le défi est d'arriver à renouveler la gouvernance territoriale pour équilibrer les rôles, les responsabilités et les liens entre une planification centralisée et les besoins locaux et régionaux.

À côté des instruments de planification imposés par l'État, la décentralisation et une relative liberté dans le droit à l'expérimentation offrent aux gouvernements locaux la possibilité de développer des visions stratégiques qui ne considèrent plus le foncier agricole comme une ressource à consommer, mais qui intègrent l'agriculture périurbaine comme composante de projets de territoire innovants (Perrin et al., 2016). Cela contribue au développement d'une forme d'intelligence collective (Maurel et al., 2014). Nous avons vu toutefois l'extrême difficulté pour les gouvernements locaux à dépasser les intérêts sectoriels et à compenser les logiques économiques bien ancrées de rente foncière autour des terres agricoles et de ressources fiscales liées à l'urbanisation.

\section{La cocréation d'informations : processus participatifs, observatoires}

Notre constat sur l'absence d'apprentissage collectif dû à des « mésusages » de l'information, du moins du point de vue de leurs concepteurs, réitère l'importance de la cocréation de l'information, afin que les acteurs négocient son sens ensemble et ainsi créent une connaissance qui puisse être considérée selon les théories de l'apprentissage comme des croyances vraies et justifiées (Nonaka et Takeuchi, 1995) et devenir ainsi des connais- 
sances actionnables. Cela a été fait avec succès, du moins ponctuellement, dans le contexte australien et français avec la modélisation du bassin alimentaire de Sydney dans le chapitre 10 (par Dana Cordell, Laura Wynne et Brent Jacobs) et la modélisation de dynamiques territoriales sur l'île de la Réunion dans le chapitre 9 (par Xavier Augusseau, Jeremy Bourgoin, Daniel David, Pascal Degenne, Erwann Lagabrielle, Guillaume Lestrelin et Danny Lo Seen). Ces deux chapitres ont démontré que la modélisation participative pouvait être le point de départ d'une médiation sur le futur du développement et de la protection des terres agricoles périurbaines sur ces deux territoires. Dans le processus participatif organisé à la Réunion, des scénarios de l'étalement urbain ont été utilisés pour co-créer, avec les acteurs, des indicateurs pertinents et légitimes pour suivre des changements d'occupation du sol sur l'île. Dans le projet de modélisation du bassin alimentaire de Sydney, des scénarios ont été utilisés pour co-créer, avec les acteurs, des trajectoires d'innovation obligeant à s'extraire, au moins temporairement, des routines planificatrices. Dans les deux cas, l'un des facteurs clés du succès a été l'utilisation de scénarios afin de (co)créer de l'information qui soit considérée comme légitime (indicateurs ou trajectoires) et qui puisse mener à l'action.

Des processus participatifs de cette nature ont l'avantage d'exposer différents groupes d'acteurs à la même information, leur permettant ainsi de discuter de leurs perspectives respectives. Cela encourage l'apprentissage collectif, permet de construire une vision partagée et de déterminer des trajectoires de changement communes. Cela peut aussi mener à un changement de perspective de la part des participants. Finalement, dans le cas de la Réunion, cela a aussi mené au développement d'une " arène » pour le travail collectif, sous la forme de l'organisation de divers ateliers et consultations.

Le principal avantage de ces processus est la légitimité qu'ils donnent aux informations créées, car ils permettent aux participants de dépasser leurs divisions traditionnelles, leurs croyances, et de prendre du recul sur leurs propres schémas de pensée (Bateson, 1972), afin de débattre ensemble et de négocier le sens de l'information.

Toutefois, les travaux en sciences politiques nous rappellent le poids et la stabilité des institutions ainsi que les routines solidement ancrées dans les processus d'apprentissage. Ils mobilisent pour cela le concept de « sentiers de dépendance », ou path dependance (Pierson, 2000), qui imprègnent sur le temps long les cultures administratives et professionnelles des acteurs socio-économiques, des fonctionnaires territoriaux, des responsables politiques, le traitement des questions d'agriculture périurbaine n'échappant pas à cette inertie (Douillet et Faure, 2010).

\section{Vers une intelligence collective}

À ce stade de la synthèse, une question clé est de savoir comment une forme d'intelligence collective concernant les terres agricoles périurbaines pourrait être développée ?

Les enseignements tirés des expériences présentées dans cet ouvrage laissent à penser que le dernier niveau de la pyramide, relevant de l'intelligence (collective), n'a pas encore été atteint et que plusieurs défis restent à relever. Le récent travail de Lehmann et al. (2017) propose des pistes intéressantes pour aller plus loin, même si leur étude ne concerne pas les terres agricoles périurbaines mais plutôt la durabilité en général, avec un intérêt particulier pour les aspects techniques liés à l'acquisition de données par télédétection. Faisant référence en introduction de leur discussion à la pyramide DIKW (Lehmann et al., 2017, p. 11), ces auteurs résument en ces termes les différents défis à relever : « La barrière $\mathrm{A}$ est d'avoir accès aux données, la barrière $\mathrm{B}$ est de transformer ces données en information, la barrière $\mathrm{C}$ est de générer de la connaissance, et 
la barrière $\mathrm{D}$ peut être considérée comme le moyen d'atteindre une sorte d'intelligence collective » (traduction par les auteurs).

Même si désormais les enjeux pour la préservation des terres agricoles périurbaines relèvent surtout des niveaux supérieurs de notre pyramide adaptée (actions et intelligence collectives), cela implique des améliorations à tous les niveaux.

\section{Niveaux données/information}

L'utilisation de bases de données cartographiques et foncières existantes ainsi que les progrès récents des technologies de l'observation de la Terre (comme par exemple le programme européen Copernicus) et du numérique (intelligence artificielle, big data, infrastructures de données spatiales interopérables) permettent d'envisager la production homogène et régulière d'informations sur l'étalement urbain aux échelles nationale, continentale, voire planétaire (Lehmann et al., 2017 ; Corbane et al., 2017). De telles données permettraient d'isoler et de mesurer plus finement les effets des instruments de régulation mis en place et l'atteinte des objectifs fixés (par exemple, stabilisation des espaces artificialisés en Europe d'ici 2050).

Concernant les données sur les sols au sens agronomique du terme, des avancées significatives sont en cours, que ce soit au niveau international (ex. : projet de la Carte mondiale des sols, Global Soil Map ${ }^{46}$, de l'Union internationale en sciences du sol), ou à des échelles intermédiaires (ex. : programme national Inventaire, gestion et conservation des sols porté par le GIS Sol en France, Laroche et al., 2014).

À l'échelon des gouvernements locaux, un autre enjeu est d'arriver à mettre en place des observatoires territoriaux (Tonneau et al., 2017), capables de s'appuyer sur des données de référence, de niveau international ou national, et de les affiner par des observations et des données complémentaires afin de disposer de représentations du phénomène reconnues comme vraies par les acteurs et les décideurs locaux. La connaissance du terrain de ces derniers permet aussi d'envisager la mise en place d'outils de cartographie collaborative afin d'enrichir ces données par leurs observations et savoirs locaux. Il peut être fait appel pour cela à des méthodologies spécifiques de conception collaborative d'observatoires territoriaux (Lemoisson et al., 2016).

Ces propositions soulèvent les questions d'interopérabilité et de subsidiarité des systèmes d'information pour la gouvernance durable des terres agricoles périurbaines, plus particulièrement entre ceux développés aux échelles globales et les observatoires des territoires locaux. Cela suppose toutefois de s'accorder, d'un point de vue ontologique, sur une conceptualisation partagée de ce que sont les terres agricoles périurbaines et leur artificialisation, et sur la manière de les objectiver par une mise en nombres et en cartes. Cela suppose aussi de surmonter les réticences des détenteurs de données à les partager.

\section{Niveau connaissance}

Des phénomènes restent encore mal connus et nécessitent des travaux académiques supplémentaires. C'est par exemple le cas des connaissances sur les effets de l'artificialisation sur la biodiversité et la physico-chimie des sols et les services qu'ils rendent. Créer des indices multifonctions de qualité des sols pour déterminer l'adéquation entre les propriétés bio-physico-chimiques et des usages possibles permettrait de prendre en compte la qualité du sol dans les décisions de conversion de terres agricoles en terrains constructibles. Les impacts de la fragmentation des paysages sur la perte d'habitats naturels et la biodiversité nécessitent également de mener davantage d'études à long terme.

46. http://globalsoilmap.net/ . 
Mais l'enjeu principal à ce niveau de la pyramide est de favoriser les apprentissages collectifs aux échelons locaux, basés sur les données et informations des observatoires territoriaux en accordant de l'importance à leur mise en forme, à la formation à leurs usages (Martin-Scholz, 2017) et au dialogue territorial via la conception de dispositifs de médiation socio-techniques (Maurel, 2012).

\section{Niveaux action collective et sagesse}

La question en suspens ici est d'arriver à construire aux échelons des gouvernements locaux des visions stratégiques partagées par l'ensemble des acteurs territoriaux, légitimées par les politiques publiques nationales et internationales, et que ces visions soient opérationnalisables en exploitant la boîte à outils de l'ingénierie territoriale, déjà très riche en instruments réglementaires et d'aménagement ainsi qu'en solutions techniques.

Comme cela a été vu dans les chapitres 9 et 10, la construction de ces visions stratégiques peut passer par des phases de modélisation participative, de scénarios prospectifs, pour comprendre les mécanismes à l'œuvre dans les dynamiques de pertes de terres agricoles, prendre conscience des impacts différenciés sur les acteurs du territoire et les habitants, et évaluer les effets potentiels d'actions d'aménagement, de changements de pratiques.

S'appuyer aussi sur l'élaboration collective de projets stratégiques, de projets de territoire qui incluraient l'agriculture périurbaine et qui seraient légitimés démocratiquement lors des élections des gouvernements locaux, constitue aussi une autre voie à explorer. Atteindre une intelligence collective reviendrait à développer l'intime conviction que les terres agricoles ne peuvent plus constituer une simple ressource foncière à urbaniser, mais deviennent une pièce centrale de nouveaux modèles de développement urbain exploitant la multifonctionnalité de l'agriculture périurbaine et permettant, par exemple, de bâtir des systèmes alimentaires locaux.

L'échelle de gouvernance adaptée pour traiter ces enjeux suppose un bon dosage pour disposer à la fois d'une légitimité démocratique pour identifier et mettre en œuvre librement des solutions efficaces et partagées pour préserver l'agriculture périurbaine, tout en étant suffisamment à l'abri des pressions liées aux intérêts locaux sectoriels. Compte tenu de son mille-feuille institutionnel, cet aspect est plus prégnant en France qu'en Australie, et l'échelon intercommunal semble le plus approprié, malgré son faible niveau actuel de légitimité démocratique.

Traiter la question des terres agricoles périurbaines soulève aussi, en creux, celle de la place des agriculteurs dans la gouvernance de ces territoires et des villes centres. Une voie d'avenir pourrait venir des nouveaux agriculteurs (péri)urbains, souvent à l'écart des structures professionnelles agricoles traditionnelles qui peinent à trouver leur place dans les gouvernances des territoires urbains (Bertrand et al., 2006).

\section{Conclusion}

En conclusion de ce chapitre de synthèse de l'ouvrage, nous allons revenir brièvement sur les trois questions centrales qui avaient été présentées en Introduction en tentant d'apporter quelques éléments de réponse.

Question 1: Quels sont les enjeux? Notre premier objectif était d'explorer la nature complexe du problème de la perte des terres agricoles périurbaines. Nous avons vu dans plusieurs chapitres que la menace de la perte rapide de terres agricoles périurbaines en France et en Australie ne relevait pas simplement d'un problème de manque de données ou de volonté politique, ou encore de chaînons intermédiaires manquants entre l'objectivation de situations territoriales par des mises en nombres, en cartes, en récits, et l'arène 
politique. Ce n'est pas non plus le simple dilemme de « la poule ou de l'œuf », qui ferait que nous n'avons pas de politiques publiques (ou de politiciens proactifs) parce que nous n'avons pas de données, et nous n'avons pas de données parce qu'il n'y a pas de politiques publiques en place qui requièrent de telles données. La complexité du phénomène réside plutôt dans la grande variété de facteurs et de leurs interactions (par exemple la croissance de la population, les politiques agricoles néolibérales, le droit de propriété, le manque de conscience au sein du grand public, l'absence de priorité donnée à la gouvernance des sols, la perception des zones périurbaines comme des banlieues en devenir, etc.). Tous ces facteurs et leurs interactions complexes devraient pouvoir être réinterrogés à l'aide de meilleures données, informations et connaissances sur tous les aspects traitant des terres agricoles périurbaines.

Question 2 : Quels sont les outils disponibles pour faire face à cet enjeu? Notre second objectif était d'évaluer les théories et méthodes utilisées pour aborder la question de la perte des terres agricoles périurbaines. Les douze chapitres de cet ouvrage ont présenté différentes approches, dans le contexte français ou australien, qui peuvent être considérées comme appartenant à trois groupes distincts : des approches techniques axées sur les données, des approches hybrides où les données, les informations et les modèles sont utilisés dans des processus participatifs, et des ouvertures sur des approches fondées sur d'autres cadres théoriques, comme par exemple les services écosystémiques. Pris ensemble, ces « outils »- associés à des retours d'expérience et à des recueils de bonnes pratiques - laissent entrevoir des progrès prometteurs. Avec l'avancée des technologies (par exemple, les nouveaux capteurs en télédétection combinés aux technologies du numérique), il est probable que de nouvelles opportunités émergeront, comme par exemple la possibilité d'accompagner en temps réel des exercices de modélisation participative pour évaluer les impacts de scénarios de développement ou des options en termes de politiques publiques. Mais il n'existe pas de solution miracle, des avancées dans ces trois catégories - les approches axées sur les données, les approches hybrides et les fondements théoriques - sont nécessaires et requerront des approches interdisciplinaires.

Question 3: Appropriation, gouvernance, résultats, changements des politiques publiques? Notre troisième objectif était de développer des perspectives pour le futur. Que peut-on dire sur les développements à venir, sur l'adoption et l'utilisation d'outils et de méthodes scientifiques pour générer des données, des informations et des connaissances sur les terres agricoles périurbaines ? Quels changements en termes de gouvernance seraient nécessaires pour cela ? Nous pouvons conclure que la réponse réside probablement dans une articulation entre deux approches de la planification spatiale, celle basée sur des preuves, des faits tangibles (evidence-based planning, Davoudi, 2006) et celle dite «communicationnelle » au sein des dispositifs de gouvernance territoriale (communicative planning, Innes, 1998). Comme cela a été vu précédemment, l'utilisation de données et d'informations qualifiées, issues de systèmes d'informations interopérables globaux et locaux, constitue une voie d'avenir. Le principal défi pour l'ingénierie territoriale sera d'imaginer des formes de gouvernance et de modèles économiques de ces systèmes d'information qui encouragent les détenteurs de données à les mutualiser. Alors que les opérateurs privés de ces systèmes chercheront des bénéfices économiques, les acteurs publics viseront surtout à améliorer l'efficacité de leurs actions tout en réduisant leurs coûts (Klievink et al., 2016). Dans l'approche communicationnelle de la planification, l'information ne se limite pas à un apport de "preuves ». Elle agira d'autant plus qu'elle sera imbriquée dans des processus d'apprentissage, des pratiques et des institutions. La manière de produire de l'information, de la valider, de lui donner collectivement 
du sens, joue un rôle crucial et devrait constituer une dimension à part entière de la planification spatiale. Enfin, les informations « objectives » ne sont pas les seules à compter, il y a toutes les autres sur lesquelles reposent les systèmes de croyances. Les théories des apprentissages organisationnels (Argyris et Schön, 1978) mériteraient aussi d'être prises en compte pour arriver à modifier durablement les référentiels cognitifs, les institutions et les pratiques chez les différentes catégories d'acteurs concernées par les terres agricoles périurbaines.

Nous espérons que les exemples et les analyses critiques présentés dans cet ouvrage constitueront une source d'inspiration pour les chercheurs et les acteurs de la planification spatiale, en France et en Australie, et que cela constituera pour eux une première étape dans leur trajectoire vers une intelligence collective pour la préservation des terres agricoles périurbaines.

\section{Références bibliographiques}

Adler M.J., 1986. A Guidebook to Learning: For the Lifelong Pursuit of Wisdom, New York, Macmillan.

Akimowicz M., Cummings H., Landman K., 2016. Green lights in the Greenbelt? A qualitative analysis of farm investment decision-making in peri-urban Southern Ontario. Land Use Policy, 55, 24-26.

Argyris C., Schön D.A., 1978. Organizational Learning: A Theory of Action Perspective, Addison Wesley Longman Publishing Co, 356 p.

Barth F., 2002. An anthropology of knowledge. Current Anthropology, 43, 1-11.

Bateson G., 1972. Steps to en Ecology of Mind: Collected Essays in Anthropology, Psychiatry, Evolution and Epistemology, University of Chicago Press.

Bertrand N., Souchard N., Rousier N., et al., 2006. Quelle contribution de l'agriculture périurbaine à la construction de nouveaux territoires: consensus ou tensions? Revue d'économie régionale et urbaine, 3 (août), 329-353.

Brand C., Bricas N., Conaré D., Daviron B., Debru J., Michel L., Soulard C.-T., 2017. Construire des politiques alimentaires urbaines : concepts et démarches, Versailles, éditions Quæ.

Bunker R., Holloway D., 2001. Fringe City and Contested Countryside: Population Trends and Policy Developments Around Sydney. Issues Paper No. 6 Urban Frontiers Program. University of Western Sydney.

Corbane C., Pesaresi M., Politis P., Syrris V., Florczyk A.J., Soille P., Maffenini L., Burger A., Vasilev V., Rodriguez D., Sabo F., Dijkstra L., Kemper T., 2017. Big earth data analytics on Sentinel-1 and Landsat imagery in support to global human settlements mapping. Big Earth Data, 1, 118-144.

Couderchet L., Amelot X., 2010. Faut-il brûler les Znieff ? Cybergeo.

Darly S., Torre A., 2013. Conflicts over farmland uses and the dynamics of agri-urban localities in the greater Paris region: An empirical analysis based on daily regional press and field interviews. Land Use Policy, 33, 90-99.

Davoudi S., 2006. Evidence-based Planning: Rhetoric and reality. DisP: The Planning Review, 42, 14-24.

Deledalle G. (ed.), 2001. Charles S. Peirce's Philosophy of Signs. Essays in Comparative Semiotics, Indiana, Indiana University Press.

Deverre C., Lamine C., 2010. Les systèmes agroalimentaires alternatifs, une revue de travaux anglophones en sciences sociales. Économie rurale, 317, 57-73.

Douillet A.C., Faure A., 2010. Périurbanité et dynamiques intercommunales : l'agriculture entre sillons de dépendance et nouvelles priorités d'action publique. In : L'agriculture dans 
la ville éclatée (Bertrand N., ed.), Montréal, Laboratoire de Développement durable et dynamique territoriale.

Graham I., Logan J., Harrison M., Straus S., Tetroe J., Caswell W., Robinson N., 2006. Lost in knowledge translation: time for a map? Journal of Continuing Education in the Health Professions, 26, 13-24.

Greiner R., Gregg D., 2011. Farmers' intrinsic motivations, barriers to the adoption of conservation practices and effectiveness of policy instruments: empirical evidence from northern Australia. Land Use Policy, 28, 257-265.

Guillou M., Matheron G., 2011. 9 milliards d'hommes à nourrir - Un défi pour demain, Paris, François Bourin Éditeur.

Innes J., 1998. Information in communicative planning. Journal of the American Planning Association, 4, 52-63.

Inwood S.M., Sharp J.S., 2012. Farm persistence and adaptation at the rural/urban interface: succession and farm adjustment. J. Rur. Stud, 28, 107-117.

Kitchin R., 2014. The Data Revolution. Big Data, Open Data, Data Infrastructures and Their Consequences, Los Angeles-London-New Delhi-Singapore-Washington DC, Sage.

Klievink B., Bharosa N., Tan Y.H., 2016. The collaborative realization of public values and business goals: Governance and infrastructure of public-private information platforms. Government Information Quarterly, 33, 1, 67-79.

Knowd I.C., 2013. Hawkesbury Harvest: panacea, paradox and the spirit of capitalism in the rural hinterlands of Sydney, Australia. Doctor of Philosophy, Western Sydney University.

Laplantine F., 2001. L'anthropologie, Paris, Éd. Payot/Rivages.

Laroche B., Richer De Forges A.C., Leménager S., Arrouays D., Schnebelen N., Eimberck M., Toutain B., Lehmann S., Tientcheu E., Hélièes F., Chenu J.P., Paros S., Desbourdes S., Girot G., Voltz M., Bardy M., 2014. Le programme Inventaire Gestion Conservation des Sols en France - Volet Référentiel Régional Pédologique. Étude et gestion des sols, 21, 125-140.

Lehmann A., Chaplin-Kramer R., Lacayo M., Giuliani G., Thau D., Koy K., Goldberg K., Sharp Jr R., 2017. Lifting the information barriers to address sustainability challenges with data from physical geography and earth observation. Sustainability, 9.

Lemoisson P., Tonneau J.P., Maurel P., 2016. L'intelligence territoriale dans le bassin de Thau : un observatoire pour penser et piloter l'action. In : Partenariats pour le développement territorial (Torre A., Vollet D., eds), Versailles, éditions Quæ.

Marshall G., 1998. A dictionary of sociology, NewYork, Oxford University Press.

Martin-Scholz A., 2017. Communiquer et organiser en échafaudant un «big data » manuel. Le cas d'un projet de formation en aménagement du territoire. Thèse de doctorat en sciences de l'information et de la communication, université de Toulouse-III.

Maurel P., 2012. Signes, données et représentations spatiales : des éléments de sens dans l'élaboration d'un projet de territoire intercommunal. Application au territoire de Thau. Thèse en sciences de l'information et de la communication, université du Sud Toulon/Var.

Maurel P., Plant R., Barreteau O., Bertacchini Y., 2014. Beyond IWRM: developing territorial intelligence at the local scales. In: River Basin Management in the Twenty-First Century: People and Place (Squires V., Milner H., Daniell K.A., eds), CRC Press, Taylor and Francis. Maury Y., Kovacs S., Marteleto R., 2014. Anthropologie des savoirs. Introduction. Études de communication, 42.

Nonaka I., Takeuchi H., 1995. The Knowledge Creating Company, New York, Oxford University Press.

Paül V., McKenzie F.H., 2013. Peri-urban farmland conservation and development of alternative food networks: insights from a case-study area in metropolitan Barcelona (Catalonia, Spain). Land Use Policy, 30, 94-105. 
Peirce C.S., 1905. What pragmatism is. The Monist, 15, 161-181.

Perrin C., Soulard C.-T., Chia E., 2016. La gouvernance du foncier agricole périurbain : entre planification urbaine et projets de développement. Revue d'économie régionale et urbaine, 2016/4, octobre, 713-736.

Pierson P., 2000. Increasing returns, path dependence, and the study of politics. The American Political Science Review, 94, 251-267.

Reckien D., Karecha J., 2007. Sprawl in European cities: the comparative background. In: Urban Sprawl in Europe: Landscapes, Land-Use Change and Policy (Couch C., Leontidou L., Petschel-Held G., eds), Oxford, Blackwell.

Rowley J., 2007. The wisdom hierarchy: representations of the DIKW hierarchy. J. Information Science, 33, 163-180.

Serrano J., 2011. La valeur patrimoniale au secours de la préservation de l'agriculture périurbaine. In: Les paysages périurbains, des héritages à une gestion différenciée des territoires, septembre 2011, Blois, France.

Soulard C.-T., Valette E., Perrin C., et al., 2018. Peri-urban agro-ecosystems in the Mediterranean: diversity, dynamics, and drivers Regional Environmental Change, 18, 651-662.

Tonneau J., Lemoisson P., Coudel E., Maurel P., Jannoyer M., Bonnal V., Bourgoin J., Cattan P., Chery J., Piraux M., Lestrelin G. 2017. Les observatoires territoriaux : des outils de la société de la connaissance ? Revue internationale de géomatique, 27, 335-354.

Untaru S., 2010. Regulatory frameworks for place-based planning. Urban Policy and Research, 20, 169-186.

Vinck D., 2009. De l'objet intermédiaire à l'objet-frontière. Revue d'anthropologie des connaissances, 3, 51-72. 


\section{Liste des auteurs}

Xavier Augusseau, UMR Tetis, Cirad, AgroParisTech, CNRS, Irstea, université de Montpellier, Montpellier, France

Maud Balestrat, UMR AGIR, Inra, Toulouse, France, et UMR Tetis, Irstea, AgroParisTech, Cirad CNRS, université de Montpellier, Montpellier, France

Éric Barbe, UMR Tetis, Irstea, AgroParisTech, Cirad, CNRS, université de Montpellier, Montpellier, France

Jeremy Bourgoin, UMR Tetis, UMR Tetis, Cirad, AgroParisTech, CNRS, Irstea, université de Montpellier, Montpellier, France

Jane Brennan, UTS-FEIT, université de technologie de Sydney, Australie

Jean-Pierre Chery, UMR Tetis, AgroParisTech, Cirad, CNRS, Irstea, université de Montpellier, Montpellier, France

Bernard Clarimont, Direction régionale de l'alimentation, de l'agriculture et de la forêt du Languedoc-Roussillon, ministère de l'Agriculture et de l'Alimentation, Draaf Occitanie, Montpellier, France

Dana Cordell, Institute for Sustainable Futures (ISF), université de technologie de Sydney, Australie

Daniel David, UMR Espace-DEV, IRD, université de Montpellier, Montpellier, France

Pascal Degenne, UMR Tetis, Cirad, AgroParisTech, CNRS, Irstea, université de Montpellier, Montpellier, France

Rakhesh Devadas, Bureau Australien d'économie et de sciences de l'agriculture et des ressources (ABARES), Canberra, Australie

Pamela Hazelton, UTS-FEIT, université de technologie de Sydney, Australie

Alfredo Huete, École des Sciences de la Vie, UTS-Science, université de Technologie de Sydney, Australie

Brent Jacobs, Institute for Sustainable Futures (ISF), université de technologie de Sydney, Australie

Erwann Lagabrielle, UMR Espace-Dev, IRD, université de la Réunion, Saint-Denis de la Réunion, France

Philippe Lagacherie, UMR Lisah, Inra, IRD, Supagro, université de Montpellier, Montpellier, France

Guillaume Lestrelin, UMR Tetis, Cirad, AgroParisTech, CNRS, Irstea, université de Montpellier, Montpellier, France

Danny Lo Seen, UMR Tetis, Cirad, AgroParisTech, CNRS, Irstea, université de Montpellier, Montpellier, France 
Anja Martin-Scholz, UMR Certop, université de Lille, France

Pierre Maurel, UMR Tetis, Irstea, AgroParisTech, Cirad, CNRS, université de Montpellier, Montpellier, France

Andrew Murrell, Service des parcs nationaux, de la faune et de la flore de NouvelleGalles du Sud, Département de l'environnement et de la planification, Australie

Roel Plant, Institute for Sustainable Futures, (ISF) Université de technologie de Sydney

Jason Prior, Institute for Sustainable Futures (ISF), université de technologie de Sydney, Australie.

Laure-Élise Ruoso, Institute for Sustainable Futures (ISF), université de technologie de Sydney, Australie

Paras Sidiqui, École des Sciences de la Vie, UTS-Science, université de technologie de Sydney, Australie

Jean-Philippe Tonneau, UMR Tetis, Cirad, AgroParisTech, CNRS, Irstea, université de Montpellier, Montpellier, France

Laura Wynne, Institute for Sustainable Futures (ISF), université de technologie de Sydney, Australie 